\title{
Therapies for Clinically Localized Prostate Cancer
}




\section{Comparative Effectiveness Review}

Number 230

\section{Therapies for Clinically Localized Prostate Cancer}

\section{Prepared for:}

Agency for Healthcare Research and Quality

U.S. Department of Health and Human Services

5600 Fishers Lane

Rockville, MD 20857

www.ahrq.gov

Contract No. 290-2015-0000-81

Prepared by:

Minnesota Evidence-based Practice Center

Minneapolis, MN

Investigators:

Philipp Dahm, M.D., M.H.S.C.

Michelle Brasure, Ph.D., M.S.P.H., M.L.I.S.

Elizabeth Ester, M.D.

Eric J. Linskens, B.S.

Roderick MacDonald, M.S.

Victoria A. Nelson, M.Sc.

Charles Ryan, M.D.

Jayati Saha, Ph.D.

Shahnaz Sultan, M.D., M.H.S.C.

Kristen E. Ullman, M.P.H.

Timothy J. Wilt, M.D., M.P.H.

AHRQ Publication No. 20-EHC022

September 2020 
This report is based on research conducted by the Minnesota Evidence-based Practice Center (EPC) under contract to the Agency for Healthcare Research and Quality (AHRQ), Rockville, MD (Contract No. 290-2015-0000-81). The findings and conclusions in this document are those of the authors, who are responsible for its contents; the findings and conclusions do not necessarily represent the views of AHRQ. Therefore, no statement in this report should be construed as an official position of AHRQ or of the U.S. Department of Health and Human Services.

\section{None of the investigators have any affiliations or financial involvement that conflicts with the material presented in this report.}

The information in this report is intended to help healthcare decision makers—patients and clinicians, health system leaders, and policymakers, among others—make well-informed decisions and thereby improve the quality of healthcare services. This report is not intended to be a substitute for the application of clinical judgment. Anyone who makes decisions concerning the provision of clinical care should consider this report in the same way as any medical reference and in conjunction with all other pertinent information, i.e., in the context of available resources and circumstances presented by individual patients.

This report is made available to the public under the terms of a licensing agreement between the author and the Agency for Healthcare Research and Quality. This report may be used and reprinted without permission except those copyrighted materials that are clearly noted in the report. Further reproduction of those copyrighted materials is prohibited without the express permission of copyright holders.

AHRQ or U.S. Department of Health and Human Services endorsement of any derivative products that may be developed from this report, such as clinical practice guidelines, other quality enhancement tools, or reimbursement or coverage policies may not be stated or implied.

This report may periodically be assessed for the currency of conclusions. If an assessment is done, the resulting surveillance report describing the methodology and findings will be found on the Effective Health Care Program Web site at www.effectivehealthcare.ahrq.gov. Search on the title of the report.

AHRQ appreciates appropriate acknowledgment and citation of its work. Suggested language for acknowledgment: This work was based on an evidence report, Therapies for Clinically Localized Prostate Cancer, by the Evidence-based Practice Center Program at the Agency for Healthcare Research and Quality (AHRQ).

Suggested citation: Dahm P, Brasure M, Ester E, Linskens EJ, MacDonald R, Nelson VA, Ryan C, Saha J, Sultan S, Ullman KE, Wilt TJ. Therapies for Clinically Localized Prostate Cancer. Comparative Effectiveness Review No. 230. (Prepared by the Minnesota Evidence-based Practice Center under Contract No. 290-2015-0000-81) AHRQ Publication No. 20-EHC022. Rockville, MD: Agency for Healthcare Research and Quality; September 2020. DOI: https://doi.org/10.23970/AHRQEPCCER230. Posted final reports are located on the Effective Health Care Program search page. 


\section{Preface}

The Agency for Healthcare Research and Quality (AHRQ), through its Evidence-based Practice Centers (EPCs), sponsors the development of systematic reviews to assist public- and private-sector organizations in their efforts to improve the quality of healthcare in the United States. These reviews provide comprehensive, science-based information on common, costly medical conditions, and new healthcare technologies and strategies.

Systematic reviews are the building blocks underlying evidence-based practice; they focus attention on the strength and limits of evidence from research studies about the effectiveness and safety of a clinical intervention. In the context of developing recommendations for practice, systematic reviews can help clarify whether assertions about the value of the intervention are based on strong evidence from clinical studies. For more information about AHRQ EPC systematic reviews, see www.effectivehealthcare.ahrq.gov/reference/purpose.cfm

AHRQ expects that these systematic reviews will be helpful to health plans, providers, purchasers, government programs, and the healthcare system as a whole. Transparency and stakeholder input are essential to the Effective Health Care Program. Please visit the website (www.effectivehealthcare.ahrq.gov) to see draft research questions and reports or to join an email list to learn about new program products and opportunities for input.

If you have comments on this systematic review, they may be sent by mail to the Task Order Officer named below at: Agency for Healthcare Research and Quality, 5600 Fishers Lane, Rockville, MD 20857, or by email to epc@ahrq.hhs.gov.

Gopal Khanna, M.B.A.

Director

Agency for Healthcare Research and Quality

Stephanie Chang, M.D., M.P.H.

Director

Evidence-based Practice Center Program

Center for Evidence and Practice Improvement Agency for Healthcare Research and Quality
Arlene Bierman, M.D., M.S.

Director

Center for Evidence and Practice Improvement

Agency for Healthcare Research and Quality

Lionel Bañez, M.D.

Task Order Officer

Center for Evidence and Practice

Improvement

Agency for Healthcare Research and Quality 


\section{Acknowledgments}

The authors gratefully acknowledge the following individuals for their contributions to this project: Jeannine Ouellette for editorial help bringing the report to completion.

\section{Technical Expert Panel}

Peter Carroll, M.D., M.P.H.*

University of California, San Francisco

San Francisco, CA

Daniel Lin, M.D.*

University of Washington

Society of Urologic Oncology

Fred Hutchinson Cancer Research Center

Seattle, WA

Nicolas Mottet, M.D., Ph.D.

Clinique Mutualiste in Saint Etienne, France

*This TEP member also served as a peer reviewer

\section{Peer Reviewers}

Mitchell S. Anscher, M.D.

Virginia Commonwealth University

Department of Radiation Oncology

Richmond, VA

Michael J. Barry, M.D.

Massachusetts General Hospital

Harvard Medical School

Boston, MA
Paul L. Nguyen, M.D.

Dana-Farber Cancer Institute

Boston, MA

Howard M. Sandler, M.D.*

Cedars-Sinai, ASTRO

Los Angeles, CA

Janice Tufte*

PCORI Sponsored Public Ambassador
Alvin Cabrera, M.D.

Kaiser Permanente

Seattle, WA

Richard M. Hoffman, M.D., M.P.H.

University of Iowa Carver College of

Medicine

Iowa City, IA

Terrence Kungel

Chairman, Emeritus of the Maine Coalition

to Fight Prostate Cancer

Woolwich, ME 


\section{Therapies for Clinically Localized Prostate Cancer}

\section{Structured Abstract}

Objective. To update findings from previous Agency for Healthcare Research and Quality (AHRQ)- and American Urological Association (AUA)-funded reviews evaluating therapies for clinically localized prostate cancer (CLPC).

Sources. Bibliographic databases (2013-January 2020); ClinicalTrials.gov; systematic reviews

Methods. Controlled studies of CLPC treatments with duration $\geq 5$ years for mortality and metastases and $\geq 1$ year for quality of life and harms. One investigator rated risk of bias (RoB), extracted data, and assessed certainty of evidence; a second checked accuracy. We analyzed English-language studies with low or medium RoB. We incorporated findings from randomized controlled trials (RCTs) identified in the prior reviews if new RCTs provided information on the same intervention comparison.

Results. We identified 67 eligible references; 17 were unique RCTs. Among clinically rather than prostate-specific antigen (PSA) detected CLPC, Watchful Waiting (WW) may increase mortality and metastases versus Radical Prostatectomy (RP) at 20+ years. Urinary and erectile dysfunction were lower with WW versus RP. WW's effect on mortality may vary by tumor risk and age but not by race, health status, comorbidities, or PSA. Active Monitoring (AM) probably results in little to no difference in mortality in PSA-detected CLPC versus RP or external beam radiation (EBR) plus Androgen Deprivation (AD) regardless of tumor risk. Metastases were slightly higher with AM. Harms were greater with RP than AM and mixed between EBR plus $A D$ versus AM. 3D-conformal EBR and AD plus low-dose-rate brachytherapy (BT) provided a small reduction in all-cause mortality versus three-dimensional conformal EBR and AD but little to no difference on metastases. EBR plus AD versus EBR alone may result in a small reduction in mortality and metastases in higher risk disease but may increase sexual harms. EBR plus neoadjuvant $A D$ versus EBR plus concurrent $A D$ may result in little to no difference in mortality and genitourinary toxicity. Conventionally fractionated EBR versus ultrahypofractionated EBR may result in little to no difference in mortality and metastases and urinary and bowel toxicity. Active Surveillance may result in fewer harms than photodynamic therapy and laparoscopic RP may result in more harms than robotic-assisted RP. Little information exists on other treatments. No studies assessed provider or hospital factors of RP comparative effectiveness.

Conclusions. RP reduces mortality versus WW in clinically detected CLPC but causes more harms. Effectiveness may be limited to younger men or to those with intermediate-risk disease and requires many years to occur. AM results in little to no mortality difference versus RP or EBR plus AD. EBR plus AD reduces mortality versus EBR alone in higher risk CLPC but may worsen sexual function. Adding low-dose-rate BT to 3D-conformal EBR and AD may reduce mortality in higher risk CLPC. RCTs in PSA-detected and MRI staged CLPC are needed. 


\section{Contents}

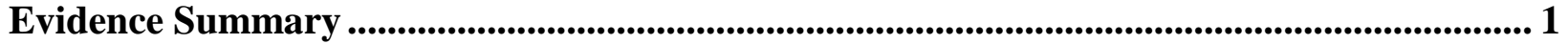

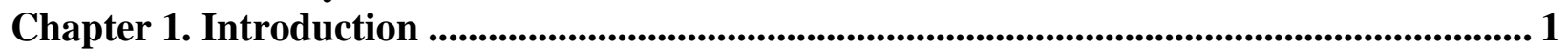

Chapter 2. Methods ..................................................................................................................................... 6

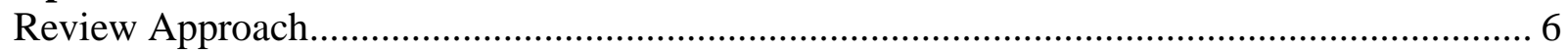

Criteria for Inclusion/Exclusion of Studies in the Review ............................................. 6

Searching for the Evidence and Updating Prior Reviews............................................ 6

Assessment of Methodological Risk of Bias of Individual Studies..................................... 7

Data Abstraction and Data Management .................................................................. 7

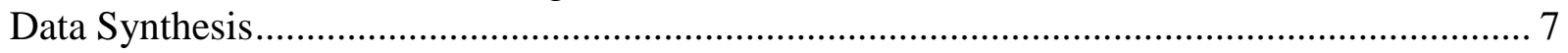

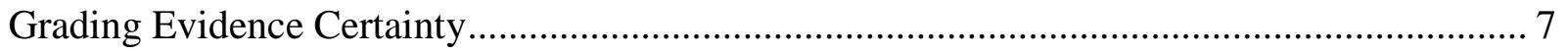

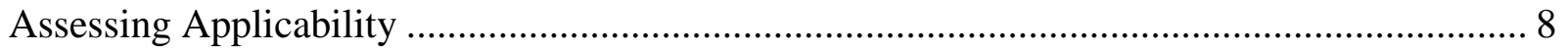

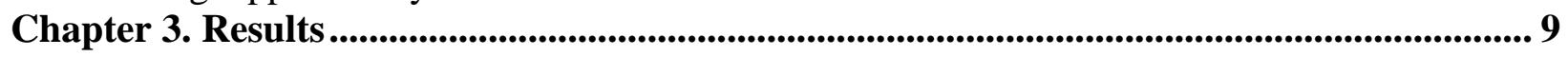

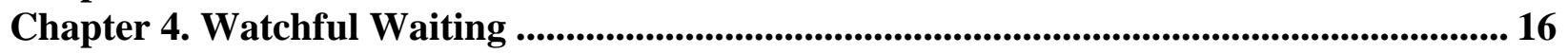

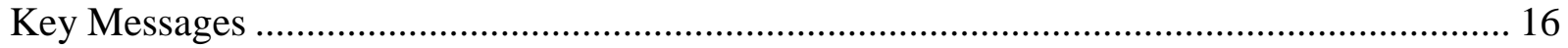

Watchful Waiting Versus Radical Prostatectomy........................................................... 16

Variation in Outcomes by Participant or Tumor Characteristics ....................................... 17

Chapter 5. Active Surveillance/Active Monitoring............................................................... 21

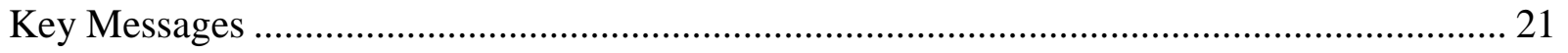

Active Monitoring Versus External Beam Radiation Therapy Plus Androgen Deprivation

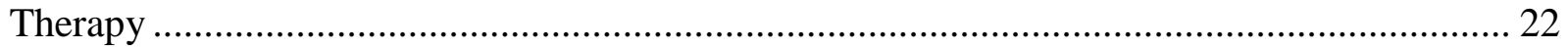

Variation in Outcomes by Participant or Tumor Characteristics ...................................... 22

Active Surveillance Versus Photodynamic Therapy ....................................................... 23

Chapter 6. Whole Gland Therapies- External Beam Radiation Therapy ............................. 26

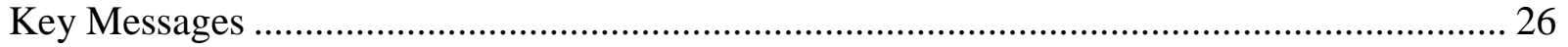

3D-Conformal Radiation Therapy and Androgen Deprivation Therapy Versus 3D-Conformal

Radiation Therapy and Androgen Deprivation Therapy Plus Low-Dose-Rate Prostate

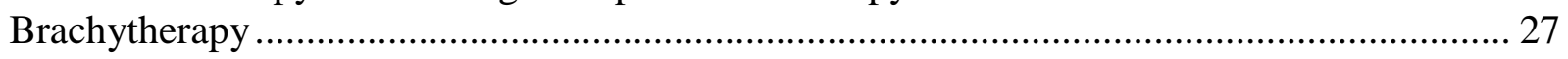

3D-Conformal Radiation Therapy Versus Intensity-Modified Radiation Therapy ................. 28

Brachytherapy With External Beam Radiation Therapy Versus Brachytherapy..................... 28

Intensity-Modified Radiation Therapy Versus Stereotactic Beam Radiation Therapy ............ 28

Radiation Therapy Versus Androgen Deprivation Therapy ............................................... 28

External Beam Radiation Therapy Plus Androgen Deprivation Therapy Versus External Beam

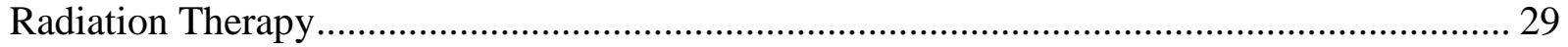

Variation in Outcomes by Participant or Tumor Characteristics ..................................... 30

External Beam Radiation Therapy Plus Androgen Deprivation Therapy Versus Androgen

Deprivation Therapy ................................................................................................. 31

External Beam Radiation Therapy Plus Neoadjuvant and Concurrent Androgen Deprivation

Therapy Versus External Beam Radiation Therapy Plus Concurrent and Adjuvant Androgen

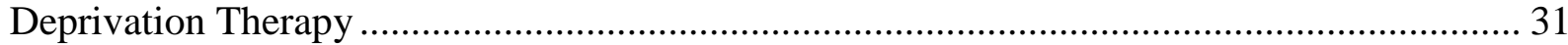

External Beam Radiation Therapy Versus Brachytherapy ................................................ 31

Conventionally Fractionated External Beam Radiation Therapy Versus Ultrahypofractionated

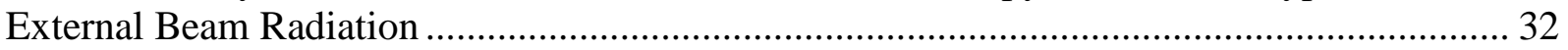

Chapter 7. Whole Gland Therapies-Radical Prostatectomy .................................................... 38

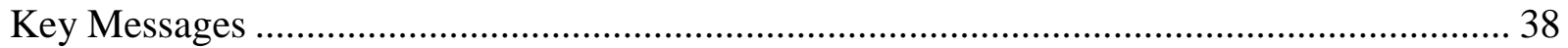

Radical Prostatectomy Versus Active Monitoring ......................................................... 39 
Variation in Outcomes by Participant or Tumor Characteristics.

Radical Prostatectomy Versus External Beam Radiation Therapy Plus Androgen Deprivation

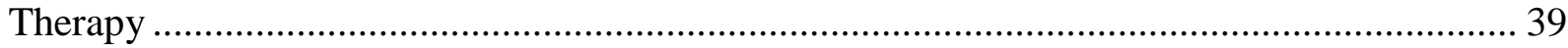

Variation in Outcomes by Participant or Tumor Characteristics.......................................... 40

Radical Prostatectomy Plus Androgen Deprivation Therapy Versus External Beam Radiation

Therapy Plus High-Dose-Rate Brachytherapy Plus Androgen Deprivation Therapy …........... 40

Laparoscopic Radical Prostatectomy Versus Robotic-Assisted Radical Prostatectomy ........... 41

Robotic-Assisted Laparoscopic Radical Prostatectomy Versus Open Retropubic Radical

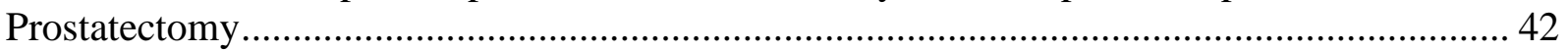

Variation in Outcomes by Participant or Tumor Characteristics........................................... 42

Radical Prostatectomy Versus Androgen Deprivation Therapy .................................................. 42

Radical Prostatectomy Versus High-Intensity Focused Ultrasound.......................................... 43

Chapter 8. Other Therapies ............................................................................................................ 48

Androgen Deprivation Therapy ………………………...................................................... 48

Focal Therapies - High-Intensity Focused Ultrasound ............................................................. 48

Focal Therapies_Photodynamic Therapy ……………..................................................... 48

Focal Therapies_Laser Ablation ....................................................................................... 48

Whole Gland Therapies_Cryotherapy …………………..................................................... 49

Whole Gland Therapies_Brachytherapy ………………..................................................... 49

Chapter 9. Key Questions 2-4........................................................................................................... 50

KQ 2: How do patient characteristics modify comparative effectiveness and harms of CLPC therapies? ....................................................................................................................... 50

KQ 3: How do tumor characteristics modify comparative effectiveness and harms of CLPC

therapies? ..................................................................................................................... 50

KQ 4: How do provider/hospital characteristics modify comparative effectiveness of RP compared with other therapies? ............................................................................................ 51

Chapter 10. Discussion ................................................................................................................................ 52

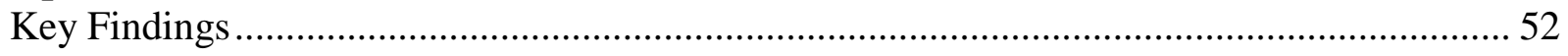

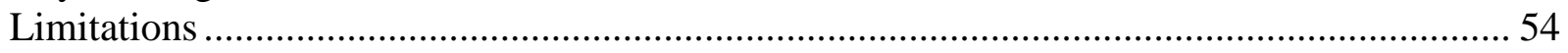

Future Research Needs .............................................................................................. 55

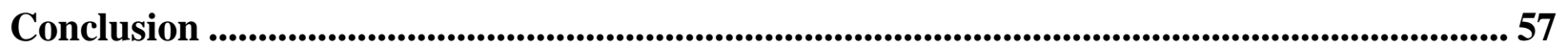

Abbreviations and Acronyms ............................................................................................... 58

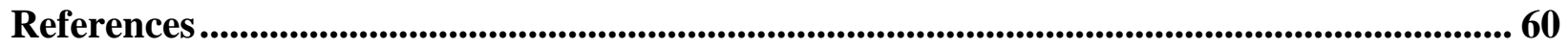

Tables

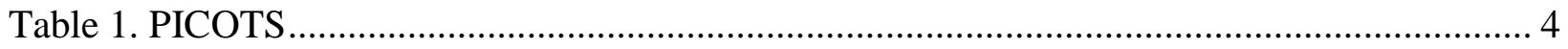

Table 2. Summary updates of comparisons between reviews ........................................................ 11

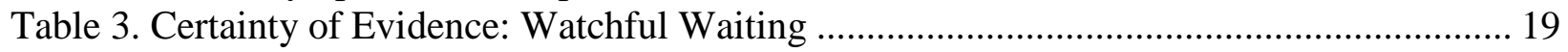

Table 4. Certainty of Evidence: Active Monitoring and Active Surveillance ................................ 24

Table 5. Certainty of Evidence: External Beam Radiation Therapy ………................................... 33

Table 6. Certainty of Evidence: Radical Prostatectomy ……......................................................... 44

\section{Figures}

Figure 1. Literature flow diagram ........................................................................................ 9

Figure 2. Plot of comparisons addressed in RCTs identified in updated literature search ............ 10

\section{Appendixes}


Appendix A. Analytic Framework

Appendix B. Search Strategies

Appendix C. Certainty of Evidence and Effect Size Language

Appendix D. Eligible Studies

Appendix E. Excluded Studies

Appendix F. Watchful Waiting

Appendix G. Active Monitoring/Active Surveillance

Appendix H. External Beam Radiation Therapy

Appendix I. Radical Prostatectomy

Appendix J. Comparisons from Past Reports

Appendix K. Ongoing RCTs for CLPC or Locally Advanced PC

Appendix L. Appendix References 


\section{Evidence Summary}

\section{Main Points}

- In men with clinically localized prostate cancer (CLPC) detected clinically rather than by prostate-specific antigen (PSA) screening, radical prostatectomy (RP) may reduce mortality and metastases more than watchful waiting (WW) but causes more harms. Mortality reductions may be limited to men age 65 and older and those with intermediate-risk disease.

- Active monitoring (AM) probably results in little to no mortality difference versus RP or external beam radiation (EBR)+androgen deprivation (AD) in PSA-detected CLPC and may result in fewer harms. Effects may not vary by patient or tumor factors.

- 3D Conformal EBR (3D-CRT)+low-dose brachytherapy+AD may slightly reduce all-cause mortality but not metastases more than 3D-CRT+AD in higher risk CLPC.

- EBR plus AD may slightly reduce mortality and metastases versus EBR alone in men with intermediate- and high-risk disease but may worsen sexual function.

- Little long-term information exists on other treatments or the effects of patient, tumor, and provider factors especially in PSA-detected and magnetic resonance imaging (MRI)-staged CLPC. We found no evidence on how biomarkers may modify treatment effects.

\section{Background and Purpose}

The American Cancer Society estimates that, in 2020, prostate cancer will be one of the most frequently diagnosed cancers among U.S. men (191,930 new cases) and the second leading cause of cancer death $(33,330) .{ }^{1}$ In 90 percent of newly diagnosed cancers, the disease is confined to the prostate gland (known as "clinically localized prostate cancer" [CLPC]). ${ }^{2}$ Most cases of CLPC grow slowly without symptoms, even if untreated. CLPC treatments thus aim to balance treatment benefits with complications, burden, and costs.

The purpose of this systematic review was to evaluate CLPC treatments by updating prior AHRQ and American Urological Association (AUA) reviews. ${ }^{3-5}$ We included controlled studies of CLPC (stages T1-T3a) treatments $\geq 5$ years duration for mortality and metastases, and $\geq 1$ year for quality of life and harms for the following interventions: WW, active surveillance (AS), AM, $\mathrm{AD}$, and focal and whole gland therapies or their combinations. We also evaluated how patient and tumor characteristics, including risk indices and biomarkers, modify treatment effects, and how provider/hospital characteristics modify effects of RP compared with other therapies. 


\section{Methods}

We employed methods consistent with the AHRQ EPC Program Methods Guidance (https://effectivehealthcare.ahrq.gov/topics/cer-methods-guide/overview). We describe these in the full report. We referenced findings from the 2014 AHRQ- and 2016 AUA-funded reviews and included them in updated analyses if randomized controlled trials (RCTs) provided additional data on similar populations, interventions, comparators, and outcomes. We summarize and compare major findings from our review with those of the prior reports. We derived a priori thresholds defining "small," “moderate," and "large” effect sizes for benefits and harms. Our searches covered publication dates from January 2013 to January 2020. We modified AHRQ methods for this review by using GRADE and EPC tools for risk of bias and certainty of evidence assessments. ${ }^{6-8}$

\section{Results}

We identified 67 eligible references (citations can be found in the full report), of which 26 were publications from 17 unique RCTs and 41 were publications from 34 unique non-RCTs. The treatment comparisons evaluated in RCTs are illustrated in Figure A.

\section{Figure A. Plot of comparisons addressed in RCTs identified in updated literature search * †‡}

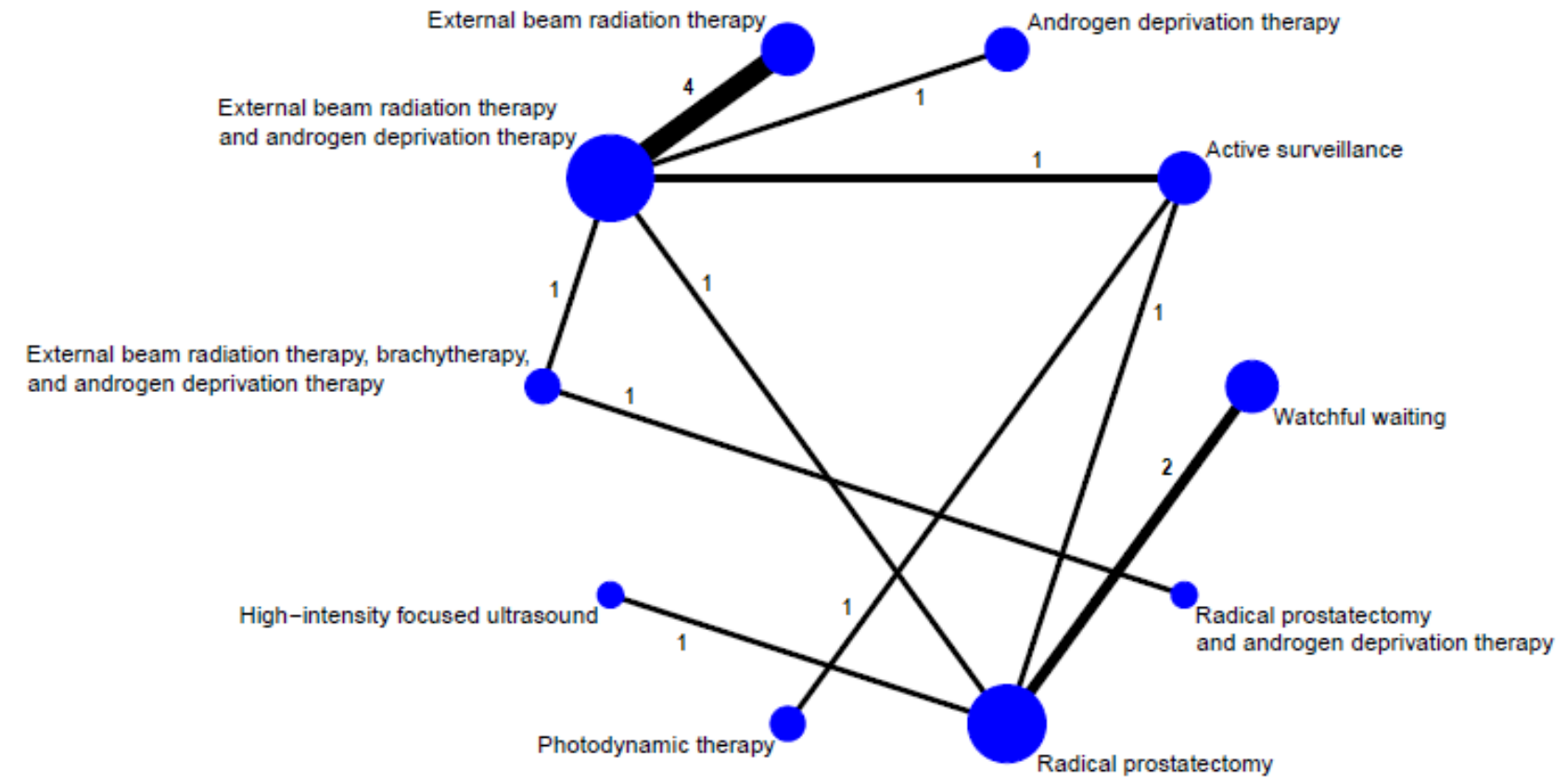

*Within-category comparisons are not shown in figure. These include RARP vs. LRP ( $\mathrm{k}=1, \mathrm{n}=120)$, 3D-CRT vs. IMRT ( $\mathrm{k}=1$, $\mathrm{n}=215)$, ultrahypofractionated EBRT vs. standard EBRT $(\mathrm{k}=2, \mathrm{n}=1,275)$, and EBRT plus neoadjuvant and concurrent ADT vs. EBRT plus concurrent and adjuvant ADT $(\mathrm{k}=1, \mathrm{n}=432)$.

†One RCT (ProtecT) was a three-arm trial. ProtecT PSA-based active monitoring group is labeled active surveillance in figure.

‡The node size reflects the sample size. The width of lines reflects the number of RCTs that evaluated that comparison. 
Watchful waiting may result in moderate to large increases in overall mortality and small to large increases in prostate cancer mortality compared with RP through 20 years among clinically, rather than PSA screen, detected CLPC. Absolute effects vary by study. WW probably results in small to large increases in metastases through 15-20 years. Effects depend on study population. WW probably results in moderately reduced urinary and erectile dysfunction. Mortality differences may be limited to men age 65 and older or those with intermediate-risk disease.

Active monitoring using PSA-based monitoring probably results in little to no difference in all-cause or prostate cancer mortality compared with RP or EBR plus AD over 10 years. Metastases were infrequent, but AM probably results in a small increase compared with RP and EBR+AD. Effects may not vary by patient or tumor risk factors. Harms were lowest with AM compared with RP or EBR plus AD or AS versus photodynamic therapy.

Radical prostatectomy probably results in little to no difference over 10 years in all-cause or prostate cancer mortality, or metastases compared with EBR plus AD. Results may not vary by patient or tumor risk characteristics. RP probably results in a large increase in urinary incontinence and a moderate increase in erectile dysfunction; fecal incontinence may be slightly decreased compared with EBR plus AD.

External beam radiation using a combination of $3 \mathrm{D}$-conformal radiation and $\mathrm{AD}$ with low-dose-rate prostate brachytherapy may slightly reduce all-cause mortality compared with 3D-conformal radiation and AD over 5 years but may make little to no difference on metastatic disease. Associated harms were unclear. EBR plus AD probably results in a small reduction in overall mortality and may result in a small reduction in prostate cancer mortality and metastases versus EBR alone over 7 years in men with intermediate- or high-risk disease. However, it may result in a moderate increase in sexual dysfunction. When comparing the sequence of add-on androgen deprivation therapy (ADT), EBR plus neoadjuvant initiation of AD compared with EBR plus concurrent initiation of AD may result in little to no difference in overall mortality and prostate cancer mortality over 12 years and late genitourinary toxicity over 3 years.

Other therapies/comparisons had too little and/or conflicting evidence to draw conclusions.

\section{Limitations}

Our review findings have several limitations including-

- Many randomized trials were too short to assess overall or prostate cancer mortality.

- We found few well-designed prospective cohort studies. Retrospective observational studies often had a high risk of bias.

- Varying thresholds to define effect size estimates may alter certainty of evidence and clinical/policy decisions.

- We found few studies of high-intensity focused ultrasound, laser ablation, or photodynamic therapy, and no eligible studies of other focal therapies.

- Few studies reported on how patient, tumor characteristics, or biomarkers modify treatment effect. No studies assessed surgeon or hospital volume treatment effects. 
- Metastases were often reported based on radiographic and PSA results in asymptomatic patients rather than as patient-reported outcomes (e.g., bone pain or ureteral obstruction) and should not be interpreted as symptomatic metastases.

- While clinical and policy decision making often incorporate patient and tumor characteristics, evidence certainty to guide decisions based on these characteristics is limited and unlikely to be greater than findings from intervention effects overall.

\section{Implications and Conclusions}

An important report contribution lies in its appraisal of longer-term data from two RCTs comparing RP with WW in clinically, rather than PSA, detected CLPC. Extended followup suggests that RP may reduce mortality and probably reduces metastases over a very extended timeframe. Age and tumor risk category may be important effect modifiers. Prostate cancer mortality is infrequent in low-risk disease, and all-cause or prostate cancer mortality reduction due to RP may be limited to intermediate-risk disease or age $<65$ years. Absolute effects are likely smaller among PSA-detected CLPC due to its more indolent course. Harms are greater with RP.

AM was compared with RP or EBR plus AD in PSA screen-detected CLPC. Prostate cancer mortality and metastases were rare in all three groups. After 10 years, overall and prostate-cancer mortality were similar across all three treatments though EBR and RP resulted in small absolute reductions in metastases. Surgery may have caused worse urinary and sexual function compared with AM, while EBR may have caused worse sexual and bowel function. No RCTs evaluated WW or AS using scheduled prostate biopsies or MRI in CLPC detected by PSA screening alone.

We found additional evidence supporting that EBR plus ADT may reduce mortality and metastases versus EBR alone in men with intermediate- and high-risk disease. However, it may also result in an increase in harms. Additionally, one newly identified RCT showed little difference between conventionally fractionated EBR versus ultra-hypofractionated EBR. Furthermore, combination 3D conformal EBR with low-dose brachytherapy plus neoadjuvant ADT may reduce mortality more than EBR plus neoadjuvant ADT in men with intermediate- to high-risk disease, but harms were unclear.

This report update was motivated, in part, by an increasing interest in focal therapies or whole prostate gland therapy that is suggested to have fewer or less serious harms than RP or EBR. For these modalities, often targeted to lower risk focal CLPC, including cryotherapy, laser ablation, and high-intensity focused ultrasound, evidence was insufficient. We found no evidence for effects of photodynamic therapy on mortality or metastases. We found little additional evidence for within-treatment comparisons between other surgical or EBR approaches.

Our findings have clinical, policy, and research implications. Our results highlight the importance of balancing treatment benefits with harms and the inclusion of patient and tumor characteristics as well as patient preferences into treatment decisions. They reinforce the need for long-term comparative effectiveness RCTs and well-designed prospective cohort studies. They highlight that the more indolent natural history of PSA-detected compared with clinically detected CLPC has important implications on net benefit of treatment options. For most men with CLPC including those with life expectancies of 15-20 years, evidence indicates that WW and AM result in little to no difference in mortality and metastases and fewer harms compared with early intent-to-cure treatments. The absolute benefit of early intervention in PSA-detected 
CLPC is likely considerably less and overtreatment greater than studies of WW and AM suggest. For men with PSA-detected CLPC who choose early treatment, RP provides similar effects through 10 years compared with EBR+AD. For men with higher risk disease who select EBR, the addition of AD reduces mortality but may increase harms compared with EBR alone. Our findings provide a cautionary note before incorporating newer treatment modalities (including refinements of RP or EBR) into clinical care as evidence on their effectiveness and harms is very limited. While AS and newer modalities hold promise, we need high-quality studies including assessment of provider, patient, and tumor characteristics on patient important outcomes.

\section{References}

1. Siegel RL, Miller KD, Jemal A. Cancer statistics, 2020. CA Cancer J Clin. 2020 Jan;70(1):730. doi: https://doi.org/10.3322/caac.21590. PMID: 30620402.

2. Buyyounouski MK, Choyke PL, McKenney JK, et al. Prostate cancer - major changes in the American Joint Committee on Cancer eighth edition cancer staging manual. CA Cancer J Clin. 2017 May 6;67(3):245-53. doi: 10.3322/caac.21391. PMID: 28222223.

3. Wilt TJ, MacDonald R, Rutks I, et al. Systematic review: comparative effectiveness and harms of treatments for clinically localized prostate cancer. Ann Intern Med. 2008 Mar 18;148(6):435-48. PMID: 18252677.

4. Sun F, Oyesanmi O, Fontanarosa J, et al. Therapies for Clinically Localized Prostate Cancer: Update of a 2008 Systematic Review. Rockville (MD): 2014. https://www.ncbi.nlm.nih.gov/pubmed/2561 $\underline{0935 .}$.
5. Fontanarosa J, Treadwell JR. Clinically Localized Prostate Cancer Evidence Report ECRI Institute,. Plymouth Meeting, PA: 2016.

6. Higgins JPT, Altman D, Sterne J. Chapter 8: Assessing risk of bias in included studies. In: Higgins JPT, Green S, eds. Cochrane Handbook for Systematic Reviews of Interventions: Version 510. The Cochrane Collaboration; 2011.

7. Sterne JA, Hernan MA, Reeves BC, et al. ROBINS-I: a tool for assessing risk of bias in non-randomised studies of interventions. BMJ. 2016 Oct 12;355:i4919. doi: https://dx.doi.org/10.1136/bmj.i4919. PMID: 27733354.

8. Guyatt GH, Oxman AD, Kunz R, et al. What is "quality of evidence" and why is it important to clinicians? BMJ. 2008 May 3;336(7651):995-8. doi: 10.1136/bmj.39490.551019.BE. PMID: 18456631. 


\section{Chapter 1. Introduction}

The American Cancer Society estimates that in 2020 prostate cancer was the most frequently diagnosed non-dermatologic malignancy (191,930 new cases) and the second leading cause of cancer death (33,330 deaths) among men in the U.S. ${ }^{1}$ Treatment-related medical costs are projected to rise to $\$ 16$ billion per year by $2020 .{ }^{1}$ In about 90 percent of men diagnosed with prostate cancer, the disease is confined to the prostate gland (referred to as clinically localized prostate cancer [CLPC]). ${ }^{2}$ Although disease progression sometimes results in morbidity and mortality, most cases of CLPC grow slowly and remain asymptomatic, even if untreated. Therefore, the potential for over-diagnosis and over-treatment is great, especially when the disease is identified through prostate specific antigen (PSA) screening.

CLPC treatments aim to balance potential benefits with complications, burden and costs. Watchful waiting (WW) monitors patients for signs or symptoms of progression and focuses on avoiding unnecessary or ineffective early interventions, while reserving treatment mainly for palliative purposes. WW is most commonly utilized in men with low-risk CLPC, or with limited life expectancy. Active surveillance and Active monitoring (AS/AM) are other options whereby tumors are not immediately removed, irradiated, or ablated, ${ }^{9,10}$ but instead monitored with delayed active treatment initiated based on varying surveillance and monitoring protocols. Although AS/AM definitions, protocols and intervention recommendations vary, further treatment is typically initiated in response to worsening tumor risk characteristics based on surveillance PSA values, prostate biopsies and/or magnetic resonance imaging (MRI) tests.

Androgen deprivation therapy (luteinizing hormone-releasing hormone [LHRH] agonists, LHRH antagonists, anti-androgens, and orchiectomy), commonly known as ADT, has historically been the first-line treatment for biochemically or clinically progressive, recurrent, and metastatic prostate cancer, even in the absence of symptoms. ADT has been used alone and in combination with radical prostatectomy (RP) or radiation therapies, though its use has declined as primary treatment particularly in men with low risk disease. ${ }^{4,11}$

Some CLPC treatments are primarily intended to cure disease. These include surgical radical prostatectomy and radiation therapy (RT). RP can be performed with an open or laparoscopic approach. Laparoscopic prostatectomy is now commonly performed with robotic-assisted (RALP) technology. Radiation therapy can be delivered either by external beams (external beam radiation therapy [EBRT]) or by internally placing radioactive sources (brachytherapy). EBRT strategies vary, and include intensity modulated radiation therapy (IMRT), three-dimensional conformal radiation therapy (3D-CRT), stereotactic body radiation therapy (SBRT), and proton beam radiation therapy. These interventions remove or treat the whole prostate gland and can have short and longer-term adverse effects including but not limited to perioperative morbidity and urinary, bowel, and erectile/sexual dysfunction.

Given the complications associated with RP and RT, and the relatively indolent nature of many PSA-screen detected CLPC, more attention is turning to potentially lower-risk focal therapies such as high-intensity focused ultrasound (HIFU) and cryotherapy, that focus treatment on the index lesion. ${ }^{12-14}$ Use of these options has also increased in response to advances in MRI technology, which now allows for better detection of limited in size local lesions potentially treatable with "lesion-targeted" interventions rather than whole-gland therapy. In addition, awareness has grown regarding the slow-growing nature of most PSA-detected tumors, and therefore the importance of weighing treatment benefits and harms relative to men's preferences to avoid treatment-related complications. ${ }^{15}$ 
The purpose of this review was to identify new information and update previous Agency for Healthcare Research and Quality (AHRQ) and American Urological Association (AUA) funded reviews $^{3-5}$ evaluating treatments for CLPC as described in our Analytic Framework (Appendix A), and to inform clinical guideline committees as they update guidelines. We updated the evidence base regarding the Key Questions (KQs) below:

KQ 1: What are the comparative effectiveness and harms of CLPC therapies?

1) Watchful waiting

2) Active surveillance/Active monitoring

3) Androgen deprivation therapy (ADT)

4) Focal therapies
a) Brachytherapy
b) Cryotherapy
c) High-intensity focused ultrasound (HIFU)
d) Laser ablation
e) Photodynamic therapy
f) Irreversible electroporation

5) Whole gland therapies
a) Brachytherapy
b) Cryotherapy
c) External beam radiation therapy
i) three-dimensional conformal radiation therapy
ii) intensity-modulated radiation therapy
iii) proton beam therapy
iv) stereotactic body radiation therapy
d) Radical prostatectomy
i) open
ii) laparoscopic

(1)without robotic assistance

(2)with robotic assistance

6) Combination of above

KQ 2: How do patient characteristics modify comparative effectiveness and harms of CLPC therapies?

1) Age

2) Race/ethnicity

3) Comorbidities

4) Health status 
KQ 3: How do tumor characteristics modify comparative effectiveness and harms of CLPC therapies?

1) Baseline PSA

2) Gleason score

3) Tumor index scores (e.g., Cancer of the Prostate Risk Assessment Score [CAPRA], D'Amico Risk Classification for Prostate Cancer, etc.)

4) Biomarker Status

a) Decipher (Genomic Classifier)

b) Oncotype Dx (Genomic Prostate Score)

c) Prolaris (Cell Cycle Progression)

KQ 4: How do provider/hospital characteristics modify comparative effectiveness of RP compared to other therapies?

1) Geographic region

2) Hospital type

3) Provider volume

4) Institutional volume 
Table 1. PICOTS

\begin{tabular}{|c|c|c|}
\hline PICOTS & KQ 1-3 & KQ 4 \\
\hline Population & $\begin{array}{l}\text { Treatment naïve men with CLPC (stages T1-T3a) } \\
\text { Studies with 15\% or more participants with T3b or } \\
\text { unspecified T3 are excluded }\end{array}$ & Same as KQ 1-3 \\
\hline Intervention & $\begin{array}{l}\text { 1) Watchful waiting (WW) } \\
\text { 2) Active surveillance/active monitoring (AS/AM) } \\
\text { 3) Androgen deprivation therapy (ADT) } \\
\text { 4) Focal therapies } \\
\text { a) Brachytherapy } \\
\text { b) Cryotherapy } \\
\text { c) High-intensity focused ultrasound (HIFU) } \\
\text { d) Laser ablation } \\
\text { e) Photodynamic therapy } \\
\text { f) Irreversible electroporation } \\
\text { 5) Whole gland therapies } \\
\text { a) Brachytherapy } \\
\text { b) Cryotherapy } \\
\text { c) External beam radiation therapy (EBRT) } \\
\text { i) Three-dimensional conformal radiation } \\
\text { ii) Intensity-modulated radiation therapy } \\
\text { iii) Proton beam therapy } \\
\text { iv Stereotactic body radiation therapy } \\
\text { d) Radical prostatectomy } \\
\text { i) Open } \\
\text { ii) Laparoscopic } \\
\text { (1) Without robotic assistance } \\
\text { (2) With robotic assistance } \\
\text { 6) Combination of above }\end{array}$ & $\begin{array}{l}\text { 1) Radical prostatectomy } \\
\text { i) Open } \\
\text { ii) Laparoscopic } \\
\text { (1) Without robotic assistance } \\
\text { (2) With robotic assistance }\end{array}$ \\
\hline Comparison & $\begin{array}{l}\text { Any other intervention of listed above except certain } \\
\text { within category comparisons (e.g., nerve-sparing vs non- } \\
\text { nerve sparing prostatectomy; different } \\
\text { dosage/frequency/timing/duration of same therapy) }\end{array}$ & Same as KQ 1-3 \\
\hline Outcomes & $\begin{array}{l}\text { Overall survival/mortality } \\
\text { Prostate cancer specific survival/mortality } \\
\text { Metastatic-progression free survival } \\
\text { Metastases (lymph nodes/distant) } \\
\text { Health status } \\
\text { Quality of life (measured with validated instruments) } \\
\text { Prostate-cancer related quality of life (measured with } \\
\text { validated instruments) } \\
\text { Harms: } \\
\text { Bowel, bladder, and sexual/erectile dysfunction } \\
\text { Serious adverse effects associated with ADT such as } \\
\text { cognitive impairment, MACE, fractures }\end{array}$ & $\begin{array}{l}\text { Overall survival/mortality } \\
\text { Prostate cancer specific survival/mortality } \\
\text { Metastatic free survival/metastases (lymph } \\
\text { nodes/distant) }\end{array}$ \\
\hline Timing & $\begin{array}{l}\text { Follow up from treatment initiation: } \\
\text { Mortality/survival outcomes/metastases: } 5 \text { years or more } \\
\text { Health status, quality of life and harms: } 1 \text { year or more }\end{array}$ & $\begin{array}{l}\text { Follow up from treatment initiation: } \\
\text { Mortality/survival outcomes/metastases: } 5 \\
\text { years or more }\end{array}$ \\
\hline Setting & All settings & Same as KQ 1-3 \\
\hline
\end{tabular}




\begin{tabular}{|l|l|l|}
\hline PICOTS & KQ 1-3 & KQ 4 \\
\hline Study & 1) RCTs & Same as KQ 1-3 \\
Design & 2) Non-RCT if: & \\
& a) Comparative & \\
& b) Concurrent \\
& c) Multicenter (enrolling patients treated at & \\
& multiple locations) & \\
& d) $\geq 500$ patients & \\
& e) Some method to control for selection bias & \\
& & (propensity scores, instrumental variables, \\
& f) Prospective data collection & \\
\hline
\end{tabular}

Abbreviations: $\mathrm{ADT}=$ androgen deprivation therapy; $\mathrm{AM}=$ active monitoring; $\mathrm{AS}=$ active surveillance; $\mathrm{CLPC}=\mathrm{clinically}$ localized prostate cancer; EBRT = external beam radiation therapy; HIFU = high-intensity focused ultrasound; KQ = Key

Question; MACE = major adverse cardiac events; PICOTS = populations, interventions, comparators, outcomes, timing, setting; $\mathrm{RCT}=$ randomized controlled trial; $\mathrm{WW}=$ watchful waiting 


\section{Chapter 2. Methods}

\section{Review Approach}

The methods for this systematic review followed the Agency for Healthcare Research Qualtiy (AHRQ) Methods Guide for Effectiveness and Comparative Effectiveness Reviews (available at https://effectivehealthcare.ahrq.gov/topics/cer-methods-guide/overview). This systematic review also reports in accordance with the Preferred Items for Reporting in Systematic Reviews and Meta-Analyses (PRISMA). ${ }^{16}$ The final protocol was posted online (https://effectivehealthcare.ahrq.gov/products/prostate-cancer-therapies/protocol) and submitted for registration in PROSPERO on October 16, 2019 (ID 154937).

\section{Criteria for Inclusion/Exclusion of Studies in the Review}

Studies were included based on the population, intervention, comparison, outcomes, timing, and setting/study design (PICOTS) study-specific inclusion criteria (Table 1).

\section{Searching for the Evidence and Updating Prior Reviews}

We searched Medline ${ }^{\circledR}$, Embase ${ }^{\circledR}$, and the Cochrane Central trials database incorporating vocabulary and natural language relevant to the Key Questions (KQs) (search strategy in Appendix B). Our search captured publications indexed between 2013 and January 2020.

Relevant studies published before 2013 were identified in the previous reports; studies published after 2013 were excluded from our review if they were analyzed in a previous report.

The evidence for this report included: 1) eligible studies published after the 2014 AHRQ and 2016 American Urological Association (AUA) funded reviews; and 2) outcomes from randomized controlled trials (RCTs) included in the 2014 AHRQ and 2016 AUA funded reviews when we also included an RCT of low-moderate risk of bias of the same comparison. It was only applicable to carry forward data for two treatment comparisons (watchful waiting [WW] versus radical prostatectomy [RP] in Chapter 4 and external beam radiation therapy [EBRT] plus androgen deprivation therapy [ADT] versus EBRT in Chapter 6). We refer the reader to findings above insufficient evidence from the 2014 AHRQ and 2016 AUA funded reviews related to treatment comparisons noted previously and that we did not address (Table 2). 4, 5

Search results were downloaded to EndNote X9 (Clarivate Analytics, 2018) and screened in DistillerSR (Evidence Partners, Ottawa, Canada). Two independent investigators reviewed titles and abstracts using predefined criteria. Two independent investigators conducted full-text screening to determine if inclusion criteria were met. Differences in screening decisions were resolved by consultation between investigators, and, if necessary, with a third investigator.

We supplemented our bibliographic database searches by citation searching relevant systematic reviews and original research. Additionally, we searched ClinicalTrials.gov to identify completed and ongoing studies. Additional grey literature was solicited through Federal Register notification. Information from grey literature was also be used to assess publication and reporting bias and inform future research needs. 


\section{Assessment of Methodological Risk of Bias of Individual Studies}

We used a hierarchical method to analyze evidence. For each comparison, we first assessed the certainty of evidence (COE) using RCTs rated as low or moderate risk of bias (ROB). If RCT data did not achieve moderate or high COE for an outcome, we next analyzed non-RCT data rated as low or moderate ROB. We did not analyze RCT data rated as high ROB and non-RCT data rated as serious or critical non-RCT data.

Eligible RCTs were assessed for ROB using the Cochrane Risk of Bias Tool ${ }^{6}$ and non-RCTs were assessed with the ROBINS-I tool. ${ }^{7}$ One investigator independently assessed risk of bias for eligible studies to be analyzed; a second investigator reviewed each risk of bias assessment. Investigators consulted to reconcile any discrepancies. Overall risk of bias assessments for RCTs were classified as low, moderate, or high based on the collective risk of bias across components and confidence that the study results were believable given the study's limitations. Overall risk of bias assessments for non-RCTs were classified as low, moderate, serious, or critical based on ROBINS-I criterion.

\section{Data Abstraction and Data Management}

From studies analyzed, we extracted inclusion and exclusion criteria; sample size; participant age, race, clinical stage, and Gleason score; tumor risk classification and score, intervention and comparator characteristics; followup duration; and results for outcomes and adverse effects. We extracted data at one year and the longest followup for quality of life, health status, and harms; we extracted data at five-year intervals for mortality and metastases or at mean/median followup if that was the only way reported. One investigator extracted data to tables with verification by a second reviewer.

\section{Data Synthesis}

We summarized results of findings in evidence tables and synthesized evidence for each unique comparison with meta-analysis when appropriate. We assessed clinical and methodological heterogeneity to determine appropriateness of pooling data. ${ }^{17}$ When metaanalysis was not appropriate, we summarized findings. We calculated risk ratios (RR) or Peto odds' ratios (OR) and absolute risk differences (RD) with the corresponding 95 percent confidence intervals (CI) for binary outcomes. Weighted mean differences (WMD) and/or standardized mean differences (SMD) with 95 percent CIs were calculated for continuous outcomes. Data were analyzed in Comprehensive Meta-Analysis version 3 (Biostat) or R software (package “meta”), version 3.6.0.

\section{Grading Evidence Certainty}

We assessed COE with Grading of Recommendations Assessment, Development and Evaluation (GRADE) ${ }^{8}$ approach for key outcomes (overall mortality; prostate-specific mortality; metastatic progression) and harms (bowel, bladder, and sexual function). For each comparison, one investigator rated the certainty of evidence for each outcome as high, moderate, low, or insufficient using GRADEpro GDT. ${ }^{18} \mathrm{COE}$ was reviewed by a second investigator. We resolved discrepancies by consensus or discussion with a third reviewer. We used suggested language ${ }^{19}$ to summarize findings and assessed effect size using prespecified thresholds (Appendix C). For 
overall and prostate cancer mortality and metastases we defined absolute risk differences of $<2 \%$ as "little to no difference", $2-4.9 \%$ as "small"; 5-9.9\% as "moderate" and $\geq 10 \%$ as "large" effects regardless of population, intervention, comparison or length of follow-up. For urinary, bowel and sexual function we defined absolute risk differences of 2-4.9\% as "small”; 5-19.9\% as "moderate" and $\geq 20 \%$ as "large."

\section{Assessing Applicability}

We assessed applicability of results by analyzing whether eligible studies reflected the relevant population according to the PICOTS framework. The population from which the study participants were enrolled, diagnostic approaches, eligibility criteria, patient and intervention characteristics, and other issues that differ from those of the population of treatment naïve men with clinically localized prostate cancer (CLPC) affect applicability. ${ }^{21}$ 


\section{Chapter 3. Results}

Our search identified 11,327 references (Figure 1). Title and abstract screening eliminated 10,564 references leaving 763 references for full text review. We identified 67 references that were eligible for inclusion to our review, of which 17 were unique randomized controlled trials (RCTs). A list of all eligible publications can be found in Appendix E. A list of all publications excluded at full-text review can be found in Appendix D. Supplemental searches of clinicaltrials.gov and other grey literature sources did not yield any additional published studies that were eligible. Comparisons addressed in eligible RCTs are illustrated in Figure 2. Table 2 summarizes our findings and major intervention and outcomes from past reports. ${ }^{4,5}$

Figure 1. Literature flow diagram

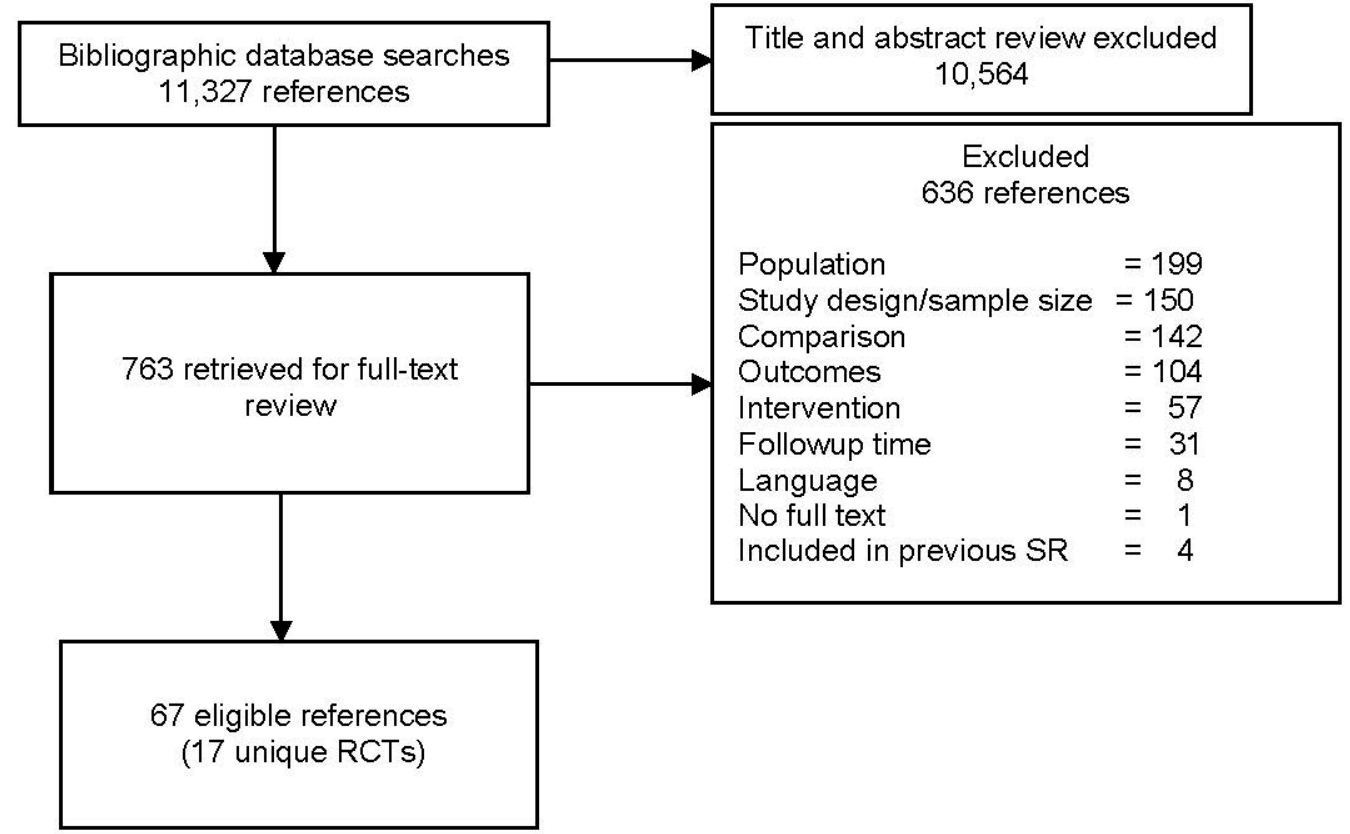

Abbreviations: RCT = randomized controlled trial; SR = systematic review 
Figure 2. Plot of comparisons addressed in RCTs identified in updated literature search ${ }^{*} \star^{\star \star} \dagger \dagger$

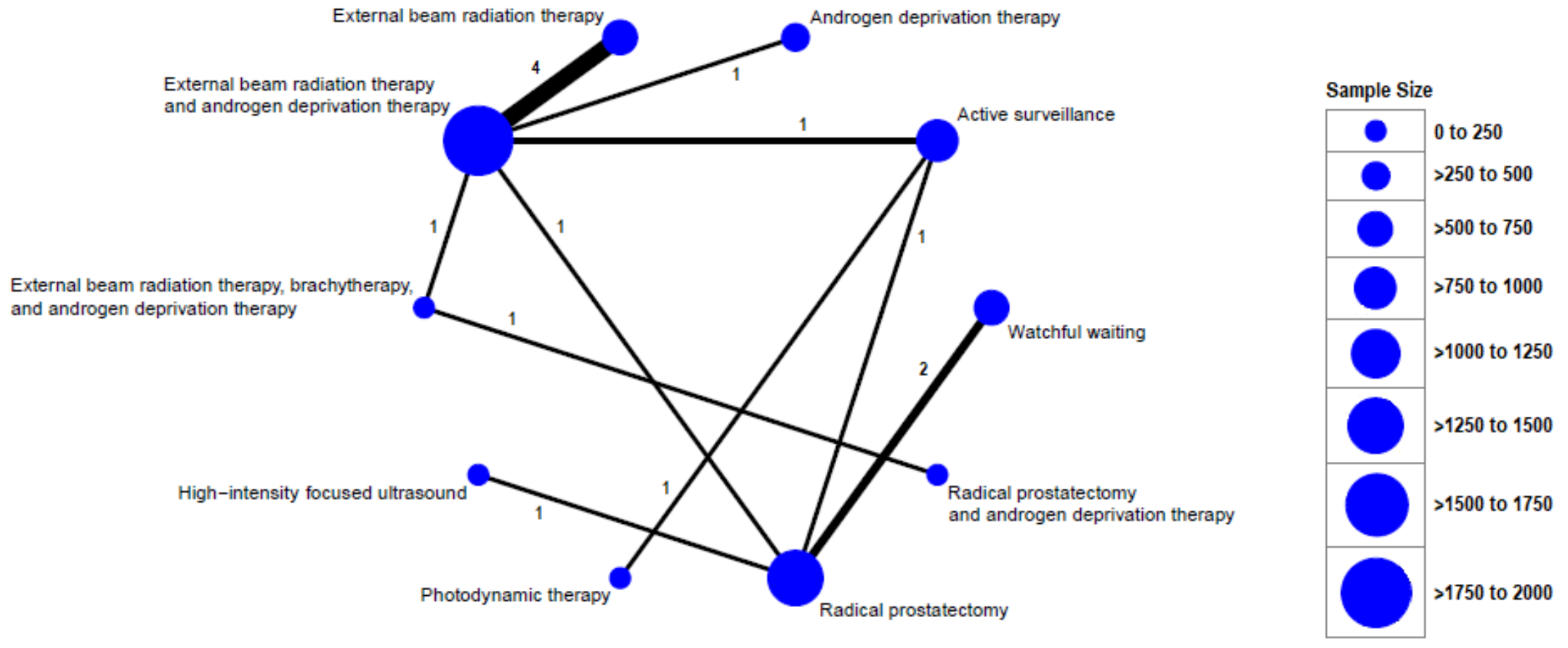

Abbreviation: RCT = randomized controlled trial

*The node size reflects the sample size. The width of lines reflects the number of RCTs that evaluated that comparison.

†Within category comparisons are not shown in figure. These include: RARP vs. LRP $(\mathrm{k}=1, \mathrm{n}=120)$, 3D-CRT vs. IMRT $(\mathrm{k}=1, \mathrm{n}=215)$, ultra-hypofractionated EBRT vs. standard EBRT $(\mathrm{k}=2, \mathrm{n}=1,275)$, and EBRT plus neoadjuvant and concurrent ADT vs. EBRT plus concurrent and adjuvant ADT ( $\mathrm{k}=1, \mathrm{n}=432)$.

‡One RCT (ProtecT) was a three-arm trial.

**The AS protocols varied. One trial evaluated biopsy-based AS vs. photodynamic therapy and a second trial evaluated PSA-based active monitoring vs. RP or EBRT plus ADT. ††We identified 4 RCTs that compared EBRT plus ADT vs. EBRT alone. The old reports identified 3 additional RCTs. 
Table 2. Summary updates of comparisons between reviews*

\begin{tabular}{|c|c|c|c|}
\hline $\begin{array}{l}\text { Interventionl } \\
\text { Comparison }\end{array}$ & Outcome(s) & $\begin{array}{l}\text { Previous Findings From } \\
2014 \text { AHRQ- or } 2016 \\
\text { AUA-Funded Reviews } \dagger\end{array}$ & $\begin{array}{l}\text { Present Findings Derived From Studies Published After the Prior } \\
\text { Reviews and by Incorporating Prior RCT Data When Applicableł }\end{array}$ \\
\hline $\begin{array}{l}\text { WW vs. RP in men } \\
\text { with clinically } \\
\text { detected (SPCG-4) } \\
\text { or mainly clinically } \\
\text { detected (PIVOT) } \\
\text { CLPC } \neq\end{array}$ & $\begin{array}{l}\text { All-cause mortality, } \\
\text { PC-specific mortality, } \\
\text { Metastases } \\
\text { Harms }\end{array}$ & $\begin{array}{l}\text { Insufficient evidence on all- } \\
\text { cause mortality, PC-specific } \\
\text { mortality, and erectile and } \\
\text { bowel harms. RP probably } \\
\text { reduces metastases. WW } \\
\text { may reduce urinary harms. } \\
\text { Insufficient evidence for } \\
\text { erectile and bowel harms.§ }\end{array}$ & $\begin{array}{l}\text { WW vS. RP in men with clinically detected CLPC: (SPCG-4) } \\
\text { - } \quad \text { probably results in moderate increases in all-cause mortality and large } \\
\text { increases in PC-specific mortality and metastases at } 25 \text { years. Mortality } \\
\text { effects may be limited to men younger than age } 65 \text { and intermediate risk } \\
\text { CLPC. } \\
\text { - No new data for harms. } \\
\text { WW versus RP in men with mainly clinically detected CLPC (PIVOT): } \\
\text { - probably results in a moderate increase in all-cause mortality and large } \\
\text { reduction in metastases and small increase in PC-specific mortality and at } \\
20 \text { years. Mortality effects may be limited to men younger than age } 65 \text { and } \\
\text { intermediate risk CLPC. } \\
\text { probably results in a moderate reduction in erectile and urinary harms at } \\
10 \text { years. }\end{array}$ \\
\hline $\begin{array}{l}\text { AM (PSA-based) vs. } \\
\text { EBRT + ADT }\end{array}$ & $\begin{array}{l}\text { All-cause mortality, PC- } \\
\text { specific mortality, } \\
\text { Metastases, } \\
\text { Harms }\end{array}$ & Not addressed & $\begin{array}{l}\text { AM versus EBRT plus ADT in men with PSA-Screen detected CLPC: } \\
\text { - } \quad \text { probably results in little to no difference in all-cause mortality, may result } \\
\text { in little to no difference in PC-specific mortality and probably results in } \\
\text { small increases in metastases at } 10 \text { years. Results may not vary by } \\
\text { patient or tumor characteristics. } \\
\text { may result in a small decrease in erectile dysfunction, probably results in } \\
\text { a small increase in urinary incontinence, and may make little to no } \\
\text { difference in fecal incontinence at } 6 \text { years. }\end{array}$ \\
\hline $\begin{array}{l}\text { AM (PSA-based) vs. } \\
\text { RP }\end{array}$ & $\begin{array}{l}\text { All-cause mortality, PC- } \\
\text { specific mortality, } \\
\text { Metastases } \\
\text { Harms }\end{array}$ & Not addressed & $\begin{array}{l}\text { AM versus RP in men with PSA-screen detected CLPC: } \\
\text { - may result in little to no difference in all-cause or PC-specific mortality but } \\
\text { probably results in a small increase in metastases at } 10 \text { years. Results } \\
\text { may not vary by patient or tumor characteristics. } \\
\text { - probably results in a large decrease in erectile dysfunction and moderate } \\
\text { decrease in urinary incontinence and may make little to no difference in } \\
\text { fecal incontinence at } 6 \text { years. }\end{array}$ \\
\hline $\begin{array}{l}\text { AS (Biopsy + PSA } \\
\text { based) vs. PDT }\end{array}$ & Harms & Not addressed & $\begin{array}{l}\text { AS versus PDT in men with PSA screen-detected low risk CLPC: } \\
\text { - } \quad \text { probably results in a large decrease in erectile dysfunction and moderate } \\
\text { decrease in urinary retention at } 2 \text { years. }\end{array}$ \\
\hline RP vs. EBRT + ADT & $\begin{array}{l}\text { All-cause mortality, PC- } \\
\text { specific mortality, } \\
\text { Metastases, } \\
\text { Harms }\end{array}$ & $\begin{array}{l}\text { Clinical outcomes not } \\
\text { addressed } \\
\text { Insufficient evidence on } \\
\text { harms. ** }\end{array}$ & $\begin{array}{l}\text { RP versus EBRT plus ADT in men with PSA-screen detected CLPC: } \\
\text { - may result in little to no difference in all-cause mortality, PC-specific } \\
\text { mortality, and metastases at } 10 \text { years. Results on PC-specific mortality } \\
\text { may not differ by age, PSA level, Gleason score or clinical stage. } \\
\text { probably results in an increase in erectile and urinary harms and a } \\
\text { decrease in bowel dysfunction at } 6 \text { years. }\end{array}$ \\
\hline
\end{tabular}




\begin{tabular}{|c|c|c|c|}
\hline $\begin{array}{l}\text { Interventionl } \\
\text { Comparison }\end{array}$ & Outcome(s) & $\begin{array}{l}\text { Previous Findings From } \\
2014 \text { AHRQ- or } 2016 \\
\text { AUA-Funded Reviews } †\end{array}$ & $\begin{array}{l}\text { Present Findings Derived From Studies Published After the Prior } \\
\text { Reviews and by Incorporating Prior RCT Data When Applicableł }\end{array}$ \\
\hline $\begin{array}{l}\text { RP + ADT vs. EBRT } \\
+ \text { HDR } \\
\text { Brachytherapy }+ \\
\text { ADT }\end{array}$ & $\begin{array}{l}\text { All-cause mortality, PC- } \\
\text { specific mortality, } \\
\text { Harms }\end{array}$ & $\begin{array}{l}\text { Insufficient evidence on } \\
\text { harms for RP vs. EBRT plus } \\
\text { brachytherapy. ** }\end{array}$ & $\begin{array}{l}\text { RP plus ADT versus EBRT plus high-dose-rate brachytherapy plus ADT in } \\
\text { men with T1b-T3a PC of any histologic grade: } \\
\text { - may result in a small increase in erectile dysfunction at } 2 \text { years. } \\
\text { - insufficient evidence on urinary or bowel harms at } 2 \text { years and all-cause } \\
\text { or PC-specific mortality through } 10 \text { years. }\end{array}$ \\
\hline RP vs. HIFU & Harms & Not addressed & $\begin{array}{l}\text { In men with Gleason score } 7,<T 2 b \text { CLPC, insufficient evidence on urinary, } \\
\text { erectile, and bowel harms at } 1 \text { year. }\end{array}$ \\
\hline $\begin{array}{l}\text { Laparoscopic RP vs. } \\
\text { Robotic Assisted RP }\end{array}$ & Harms & $\begin{array}{l}\text { Insufficient evidence on } \\
\text { urinary and erectile harms at } \\
1 \text { year. } \S\end{array}$ & $\begin{array}{l}\text { Laparoscopic RP versus robotic RP in men with PSA detected predominately } \\
\text { low-intermediate risk CLPC: } \\
\text { - may result in a moderate increase in urinary incontinence and a large } \\
\text { increase in erectile dysfunction at } 5 \text { years. }\end{array}$ \\
\hline $\begin{array}{l}\text { Robotic-assisted } \\
\text { Laparoscopic RP vs. } \\
\text { Open Retropubic RP }\end{array}$ & $\begin{array}{l}\text { All-cause mortality, PC- } \\
\text { specific mortality, } \\
\text { Metastases } \\
\text { Harms }\end{array}$ & $\begin{array}{l}\text { Insufficient evidence on all- } \\
\text { cause mortality, PC-specific } \\
\text { mortality, metastases, and } \\
\text { harms. *夫 }\end{array}$ & $\begin{array}{l}\text { In men with predominately low and intermediate D'Amico risk CLPC, } \\
\text { insufficient evidence on erectile dysfunction. No data for mortality/metastases. }\end{array}$ \\
\hline $\begin{array}{l}\text { EBRT vs. } \\
\text { Brachytherapy }\end{array}$ & $\begin{array}{l}\text { All-cause mortality, PC- } \\
\text { specific mortality, } \\
\text { Metastasis-free survival }\end{array}$ & $\begin{array}{l}\text { Insufficient evidence on all- } \\
\text { cause mortality and PC- } \\
\text { specific mortality. } \S\end{array}$ & $\begin{array}{l}\text { In men with Gleason } 6 \text { or } 7 \text { CLPC, insufficient evidence on overall survival, } \\
\text { PC-specific survival, and metastasis-free survival. }\end{array}$ \\
\hline $\begin{array}{l}\text { EBRT + } \\
\text { Brachytherapy vs. } \\
\text { Brachytherapy }\end{array}$ & $\begin{array}{l}\text { All-cause mortality, PC- } \\
\text { specific mortality }\end{array}$ & $\begin{array}{l}\text { Insufficient evidence on PC- } \\
\text { specific mortality. § }\end{array}$ & $\begin{array}{l}\text { In men with intermediate NCCN risk CLPC, insufficient evidence on all-cause } \\
\text { mortality in men. }\end{array}$ \\
\hline IMRT vs. SBRT & All-cause Mortality & Not addressed & $\begin{array}{l}\text { In men with predominately Gleason } 6-7, \mathrm{PSA}<10 \text {, and T1C CLPC, insufficient } \\
\text { evidence on all-cause mortality. }\end{array}$ \\
\hline $\begin{array}{l}\text { Conventionally } \\
\text { fractionated EBRT } \\
\text { vs. ultra- } \\
\text { hypofractionated } \\
\text { EBRT }\end{array}$ & $\begin{array}{l}\text { All-cause mortality, } \\
\text { PC-specific mortality, } \\
\text { Metastasis } \\
\text { Harms }\end{array}$ & Not addressed & $\begin{array}{l}\text { Conventionally fractionated EBRT versus ultra-hypofractionated EBRT in men } \\
\text { with predominantly intermediate-risk CLPC: } \\
\text { - probably results in little to no difference in all-cause mortality and may } \\
\text { result in little to no difference in PC-specific mortality and metastasis at } 5 \\
\text { years. } \\
\text { may result in little to no differences on urinary and bowel harms (except } \\
\text { urinary harms at } 1 \text { year). Insufficient evidence on erectile function. }\end{array}$ \\
\hline $\begin{array}{l}\text { 3D-CRT + ADT + } \\
\text { low-dose-rate } \\
\text { Brachytherapy vs. } \\
\text { 3D-CRT + ADT }\end{array}$ & $\begin{array}{l}\text { All-cause mortality, PC- } \\
\text { specific mortality, } \\
\text { Metastases } \\
\text { Harms }\end{array}$ & $\begin{array}{l}\text { Insufficient evidence on PC- } \\
\text { specific mortality for EBRT } \\
\text { plus BT vs. EBRT. } \S\end{array}$ & $\begin{array}{l}\text { 3D-CRT and ADT plus low-dose-rate brachytherapy versus 3D-CRT and ADT } \\
\text { in men with intermediate and high NCCN risk CLPC: } \\
\text { - may result in a small decrease in all-cause mortality and little to no } \\
\text { difference in metastases at } 5 \text { years. } \\
\text { insufficient evidence on PC-specific mortality, urinary incontinence, and } \\
\text { erectile function. }\end{array}$ \\
\hline
\end{tabular}




\begin{tabular}{|c|c|c|c|}
\hline $\begin{array}{l}\text { Interventionl } \\
\text { Comparison }\end{array}$ & Outcome(s) & $\begin{array}{l}\text { Previous Findings From } \\
2014 \text { AHRQ- or } 2016 \\
\text { AUA-Funded Reviews } \dagger\end{array}$ & $\begin{array}{l}\text { Present Findings Derived From Studies Published After the Prior } \\
\text { Reviews and by Incorporating Prior RCT Data When Applicableł }\end{array}$ \\
\hline $\begin{array}{l}\text { EBRT + ADT vs. } \\
\text { EBRT } \ddagger\end{array}$ & $\begin{array}{l}\text { All-cause mortality, PC- } \\
\text { specific mortality, } \\
\text { Metastases } \\
\text { Harms }\end{array}$ & $\begin{array}{l}\text { Inconsistent findings on all- } \\
\text { cause mortality/survival and } \\
\text { metastases but evidence } \\
\text { consistently favored } \\
\text { combination therapy on PC- } \\
\text { mortality. }{ }^{* *}\end{array}$ & $\begin{array}{l}\text { EBRT plus ADT versus EBRT in men with predominately intermediate to high } \\
\text { risk CLPC (using different risk classifications): } \\
\text { - } \quad \text { probably results in a small reduction in all-cause mortality and may result } \\
\text { in a small reduction in PC-mortality and metastasis at } 5 \text { to } 10 \text { years. } \\
\text { Mortality effects may be limited to intermediate-high risk men and men } \\
\text { with no or minimal co-morbidity. } \\
\text { may moderately increase sexual dysfunction. Insufficient evidence on } \\
\text { urinary incontinence and rectal bleeding. }\end{array}$ \\
\hline $\begin{array}{l}\text { EBRT + neoadjuvant } \\
\text { and concurrent ADT } \\
\text { vs. EBRT plus } \\
\text { concurrent and } \\
\text { adjuvant ADT }\end{array}$ & $\begin{array}{l}\text { All-cause mortality, PC- } \\
\text { specific mortality, } \\
\text { Metastasis, } \\
\text { Harms }\end{array}$ & Not addressed & $\begin{array}{l}\text { EBRT plus neoadjuvant and concurrent ADT versus EBRT plus concurrent } \\
\text { and adjuvant ADT in men with predominantly intermediate-risk CLPC: } \\
\text { - may result in little to no difference in all-cause mortality and PC-specific } \\
\text { mortality at } 12 \text { years. Insufficient evidence on metastasis. } \\
\text { - may result in little to no difference in genitourinary toxicity at } 3 \text { years. }\end{array}$ \\
\hline RP vs. EBRT & $\begin{array}{l}\text { All-cause mortality, PC- } \\
\text { specific mortality }\end{array}$ & $\begin{array}{l}\text { RP may reduce all-cause } \\
\text { mortality and PC-specific } \\
\text { mortality vs. EBRT. } \S\end{array}$ & No new data \\
\hline $\begin{array}{l}\text { Retropubic RP vs. } \\
\text { Brachytherapy }\end{array}$ & Harms & $\begin{array}{l}\text { Results were similar on } \\
\text { erectile and urinary function } \\
\text { at } 5 \text { years. Retropubic RP } \\
\text { may reduce short-term } \\
\text { urinary symptoms versus } \\
\text { brachytherapy. } * \star\end{array}$ & No new data \\
\hline $\begin{array}{l}\text { Retropubic RP vs. } \\
\text { Perineal RP }\end{array}$ & Harms & $\begin{array}{l}\text { Retropubic RP may improve } \\
\text { erectile function at } 2 \text { years } \\
\text { versus Perineal RP, but no } \\
\text { between-group difference at } \\
6 \text { months. No difference on } \\
\text { urinary function. }{ }^{\star}\end{array}$ & No new data \\
\hline $\begin{array}{l}\text { Transperitoneal } \\
\text { Robotic-Assisted } \\
\text { Laparoscopic RP vs. } \\
\text { Extraperitoneal } \\
\text { Robotic-Assisted } \\
\text { Laparoscopic RP } \\
\end{array}$ & Harms & $\begin{array}{l}\text { Results on incontinence or } \\
\text { erection rates at } 6 \text { months } \\
\text { were similar. }{ }^{\star *}\end{array}$ & No new data \\
\hline $\begin{array}{l}\text { EBRT vs. } \\
\text { Observation }\end{array}$ & PC-specific mortality & $\begin{array}{l}\text { EBRT may reduce PC- } \\
\text { specific mortality versus } \\
\text { observation. ** }\end{array}$ & No new data \\
\hline
\end{tabular}




\begin{tabular}{|c|c|c|c|}
\hline $\begin{array}{l}\text { Interventionl } \\
\text { Comparison }\end{array}$ & Outcome(s) & $\begin{array}{l}\text { Previous Findings From } \\
2014 \text { AHRQ- or } 2016 \\
\text { AUA-Funded Reviews } \dagger\end{array}$ & $\begin{array}{l}\text { Present Findings Derived From Studies Published After the Prior } \\
\text { Reviews and by Incorporating Prior RCT Data When Applicableł }\end{array}$ \\
\hline $\begin{array}{l}\text { EBRT vs. } \\
\text { Cryotherapy }\end{array}$ & $\begin{array}{l}\text { All-cause mortality } \\
\text { PC-specific mortality, } \\
\text { harms }\end{array}$ & $\begin{array}{l}\text { No between-group } \\
\text { difference on overall survival } \\
\text { or PC-specific mortality. } \\
\text { Safety outcomes were } \\
\text { inconsistent. ** }\end{array}$ & No new data \\
\hline $\begin{array}{l}\text { EBRT + ADT vs. } \\
\text { ADT }\end{array}$ & PC-specific mortality & $\begin{array}{l}\text { EBRT plus ADT may reduce } \\
\text { PC-specific mortality versus } \\
\text { ADT. ** }\end{array}$ & No new data \\
\hline $\begin{array}{l}\text { IMRT vS. Proton } \\
\text { beam }\end{array}$ & Harms & $\begin{array}{l}\text { IMRT may reduce } \mathrm{GI} \\
\text { adverse events versus } \\
\text { proton beam. }{ }^{\star \star}\end{array}$ & No new data \\
\hline $\begin{array}{l}\text { 3D-CRT } \\
\text { conventional vs. 3D- } \\
\text { CRT high dose }\end{array}$ & $\begin{array}{l}\text { All-cause mortality, PC- } \\
\text { specific mortality, } \\
\text { metastases, harms }\end{array}$ & $\begin{array}{l}\text { No between-group } \\
\text { differences. }{ }^{*}\end{array}$ & Out of scope for this review \\
\hline $\begin{array}{l}\text { Hypofractionated RT } \\
\text { vs. conventionally- } \\
\text { fractionated RT }\end{array}$ & $\begin{array}{l}\text { All-cause mortality, PC- } \\
\text { specific mortality, } \\
\text { Harms }\end{array}$ & $\begin{array}{l}\text { No between-group } \\
\text { differences. }{ }^{* \star}\end{array}$ & Out of scope for this review \\
\hline $\begin{array}{l}\text { Brachytherapy } \\
\text { conventional dose } \\
\text { vs. Brachytherapy } \\
\text { low dose }\end{array}$ & $\begin{array}{l}\text { All-cause mortality, } \\
\text { Urinary symptoms }\end{array}$ & $\begin{array}{l}\text { No between-group } \\
\text { difference (except in short- } \\
\text { term urinary symptoms). }{ }^{* *}\end{array}$ & Out of scope for this review \\
\hline ADT plus RP vs. RP & $\begin{array}{l}\text { All-cause mortality, PC- } \\
\text { specific mortality, } \\
\text { Metastasis }\end{array}$ & $\begin{array}{l}\text { No between-group } \\
\text { differences. }{ }^{* *}\end{array}$ & No new data \\
\hline $\begin{array}{l}\text { ADT plus SOC (RP } \\
\text { or RT) vs. SOC (RP } \\
\text { or RT) }\end{array}$ & $\begin{array}{l}\text { All-cause mortality, } \\
\text { metastases }\end{array}$ & $\begin{array}{l}\text { No between-group } \\
\text { difference. ** }\end{array}$ & Out of scope for this review \\
\hline $\begin{array}{l}\text { ADT plus SOC } \\
\text { (WW) vs. SOC } \\
\text { (WW) alone }\end{array}$ & $\begin{array}{l}\text { All-cause mortality, } \\
\text { metastases }\end{array}$ & Inconsistent results. ** & No new data \\
\hline $\begin{array}{l}\text { ADT short-term plus } \\
\text { RT vs. ADT long- } \\
\text { term plus RT }\end{array}$ & $\begin{array}{l}\text { All-cause mortality, PC- } \\
\text { specific mortality }\end{array}$ & $\begin{array}{l}\text { No between-group } \\
\text { difference on mortality. } \\
\text { Inconsistent results on PC- } \\
\text { specific mortality. }{ }^{* *}\end{array}$ & Out of scope for this review \\
\hline $\begin{array}{l}\text { ADT vs. ADT plus } \\
\text { docetaxel and } \\
\text { estramustine }\end{array}$ & $\begin{array}{l}\text { All-cause mortality, PC- } \\
\text { specific mortality, } \\
\text { Metastases }\end{array}$ & $\begin{array}{l}\text { Combination therapy may } \\
\text { reduce mortality, PC-specific } \\
\text { mortality, and metastases } \\
\text { versus ADT, but results } \\
\text { considered "inconclusive" in } \\
2016 \text { AUA-funded report. ** }\end{array}$ & No new data \\
\hline
\end{tabular}


Abbreviations: 3D-CRT = three-dimensional conformal radiation therapy; ADT = androgen deprivation therapy; AHRQ = Agency for Healthcare Research and Quality;

AM = active monitoring; AS = active surveillance/ AUA = American Urological Association; BT = brachytherapy; CLPC = clinically localized prostate cancer; EBRT = external beam radiation therapy; GI = gastrointestinal; HDR = high-dose rate; HIFU = high-intensity focused ultrasound; IMRT = intensity-modulated radiation therapy; NCCN = National Comprehensive Cancer Network; PC = prostate cancer; PDT = photodynamic therapy; PIVOT = Prostate Cancer Intervention Versus Observation Trial; PSA = prostate-specific antigen; RCT = randomized controlled trial; RP = radical prostatectomy; RT = radiation therapy; SBRT = stereotactic body radiation therapy; SOC = standard of care; SPCG-4 = Scandinavian Prostate Cancer Group Study Number 4; WW = watchful waiting

*This table shows findings on mortality, PC-specific mortality, metastases, sexual, urinary, and bowel harms from treatment comparisons analyzed in this current systematic review and any comparisons from the 2014 AHRQ-funded and 2016 AUA-funded systematic reviews with findings above insufficient evidence.

†We interpreted findings from the 2016 AUA-funded report with "Level C" evidence to be equivalent to "insufficient evidence".

$\ddagger$ For select treatment comparisons (WW vs. RP and EBRT plus ADT vs. EBRT), our findings incorporate data/outcomes from the prior reviews (see methods).

$\S$ Findings from the 2014 AHRQ-funded systematic review

**Findings from the 2016 AUA-funded systematic review 


\section{Chapter 4. Watchful Waiting}

\section{Key Messages}

- Watchful waiting (WW) versus radical prostatectomy (RP) in men with predominately clinically, rather than prostate specific antigen (PSA) screen-detected clinically localized prostate cancer (CLPC):

o May result in a moderate to large increase in all-cause mortality and a small to large increase in prostate cancer mortality and metastases through 20 years. Absolute effects varied between studies. (Low to moderate certainty of evidence [COE])

o Mortality effects may not vary based on race or PSA levels but may be limited to men with D'Amico intermediate tumor risk and men younger than age 65

0 Probably results in a moderate reduction in erectile dysfunction and urinary incontinence at 10 years versus $\mathrm{RP}$ (moderate $\mathrm{COE}$ ).

- No RCTs evaluated WW among men with CLPC detected by PSA screening alone.

We identified three reports of two unique RCTs ${ }^{20-22}$ and five reports of four unique non$\mathrm{RCTs}^{23-27}$ comparing WW to other therapies. Serious risk of bias (ROB) precluded inclusion of non-RCTs in the analysis. Some comparisons were only evaluated in studies rated high ROB (e.g., WW vs. external beam radiation therapy [EBRT][k=3 non-RCTs], ${ }^{23,}{ }^{25,}{ }^{26} \mathrm{WW}$ vs. radiation therapy [either EBRT and/or brachytherapy][k=1 non-RCT], ${ }^{24,}{ }^{27} \mathrm{WW}$ vs. active surveillance $\left[\mathrm{k}=1\right.$ non-RCT], ${ }^{24,27} \mathrm{WW}$ vs. androgen deprivation therapy [ADT] [k=1 non-RCT]). ${ }^{24,27}$

ROB assessments, population characteristics of the analyzed studies, outcomes data, and detailed GRADE rating tables are in Appendix F. Summary of Findings appears in Table 3.

There were several comparisons of WW to other therapies addressed in the 2016 evidence report commissioned by the American Urological Association (AUA) in which we did not identify additional studies that met analysis criteria. ${ }^{5} \mathrm{~A}$ list of these comparisons can be found in Appendix J. The 2016 evidence report contains detailed results, strength of evidence, and evidence tables for these comparisons. Table 3 summarizes major findings of WW versus other comparisons.

\section{Watchful Waiting Versus Radical Prostatectomy}

We identified two RCTs that compared WW to RP and reported long-term results. ${ }^{20-22}$ The Scandinavian Prostate Cancer Group 4 (SPCG-4) study was conducted in Scandinavia prior to PSA screening, and enrolled men with clinically detected disease. The U.S. Prostate Cancer Intervention Versus Observation Trial (PIVOT) study began during the early period of PSA screening and enrolled approximately 50 percent of men with T1C disease. Because of clinical heterogeneity in the enrolled populations, we did not think pooling of results was appropriate. Instead, we describe and evaluate findings from each study and attempt to note implications for patients with T1C tumors diagnosed primarily through PSA screening. Shorter followup times, development of metastatic disease, and harms have been reported in earlier publications of these trials, and were included in the previous reviews though we note some data here. ${ }^{28-41}$ Both trials enrolled men under 75 with clinical T1 or T2 and life expectancy greater than 10 years. Results 
reported since the previous review had longer followup periods for mortality and metastases and provided additional information about harms.

At nearly 20 years, prostate cancer mortality as well as the absolute overall and prostate cancer mortality differences in both the RP and WW groups were much larger in SPCG-4 versus PIVOT. Overall mortality and distant metastases were higher with WW versus RP in SPCG-4, but not PIVOT, likely reflecting the greater absolute risk of metastases and prostate cancer death in SPCG-4 compared to PIVOT.

Based on combined results from these two studies, WW may increase overall and prostatespecific mortality (low COE) among men whose prostate disease was mostly detected clinically rather than through PSA screening. Based on findings from SPCG-4, WW probably results in a moderate increase in overall mortality at 25 years (moderate COE) and a large increase in prostate-specific mortality (moderate $\mathrm{COE}$ ). While the relative effects were similar between the two studies, the absolute effect varied considerably: 12 percentage points in SPCG-4 and 4 percentage points in PIVOT for overall mortality. Based on SPCG-4, at both 20 and 25 years, WW probably resulted in a large increase in metastases (moderate COE). Based on findings from PIVOT, WW may result in a small increase in metastatic disease, defined as systemic progression (low COE). The prior systematic reviews noted that in PIVOT at 10 years followup, incidences of bone metastases were much less frequent overall compared to SPCG-4 but still lower in the RP group (4.7\%) compared with WW (10.6\%) with an absolute difference of approximately 6 percentage points. ${ }^{4,5}$ No RCTs have assessed WW versus radiation therapy or other interventions among men whose disease was detected solely through PSA screening.

In the PIVOT trial, WW probably resulted in moderately lower erectile dysfunction (defined as unable to have an erection or able to have an erection but the erection was not sufficient for vaginal penetration) and urinary incontinence (defined as $>1$ pad use per day) versus RP at 2, 5 and 10 years (moderate COE) (personal communication from the study lead author). Satisfaction with sexual functioning was moderately lower with RP vs. WW at two, five and 10 years. While sexual function was low in both groups at 10 years more men in the RP group reported poor sexual functioning compared with men in the WW group at two, five and 10 years. There may be a small increase bowel dysfunction with WW, defined as patient reported dysfunction as a "moderate" or "big” problem, versus RP at 10 years (low COE) (personal communication from the study lead author). No harms data were reported for SPCG-4 trial at the longest-term followup. Prior reviews reported that at 8-year followup, men allocated to WW regularly reported less erection dysfunction and urinary leakage than men allocated to RP. ${ }^{4,5}$ Quality of life data has been previously reported but indicates that WW does not result in worse quality of life.

\section{Variation in Outcomes by Participant or Tumor Characteristics}

Outcomes specific to several subgroups were analyzed. Both trials analyzed subgroups defined by age and tumor characteristics at nearly 20 years followup. ${ }^{20-22}$ Wilt et al. also analyzed race as a potential effect modifier. ${ }^{22}$

Overall mortality was higher with WW than RP in men younger than 65; this difference was not significant in men 65 and older. Race did not modify treatment effects based on PIVOT results. Age was an important effect modifier for prostate-cancer-specific mortality on SPCG-4, but not PIVOT. SPCG-4 analyzed the effect of age on distant metastases. Distant metastases were higher with WW versus RP in both age groups.

Two tumor characteristics, namely PSA and D’Amico classified prostate cancer risk category, were analyzed for effect modification. The effect of treatment on overall mortality or 
prostate-specific mortality did not vary by PSA level ( $<10$ vs. $>=10 \mathrm{ng} / \mathrm{mL}$ ) in PIVOT. Both trials found that D'Amico tumor risk category modified the effect of treatment. In both trials at 20 years followup, WW versus RP was associated with higher mortality among men at intermediate risk but not low- or high-risk disease. 
Table 3. Certainty of evidence: watchful waiting

\begin{tabular}{|c|c|c|c|c|c|c|c|}
\hline Comparison & $\begin{array}{l}\text { Outcome } \\
\text { № of Participants } \\
\text { (studies) }\end{array}$ & $\begin{array}{l}\text { Relative Effect } \\
(95 \% \mathrm{Cl})\end{array}$ & $\begin{array}{l}\text { Absolute } \\
\text { Effects } \\
\text { WW }\end{array}$ & $\begin{array}{l}\text { Absolute } \\
\text { Effects } \\
\text { Comparator }\end{array}$ & $\begin{array}{l}\text { Absolute Effects } \\
\text { Difference } \\
(95 \% \mathrm{Cl})\end{array}$ & $\begin{array}{l}\text { Certainty of } \\
\text { Evidence: }\end{array}$ & What Happens \\
\hline \multirow[t]{9}{*}{$\begin{array}{l}\text { WW versus } \\
\text { RP }^{20-22}\end{array}$} & $\begin{array}{l}\text { All-cause mortality } \\
\sim 20 \text {-years followup } \\
2 \text { RCTs }(n=1426)\end{array}$ & $\begin{array}{l}\text { SPCG-4: } \\
\text { RR } 1.23 \\
(1.10 \text { to } 1.38)\end{array}$ & $\begin{array}{l}70.9 \% \\
(247 / 348)\end{array}$ & $\begin{array}{l}57.6 \% \\
(200 / 347)\end{array}$ & $\begin{array}{l}13.3 \% \\
\text { (6.3 to } 20.4)\end{array}$ & $\begin{array}{l}\oplus \oplus \bigcirc \bigcirc \\
\mathrm{LOW} a, b\end{array}$ & $\begin{array}{l}\text { WW may result in a moderate to } \\
\text { large increase in all-cause } \\
\text { mortality versus RP }\end{array}$ \\
\hline & & $\begin{array}{l}\text { PIVOT: } \\
\text { RR } 1.09 \\
\text { (0.98 to1.22) }\end{array}$ & $\begin{array}{l}66.7 \% \\
(245 / 367)\end{array}$ & $\begin{array}{l}61.3 \% \\
(223 / 364)\end{array}$ & $\begin{array}{l}5.5 \% \\
(-1.45 \text { to } 12.4)\end{array}$ & & \\
\hline & $\begin{array}{l}\text { All-cause mortality } \\
\sim 25 \text {-years followup } \\
1 \text { RCT ( } n=695)\end{array}$ & $\begin{array}{l}\text { RR } 1.12 \\
(1.03 \text { to1.2) }\end{array}$ & $\begin{array}{l}83.9 \% \\
(292 / 348)\end{array}$ & $\begin{array}{l}75.2 \% \\
(261 / 347)\end{array}$ & $\begin{array}{l}8.7 \\
(2.7 \text { to14.6) }\end{array}$ & $\begin{array}{l}\oplus \oplus \oplus \bigcirc \\
\text { MODERATE } b\end{array}$ & $\begin{array}{l}\text { WW probably results in a moderate } \\
\text { increase in all-cause mortality } \\
\text { versus RP }\end{array}$ \\
\hline & $\begin{array}{l}\text { PC-specific mortality } \\
\sim 20 \text {-years followup } \\
2 \text { RCT ( } n=1426)\end{array}$ & $\begin{array}{l}\text { SPCG-4: } \\
\text { RR } 1.57 \\
\text { (1.19 to } 2.07)\end{array}$ & $\begin{array}{l}28.4 \% \\
(99 / 348)\end{array}$ & $\begin{array}{l}18.1 \% \\
(63 / 347)\end{array}$ & $\begin{array}{l}10.3 \% \\
(4.05 \text { to } 16.5)\end{array}$ & $\begin{array}{l}\oplus \oplus \bigcirc \bigcirc \\
\mathrm{LOW} a, b\end{array}$ & $\begin{array}{l}\text { WW may result in a small to large } \\
\text { increase in PC-specific mortality } \\
\text { versus RP }\end{array}$ \\
\hline & & $\begin{array}{l}\text { PIVOT: } \\
\text { RR } 1.54 \\
(0.97 \text { to } 2.45)\end{array}$ & $\begin{array}{l}11.4 \% \\
(42 / 367)\end{array}$ & $\begin{array}{l}7.4 \% \\
(27 / 364)\end{array}$ & $\begin{array}{l}4.0 \% \\
(-0.19 \text { to } 8.25)\end{array}$ & & \\
\hline & $\begin{array}{l}\text { PC-specific mortality } \\
\sim 25-y e a r s \text { followup } \\
1 \text { RCT ( } n=695)\end{array}$ & $\begin{array}{l}\text { RR } 1.54 \\
\text { (1.19 to } 2.00)\end{array}$ & $\begin{array}{l}31.6 \% \\
(110 / 348)\end{array}$ & $\begin{array}{l}20.5 \% \\
(71 / 347)\end{array}$ & $\begin{array}{l}11.1 \% \\
(4.7 \text { to } 17.6)\end{array}$ & $\begin{array}{l}\oplus \oplus \oplus \bigcirc \\
\text { MODERATE }\end{array}$ & $\begin{array}{l}\text { WW probably results in a large } \\
\text { increase in PC-specific mortality } \\
\text { versus RP }\end{array}$ \\
\hline & $\begin{array}{l}\text { Metastases } \\
\sim 20 \text {-years followup } \\
1 \text { RCT }(n=695)\end{array}$ & $\begin{array}{l}\text { RR } 1.54 \\
\text { (1.24 to1.93) }\end{array}$ & $\begin{array}{l}39.7 \% \\
(138 / 348)\end{array}$ & $\begin{array}{l}25.6 \% \\
(89 / 347)\end{array}$ & $\begin{array}{l}14 \% \\
\text { (7.1 to } 20.9)\end{array}$ & $\begin{array}{l}\oplus \oplus \oplus \bigcirc \\
\text { MODERATE } b\end{array}$ & $\begin{array}{l}\text { WW probably results in a large } \\
\text { increase in metastases versus RP }\end{array}$ \\
\hline & $\begin{array}{l}\text { Metastases } \\
\sim 25 \text {-years followup } \\
1 \text { RCT }(n=695)\end{array}$ & $\begin{array}{l}\text { RR } 1.63 \\
(1.3 \text { to } 2.00)\end{array}$ & $\begin{array}{l}43.1 \% \\
(150 / 348)\end{array}$ & $\begin{array}{l}26.5 \% \\
(92 / 347)\end{array}$ & $\begin{array}{l}16.6 \% \\
(9.6 \text { to } 23.6)\end{array}$ & $\begin{array}{l}\oplus \oplus \oplus \bigcirc \\
\text { MODERATE }\end{array}$ & $\begin{array}{l}\text { WW probably results in a large } \\
\text { increase in metastases versus RP }\end{array}$ \\
\hline & $\begin{array}{l}\text { Metastases (Systemic } \\
\text { progression) } \\
\sim 20 \text {-years followup } \\
1 \text { RCT }(n=731)\end{array}$ & $\begin{array}{l}\text { RR } 1.45 \\
(0.98 \text { to } 2.14)\end{array}$ & $\begin{array}{l}14.7 \% \\
(54 / 367)\end{array}$ & $\begin{array}{l}10.2 \% \\
(37 / 364)\end{array}$ & $\begin{array}{l}4.5 \% \\
(-0.3 \text { to } 9.4)\end{array}$ & $\begin{array}{l}\oplus \oplus \bigcirc \bigcirc \\
\text { LOW }\end{array}$ & $\begin{array}{l}\text { WW may result in a small increase } \\
\text { in metastases (systemic } \\
\text { progression) versus RP }\end{array}$ \\
\hline
\end{tabular}




\begin{tabular}{|c|c|c|c|c|c|c|c|}
\hline Comparison & $\begin{array}{l}\text { Outcome } \\
\text { № of Participants } \\
\text { (studies) }\end{array}$ & $\begin{array}{l}\text { Relative Effect } \\
(95 \% \mathrm{Cl})\end{array}$ & $\begin{array}{l}\text { Absolute } \\
\text { Effects } \\
\text { WW }\end{array}$ & $\begin{array}{l}\text { Absolute } \\
\text { Effects } \\
\text { Comparator }\end{array}$ & $\begin{array}{l}\text { Absolute Effects } \\
\text { Difference } \\
(95 \% \mathrm{Cl})\end{array}$ & $\begin{array}{l}\text { Certainty of } \\
\text { Evidence: }\end{array}$ & What Happens \\
\hline & $\begin{array}{l}\text { Erectile dysfunction } \\
10 \text { years followup } \\
1 \text { RCT }(n=293)\end{array}$ & $\begin{array}{l}\text { RR } 0.82 \\
(0.72 \text { to } 0.93)\end{array}$ & $\begin{array}{l}69.9 \% \\
(102 / 146)\end{array}$ & $\begin{array}{l}85.0 \% \\
(125 / 147)\end{array}$ & $\begin{array}{l}-15.2 \% \\
(-24.6 \text { to }-5.8)\end{array}$ & $\begin{array}{l}\oplus \oplus \oplus \bigcirc \\
\text { MODERATE } b\end{array}$ & $\begin{array}{l}\text { WW probably results in a moderate } \\
\text { reduction of erectile dysfunction } \\
\text { versus RP }\end{array}$ \\
\hline & $\begin{array}{l}\text { Urinary incontinence (>1 pad } \\
\text { per day) } \\
\text { 10-years followup } \\
1 \text { RCT }(n=295)\end{array}$ & $\begin{array}{l}\text { RR } 0.25 \\
(0.12 \text { to } 0.53)\end{array}$ & $\begin{array}{l}5.4 \% \\
(8 / 147)\end{array}$ & $\begin{array}{l}21.6 \% \\
(32 / 148)\end{array}$ & $\begin{array}{l}-16.2 \% \\
(-23.8 \text { to }-8.6)\end{array}$ & $\begin{array}{l}\oplus \oplus \oplus \bigcirc \\
\text { MODERATE } b\end{array}$ & $\begin{array}{l}\text { WW probably results in a moderate } \\
\text { reduction in urinary incontinence } \\
\text { versus RP }\end{array}$ \\
\hline & $\begin{array}{l}\text { Bowel dysfunction } \\
\text { 10-years followup } \\
1 \text { RCT }(n=299)\end{array}$ & $\begin{array}{l}\text { RR } 1.21 \\
(0.77 \text { to } 1.88)\end{array}$ & $\begin{array}{l}22.7 \% \\
(34 / 150)\end{array}$ & $\begin{array}{l}18.8 \% \\
(28 / 149)\end{array}$ & $\begin{array}{l}3.9 \% \\
(-5.3 \text { to } 13.1)\end{array}$ & $\begin{array}{l}\oplus \oplus \bigcirc \bigcirc \\
\text { LOW }^{c}\end{array}$ & $\begin{array}{l}\text { WW may result in a small increase } \\
\text { in bowel dysfunction versus RP }\end{array}$ \\
\hline
\end{tabular}

Abbreviations: $\mathrm{CI}$ = confidence interval; $\mathrm{n}$ = sample size; $\mathrm{PC}=$ prostate cancer; PIVOT = Prostate Cancer Intervention Versus Observation Trail; RCT = randomized controlled trial;

$\mathrm{RP}$ = radical prostatectomy; RR = relative risk; SPCG-4 = Scandinavian Prostate Cancer Group Study Number 4; WW = watchful waiting

\section{GRADE Working Group grades of evidence}

High certainty: We are very confident that the true effect lies close to that of the estimate of the effect

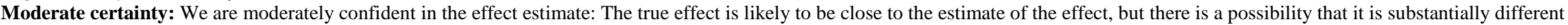

Low certainty: Our confidence in the effect estimate is limited: The true effect may be substantially different from the estimate of the effect

Insufficient: We have very little confidence in the effect estimate: The true effect is likely to be substantially different from the estimate of effect

Explanations
a. Rated down one level for inconsistency
b. Rated down one level for imprecision
c. Rated down two levels for imprecision




\section{Chapter 5. Active Surveillance/Active Monitoring}

\section{Key Messages}

- Prostate specific antigen (PSA) -based Active Monitoring (AM) versus external beam radiation therapy (EBRT) plus androgen deprivation therapy (ADT) in men with PSA detected clinically localized prostate cancer (CLPC):

o There may be little to no difference in all-cause mortality (moderate [certainty of evidence] COE) or prostate-specific mortality (low COE) over 10 years with AM versus EBRT plus ADT among men with prostate cancer detected by PSA screening. Metastases were infrequent, but probably slightly higher with AM (moderate COE).

o Results may not vary by age, PSA level, tumor stage or Gleason score

o No studies evaluated biopsy based active surveillance/active monitoring (AS/AM) versus radical prostatectomy (RP) or EBRT.

o Urinary incontinence was higher with AM than with EBRT+ADT.

- AM versus RP over 10 years in men with PSA detected CLPC:

0 There was little to no difference in all-cause (moderate COE) and prostate-cancerspecific mortality (low COE). Metastases were infrequent but slightly increased with AM (moderate COE)

o Erectile dysfunction (ED) and urinary incontinence were moderately lower with AM versus RP over 6 years (moderate COE)

- Biopsy and PSA-based AS versus Photodynamic Therapy (PDT) over 2 years

o Data are insufficient to assess the effect of biopsy-based AS versus PDT on all-cause or prostate cancer specific mortality or metastasis in men with low-risk disease.

o Urinary retention was moderately lower, and hematuria was largely lower with AS than with PDT among men with low risk disease (moderate COE). AS probably results in a large reduction in ED and a moderate reduction in perineal pain with AS versus PTD (moderate COE).

We identified six reports of two unique randomized controlled trials (RCTs) ${ }^{12,42-46}$ and nine reports of four unique non-RCTs ${ }^{24,27,47-53}$ that compared AS/AM to other therapies. Serious or critical risk of bias (ROB) precluded the inclusion of non-RCTs in the analysis. Some comparisons were only evaluated in studies rated high ROB (e.g., AS vs. watchful waiting $[\mathrm{WW}][\mathrm{k}=1$ non-RCT $],{ }^{24,27}$ AS vs. EBRT $[\mathrm{k}=1$ non-RCT $],{ }^{52}$ AS vs. brachytherapy $[\mathrm{k}=1$ nonRCT],$^{52}$ AS vs. radiation therapy [either EBRT and/or brachytherapy][k=1 non-RCT], ${ }^{24,27}$ and AS vs. ADT[k=1 non-RCT]. ${ }^{24,27}$

ROB assessments, population characteristics of the analyzed studies, outcomes data, and detailed GRADE rating tables are in Appendix G. Summary of Findings appears in Table 4. Information on AS/AM versus radical prostatectomy can be found in Chapter 7.

There were several comparisons of AS/AM to other therapies addressed in the 2016 evidence report commissioned by the American Urological Association (AUA) in which we did not identify any additional studies that met our analysis criteria. ${ }^{5} \mathrm{~A}$ list of these comparisons can be found in Appendix J. The 2016 evidence report and appendices contain detailed results, strength of evidence, and evidence tables for these comparisons. Table 2 summarizes major findings of AS/AM versus other comparisons. 


\section{Active Monitoring Versus External Beam Radiation Therapy Plus Androgen Deprivation Therapy}

Four reports of one eligible RCT (ProtecT) compared PSA-based AM to EBRT plus ADT or to RP and reported results for survival, metastases, quality of life, or harms. ${ }^{42-44,46}$ Men with PSA-screen-detected T1c-T2 CLPC were randomized to PSA-based monitoring (which were included under the intervention category of active surveillance) AM ( $n=545), R P(n=553)$ or EBRT plus ADT ( $n=545)$. Most men had a Gleason score of $6(77 \%)$, followed by scores of 7 (21\%) and 8-10 (2\%). Eighty-eight percent of men allocated to AM, 71 percent to RP and 74 percent to EBRT received the assigned treatment within 9 months after randomization.

Participants assigned to AM had serum PSA levels measured every 3 months in the first year and every 6 to 12 months thereafter. A 50 percent or greater increase in PSA level initiated a review. Following review, participants could continue AM, undergo further testing (including rebiopsy), or receive radical or palliative interventions as needed. At the 10-year followup, 53 percent $(n=291)$ of men assigned to AM had received radical treatment (surgery 49\%, perprotocol EBRT 33\%, 8\% brachytherapy, 9\% non-protocol EBRT, 1\% high intensity focused ultrasound). Participants assigned to EBRT received 74 Gy in 37 fractions with neoadjuvant androgen suppression (ADT) given for 3 to 6 months before and concomitantly. ${ }^{44}$ Median age was 62 years and the majority were white (98\%). ${ }^{44}$ ProtecT was conducted in the UK, was nonindustry funded, and rated low risk of bias. Prior reviews included no randomized trials that directly compared AS/AM or PSA plus biopsy-based AS/AM to EBRT. No studies enrolled patients based on evaluation, monitoring, or targeted biopsies with MRI.

There probably was little to no difference in all-cause (moderate COE) and prostate-cancerspecific mortality (low COE) with AM versus EBRT plus ADT at 10 -years (Table 4). ${ }^{43}$ Deaths attributable to prostate cancer were few; 8 (1.5\%) and 4 (0.7\%) in the AM and EBRT plus ADT groups, respectively.

Metastases were infrequent but probably slightly higher with AM than with EBRT plus ADT over 10 years (33 (6.0\%) versus 16 (2.9\%)) (moderate COE) (Table 4$){ }^{43}$ There were no differences in the Medical Outcomes Study 12-Item Short-Form General Health Survey (SF-12) physical and mental health subscales and the European Organization for Research and Treatment of Cancer Quality-of-Life Questionnaire-Core 30 module (EORTC QLQ-C30) with AM versus EBRT plus ADT at 12 and 72 month followups. ${ }^{42}$

Erectile dysfunction was slightly lower with AM versus EBRT plus ADT (low COE). ${ }^{42}$ However, urinary incontinence was higher with AM than with EBRT plus ADT. At 72 months, urinary incontinence (defined as any use of absorbent pads) was reported by 38 of 453 (8.4\%) men randomized to AM versus 16 of 452 (3.5\%) randomized to EBRT plus ADT (moderate $\mathrm{COE}$ ). Fecal incontinence at least one time per week was reported for $2.6 \%$ with AM versus 4.1\% with EBRT plus ADT group at 72 months. The interventions differed little for the outcome of fecal incontinence (low COE).

\section{Variation in Outcomes by Participant or Tumor Characteristics}

Pre-specified subgroup analyses found no differences between groups in prostate-cancerspecific mortality when stratified by age, PSA level, Gleason score, or clinical stage, though few events occurred. 


\section{Active Surveillance Versus Photodynamic Therapy}

One multicenter RCT compared biopsy and PSA-based AS versus PDT in men with low but not very low-risk disease. ${ }^{12}$ Azzouzi et al. enrolled men $(n=413)$ with low-risk T1a through T2a CLPC with up to 24 months followup. Men were eligible if one core of cancer that was free of Gleason patterns 4 or 5 was present, provided that the cancer core length was between $3 \mathrm{~mm}$ and $5 \mathrm{~mm}$. Eighty-six percent of men had T1c tumors, more than three quarters had unilateral prostate cancer; the baseline PSA was approximately $6 \mathrm{ng} / \mathrm{mL}$. The mean age of enrollees was 63 years. AS included protocol-directed prostate biopsies at 12-month intervals and PSA measurements every 3 months. Photodynamic therapy involved a dedicated magnetic resonance imaging (MRI), intravenous padeliporfin, and transurethral administration of laser light. The coprimary study outcomes were "treatment failure (defined by biopsy determined histological progression of cancer from low to moderate or high risk or death) and absence of definite cancer for 24 months). We did not extract data on mortality or metastases because of the short followup. No eligible non-RCTs or studies in previous reports addressed this comparison.

Certainty was very low for both urinary incontinence and erectile function (Table 4) assessed with the International Index of Erectile Function (IIEF-5) scale (insufficient COE). Urinary retention was probably moderately lower, and hematuria was largely lower with AS (moderate $\mathrm{COE}$ ). AS probably results in a large reduction in erectile dysfunction (ED) and a moderate reduction in perineal pain versus PDT (moderate COE). 
Table 4. Certainty of evidence: active monitoring and active surveillance

\begin{tabular}{|c|c|c|c|c|c|c|c|}
\hline Comparison & $\begin{array}{l}\text { Outcome } \\
\text { № of Participants } \\
\text { (studies) }\end{array}$ & $\begin{array}{l}\text { Relative Effect } \\
(95 \% \mathrm{Cl})\end{array}$ & $\begin{array}{l}\text { Absolute } \\
\text { Effects } \\
\text { ASIAM }\end{array}$ & $\begin{array}{l}\text { Absolute } \\
\text { Effects } \\
\text { Comparator }\end{array}$ & $\begin{array}{l}\text { Absolute } \\
\text { Effects } \\
\text { Difference } \\
(95 \% \mathrm{Cl})\end{array}$ & $\begin{array}{l}\text { Certainty of } \\
\text { Evidence: }\end{array}$ & What Happens \\
\hline \multirow{6}{*}{$\begin{array}{l}\text { PSA-based } \\
\text { AM versus } \\
\text { EBRT + } \\
\text { ADT }^{42-44}\end{array}$} & $\begin{array}{l}\text { All-cause mortality } \\
10 \text { years followup } \\
1 \text { RCT }(n=1090)\end{array}$ & $\begin{array}{l}\text { RR } 1.07 \\
\text { (0.8 to } 1.5)\end{array}$ & $\begin{array}{l}10.8 \% \\
(59 / 545)\end{array}$ & $\begin{array}{l}10.1 \% \\
(55 / 545)\end{array}$ & $\begin{array}{l}0.7 \% \\
(-2.9 \text { to } 4.4)\end{array}$ & $\begin{array}{l}\oplus \oplus \oplus \bigcirc \\
\text { MODERATE a }\end{array}$ & $\begin{array}{l}\text { AM probably results in little to no } \\
\text { difference in all-cause mortality versus } \\
\text { EBRT + ADT }\end{array}$ \\
\hline & $\begin{array}{l}\text { PC-specific mortality } \\
10 \text { years followup } \\
1 \text { RCT }(n=1090)\end{array}$ & $\begin{array}{l}\text { Peto OR } 1.96 \\
(0.63 \text { to } 6.12)\end{array}$ & $\begin{array}{l}1.5 \% \\
(8 / 545)\end{array}$ & $\begin{array}{l}0.7 \% \\
(4 / 545)\end{array}$ & $\begin{array}{l}0.7 \% \\
(-0.5 \text { to } 1.9)\end{array}$ & $\begin{array}{l}\oplus \oplus \bigcirc \bigcirc \\
\text { LOW }^{\mathrm{b}}\end{array}$ & $\begin{array}{l}\text { AM may result in little to no difference in } \\
\text { PC-specific mortality versus EBRT + } \\
\text { ADT }\end{array}$ \\
\hline & $\begin{array}{l}\text { Metastases } \\
10 \text { years followup } \\
1 \text { RCT ( } n=1090)\end{array}$ & $\begin{array}{l}\text { RR } 2.1 \\
\text { (1.15 to } 3.7)\end{array}$ & $\begin{array}{l}6.0 \% \\
(33 / 545)\end{array}$ & $\begin{array}{l}2.9 \% \\
(16 / 545)\end{array}$ & $\begin{array}{l}3.1 \% \\
(0.67 \text { to } 5.6)\end{array}$ & $\begin{array}{l}\oplus \oplus \oplus \bigcirc \\
\text { MODERATE }^{a}\end{array}$ & $\begin{array}{l}\text { AM probably results in a small increase } \\
\text { of metastases versus EBRT + ADT }\end{array}$ \\
\hline & $\begin{array}{l}\text { Erectile dysfunction } \\
6 \text { years followup } \\
1 \text { RCT ( } n=908)\end{array}$ & $\begin{array}{l}\text { RR } 0.97 \\
(0.89 \text { to } 1.05)\end{array}$ & $\begin{array}{l}70.4 \% \\
(318 / 452)\end{array}$ & $\begin{array}{l}72.6 \% \\
(331 / 456)\end{array}$ & $\begin{array}{l}-2.4 \% \\
(-8.2 \text { to } 3.5)\end{array}$ & $\begin{array}{l}\oplus \oplus \bigcirc \bigcirc \\
\text { LOW }^{\mathrm{b}}\end{array}$ & $\begin{array}{l}\text { AM may result in a small decrease in } \\
\text { erectile dysfunction versus EBRT + } \\
\text { ADT }\end{array}$ \\
\hline & $\begin{array}{l}\text { Urinary incontinence } \\
6 \text { years followup } \\
1 \text { RCT }(n=903)\end{array}$ & $\begin{array}{l}\text { RR } 2.37 \\
\text { (1.34 to } 4.19)\end{array}$ & $\begin{array}{l}8.4 \% \\
(38 / 453)\end{array}$ & $\begin{array}{l}3.5 \% \\
(16 / 452)\end{array}$ & $\begin{array}{l}4.8 \% \\
(1.8 \text { to } 7.9)\end{array}$ & $\begin{array}{l}\oplus \oplus \oplus \bigcirc \\
\text { MODERATE }^{a}\end{array}$ & $\begin{array}{l}\text { AM probably results in a small increase } \\
\text { in urinary incontinence versus EBRT + } \\
\text { ADT }\end{array}$ \\
\hline & $\begin{array}{l}\text { Fecal incontinence } \\
6 \text { years followup } \\
1 \text { RCT }(n=927)\end{array}$ & $\begin{array}{l}\text { RR } 0.64 \\
\text { (0.3 to } 1.3 \text { ) }\end{array}$ & $\begin{array}{l}2.6 \% \\
(12 / 462)\end{array}$ & $\begin{array}{l}4.1 \% \\
(19 / 465)\end{array}$ & $\begin{array}{l}-1.5 \% \\
(-3.8 \text { to } 0.82)\end{array}$ & $\begin{array}{l}\oplus \oplus \bigcirc \bigcirc \\
\text { LOW }^{\mathrm{b}}\end{array}$ & $\begin{array}{l}\text { AM may result in little to no difference in } \\
\text { fecal incontinence versus EBRT + ADT }\end{array}$ \\
\hline \multirow[t]{3}{*}{$\begin{array}{l}\text { AS versus } \\
\text { PDT }^{12}\end{array}$} & $\begin{array}{l}\text { Erectile dysfunction } \\
24 \text {-month followup } \\
1 \text { RCT }(n=404)\end{array}$ & $\begin{array}{l}\text { RR } 0.31 \\
(0.2 \text { to } 0.5)\end{array}$ & $\begin{array}{l}11.6 \% \\
(24 / 207)\end{array}$ & $\begin{array}{l}37.6 \% \\
(74 / 197)\end{array}$ & $\begin{array}{l}-26 \% \\
(-34 \text { to }-18)\end{array}$ & $\begin{array}{l}\oplus \oplus \oplus \bigcirc \\
\text { MODERATE }\end{array}$ & $\begin{array}{l}\text { AS probably results in a large decrease } \\
\text { in erectile dysfunction versus PDT }\end{array}$ \\
\hline & $\begin{array}{l}\text { Urinary incontinence } \\
24 \text { months followup } \\
1 \text { RCT }(n=404)\end{array}$ & $\begin{array}{l}\text { RR } 0.5 \\
(0.24 \text { to } 1.05)\end{array}$ & $\begin{array}{l}4.8 \% \\
(10 / 207)\end{array}$ & $\begin{array}{l}9.6 \% \\
(19 / 197)\end{array}$ & $\begin{array}{l}-4.8 \% \\
(-9.9 \text { to } 2.4)\end{array}$ & $\begin{array}{l}\oplus \bigcirc \bigcirc \bigcirc \\
\text { INSUFFICIENT b, c }\end{array}$ & $\begin{array}{l}\text { The evidence is very uncertain about } \\
\text { the effect of AS on urinary incontinence } \\
\text { versus PDT }\end{array}$ \\
\hline & $\begin{array}{l}\text { Urinary retention } \\
24 \text { months followup } \\
1 \text { RCT }(n=404)\end{array}$ & $\begin{array}{l}\text { RR } 0.06 \\
(0.01 \text { to } 0.24)\end{array}$ & $\begin{array}{l}1.0 \% \\
(2 / 207)\end{array}$ & $\begin{array}{l}16.2 \% \\
(32 / 197)\end{array}$ & $\begin{array}{l}-15.3 \% \\
(-20.6 \text { to }-10)\end{array}$ & $\begin{array}{l}\oplus \oplus \oplus \bigcirc \\
\text { MODERATE }^{c}\end{array}$ & $\begin{array}{l}\text { AS probably results in a moderate } \\
\text { reduction of urinary retention versus } \\
\text { PDT }\end{array}$ \\
\hline
\end{tabular}

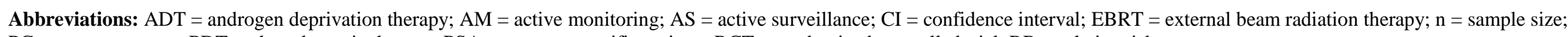
$\mathrm{PC}=$ prostate cancer; $\mathrm{PDT}=$ photodynamic therapy; $\mathrm{PSA}=$ prostate-specific antigen; $\mathrm{RCT}=$ randomized controlled trial; $\mathrm{RR}=$ relative risk 


\begin{tabular}{|c|c|c|c|c|c|c|c|}
\hline Com & $\begin{array}{l}\text { Outcome } \\
\text { № of Participants } \\
\text { (studies) }\end{array}$ & $\begin{array}{l}\text { Relative Effect } \\
(95 \% \mathrm{Cl})\end{array}$ & $\begin{array}{l}\text { Absolute } \\
\text { Effects } \\
\text { ASIAM }\end{array}$ & $\begin{array}{l}\text { Absolute } \\
\text { Effects } \\
\text { Comparator }\end{array}$ & $\begin{array}{l}\text { Absolute } \\
\text { Effects } \\
\text { Difference } \\
(95 \% \mathrm{Cl})\end{array}$ & $\begin{array}{l}\text { Certainty of } \\
\text { Evidence: }\end{array}$ & What Happens \\
\hline
\end{tabular}

GRADE Working Group grades of evidence

High certainty: We are very confident that the true effect lies close to that of the estimate of the effect

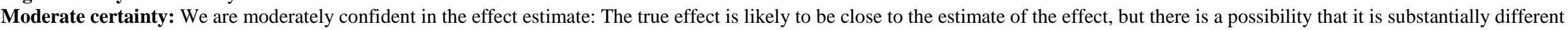

Low certainty: Our confidence in the effect estimate is limited: The true effect may be substantially different from the estimate of the effect

Insufficient: We have very little confidence in the effect estimate: The true effect is likely to be substantially different from the estimate of effect
Explanations
a. Rated down one level for imprecision
b. Rated down two levels for imprecision
c. Rated down one level for risk of bias 


\section{Chapter 6. Whole Gland Therapies- External Beam Radiation Therapy}

\section{Key Messages}

- 3D-conformal radiation therapy (3D-CRT) and androgen deprivation therapy (ADT) plus low-dose-rate prostate brachytherapy (LDR-prostate brachytherapy) versus 3D-CRT and ADT in men with predominately high National Comprehensive Cancer Network (NCCN) classified risk disease over 5 years:

o may provide a small reduction in all-cause mortality (low certainty of evidence [COE])

o may make little to no difference on metastatic disease (low COE).

- External beam radiation therapy (EBRT) plus ADT versus EBRT alone in men with predominantly intermediate or high-risk disease:

o Probably results in a small reduction in overall mortality over 6 to 9 years (moderate COE)

o May result in a small reduction in prostate cancer specific mortality over 7 to 9 years (low COE)

o May result in a small reduction in metastasis over 5 to 10 years (low COE)

o May result in a moderate increase in sexual impairment over 7 years (low COE)

o Appears to vary by patient comorbidities for overall mortality.

- EBRT plus neoadjuvant and concurrent ADT versus EBRT plus concurrent and adjuvant ADT in men with predominantly intermediate-risk disease:

o May result in little to no difference in overall mortality over 12 years (low COE)

o May result in little to no difference in prostate cancer specific mortality over 12 years (low COE)

o May result in little to no difference in late genitourinary toxicity grade $\geq 3$ over 3 years (low COE).

- Conventionally fractionated EBRT versus ultra-hypofractionated EBRT in men with predominantly intermediate risk disease:

o Probably results in little to no difference in overall mortality over 5 years (moderate COE)

o May result in little to no difference in prostate cancer specific mortality over 5 years (low COE)

o May result in little to no difference in metastasis over 5 years (low COE)

0 May result in a small reduction in urinary toxicity grade $\geq 2$ at 1 year, but little to no difference at 2 years (low COE)

0 May result in little to no difference in bowel toxicity grade $\geq 2$ at 2 years (low COE)

We identified 12 randomized controlled trials (RCTs) and 18 observational studies comparing EBRT to other therapies or different types of EBRT. ${ }^{24,27,42,43,45,52,54-84}$ Among the RCTs, one compared 3D-CRT versus intensity-modulated radiation therapy (IMRT). ${ }^{70}$ One RCT compared 3D-CRT and ADT versus 3D-CRT and ADT plus low-dose-rate (LDR) prostate brachytherapy boost. ${ }^{61,64,65}$ Four RCTs (6 publications) compared EBRT plus ADT versus EBRT alone. ${ }^{69,}$ 74, 76, 78-80 One RCT compared EBRT plus ADT versus ADT alone. ${ }^{75}$ Two RCTs 
compared ultra-hypofractionated EBRT versus standard fractionations. ${ }^{68,82}$ One RCT compared EBRT plus neoadjuvant and concurrent ADT versus EBRT plus concurrent and adjuvant ADT. ${ }^{81}$ Three of the aforementioned RCTs were rated high risk of bias (ROB) and therefore not analyzed. ${ }^{68,69,75}$ Two RCTs involving EBRT are described in other sections of the report. Serious or critical ROB precluded the inclusion of most non-RCTs in the analysis.

ROB assessments, population characteristics of the analyzed studies, outcomes data, and detailed GRADE rating tables are in Appendix H. Summary of Findings appears in Table 5.

Information about AS versus EBRT plus ADT can be found in Chapter 5. Information about RP versus EBRT plus ADT and RP plus ADT versus EBRT plus high-dose brachytherapy plus ADT can be found in Chapter 7.

There were several comparisons of radiation therapy to other therapies addressed in the 2016 evidence report commissioned by the American Urological Association (AUA) in which we did not identify any additional studies that met our analysis criteria published after this report. ${ }^{5} \mathrm{~A}$ list of these comparisons can be found in Appendix J. The 2016 evidence report and appendices contain detailed results, strength of evidence, and evidence tables for these comparisons. Table 2 summarizes major findings of EBRT versus other comparisons.

\section{D-Conformal Radiation Therapy and Androgen Deprivation Therapy Versus 3D-Conformal Radiation Therapy and Androgen Deprivation Therapy Plus Low-Dose-Rate Prostate Brachytherapy}

The Androgen Suppression Combined with Elective Nodal and Dose Escalated Radiation Therapy (ASCENDE-RT) Trial $(\mathrm{n}=398)$ compared 3D-CRT and ADT with 3D-CRT and ADT plus low-dose-rate prostate brachytherapy (LDR-PB) boost. ${ }^{61,64,65}$ The trial compared 46 Gray of dose-escalated EBRT delivered in 23 fractions plus an additional 32 Gray/16 fractions 3dimensional conformal boost versus the same 46 Gray of EBRT plus a low-dose-rate brachytherapy boost using Iodine-125. Approximately two-thirds of patients had high-risk disease; the remainder had intermediate risk disease as per NCCN risk categories. Median followup was 6.5 years. Both arms received 12 months of neoadjuvant ADT initiated 8 months prior to pelvic irradiation. At baseline mean age was 68 years. Race was not reported.

At five years, 3D-CRT and ADT with LDR-PB boost may result in a small reduction in overall mortality versus 3D-CRT and ADT (low COE) (Table 5). 3D-CRT and ADT with LDRPB boost may result in little to no difference in metastatic disease compared to 3D-CRT and ADT (low COE), while effects on prostate cancer specific mortality were very uncertain (insufficient COE). These outcomes were also reported at 7- and 9-year followup, but groups were not statistically compared or reported in enough detail for us to evaluate.

Evidence was very uncertain about the effect of 3D-CRT plus ADT with or without LDR-PB on urinary incontinence or erectile function (both insufficient COE) after 5 years.

Quality of life (QOL) not extracted due to high ROB of the reporting study ${ }^{64}$ 


\section{D-Conformal Radiation Therapy Versus Intensity-Modified Radiation Therapy}

One RCT ( $\mathrm{n}=215)$ compared 3D-CRT with 70 Gray delivered in 25 fractions versus the same target dose and fractions of IMRT. Followup was over 3 years ${ }^{70}$ Mean age was 72 years and 79 percent had clinical stage T1a-T2a tumors. Approximately half of patients had low-risk prostate cancer based on NCCN risk groups, a third had high-risk, and the remainder had intermediate risk disease. Patients with intermediate and high-risk disease received 6 and 24 months of systematic androgen deprivation therapy, respectively. Race was not reported. The only eligible outcome reported was QOL based on the EORTC QLQ-PR25. At 1 year, QOL scores were statistically worse with 3D-CRT versus IMRT for urinary symptoms, bowel symptoms, and treatment-related symptoms, though the clinical significance of these differences is unclear. Neither sexual function nor activity differed between groups. At 3 years, groups did not differ in any QOL domain.

\section{Brachytherapy With External Beam Radiation Therapy Versus Brachytherapy}

No randomized trial evidence informed this question. One observational study used propensity score matching to retrospectively analyze a subset of the National Cancer Database $(n=5,858) .{ }^{55}$ EBRT types were not specified, but EBRT doses ranged from 40 to 50.4 Gy in 1.8$2.0 \mathrm{~Gy}$ fractionations. Brachytherapy doses were not reported. In the brachytherapy group, $34.3 \%$ of patients received ADT and $48.4 \%$ in the combination therapy group received ADT. The duration of ADT was not reported. All patients had intermediate-risk disease per NCCN risk categories. Mean age was 69 years, 61 percent had clinical stage T1, and 83 percent were white. ROB was medium for one analysis which was propensity score matched (overall survival at 7 years). The evidence was very uncertain about the effect of brachytherapy with EBRT on overall survival versus brachytherapy alone (insufficient COE). No effect modifiers were reported for the propensity-score-matched analysis.

\section{Intensity-Modified Radiation Therapy Versus Stereotactic Beam Radiation Therapy}

No randomized trial evidence evaluated these interventions, but we identified a propensityscore-matched observational study that retrospectively analyzed the National Cancer Database $(n=5,430) .{ }^{63}$ and compared overall survival between IMRT versus stereotactic body radiation therapy (SBRT). Subjects were excluded if they received more or less than 72-86.4 Gy IMRT or 35-50 Gy SBRT. Mean age was 69; 87 percent were white. Most men had T1 tumor (80\%), followed by T2 (19\%). The majority had a prostate specific antigen (PSA) level $<10$ (82\%) and a Gleason score of 6 (56\%) or 7 (38\%). Approximately $8 \%$ had a PSA level $>20$ and 5\% and a Gleason score between 8 and 10. The only outcome reported was overall survival at 8 years. The evidence was very uncertain about the effect of IMRT on overall survival versus SBRT (insufficient COE).

\section{Radiation Therapy Versus Androgen Deprivation Therapy}

We identified no RCTs and two references of one non-RCT that evaluated radiation therapy (either EBRT and/or brachytherapy) versus ADT. ${ }^{24,27}$ The non-RCT (Hormonal therapy, Active 
Surveillance, Radiation, Operation, Watchful Waiting Study [HAROW] study) was rated high ROB based on the ROBINS-I tool. The previous 2014 and 2016 systematic reviews included no RCTs and three non-RCTs for this comparison. ${ }^{85-87}$ All three non-RCTs were previously rated low quality.

\section{External Beam Radiation Therapy Plus Androgen Deprivation Therapy Versus External Beam Radiation Therapy}

Seven RCTs compared EBRT plus ADT versus EBRT alone (four RCTs in past reviews and three RCTs newly identified). ${ }^{69,74,76,78 ~ 88-90 ~ 91,92 ~ A m o n g ~ t h e ~ s e v e n ~ t o t a l ~ t r i a l s, ~ o n e ~ w a s ~ r a t e d ~ h i g h ~}$ ROB ${ }^{69}$ The analysis focuses on the remaining six. In one trial, the EBRT examined was IMRT, ${ }^{76}$ two trials predominantly used three-dimensional conformal radiation therapy (3D-CRT), ${ }^{74,91}$ and two trials did not specify EBRT type. ${ }^{88,89}$ The sixth trial allowed different EBRT techniques to be used across trial centers. ${ }^{90}$ The ADT in four trials consisted of an antiandrogen (flutamide or bicalutamide) with a luteinizing hormone-releasing hormone (LHRH) agonist (goserelin or leuprolide $)^{74,88,89,91}$ and two trials used antiandrogen monotherapy with bicalutamide. ${ }^{76,90}$ In five trials, the duration of ADT ranged from 3 to 6 months. The sixth administered ADT for 2 years or until disease progression (maximum 5 years).$^{90}$ Most participants had intermediate-risk disease, high-risk was the next common, and low-risk was least common (defined variably across trials). Tumor stage varied across trials with four only including T1-T2 patients ${ }^{74,76, ~ 89, ~} 92$ and two also enrolling patients with higher tumor stages. Patients were eligible for two trials in part by Gleason $\geq 7$ and a third Gleason 6-8 (three trials specified Gleason in eligibility criteria). ${ }_{74,76,92}$ The median PSA at baseline ranged from $7.6 \mathrm{ng} / \mathrm{mL}$ to 16.4 (five trials reporting). ${ }^{74,76,88,}$ 90, 92 At baseline, mean/median age ranged from 67 to 73 years (all six trials reporting). Only two trials reported race, and most participants were white. ${ }^{89,90}$ The longest mean/median followups ranged from 5.4 to 18.2 years. We also identified one non- $\mathrm{RCT}^{93}$ that reported overall mortality/survival. The 2016 systemic review also included two non-RCTs ${ }^{85}, 94$ that were previously rated low quality.

After 5.9 to 9.1 years, pooled analysis showed EBRT plus ADT versus EBRT alone probably results in a small reduction in overall mortality (moderate COE) ${ }^{74,76,88,89,91}$ The pooled analysis had minimal heterogeneity (relative effect: $\mathrm{I}^{2}=0 \%$; absolute effect: $\mathrm{I}^{2}=20 \%$ ). When stratified by type of EBRT, combination therapy reduced overall mortality with a magnitude ranging from small to large based on two trials of predominantly 3D-CRT plus ADT versus predominantly 3D-CRT alone reporting at 7.2 to 7.6 years (risk difference [RD] $-3.5 \%$ and RD $-12.9 \%$, respectively) ${ }^{74,91}$ Mortality reduction persisted at 16.6 years with combination therapy in one 3D-CRT trial that reported longer followup (RD -2.4\%) ${ }^{78}$ In contrast, a single trial reported a small increase in overall mortality for IMRT plus ADT versus IMRT alone at 9.1 years (RD 4.6\%). ${ }^{76}$ Pooled analysis showed EBRT plus ADT versus EBRT alone may result in a small reduction in prostate cancer specific mortality after 7.2 to 9.1 years (low COE) ${ }^{74,89,91}$ The pooled analysis had minimal to moderate heterogeneity (relative effect: $\mathrm{I}^{2}=0 \%$; absolute effect: $\left.\mathrm{I}^{2}=55 \%\right)$. In the predominant 3D-CRT trials, one found a moderate reduction in prostate cancer mortality with combination therapy at median followup of 7.6 years (RD -9.5\%), with a reduction remaining at 16.6 years ( $\mathrm{RD}-16.2 \%),{ }^{78}$ while another reported little to no difference between predominantly 3D-CRT plus ADT versus predominantly 3D-CRT alone at median follow up of 7.2 years (RD -1.7\%). ${ }^{74}$ The IMRT trial did not report prostate cancer mortality.

After 5 to 10 years, pooled analysis showed EBRT plus ADT versus EBRT alone may result in a small reduction in metastasis (low COE). ${ }^{74,88-90}$. A trial that predominantly used 3D- 
CRT also reported a small magnitude reduction with combination therapy (RD -3.2\%). ${ }^{74}$ For IMRT, distant metastasis was only reported among patients who experienced biochemical relapse and occurred in 51 percent treated with IMRT plus ADT and 68.6 percent with IMRT. ${ }^{76}$

From two trials reporting quality of life, there was generally little to no difference between groups especially at longer followup times, though the results varied by specific scales. McPartlin et al. reported "no marked effect" on the European Organization for Research and Treatment of Cancer (EORTC) quality of life questionnaire for EBRT plus ADT versus EBRT alone. However, no data were reported. ${ }^{76}$ A second trial reported little to no difference between treatment groups in mean change on the global health status/quality of life scale of the EORTC quality of life questionnaire at 1 and 3 years. ${ }^{74}$ EBRT plus ADT versus EBRT alone resulted in significant impairment at 1 year in sexual functioning and sexual activity subscales of the EORTC questionnaire, but by three years, groups differed little to not at all on sexual functioning and sexual activity scales. However, also at 3 years, statistically significant impairment remained on the hormonal symptoms scale for EBRT plus ADT versus EBRT alone.

Sexual function may be worse with EBRT plus ADT versus EBRT alone (reported differently across trials). EBRT plus ADT versus EBRT alone may result in a moderate increase in severe impairment in sexual function, based on toxicity scores measured from 6 months until end of followup (low COE). ${ }^{74}$ From a second trial, the evidence was insufficient on the effect of EBRT plus ADT versus EBRT alone on impotence grades 2 to 4 (insufficient COE). ${ }^{92}$ Evidence was insufficient for adverse effects of EBRT plus ADT versus EBRT alone on urinary incontinence (stress) grades 2 to 4 and rectal bleeding (insufficient COE). ${ }^{92}$ One trial also reported that fewer patients who received EBRT plus ADT versus EBRT alone at 1 year were “always or almost always able to have an erection" assessed by the sexual adjustment questionnaire. ${ }^{89}$ One trial reported little to no difference between groups in hematuria grades 2 to 4, diarrhea, and "complete urinary incontinence". ${ }^{92}$ Two trials reported a small increase in genitourinary late toxicity for EBRT plus ADT versus RT (RD 2.2\% for grades 3 to 4; RD 3.0\% for grades 2 to 3). ${ }^{74,76}$ One trial reported a small decrease in gastrointestinal late toxicity grades 2 to 3 with combination therapy (RD $-2.4 \%){ }^{76}$

\section{Variation in Outcomes by Participant or Tumor Characteristics}

A post hoc analysis by D'Amico and colleagues suggested that the benefit of EBRT plus ADT versus EBRT alone in D'Amico classified intermediate risk disease on mortality may only be in men with no or minimal comorbidity (mortality interaction test, $\mathrm{p}<.001$ at 7.6 years and $\mathrm{p}=.01$ at 16.6 years). ${ }^{78,91}$ While the effect modification on prostate cancer mortality appeared similar, the eligible references reported no test for interaction. Results from a second RCT reporting a post hoc analysis showed possible effect modification by tumor risk level on prostate cancer mortality at 9.1 years (interaction test, $\mathrm{p}=.08$ ). ${ }^{89}$ There were moderate reductions in men with intermediate and high-risk disease with combination treatment versus EBRT alone, but little to no difference in low-risk men. The same RCT reported that the effect on overall survival due to EBRT plus ADT versus EBRT alone did not significantly vary by tumor risk level (interaction test, $\mathrm{p}=.71$ ), between white and black men (interaction test, $\mathrm{p}=0.79$ ) or among men aged $\leq 70$ years and $>70$ years (interaction test, $\mathrm{p}=0.47$ ). Only the race subgroup analysis was pre-specified. 


\section{External Beam Radiation Therapy Plus Androgen Deprivation Therapy Versus Androgen Deprivation Therapy}

We identified one publication of the Scandinavian Prostate Cancer Group-7 (SPCG-7). ${ }^{75}$ The 2016 systematic review included an earlier followup publication of SPCG-7. ${ }^{95}$ That trial randomized mostly men with high-risk disease to total androgen blockade with EBRT versus without EBRT. Based on the prior report, SPCG-7 showed a reduction in 10-year prostate cancer mortality with EBRT plus ADT versus ADT alone in T1b-T2 patients. ${ }^{95}$ At median followup of 13.6 years, there was a suggested benefit with combination treatment on prostate-cancer mortality in the T1-T2 patients and no difference on overall mortality. ${ }^{75}$ We did not extract the data or rate the COE because the previous report rated the trial as low quality. We identified one non-RCT for this comparison. ${ }^{73}$ It was rated serious ROB based on the ROBINS-I tool. The 2016 systematic review included one non-RCT. ${ }^{85}$ They rated it low quality.

\section{External Beam Radiation Therapy Plus Neoadjuvant and Concurrent Androgen Deprivation Therapy Versus External Beam Radiation Therapy Plus Concurrent and Adjuvant Androgen Deprivation Therapy}

One RCT compared EBRT plus neoadjuvant and concurrent ADT versus EBRT plus concurrent and adjuvant ADT and reported mortality, metastases, and harms. ${ }^{81}$ The EBRT approach was image-guided 3D-CRT over 7.5 weeks. Participants assigned to neoadjuvant and concurrent ADT received 6 months ADT starting 4 months before EBRT. Patients in the concurrent and adjuvant ADT treatment group received 6 months ADT starting simultaneously with EBRT. The ADT consisted of an oral antiandrogen (e.g. bicalutamide) and goserelin. Participants were required to have a Gleason score $\leq 7$, clinical tumor stage of T1b to T3a, and serum PSA $<30 \mathrm{ng} / \mathrm{mL}$. Patients were excluded if they had low-risk disease (Gleason score $\leq 6$, T1-T2a, and PSA $\leq 10 \mathrm{ng} / \mathrm{mL}$ ) or had radiologic evidence of nodal or distant metastasis. At baseline, $95 \%$ of men had intermediate risk disease and mean serum PSA was $10.3 \mathrm{ng} / \mathrm{mL}$. Mean age at baseline was 69 years. The trial was conducted at two institutions in Canada and was rated medium risk of bias. We also identified one non-RCT that compared EBRT plus neoadjuvant ADT versus EBRT plus adjuvant ADT. ${ }^{77}$ The non-RCT was rated serious ROB. The prior 2014 and 2016 systematic reviews did not identify any studies addressing this comparison.

At a median 12.2 years followup, there may be little to no difference in all-cause mortality (34.9\% vs. 33.2\% [low COE]). Seven deaths in each treatment group were attributable to prostate cancer. There may be little to no difference in prostate cancer mortality (low COE). The evidence is very uncertain whether metastasis differs for EBRT plus neoadjuvant and concurrent ADT versus EBRT plus concurrent and adjuvant ADT.

Regarding harms, there may be little to no difference in late genitourinary toxicity grade 3 or higher after 3 years (low COE). Lastly, the RCT reported no difference in late gastrointestinal toxicity grade 3 or higher after 3 years (2.5\% vs. $3.9 \%)$.

\section{External Beam Radiation Therapy Versus Brachytherapy}

No randomized trial evidence informed this question. One observational study used propensity score matching to retrospectively analyze data from a multifacility health care system 
$(n=684) .{ }^{83}$ The EBRT patients $(n=574)$ received 3D-CRT with a median dose of 75.3 Gray (range 73.5 to 77.1$)$ over 8.5 weeks. Brachytherapy $(n=110)$ was prescribed as Iodine-125 radioactive seeds with a minimum peripheral dose of 145 Gray. Neoadjuvant ADT (Leuprolide) was administered for a median of 6 months in 59\% of the EBRT patients and a median of 4 months in $13 \%$ of the brachytherapy patients. Patients in the brachytherapy group were younger compared with the EBRT group with median ages of 65 versus 71, respectively. Most patients had clinical stage T1c (69\%). All patients had a Gleason score of 6 (30\%) or 7 (70\%), mostly $3+4$ (48\% of all patients). Nearly half were white (49\%) followed by black race (25\%). ROB was medium. Over a median followup of 10 years, the evidence was uncertain about the effect of EBRT on overall, prostate cancer-specific, and metastases-free survival versus brachytherapy (insufficient COE). Observed deaths and metastases were not reported over the 10-year followup period. No effect modifiers were reported for the propensity-score-matched analysis.

\section{Conventionally Fractionated External Beam Radiation Therapy Versus Ultrahypofractionated External Beam Radiation}

Two RCTs compared conventionally fractionated EBRT versus ultra-hypofractionated EBRT. ${ }^{68,82}$ One RCT was rated high risk of bias ${ }^{68}$ and hereafter, our analysis only focuses on the second trial. ${ }^{82}$ In the trial we analyzed, the EBRT approach was 3D-CRT, volumetric-modulated arc therapy (VMAT), or IMRT (80\% of participants received 3D-CRT and 20\% VMAT/IMRT). Patients assigned to conventionally fractioned EBRT received $78.0 \mathrm{~Gy}$ in 39 fractions 5 days per week for 8 weeks. Patients assigned ultra-hypofractionated EBRT received 42.7 Gy in 7 fractions 3 days per week for 2.5 weeks. No ADT was permitted. At baseline, $89 \%$ of men had intermediate risk disease and 11\% had high risk. The median PSA values were $8.6 \mathrm{ng} / \mathrm{mL}$ and 8.7 in the two arms. Most patients had Gleason score of 7 (76\%). The median ages were 69 years and 68 in the two arms. The trial was conducted in Sweden and Denmark. The prior 2016 systematic review did not identify any studies addressing this comparison.

At a median 5-year followup, there is probably little to no difference in all-cause mortality between conventionally fractionated EBRT and ultra-hypofractionated EBRT (7.3\% vs. 7.8\% [moderate COE]). There may be little to no difference in prostate cancer specific mortality (1.4\% vs. $1.9 \%$ ) and metastasis (low COE).

There was generally little to no difference in harms between conventionally fractionated EBRT and ultra-hypofractionated EBRT, except in urinary toxicity at 1-year followup. Conventionally fractionated EBRT may result in a small reduction in physician-evaluated urinary toxicity grade $\geq 2$ at 1 year, but little to no difference at 2 years versus ultrahypofractionated EBRT (low COE). There may be little to no difference in physician-evaluated bowel toxicity grade $\geq 2$ at 2 years between treatment groups (low COE). The trial also reported patient-reported urinary and bowel problems with results in line with physician-recorded toxicity. The evidence is very uncertain about the effect of conventionally fractionated EBRT on erectile function versus ultra-hypofractionated EBRT (insufficient COE). Harms reporting from longer-term followup, had substantial missing data and was considered to be at a high ROB. 
Table 5. Certainty of evidence: external beam radiation therapy

\begin{tabular}{|c|c|c|c|c|c|c|c|}
\hline Comparison & $\begin{array}{l}\text { Outcome } \\
\text { № of Participants } \\
\text { (studies) }\end{array}$ & $\begin{array}{l}\text { Relative } \\
\text { Effect } \\
(95 \% \mathrm{Cl})\end{array}$ & $\begin{array}{l}\text { Anticipated } \\
\text { Absolute } \\
\text { Effects } \\
\text { EBRT }\end{array}$ & $\begin{array}{l}\text { Anticipated } \\
\text { Absolute } \\
\text { Effects } \\
\text { Comparator }\end{array}$ & $\begin{array}{l}\text { Anticipated } \\
\text { Absolute } \\
\text { Effects } \\
\text { Difference } \\
(95 \% \mathrm{Cl})\end{array}$ & $\begin{array}{l}\text { Certainty of } \\
\text { Evidence }\end{array}$ & What Happens \\
\hline \multirow[t]{5}{*}{$\begin{array}{l}\text { 3D-CRT and } \\
\text { ADT vs. 3D-CRT } \\
\text { and ADT with } \\
\text { LDR-PB boost } \\
64,65\end{array}$} & $\begin{array}{l}\text { Mortality 5-year } \\
\text { followup } \\
1 \text { RCT study }(n=398)\end{array}$ & $\begin{array}{l}\text { RR } 1.25 \\
(0.81 \text { to } 1.94)\end{array}$ & $\begin{array}{l}18.9 \% \\
(38 / 200)\end{array}$ & $\begin{array}{l}15.2 \% \\
(30 / 198)\end{array}$ & $\begin{array}{l}3.8 \% \\
(-3.5 \text { to } 11.2)\end{array}$ & $\begin{array}{l}\oplus \oplus \bigcirc \bigcirc \\
L O W \\
\text { a,b }\end{array}$ & $\begin{array}{l}3 \mathrm{D}-\mathrm{CRT} \text { and } \mathrm{ADT} \text { may result in a small } \\
\text { increase in mortality versus } 3 \mathrm{D}-\mathrm{CRT} \\
\text { and ADT with LDR-PB boost in higher } \\
\text { risk disease }\end{array}$ \\
\hline & $\begin{array}{l}\text { Prostate-specific } \\
\text { mortality 5-year } \\
\text { followup } \\
1 \text { RCT study }(n=398)\end{array}$ & $\begin{array}{l}\text { RR } 1.56 \\
(0.62 \text { to } 3.93)\end{array}$ & $\begin{array}{l}5.5 \% \\
(11 / 200)\end{array}$ & $\begin{array}{l}3.5 \% \\
(7 / 198)\end{array}$ & $\begin{array}{l}2.0 \% \\
(-2.1 \text { to } 6.0)\end{array}$ & $\begin{array}{l}\oplus \bigcirc \bigcirc \bigcirc \\
\text { INSUFFICIENT a, c }\end{array}$ & $\begin{array}{l}\text { The evidence is very uncertain about } \\
\text { the effect of 3D-CRT and ADT with } \\
\text { LDR-PB boost on prostate-specific } \\
\text { mortality versus 3D-CRT and ADT in } \\
\text { higher risk disease }\end{array}$ \\
\hline & $\begin{array}{l}\text { Metastatic disease } 5- \\
\text { year followup } \\
1 \text { RCT study }(n=398)\end{array}$ & $\begin{array}{l}\text { RR } 1.05 \\
(0.56 \text { to } 1.97)\end{array}$ & $\begin{array}{l}9.0 \% \\
(18 / 200)\end{array}$ & $\begin{array}{l}8.6 \% \\
(17 / 198)\end{array}$ & $\begin{array}{l}0.4 \% \\
(-5.1 \text { to } 6.0)\end{array}$ & $\begin{array}{l}\oplus \oplus \bigcirc \bigcirc \\
L O W a, b\end{array}$ & $\begin{array}{l}\text { 3D-CRT and ADT with LDR-PB boost } \\
\text { may result in little to no difference in } \\
\text { metastatic disease versus 3D-CRT and } \\
\text { ADT in higher risk disease }\end{array}$ \\
\hline & $\begin{array}{l}\text { Urinary incontinence } \\
\text { 5-year followup } \\
1 \text { RCT study }(n=383)\end{array}$ & not estimable & - & - & - & $\begin{array}{l}\oplus \bigcirc \bigcirc \bigcirc \\
\text { INSUFFICIENT a, d }\end{array}$ & $\begin{array}{l}\text { The evidence is very uncertain about } \\
\text { the effect of 3D-CRT and ADT with } \\
\text { LDR-PB boost on urinary incontinence } \\
\text { versus 3D-CRT and ADT }\end{array}$ \\
\hline & $\begin{array}{l}\text { Erectile function } 5- \\
\text { year followup } \\
1 \text { RCT study }(n=383)\end{array}$ & not estimable & - & - & - & $\begin{array}{l}\oplus \bigcirc \bigcirc \bigcirc \\
\text { INSUFFICIENT a, c, } \\
d\end{array}$ & $\begin{array}{l}\text { The evidence is very uncertain about } \\
\text { the effect of 3D-CRT and ADT with } \\
\text { LDR-PB boost on erectile function } \\
\text { versus 3D-CRT and ADT }\end{array}$ \\
\hline $\begin{array}{l}\text { Brachytherapy + } \\
\text { EBRT vs. } \\
\text { Brachytherapy }^{55}\end{array}$ & $\begin{array}{l}\text { Overall mortality } 7- \\
\text { year followup } \\
1 \text { observational study } \\
(n=5858)\end{array}$ & not estimable & - & - & - & $\begin{array}{l}\oplus \bigcirc \bigcirc \bigcirc \\
\text { INSUFFICIENT a, d }\end{array}$ & $\begin{array}{l}\text { The evidence is very uncertain about } \\
\text { the effect of brachytherapy with EBRT } \\
\text { on overall survival versus } \\
\text { brachytherapy alone }\end{array}$ \\
\hline IMRT vs. SBRT ${ }^{63}$ & $\begin{array}{l}\text { Overall mortality } 9- \\
\text { year followup } \\
1 \text { observational study } \\
(n=5430)\end{array}$ & not estimable & - & - & - & $\begin{array}{l}\oplus \bigcirc \bigcirc \bigcirc \\
\text { INSUFFICIENT a, c, } \\
d\end{array}$ & $\begin{array}{l}\text { The evidence is very uncertain about } \\
\text { the effect of IMRT on overall survival } \\
\text { versus SBRT }\end{array}$ \\
\hline
\end{tabular}




\begin{tabular}{|c|c|c|c|c|c|c|c|}
\hline Comparison & $\begin{array}{l}\text { Outcome } \\
\text { № of Participants } \\
\text { (studies) }\end{array}$ & $\begin{array}{l}\text { Relative } \\
\text { Effect } \\
(95 \% \mathrm{Cl})\end{array}$ & $\begin{array}{l}\text { Anticipated } \\
\text { Absolute } \\
\text { Effects } \\
\text { EBRT }\end{array}$ & $\begin{array}{l}\text { Anticipated } \\
\text { Absolute } \\
\text { Effects } \\
\text { Comparator }\end{array}$ & $\begin{array}{l}\text { Anticipated } \\
\text { Absolute } \\
\text { Effects } \\
\text { Difference } \\
(95 \% \mathrm{Cl})\end{array}$ & $\begin{array}{l}\text { Certainty of } \\
\text { Evidence }\end{array}$ & What Happens \\
\hline \multirow[t]{7}{*}{$\begin{array}{l}\text { EBRT plus ADT } \\
\text { versus EBRT } \\
76,88-92\end{array}$} & $\begin{array}{l}\text { Overall mortality- } 5.9 \\
\text { to } 9.1 \text { years } \\
5 \text { RCTs }(n=4047)\end{array}$ & $\begin{array}{l}\text { RR } 0.86 \\
(0.69 \text { to } 1.06)\end{array}$ & $\begin{array}{l}587 / 2150 \\
(27.3 \%)\end{array}$ & $\begin{array}{l}615 / 1897 \\
(32.4 \%)\end{array}$ & $\begin{array}{l}-3.7 \% \\
(-9.8 \text { to } 2.4)\end{array}$ & $\begin{array}{l}\oplus \oplus \oplus \bigcirc \\
\text { MODERATE b }\end{array}$ & $\begin{array}{l}\text { EBRT plus ADT probably results in a } \\
\text { small reduction in overall mortality } \\
\text { versus EBRT in higher risk disease }\end{array}$ \\
\hline & $\begin{array}{l}\text { Prostate cancer } \\
\text { mortality- } 7.2 \text { to } 9.1 \\
\text { years } \\
3 \text { RCTs } \\
(n=3004)\end{array}$ & $\begin{array}{l}\text { Peto OR } 0.51 \\
(0.37 \text { to } 0.70)\end{array}$ & $\begin{array}{l}53 / 1499 \\
(3.53 \%)\end{array}$ & $\begin{array}{l}104 / 1505 \\
(6.9 \%)\end{array}$ & $\begin{array}{l}-3.4 \% \\
(-4.95 \text { to }- \\
1.8)\end{array}$ & $\begin{array}{l}\oplus \oplus \bigcirc \bigcirc \\
\text { LOW a,b }\end{array}$ & $\begin{array}{l}\text { EBRT and ADT may result in a small } \\
\text { reduction in prostate cancer mortality } \\
\text { versus EBRT in higher risk disease }\end{array}$ \\
\hline & $\begin{array}{l}\text { Metastasis- } 5 \text { to } 10 \\
\text { years } \\
4 \text { RCTs }(n=4664)\end{array}$ & $\begin{array}{l}\text { RR } 0.83 \\
(0.71 \text { to } 0.97)\end{array}$ & $\begin{array}{l}284 / 2461 \\
(11.5 \%)\end{array}$ & $\begin{array}{l}289 / 2203 \\
(13.1 \%)\end{array}$ & $\begin{array}{l}-2.3 \% \\
(-4.1 \text { to }-0.4)\end{array}$ & $\begin{array}{l}\oplus \oplus \bigcirc \bigcirc \\
\text { LOW a,b }\end{array}$ & $\begin{array}{l}\text { EBRT and ADT may result in a small } \\
\text { reduction in metastasis versus EBRT in } \\
\text { higher risk disease }\end{array}$ \\
\hline & $\begin{array}{l}\text { Sexual function: } \\
\text { severe impairment } \\
\text { based on late toxicity } \\
\text { scores-measured } \\
\text { from six months until } \\
\text { end of followup ( } 7.2 \\
\text { years) } \\
1 \text { RCT ( } n=813)\end{array}$ & $\begin{array}{l}\text { RR } 1.40 \\
\text { (1.08 to } 1.80)\end{array}$ & $\begin{array}{l}110 / 406 \\
(27.0 \%)\end{array}$ & $\begin{array}{l}79 / 407 \\
(19.4 \%)\end{array}$ & $\begin{array}{l}7.7 \% \\
(1.9 \text { to } 13.5)\end{array}$ & $\begin{array}{l}\oplus \oplus \bigcirc \bigcirc \\
\text { LOW a,b }\end{array}$ & $\begin{array}{l}\text { EBRT and ADT may result in a } \\
\text { moderate increase in severe } \\
\text { impairment in sexual function versus } \\
\text { EBRT in higher risk disease }\end{array}$ \\
\hline & $\begin{array}{l}\text { Sexual function: } \\
\text { impotence grade } 2-4- \\
4.5 \text { years } \\
1 \text { RCT }(n=201)\end{array}$ & $\begin{array}{l}\text { RR } 1.20 \\
(0.79 \text { to } 1.84)\end{array}$ & $\begin{array}{l}32 / 98 \\
(32.7 \%)\end{array}$ & $\begin{array}{l}28 / 103 \\
(27.2 \%)\end{array}$ & $\begin{array}{l}5.5 \% \\
(-7.2 \% \text { to } \\
18.1 \%)\end{array}$ & $\begin{array}{l}\oplus \bigcirc \bigcirc \bigcirc \\
\text { INSUFFICIENT a, c }\end{array}$ & $\begin{array}{l}\text { The evidence is very uncertain about } \\
\text { the effect of EBRT plus ADT on } \\
\text { impotence grade } 2-4 \text { versus EBRT } \\
\text { alone }\end{array}$ \\
\hline & $\begin{array}{l}\text { Urinary incontinence } \\
\text { (stress) grades 2-4- } \\
4.5 \text { years } \\
1 \text { RCT }(n=201)\end{array}$ & $\begin{array}{l}\text { RR } 0.90 \\
(0.31 \text { to } 2.59)\end{array}$ & $\begin{array}{l}6 / 98 \\
(6.1 \%)\end{array}$ & $\begin{array}{l}7 / 103 \\
(6.8 \%)\end{array}$ & $\begin{array}{l}-0.7 \% \\
(-7.5 \text { to } 6.1)\end{array}$ & $\begin{array}{l}\oplus \bigcirc \bigcirc \bigcirc \\
\text { INSUFFICIENT a, c }\end{array}$ & $\begin{array}{l}\text { The evidence is very uncertain about } \\
\text { the effect of EBRT plus ADT on urinary } \\
\text { incontinence versus EBRT alone }\end{array}$ \\
\hline & $\begin{array}{l}\text { Rectal bleeding } \\
\text { grades } 2-4-4.5 \text { years } \\
1 \text { RCT }(n=201)\end{array}$ & $\begin{array}{l}\text { RR } 1.00 \\
(0.57 \text { to } 1.75)\end{array}$ & $\begin{array}{l}19 / 98 \\
(19.4 \%)\end{array}$ & $\begin{array}{l}20 / 103 \\
(19.4 \%)\end{array}$ & $\begin{array}{l}0.0 \% \\
(-11.0 \text { to } \\
10.9)\end{array}$ & $\begin{array}{l}\oplus \bigcirc \bigcirc \bigcirc \\
\text { INSUFFICIENT a, c }\end{array}$ & $\begin{array}{l}\text { The evidence is very uncertain about } \\
\text { the effect of EBRT plus ADT on rectal } \\
\text { bleeding versus EBRT alone }\end{array}$ \\
\hline
\end{tabular}




\begin{tabular}{|c|c|c|c|c|c|c|c|}
\hline Comparison & $\begin{array}{l}\text { Outcome } \\
\text { № of Participants } \\
\text { (studies) }\end{array}$ & $\begin{array}{l}\text { Relative } \\
\text { Effect } \\
(95 \% \mathrm{Cl})\end{array}$ & $\begin{array}{l}\text { Anticipated } \\
\text { Absolute } \\
\text { Effects } \\
\text { EBRT }\end{array}$ & $\begin{array}{l}\text { Anticipated } \\
\text { Absolute } \\
\text { Effects } \\
\text { Comparator }\end{array}$ & $\begin{array}{l}\text { Anticipated } \\
\text { Absolute } \\
\text { Effects } \\
\text { Difference } \\
(95 \% \mathrm{Cl})\end{array}$ & $\begin{array}{l}\text { Certainty of } \\
\text { Evidence }\end{array}$ & What Happens \\
\hline \multirow{4}{*}{$\begin{array}{l}\text { EBRT plus } \\
\text { neoadjuvant and } \\
\text { concurrent ADT } \\
\text { vs. EBRT plus } \\
\text { concurrent and } \\
\text { adjuvant } A D T^{81}\end{array}$} & $\begin{array}{l}\text { Overall mortality-12.2 } \\
\text { years } \\
1 \text { RCT }(n=432)\end{array}$ & $\begin{array}{l}\text { RR } 1.05 \\
\text { (0.81 to } 1.37)\end{array}$ & $\begin{array}{l}75 / 215 \\
(34.9 \%)\end{array}$ & $\begin{array}{l}72 / 217 \\
(33.2 \%)\end{array}$ & $\begin{array}{l}1.7 \% \\
(-7.2 \% \text { to } \\
10.6 \%)\end{array}$ & $\begin{array}{l}\oplus \oplus \bigcirc \bigcirc \\
L^{c} W^{c}\end{array}$ & $\begin{array}{l}\text { EBRT plus neoadjuvant and concurrent } \\
\text { ADT may result in little to no difference } \\
\text { in overall mortality versus EBRT plus } \\
\text { concurrent and adjuvant ADT }\end{array}$ \\
\hline & $\begin{array}{l}\text { Prostate cancer } \\
\text { mortality-12.2 years } \\
1 \text { RCT }(n=432)\end{array}$ & $\begin{array}{l}\text { Peto OR } 1.01 \\
\text { (0.35 to } 2.93)\end{array}$ & $\begin{array}{l}7 / 215 \\
(3.3 \%)\end{array}$ & $\begin{array}{l}7 / 217 \\
(3.2 \%)\end{array}$ & $\begin{array}{l}0 \% \\
(-3.3 \% \text { to } \\
3.4 \%)\end{array}$ & $\begin{array}{l}\oplus \oplus \bigcirc \bigcirc \\
L O W a, b\end{array}$ & $\begin{array}{l}\text { EBRT plus neoadjuvant and concurrent } \\
\text { ADT may result in little to no difference } \\
\text { in prostate cancer mortality versus } \\
\text { EBRT plus concurrent and adjuvant } \\
\text { ADT }\end{array}$ \\
\hline & $\begin{array}{l}\text { Metastasis distant } \\
\text { progression-12.2 } \\
\text { years } \\
1 \text { RCT }(n=432)\end{array}$ & $\begin{array}{l}\text { Peto OR } 1.36 \\
(0.57 \text { to } 3.27)\end{array}$ & $\begin{array}{l}12 / 215 \\
(5.6 \%)\end{array}$ & $\begin{array}{l}9 / 217 \\
(4.1 \%)\end{array}$ & $\begin{array}{l}1.4 \% \\
(-2.6 \% \text { to } \\
5.5 \%)\end{array}$ & $\begin{array}{l}\oplus \bigcirc \bigcirc \bigcirc \\
\text { INSUFFICIENT }^{\text {a, c }}\end{array}$ & $\begin{array}{l}\text { The evidence is very uncertain about } \\
\text { the effect of EBRT plus neoadjuvant } \\
\text { and concurrent ADT on metastasis } \\
\text { versus EBRT plus concurrent and } \\
\text { adjuvant ADT }\end{array}$ \\
\hline & $\begin{array}{l}\text { Late genitourinary } \\
\text { toxicity grade } \geq 3-3 \\
\text { years } \\
1 \text { RCT (428) }\end{array}$ & $\begin{array}{l}\text { Peto OR } 1.01 \\
(0.32 \text { to } 3.18)\end{array}$ & $\begin{array}{l}6 / 213 \\
(2.8 \%)\end{array}$ & $\begin{array}{l}6 / 215 \\
(2.8 \%)\end{array}$ & $\begin{array}{l}0 \% \\
(-3.1 \% \text { to } \\
3.2 \%)\end{array}$ & $\begin{array}{l}\oplus \oplus \bigcirc \bigcirc \\
\text { LOW a,b }\end{array}$ & $\begin{array}{l}\text { EBRT plus neoadjuvant and concurrent } \\
\text { ADT may result in little to no difference } \\
\text { in late genitourinary toxicity versus } \\
\text { EBRT plus concurrent and adjuvant } \\
\text { ADT }\end{array}$ \\
\hline \multirow[t]{2}{*}{$\begin{array}{l}\text { EBRT vs. } \\
\text { Brachytherapy }{ }^{83}\end{array}$} & $\begin{array}{l}\text { Overall survival } \\
\text { Median } 10 \text { years } \\
1 \text { observational study } \\
(n=684)\end{array}$ & not estimable & $\begin{array}{l}\text { Propensity } \\
\text { score adjusted } \\
\text { probability } \\
75.5 \%(\mathrm{Cl} 71.8 \\
\text { to } 79.4)\end{array}$ & $\begin{array}{l}\text { Propensity } \\
\text { score } \\
\text { adjusted } \\
\text { probability } \\
78.3 \%(\mathrm{Cl} \\
70.1 \text { to } 87.4)\end{array}$ & $\begin{array}{l}\sim-2.8 \% \\
\text { (not } \\
\text { estimable) }\end{array}$ & $\begin{array}{l}\oplus \bigcirc \bigcirc \bigcirc \\
\text { INSUFFICIENT e, f }\end{array}$ & $\begin{array}{l}\text { The evidence is uncertain about the } \\
\text { effect of EBRT on overall survival } \\
\text { versus brachytherapy }\end{array}$ \\
\hline & $\begin{array}{l}\text { Prostate cancer- } \\
\text { specific survival } \\
\text { Median } 10 \text { years } \\
1 \text { observational study } \\
(\mathrm{n}=684)\end{array}$ & not estimable & $\begin{array}{l}\text { Propensity } \\
\text { score adjusted } \\
\text { probability } \\
96.2 \%(\mathrm{Cl} 94.3 \\
\text { to } 98.1)\end{array}$ & $\begin{array}{l}\text { Propensity } \\
\text { score } \\
\text { adjusted } \\
\text { probability } \\
95.4 \%(\mathrm{Cl} \\
91.1 \text { to } 100)\end{array}$ & $\begin{array}{l}\sim 0.8 \% \\
\text { (not } \\
\text { estimable) }\end{array}$ & $\begin{array}{l}\oplus \bigcirc \bigcirc \bigcirc \\
\text { INSUFFICIENT e, f }\end{array}$ & $\begin{array}{l}\text { The evidence is uncertain about the } \\
\text { effect of EBRT on prostate cancer- } \\
\text { specific survival versus brachytherapy }\end{array}$ \\
\hline
\end{tabular}




\begin{tabular}{|c|c|c|c|c|c|c|c|}
\hline Comparison & $\begin{array}{l}\text { Outcome } \\
\text { № of Participants } \\
\text { (studies) }\end{array}$ & $\begin{array}{l}\text { Relative } \\
\text { Effect } \\
(95 \% \mathrm{Cl})\end{array}$ & $\begin{array}{l}\text { Anticipated } \\
\text { Absolute } \\
\text { Effects } \\
\text { EBRT }\end{array}$ & $\begin{array}{l}\text { Anticipated } \\
\text { Absolute } \\
\text { Effects } \\
\text { Comparator }\end{array}$ & $\begin{array}{l}\text { Anticipated } \\
\text { Absolute } \\
\text { Effects } \\
\text { Difference } \\
(95 \% \mathrm{Cl})\end{array}$ & $\begin{array}{l}\text { Certainty of } \\
\text { Evidence }\end{array}$ & What Happens \\
\hline & $\begin{array}{l}\text { Metastasis-free } \\
\text { survival } \\
\text { Median } 10 \text { years } \\
1 \text { observational study } \\
(\mathrm{n}=684)\end{array}$ & not estimable & $\begin{array}{l}\text { Propensity } \\
\text { score adjusted } \\
\text { probability } \\
90.6 \%(\mathrm{Cl} 87.9 \\
\text { to } 93.3)\end{array}$ & $\begin{array}{l}\text { Propensity } \\
\text { score } \\
\text { adjusted } \\
\text { probability } \\
94.1 \%(\mathrm{Cl} \\
89.5 \text { to } 98.9)\end{array}$ & $\begin{array}{l}\sim-3.5 \% \\
\text { (not } \\
\text { estimable) }\end{array}$ & $\begin{array}{l}\oplus \bigcirc \bigcirc \bigcirc \\
\text { INSUFFICIENT e, f }\end{array}$ & $\begin{array}{l}\text { The evidence is uncertain about the } \\
\text { effect of EBRT on metastasis-free } \\
\text { survival versus brachytherapy }\end{array}$ \\
\hline \multirow[t]{5}{*}{$\begin{array}{l}\text { Conventionally } \\
\text { fractionated } \\
\text { EBRT vs. ultra- } \\
\text { hypofractionated } \\
\text { EBRT }^{82}\end{array}$} & $\begin{array}{l}\text { Mortality-5 years } \\
1 \text { RCT }(n=1180)\end{array}$ & $\begin{array}{l}\text { RR } 0.93 \\
\text { (0.63 to 1.39) }\end{array}$ & $\begin{array}{l}7.3 \% \\
(43 / 591)\end{array}$ & $\begin{array}{l}7.8 \% \\
(46 / 589)\end{array}$ & $\begin{array}{l}-0.5 \% \\
(-3.5 \text { to } 2.5)\end{array}$ & $\begin{array}{l}\oplus \oplus \oplus \bigcirc \\
\text { MODERATE } b\end{array}$ & $\begin{array}{l}\text { Conventionally fractionated EBRT } \\
\text { probably results in little to no difference } \\
\text { in all-cause mortality versus ultra- } \\
\text { hypofractionated EBRT }\end{array}$ \\
\hline & $\begin{array}{l}\text { Prostate cancer } \\
\text { mortality-5 years } \\
1 \text { RCT }(n=1180)\end{array}$ & $\begin{array}{l}\text { Peto OR } 0.72 \\
(0.29 \text { to } 1.79)\end{array}$ & $\begin{array}{l}1.4 \% \\
(8 / 591)\end{array}$ & $\begin{array}{l}1.9 \% \\
(11 / 589)\end{array}$ & $\begin{array}{l}-0.5 \% \\
(-2.0 \text { to } 0.9)\end{array}$ & $\begin{array}{l}\oplus \oplus \bigcirc \bigcirc \\
L^{a} W^{a, b}\end{array}$ & $\begin{array}{l}\text { Conventionally fractionated EBRT may } \\
\text { result in little to no difference in } \\
\text { prostate cancer mortality versus ultra- } \\
\text { hypofractionated EBRT }\end{array}$ \\
\hline & $\begin{array}{l}\text { Metastasis- } 5 \text { years } \\
1 \text { RCT }(n=1180)\end{array}$ & $\begin{array}{l}\text { RR } 1.02 \\
\text { (0.66 to } 1.58)\end{array}$ & $\begin{array}{l}6.6 \% \\
(39 / 591)\end{array}$ & $\begin{array}{l}6.5 \% \\
(38 / 589)\end{array}$ & $\begin{array}{l}0.1 \% \\
(-2.7 \text { to } 3.0)\end{array}$ & $\begin{array}{l}\oplus \oplus \bigcirc \bigcirc \\
\text { LOW a,b }\end{array}$ & $\begin{array}{l}\text { Conventionally fractionated EBRT may } \\
\text { result in little to no difference in } \\
\text { metastasis versus ultra- } \\
\text { hypofractionated EBRT }\end{array}$ \\
\hline & $\begin{array}{l}\text { Urinary toxicity grade } \\
\geq 2 \text { based on RTOG } \\
\text { morbidity scale- } 1 \text { and } \\
2 \text { years } \\
1 \text { RCT ( } n=989 \text { to } \\
1057 \text { ) }\end{array}$ & $\begin{array}{l}\frac{1 \text { year }}{\text { RR } 0.41} \\
(0.22 \text { to } 0.76) \\
\frac{2 \text { years }}{\text { RR } 1.11} \\
(0.66 \text { to } 1.87)\end{array}$ & $\begin{array}{l}\frac{1 \text { year }}{2.5 \%} \\
(13 / 529) \\
\frac{2 \text { years }}{5.6 \%} \\
(28 / 497)\end{array}$ & $\begin{array}{l}\frac{1 \text { year }}{6.1 \%} \\
(32 / 528) \\
\frac{2 \text { years }}{5.1 \%} \\
(25 / 492)\end{array}$ & $\begin{array}{l}\frac{1 \text { year }}{-3.6 \%} \\
(-6.0 \text { to }-1.2) \\
\frac{2 \text { years }}{0.6 \%} \\
(-2.3 \text { to } 3.4)\end{array}$ & $\begin{array}{l}\oplus \oplus \bigcirc \bigcirc \\
\text { LOW }^{a, b}\end{array}$ & $\begin{array}{l}\text { Conventionally fractionated EBRT may } \\
\text { result in a small reduction in urinary } \\
\text { toxicity at } 1 \text { year, but little to no } \\
\text { difference at } 2 \text { years versus ultra- } \\
\text { hypofractionated EBRT }\end{array}$ \\
\hline & $\begin{array}{l}\text { Bowel toxicity grade } \\
\geq 2 \text { based on RTOG } \\
\text { morbidity scale- } 2 \\
\text { years } \\
1 \text { RCT }(n=991)\end{array}$ & $\begin{array}{l}\text { Peto OR } 1.77 \\
(0.80 \text { to } 3.92)\end{array}$ & $\begin{array}{l}3.2 \% \\
(16 / 496)\end{array}$ & $\begin{array}{l}1.8 \% \\
(9 / 495)\end{array}$ & $\begin{array}{l}1.4 \% \\
(-0.5 \text { to } 3.4)\end{array}$ & $\begin{array}{l}\oplus \oplus \bigcirc \bigcirc \\
L^{\circ} W^{a, b}\end{array}$ & $\begin{array}{l}\text { Conventionally fractionated EBRT may } \\
\text { result in little to no difference in bowel } \\
\text { toxicity versus ultra-hypofractionated } \\
\text { EBRT }\end{array}$ \\
\hline
\end{tabular}




\begin{tabular}{|c|c|c|c|c|c|c|c|}
\hline Comparison & $\begin{array}{l}\text { Outcome } \\
\text { № of Participants } \\
\text { (studies) }\end{array}$ & $\begin{array}{l}\text { Relative } \\
\text { Effect } \\
(95 \% \mathrm{Cl})\end{array}$ & $\begin{array}{l}\text { Anticipated } \\
\text { Absolute } \\
\text { Effects } \\
\text { EBRT }\end{array}$ & $\begin{array}{l}\text { Anticipated } \\
\text { Absolute } \\
\text { Effects } \\
\text { Comparator }\end{array}$ & $\begin{array}{l}\text { Anticipated } \\
\text { Absolute } \\
\text { Effects } \\
\text { Difference } \\
(95 \% \mathrm{Cl})\end{array}$ & $\begin{array}{l}\text { Certainty of } \\
\text { Evidence }\end{array}$ & What Happens \\
\hline & $\begin{array}{l}\text { Erectile function-1 } \\
\text { and } 2 \text { years } \\
1 \text { RCT ( } n=944 \text { to } \\
1001)\end{array}$ & not estimable & NR & NR & $\begin{array}{l}\text { Not } \\
\text { significantly } \\
\text { different } \\
(p=0.59-0.60)\end{array}$ & $\begin{array}{l}\oplus \bigcirc \bigcirc \bigcirc \\
\text { INSUFFICIENT a, c }\end{array}$ & $\begin{array}{l}\text { The evidence is very uncertain about } \\
\text { the effect of conventionally fractionated } \\
\text { EBRT on erectile function versus ultra- } \\
\text { hypofractionated EBRT }\end{array}$ \\
\hline
\end{tabular}

Abbreviations: 3D-CRT =3-dimensional conformal radiation therapy; ADT = androgen deprivation therapy; CI = confidence interval; EBRT = external beam radiation therapy;

IMRT = intensity modulated radiation therapy; LDR-PB = low dose rate prostate brachytherapy; NR = not reported; OR = odds ratio; RCT = randomized controlled trial; RR = relative risk; RTOG = Radiation Therapy Oncology Group; SBRT = stereotactic body radiation therapy

\section{GRADE Working Group grades of evidence}

High certainty: We are very confident that the true effect lies close to that of the estimate of the effect

Moderate certainty: We are moderately confident in the effect estimate: The true effect is likely to be close to the estimate of the effect, but there is a possibility that it is substantially different

Low certainty: Our confidence in the effect estimate is limited: The true effect may be substantially different from the estimate of the effect

Insufficient: We have very little confidence in the effect estimate: The true effect is likely to be substantially different from the estimate of effect

Explanations
a. Rated down one level for risk of bias
b. Rated down one level imprecision
c. Rated down two levels for imprecision
d. Rated down for suspected publication bias
e. Rated down two levels for risk of bias
f. Rated down one level for imprecision (unable to estimate based on data presented)




\section{Chapter 7. Whole Gland Therapies-Radical Prostatectomy}

\section{Key Messages}

- Radical prostatectomy (RP) versus prostate specific antigen (PSA)-based active monitoring (AM) over 10 years showed:

o Little to no difference in all-cause (moderate certainty of evidence [COE]) and prostate-cancer-specific mortality (low COE)

o Small reduction in metastases (moderate COE)

o Moderate increases in erectile dysfunction (ED) and urinary incontinence over 6 years (moderate COE)

o Prostate cancer mortality may not vary by age, PSA, tumor stage or Gleason score

- RP versus external beam radiation therapy (EBRT) plus androgen deprivation therapy

(ADT) over 10 years showed:

o Little to no difference in all-cause (moderate $\mathrm{COE}$ ) or prostate-cancer-specific mortality (low COE) or metastases (low COE).

o Moderate increases in erectile dysfunction and urinary incontinence at 6-year followup (moderate COE).

o Small reduction in fecal incontinence over 6 years (low COE)

o Prostate cancer mortality may not vary by age, PSA, tumor stage or Gleason score

- RP plus ADT versus EBRT plus High-dose-rate Brachytherapy (BT) plus ADT over 2 years showed:

o Small increase in erectile dysfunction (low COE).

- Laparoscopic RP versus robotic-assisted RP over 5 years showed:

o Moderate increase in urinary incontinence and large increase in erectile dysfunction and (low COE).

o Results did not vary by patient or tumor characteristics, but events were few.

We identified seven reports of four eligible randomized controlled trials (RCTs) 42-45, 84, 96, 97 and one non-RCT ${ }^{98}$ that compared RP to other therapies. Serious or critical risk of bias (ROB) precluded the inclusion of eight non-RCTs in the analysis. ${ }^{24,27,52,99-103}$ Several comparisons were only evaluated in studies rated high ROB (see Appendix I). We identified six articles which were not analyzed due to the inclusion of articles with lower risk of bias of the same comparisons. ${ }^{104-}$ 109

ROB assessments, population characteristics of the analyzed studies, outcomes data, and detailed GRADE rating tables are in Appendix I. Summary of Findings appears in Table 6.

Information on watchful waiting versus RP can be found in Chapter 4.

There were several comparisons of RP to other therapies addressed in the 2016 evidence report commissioned by the American Urological Association (AUA) in which we did not identify any additional studies of low to moderate ROB published after this report. ${ }^{5} \mathrm{~A}$ list of these comparisons can be found in Appendix J. The 2016 evidence report and appendices contain detailed results, strength of evidence, and evidence tables for these comparisons. Table 2 summarizes major findings of whole gland therapies versus other comparisons. 


\section{Radical Prostatectomy Versus Active Monitoring}

Four reports of one eligible RCT (ProtecT) compared PSA-based AM, to RP or EBRT plus ADT in men with PSA-screen detected clinically localized prostate cancer (CLPC) and reported results for survival, metastases, quality of life, or harms. ${ }^{42-45}$ Men with T1c-T2 CLPC were randomized to AM ( $\mathrm{n}=545)$, RP $(\mathrm{n}=553)$ or radiation therapy $(\mathrm{RT})(\mathrm{n}=545)$. Eighty-eight percent of men allocated to AM, 71 percent to RP and 74 percent to EBRT received the assigned treatment within 9 months after randomization. Most men had a Gleason score of $6(77 \%)$, followed by scores of 7 (21\%) and 8-10 (2\%). Primary RP approach was open retropubic radical. For participants assigned to AM, serum PSA levels were measured every 3 months in the first year and every 6 to 12 months thereafter. Surveillance prostate biopsies were permitted but not performed on a standard protocol. Increases of 50 percent or greater initiated review. Following review, participants could continue monitoring or further testing or receive radical or palliative interventions as needed. At the 10-year followup, 53 percent $(n=291)$ of men assigned AM had received radical treatment (surgery 49\%, per-protocol RT 33\%, 8\% BT, 9\% non-protocol RT, $1 \%$ high intensity focused ultrasound [HIFU]). Median age was 62 years and the majority were white (98\%). ${ }^{44}$ ProtecT was conducted in the UK, non-industry funded, and rated low risk of bias. Prior reviews included no randomized trials directly comparing RP with AM.

At 10-year followup there probably was little to no difference in all-cause (moderate COE) and prostate-cancer-specific mortality (low COE) for RP versus AM. ${ }^{43}$ Few deaths were attributable to prostate cancer; five and eight in the RP and AM groups, respectively.

There was probably was a small reduction in the development of metastases with RP compared with AM over 10 years (moderate COE). ${ }^{43}$ At 12 - and 72-months, AM and surgery did not differ in the Medical Outcomes Study 12-Item Short-Form General Health Survey (SF-12) physical and mental health subscales and the European Organization for Research and Treatment of Cancer Quality-of-Life Questionnaire-Core 30 module (EORTC QLQ-C30). ${ }^{42}$

Harms associated with urinary and sexual function were worse with RP than AM. ${ }^{42}$ Incontinence (defined as any use of absorbent pads) at 12 and 72 months was reported for 26 percent and 17 percent of the participants in the RP group versus 4 percent and 8 percent for AM (moderate COE). Erectile dysfunction (defined as an erection not firm enough for intercourse) was also greater for RP versus AM at both followup periods. At 72 months, 83.5 percent allocated to RP reported ED versus 70 percent allocated to AM (moderate COE). There may be little to no difference for harms associated with bowel function for RP versus AM (low COE). At 12 months, fecal incontinence at least one time per week was reported by approximately 1 percent in both groups; long-term it was reported by 2 percent for RP versus 3 percent for AM.

\section{Variation in Outcomes by Participant or Tumor Characteristics}

Prespecified subgroup analyses found no differences between groups in prostate-cancerspecific mortality according to age, PSA level, Gleason score, or clinical stage.

\section{Radical Prostatectomy Versus External Beam Radiation Therapy Plus Androgen Deprivation Therapy}

Four reports of one eligible RCT (ProtecT) compared PSA-based AM, RP, and EBRT plus ADT and reported results for survival, metastases, quality of life, or harms; ${ }^{42-45}$ Men with PSA screen detected T1c-T2 of any histologic grade CLPC were randomized to AM ( $\mathrm{n}=545)$, RP $(n=553)$ or EBRT plus ADT $(n=545)$. Most men had a Gleason score of $6(77 \%)$, followed by 
scores of 7 (21\%) and 8-10 (2\%). The primary RP approach was open retropubic radical. Participants assigned EBRT plus ADT received 74 Gy in 37 fractions with neoadjuvant ADT given 3 to 6 months before and concomitantly. ${ }^{44}$ Median age was 62 years, nearly all were white (98\%). ${ }^{44}$ ProtecT was conducted in the UK, non-industry funded, and rated low risk of bias.

Prior reviews included no randomized trials that directly compared RP to EBRT alone or in combination with ADT and reported mortality or metastases outcomes with a followup longer than 5 years. The 2016 evidence report commissioned by the AUA identified one small RCT $(n=97)$ deemed high risk of bias that reported no difference in deaths or measures of metastases between RP versus RT at 5 years. ${ }^{5}$ The 2014 Agency for Healthcare Research and Quality (AHRQ) systematic review authors found low-strength evidence favoring RP versus RT for allcause mortality and prostate-cancer-specific over followup periods ranging from 3 to 15 years, but this was based on nonrandomized studies of mostly high risk of bias. ${ }^{4}$ The prior AHRQ review also concluded that, in general, urinary incontinence and erectile dysfunction were commonly reported adverse events among men who underwent RP, and gastrointestinal/genitourinary toxicity and erectile dysfunction were commonly reported harms for men who received RT.

The 10-year followup probably showed little to no difference in all-cause (moderate COE) and prostate-cancer-specific mortality (low COE) for RP versus EBRT plus ADT. ${ }^{43}$ Few deaths were attributable to prostate cancer in the RP and RT groups, respectively.

The number of participants who developed metastases may not differ between RP and EBRT plus ADT over 10 years (low COE). ${ }^{43}$

At 12 and 72 months, incontinence (defined as any use of absorbent pads) was reported by 26 percent and 17 percent of participants in the RP group versus approximately 4 percent for RT at both followups (moderate COE). Erectile dysfunction (defined as an erection not firm enough for intercourse) was also greater for RP than EBRT plus ADT at both followup periods. At 72 months, erectile dysfunction was reported by 83.5 percent in the RP group versus 73 percent for RT (moderate COE). Harms associated with bowel function were generally worse with EBRT plus ADT versus RP. At 12 months, fecal incontinence at least once per week was reported for 4 percent in the EBRT plus ADT group and 0.8 percent for the RP group. At 72 months, fecal incontinence did not statistically differ between groups (low COE). Bloody stools half of the time or more were reported for nearly 6 percent of EBRT plus ADT participants versus one percent in the RP group (absolute risk difference [ARD] -5\% [95\% CI -7 to -2]).

\section{Variation in Outcomes by Participant or Tumor Characteristics}

Pre-specified subgroup analyses found no differences between groups in prostate-cancerspecific mortality when stratified by age, PSA level, Gleason score, or clinical stage, but the small number of events limits our interpretation of these findings.

\section{Radical Prostatectomy Plus Androgen Deprivation Therapy Versus External Beam Radiation Therapy Plus High-Dose- Rate Brachytherapy Plus Androgen Deprivation Therapy}

We identified one eligible small RCT conducted in Sweden that compared RP plus ADT to high-dose radiation (EBRT plus high dose rate brachytherapy [HDR-BT]) plus ADT and reported results for survival, quality of life, or harms through 10 years. ${ }^{84}$ Men with clinically 
localized/locally advanced T1b-T3a PC of any histologic grade and a PSA $<50 \mathrm{ng} / \mathrm{mL}$ were randomized to primarily nerve sparing RP $(\mathrm{n}=45)$ or EBRT plus HDR-BT $(\mathrm{n}=44)$. Participants assigned to EBRT received EBRT ( 25 x 2 Gy) plus HDR-BT (2 x 10 Gy). All patients were treated with neoadjuvant ADT that continued for six months. Median ages ranged from 64 to 66 years. T1 tumors were present in $40 \%$ and T2 in 37\% of individuals though information was not provided to assess tumor risk status. The trial was originally designed to enroll 360 men but due to recruitment difficulties the study only included 89 and focused on outcomes other than mortality. The trial was non-industry funded and was rated moderate risk of bias.

Prior reviews included no randomized trials that directly compared RP with combined EBRT and HDR-BT. The 2016 evidence report commissioned by the AUA identified three observational studies deemed high risk of bias comparing RP to combination EBRT plus BT that did not report death or metastases outcomes. ${ }^{5}$ One study reported a higher rate of urinary incontinence with RP and one study reported that the results for urinary, bowel, and sexual harms were inconclusive for this comparison.

Mortality outcomes were reported at 10-years. The evidence is uncertain whether all-cause and prostate-cancer-specific mortality differ for RP plus ADT versus EBRT plus HDR-BT plus ADT (insufficient COE). The number of participants who developed metastases was not reported. Versus EBRT plus HDR-BT plus ADT, there were no differences in the European Organization for Research and Treatment of Cancer Quality-of-Life Questionnaire C33 (EORTC QLQ-C33) with surgery plus ADT at 12- and 24-month followup periods. Harms associated with urinary, bowel, sexual function were reported at 12 and 24 months. Erectile dysfunction, defined as occurring "quite a bit" to "very much" may be slightly higher in the RP plus ADT group (Low $\mathrm{COE}$ ). It is uncertain whether urinary or fecal incontinence differ for RP plus ADT versus EBRT plus HDR-BT plus ADT (insufficient COE).

\section{Laparoscopic Radical Prostatectomy Versus Robotic- Assisted Radical Prostatectomy}

We identified a publication ${ }^{97}$ with longer (5-year) followup of a small RCT previously included in both the 2016 evidence report commissioned by the $\mathrm{AUA}^{5}$ and the 2014 systematic review conducted by AHRQ. ${ }^{4}$, that compared laparoscopic radical prostatectomy (LRP) to roboticassisted radical prostatectomy (RARP) and reported results for quality of life and harms. ${ }^{97}$ Men with T1-T2N0M0 PC were randomized to either LRP $(n=60)$ or RARP $(n=60)$. Half of the men had a Gleason score ranging from 2 to $6,43 \%$ had a score of 7 , and $7 \%$ had a score of 8 to 10 . Nerve-sparing procedures were performed in all potent patients with a PSA $<10 \mathrm{ng} / \mathrm{ml}$, Gleason score $<7$, and a positive core (on the same side as the bundle preservation) $<30 \% .{ }^{110}$ Extended lymph node dissections were indicated in men with a preoperative PSA $>10 \mathrm{ng} / \mathrm{ml}$, Gleason score $\geq 7 \mathrm{~b}(4+3)$, and/or a lymph node involvement risk $>5 \%$ according to Partin tables. ${ }^{111}$ Lymph node dissection procedures were conducted for 13 men in each arm (22\%). Mean age was 64 years. The trial was conducted in Italy, was non-industry funded, and rated moderate risk of bias. The earlier results indicated higher rates of recovered urinary continence and potency (among potent patients undergoing nerve-sparing techniques) with RARP versus LRP through 1 year of followup. ${ }^{110}$ Neither of the previous reports included observational studies directly comparing LRP with RARP.

Authors did not report mortality and metastases outcomes. Participants allocated to LRP were less likely to rate their health status as excellent, very good, or good versus RARP, 86 percent compared with 100 percent, respectively $(\mathrm{p}=0.003)$. Harms associated with urinary, 
bowel, and sexual function were reported at 12 and 60 months. At 60 months, erectile dysfunction (defined as the inability to achieve an erection sufficient for penetration) may be much higher with LRP versus RARP (low COE). Urinary incontinence, defined as use of any pads or used one safety pad per day, may be moderately higher in the LRP group versus RARP (low COE).

\section{Robotic-Assisted Laparoscopic Radical Prostatectomy Versus Open Retropubic Radical Prostatectomy}

We identified one eligible observational study, LAPPRO $(n=2,545)$, that compared roboticassisted laparoscopic radical prostatectomy (RALRP) to open retropubic radical prostatectomy (open RRP) and reported results for harms. ${ }^{98}$ LAPRO recruited men with T1-T3 (T3 3\%) PC who underwent RALRP $(n=1,792)$ or open RRP $(n=753)$. Median age was approximately 63 years. The trial was conducted in Sweden, non-industry funded, and rated moderate risk of bias.

One additional RCT among men with CLPC reported findings for urinary, sexual, and erectile function as well as quality of life. This study was excluded because authors did not provide information on tumor stage inclusion or baseline criteria. ${ }^{12,}, 113$

Prior reviews included no randomized trials that directly compared RALRP with open RRP. A 2016 evidence report commissioned by the AUA identified four observational studies with mainly inconclusive findings. ${ }^{5}$ The 2014 systematic review conducted by AHRQ indicated that RALRP versus RRP was the most common comparison. ${ }^{4}$ However, most studies were assessed as high risk of bias and did not report long-term ( $\geq 5$ years) results for mortality and metastases outcomes. Evidence was insufficient for all outcomes.

Among the subset of men who had preoperative erectile function ( $n=1702)$, recovery of erectile function was assessed at 12 and 24 months. At 24 months, more men in the RRP group were classified as having recovered erectile function (defined as being unable to achieve a stiff erection at any time or an erection stiff enough for intercourse at any time) compared with open RALP, 61 percent versus 49 percent $(\mathrm{P} \leq .0014)$ However, we rated this evidence as insufficient because data were not presented in a useable manner (denominators for each group could not be calculated).

\section{Variation in Outcomes by Participant or Tumor Characteristics}

When stratified by D'Amico risk categories, rates for non-recovery of erectile dysfunction in men with low- to moderate-risk disease were comparable to the overall findings, but treatment groups differed little to none for men with high-risk disease (test for subgroup differences not reported).

\section{Radical Prostatectomy Versus Androgen Deprivation Therapy}

We identified no RCTs and two references of one non-RCT that evaluated RP versus ADT. ${ }^{24,}$ 27 The non-RCT (HAROW study) was rated serious ROB based on the ROBINS-I tool. The previous 2014 and 2016 systematic reviews included no RCTs and five non-RCTs for this comparison. ${ }^{4,5}$ All five non-RCTs were previously rated low quality. 


\section{Radical Prostatectomy Versus High-Intensity Focused Ultrasound}

We identified one small RCT (Partial prostate Ablation versus Radical prosTatectomy [PART], n=82), that compared conventional open, laparoscopic, or robot-assisted laparoscopic radical prostatectomy (RP) to partial ablation using high-intensity focused ultrasound (HIFU) and reported results for harms. ${ }^{96}$ PART aimed to assess the feasibility of conducting a similar RCT on a larger scale. PART recruited men with a Gleason score of 7 (3+4 or $4+3)$ or clinically staged $\leq \mathrm{T} 2 \mathrm{~b}$ disease from five United Kingdom healthcare centers. Men were randomized to RP $(n=41)$ or HIFU $(n=41)$ and followed for 12 months for harms outcomes. Median age was approximately 66 years. The trial was non-industry funded and rated moderate risk of bias.

Urinary incontinence, erectile dysfunction, and fecal incontinence were assessed at 12 months. Data were not presented in useable manner as only approximate percentages of men reporting each outcome were provided (nominators and denominators for each group could not be calculated). There was an increase in the need to use an absorbent pad at least once per day compared to baseline in the RP group. The percentage of men reporting erectile dysfunction and fecal incontinence was higher in the RP group compared to the HIFU group (insufficient COE). 
Table 6. Certainty of evidence: radical prostatectomy

\begin{tabular}{|c|c|c|c|c|c|c|c|}
\hline Comparison & $\begin{array}{l}\text { Outcome } \\
\text { № of Participants } \\
\text { (studies) }\end{array}$ & $\begin{array}{l}\text { Relative Effect } \\
(95 \% \mathrm{Cl})\end{array}$ & $\begin{array}{l}\text { Absolute } \\
\text { Effects } \\
\text { RP }\end{array}$ & $\begin{array}{l}\text { Absolute } \\
\text { Effects } \\
\text { Comparator }\end{array}$ & $\begin{array}{l}\text { Absolute } \\
\text { Effects } \\
\text { Difference } \\
(95 \% \mathrm{Cl})\end{array}$ & $\begin{array}{l}\text { Certainty of } \\
\text { Evidence }\end{array}$ & What Happens \\
\hline \multirow[t]{6}{*}{$\begin{array}{l}\text { RP versus } \\
\mathrm{AM}^{42,43}\end{array}$} & $\begin{array}{l}\text { All-cause mortality } \\
10 \text {-year followup } \\
1 \text { RCT ( } n=1098)\end{array}$ & $\begin{array}{l}\text { RR } 0.92 \\
(0.65 \text { to } 1.30)\end{array}$ & $\begin{array}{l}9.9 \% \\
(55 / 553)\end{array}$ & $\begin{array}{l}10.8 \% \\
(59 / 545)\end{array}$ & $\begin{array}{l}-0.9 \% \\
(-4.5 \text { to } 2.7)\end{array}$ & $\begin{array}{l}\oplus \oplus \oplus \bigcirc \\
\text { MODERATE }\end{array}$ & $\begin{array}{l}\text { RP probably results in little to no } \\
\text { difference in all-cause mortality versus } \\
\text { AM }\end{array}$ \\
\hline & $\begin{array}{l}\text { PC-specific mortality } \\
\text { 10-year followup } \\
1 \text { RCT ( } n=1098)\end{array}$ & $\begin{array}{l}\text { Peto OR } 0.62 \\
(0.20 \text { to } 1.87)\end{array}$ & $\begin{array}{l}0.9 \% \\
(5 / 553)\end{array}$ & $\begin{array}{l}1.5 \% \\
(8 / 545)\end{array}$ & $\begin{array}{l}-0.6 \% \\
(-1.8 \text { to } 0.7)\end{array}$ & $\begin{array}{l}\oplus \oplus \bigcirc \bigcirc \\
\text { LOW }\end{array}$ & $\begin{array}{l}\text { RP may result in little to no difference in } \\
\text { PC-specific mortality versus AM }\end{array}$ \\
\hline & $\begin{array}{l}\text { Metastases } \\
10-\text { year followup. } \\
1 \text { RCT ( } n=1098)\end{array}$ & $\begin{array}{l}\text { Peto OR } 0.40 \\
(0.22 \text { to } 0.72)\end{array}$ & $\begin{array}{l}2.4 \% \\
(13 / 553)\end{array}$ & $\begin{array}{l}6.4 \% \\
(33 / 545)\end{array}$ & $\begin{array}{l}-4.0 \% \\
(-6.1 \text { to } \\
-1.3) ; \\
\text { NNT=25 }\end{array}$ & $\begin{array}{l}\oplus \oplus \oplus \bigcirc \\
\text { MODERATE }\end{array}$ & $\begin{array}{l}\text { RP probably results in a small reduction } \\
\text { in metastases versus AM }\end{array}$ \\
\hline & $\begin{array}{l}\text { Urinary incontinence } \\
\text { (pad use) } \\
\text { 72-month followup } \\
1 \text { RCT (n=908) }\end{array}$ & $\begin{array}{l}\text { RR } 2.07 \\
\text { (1.44 to } 2.98)\end{array}$ & $\begin{array}{l}17.4 \% \\
(79 / 455)\end{array}$ & $\begin{array}{l}8.4 \% \\
(38 / 453)\end{array}$ & $\begin{array}{l}9 \% \\
\text { (5 to } 13) ; \\
\mathrm{NNH}=11\end{array}$ & $\begin{array}{l}\oplus \oplus \oplus \bigcirc \\
\text { MODERATE }\end{array}$ & $\begin{array}{l}\text { RP probably results in a moderate } \\
\text { increase in urinary incontinence versus } \\
\text { AM }\end{array}$ \\
\hline & $\begin{array}{l}\text { Erectile dysfunction } \\
\text { 72-month followup } \\
1 \text { RCT }(n=913)\end{array}$ & $\begin{array}{l}\text { RR } 1.19 \\
\text { (1.10 to } 1.28)\end{array}$ & $\begin{array}{l}83.5 \% \\
(385 / 461)\end{array}$ & $\begin{array}{l}70.4 \% \\
(318 / 452)\end{array}$ & $\begin{array}{l}13 \% \\
(8 \text { to } 19) \\
\mathrm{NNH}=9\end{array}$ & $\begin{array}{l}\oplus \oplus \oplus \bigcirc \\
\text { MODERATE }\end{array}$ & $\begin{array}{l}\text { RP probably results in a large increase } \\
\text { in erectile dysfunction versus AM }\end{array}$ \\
\hline & $\begin{array}{l}\text { Fecal incontinence } \\
72-\text { month followup } \\
1 \mathrm{RCT}(\mathrm{n}=930)\end{array}$ & $\begin{array}{l}\text { Peto OR } 0.74 \\
(0.31 \text { to } 1.75)\end{array}$ & $\begin{array}{l}1.9 \% \\
(9 / 468)\end{array}$ & $\begin{array}{l}2.6 \% \\
(12 / 462)\end{array}$ & $\begin{array}{l}-0.7 \% \\
(-2.6 \text { to } 1.2)\end{array}$ & $\begin{array}{l}\oplus \oplus \bigcirc \bigcirc \\
\text { LOW a,c }\end{array}$ & $\begin{array}{l}\text { RP may result in little to no difference in } \\
\text { fecal incontinence versus AM }\end{array}$ \\
\hline \multirow[t]{3}{*}{$\begin{array}{l}\text { RP versus EBRT } \\
\text { plus } \mathbf{A D T}^{42,43}\end{array}$} & $\begin{array}{l}\text { All-cause mortality } \\
10 \text {-year followup } \\
1 \text { RCT ( } n=1098)\end{array}$ & $\begin{array}{l}\text { RR } 0.99 \\
(0.69 \text { to } 1.04)\end{array}$ & $\begin{array}{l}9.9 \% \\
(55 / 553)\end{array}$ & $\begin{array}{l}10.1 \% \\
(55 / 545)\end{array}$ & $\begin{array}{l}-0.1 \% \\
(-3.7 \text { to } 3.7)\end{array}$ & $\begin{array}{l}\oplus \oplus \oplus \bigcirc \\
\text { MODERATE a }\end{array}$ & $\begin{array}{l}\text { RP probably results in little to no } \\
\text { difference in all-cause mortality versus } \\
\text { AM }\end{array}$ \\
\hline & $\begin{array}{l}\text { PC-specific mortality } \\
10 \text {-year followup } \\
1 \text { RCT ( } n=1098)\end{array}$ & $\begin{array}{l}\text { Peto OR } 1.23 \\
(0.33 \text { to } 4.58)\end{array}$ & $0.9 \%(5 / 553)$ & $\begin{array}{l}0.7 \% \\
(4 / 545)\end{array}$ & $\begin{array}{l}0.2 \% \\
(-0.9 \text { to } 1.2)\end{array}$ & $\begin{array}{l}\oplus \oplus \bigcirc \bigcirc \\
\text { LOW }^{\mathrm{b}}\end{array}$ & $\begin{array}{l}\text { RP may result in little to no difference in } \\
\text { PC-specific mortality versus EBRT plus } \\
\text { ADT }\end{array}$ \\
\hline & $\begin{array}{l}\text { Metastases } \\
\text { 10- year followup. } \\
1 \text { RCT ( } n=1098)\end{array}$ & $\begin{array}{l}\text { Peto OR } 0.80 \\
(0.38 \text { to } 1.67)\end{array}$ & $\begin{array}{l}2.4 \% \\
(13 / 553)\end{array}$ & $\begin{array}{l}2.9 \% \\
(16 / 545)\end{array}$ & $\begin{array}{l}-0.6 \% \\
(-2.5 \text { to } 1.3)\end{array}$ & $\begin{array}{l}\oplus \oplus \bigcirc \bigcirc \\
\text { LOW }^{b}\end{array}$ & $\begin{array}{l}\text { RP may result in little to no difference in } \\
\text { metastases versus EBRT plus ADT }\end{array}$ \\
\hline
\end{tabular}




\begin{tabular}{|c|c|c|c|c|c|c|c|}
\hline \multirow[t]{3}{*}{ Comparison } & $\begin{array}{l}\text { Outcome } \\
\text { № of Participants } \\
\text { (studies) }\end{array}$ & $\begin{array}{l}\text { Relative Effect } \\
(95 \% \mathrm{Cl})\end{array}$ & $\begin{array}{l}\text { Absolute } \\
\text { Effects } \\
\text { RP }\end{array}$ & $\begin{array}{l}\text { Absolute } \\
\text { Effects } \\
\text { Comparator }\end{array}$ & $\begin{array}{l}\text { Absolute } \\
\text { Effects } \\
\text { Difference } \\
(95 \% \mathrm{Cl})\end{array}$ & $\begin{array}{l}\text { Certainty of } \\
\text { Evidence }\end{array}$ & What Happens \\
\hline & $\begin{array}{l}\text { Urinary incontinence } \\
\text { (pad use) } \\
\text { 72-month followup } \\
1 \text { RCT }(n=907)\end{array}$ & $\begin{array}{l}\text { RR } 4.90 \\
\text { (2.91 to } 8.26 \text { ) }\end{array}$ & $\begin{array}{l}17.4 \% \\
(79 / 455)\end{array}$ & $\begin{array}{l}3.5 \% \\
(16 / 452)\end{array}$ & $\begin{array}{l}14 \% \\
(10 \text { to } 18) \\
\mathrm{NNH}=7\end{array}$ & $\begin{array}{l}\oplus \oplus \oplus \bigcirc \\
\text { MODERATE c }\end{array}$ & $\begin{array}{l}\text { RP probably results in a large increase } \\
\text { in urinary incontinence versus EBRT } \\
\text { plus ADT }\end{array}$ \\
\hline & $\begin{array}{l}\text { Fecal incontinence-72- } \\
\text { month followup } \\
1 \text { RCT }(n=933)\end{array}$ & $\begin{array}{l}\text { Peto OR } 0.48 \\
(0.22 \text { to } 1.01)\end{array}$ & $\begin{array}{l}1.9 \% \\
(9 / 468)\end{array}$ & $\begin{array}{l}4.1 \% \\
(19 / 465)\end{array}$ & $\begin{array}{l}-2.2 \\
(-4.4 \text { to } 0.02)\end{array}$ & $\begin{array}{l}\oplus \oplus \bigcirc \bigcirc \\
L O W \text { a,c }\end{array}$ & $\begin{array}{l}\text { RP may result in a small reduction in } \\
\text { fecal incontinence versus EBRT plus } \\
\text { ADT }\end{array}$ \\
\hline \multirow[t]{5}{*}{$\begin{array}{l}\text { RP plus ADT } \\
\text { versus } \\
\text { EBRT plus HDR- } \\
\text { BT plus ADT }{ }^{84}\end{array}$} & $\begin{array}{l}\text { All-cause mortality-10- } \\
\text { year followup } \\
1 \text { RCT }(n=89)\end{array}$ & $\begin{array}{l}\text { RR } 1.30 \\
\text { (0.61 to } 2.78)\end{array}$ & $\begin{array}{l}26.7 \% \\
(12 / 45)\end{array}$ & $\begin{array}{l}20.5 \% \\
(9 / 44)\end{array}$ & $\begin{array}{l}6.2 \% \\
(-11.4 \text { to } 23.8)\end{array}$ & $\begin{array}{l}\oplus \bigcirc \bigcirc \bigcirc \\
\text { INSUFFICIENT a, b }\end{array}$ & $\begin{array}{l}\text { The evidence is very uncertain about } \\
\text { the effect of RP plus ADT on all-cause } \\
\text { mortality versus EBRT plus HDR-BT } \\
\text { plus ADT }\end{array}$ \\
\hline & $\begin{array}{l}\text { PC-specific mortality } \\
\text { 10-year followup } \\
1 \text { RCT ( } n=89)\end{array}$ & $\begin{array}{l}\text { Peto OR } 2.89 \\
\text { (0.68 to } 12.27)\end{array}$ & $\begin{array}{l}13.3 \% \\
(6 / 45)\end{array}$ & $\begin{array}{l}4.5 \% \\
(2 / 44)\end{array}$ & $\begin{array}{l}8.8 \% \\
(-2.9 \text { to } 20.5)\end{array}$ & $\begin{array}{l}\oplus \bigcirc \bigcirc \bigcirc \\
\text { INSUFFICIENT a, b }\end{array}$ & $\begin{array}{l}\text { The evidence is very uncertain about } \\
\text { the effect of RP plus ADT on PC- } \\
\text { specific mortality versus EBRT plus } \\
\text { HDR- BT plus ADT }\end{array}$ \\
\hline & $\begin{array}{l}\text { Urinary incontinence } \\
\left.\text { (Grade } 3-4^{*}\right) \\
\text { 24-month followup } \\
1 \text { RCT }(n=55)\end{array}$ & $\begin{array}{l}\text { Peto OR } 1.70 \\
(0.35 \text { to } 8.23)\end{array}$ & $\begin{array}{l}16 \% \\
(4 / 25)\end{array}$ & $\begin{array}{l}10 \% \\
(3 / 30)\end{array}$ & $\begin{array}{l}6 \% \\
(-11.9 \text { to } 23.9)\end{array}$ & $\begin{array}{l}\oplus \bigcirc \bigcirc \bigcirc \\
\text { INSUFFICIENT a, b }\end{array}$ & $\begin{array}{l}\text { The evidence is very uncertain about } \\
\text { the effect of RP plus ADT on urinary } \\
\text { incontinence versus EBRT plus HDR- } \\
\text { BT plus ADT }\end{array}$ \\
\hline & $\begin{array}{l}\text { Erectile dysfunction } \\
\left(\text { Grade } 3-4^{\star}\right) \\
24-\text { month followup } \\
1 \text { RCT }(n=71)\end{array}$ & $\begin{array}{l}\text { RR } 1.05 \\
\text { (0.87 to } 1.25 \text { ) }\end{array}$ & $\begin{array}{l}89 \% \\
(33 / 37)\end{array}$ & $\begin{array}{l}85 \% \\
(29 / 34)\end{array}$ & $\begin{array}{l}4 \% \\
(-12 \text { to } 19)\end{array}$ & $\begin{array}{l}\oplus \oplus \bigcirc \bigcirc \\
L O W \\
\text { a,d }\end{array}$ & $\begin{array}{l}\text { RP plus ADT may result in a small } \\
\text { increase of erectile dysfunction versus } \\
\text { EBRT plus HDR- BT plus ADT }\end{array}$ \\
\hline & $\begin{array}{l}\text { Fecal incontinence } \\
\left.\text { (Grade } 2^{\star}\right) \\
\text { 24-month followup } \\
1 \text { RCT }(n=54)\end{array}$ & $\begin{array}{l}\text { Peto OR } 0.32 \\
(0.08 \text { to } 1.33)\end{array}$ & $\begin{array}{l}8 \% \\
(2 / 25)\end{array}$ & $\begin{array}{l}24.1 \% \\
(7 / 29)\end{array}$ & $\begin{array}{l}-16.1 \% \\
(-35 \text { to } 27)\end{array}$ & $\begin{array}{l}\oplus \bigcirc \bigcirc \bigcirc \\
\text { INSUFFICIENT a, b }\end{array}$ & $\begin{array}{l}\text { The evidence is very uncertain about } \\
\text { the effect of RP plus ADT on fecal } \\
\text { incontinence versus EBRT plus HDR- } \\
\text { BT plus ADT }\end{array}$ \\
\hline
\end{tabular}




\begin{tabular}{|c|c|c|c|c|c|c|c|}
\hline Comparison & $\begin{array}{l}\text { Outcome } \\
\text { № of Participants } \\
\text { (studies) }\end{array}$ & $\begin{array}{l}\text { Relative Effect } \\
(95 \% \mathrm{Cl})\end{array}$ & $\begin{array}{l}\text { Absolute } \\
\text { Effects } \\
\text { RP }\end{array}$ & $\begin{array}{l}\text { Absolute } \\
\text { Effects } \\
\text { Comparator }\end{array}$ & $\begin{array}{l}\text { Absolute } \\
\text { Effects } \\
\text { Difference } \\
(95 \% \mathrm{Cl})\end{array}$ & $\begin{array}{l}\text { Certainty of } \\
\text { Evidence }\end{array}$ & What Happens \\
\hline \multirow[t]{2}{*}{$\begin{array}{l}\text { LRP versus } \\
\text { RARP }^{97}\end{array}$} & $\begin{array}{l}\text { Urinary incontinence } \\
\text { (pad use), 60-month } \\
\text { followup } \\
1 \text { RCT }(n=115)\end{array}$ & $\begin{array}{l}\text { Peto OR } 3.96 \\
(1.15 \text { to } 13.65)\end{array}$ & $\begin{array}{l}15.5 \% \\
(9 / 58)\end{array}$ & $\begin{array}{l}3.5 \% \\
(2 / 57)\end{array}$ & $\begin{array}{l}12 \% \\
(1.5 \text { to } 23)\end{array}$ & $\begin{array}{l}\oplus \oplus \bigcirc \bigcirc \\
\mathrm{LOW} \text { a, b }\end{array}$ & $\begin{array}{l}\text { LRP may result in a moderate increase } \\
\text { in urinary incontinence versus RARP }\end{array}$ \\
\hline & $\begin{array}{l}\text { Erectile dysfunction } \\
\text { (insufficient erections), } \\
60 \text {-month followup } \\
1 \text { RCT }(n=70)\end{array}$ & $\begin{array}{l}\text { RR } 1.89 \\
(0.98 \text { to } 3.65)\end{array}$ & $\begin{array}{l}49 \% \\
(17 / 35)\end{array}$ & $\begin{array}{l}26 \% \\
(9 / 35)\end{array}$ & $\begin{array}{l}23 \% \\
\text { (1 to } 45)\end{array}$ & $\begin{array}{l}\oplus \oplus \bigcirc \bigcirc \\
L O W a, b\end{array}$ & $\begin{array}{l}\text { LRP may result in a large increase in } \\
\text { erectile dysfunction versus RARP }\end{array}$ \\
\hline $\begin{array}{l}\text { RALRP versus } \\
\text { Open RRP }\end{array}$ & $\begin{array}{l}\text { Erectile dysfunction } \\
\text { (unrecovered erectile } \\
\text { function), 24-month } \\
\text { followup } \\
1 \text { Obs (n=1702) }\end{array}$ & $\begin{array}{l}\text { Data not } \\
\text { presented in } \\
\text { usable manner }\end{array}$ & $49 \%$ & $61 \%$ & $\begin{array}{l}-12 \% \\
(\mathrm{Cl} N A)\end{array}$ & $\begin{array}{l}\oplus \bigcirc \bigcirc \bigcirc \\
\text { INSUFFICIENT c, d }\end{array}$ & $\begin{array}{l}\text { The evidence is very uncertain about } \\
\text { the effect of RALRP on erectile } \\
\text { dysfunction versus open RRP }\end{array}$ \\
\hline \multirow[t]{3}{*}{$\begin{array}{l}\text { RP versus } \\
\text { HIFU }\end{array}$} & $\begin{array}{l}\text { Urinary incontinence } \\
\text { 12-month followup } \\
1 \text { RCT }(n=82)\end{array}$ & $\begin{array}{l}\text { Data not } \\
\text { presented in } \\
\text { usable manner }\end{array}$ & $58 \%$ & $0 \%$ & $\begin{array}{l}-58 \% \\
(\mathrm{Cl} \mathrm{NA})\end{array}$ & $\begin{array}{l}\oplus \bigcirc \bigcirc \bigcirc \\
\text { INSUFFICIENT c, d }\end{array}$ & $\begin{array}{l}\text { The evidence is very uncertain about } \\
\text { the effect of RP on urinary incontinence } \\
\text { versus HIFU }\end{array}$ \\
\hline & $\begin{array}{l}\text { Erectile dysfunction } \\
\text { 12-month followup } \\
1 \text { RCT }(n=82)\end{array}$ & $\begin{array}{l}\text { Data not } \\
\text { presented in } \\
\text { usable manner }\end{array}$ & $50 \%$ & $20 \%$ & $\begin{array}{l}-30 \% \\
(\mathrm{Cl} \mathrm{NA})\end{array}$ & $\begin{array}{l}\oplus \bigcirc \bigcirc \bigcirc \\
\text { INSUFFICIENT c, d }\end{array}$ & $\begin{array}{l}\text { The evidence is very uncertain about } \\
\text { the effect of RP on erectile dysfunction } \\
\text { versus HIFU }\end{array}$ \\
\hline & $\begin{array}{l}\text { Fecal incontinence } \\
\text { 12-month followup } \\
1 \mathrm{RCT}(\mathrm{n}=82)\end{array}$ & $\begin{array}{l}\text { Data not } \\
\text { presented in } \\
\text { usable manner }\end{array}$ & $22 \%$ & $15 \%$ & $\begin{array}{l}-7 \% \\
(\mathrm{Cl} \mathrm{NA})\end{array}$ & $\begin{array}{l}\oplus \bigcirc \bigcirc \bigcirc \\
\text { INSUFFICIENT c, d }\end{array}$ & $\begin{array}{l}\text { The evidence is very uncertain about } \\
\text { the effect of RP on fecal incontinence } \\
\text { versus HIFU }\end{array}$ \\
\hline \multicolumn{8}{|c|}{$\begin{array}{l}\text { Abbreviations: } \mathrm{ADT}=\text { androgen deprivation therapy; } \mathrm{AM}=\text { active monitoring; } \mathrm{CI}=\text { confidence interval; } \mathrm{EBRT}=\text { external beam radiation therapy; HDR-BT = high dose rate brachytherapy; } \\
\text { HIFU = high-intensity focused ultrasound; LRP = laparoscopic radical prostatectomy; } \mathrm{n}=\text { number; } \mathrm{NA}=\text { not available; } \mathrm{NNH}=\text { number needed to harm; } \mathrm{NNT}=\text { number needed to treat; Obs = } \\
\text { observational study; OR = odds ratio; } \mathrm{PC}=\text { prostate cancer; RALRP = robotic-assisted laparoscopic radical prostatectomy; RARP = robotic-assisted radical prostatectomy; RCT = randomized } \\
\text { controlled trial; RP = radical prostatectomy; RR = relative risk; RRP = retropubic radical prostatectomy }\end{array}$} \\
\hline
\end{tabular}




\begin{tabular}{|c|c|c|c|c|c|c|c|}
\hline Comparison & $\begin{array}{l}\text { Outcome } \\
\text { № of Participants } \\
\text { (studies) }\end{array}$ & $\begin{array}{l}\text { Relative Effect } \\
(95 \% \mathrm{Cl})\end{array}$ & $\begin{array}{l}\text { Absolute } \\
\text { Effects } \\
\text { RP }\end{array}$ & $\begin{array}{l}\text { Absolute } \\
\text { Effects } \\
\text { Comparator }\end{array}$ & $\begin{array}{l}\text { Absolute } \\
\text { Effects } \\
\text { Difference } \\
(95 \% \mathrm{Cl})\end{array}$ & $\begin{array}{l}\text { Certainty of } \\
\text { Evidence }\end{array}$ & What Happens \\
\hline
\end{tabular}

\section{GRADE Working Group grades of evidence}

High certainty: We are very confident that the true effect lies close to that of the estimate of the effect

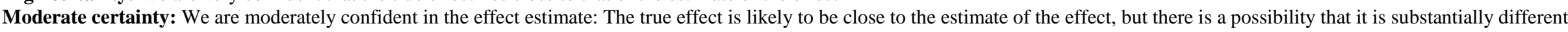

Low certainty: Our confidence in the effect estimate is limited: The true effect may be substantially different from the estimate of the effect

Insufficient: We have very little confidence in the effect estimate: The true effect is likely to be substantially different from the estimate of effect

\footnotetext{
Explanations

a. Rated down by one level for imprecision

b. Rated down by two levels for imprecision and sparse data.

c. Rated down by one level for risk of bias

d. Rated down one level for unknown precision
} 


\section{Chapter 8. Other Therapies}

\section{Androgen Deprivation Therapy}

Information about active monitoring (AM) versus external beam radiation therapy (EBRT) plus androgen deprivation therapy (ADT) can be found in Chapter 5.

Information about the following comparisons can be found in Chapter 6:

- 3D-conformal radiation therapy (3D-CRT) and ADT versus 3D-CRT and ADT plus lowdose-rate brachytherapy (LDR-BT)

- EBRT plus ADT versus EBRT

- EBRT plus neoadjuvant and concurrent ADT versus EBRT plus concurrent and adjuvant ADT

Information about the following comparisons can be found in Chapter 7:

- Radical prostatectomy (RP) versus EBRT plus ADT

- RP plus ADT versus EBRT plus high-dose-rate brachytherapy plus ADT

Some comparisons of ADT to other therapies were only evaluated in studies rated high risk of bias (ROB) (e.g., ADT versus EBRT plus ADT [ $k=1$ randomized controlled trial [RCT] and 1 non-RCT $],{ }^{73,75} \mathrm{ADT}$ versus RP [ $\mathrm{k}=1$ non-RCT $],{ }^{24,27} \mathrm{ADT}$ versus radiation therapy [either EBRT and/or brachytherapy][k=1 non-RCT], ${ }^{24,27}$ ADT versus AS [k=1 non-RCT], ${ }^{24,27}$ ADT versus watchful waiting [k=1 non-RCT]). ${ }^{24,27}$

There were several comparisons of ADT to other therapies addressed in the 2016 evidence report commissioned by the American Urological Association (AUA in which we did not identify any additional studies that met our analysis criteria. ${ }^{5} \mathrm{~A}$ list of these comparisons can be found in Appendix J.

The 2016 evidence report and appendices contains detailed results, strength of evidence, and evidence tables for these comparisons. Table 2 summarizes major findings of whole gland therapies versus other comparisons.

\section{Focal Therapies-High-Intensity Focused Ultrasound}

Information about radical prostatectomy versus high-intensity focused ultrasound (HIFU) can be found in Chapter 7.

HIFU versus HIFU plus ADT was addressed in the 2016 evidence report commissioned by the AUA. ${ }^{5}$ The 2016 evidence report and appendices contains detailed results, strength of evidence, and evidence tables for this comparison.

\section{Focal Therapies-Photodynamic Therapy}

Information about active surveillance (AS) versus photodynamic therapy (PDT) can be found in Chapter 5.

The 2016 evidence report commissioned by the AUA did not report on any eligible comparisons of photodynamic therapy. ${ }^{5}$

\section{Focal Therapies-Laser Ablation}

Laser ablation versus RP was addressed in one non-RCT rated as critical ROB. ${ }^{102}$ The 2016 evidence report commissioned by the AUA did not report on any eligible comparisons of laser ablation. ${ }^{5}$ 


\section{Whole Gland Therapies-Cryotherapy}

No eligible studies of cryotherapy were identified.

There were several comparisons of cryotherapy to other therapies addressed in the 2016 evidence report commissioned by the AUA in which we did not identify any additional studies of low to moderate ROB published after this report. ${ }^{5} \mathrm{~A}$ list of these comparisons can be found in Appendix J. The 2016 evidence report and appendices contains detailed results, strength of evidence, and evidence tables for these comparisons.

\section{Whole Gland Therapies-Brachytherapy}

Information about the following comparisons can be found in Chapter 6:

- 3D-CRT plus ADT versus 3D-CRT plus low-dose-rate brachytherapy plus ADT

- Brachytherapy with EBRT versus brachytherapy alone

- EBRT versus brachytherapy

Information about the following comparison can be found in Chapter 7:

- RP plus ADT versus EBRT plus high-dose-rate brachytherapy plus ADT

Some comparisons of brachytherapy to other therapies were only evaluated in studies rated high ROB (e.g., AS vs. brachytherapy [k=1 non-RCT], ${ }^{52}$ AS vs. radiation [either EBRT and/or brachytherapy][k=1 non-RCT] ${ }^{24,}{ }^{27} \mathrm{RP}$ vs. brachytherapy [k=2 non-RCTs], ${ }^{52,}{ }^{99} \mathrm{RP}$ vs. radiation [either EBRT and/or brachytherapy][k=1 non-RCT], ${ }^{24,}{ }^{27}$ ADT vs. radiation [either EBRT and/or brachytherapy] $\left[\mathrm{k}=1\right.$ non-RCT] ${ }^{24,27}$ and watchful waiting (WW) vs. radiation [either EBRT and/or brachytherapy][k=1 non-RCT]). ${ }^{24,27}$

There were several comparisons of brachytherapy to other therapies addressed in the 2016 evidence report commissioned by the AUA in which we did not identify any additional studies that met our analysis criteria published after this report. ${ }^{5}$ A list of these comparisons can be found in Appendix J. The 2016 evidence report and appendices contains detailed results, strength of evidence, and evidence tables for these comparisons. 


\section{Chapter 9. Key Questions 2-4}

\section{KQ 2: How do patient characteristics modify comparative effectiveness and harms of CLPC therapies?}

We systematically searched for evidence on the patient characteristics that might impact the relative effectiveness of the treatment modalities of interest. We found limited information that met our predefined inclusion criteria related to the characteristic of patients' age. We found limited information from one RCT of watchful waiting (WW) vs. radical prostatectomy (RP) in men with mainly clinically detected clinically localized prostate cancer (CLPC) that the effect of interventions may have varied by age but did not vary by race/ethnicity, comorbidity status or health status. We found limited information that the effects of active monitoring (AM) versus either RP or external beam radiation therapy (EBRT) plus androgen deprivation therapy (ADT) did not vary by age. We found limited information that the benefit of EBRT plus ADT versus EBRT alone on mortality in intermediate risk disease may only be in men with no or minimal comorbidity. Available information for these secondary analyses is presented in the primary analyses of the specific comparisons.

\section{KQ 3: How do tumor characteristics modify comparative effectiveness and harms of CLPC therapies?}

We searched for evidence on a potential effect modifying effect of several tumor related prognostic variables including baseline prostate specific antigen (PSA), Gleason score, tumor index scores (such as D’Amico and National Comprehensive Cancer Network [NCCN] risk categories) and biomarkers. We also provide specific information, where available, regarding tumor eligibility criteria and baseline risk status among enrollees from studies to permit interpretation of applicability of the overall study findings. Additionally, when available, findings of secondary analyses were presented in the context of the primary analyses. We found no evidence that met our predefined inclusion criteria for the newer prognostic (proprietary) biomarkers such as Decipher, Oncotype Dx and Prolaris as it relates to comparative effectiveness modification. Evidence suggested that the effect of radical prostatectomy versus watchful waiting on all-cause and prostate cancer mortality among men with mainly clinically detected CLPC may be limited to men with D'Amico intermediate risk disease but that the effect of either RP or EBRT versus AM in men with mostly lower risk PSA-screen detected disease did not vary by baseline tumor stage, PSA level or Gleason score. There was wide variation in the absolute risk of prostate cancer death and the absolute treatment effect overall as well as across similar D'Amico tumor risk characteristics between the two studies. Absolute prostate-cancer and metastatic events and absolute risk differences between WW and RP were much greater in the Scandinavian Prostate Cancer Group-4 (SPCG-4) trial than the Prostate Intervention Versus Observation Trial (PIVOT). Many trials of whole gland therapy with radiation enrolled men with higher risk CLPC but rarely reported subgroup findings by tumor (or patient) factors. Post-hoc analysis suggested the benefit of EBRT plus ADT versus EBRT on prostate-cancer mortality may only be in men with intermediate and high-risk disease, but not in low-risk men. 


\section{KQ 4: How do provider/hospital characteristics modify comparative effectiveness of RP compared with other therapies?}

We found no information about potential effect modification for variables such as geographic region, hospital type, provider volume and institutional volume to inform this review. 


\section{Chapter 10. Discussion}

\section{Key Findings}

We provide information newly published since the previous Agency for Healthcare Research and Quality (AHRQ) and American Urological Association (AUA) funded reviews. We summarize key findings from newly published reports, incorporate information from past reviews when applicable, and refer readers to key findings from prior reports for intervention comparisons addressed previously and not by our report. With few exceptions, these new findings provide little additional information on previously reported comparisons and outcomes to alter previously assessed effect magnitude or certainty. Our report provides new information on longer followup or other outcomes from comparisons published earlier or from different intervention/comparison combinations.

An important contribution of this updated report lies in its' critical appraisal of newer and longer-term data from two trials (SPCG-4 and PIVOT) that have informed the comparison of radical prostatectomy (RP) to watchful waiting (WW). Extended follow-up suggests that RP may reduce mortality and probably reduces metastases over a very extended time frame. Age and tumor risk category may be important effect modifiers. However, these benefits are only realized over a very extended time frame, and tumor risk category appears to be an important effect modifier. Specifically, prostate cancer mortality is infrequent or rare in men with low-risk disease, and the effect of RP on all-cause or prostate cancer mortality may be limited to men with D’Amico intermediate risk disease. Many patients with clinically localized prostate cancer (CLPC) who are treated with WW may avoid prostate-cancer-related morbidity or mortality for an extended period time, thereby also avoiding treatment-related side-effects. Supporting these findings is new information from the ProtecT trial that enrolled prostate specific antigen (PSA)screen-detected men and, irrespective of treatment arm, observed few prostate cancer related events. ProtecT found that in men with PSA screen detected and primarily lower risk disease, active monitoring (AM) resulted in similar all-cause and prostate-cancer mortality versus RP or external beam radiation therapy (EBRT). Differences in metastases were small in absolute terms.

Comparisons of SPCG-4, PIVOT and ProtecT from the pre-PSA, early PSA, and late PSA era, respectively, illustrate the increasing impact of lead time on baseline risk, as well as the increasing concern related to overdiagnosis and overtreatment. Given prostate cancer is now increasingly diagnosed not by digital rectal examination (as in SPCG-4) but through a combination of PSA and its derivatives, other biochemical markers and magnetic resonance imaging (MRI), the absolute benefits derived from treatment are likely smaller than those observed in the three existing trials that have informed this report. Increasing life expectancy is commonly cited as a reason for aggressive local treatment, but recent epidemiological data for the U.S. population contradicts this reasoning, since average male life expectancy is in fact declining. ${ }^{114}$ We recognize that today's surgical approach to prostate cancer has evolved dramatically from past practices, with most patients now undergoing robotic assisted prostatectomy. However, little to no high-quality evidence supports the notion that the benefit-torisk ratio of radical surgery has fundamentally changed with the widespread adoption of robotic surgery. The only published randomized trial of robotic assisted laparoscopic prostatectomy (RALP) versus open RP reported no oncological outcomes, found little to no difference in urinary and sexual quality of life but did demonstrate lower rates of transfusion and a shorter length of stay. ${ }^{113}$ Functional outcomes at 24 months were also similar. ${ }^{112}$ These findings stand in contrast to recent developments in field of medical treatment for advanced prostate cancer with 
high quality trial evidence supporting an increasing role for newer agents such as apalutamide, enzalutamide and abiraterone as highly effective in prolonging progression and/or all-cause and disease-specific survival. ${ }^{115}$

As noted above, this report is the first to include data on PSA-based AM compared to radiation therapy (RT) and RP in the ProtecT trial. AM included routine PSA measurements but no protocol driven surveillance biopsies or MRI. AM is in the middle of spectrum of monitoring intensity options between WW with additional treatments primarily for palliative/symptomatic care and more intensive approaches that involve surveillance MRI and prostate biopsies. After 10 years, a newly expanded definition of metastatic disease and clinical progression favored surgery and radiation by small absolute amounts, but all-cause and prostate-cancer-specific mortality did not differ. However, surgery resulted in worse urinary and sexual function. A main finding of this study was that prostate cancer mortality was rare irrespective of treatment assignment, and that patients were ten times more likely to die of competing other causes than prostate cancer, thereby again emphasizing the importance of appropriate patient selection and meaningful counseling about short- and long-term treatment outcomes. Furthermore, the effect of AM versus RP or EBRT did not vary by baseline age, PSA, tumor stage or Gleason score.

For radiation therapy, we found similar functional outcomes as AM in the ProtecT trial, yet superior functional outcomes compared with RP. This updated report also provides further support that the addition of systemic androgen deprivation therapy (ADT) to EBRT improves oncological outcomes in men with higher risk disease. However, it may also result in a moderate increase in sexual dysfunction. The duration of ADT varied by study, which could confound generalizability of toxicity data. We also found that 3D-CRT plus low-dose rate brachytherapy plus ADT may slightly reduce all-cause mortality but not metastases more than 3D-CRT plus ADT in higher risk CLPC. We found no eligible studies comparing proton beam therapy to other forms of radiotherapy.

This report update was motivated in part by an increasing interest in novel treatment modalities (other than surgery and radiation) applied as whole prostate gland therapy or as focal therapy. For these modalities, which include cryotherapy of index lesion, which is quite widely used in the U.S., as well as high-intensity focused ultrasound (HIFU), which was cleared for use in 2016 for prostate cancer by the Food and Drug Administration (FDA), ${ }^{116}$ we found either no eligible or insufficient evidence. For photodynamic therapy, we found no evidence for oncological outcomes. Although these and other newer modalities hold promise, we need higher quality studies to assess patient important outcomes to guide evidence-based clinical practice.

Our findings have clinical, policy and research implications. Our results highlight the importance of balancing treatment benefits with harms and the inclusion of patient and tumor characteristics as well as patient preferences into treatment decisions. They reinforce the need for long-term comparative effectiveness RCTs and well-designed prospective cohort studies. They highlight that the more indolent natural history of PSA detected compared with clinically detected CLPC has important implications on net benefit of treatment options. For most men with CLPC including those with life expectancies of 15-20 years, evidence indicates that WW and AM result in little to no difference in mortality and metastases and fewer harms compared with early intent-to-cure treatments. Any mortality benefit due to early intervention may be limited to men age $<65$ years and men with intermediate risk disease. Few men with low risk disease develop systemic spread or die from prostate cancer. Overtreatment and harms could be avoided with greater implementation of WW and/or AM. The absolute benefit of early intervention in PSA detected CLPC is likely considerably less and overtreatment greater than 
studies of WW and AM suggest. This is particularly important for practice and policy decisions because most men currently diagnosed with CLPC have PSA-screen detected disease and most are over age $65 .{ }^{117}$ Many of these men have lower risk disease or have comorbidities that limit life expectancy to less than 20 years. Furthermore, trials of WW and AM were conducted prior to development of effective pharmacologic treatments for men who develop advanced prostate cancer and thus the net benefit from early intervention may be currently lower than that observed prior to the development of these therapies. ${ }^{118}$ For men with PSA detected CLPC who would like to undergo early treatment and who have a long-life expectancy, RP provides similar effects through 10 years compared with EBRT + ADT. For men with higher risk disease who select EBRT, the addition of ADT reduces mortality but may increase harms compared to EBRT alone. Our findings provide a cautionary note before incorporating newer treatment modalities (including refinements of RP or EBRT) into clinical care as evidence on their effectiveness and harms is very limited. While active surveillance (AS) and newer modalities hold promise, we need high quality studies that include an assessment of provider, patient, and tumor characteristics on patient important outcomes.

For men with higher risk disease, while RP may not reduce mortality versus WW, EBRT plus ADT compared to EBRT alone probably reduces mortality but may increase harms. Our updated report also emphasizes the relative lack of, and need for, long-term comparative effectiveness randomized trials and well-designed prospective cohort studies. Particular emphasis should be directed towards evaluating whether known patient and tumor prognostic factors modify comparative treatment outcomes to more accurately guide practice and policy decisions. Our findings also indicate that the incorporation of newer treatment modalities (including refinements of RP or EBRT) into routine clinical care are not convincingly supported by evidence. While AS and newer modalities hold promise, we need additional higher quality studies including assessment of the effect of provider, patient, tumor and biomarker characteristics of these options on patient important outcomes.

\section{Limitations}

A central limitation of this updated systematic review lies in the lack of relevant studies. When studies did exist, their value was frequently limited by methodological and clinical limitations. For many important comparisons, especially as related to newer treatment modalities such as HIFU or photodynamic therapy, we found no evidence for oncological outcomes. For comparisons informed by RCTs, followup was often too short to adequately assess long-term prostate-specific and overall mortality as key outcomes. Whereas metastatic disease was assessed more frequently, this outcome was typically a composite of asymptomatic radiographic findings and PSA elevations (> $100 \mathrm{ng} / \mathrm{ml}$ ) rather than patient-reported, metastases-related complications (such as bone pain or ureteral obstruction). Despite a major interest in focal therapy, we were unable to identify studies that met inclusion criteria for this review. Although we planned to include nonrandomized studies as supplemental evidence for Key Questions since they are usually easier to conduct, most studies were deemed high risk of bias studies, thereby highlighting the importance for future well-designed prospective cohort studies.

Clinical decision-making in the treatment of CLPC is highly influenced by both patient and tumor characteristics, in particular age and comorbidity serving to estimate life expectancy and disease stage most commonly, the D’Amico and National Comprehensive Cancer Network (NCCN) risk categories or the Cancer of the Prostate Risk Assessment Score (CAPRA) score, to predict the natural history of the disease. Evidence for or against any effect modification by these 
variables was included in this report as specific Key Questions whenever it was available; however, in accordance with our predefined methods we did not include a formal assessment of the strength of evidence. All subgroup analyses need to be interpreted with caution however, especially those performed post-hoc. Furthermore, it was not possible to construct a treatment flow pattern for a given index patient based off existing data. Patient agreement to enroll in randomized trials was likely often influenced by patient and provider preference for various treatment options. The best that the data can inform relates mainly to the patient and clinical characteristics commonly seen in men with newly diagnosed prostate cancer. That includes men in their 60s in good to excellent health and with low to moderate risk PSA-screen detected prostate cancer. For these men, WW or AM provides similar long-term overall and prostate cancer mortality and metastatic disease spread with fewer harms compared to early intervention. For older men or those with limited life expectancy due to comorbidities or those wishing to avoid harms of early intervention, WW or AM provides even greater net benefit. For younger men or those desiring early intervention for a potential small reduction in mortality despite harms, than either surgery or EBRT + ADT has supporting evidence. For healthy men with longlife expectancy and with higher risk disease EBRT +ADT appears to have benefits that exceed harms versus EBRT alone. Both EBRT + ADT and surgery probably reduced metastases but may not $r$ reduce overall or prostate cancer mortality through at least 10 years. For men with higher risk clinically detected rather than PSA detected CLPC and with long life expectancy surgery may have mortality and metastases benefits that exceed harms. Importantly, we defined effect sizes as small, moderate, or large based on consensus derived thresholds. Varying absolute risk differences to define benefit and harms thresholds as well as patient and provider values on the magnitude of these differences to determine clinical importance may alter certainty of evidence, assessment of net benefit as well as clinical and policy decisions. Furthermore, while clinical and policy decision making often rely on the effects of treatments based on patient and tumor characteristics, evidence certainty to guide these decisions is limited and unlikely to be greater than findings from intervention effects overall. Similar to the 2014 AHRQ report we found no evidence on the impact of geographic region, surgeon and hospital volume for RP versus other treatments modalities.

\section{Future Research Needs}

This review update highlights the lack of high-quality research that meet the evidentiary standards predefined in this and prior AHRQ reports protocols. New and updated evidence summarized here stems mainly from a few carefully planned RCTs, in particular SPCG-4, PIVOT and ProtecT, which include long-term followup of $10+$ years. Whereas much has there are known challenges of performing clinical trials in CLPC due to its protracted and relatively indolent disease course and the lack of widely accepted surrogate outcome measures, these issues are inherent to the disease itself and therefore relevant to clinical decision making. A search of clinicaltrials.gov failed to find completed trials whose results have not been published in peerreviewed journals. We also searched for large (planned enrollment $>300$ ) ongoing RCTs of nonpharmacological interventions. We identified approximately 30 ongoing trials that may be of sufficient size and duration to provide oncological outcomes in addition to harms and quality of life information (Appendix Table K-1). Fewer than 10 of these studies are scheduled for completion prior to 2025. However, ongoing studies are likely to contribute greatly to our understanding and include comparative effectiveness studies of surgery versus percutaneous radiation implant versus active surveillance for low to intermediate risk CLPC; radical versus 
focal therapy plus pharmacological therapies; proton versus photon EBRT; laparoscopic versus conventional RP and other comparative treatments of radical treatments. Almost all are being conducted outside of the United States. Large studies within the US are needed and should include AS and recruit sufficiently to report on subgroups or prognostic factors current interest.

Specific issues for future research include the following:

- What is long-term comparative effectiveness of RP and RT for treating screen-detected men with prostate cancer stratified by tumor risk category and patients' characteristics (such as competing medical comorbidities), and how do outcomes compare with WW?

- What is the comparative effectiveness of contemporary AS, including surveillance biopsies and MRI-imaging, compared with WW stratified by tumor risk category and patient characteristics? Whereas the therapeutic burden of AS for patients should be less than that of surgery and radiation, it may nevertheless contribute to the issue of overtreatment in those men who are unlikely to experience prostate-cancer related morbidity and mortality during their lifetime.

- What is the comparative effectiveness, harms and costs of different radiation therapies, including proton beam therapy, given the variation in treatment time and capital expense of various therapies.

- Evolving newer treatment modalities for CLPC, especially as they relate to the paradigm of focal therapy, should undergo more formal research evaluation up front. Despite the promise of similar outcomes and a potentially more favorable side-effect profile, their current role remains poorly defined.

- Defining clinically meaningful absolute risk difference to set thresholds for small, moderate and large effects and how these might alter clinical and policy decisions.

- A number of commercially available blood, urine, and tissue-based biomarkers have been proposed not only as prognostic tools but also to guide to treatment management decisions and determine comparative effectiveness. However, none met inclusion criteria, thereby emphasizing the importance of their rigorous, prospective evaluation.

- Given the favorable long-term outcomes of deferred management in the form of AS or WW, future research should focus on identifying those men with intermediate- and highrisk disease who are most likely to benefit from treatment.

- Given the importance of patient and tumor characteristics on clinical decision-making, these should be routinely reported in a standardized manner and studies either adequately powered to assess these subgroups or specifically focus on high-priority groups. Secondary analyses based on these variables should considered $a$ priori.

- There is an imperative for high quality studies that would meet inclusion criteria of this report to assess the plausible impact of geographic region, provider and institution volume on comparative treatment outcomes. 


\section{Conclusion}

This systematic review update focused on information newly published since prior Agency for Healthcare Research and Quality (AHRQ) and American Urological Association (AUA) funded reviews. As applicable, we incorporated findings from prior reviews when they and our report identified RCTs that addressed the same comparison and refer readers to past reviews for intervention comparisons only addressed in prior reviews. We also describe how are findings compare and contrast to these two reviews, provide practice and policy implications of the results, and targeted suggestions for future research. We found that compared to watchful waiting, radical prostatectomy may reduce overall and prostate cancer mortality and metastatic spread at 20+ years followup in men with clinically localized prostate cancer not detected by prostate specific antigen (PSA) screening. Mortality benefits may be limited to men under age 65 years and those with intermediate risk disease. Radical prostatectomy probably resulted in increased urinary, sexual, and erectile dysfunction. There is no information on the effect of any early intervention strategies versus watchful waiting in men with PSA-detected prostate cancer. Compared to PSA-based active monitoring, neither radical prostatectomy nor external beam radiation reduce overall or prostate cancer mortality through 10 years regardless of patient or tumor risk characteristic, and both are associated with increased harms. External beam radiation therapy plus androgen deprivation therapy reduces mortality and metastases versus EBRT alone but is associated with worse sexual function. Treatment with three-dimensional conformal radiation therapy (3D-CRT) and androgen deprivation therapy (ADT) with low-dose-rate prostate brachytherapy (LDR-BT) may provide a small reduction in all-cause mortality versus 3D-CRT and ADT in higher risk disease but may have little to no effect on metastatic disease. The evidence is absent or insufficient to assess the comparative effectiveness and harms of many other therapies and comparisons, particularly their effect on long-term outcomes including overall and prostate cancer mortality and metastatic disease. Patient age and tumor risk may modify the effect of radical prostatectomy versus watchful waiting (WW) in men with clinically localized prostate cancer (CLPC) not detected by PSA screening with mortality benefits limited to younger men and those with intermediate-risk disease. The information on comparative effectiveness and harms should be incorporated into practice and policy decisions and patient informed decision materials. Large, long-term randomized trials in men with PSA detected CLPC are needed, particularly in light of the known more indolent nature of PSA detected CLPC, the widespread use of MRI assessment for tumor identification, and characterization, and the availability of effective medical treatments for the minority of men with CLPC who develop advanced disease if not treated with early options. 
3D-CRT

ADT

AHRQ

AM

ARD

AS

ASCENDE-RT

AUA

CAPRA

CI

CLPC

COE

EBRT

EORTC

EORTC QLQ-C2

EORTC QLQ-C30

EORTC QLQ-C33

FDA

GRADE

HAROW

HDR-BT

HIFU

IIEF-5

IMRT

$\mathrm{KQ}$

LDR

LDR-PB

LHRH

LRP

MRI

NCCN

OR

\section{Abbreviations and Acronyms}

three-dimensional conformal radiation therapy

androgen deprivation therapy

Agency for Healthcare Research and Quality

Active Monitoring

absolute risk difference

Active Surveillance

Androgen Suppression Combined with Elective Nodal and Dose Escalated Radiation Therapy

American Urological Association BTinterstitial brachytherapy

Cancer of the Prostate Risk Assessment Score

confidence interval

clinically localized prostate cancer

certainty of evidence

external beam radiation therapy

European Organization for Research and Treatment of Cancer

European Organization for Research and Treatment of Cancer Quality-ofLife Questionnaire-Core 25 module

European Organization for Research and Treatment of Cancer Quality-ofLife Questionnaire-Core 30 module

European Organization for Research and Treatment of Cancer Quality-ofLife Questionnaire-Core 33 module

U.S. Food and Drug Administration

Grading of Recommendations Assessment, Development and Evaluation

Hormonal therapy, Active Surveillance, Radiation, Operation, Watchful Waiting Study

high dose rate brachytherpay

high-intensity focused ultrasound

International Index of Erectile Function

intensity modulated radiation therapy

Key Question

low dose rate

low-dose-rate prostate brachytherapy

luteinizing hormone-releasing hormone

laparoscopic radical prostatectomy

magnetic resonance imaging

National Comprehensive Cancer Network

odds ratio 
PART

PDT

PICOTS

PIVOT

PRIMSA

PSA

QOL

RALP

RALRP

RARP

RCT

RD

ROB

RP

RR

RT

RRP

SBRT

SF-12

SMD

SPCG

T1

T2

T3

T4

VMAT

WMD

WW
Partial prostate Ablation versus Radical prosTatectomy

photodynamic therapy

population, intervention, comparison, outcomes, timing, and setting/study design

Prostate Cancer Intervention Versus Observation Trial

Preferred Items for Reporting in Systematic Reviews and Meta-Analyses

prostate-specific antigen

quality of life

Robotic assisted laparoscopic prostatectomy

robotic-assisted laparoscopic radical prostatectomy

Robotic assisted radical prostatectomy

randomized controlled trial

risk difference

risk of bias

radical prostatectomy

risk ratios

radiation therapy

open retropubic radical prostatectomy

stereotactic body radiation therapy

Medical Outcomes Study 12-Item Short-Form General Health Survey

standardized mean difference

Scandinavian Prostate Cancer Group

tumor Stage 1

tumor Stage 2

tumor Stage 3

tumor Stage 4

volumetric-modulated arc therapy

weighted mean differences

watchful waiting 


\section{References}

1. Siegel RL, Miller KD, Jemal A. Cancer statistics, 2020. CA Cancer J Clin. 2020 Jan;70(1):730. doi: https://doi.org/10.3322/caac.21590. PMID: 30620402.

2. Buyyounouski MK, Choyke PL, McKenney JK, et al. Prostate cancer - major changes in the American Joint Committee on Cancer eighth edition cancer staging manual. CA Cancer J Clin. 2017 May 6;67(3):245-53. doi: 10.3322/caac.21391. PMID: 28222223.

3. Wilt TJ, MacDonald R, Rutks I, et al. Systematic review: comparative effectiveness and harms of treatments for clinically localized prostate cancer. Ann Intern Med. 2008 Mar 18;148(6):435-48. PMID: 18252677.

4. Sun F, Oyesanmi O, Fontanarosa J, et al. Therapies for Clinically Localized Prostate Cancer: Update of a 2008 Systematic Review. Rockville (MD): 2014. https://www.ncbi.nlm.nih.gov/pubmed/2561 $\underline{0935}$

5. Fontanarosa J, Treadwell JR. Clinically Localized Prostate Cancer Evidence Report ECRI Institute,. Plymouth Meeting, PA: 2016.

6. Higgins JPT, Altman D, Sterne J. Chapter 8: Assessing risk of bias in included studies. In: Higgins JPT, Green S, eds. Cochrane Handbook for Systematic Reviews of Interventions: Version 510. The Cochrane Collaboration; 2011. Available from www.training.cochrane.org/handbook.

7. Sterne JA, Hernan MA, Reeves BC, et al. ROBINS-I: a tool for assessing risk of bias in non-randomised studies of interventions. BMJ. 2016 Oct 12;355:i4919. doi: https://dx.doi.org/10.1136/bmj.i4919. PMID: 27733354.

8. Guyatt GH, Oxman AD, Kunz R, et al. What is "quality of evidence" and why is it important to clinicians? BMJ. 2008 May 3;336(7651):995-8. doi: 10.1136/bmj.39490.551019.BE. PMID: 18456631.

9. Cornford P, Bellmunt J, Bolla M, et al. EAUESTRO-SIOG Guidelines on Prostate Cancer. Part II: Treatment of Relapsing, Metastatic, and Castration-Resistant Prostate Cancer. Eur Urol. 2017 Apr;71(4):630-42. doi: 10.1016/j.eururo.2016.08.002. PMID: 27591931.
10. Sanda MG, Cadeddu JA, Kirkby E, et al. Clinically Localized Prostate Cancer: AUA/ASTRO/SUO Guideline. Part I: Risk Stratification, Shared Decision Making, and Care Options. J Urol. 2018 Mar;199(3):68390. doi: 10.1016/j.juro.2017.11.095. PMID: 29203269.

11. Lee DJ, Barocas DA, Zhao Z, et al. Contemporary prostate cancer radiation therapy in the United States: Patterns of care and compliance with quality measures. Pract Radiat Oncol. 2018 Sep - Oct;8(5):307-16. doi: 10.1016/j.prro.2018.04.009. PMID: 30177030.

12. Azzouzi AR, Vincendeau S, Barret E, et al. Padeliporfin vascular-targeted photodynamic therapy versus active surveillance in men with low-risk prostate cancer (CLIN1001 PCM301): an open-label, phase 3, randomised controlled trial. Lancet Oncol. 2017 Feb;18(2):181-91. doi: 10.1016/S1470-2045(16)30661-1. PMID: 28007457.

13. Jung JH, Risk MC, Goldfarb R, et al. Primary cryotherapy for localised or locally advanced prostate cancer. Cochrane Database Syst Rev. 2018 May 30;5:CD005010. doi: 10.1002/14651858.CD005010.pub3. PMID: 29845595.

14. Warmuth M, Johansson T, Mad P. Systematic review of the efficacy and safety of highintensity focussed ultrasound for the primary and salvage treatment of prostate cancer. Eur Urol. 2010 Dec;58(6):803-15. doi: 10.1016/j.eururo.2010.09.009. PMID: 20864250.

15. Ahmed HU, El-Shater Bosaily A, Brown LC, et al. Diagnostic accuracy of multi-parametric MRI and TRUS biopsy in prostate cancer (PROMIS): a paired validating confirmatory study. Lancet. 2017 Feb 25;389(10071):81522. doi: 10.1016/S0140-6736(16)32401-1. PMID: 28110982.

16. Moher D, Liberati A, Tetzlaff J, et al. Preferred reporting items for systematic reviews and meta-analyses: the PRISMA statement. Ann Intern Med. 2009 Aug 18;151(4):264-9, W64. doi: 10.7326/0003-4819-151-4200908180-00135. PMID: 19622511. 
17. Fu R, Gartlehner G, Grant M, et al. Conducting quantitative synthesis when comparing medical interventions: AHRQ and the Effective Health Care Program. J Clin Epidemiol. 2011 Nov;64(11):1187-97. PMID: 21477993.

18. [Software]. GGGGDT. McMaster University: (devleoped by Evidence Prime, Inc.) Available from gradepro.org; 2015.

19. Santesso N, Glenton C, Dahm P, et al. GRADE guidelines 26: Informative statements to communicate the findings of systematic reviews of interventions. J Clin Epidemiol. 2019 Nov 08;08:08. doi: https://dx.doi.org/10.1016/j.jclinepi.2019.10. 014. PMID: 31711912.

20. Bill-Axelson A, Holmberg L, Garmo H, et al. Radical prostatectomy or watchful waiting in early prostate cancer. N Engl J Med. 2014 Mar 06;370(10):932-42. PMID: 24597866.

21. Bill-Axelson A, Holmberg L, Garmo H, et al. Radical Prostatectomy or Watchful Waiting in Prostate Cancer - 29-Year Follow-up. N Engl J Med. 201812 13;379(24):2319-29. PMID: 30575473.

22. Wilt TJ, Jones KM, Barry MJ, et al. Follow-up of Prostatectomy versus Observation for Early Prostate Cancer. N Engl J Med. 201707 13;377(2):132-42. PMID: 28700844.

23. Dell'Oglio P, Boehm K, Trudeau V, et al. Survival After Conservative Management Versus External Beam Radiation Therapy in Elderly Patients With Localized Prostate Cancer. International Journal of Radiation Oncology Biology Physics. 201601 Dec;96(5):1037-45. PMID: 611451436.

24. Herden J, Ansmann L, Ernstmann N, et al. The Treatment of Localized Prostate Cancer in Everyday Practice in Germany. Dtsch. 2016 May 13;113(19):329-36. PMID: 27232362.

25. Hoffman RM, Lo M, Clark JA, et al. Treatment Decision Regret Among Long-Term Survivors of Localized Prostate Cancer: Results From the Prostate Cancer Outcomes Study. Journal of Clinical Oncology. 2017 Jul 10;35(20):2306-14. PMID: 28493812.
26. Lu-Yao GL, Kim S, Moore DF, et al. Primary radiotherapy vs conservative management for localized prostate cancer - A populationbased study. Prostate Cancer and Prostatic Diseases. 201501 Dec;18(4):317-24. PMID: 604966551.

27. Weissbach L, Stuerzebecher S, Mumperow E, et al. HAROW: the first comprehensive prospective observational study comparing treatment options in localized prostate cancer. World J Urol. 2016 May;34(5):6417. PMID: 26373955.

28. Wilt TJ. Management of low risk and low PSA prostate cancer: long term results from the prostate cancer intervention versus observation trial. Recent Results Cancer Res. 2014;202:149-69. doi: https://dx.doi.org/10.1007/978-3-64245195-9_18. PMID: 24531789.

29. Bill-Axelson A, Garmo H, Holmberg L, et al. Long-term distress after radical prostatectomy versus watchful waiting in prostate cancer: a longitudinal study from the Scandinavian Prostate Cancer Group-4 randomized clinical trial. European Urology. 2013 Dec;64(6):920-8. doi: https://dx.doi.org/10.1016/j.eururo.2013.02. 025. PMID: 23465517.

30. Wilt TJ, Brawer MK, Jones KM, et al. Radical prostatectomy versus observation for localized prostate cancer. N Engl J Med. 2012 Jul 19;367(3):203-13. doi: https://dx.doi.org/10.1056/NEJMoa1113162. PMID: 22808955.

31. Wilt TJ. The Prostate Cancer Intervention Versus Observation Trial: VA/NCI/AHRQ Cooperative Studies Program \#407 (PIVOT): design and baseline results of a randomized controlled trial comparing radical prostatectomy with watchful waiting for men with clinically localized prostate cancer. J Natl Cancer Inst Monogr. 2012 Dec;2012(45):184-90. doi: https://dx.doi.org/10.1093/jncimonographs/l gs041. PMID: 23271771. 
32. Holmberg L, Bill-Axelson A, Steineck G, et al. Results from the Scandinavian Prostate Cancer Group Trial Number 4: a randomized controlled trial of radical prostatectomy versus watchful waiting. $\mathrm{J}$

Natl Cancer Inst Monogr. 2012 Dec;2012(45):230-3. doi: https://dx.doi.org/10.1093/jncimonographs/l gs025. PMID: 23271778.

33. Johansson E, Steineck G, Holmberg L, et al. Long-term quality-of-life outcomes after radical prostatectomy or watchful waiting: the Scandinavian Prostate Cancer Group-4 randomised trial. Lancet Oncology. 2011 Sep;12(9):891-9. doi: https://dx.doi.org/10.1016/S14702045(11)70162-0. PMID: 21821474.

34. Bill-Axelson A, Holmberg L, Ruutu M, et al. Radical prostatectomy versus watchful waiting in early prostate cancer. $\mathrm{N}$ Engl J Med. 2011 May 05;364(18):1708-17. doi: https://dx.doi.org/10.1056/NEJMoa1011967. PMID: 21542742.

35. Wilt TJ, Brawer MK, Barry MJ, et al. The Prostate cancer Intervention Versus Observation Trial:VA/NCI/AHRQ Cooperative Studies Program \#407 (PIVOT): design and baseline results of a randomized controlled trial comparing radical prostatectomy to watchful waiting for men with clinically localized prostate cancer. Contemp Clin Trials. 2009 Jan;30(1):81-7. doi: https://dx.doi.org/10.1016/j.cct.2008.08.002. PMID: 18783735.

36. Johansson E, Bill-Axelson A, Holmberg L, et al. Time, symptom burden, androgen deprivation, and self-assessed quality of life after radical prostatectomy or watchful waiting: the Randomized Scandinavian Prostate Cancer Group Study Number 4 (SPCG-4) clinical trial. European Urology. 2009 Feb;55(2):422-30. doi: https://dx.doi.org/10.1016/j.eururo.2008.08. 054. PMID: 18783877.
37. Bill-Axelson A, Holmberg L, Filen F, et al. Radical prostatectomy versus watchful waiting in localized prostate cancer: the Scandinavian prostate cancer group-4 randomized trial. Journal of the National Cancer Institute. 2008 Aug 20;100(16):1144-54. doi: https://dx.doi.org/10.1093/jnci/djn255. PMID: 18695132.

38. Bill-Axelson A, Holmberg L, Ruutu M, et al. Radical prostatectomy versus watchful waiting in early prostate cancer. N Engl J Med. 2005 May 12;352(19):1977-84. PMID: 15888698.

39. Wilt TJ, Brawer MK. The Prostate Cancer Intervention Versus Observation Trial (PIVOT). Oncology (Williston). 1997 Aug;11(8):1133-9; discussion 9-40, 43. PMID: 9268976.

40. Wilt TJ, Brawer MK. Early intervention or expectant management for prostate cancer. The Prostate Cancer Intervention Versus Observation Trial (PIVOT): a randomized trial comparing radical prostatectomy with expectant management for the treatment of clinically localized prostate cancer. Semin Urol. 1995 May;13(2):130-6. PMID: 7638470 .

41. Wilt TJ, Brawer MK. The Prostate Cancer Intervention Versus Observation Trial: a randomized trial comparing radical prostatectomy versus expectant management for the treatment of clinically localized prostate cancer. Journal of Urology. 1994 Nov;152(5 Pt 2):1910-4. PMID: 7523736.

42. Donovan JL, Hamdy FC, Lane JA, et al. PatientReported Outcomes after Monitoring, Surgery, or Radiotherapy for Prostate Cancer. N Engl J Med. 2016 Oct 13;375(15):1425-37. doi: https://dx.doi.org/10.1056/NEJMoa1606221. PMID: 27626365.

43. Hamdy FC, Donovan JL, Lane JA, et al. 10-Year Outcomes after Monitoring, Surgery, or Radiotherapy for Localized Prostate Cancer. N Engl J Med. 201610 13;375(15):1415-24. PMID: 27626136. 
44. Lane JA, Donovan JL, Davis M, et al. Active monitoring, radical prostatectomy, or radiotherapy for localised prostate cancer: study design and diagnostic and baseline results of the ProtecT randomised phase 3 trial. Lancet Oncology. 2014

Sep;15(10):1109-18. doi: https://dx.doi.org/10.1016/S14702045(14)70361-4. PMID: 25163905.

45. Lane A, Metcalfe C, Young GJ, et al. Patientreported outcomes in the ProtecT randomized trial of clinically localized prostate cancer treatments: study design, and baseline urinary, bowel and sexual function and quality of life. BJU Int. 2016 Dec;118(6):869-79. doi: 10.1111/bju.13582. PMID: 27415448.

46. Neal DE, Metcalfe C, Donovan JL, et al. Tenyear Mortality, Disease Progression, and Treatment-related Side Effects in Men with Localised Prostate Cancer from the ProtecT Randomised Controlled Trial According to Treatment Received. Eur Urol. 2020 Mar;77(3):320-30. doi: 10.1016/j.eururo.2019.10.030. PMID: 31771797.

47. Barocas DA, Chen V, Cooperberg M, et al. Using a population-based observational cohort study to address difficult comparative effectiveness research questions: The CEASAR study. Journal of Comparative Effectiveness Research. 2013 July;2(4):44560. doi: http://dx.doi.org/10.2217/cer.13.34. PMID: 369311498.

48. Tosoian JJ, Sundi D, Trock BJ, et al. Pathologic Outcomes in Favorable-risk Prostate Cancer: Comparative Analysis of Men Electing Active Surveillance and Immediate Surgery. European Urology. 2016 Apr;69(4):576-81. PMID: 26456680.

49. Tyson MD, Alvarez J, Koyama T, et al. Racial Variation in Patient-Reported Outcomes Following Treatment for Localized Prostate Cancer: Results from the CEASAR Study. European Urology. 2017 08;72(2):307-14. PMID: 27816300.
50. Ansmann L, Winter N, Ernstmann N, et al. Health-related quality of life in active surveillance and radical prostatectomy for low-risk prostate cancer: a prospective observational study (HAROW - Hormonal therapy, Active Surveillance, Radiation, Operation, Watchful Waiting). BJU Int. 2018 Sep;122(3):401-10. PMID: 29603553.

51. Barocas DA, Alvarez J, Resnick MJ, et al. Association Between Radiation Therapy, Surgery, or Observation for Localized Prostate Cancer and Patient-Reported Outcomes After 3 Years. Jama. 201703 21;317(11):1126-40. PMID: 28324093.

52. Hoffman KE, Penson DF, Zhao Z, et al. PatientReported Outcomes Through 5 Years for Active Surveillance, Surgery, Brachytherapy, or External Beam Radiation With or Without Androgen Deprivation Therapy for Localized Prostate Cancer. Jama. 2020 Jan 14;323(2):149-63. doi: 10.1001/jama.2019.20675. PMID: 31935027.

53. Thomsen FB, Roder MA, Jakobsen H, et al. Active Surveillance Versus Radical Prostatectomy in Favorable-risk Localized Prostate Cancer. Clin Genitourin Cancer. 2019 Aug;17(4):e814-e21. doi: 10.1016/j.clgc.2019.05.005. PMID: 31196798.

54. Abugharib AE, Dess RT, Soni PD, et al. External beam radiation therapy with or without lowdose-rate brachytherapy: Analysis of favorable and unfavorable intermediate-risk prostate cancer patients. Brachytherapy. 2017 Jul - Aug;16(4):782-9. PMID: 28499487.

55. Amini A, Jones BL, Jackson MW, et al. Survival outcomes of combined external beam radiotherapy and brachytherapy vs. brachytherapy alone for intermediate-risk prostate cancer patients using the National Cancer Data Base. Brachytherapy. 2016 Mar-Apr;15(2):136-46. PMID: 26825856.

56. Ashamalla H, Guirguis A, McCool K, et al. Brachytherapy improves outcomes in young men ( $<=60$ years) with prostate cancer: A SEER analysis. Brachytherapy. 201701 Mar;16(2):323-9. PMID: 614251483. 
57. Evans JR, Zhao S, Daignault S, et al. Patientreported quality of life after stereotactic body radiotherapy (SBRT), intensity modulated radiotherapy (IMRT), and brachytherapy. Radiother Oncol. 2015 Aug;116(2):179-84. PMID: 26276528.

58. Jackson MW, Amini A, Jones BL, et al. Prostate brachytherapy, either alone or in combination with external beam radiation, is associated with longer overall survival in men with favorable pathologic Group 4 (Gleason score 8) prostate cancer. Brachytherapy. 2017 July;16(4):790-6. PMID: 615636725.

59. Jiang R, Tomaszewski JJ, Ward KC, et al. The burden of overtreatment: comparison of toxicity between single and combined modality radiation therapy among low risk prostate cancer patients. The Canadian journal of urology. 201501 Feb;22(1):764855. PMID: 607086080.

60. Lee DJ, Barocas DA, Zhao Z, et al. Comparison of Patient-reported Outcomes After External Beam Radiation Therapy and Combined External Beam With Low-dose-rate Brachytherapy Boost in Men With Localized Prostate Cancer. International Journal of Radiation Oncology, Biology, Physics. 2018 Sep 01;102(1):116-26. PMID: 30102188.

61. Morris WJ, Tyldesley S, Rodda S, et al. Androgen Suppression Combined with Elective Nodal and Dose Escalated Radiation Therapy (the ASCENDE-RT Trial): An Analysis of Survival Endpoints for a Randomized Trial Comparing a LowDose-Rate Brachytherapy Boost to a DoseEscalated External Beam Boost for Highand Intermediate-risk Prostate Cancer. International Journal of Radiation Oncology, Biology, Physics. 201706 01;98(2):275-85. PMID: 28262473.

62. Muralidhar V, Xiang M, Orio PF, et al. Brachytherapy boost and cancer-specific mortality in favorable high-risk versus other high-risk prostate cancer. J. 2016 Feb;8(1):1-6. PMID: 26985191.
63. Ricco A, Hanlon A, Lanciano R. Propensity score matched comparison of intensity modulated radiation therapy vs stereotactic body radiation therapy for localized prostate cancer: A survival analysis from the national cancer database. Frontiers in Oncology. 201731 Aug;7 (AUG) (no pagination)(185). PMID: 618033192.

64. Rodda S, Morris WJ, Hamm J, et al. ASCENDERT: An Analysis of Health-Related Quality of Life for a Randomized Trial Comparing Low-Dose-Rate Brachytherapy Boost With Dose-Escalated External Beam Boost for High- and Intermediate-Risk Prostate Cancer. International Journal of Radiation Oncology, Biology, Physics. 201707 01;98(3):581-9. PMID: 28581398.

65. Rodda S, Tyldesley S, Morris WJ, et al. ASCENDE-RT: An Analysis of TreatmentRelated Morbidity for a Randomized Trial Comparing a Low-Dose-Rate Brachytherapy Boost with a Dose-Escalated External Beam Boost for High- and Intermediate-Risk Prostate Cancer. International Journal of Radiation Oncology, Biology, Physics. 2017 06 01;98(2):286-95. PMID: 28433432.

66. Smith GD, Pickles T, Crook J, et al. Brachytherapy improves biochemical failure-free survival in low- and intermediate-risk prostate cancer compared with conventionally fractionated external beam radiation therapy: A propensity score matched analysis. International Journal of Radiation Oncology Biology Physics. 2015 01 Mar;91(3):505-16. PMID: 601554874.

67. Tward JD, Jarosek S, Chu H, et al. Time Course and Accumulated Risk of Severe Urinary Adverse Events After High- Versus LowDose-Rate Prostate Brachytherapy With or Without External Beam Radiation Therapy. International Journal of Radiation Oncology, Biology, Physics. 201608 01;95(5):144353. PMID: 27325475.

68. Vargas C, Schmidt M, Jr H, et al. Initial toxicity, quality-of-life outcomes, and dosimetric impact in a randomized phase 3 trial of hypofractionated versus standard fractionated proton therapy for low-risk prostate cancer. Advances in radiation oncology. 2018;3(3):322-30. PMID: CN01611807. 
69. Vargas CE, Alam NB, Terk M, et al. Initial results of a randomized phase III trial of high dose image guided radiation with or without androgen deprivation therapy for intermediate-risk prostate cancer. Cancer Treatment and Research Communications. 201901 Jan;19 (no pagination)(100119). PMID: 2001573236.

70. Viani GA, Viana BS, Martin JE, et al. Intensitymodulated radiotherapy reduces toxicity with similar biochemical control compared with 3-dimensional conformal radiotherapy for prostate cancer: A randomized clinical trial. Cancer. 2016 Jul 01;122(13):2004-11. PMID: 27028170.

71. Xiang M, Nguyen PL. Significant association of brachytherapy boost with reduced prostate cancer-specific mortality in contemporary patients with localized, unfavorable-risk prostate cancer. Brachytherapy. 2015 NovDec;14(6):773-80. PMID: 26489921.

72. Yang DD, Muralidhar V, Nguyen PL, et al. Lack of Benefit From the Addition of External Beam Radiation Therapy to Brachytherapy for Intermediate- and High-risk Prostate Cancer. International Journal of Radiation Oncology, Biology, Physics. 201711 15;99(4):904-11. PMID: 29063853.

73. Bekelman JE, Mitra N, Handorf EA, et al. Effectiveness of androgen-deprivation therapy and radiotherapy for older men with locally advanced prostate cancer. Journal of Clinical Oncology. 201501 Mar;33(7):71622. PMID: 602911329.

74. Bolla M, Maingon P, Carrie C, et al. Short Androgen Suppression and Radiation Dose Escalation for Intermediate- and High-Risk Localized Prostate Cancer: Results of EORTC Trial 22991. Journal of Clinical Oncology. 201605 20;34(15):1748-56. PMID: 26976418.

75. Fossa SD, Wiklund F, Klepp O, et al. Ten- and 15-yr Prostate Cancer-specific Mortality in Patients with Nonmetastatic Locally Advanced or Aggressive Intermediate Prostate Cancer, Randomized to Lifelong Endocrine Treatment Alone or Combined with Radiotherapy: Final Results of The Scandinavian Prostate Cancer Group-7. European Urology. 2016 10;70(4):684-91. PMID: 27025586.
76. McPartlin AJ, Glicksman R, Pintilie M, et al. PMH 9907: Long-term outcomes of a randomized phase 3 study of short-term bicalutamide hormone therapy and doseescalated external-beam radiation therapy for localized prostate cancer. Cancer. 2016 Aug 15;122(16):2595-603. PMID: 27219522.

77. Weller MA, Kupelian PA, Reddy CA, et al. Adjuvant versus neoadjuvant androgen deprivation with radiotherapy for prostate cancer: Does sequencing matter? Clinical Genitourinary Cancer. 201501 Jun;13(3):e183-e9. PMID: 602262362.

78. Giacalone NJ, Wu J, Chen MH, et al. Prostatespecific antigen failure and risk of death within comorbidity subgroups among men with unfavorable-risk prostate cancer treated in a randomized trial. Journal of Clinical Oncology. 201601 Nov;34(31):3781-6. PMID: 612965014.

79. McDuff SGR, Chen MH, Renshaw AA, et al. Impact of time to testosterone rebound and comorbidity on the risk of cause-specific mortality in men with unfavorable-risk prostate cancer. Cancer. $2018 \mathrm{Apr}$ 01;124(7):1391-9. PMID: 29338073.

80. Phillips JG, Chen MH, Zhang D, et al. Percent positive biopsy cores and the risk of death from prostate cancer in men with unfavorable-risk prostate cancer. Journal of Radiation Oncology. 201401 Sep;3(3):30712. PMID: 603283685.

81. Malone S, Roy S, Eapen L, et al. Sequencing of Androgen-Deprivation Therapy With External-Beam Radiotherapy in Localized Prostate Cancer: A Phase III Randomized Controlled Trial. J Clin Oncol. 2020 Feb 20;38(6):593-601. doi: 10.1200/JCO.19.01904. PMID: 31829912.

82. Widmark A, Gunnlaugsson A, Beckman L, et al. Ultra-hypofractionated versus conventionally fractionated radiotherapy for prostate cancer: 5-year outcomes of the HYPO-RT-PC randomised, non-inferiority, phase 3 trial. Lancet. 2019 Aug 3;394(10196):385-95. doi: 10.1016/S01406736(19)31131-6. PMID: 31227373. 
83. Goy BW, Burchette R, Soper MS, et al. Ten-Year Treatment Outcomes of Radical Prostatectomy Vs External Beam Radiation Therapy Vs Brachytherapy for 1503 Patients With Intermediate-risk Prostate Cancer. Urology. 2020 Feb;136:180-9. doi: 10.1016/j.urology.2019.09.040. PMID: 31704459 .

84. Lennernas B, Majumder K, Damber JE, et al. Radical prostatectomy versus high-dose irradiation in localized/locally advanced prostate cancer: A Swedish multicenter randomized trial with patient-reported outcomes. Acta Oncologica. 201501 Jun;54(6):875-81. PMID: 604399742.

85. Carlsson S, Drevin L, Loeb S, et al. Populationbased study of long-term functional outcomes after prostate cancer treatment. BJU Int. 2016 Jun;117(6B):E36-45. doi: 10.1111/bju.13179. PMID: 25959859.

86. Cooperberg MR, Vickers AJ, Broering JM, et al. Comparative risk-adjusted mortality outcomes after primary surgery, radiotherapy, or androgen-deprivation therapy for localized prostate cancer. Cancer. 2010 Nov 15;116(22):5226-34. doi: 10.1002/cncr.25456. PMID: 20690197.

87. Prasad SM, Eggener SE, Lipsitz SR, et al. Effect of depression on diagnosis, treatment, and mortality of men with clinically localized prostate cancer. J Clin Oncol. 2014 Aug 10;32(23):2471-8. doi: 10.1200/JCO.2013.51.1048. PMID: 25002728.

88. Denham JW, Steigler A, Lamb DS, et al. Shortterm androgen deprivation and radiotherapy for locally advanced prostate cancer: results from the Trans-Tasman Radiation Oncology Group 96.01 randomised controlled trial. Lancet Oncol. 2005 Nov;6(11):841-50. doi: 10.1016/S1470-2045(05)70348-X. PMID: 16257791.

89. Jones CU, Hunt D, McGowan DG, et al. Radiotherapy and short-term androgen deprivation for localized prostate cancer. $\mathrm{N}$ Engl J Med. 2011 Jul 14;365(2):107-18. doi: 10.1056/NEJMoa1012348. PMID: 21751904.
90. Wirth MP, See WA, McLeod DG, et al. Bicalutamide $150 \mathrm{mg}$ in addition to standard care in patients with localized or locally advanced prostate cancer: results from the second analysis of the early prostate cancer program at median followup of 5.4 years. J Urol. 2004 Nov;172(5 Pt 1):1865-70. doi: 10.1097/01.ju.0000140159.94703.80. PMID: 15540740.

91. D'Amico AV, Chen MH, Renshaw AA, et al. Androgen suppression and radiation vs radiation alone for prostate cancer: a randomized trial. Jama. 2008 Jan 23;299(3):289-95. doi: https://dx.doi.org/10.1001/jama.299.3.289. PMID: 18212313.

92. D'Amico AV, Manola J, Loffredo M, et al. 6month androgen suppression plus radiation therapy vs radiation therapy alone for patients with clinically localized prostate cancer: a randomized controlled trial. Jama. 2004 Aug 18;292(7):821-7. doi: 10.1001/jama.292.7.821. PMID: 15315996.

93. Falchook AD, Basak R, Mohiuddin JJ, et al. Evaluation of the effectiveness of adding androgen deprivation to modern doseescalated radiotherapy for men with favorable intermediate-risk prostate cancer. Cancer. 201608 01;122(15):2341-9. PMID: 27191936.

94. Wu AK, Cooperberg MR, Sadetsky N, et al. Health related quality of life in patients treated with multimodal therapy for prostate cancer. J Urol. 2008 Dec;180(6):2415-22; discussion 22. doi: 10.1016/j.juro.2008.08.015. PMID: 18930279.

95. Widmark A, Klepp O, Solberg A, et al. Endocrine treatment, with or without radiotherapy, in locally advanced prostate cancer (SPCG-7/SFUO-3): an open randomised phase III trial. Lancet. 2009 Jan 24;373(9660):301-8. doi: https://dx.doi.org/10.1016/S01406736(08)61815-2. PMID: 19091394.

96. Hamdy FC, Elliott D, le Conte S, et al. Partial ablation versus radical prostatectomy in intermediate-risk prostate cancer: the PART feasibility RCT. Health Technol Assess. 2018 09;22(52):1-96. PMID: 30264692. 
97. Porpiglia F, Fiori C, Bertolo R, et al. Five-year Outcomes for a Prospective Randomised Controlled Trial Comparing Laparoscopic and Robot-assisted Radical Prostatectomy. Eur Urol Focus. 2018 01;4(1):80-6. PMID: 28753822.

98. Sooriakumaran P, Pini G, Nyberg T, et al. Erectile Function and Oncologic Outcomes Following Open Retropubic and Robotassisted Radical Prostatectomy: Results from the LAParoscopic Prostatectomy Robot Open Trial. European Urology. 2018 04;73(4):618-27. PMID: 28882327.

99. Chang P, Regan MM, Ferrer M, et al. Relief of Urinary Symptom Burden after Primary Prostate Cancer Treatment. Journal of Urology. 2017 02;197(2):376-84. PMID: 27593476.

100. Herlemann A, Cowan JE, Carroll PR, et al. Community-based Outcomes of Open versus Robot-assisted Radical Prostatectomy. European Urology. 2018 02;73(2):215-23. PMID: 28499617.

101. Loeb S, Meyer CP, Krasnova A, et al. Risk of Small Bowel Obstruction After RobotAssisted vs Open Radical Prostatectomy. Journal of Endourology. 2016 12;30(12):1291-5. PMID: 27615204.

102. Zheng X, Jin K, Qiu S, et al. Focal Laser Ablation Versus Radical Prostatectomy for Localized Prostate Cancer: Survival Outcomes From a Matched Cohort. Clin Genitourin Cancer. 2019 Dec;17(6):464-9 e3. doi: 10.1016/j.clgc.2019.08.008. PMID: 31594734.

103. Knipper S, Pecoraro A, Palumbo C, et al. A 25year Period Analysis of Other-cause Mortality in Localized Prostate Cancer. Clin Genitourin Cancer. 2019 Oct;17(5):395-401. doi: 10.1016/j.clgc.2019.07.008. PMID: 31416752.

104. Berlin A, Ahmad AE, Chua MLK, et al. Curative Radiation Therapy at Time of Progression Under Active Surveillance Compared With Up-front Radical Radiation Therapy for Prostate Cancer. International Journal of Radiation Oncology, Biology, Physics. 201803 01;100(3):702-9. PMID: 29249526.
105. Fossa SD, Nilssen Y, Kvale R, et al. Treatment and 5-year survival in patients with nonmetastatic prostate cancer: The Norwegian experience. Urology. 2014 January;83(1):146-52. PMID: 52860957.

106. Fridriksson JO, Folkvaljon Y, Lundstrom KJ, et al. Long-term adverse effects after retropubic and robot-assisted radical prostatectomy. Nationwide, populationbased study. Journal of Surgical Oncology. 201715 Sep;116(4):500-6. PMID: 616678085

107. Gershman B, Psutka SP, McGovern FJ, et al. Patient-reported Functional Outcomes Following Open, Laparoscopic, and Robotic Assisted Radical Prostatectomy Performed by High-volume Surgeons at High-volume Hospitals. Eur Urol Focus. 2016 Jun;2(2):172-9. PMID: 28723533.

108. Robinson D, Garmo H, Lissbrant IF, et al. Prostate Cancer Death After Radiotherapy or Radical Prostatectomy: A Nationwide Population-based Observational Study. European Urology. 2018 04;73(4):502-11. PMID: 29254629.

109. Tyson MD, Koyama, T L, et al. Effect of Prostate Cancer Severity on Functional Outcomes After Localized Treatment: Comparative Effectiveness Analysis of Surgery and Radiation Study Results. European Urology. 2018 07;74(1):26-33. PMID: 29501451.

110. Porpiglia F, Morra I, Lucci Chiarissi M, et al. Randomised controlled trial comparing laparoscopic and robot-assisted radical prostatectomy. Eur Urol. 2013 Apr;63(4):606-14. doi: 10.1016/j.eururo.2012.07.007. PMID: 22840353.

111. Partin AW, Mangold LA, Lamm DM, et al. Contemporary update of prostate cancer staging nomograms (Partin Tables) for the new millennium. Urology. 2001 Dec;58(6):843-8. doi: 10.1016/s00904295(01)01441-8. PMID: 11744442. 
112. Coughlin GD, Yaxley JW, Chambers SK, et al. Robot-assisted laparoscopic prostatectomy versus open radical retropubic prostatectomy: 24-month outcomes from a randomised controlled study. Lancet Oncol. 2018 Aug;19(8):1051-60. doi: 10.1016/S1470-2045(18)30357-7. PMID: 30017351.

113. Yaxley JW, Coughlin GD, Chambers SK, et al. Robot-assisted laparoscopic prostatectomy versus open radical retropubic prostatectomy: early outcomes from a randomised controlled phase 3 study. Lancet. 2016 Sep 10;388(10049):1057-66. doi: 10.1016/S0140-6736(16)30592-X. PMID: 27474375.

114. Woolf SH, Schoomaker H. Life Expectancy and Mortality Rates in the United States, 19592017. Jama. 2019 Nov 26;322(20):19962016. doi: 10.1001/jama.2019.16932. PMID: 31769830.
115. Lowrance WT, Murad MH, Oh WK, et al. Castration-Resistant Prostate Cancer: AUA Guideline Amendment 2018. J Urol. 2018 Dec;200(6):1264-72. doi: 10.1016/j.juro.2018.07.090. PMID: 30086276.

116. Babalola O, Lee T-HJ, Viviano CJ. Prostate ablation using high intensity focused ultrasound: A literature review of the potential role for patient preference information. The Journal of urology. 2018;200(3):512-9. doi: 10.1016/j.juro.2018.04.066. PMID: 29702099.

117. Cancer.net Editorial Board. Prostate Cancer: Statistics. 2020.

https://www.cancer.net/cancertypes/prostate-cancer/statistics. Accessed on 5/19/2020.

118. Welch HG, Albertsen PC. Reconsidering Prostate Cancer Mortality - The Future of PSA Screening. N Engl J Med. 2020 Apr 16;382(16):1557-63. doi: 10.1056/NEJMms1914228. PMID: 32294352. 


\section{Appendix A. Analytic Framework}

Figure A-1. Analytical framework for therapies for clinically localized prostate cancer

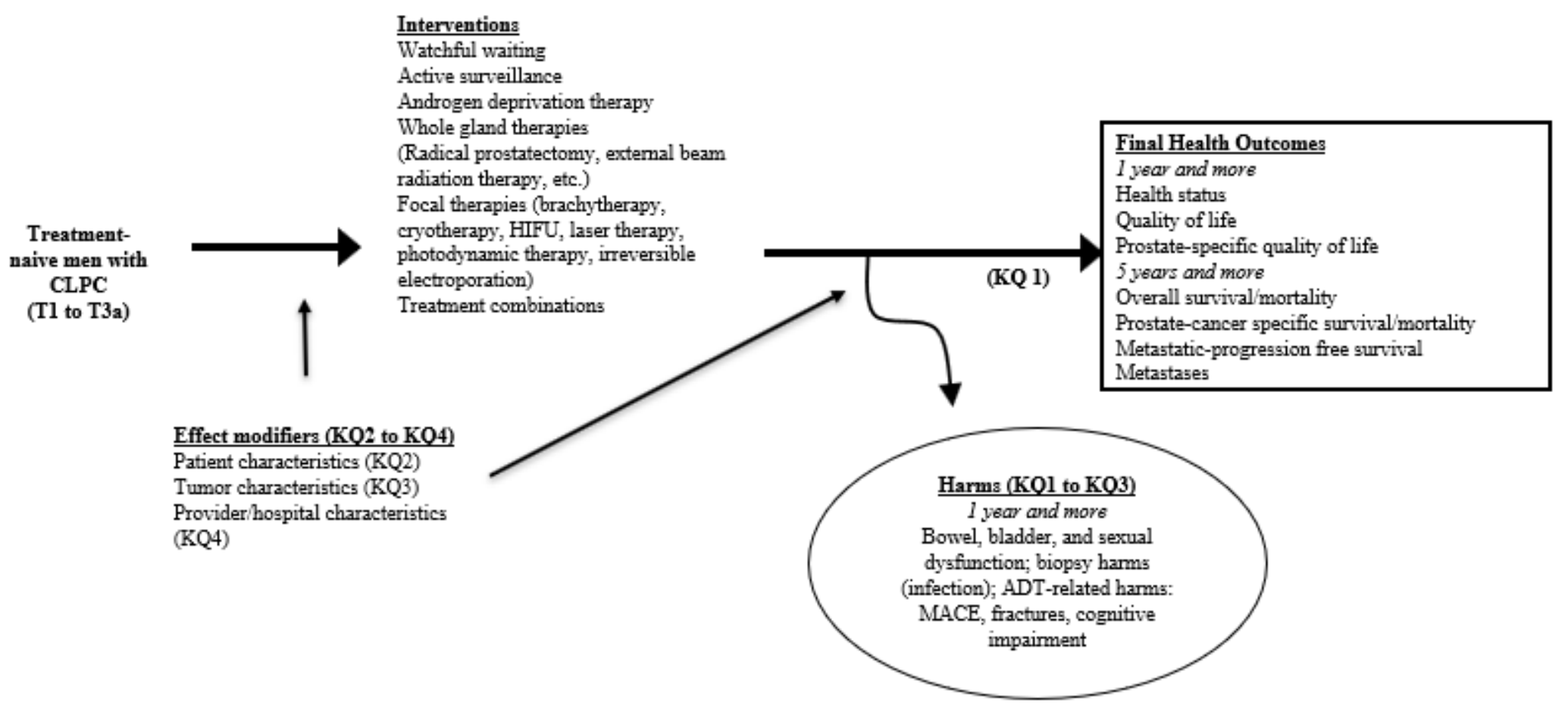




\section{Ovid MEDLINE(R)}

\section{Appendix B. Search Strategies}

1 exp Prostatic Neoplasms/ (118593)

2 (prostat* and (neoplasm* or cancer* or carcinoma*)).ti,ab. (138807)

3 watchful waiting.ti,ab. (2358)

4 active surveillance.ti,ab. (6438)

5 LRP.ti,ab. (3633)

6 RLRP.ti,ab. (48)

7 prostatectom*.ti,ab. (28151)

8 radiotherap*.ti,ab. (156325)

9 EBRT.ti,ab. (2794)

10 IMRT.ti,ab. (8646)

11 proton.ti,ab. (94523)

12 (intensity and modulated and therap*).ti,ab. (7296)

13 brachytherap*.ti,ab. (16219)

14 curietherap*.ti,ab. (455)

15 cryosurger*.ti,ab. (3377)

16 cryotherap*.ti,ab. (6755)

17 cryoablat*.ti,ab. (3229)

18 Cyberknife.ti,ab. (1201)

19 freezing.ti,ab. (32530)

20 androgen deprivation.ti,ab. (6870)

21 HIFU.ti,ab. (2087)

22 (high and intensity and focused and ultrasound*).ti,ab. (2812)

231 or $2(160965)$

243 or 4 or 5 or 6 or 7 or 8 or 9 or 10 or 11 or 12 or 13 or 14 or 15 or 16 or 17 or 18 or 19 or 20 or 21 or 22 (350793)

2523 and 24 (39445)

26 limit 25 to (clinical trial, all or clinical trial, phase i or clinical trial, phase ii or clinical trial, phase iii or clinical trial, phase iv or clinical trial or comparative study or evaluation studies or meta analysis or multicenter study or observational study or pragmatic clinical trial or randomized controlled trial) (8330)

27 (clinical trial* or comparative stud* evaluation stud*).tw. (326592)

28 ((sing ${ }^{*}$ or doubl* or trebl* or trip|*) and (mask* or blind $\left.\left.{ }^{\star}\right)\right)$.tw. (181405)

29 (latin square or placebo or random or control group or prospective* or retrospective* or volunteer* or sham).tw. (2158358)

30 (meta?analysis or cohort or ISRCTN* or ACTRN* or NCT*).tw. (516377)

3127 or 28 or 29 or 30 (2702617) 
$32 \quad 25$ and $31(12356)$

3326 or $32(17063)$

34 limit 33 to years="2014 -Current" (6117)

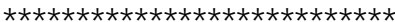

\section{Embase Classic + Embase}

1 Prostatic Neoplasms/ (8570)

2 (prostat\$.ti,ab. or Prostate/) and (cancer.ti,ab. or Neoplasms/ or neoplasm\$.mp. or carcinoma\$.mp.) [mp=title, abstract, heading word, drug trade name, original title, device manufacturer, drug manufacturer, device trade name, keyword, floating subheading word, candidate term word] (209978)

31 or $2(212135)$

4 watchful waiting.ti,ab. or Watchful Waiting/ or active surveillance.ti,ab. or prostatectom\$.ti,ab. or Prostatectomy/ or LRP.ti,ab. or RLRP.ti,ab. or exp Radiation therapy/ or radiotherap\$.ti,ab. or EBRT.ti,ab. or IMRT.ti,ab. or proton.ti,ab. or brachytherap\$.ti,ab. or Brachytherapy/ or curietherap\$.ti,ab. or cryosurger\$.ti,ab. or Cryosurgery/ or cryotherap\$.ti,ab. or Cyberknife.ti,ab. or Cryotherapy/ or cryoablat\$.ti,ab. or Freezing/ or freez\$.ti,ab. or androgen deprivation.ti,ab. or High-Intensity Focused Ultrasound Ablation/ or high intensity focused ultrasound.ti,ab. or HIFU.ti,ab. or (high and intensity and focused and ultrasound).ti,ab. (904076)

5 Randomized controlled trial/ or random allocation/ or double-blind method/ or single-blind method/ or placebos/ or cross-over studies/ or crossover procedure/ or cross over studies/ or double blind procedure/ or single blind procedure/ or placebo/ or latin square design/ or crossover design/ or doubleblind studies/ or single-blind studies/ or triple-blind studies/ or random assignment/ or exp clinical trial/ or exp comparative study/ or cohort analysis.mp. or followup studies/ or intermethod comparison/ or parallel design/ or control group/ or prospective study/ or retrospective study/ or case control study/ or major clinical study/ or evaluation studies/ or followup studies/ or case series.ti,ab. or random\$.hw. or random $\$$.ti. or placebo \$.ti,ab. or ((singl\$ or doubl\$ or tripl\$ or trebl\$) and (dummy or blind or sham)).ti,ab. or latin square.ti,ab. or ISRCTN\$.ti,ab. or ACTRN\$.ti,ab. or (NCT\$ not NCT).ti,ab. [mp=title, abstract, heading word, drug trade name, original title, device manufacturer, drug manufacturer, device trade name, keyword, floating subheading word, candidate term word] (6606139)

$6 \quad 3$ and 4 and 5 (33678)

76 not ((letter or editorial or news or comment or case reports or note or conference paper).de. or (letter or editorial or news or comment or case reports).pt.) (32195)

87 not (book/ or edited book/ or case report/ or case reports/ or comment/ or conference abstract/ or conference paper/ or conference review/ or editorial/ or letter/ or news/ or note/ or proceeding/ or (book or edited book or case report or case reports or comment or conference or editorial or letter or news or note or proceeding).pt.) (23732)

98 not (case report.de. or case reports.pt. or case report.ti. or (year adj old).ti,ab.) (23699) 
10 limit 9 to (english language and years="2014 -Current") (8173)

1110 and compar\$.ti,hw. (1454)

1210 and (clinically adj local\$).mp. [mp=title, abstract, heading word, drug trade name, original title, device manufacturer, drug manufacturer, device trade name, keyword, floating subheading word, candidate term word] (387)

1310 and (stage 1 or stage one).mp. [mp=title, abstract, heading word, drug trade name, original title, device manufacturer, drug manufacturer, device trade name, keyword, floating subheading word, candidate term word] (9)

1410 and (early adj3 stage).mp. [mp=title, abstract, heading word, drug trade name, original title, device manufacturer, drug manufacturer, device trade name, keyword, floating subheading word, candidate term word] (102)

1510 and (nonmetastatic or non-metastatic).mp. [mp=title, abstract, heading word, drug trade name, original title, device manufacturer, drug manufacturer, device trade name, keyword, floating subheading word, candidate term word] (234)

1610 and (gleason 7 or gleason score 7 or gleason 6 or gleason score 6).mp. [mp=title, abstract, heading word, drug trade name, original title, device manufacturer, drug manufacturer, device trade name, keyword, floating subheading word, candidate term word] (490)

1710 and (local\$ adj advanced).mp. [mp=title, abstract, heading word, drug trade name, original title, device manufacturer, drug manufacturer, device trade name, keyword, floating subheading word, candidate term word] (239)

1810 and (9T3 or T4).mp. [mp=title, abstract, heading word, drug trade name, original title, device manufacturer, drug manufacturer, device trade name, keyword, floating subheading word, candidate term word] (86)

1910 and (high adj risk).mp. [mp=title, abstract, heading word, drug trade name, original title, device manufacturer, drug manufacturer, device trade name, keyword, floating subheading word, candidate term word] (1484)

2011 or 12 or 13 or 14 or 15 or 16 or 17 or 18 or $19(3580)$

\section{Cochrane}

1-prostat* AND (neoplasm* OR cancer* OR carcinoma*)

2-"watchful waiting" OR "active surveillance" OR LRP OR RLRP OR prostatectom* OR radiotherap* OR EBRT OR IMRT OR proton OR (intensity AND modulated AND therap*) OR brachytherap* OR curietherap* OR cryosurger* OR cryotherap* OR cryoablat* OR Cyberknife OR freezing OR "androgen deprivation" OR HIFU OR (high AND intensity AND focused AND ultrasound*)

3-1 and 2

4- limit 3 to: publication date from 2014 to 2019 


\section{Appendix C. Certainty of Evidence Effect Size Language}

Table C-1. Effect size and certainty of evidence narrative language

\begin{tabular}{|c|c|c|c|c|}
\hline $\begin{array}{l}\text { Certainty of } \\
\text { Evidence }\end{array}$ & $\begin{array}{l}\text { Size of the } \\
\text { Effect } \\
\text { Estimate }\end{array}$ & $\begin{array}{l}\text { Absolute } \\
\text { Risk } \\
\text { Difference } \\
\text { Between } \\
\text { Groups: } \\
\text { Mortality } \\
\text { PC Specific } \\
\text { Mortality } \\
\text { Mets }\end{array}$ & $\begin{array}{l}\text { Absolute } \\
\text { Risk } \\
\text { Difference } \\
\text { Between } \\
\text { Groups: } \\
\text { Harms } \\
\text { (urinary, } \\
\text { bowel, } \\
\text { sexual } \\
\text { dysfunction) }\end{array}$ & $\begin{array}{l}\text { Suggested Statements } \\
\text { (replace } X \text { with intervention, replace } \\
\text { 'reduce/increase' with direction of } \\
\text { effect, replace 'outcome' with name of } \\
\text { outcome, include 'when compared } \\
\text { with } Y \text { ' when needed) }\end{array}$ \\
\hline \multirow[t]{4}{*}{$\begin{array}{l}\text { HIGH Certainty } \\
\text { of Evidence }\end{array}$} & Large effect & $\geq 10 \%$ & $\geq 20 \%$ & $\begin{array}{l}\mathrm{X} \text { results in a large reduction/increase in } \\
\text { outcome }\end{array}$ \\
\hline & Moderate effect & $5-9.9 \%$ & $5-19.9 \%$ & $\begin{array}{l}\mathrm{X} \text { results in a moderate reduction/increase in } \\
\text { outcome }\end{array}$ \\
\hline & $\begin{array}{l}\text { Small important } \\
\text { effect }\end{array}$ & $2-4.9 \%$ & $2-4.9 \%$ & $\begin{array}{l}\mathrm{X} \text { results in a small reduction/increase in } \\
\text { outcome }\end{array}$ \\
\hline & $\begin{array}{l}\text { Small } \\
\text { unimportant } \\
\text { effect }\end{array}$ & $<2.0 \%$ & $<2.0 \%$ & $\mathrm{X}$ results in little to no difference in outcome \\
\hline \multirow{4}{*}{$\begin{array}{l}\text { MODERATE } \\
\text { Certainty of } \\
\text { Evidence }\end{array}$} & Large effect & $\geq 10 \%$ & $\geq 20 \%$ & $\begin{array}{l}\mathrm{X} \text { probably results in a large } \\
\text { reduction/increase in outcome }\end{array}$ \\
\hline & Moderate effect & $5-9.9 \%$ & $5-19.9 \%$ & $\begin{array}{l}\mathrm{X} \text { probably results in a moderate } \\
\text { reduction/increase in outcome }\end{array}$ \\
\hline & $\begin{array}{l}\text { Small important } \\
\text { effect }\end{array}$ & $2-4.9 \%$ & $2-4.9 \%$ & $\begin{array}{l}\mathrm{X} \text { probably results in a small } \\
\text { reduction/increase in outcome }\end{array}$ \\
\hline & $\begin{array}{l}\text { Small } \\
\text { unimportant } \\
\text { effect }\end{array}$ & $<2.0 \%$ & $<2.0 \%$ & $\begin{array}{l}X \text { probably results in little to no difference in } \\
\text { outcome }\end{array}$ \\
\hline \multirow[t]{4}{*}{$\begin{array}{l}\text { LOW Certainty } \\
\text { of Evidence }\end{array}$} & Large effect & $\geq 10 \%$ & $\geq 20 \%$ & $\begin{array}{l}\mathrm{X} \text { may result in a large reduction/increase in } \\
\text { outcome }\end{array}$ \\
\hline & Moderate effect & $5-9.9 \%$ & $5-19.9 \%$ & $\begin{array}{l}\mathrm{X} \text { may result in a moderate reduction/increase } \\
\text { in outcome }\end{array}$ \\
\hline & $\begin{array}{l}\text { Small important } \\
\text { effect }\end{array}$ & $2-4.9 \%$ & $2-4.9 \%$ & $\begin{array}{l}\mathrm{X} \text { may result in a small reduction/increase in } \\
\text { outcome }\end{array}$ \\
\hline & $\begin{array}{l}\text { Small } \\
\text { unimportant } \\
\text { effect }\end{array}$ & $<2.0 \%$ & $<2.0 \%$ & $\begin{array}{l}\mathrm{X} \text { may result in little to no difference in } \\
\text { outcome }\end{array}$ \\
\hline $\begin{array}{l}\text { INSUFFICIENT } \\
\text { Certainty of } \\
\text { Evidence }\end{array}$ & \multicolumn{4}{|c|}{ The evidence is very uncertain about the effect of $X$ on outcome } \\
\hline
\end{tabular}




\section{Appendix D. Eligible Studies}

1. Abugharib AE, Dess RT, Soni PD, et al. External beam radiation therapy with or without lowdose-rate brachytherapy: Analysis of favorable and unfavorable intermediate-risk prostate cancer patients. Brachytherapy. 2017 Jul - Aug;16(4):782-9. PMID: 28499487.

2. Amini A, Jones BL, Jackson MW, et al. Survival outcomes of combined external beam radiotherapy and brachytherapy vs. brachytherapy alone for intermediate-risk prostate cancer patients using the National Cancer Data Base. Brachytherapy. 2016 Mar-Apr;15(2):136-46. PMID: 26825856.

3. Ansmann L, Winter N, Ernstmann N, et al. Healthrelated quality of life in active surveillance and radical prostatectomy for low-risk prostate cancer: a prospective observational study (HAROW - Hormonal therapy, Active Surveillance, Radiation, Operation, Watchful Waiting). BJU Int. 2018 Sep;122(3):401-10. PMID: 29603553.

4. Ashamalla H, Guirguis A, McCool K, et al. Brachytherapy improves outcomes in young men ( $<=60$ years) with prostate cancer: A SEER analysis. Brachytherapy. 201701 Mar;16(2):323-9. PMID: 614251483.

5. Azzouzi AR, Vincendeau S, Barret E, et al. Padeliporfin vascular-targeted photodynamic therapy versus active surveillance in men with low-risk prostate cancer (CLIN1001 PCM301): an open-label, phase 3, randomised controlled trial. Lancet Oncol. 2017 Feb;18(2):181-91. doi: 10.1016/S1470-2045(16)30661-1. PMID: 28007457.

6. Barocas DA, Alvarez J, Resnick MJ, et al. Association Between Radiation Therapy, Surgery, or Observation for Localized Prostate Cancer and Patient-Reported Outcomes After 3 Years. Jama. 201703 21;317(11):1126-40. PMID: 28324093.
7. Barocas DA, Chen V, Cooperberg M, et al. Using a population-based observational cohort study to address difficult comparative effectiveness research questions: The CEASAR study. Journal of Comparative Effectiveness Research. 2013 July;2(4):44560. doi: http://dx.doi.org/10.2217/cer.13.34. PMID: 369311498.

8. Bekelman JE, Mitra N, Handorf EA, et al. Effectiveness of androgen-deprivation therapy and radiotherapy for older men with locally advanced prostate cancer. Journal of Clinical Oncology. 201501 Mar;33(7):71622. PMID: 602911329.

9. Berlin A, Ahmad AE, Chua MLK, et al. Curative Radiation Therapy at Time of Progression Under Active Surveillance Compared With Up-front Radical Radiation Therapy for Prostate Cancer. International Journal of Radiation Oncology, Biology, Physics. 2018 03 01;100(3):702-9. PMID: 29249526.

10. Bill-Axelson A, Holmberg L, Garmo H, et al. Radical prostatectomy or watchful waiting in early prostate cancer. N Engl J Med. 2014 Mar 06;370(10):932-42. PMID: 24597866.

11. Bill-Axelson A, Holmberg L, Garmo H, et al. Radical Prostatectomy or Watchful Waiting in Prostate Cancer - 29-Year Follow-up. N Engl J Med. 201812 13;379(24):2319-29. PMID: 30575473.

12. Bolla M, Maingon P, Carrie C, et al. Short Androgen Suppression and Radiation Dose Escalation for Intermediate- and High-Risk Localized Prostate Cancer: Results of EORTC Trial 22991. Journal of Clinical Oncology. 201605 20;34(15):1748-56. PMID: 26976418.

13. Chang P, Regan MM, Ferrer M, et al. Relief of Urinary Symptom Burden after Primary Prostate Cancer Treatment. Journal of Urology. 2017 02;197(2):376-84. PMID: 27593476. 
14. Dell'Oglio P, Boehm K, Trudeau V, et al. Survival After Conservative Management Versus External Beam Radiation Therapy in Elderly Patients With Localized Prostate Cancer. International Journal of Radiation Oncology Biology Physics. 201601 Dec;96(5):1037-45. PMID: 611451436.

15. Donovan JL, Hamdy FC, Lane JA, et al. PatientReported Outcomes after Monitoring, Surgery, or Radiotherapy for Prostate Cancer. N Engl J Med. 2016 Oct 13;375(15):1425-37. doi: https://dx.doi.org/10.1056/NEJMoa1606221. PMID: 27626365.

16. Evans JR, Zhao S, Daignault S, et al. Patientreported quality of life after stereotactic body radiotherapy (SBRT), intensity modulated radiotherapy (IMRT), and brachytherapy. Radiother Oncol. 2015 Aug;116(2):179-84. PMID: 26276528.

17. Falchook AD, Basak R, Mohiuddin JJ, et al. Evaluation of the effectiveness of adding androgen deprivation to modern doseescalated radiotherapy for men with favorable intermediate-risk prostate cancer. Cancer. 201608 01;122(15):2341-9. PMID: 27191936.

18. Fossa SD, Nilssen Y, Kvale R, et al. Treatment and 5-year survival in patients with nonmetastatic prostate cancer: The Norwegian experience. Urology. 2014 January;83(1):146-52. PMID: 52860957.

19. Fossa SD, Wiklund F, Klepp O, et al. Ten- and 15-yr Prostate Cancer-specific Mortality in Patients with Nonmetastatic Locally Advanced or Aggressive Intermediate Prostate Cancer, Randomized to Lifelong Endocrine Treatment Alone or Combined with Radiotherapy: Final Results of The Scandinavian Prostate Cancer Group-7. European Urology. 2016 10;70(4):684-91. PMID: 27025586.

20. Fridriksson JO, Folkvaljon Y, Lundstrom KJ, et al. Long-term adverse effects after retropubic and robot-assisted radical prostatectomy. Nationwide, populationbased study. Journal of Surgical Oncology. 201715 Sep;116(4):500-6. PMID: 616678085.
21. Gershman B, Psutka SP, McGovern FJ, et al. Patient-reported Functional Outcomes Following Open, Laparoscopic, and Robotic Assisted Radical Prostatectomy Performed by High-volume Surgeons at High-volume Hospitals. Eur Urol Focus. 2016 Jun;2(2):172-9. PMID: 28723533.

22. Giacalone NJ, Wu J, Chen MH, et al. Prostatespecific antigen failure and risk of death within comorbidity subgroups among men with unfavorable-risk prostate cancer treated in a randomized trial. Journal of Clinical Oncology. 201601 Nov;34(31):3781-6. PMID: 612965014.

23. Hamdy FC, Donovan JL, Lane JA, et al. 10-Year Outcomes after Monitoring, Surgery, or Radiotherapy for Localized Prostate Cancer. N Engl J Med. 201610 13;375(15):1415-24. PMID: 27626136.

24. Hamdy FC, Elliott D, le Conte S, et al. Partial ablation versus radical prostatectomy in intermediate-risk prostate cancer: the PART feasibility RCT. Health Technol Assess. 2018 09;22(52):1-96. PMID: 30264692.

25. Herden J, Ansmann L, Ernstmann N, et al. The Treatment of Localized Prostate Cancer in Everyday Practice in Germany. Dtsch. 2016 May 13;113(19):329-36. PMID: 27232362.

26. Herlemann A, Cowan JE, Carroll PR, et al. Community-based Outcomes of Open versus Robot-assisted Radical Prostatectomy. European Urology. 2018 02;73(2):215-23. PMID: 28499617.

27. Hoffman RM, Lo M, Clark JA, et al. Treatment Decision Regret Among Long-Term Survivors of Localized Prostate Cancer: Results From the Prostate Cancer Outcomes Study. Journal of Clinical Oncology. 2017 Jul 10;35(20):2306-14. PMID: 28493812.

28. Jackson MW, Amini A, Jones BL, et al. Prostate brachytherapy, either alone or in combination with external beam radiation, is associated with longer overall survival in men with favorable pathologic Group 4 (Gleason score 8) prostate cancer. Brachytherapy. 2017 July;16(4):790-6. PMID: 615636725. 
29. Jiang R, Tomaszewski JJ, Ward KC, et al. The burden of overtreatment: comparison of toxicity between single and combined modality radiation therapy among low risk prostate cancer patients. The Canadian journal of urology. 201501 Feb;22(1):764855. PMID: 607086080.

30. Lane A, Metcalfe C, Young GJ, et al. Patientreported outcomes in the ProtecT randomized trial of clinically localized prostate cancer treatments: study design, and baseline urinary, bowel and sexual function and quality of life. BJU Int. 2016 Dec;118(6):869-79. doi: 10.1111/bju.13582. PMID: 27415448.

31. Lane JA, Donovan JL, Davis M, et al. Active monitoring, radical prostatectomy, or radiotherapy for localised prostate cancer: study design and diagnostic and baseline results of the ProtecT randomised phase 3 trial. Lancet Oncology. 2014 Sep;15(10):1109-18. doi: https://dx.doi.org/10.1016/S14702045(14)70361-4. PMID: 25163905.

32. Lee DJ, Barocas DA, Zhao Z, et al. Comparison of Patient-reported Outcomes After External Beam Radiation Therapy and Combined External Beam With Low-dose-rate Brachytherapy Boost in Men With Localized Prostate Cancer. International Journal of Radiation Oncology, Biology, Physics. 2018 Sep 01;102(1):116-26. PMID: 30102188.

33. Lennernas B, Majumder K, Damber JE, et al. Radical prostatectomy versus high-dose irradiation in localized/locally advanced prostate cancer: A Swedish multicenter randomized trial with patient-reported outcomes. Acta Oncologica. 201501 Jun;54(6):875-81. PMID: 604399742.

34. Loeb S, Meyer CP, Krasnova A, et al. Risk of Small Bowel Obstruction After RobotAssisted vs Open Radical Prostatectomy. Journal of Endourology. 2016 12;30(12):1291-5. PMID: 27615204.

35. Lu-Yao GL, Kim S, Moore DF, et al. Primary radiotherapy vs conservative management for localized prostate cancer - A populationbased study. Prostate Cancer and Prostatic Diseases. 201501 Dec;18(4):317-24. PMID: 604966551.
36. McDuff SGR, Chen MH, Renshaw AA, et al. Impact of time to testosterone rebound and comorbidity on the risk of cause-specific mortality in men with unfavorable-risk prostate cancer. Cancer. 2018 Apr 01;124(7):1391-9. PMID: 29338073.

37. McPartlin AJ, Glicksman R, Pintilie M, et al. PMH 9907: Long-term outcomes of a randomized phase 3 study of short-term bicalutamide hormone therapy and doseescalated external-beam radiation therapy for localized prostate cancer. Cancer. 2016 Aug 15;122(16):2595-603. PMID: 27219522.

38. Morris WJ, Tyldesley S, Rodda S, et al. Androgen Suppression Combined with Elective Nodal and Dose Escalated Radiation Therapy (the ASCENDE-RT Trial): An Analysis of Survival Endpoints for a Randomized Trial Comparing a LowDose-Rate Brachytherapy Boost to a DoseEscalated External Beam Boost for Highand Intermediate-risk Prostate Cancer. International Journal of Radiation Oncology, Biology, Physics. 201706 01;98(2):275-85. PMID: 28262473.

39. Muralidhar V, Xiang M, Orio PF, et al. Brachytherapy boost and cancer-specific mortality in favorable high-risk versus other high-risk prostate cancer. J. 2016 Feb;8(1):1-6. PMID: 26985191.

40. Phillips JG, Chen MH, Zhang D, et al. Percent positive biopsy cores and the risk of death from prostate cancer in men with unfavorable-risk prostate cancer. Journal of Radiation Oncology. 201401 Sep;3(3):30712. PMID: 603283685.

41. Porpiglia F, Fiori C, Bertolo R, et al. Five-year Outcomes for a Prospective Randomised Controlled Trial Comparing Laparoscopic and Robot-assisted Radical Prostatectomy. Eur Urol Focus. 2018 01;4(1):80-6. PMID: 28753822.

42. Ricco A, Hanlon A, Lanciano R. Propensity score matched comparison of intensity modulated radiation therapy vs stereotactic body radiation therapy for localized prostate cancer: A survival analysis from the national cancer database. Frontiers in Oncology. 201731 Aug;7 (AUG) (no pagination)(185). PMID: 618033192. 
43. Robinson D, Garmo H, Lissbrant IF, et al. Prostate Cancer Death After Radiotherapy or Radical Prostatectomy: A Nationwide Population-based Observational Study. European Urology. 2018 04;73(4):502-11. PMID: 29254629.

44. Rodda S, Morris WJ, Hamm J, et al. ASCENDERT: An Analysis of Health-Related Quality of Life for a Randomized Trial Comparing Low-Dose-Rate Brachytherapy Boost With Dose-Escalated External Beam Boost for High- and Intermediate-Risk Prostate Cancer. International Journal of Radiation Oncology, Biology, Physics. 201707 01;98(3):581-9. PMID: 28581398.

45. Rodda S, Tyldesley S, Morris WJ, et al. ASCENDE-RT: An Analysis of TreatmentRelated Morbidity for a Randomized Trial Comparing a Low-Dose-Rate Brachytherapy Boost with a Dose-Escalated External Beam Boost for High- and Intermediate-Risk Prostate Cancer. International Journal of Radiation Oncology, Biology, Physics. 2017 06 01;98(2):286-95. PMID: 28433432.

46. Smith GD, Pickles T, Crook J, et al. Brachytherapy improves biochemical failure-free survival in low- and intermediate-risk prostate cancer compared with conventionally fractionated external beam radiation therapy: A propensity score matched analysis. International Journal of Radiation Oncology Biology Physics. 2015 01 Mar;91(3):505-16. PMID: 601554874.

47. Sooriakumaran P, Pini G, Nyberg T, et al. Erectile Function and Oncologic Outcomes Following Open Retropubic and Robotassisted Radical Prostatectomy: Results from the LAParoscopic Prostatectomy Robot Open Trial. European Urology. 2018 04;73(4):618-27. PMID: 28882327.

48. Tosoian JJ, Sundi D, Trock BJ, et al. Pathologic Outcomes in Favorable-risk Prostate Cancer: Comparative Analysis of Men Electing Active Surveillance and Immediate Surgery. European Urology. 2016 Apr;69(4):576-81. PMID: 26456680.
49. Tward JD, Jarosek S, Chu H, et al. Time Course and Accumulated Risk of Severe Urinary Adverse Events After High- Versus LowDose-Rate Prostate Brachytherapy With or Without External Beam Radiation Therapy. International Journal of Radiation Oncology, Biology, Physics. 201608 01;95(5):144353. PMID: 27325475.

50. Tyson MD, Alvarez J, Koyama T, et al. Racial Variation in Patient-Reported Outcomes Following Treatment for Localized Prostate Cancer: Results from the CEASAR Study. European Urology. 2017 08;72(2):307-14. PMID: 27816300.

51. Tyson MD, Koyama, T L, et al. Effect of Prostate Cancer Severity on Functional Outcomes After Localized Treatment: Comparative Effectiveness Analysis of Surgery and Radiation Study Results. European Urology. 2018 07;74(1):26-33. PMID: 29501451.

52. Vargas C, Schmidt M, Jr H, et al. Initial toxicity, quality-of-life outcomes, and dosimetric impact in a randomized phase 3 trial of hypofractionated versus standard fractionated proton therapy for low-risk prostate cancer. Advances in radiation oncology. 2018;3(3):322-30. PMID: CN01611807.

53. Vargas CE, Alam NB, Terk M, et al. Initial results of a randomized phase III trial of high dose image guided radiation with or without androgen deprivation therapy for intermediate-risk prostate cancer. Cancer Treatment and Research Communications. 201901 Jan;19 (no pagination)(100119). PMID: 2001573236.

54. Viani GA, Viana BS, Martin JE, et al. Intensitymodulated radiotherapy reduces toxicity with similar biochemical control compared with 3-dimensional conformal radiotherapy for prostate cancer: A randomized clinical trial. Cancer. 2016 Jul 01;122(13):2004-11. PMID: 27028170.

55. Weissbach L, Stuerzebecher S, Mumperow E, et al. HAROW: the first comprehensive prospective observational study comparing treatment options in localized prostate cancer. World J Urol. 2016 May;34(5):6417. PMID: 26373955. 
56. Weller MA, Kupelian PA, Reddy CA, et al. Adjuvant versus neoadjuvant androgen deprivation with radiotherapy for prostate cancer: Does sequencing matter? Clinical Genitourinary Cancer. 201501 Jun;13(3):e183-e9. PMID: 602262362.

57. Wilt TJ, Jones KM, Barry MJ, et al. Follow-up of Prostatectomy versus Observation for Early Prostate Cancer. N Engl J Med. 201707 13;377(2):132-42. PMID: 28700844.

58. Xiang M, Nguyen PL. Significant association of brachytherapy boost with reduced prostate cancer-specific mortality in contemporary patients with localized, unfavorable-risk prostate cancer. Brachytherapy. 2015 NovDec;14(6):773-80. PMID: 26489921.

59. Yang DD, Muralidhar V, Nguyen PL, et al. Lack of Benefit From the Addition of External Beam Radiation Therapy to Brachytherapy for Intermediate- and High-risk Prostate Cancer. International Journal of Radiation Oncology, Biology, Physics. 201711 15;99(4):904-11. PMID: 29063853.

60. Goy BW, Burchette R, Soper MS, et al. Ten-Year Treatment Outcomes of Radical Prostatectomy Vs External Beam Radiation Therapy Vs Brachytherapy for 1503 Patients With Intermediate-risk Prostate Cancer. Urology. 2020 Feb;136:180-9. doi: 10.1016/j.urology.2019.09.040. PMID: 31704459.

61. Hoffman KE, Penson DF, Zhao Z, et al. PatientReported Outcomes Through 5 Years for Active Surveillance, Surgery, Brachytherapy, or External Beam Radiation With or Without Androgen Deprivation Therapy for Localized Prostate Cancer. Jama. 2020 Jan 14;323(2):149-63. doi: 10.1001/jama.2019.20675. PMID: 31935027.
62. Knipper S, Pecoraro A, Palumbo C, et al. A 25year Period Analysis of Other-cause Mortality in Localized Prostate Cancer. Clinical Genitourinary Cancer. 2019 17(5): 395-401. PMID: 31416752.

63. Malone S, Roy S, Eapen L, et al. Sequencing of Androgen-Deprivation Therapy With External-Beam Radiotherapy in Localized Prostate Cancer: A Phase III Randomized Controlled Trial. J Clin Oncol. 2020 Feb 20;38(6):593-601. doi: 10.1200/JCO.19.01904. PMID: 31829912.

64. Neal DE, Metcalfe C, Donovan JL, et al. Tenyear Mortality, Disease Progression, and Treatment-related Side Effects in Men with Localised Prostate Cancer from the ProtecT Randomised Controlled Trial According to Treatment Received. Eur Urol. 2020 Mar;77(3):320-30. doi: 10.1016/j.eururo.2019.10.030. PMID: 31771797.

65. Thomsen FB, Roder MA, Jakobsen H, et al. Active Surveillance Versus Radical Prostatectomy in Favorable-risk Localized Prostate Cancer. Clin Genitourin Cancer. 2019 Aug;17(4):e814-e21. doi: 10.1016/j.clgc.2019.05.005. PMID: 31196798.

66. Widmark A, Gunnlaugsson A, Beckman L, et al. Ultra-hypofractionated versus conventionally fractionated radiotherapy for prostate cancer: 5-year outcomes of the HYPO-RT-PC randomised, non-inferiority, phase 3 trial. Lancet. 2019 Aug 3;394(10196):385-95. doi: 10.1016/S01406736(19)31131-6. PMID: 31227373.

67. Zheng X, Jin K, Qiu S, et al. Focal Laser Ablation Versus Radical Prostatectomy for Localized Prostate Cancer: Survival Outcomes From a Matched Cohort. Clin Genitourin Cancer. 2019 Dec;17(6):464-9 e3. doi: 10.1016/j.clgc.2019.08.008. PMID: 31594734. 


\section{Appendix E. Excluded Studies}

1. Aarts MJ, Koldewijn EL, Poortmans PM, et al. The impact of socioeconomic status on prostate cancer treatment and survival in the Southern Netherlands. Urology. 2013 March;81(3):593-600. PMID: 52388383. Ineligible comparison

2. Aas K, Axcrona K, Kvale R, et al. Ten-year Mortality in Men With Nonmetastatic Prostate Cancer in Norway. Urology. 2017 Dec;110:140-7. PMID: 28823634. Ineligible population

3. Abdel-Rahman O. Outcomes of Prostatectomy versus Radiation Therapy in the Management of Clinically Localized Prostate Cancer Patients Within the PLCO Trial. Clinical Genitourinary Cancer. 2019 Jan 04;04:04. PMID: 30704795. Ineligible population

4. Abdollah F, Karnes RJ, Suardi N, et al. Impact of adjuvant radiotherapy on survival of patients with node-positive prostate cancer. Journal of Clinical Oncology. 201410 Dec;32(35):3939-47. PMID: 600782396. Ineligible population

5. Abdollah F, Sammon JD, Reznor G, et al. Medical androgen deprivation therapy and increased non-cancer mortality in non-metastatic prostate cancer patients aged $>=66$ years. Eur J Surg Oncol. 2015 Nov;41(11):152939. PMID: 26210655. Ineligible comparison

6. Abdollah F, Suardi N, Cozzarini C, et al. Selecting the optimal candidate for adjuvant radiotherapy after radical prostatectomy for prostate cancer: a long-term survival analysis. European Urology. 2013 Jun;63(6):998-1008. PMID: 23122664. Ineligible population

7. Abelson B, Reddy CA, Ciezki JP, et al. Outcomes after photoselective vaporization of the prostate and transurethral resection of the prostate in patients who develop prostatic obstruction after radiation therapy. Urology. 2014 Feb;83(2):422-7. PMID: 24315301. Ineligible intervention

8. Abern MR, Dude AM, Tsivian M, et al. The characteristics of bladder cancer after radiotherapy for prostate cancer. Urol. 2013 Nov;31(8):1628-34. PMID: 22575239. No eligible outcomes reported
9. Adam M, Tennstedt P, Lanwehr D, et al. Functional Outcomes and Quality of Life After Radical Prostatectomy Only Versus a Combination of Prostatectomy with Radiation and Hormonal Therapy. European Urology. 2017 03;71(3):330-6. PMID: 27887941. Ineligible population

10. Adejoro O, Gupta P, Ziegelmann M, et al. Effect of minimally invasive radical prostatectomy in older men. Urol. 2016 May;34(5):234.e111. PMID: 26795606. Ineligible population

11. Ahn S, Lee M, Jeong CW. Comparative qualityadjusted survival analysis between radiation therapy alone and radiation with androgen deprivation therapy in patients with locally advanced prostate cancer: a secondary analysis of Radiation Therapy Oncology Group 85-31 with novel decision analysis methods. Prostate International. 2018 December;6(4):140-4. PMID: 620811351. No eligible outcomes reported

12. Aizer AA, Chen MH, Hattangadi J, et al. Initial management of prostate-specific antigendetected, low-risk prostate cancer and the risk of death from prostate cancer. BJU International. 2014 January;113(1):43-50. Ineligible comparison

13. Akitake N, Shiota M, Obata H, et al. Neoadjuvant androgen-deprivation therapy with radical prostatectomy for prostate cancer in association with age and serum testosterone. Prostate International. 2018 September;6(3):104-9. PMID: 619465299. No eligible outcomes reported

14. Alayed Y, Cheung P, Vesprini D, et al. SABR in High-Risk Prostate Cancer: Outcomes From 2 Prospective Clinical Trials With and Without Elective Nodal Irradiation. International Journal of Radiation Oncology Biology Physics. 2019. Ineligible study design

15. Albertsen P. Randomised controlled trial: radical prostatectomy reduces prostate cancerspecific mortality among men with intermediate-grade disease, but provides minimal benefit for men with low-grade and high-grade disease. Evidence-based medicine. 2014;19(5):176. PMID: CN01050592. Ineligible study design 
16. Albertsen PC, Klotz L, Tombal B, et al. Cardiovascular morbidity associated with gonadotropin releasing hormone agonists and an antagonist. European Urology. 2014 Mar;65(3):565-73. PMID: 24210090. Ineligible comparison

17. Albisinni S, Aoun F, Diamand R, et al. Cytoreductive prostatectomy: what is the evidence? A systematic review. Minerva Urologica e Nefrologica. 2019 Feb;71(1):18. PMID: 30547907. Ineligible study design

18. Albisinni S, Grosman J, Aoun F, et al. Exploring positive surgical margins after minimally invasive radical prostatectomy: Does body habitus really make a difference ? Progres en Urologie. 2018 Jun;28(8-9):434-41. PMID: 29789234. Ineligible population

19. Alexidis P, Guo W, Bekelman JE, et al. Use of high and very high dose radiotherapy after radical prostatectomy for prostate cancer in the United States. Prostate Cancer Prostatic Dis. 2018 11;21(4):584-93. PMID: 30087427. Ineligible population

20. Altay B, Erkurt B, Kiremit MC, et al. A comparison of $120 \mathrm{~W}$ laser photoselective vaporization versus transurethral resection of the prostate for bladder outlet obstruction by prostate cancer. Urol Int. 2015;94(3):326-9. PMID: 25721931. Ineligible population

21. Altok M, Babaian K, Achim MF, et al. Surgeonled prostate cancer lymph node staging: pathological outcomes stratified by robotassisted dissection templates and patient selection. BJU International. 2018 07;122(1):66-75. PMID: 29446205. Ineligible comparison

22. Aluwini S, Pos F, Schimmel E, et al. Hypofractionated versus conventionally fractionated radiotherapy for patients with prostate cancer (HYPRO): late toxicity results from a randomised, non-inferiority, phase 3 trial. Lancet Oncol. 2016 Apr;17(4):464-74. PMID: 26968359. Ineligible population
23. Aluwini S, Pos F, Schimmel E, et al. Hypofractionated versus conventionally fractionated radiotherapy for patients with prostate cancer (HYPRO): Acute toxicity results from a randomised non-inferiority phase 3 trial. The Lancet Oncology. 201501 Mar;16(3):274-83. PMID: 602005707. Ineligible population

24. Amini A, Jones B, Jackson MW, et al. Survival Outcomes of Dose-Escalated External Beam Radiotherapy versus Combined Brachytherapy for Intermediate and High Risk Prostate Cancer Using the National Cancer Data Base. Journal of Urology. 2016 May;195(5):1453-8. PMID: 26576709. Ineligible population

25. Amini A, Jones BL, Yeh N, et al. Survival outcomes of whole-pelvic versus prostateonly radiation therapy for high-risk prostate cancer patients with use of the National Cancer Data Base. International Journal of Radiation Oncology Biology Physics. 2015 01 Dec;93(5):1052-63. PMID: 606885844. Ineligible population

26. Amini A, Rusthoven CG, Jones BL, et al. Survival outcomes of radiotherapy with or without androgen-deprivation therapy for patients with intermediate-risk prostate cancer using the National Cancer Data Base. Urol. 2016 Apr;34(4):165.e1-9. PMID: 26699831. Ineligible population

27. Aoun F, Limani K, Peltier A, et al. High Intensity Focused Ultrasound versus Brachytherapy for the Treatment of Localized Prostate Cancer: A Matched-Pair Analysis. Adv. 2015;2015:350324. PMID: 26357511. Ineligible study design

28. Arcangeli G, Saracino B, Arcangeli S, et al. Moderate Hypofractionation in High-Risk, Organ-Confined Prostate Cancer: Final Results of a Phase III Randomized Trial. Journal of Clinical Oncology. 2017 Jun 10;35(17):1891-7. PMID: 28355113. Ineligible comparison

29. Asawabharuj K, Ramart P, Nualyong C, et al. Comparison of urinary continence outcome between robotic assisted laparoscopic prostatectomy versus laparoscopic radical prostatectomy. J Med Assoc Thai. 2014 Apr;97(4):393-8. PMID: 24964681. Ineligible study design 
30. Asimakopoulos AD, Topazio L, De Angelis M, et al. Retzius-sparing versus standard robotassisted radical prostatectomy: a prospective randomized comparison on immediate continence rates. Surgical Endoscopy. 2018 Nov 13;13:13. PMID: 30426256. Insufficient follow-up time

31. Astrom L, Grusell E, Sandin F, et al. Two decades of high dose rate brachytherapy with external beam radiotherapy for prostate cancer. Radiotherapy and Oncology. 2018 April;127(1):81-7. PMID: 2000469962. Ineligible study design

32. Astrom L, Sandin F, Holmberg L. Good prognosis following a PSA bounce after high dose rate brachytherapy and external radiotherapy in prostate cancer. Radiother Oncol. 2018 Dec;129(3):561-6. PMID: 30193693. Ineligible study design

33. Auffenberg GB, Linsell S, Dhir A, et al. Comparison of Pathological Outcomes for Men with Low Risk Prostate Cancer from Diverse Practice Settings: Similar Results from Immediate Prostatectomy or Initial Surveillance with Delayed Prostatectomy. Journal of Urology. 2016 11;196(5):141521. PMID: 27256204. Insufficient follow-up time

34. Avulova S, Zhao Z, Lee D, et al. The Effect of Nerve Sparing Status on Sexual and Urinary Function: 3-Year Results from the CEASAR Study. Journal of Urology. 2018 May;199(5):1202-9. PMID: 29253578. Ineligible population

35. Baker C, McDonald A, Yang E, et al. Pelvic radiotherapy versus radical prostatectomy with limited lymph node sampling for highgrade prostate adenocarcinoma. Prostate cancer. 2016;2016(no pagination). PMID: $\mathrm{CN}-01265144$. Ineligible study design

36. Baldwin LM, Andrilla CHA, Porter MP, et al. Treatment of early-stage prostate cancer among rural and urban patients. Cancer. 2013;119(16):3067-75. PMID: 52634939. No eligible outcomes reported

37. Bandini M, Marchioni M, Preisser F, et al. Survival after radical prostatectomy or radiotherapy for locally advanced (cT3) prostate cancer. World Journal of Urology. 2018 Sep;36(9):1399-407. PMID: 29717358. Ineligible population
38. Bandini M, Pompe RS, Marchioni M, et al. Radical prostatectomy or radiotherapy reduce prostate cancer mortality in elderly patients: a population-based propensity score adjusted analysis. World Journal of Urology. 2018 Jan;36(1):7-13. PMID: 29063268. Ineligible comparison

39. Banerji JS, Hurwitz LM, Cullen J, et al. A prospective study of health-related qualityof-life outcomes for patients with low-risk prostate cancer managed by active surveillance or radiation therapy. Urol. 2017 05;35(5):234-42. PMID: 28110975. Ineligible study design

40. Barry MJ, Andriole GL, Culkin DJ, et al. Ascertaining cause of death among men in the prostate cancer intervention versus observation trial. Clin. 2013;10(6):907-14. PMID: 23988464. Ineligible study design

41. Beauval JB, Roumiguie M, Ouali M, et al. A prospective trial comparing consecutive series of open retropubic and robot-assisted laparoscopic radical prostatectomy in a centre: oncologic and functional outcomes. Progres en urologie. 2015;25(7):370-8. PMID: CN-01248904. No pdf available.

42. Becker A, Seiler D, Kwiatkowski M, et al. A comparative assessment of active surveillance for localized prostate cancer in the community versus tertiary care referral center. World Journal of Urology. 2014 Aug;32(4):891-7. PMID: 24820259. Ineligible study design

43. Beckmann KR, O'Callaghan ME, Ruseckaite R, et al. Prostate cancer outcomes for men who present with symptoms at diagnosis. BJU International. 2017 06;119(6):862-71. PMID: 27489140. Ineligible comparison

44. Beckmann KR, Vincent AD, O'Callaghan ME, et al. Oncological outcomes in an Australian cohort according to the new prostate cancer grading groupings. BMC Cancer. 2017 Aug 10;17(1):537. PMID: 28797228. Ineligible comparison

45. Beesley LJ, Morgan TM, Spratt DE, et al. Individual and Population Comparisons of Surgery and Radiotherapy Outcomes in Prostate Cancer Using Bayesian Multistate Models. JAMA netw. 2019 Feb 01;2(2):e187765. PMID: 30707231. Ineligible study design 
46. Benelli A, Varca V, Rosso M, et al. 3D versus 2D laparoscopic radical prostatectomy for organ confined prostate cancer: Our experience. Journal of Clinical Urology. 2018. PMID: 625668505. Ineligible study design

47. Berg S, Cole AP, Krimphove MJ, et al. Comparative Effectiveness of Radical Prostatectomy Versus External Beam Radiation Therapy Plus Brachytherapy in Patients with High-risk Localized Prostate Cancer. European Urology. 2018. PMID: 2001261073. Ineligible population

48. Bjorklund J, Folkvaljon Y, Cole A, et al. Postoperative mortality 90 days after robotassisted laparoscopic prostatectomy and retropubic radical prostatectomy: a nationwide population-based study. BJU International. 2016 08;118(2):302-6. PMID: 26762928. Insufficient follow-up time

49. Blanchard P, Pugh TJ, Swanson DA, et al. Patient-reported health-related quality of life for men treated with low-dose-rate prostate brachytherapy as monotherapy with 125iodine, 103-palladium, or 131-cesium: Results of a prospective phase II study. Brachytherapy. 2018 Mar - Apr;17(2):26576. PMID: 29269207. Ineligible study design

50. Bock D, Angenete E, Bjartell A, et al. Habits and self-assessed quality of life, negative intrusive thoughts and depressed mood in patients with prostate cancer: a longitudinal study. Scandinavian Journal of Urology. 2017 Oct;51(5):353-9. PMID: 28621209. Ineligible comparison

51. Boehm K, Schiffmann J, Tian Z, et al. Five-year biochemical recurrence-free and overall survival following high-dose-rate brachytherapy with additional external beam or radical prostatectomy in patients with clinically localized prostate cancer. Urol. 2016 Mar;34(3):119.e11-8. PMID: 26602027. Ineligible study design

52. Bokhorst L, Kranse R, Venderbos L, et al. Differences in Treatment and Outcome After Treatment with Curative Intent in the Screening and Control Arms of the ERSPC Rotterdam. European urology. 2015;68(2):179-82. PMID: CN-01254843. Ineligible intervention Pubmed 25457496.
53. Bokhorst LP, Venderbos LDF, Schroder FH, et al. Do Treatment Differences between Arms Affect the Main Outcome of ERSPC Rotterdam? Journal of Urology. 2015;194(2):336-42. PMID: 607453442. Ineligible intervention

54. Bolch CA, Chu H, Jarosek S, et al. Inverse probability of treatment-weighted competing risks analysis: an application on long-term risk of urinary adverse events after prostate cancer treatments. BMC Med Res Methodol. 2017 Jul 10;17(1):93. PMID: 28693428. Ineligible population

55. Bosco C, Garmo H, Adolfsson J, et al. Prostate Cancer Radiation Therapy and Risk of Thromboembolic Events. International Journal of Radiation Oncology, Biology, Physics. 201704 01;97(5):1026-31. PMID: 28332985. No eligible outcomes reported

56. Bottke D, Golz R, Storkel S, et al. Phase 3 study of adjuvant radiotherapy versus wait and see in pT3 prostate cancer: impact of pathology review on analysis. European Urology. 2013 Aug;64(2):193-8. PMID: 23522911. Ineligible population

57. Bradley CJ, Dahman B, Anscher M. Prostate cancer treatment and survival: Evidence for men with prevalent comorbid conditions. Medical Care. 2014 June;52(6):482-9. PMID: 373142709. Ineligible comparison

58. Bradley MC, Zhou Y, Freedman AN, et al. Risk of diabetes complications among those with diabetes receiving androgen deprivation therapy for localized prostate cancer. Cancer Causes Control. 2018 Aug;29(8):785-91. PMID: 29959604. Ineligible population

59. Bratt O, Folkvaljon Y, Hjalm Eriksson M, et al. Undertreatment of Men in Their Seventies with High-risk Nonmetastatic Prostate Cancer. European Urology. 2015 Jul;68(1):53-8. PMID: 25813688. Ineligible comparison

60. Braunstein LZ, Chen MH, Dosoretz DE, et al. Whole Pelvis Versus Prostate-Only Radiotherapy With or Without Short-Course Androgen Deprivation Therapy and Mortality Risk. Clinical Genitourinary Cancer. 2015 Dec;13(6):555-61. PMID: 26003267. Insufficient follow-up time 
61. Brodszky V, Varga P, Gimesi-Orszagh J, et al. Long-term costs and survival of prostate cancer: a population-based study. Int Urol Nephrol. 2017 Oct;49(10):1707-14. PMID: 28762117. Ineligible population

62. Brundage M, Sydes MR, Parulekar WR, et al. Impact of Radiotherapy When Added to Androgen-Deprivation Therapy for Locally Advanced Prostate Cancer: Long-Term Quality-of-Life Outcomes From the NCIC CTG PR3/MRC PR07 Randomized Trial. Journal of Clinical Oncology. $2015 \mathrm{Jul}$ 01;33(19):2151-7. PMID: 26014295. Ineligible population

63. Bruner DW, Hunt D, Michalski JM, et al. Preliminary patient-reported outcomes analysis of 3-dimensional radiation therapy versus intensity-modulated radiation therapy on the high-dose arm of the Radiation Therapy Oncology Group (RTOG) 0126 prostate cancer trial. Cancer. $2015 \mathrm{Jul}$ 15;121(14):2422-30. PMID: 25847819. No eligible outcomes reported

64. Bruner DW, Pugh SL, Lee WR, et al. Quality of Life in Patients With Low-Risk Prostate Cancer Treated With Hypofractionated vs Conventional Radiotherapy: A Phase 3 Randomized Clinical Trial. JAMA Oncol. 2019 Feb 14;14:14. PMID: 30763425. Ineligible population

65. Bryant AK, Kader AK, McKay RR, et al. Definitive Radiation Therapy and Survival in Clinically Node-Positive Prostate Cancer. International Journal of Radiation Oncology, Biology, Physics. 2018 Aug 01;101(5):1188-93. PMID: 29891203. Ineligible population

66. Bryant AK, McKay RR, Kader AK, et al. Subcastrate testosterone nadir and clinical outcomes in intermediate or high-risk localized prostate cancer. International Journal of Radiation Oncology, Biology, Physics. 2018 Dec 10;10:10. PMID: 30543857. Ineligible comparison

67. Burt LM, Shrieve DC, Tward JD. Factors influencing prostate cancer patterns of care: An analysis of treatment variation using the SEER database. Advances in Radiation Oncology. 2018 April - June;3(2):170-80. PMID: 620708373. No eligible outcomes reported
68. Buscariollo DL, Drumm M, Niemierko A, et al. Long-term results of adjuvant versus early salvage postprostatectomy radiation: A large single-institutional experience. Practical Radiation Oncology. 2017 Mar Apr;7(2):e125-e33. PMID: 28274403. Ineligible study design

69. Busch J, Gonzalgo ML, Leva N, et al. Matched comparison of robot-assisted, laparoscopic and open radical prostatectomy regarding pathologic and oncologic outcomes in obese patients. World journal of urology. 201501 Mar;33(3):397-402. PMID: 612550606. Insufficient follow-up time

70. Busch J, Magheli A, Leva N, et al. Matched comparison of outcomes following open and minimally invasive radical prostatectomy for high-risk patients. World Journal of Urology. 2014 Dec;32(6):1411-6. PMID: 24609219. Ineligible study design

71. Canter DJ, Reid J, Latsis M, et al. Comparison of the Prognostic Utility of the Cell Cycle Progression Score for Predicting Clinical Outcomes in African American and NonAfrican American Men with Localized Prostate Cancer. European Urology. 2019 Mar;75(3):515-22. PMID: 30391079. No eligible outcomes reported

72. Carles J, Gallardo E, Domenech M, et al. Phase 2 Randomized Study of Radiation Therapy and 3-Year Androgen Deprivation With or Without Concurrent Weekly Docetaxel in High-Risk Localized Prostate Cancer Patients. International Journal of Radiation Oncology Biology Physics. 20191 February;103(2):344-52. PMID: 2001389177. Ineligible population

73. Carrie C, Hasbini A, e Laroche G, et al. Salvage radiotherapy with or without short-term hormone therapy for rising prostate-specific antigen concentration after radical prostatectomy (GETUG-AFU 16): a randomised, multicentre, open-label phase 3 trial. Lancet Oncol. 2016 Jun;17(6):747-56. PMID: 27160475. Ineligible population

74. Carter SC, Lipsitz S, Shih YC, et al. Populationbased determinants of radical prostatectomy operative time. BJU International. 2014 May;113(5b):E112-8. PMID: 24053198. No eligible outcomes reported 
75. Cary KC, Punnen S, Odisho AY, et al. Nationally representative trends and geographic variation in treatment of localized prostate cancer: The Urologic Diseases in America project. Prostate Cancer and Prostatic Diseases. 201514 Jun;18(2):149-54. PMID: 602237685. Ineligible study design

76. Cary KC, Singla N, Cowan JE, et al. Impact of androgen deprivation therapy on mental and emotional well-being in men with prostate cancer: analysis from the CaPSURETM registry. Journal of Urology. 2014 Apr;191(4):964-70. PMID: 24184370. Ineligible population

77. Casas F, Henriquez I, Bejar A, et al. Intermittent versus continuous androgen deprivation therapy to biochemical recurrence after external beam radiotherapy: a phase 3 GICOR study. Clin Transl Oncol. 2017 Mar;19(3):373-8. PMID: 27770397. Ineligible comparison

78. Catton CN, Lukka H, Gu CS, et al. Randomized Trial of a Hypofractionated Radiation Regimen for the Treatment of Localized Prostate Cancer. Journal of Clinical Oncology. 2017 Jun 10;35(17):1884-90. PMID: 28296582. Ineligible intervention

79. Cazzaniga W, Garmo H, Robinson D, et al. Mortality after radical prostatectomy in a matched contemporary cohort in Sweden compared to the Scandinavian Prostate Cancer Group 4 (SPCG-4) study. BJU International. 2018 Sep 25;25:25. PMID: 30253031. Ineligible study design

80. Chang K, Qin XJ, Zhang HL, et al. Comparison of two adjuvant hormone therapy regimens in patients with high-risk localized prostate cancer after radical prostatectomy: primary results of study CU1005. Asian Journal of Andrology. 2016 May-Jun;18(3):452-5. PMID: 26323560. Ineligible population

81. Chang KD, Abdel Raheem A, Santok GDR, et al. Anatomical Retzius-space preservation is associated with lower incidence of postoperative inguinal hernia development after robot-assisted radical prostatectomy. Hernia. 2017 08;21(4):555-61. PMID: 28160111. Ineligible comparison
82. Chao M, Lim Joon D, Khoo V, et al. The use of hydrogel spacer in men undergoing highdose prostate cancer radiotherapy: results of a prospective phase 2 clinical trial. World Journal of Urology. 2018 Sep 24;24:24. PMID: 30251049. Ineligible intervention

83. Chen $\mathrm{CH}, \mathrm{Pu}$ YS. Adjuvant androgen-deprivation therapy following prostate total cryoablation in high-risk localized prostate cancer patients - Open-labeled randomized clinical trial. Cryobiology. 2018 06;82:88-92. PMID: 29626465. Ineligible population

84. Chen RC, Basak R, Meyer AM, et al. Association Between Choice of Radical Prostatectomy, External Beam Radiotherapy, Brachytherapy, or Active Surveillance and Patient-Reported Quality of Life Among Men With Localized Prostate Cancer. Jama. 201703 21;317(11):1141-50. PMID: 28324092. Ineligible intervention

85. Chen S, Zhu L, Cai J, et al. Plasmakinetic enucleation of the prostate compared with open prostatectomy for prostates larger than 100 grams: a randomized noninferiority controlled trial with long-term results at 6 years. European urology. 2014;66(2):28491. PMID: CN-00994669. Ineligible population Pubmed 24502959.

86. Chen YW, Muralidhar V, Mahal BA, et al. Factors associated with the omission of androgen deprivation therapy in radiationmanaged high-risk prostate cancer. Brachytherapy. 201601 Nov;15(6):695-700. PMID: 613276184. Ineligible population

87. Chiang IN, Huang CY, Pu YS, et al. Association between ischaemic bowel syndromes and androgen deprivation therapy in patients with prostate cancer: a retrospective cohort study. BMJ Open. 201702 28;7(2):e012950. PMID: 28246133. Ineligible comparison

88. Chien GW, Slezak JM, Harrison TN, et al. Health-related quality of life outcomes from a contemporary prostate cancer registry in a large diverse population. BJU International. 2017 10;120(4):520-9. PMID: 28425193. Ineligible population

89. Chinenov DV, Rapoport LM, Shpot EV, et al. Comparative results of cryoablation and laparoscopic radical prostatectomy in the treatment of localized prostate cancer. Urologia. 2018 May;85(2):68-72. PMID: 30043713. Ineligible study design 
90. Choo MS, Cho SY, Jeong CW, et al. Predictors of positive surgical margins and their location in Korean men undergoing radical prostatectomy. International Journal of Urology. 2014 Sep;21(9):894-8. PMID: 24807736. Ineligible population

91. Choo R, Lukka H, Cheung P, et al. Randomized, double-blinded, placebo-controlled, trial of risedronate for the prevention of bone mineral density loss in nonmetastatic prostate cancer patients receiving radiation therapy plus androgen deprivation therapy. International Journal of Radiation Oncology, Biology, Physics. 2013 Apr 01;85(5):123945. PMID: 23265571. Ineligible intervention

92. Chu FM, Sartor O, Gomella L, et al. A randomised, double-blind study comparing the addition of bicalutamide with or without dutasteride to GnRH analogue therapy in men with non-metastatic castrate-resistant prostate cancer. Eur J Cancer. 2015 Aug;51(12):1555-69. PMID: 26048455. Ineligible intervention

93. Chung SD, Kao LT, Lin HC, et al. Patients receiving androgen deprivation therapy for prostate cancer have an increased risk of depressive disorder. PLoS ONE. 2017;12(3):e0173266. PMID: 28253340. Ineligible population

94. Ciezki JP, Weller M, Reddy CA, et al. A Comparison Between Low-Dose-Rate Brachytherapy With or Without Androgen Deprivation, External Beam Radiation Therapy With or Without Androgen Deprivation, and Radical Prostatectomy With or Without Adjuvant or Salvage Radiation Therapy for High-Risk Prostate Cancer. International Journal of Radiation Oncology, Biology, Physics. 201704 01;97(5):962-75. PMID: 28333019. Ineligible comparison

95. Close A, Robertson C, Rushton S, et al. Comparative cost-effectiveness of robotassisted and standard laparoscopic prostatectomy as alternatives to open radical prostatectomy for treatment of men with localised prostate cancer: a health technology assessment from the perspective of the UK National Health Service. European Urology. 2013 Sep;64(3):361-9. PMID: 23498062. Ineligible study design
96. Cole E, Margel D, Greenspan M, et al. Is there a role for anterior zone sampling as part of saturation trans-rectal ultrasound guided prostate biopsy? BMC Urology. 201403 May;14 (1) (no pagination)(34). PMID: 53126359. Ineligible study design

97. Collettini F, Enders J, Stephan C, et al. Imageguided Irreversible Electroporation of Localized Prostate Cancer: Functional and Oncologic Outcomes. Radiology. 2019 Jul;292(1):250-7. PMID: 31161973. Ineligible study design

98. Cooperberg MR, Ramakrishna NR, Duff SB, et al. Primary treatments for clinically localised prostate cancer: A comprehensive lifetime cost-utility analysis. BJU International. 2013 March;111(3):437-50. Ineligible study design

99. Cormie P, Galvão D, Spry N, et al. Can supervised exercise prevent treatment toxicity in patients with prostate cancer initiating androgen-deprivation therapy: a randomised controlled trial. BJU international. 2015;115(2):256-66. PMID: CN-01052001. Ineligible intervention Pubmed 24467669.

100. Coughlin GD, Yaxley JW, Chambers SK, et al. Robot-assisted laparoscopic prostatectomy versus open radical retropubic prostatectomy: 24-month outcomes from a randomised controlled study. Lancet Oncol. 2018 Aug;19(8):1051-60. PMID: 30017351. Ineligible population

101. Crandley EF, Hegarty SE, Hyslop T, et al. Treatment-related complications of radiation therapy after radical prostatectomy: comparative effectiveness of intensitymodulated versus conformal radiation therapy. Cancer Medicine. 2014 Apr;3(2):397-405. PMID: 24519910. Ineligible population

102. Dalela D, Karabon P, Sammon J, et al. Generalizability of the Prostate Cancer Intervention Versus Observation Trial (PIVOT) Results to Contemporary North American Men with Prostate Cancer. European Urology. 2017 04;71(4):511-4. PMID: 27638094. No eligible outcomes reported 
103. D'Amico AV, Chen MH, Renshaw A, et al. Long-term Follow-up of a Randomized Trial of Radiation With or Without Androgen Deprivation Therapy for Localized Prostate Cancer. Jama. 2015 Sep 2229;314(12):1291-3. PMID: 26393854. Ineligible study design

104. Daskivich TJ, Chamie K, Kwan L, et al. Matching tumor risk with aggressiveness of treatment in men with multiple comorbidities and early-stage prostate cancer. Cancer. 201301 Oct;119(19):344653. PMID: 52688994. Ineligible comparison

105. Daskivich TJ, Lai J, Dick AW, et al. Comparative effectiveness of aggressive versus nonaggressive treatment among men with early-stage prostate cancer and differing comorbid disease burdens at diagnosis. Cancer. 201415 Aug;120(16):2432-9. PMID: 373709135. Ineligible comparison

106. Daskivich TJ, Lai J, Dick AW, et al. Questioning the 10-year Life Expectancy Rule for High-grade Prostate Cancer: Comparative Effectiveness of Aggressive vs Nonaggressive Treatment of High-grade Disease in Older Men With Differing Comorbid Disease Burdens. Urology. 2016 07;93:68-76. PMID: 27079130. Ineligible comparison

107. Davis JW, Kreaden US, Gabbert J, et al. Learning curve assessment of robot-assisted radical prostatectomy compared with opensurgery controls from the premier perspective database. Journal of Endourology. 2014 May;28(5):560-6. PMID: 24350787. Ineligible study design

108. Dayes IS, Parpia S, Gilbert J, et al. Long-Term Results of a Randomized Trial Comparing Iridium Implant Plus External Beam Radiation Therapy With External Beam Radiation Therapy Alone in Node-Negative Locally Advanced Cancer of the Prostate. International Journal of Radiation Oncology, Biology, Physics. 201709 01;99(1):90-3. PMID: 28816169. Ineligible population
109. Dearnaley D, Griffin CL, Lewis R, et al. Toxicity and Patient-Reported Outcomes of a Phase 2 Randomized Trial of Prostate and Pelvic Lymph Node Versus Prostate only Radiotherapy in Advanced Localised Prostate Cancer (PIVOTAL). International Journal of Radiation Oncology Biology Physics. 20191 March;103(3):605-17. PMID: 2001502359. Ineligible intervention

110. Dearnaley D, Syndikus I, Mossop H, et al. Conventional versus hypofractionated highdose intensity-modulated radiotherapy for prostate cancer: 5-year outcomes of the randomised, non-inferiority, phase $3 \mathrm{CHHiP}$ trial. Lancet Oncol. 2016 Aug;17(8):104760. PMID: 27339115. Ineligible intervention

111. Dell'Oglio P, Bandini M, Leyh-Bannurah SR, et al. External beam radiotherapy with or without androgen deprivation therapy in elderly patients with high metastatic risk prostate cancer. Urol. 2018 May;36(5):239.e9-.e15. PMID: 29426698. Ineligible population

112. Dell'Oglio P, Suardi N, Boorjian SA, et al. Predicting survival of men with recurrent prostate cancer after radical prostatectomy. Eur J Cancer. 2016 Feb;54:27-34. PMID: 26707594. Ineligible population

113. Delobel JB, Gnep K, Ospina JD, et al. Nomogram to predict rectal toxicity following prostate cancer radiotherapy. PLoS ONE. 2017;12(6):e0179845. PMID: 28640871. Ineligible study design

114. Denham JW, Joseph D, Lamb DS, et al. Shortterm androgen suppression and radiotherapy versus intermediate-term androgen suppression and radiotherapy, with or without zoledronic acid, in men with locally advanced prostate cancer (TROG 03.04 RADAR): an open-label, randomised, phase 3 factorial trial. Lancet Oncol. 2014 Sep;15(10):1076-89. PMID: 25130995. Ineligible comparison

115. Denham JW, Nowitz M, Joseph D, et al. Impact of androgen suppression and zoledronic acid on bone mineral density and fractures in the Trans-Tasman Radiation Oncology Group (TROG) 03.04 Randomised Androgen Deprivation and Radiotherapy (RADAR) randomized controlled trial for locally advanced prostate cancer. BJU International. 2014 Sep;114(3):344-53. PMID: 24512527. Ineligible intervention 
116. Denham JW, Steigler A, Joseph D, et al. Radiation dose escalation or longer androgen suppression for locally advanced prostate cancer? Data from the TROG 03.04 RADAR trial. Radiother Oncol. 2015 Jun;115(3):301-7. PMID: 26072289. Ineligible population

117. Dess RT, Jackson WC, Suy S, et al. Predictors of multidomain decline in health-related quality of life after stereotactic body radiation therapy (SBRT) for prostate cancer. Cancer. 201705 01;123(9):1635-42. PMID: 28001303. Ineligible intervention

118. Di LG, Autorino R, Sonpavde G. Re: androgen Deprivation Therapy plus Docetaxel and Estramustine Versus Androgen Deprivation Therapy Alone for High-risk Localised Prostate Cancer (GETUG 12): a Phase 3 Randomised Controlled Trial. European urology. 2015;68(6):1098-9. PMID: CN01162017. Ineligible study design

119. Dieperink KB, Johansen C, Hansen S, et al. Male coping through a long-term cancer trajectory. Secondary outcomes from a RTC examining the effect of a multidisciplinary rehabilitation program (RePCa) among radiated men with prostate cancer. Acta Oncol. 2017 Feb;56(2):254-61. PMID: 28093012. Ineligible intervention

120. Dignam JJ, Hamstra DA, Lepor H, et al. Time Interval to Biochemical Failure as a Surrogate End Point in Locally Advanced Prostate Cancer: Analysis of Randomized Trial NRG/RTOG 9202. Journal of Clinical Oncology. 2019 Jan 20;37(3):213-21. PMID: 30526194. Ineligible comparison

121. Ding XF, Huang TB, Gao Y, et al. Permanent $<$ sup $>125<$ /sup $>$ I prostate brachytherapy for castration-resistant prostate cancer. International Journal of Urology. 2019 02;26(2):278-83. PMID: 30515888. Ineligible study design

122. Dinh KT, Yang DD, Nead KT, et al. Association between androgen deprivation therapy and anxiety among 78000 patients with localized prostate cancer. International Journal of Urology. 2017 10;24(10):743-8. PMID: 28734019. No eligible outcomes reported
123. Diniz CP, Landis P, Carter HB, et al. Comparison of Biochemical RecurrenceFree Survival after Radical Prostatectomy Triggered by Grade Reclassification during Active Surveillance and in Men Newly Diagnosed with Similar Grade Disease. Journal of Urology. 2017 Sep;198(3):60813. PMID: 28347771. Ineligible population

124. Dolezel M, Odrazka K, Zouhar M, et al. Comparing morbidity and cancer control after 3D-conformal (70/74 Gy) and intensity modulated radiotherapy (78/82 Gy) for prostate cancer. Strahlentherapie und Onkologie. 2015;191(4):338-46. PMID: $\mathrm{CN}-01111085$. Ineligible population Pubmed 25589224.

125. Dong Y, Ruth KJ, Churilla TM, et al. The need for androgen deprivation therapy in patients with intermediate-risk prostate cancer treated with dose-escalated external beam radiation therapy. Can J Urol. 2017 Feb;24(1):8656-62. PMID: 28263132. Ineligible study design

126. Dong Y, Zaorsky NG, Li T, et al. Effects of interruptions of external beam radiation therapy on outcomes in patients with prostate cancer. Journal of Medical Imaging and Radiation Oncology. 2018 Februaryy;62(1):116-21. PMID: 618783317. Ineligible comparison

127. Dosani M, Morris WJ, Tyldesley S, et al. The Relationship between Hot Flashes and Testosterone Recovery after 12 Months of Androgen Suppression for Men with Localised Prostate Cancer in the ASCENDE-RT Trial. Clin Oncol (R Coll Radiol). 2017 10;29(10):696-701. PMID: 28712786. No eligible outcomes reported

128. Duchesne G, Woo H, Bassett J, et al. Timing of androgen-deprivation therapy in patients with prostate cancer with a rising PSA (TROG 03.06 and VCOG PR 01-03 ): a randomised, multicentre, non-blinded, phase 3 trial. The lancet Oncology. 2016;17(6):727-37. PMID: CN-01165445. Ineligible population Pubmed 27155740. 
129. Duchesne G, Woo H, King M, et al. Healthrelated quality of life for immediate versus delayed androgen-deprivation therapy in patients with asymptomatic, non-curable prostate cancer (TROG 03.06 and VCOG PR 01-03 ): a randomised, multicentre, nonblinded, phase 3 trial. The lancet Oncology. 2017;18(9):1192-201. PMID: CN01412973. Ineligible population Pubmed 28760403.

130. e Crevoisier R, Bayar MA, Pommier P, et al. Daily Versus Weekly Prostate Cancer Image Guided Radiation Therapy: Phase 3 Multicenter Randomized Trial. International Journal of Radiation Oncology Biology Physics. 20181 December;102(5):1420-9. PMID: 2001254666. Ineligible comparison

131. Ebell MH. Active Surveillance for Localized Prostate Cancer: No Increased Mortality, but Higher Rates of Clinical Progression. Am Fam Physician. 2017 Feb 01;95(3):196. PMID: 28145675. Ineligible study design

132. Ebert M, Foo K, Haworth A, et al. Gastrointestinal dose-histogram effects in the context of dose-volume-constrained prostate radiation therapy: analysis of data from the RADAR prostate radiation therapy trial. International journal of radiation oncology, biology, physics. 2015;91(3):595603. PMID: CN-01111039. No eligible outcomes reported Pubmed 25596108.

133. Eccles BK, Cross W, Rosario DJ, et al. SABRE 1 (Surgery Against Brachytherapy - a Randomised Evaluation): feasibility randomised controlled trial (RCT) of brachytherapy vs radical prostatectomy in low-intermediate risk clinically localised prostate cancer. BJU International. 2013 Aug;112(3):330-7. PMID: 23826842. No eligible outcomes reported

134. Eggener S. Commentary on: "Long-term functional outcomes after treatment for localized prostate cancer.". Urologic Oncology: Seminars and Original Investigations. 2014 May;32(4):513-4. PMID: 372927495. Ineligible study design

135. Egger SJ, Calopedos RJ, O'Connell DL, et al. Long-term Psychological and Quality-of-life Effects of Active Surveillance and Watchful Waiting After Diagnosis of Low-risk Localised Prostate Cancer. European Urology. 2018 06;73(6):859-67. PMID: 28851582. Ineligible study design
136. Eifler JB, Alvarez J, Koyama T, et al. More Judicious Use of Expectant Management for Localized Prostate Cancer during the Last 2 Decades. Journal of Urology. 201701 Mar;Part 1. 197(3):614-20. PMID: 614251526. Ineligible comparison

137. El-Ghamrawi K, El-Haddad M, Hanna S, et al. Hypofractionated Simultaneous Integrated Boost (SIB) versus Conventional Fractionation in Localized Prostate Cancer: A Randomized Pilot Study. Gulf J Oncolog. 2015 May;1(18):44-53. PMID: 26003105. Insufficient follow-up time

138. Ellett JD, Rosoff JS, Prasad SM. Long-term differences in urinary, bowel and sexual function among men treated with surgery versus radiation for prostate cancer. Asian Journal of Andrology. 2013 July;15(4):4434. PMID: 369307995. Ineligible study design

139. Ellimoottil C, Roghmann F, Blackwell R, et al. Open versus robotic radical prostatectomy in obese men. Current Urology. 201504 Sep;8:156-61. PMID: 606124779. Insufficient follow-up time

140. Emery J, Jefford M, King M, et al. ProCare Trial: a phase II randomized controlled trial of shared care for follow-up of men with prostate cancer. BJU international. 2017;119(3):381-9. PMID: CN-01339892. Ineligible intervention Pubmed 27431584.

141. Eriguchi T, Yorozu A, Kuroiwa N, et al. Predictive factors for urinary toxicity after iodine-125 prostate brachytherapy with or without supplemental external beam radiotherapy. Brachytherapy. 2016 MayJun;15(3):288-95. PMID: 26924022. Ineligible study design

142. Ettel M, Kong M, Lee P, et al. Modification of the pT2 substage classification in prostate adenocarcinoma. Hum Pathol. 2016 10;56:57-63. PMID: 27251951. Ineligible study design

143. Evans SM, Millar JL, Davis ID, et al. Patterns of care for men diagnosed with prostate cancer in Victoria from 2008 to 2011. Medical Journal of Australia. 2013 June;198(10):540-5. PMID: 369053155. No eligible outcomes reported 
144. Faiena I, Dombrovskiy VY, Modi PK, et al. Regional Cost Variations of Robot-Assisted Radical Prostatectomy Compared With Open Radical Prostatectomy. Clinical Genitourinary Cancer. 2015 Oct;13(5):44752. PMID: 26065923. No eligible outcomes reported

145. Feldman AS, Meyer CP, Sanchez A, et al. Morbidity and Mortality of Locally Advanced Prostate Cancer: A Population Based Analysis Comparing Radical Prostatectomy versus External Beam Radiation. Journal of Urology. 2017 Nov;198(5):1061-8. PMID: 28552709. Ineligible population

146. Feng FY, Blas K, Olson K, et al. Retrospective evaluation reveals that long-term androgen deprivation therapy improves cause-specific and overall survival in the setting of doseescalated radiation for high-risk prostate cancer. International Journal of Radiation Oncology, Biology, Physics. 2013 May 01;86(1):64-71. PMID: 23462420. Ineligible population

147. Fenoglietto P, Khodri M, Nguyen D, et al. Twin machines validation for VMAT treatments using electronic portal-imaging device: a multicenter study. Radiation oncology (london, england). 2016;11(1) (no pagination). PMID: CN-01134200. Ineligible study design

148. Ferreira AS, Guerra MR, Lopes HE, et al. Brachytherapy and radical prostatectomy in patients with early prostate cancer. Rev Assoc Med Bras. 2015 Sep-Oct;61(5):431-9. PMID: 26603006. Ineligible study design

149. Ferris MJ, Liu Y, Ao J, et al. The addition of chemotherapy in the definitive management of high risk prostate cancer. Urol. 2018 11;36(11):475-87. PMID: 30309766. Ineligible intervention

150. Fersino S, Fiorentino A, Giaj Levra N, et al. Impact of Ialuril Soft Gels in reducing urinary toxicity during radical hypofractionated radiotherapy in prostate cancer: a preliminary experience. Minerva Urologica e Nefrologica. 2016 Feb;68(1):913. PMID: 26491889. Insufficient follow-up time
151. Filson CP, Schroeck FR, Ye Z, et al. Variation in use of active surveillance among men undergoing expectant treatment for early stage prostate cancer. Journal of Urology. 2014;192(1):75-80. PMID: 602678947. No eligible outcomes reported

152. Finazzi T, Guckenberger M. Image-guided intensity-modulated radiation therapy decreases late gastrointestinal side effects after radiation therapy for prostate cancer. Strahlentherapie und Onkologie. 2017;193(2):162-4. PMID: CN-01342270. Not available in English Pubmed 28004132.

153. Fizazi K, Scher H, Miller K, et al. Effect of enzalutamide on time to first skeletal-related event, pain, and quality of life in men with castration-resistant prostate cancer: results from the randomised, phase 3 AFFIRM trial. The lancet Oncology. 2014;15(10):1147-56. PMID: CN-01002026. Ineligible population Pubmed 25104109.

154. Fizazi K, Shore N, Tammela TL, et al. Darolutamide in Nonmetastatic, CastrationResistant Prostate Cancer. N Engl J Med. 201902 14;02:14. PMID: 30763142. Insufficient follow-up time

155. Fossati N, Karnes RJ, Boorjian SA, et al. Longterm Impact of Adjuvant Versus Early Salvage Radiation Therapy in pT3N0 Prostate Cancer Patients Treated with Radical Prostatectomy: Results from a Multi-institutional Series. European Urology. 2017 06;71(6):886-93. PMID: 27484843. Ineligible comparison

156. Freeman D, Dickerson G, Perman M. Multiinstitutional registry for prostate cancer radiosurgery: a prospective observational clinical trial. Frontiers in Oncology. 2014;4:369. PMID: 25657929. Ineligible population

157. Freytag SO, Stricker H, Lu M, et al. Prospective randomized phase 2 trial of intensity modulated radiation therapy with or without oncolytic adenovirus-mediated cytotoxic gene therapy in intermediate-risk prostate cancer. International Journal of Radiation Oncology, Biology, Physics. 2014 Jun 01;89(2):268-76. PMID: 24837889. Ineligible comparison 
158. Fridriksson JO, Folkvaljon Y, Nilsson P, et al. Long-term adverse effects after curative radiotherapy and radical prostatectomy: population-based nationwide register study. Scandinavian Journal of Urology. 201602 Sep;50(5):338-45. PMID: 610941656. Ineligible population

159. Fujimura T, Fukuhara H, Taguchi S, et al. Robot-assisted radical prostatectomy significantly reduced biochemical recurrence compared to retro pubic radical prostatectomy. BMC Cancer. 201729 Jun;17 (1) (no pagination)(454). PMID: 616999852. Ineligible study design

160. Fuller A, Vanderhaeghe L, Nott L, et al. Intravesical ropivacaine as a novel means of analgesia post-robot-assisted radical prostatectomy: a randomized, double-blind, placebo-controlled trial. Journal of Endourology. 2013 Mar;27(3):313-7. PMID: 22967208. Ineligible intervention

161. Gandaglia G, Abdollah F, Hu J, et al. Is robotassisted radical prostatectomy safe in men with high-risk prostate cancer? Assessment of perioperative outcomes, positive surgical margins, and use of additional cancer treatments. Journal of Endourology. 2014 Jul;28(7):784-91. PMID: 24499306. Insufficient follow-up time

162. Gandaglia G, Fossati N, Karnes RJ, et al. Use of Concomitant Androgen Deprivation Therapy in Patients Treated with Early Salvage Radiotherapy for Biochemical Recurrence After Radical Prostatectomy: Long-term Results from a Large, Multiinstitutional Series. European Urology. 2018 04;73(4):512-8. PMID: 29229176. Ineligible population

163. Gandaglia G, Sammon JD, Chang SL, et al. Comparative effectiveness of robot-assisted and open radical prostatectomy in the postdissemination era. Journal of Clinical Oncology. 2014 May 10;32(14):1419-26. PMID: 24733797. Insufficient follow-up time

164. Gandaglia G, Sun M, Popa I, et al. The impact of androgen-deprivation therapy (ADT) on the risk of cardiovascular $(\mathrm{CV})$ events in patients with non-metastatic prostate cancer: a population-based study. BJU International. 2014 Dec;114(6b):E82-E9. PMID: 24612110. Ineligible comparison
165. Gandaglia G, Sun M, Trinh QD, et al. Survival benefit of definitive therapy in patients with clinically advanced prostate cancer: Estimations of the number needed to treat based on competing-risks analysis. BJU International. 201401 Dec;Part B. 114(6):E62-E9. PMID: 53245045. Ineligible population

166. Garbens A, Wallis CJD, Matta R, et al. The cost of treatment and its related complications for men who receive surgery or radiation therapy for prostate cancer. Can Urol Assoc J. 2018 Dec 03;03:03. PMID: 30526806. No eligible outcomes reported

167. Garcia-Barreras S, Sanchez-Salas R, Sivaraman A, et al. Comparative Analysis of Partial Gland Ablation and Radical Prostatectomy to Treat Low and Intermediate Risk Prostate Cancer: Oncologic and Functional Outcomes. Journal of Urology. 2018 01;199(1):140-6. PMID: 28823768. Ineligible study design

168. Gardiner RA, Coughlin GD, Yaxley JW, et al. A progress report on a prospective randomised trial of open and robotic prostatectomy. European Urology. 2014 Mar;65(3):512-5. PMID: 24215940. No eligible outcomes reported

169. Gatti L, Antonelli A, Gritti A, et al. [Short and medium term oncological results after robotassisted prostatectomy: a comparative prospective non randomized study]. Urologia. 2013 Apr-Jun;80(2):135-9. PMID: 23504861. Not available in English

170. Gay HA, Sanda MG, Liu J, et al. External Beam Radiation Therapy or Brachytherapy With or Without Short-course Neoadjuvant Androgen Deprivation Therapy: Results of a Multicenter, Prospective Study of Quality of Life. International Journal of Radiation Oncology, Biology, Physics. 201706 01;98(2):304-17. PMID: 28463150. Ineligible study design

171. Geavlete B, Bulai C, Ene C, et al. Bipolar vaporization, resection, and enucleation versus open prostatectomy: optimal treatment alternatives in large prostate cases? Journal of endourology / Endourological Society. 2015;29(3):323-31. PMID: CN-01051803. Ineligible population Pubmed 25111385. 
172. Giberti C, Gallo F, Schenone M, et al. Robotic prostatectomy versus brachytherapy for the treatment of low risk prostate cancer. Can J Urol. 2017 Apr;24(2):8728-33. PMID: 28436359. Ineligible study design

173. Giganti F, Moore CM, Robertson NL, et al. MRI findings in men on active surveillance for prostate cancer: does dutasteride make MRI visible lesions less conspicuous? Results from a placebo-controlled, randomised clinical trial. Eur Radiol. 2017 Nov;27(11):4767-74. PMID: 28523355. Ineligible comparison

174. Gilbert D, Duong T, Kynaston H, et al. Qualityof-life outcomes from the Prostate Adenocarcinoma: transCutaneous Hormones (PATCH) trial evaluating luteinising hormone-releasing hormone agonists versus transdermal oestradiol for androgen suppression in advanced prostate cancer. BJU international. 2016;(no pagination). PMID: CN-01291300. Ineligible population

175. Gilbert SM, Dunn RL, Miller DC, et al. Functional Outcomes Following Nerve Sparing Prostatectomy Augmented with Seminal Vesicle Sparing Compared to Standard Nerve Sparing Prostatectomy: Results from a Randomized Controlled Trial. Journal of Urology. 2017 September;198(3):600-7. PMID: 617478501. Ineligible comparison

176. Gill IS, Azzouzi AR, Emberton M, et al. Randomized Trial of Partial Gland Ablation with Vascular Targeted Phototherapy versus Active Surveillance for Low Risk Prostate Cancer: Extended Followup and Analyses of Effectiveness. Journal of Urology. 2018 Oct;200(4):786-93. PMID: 29864437. Insufficient follow-up time

177. Glaser SM, Dohopolski MJ, Balasubramani GK, et al. Brachytherapy boost for prostate cancer: Trends in care and survival outcomes. Brachytherapy. 201701 Mar;16(2):330-41. PMID: 614278119. Ineligible population

178. Glowacki G, Majewski W, Wojcieszek P, et al. Ultrahypofractionated CyberKnifeTM based stereotactic radiotherapy versus conventional radiotherapy in patients with prostate cancer - acute toxicity evaluation in two phase II prospective studies. Neoplasma. 2017;64(4):599-604. PMID: 28699351. Ineligible study design
179. Godtman R, Holmberg E, Khatami A, et al. Long-term Results of Active Surveillance in the Göteborg Randomized, Population-based Prostate Cancer Screening Trial. European urology. 2016;70(5):760-6. PMID: CN01290512. Ineligible study design Pubmed 27090975.

180. Godtman RA, Holmberg E, Khatami A, et al. Long-term Results of Active Surveillance in the Goteborg Randomized, Population-based Prostate Cancer Screening Trial. European Urology. 2016 11;70(5):760-6. PMID: 27090975. Ineligible population

181. Golan R, Patel NA, Sun T, et al. Impact of Pelvic Radiation Therapy on Inflatable Penile Prosthesis Reoperation Rates. J Sex Med. 2018 Nov;15(11):1653-8. PMID: 30415817. No eligible outcomes reported

182. Goldin GH, Sheets NC, Meyer AM, et al. Comparative effectiveness of intensitymodulated radiotherapy and conventional conformal radiotherapy in the treatment of prostate cancer after radical prostatectomy. JAMA Intern Med. 2013 Jun 24;173(12):1136-43. PMID: 23689844. Ineligible population

183. Gottschalk A. Commentary on "Patient-reported outcomes after 3-dimensional conformal, intensity-modulated, or proton beam radiotherapy for localized prostate cancer." Gray PJ, Paly JJ, Yeap BY, Sanda MG, Sandler HM, Michalski JM, Talcott JA, Coen JJ, Hamstra DA, Shipley WU, Hahn SM, Zietman AL, Bekelman JE, Efstathiou JA. Harvard Radiation Oncology Program, Boston, MA.: Cancer 2013;119(9):1729-35. doi: 10.1002/cncr.27956. Urol. 2014 Apr;32(3):373-4. PMID: 24679463. Ineligible study design

184. Goy BW, Soper MS, Chang T, et al. Treatment results of brachytherapy vs. external beam radiation therapy for intermediate-risk prostate cancer with 10-year followup. Brachytherapy. 2016 Nov - Dec;15(6):68794. PMID: 27600607. Ineligible study design 
185. Gravis G, Boher JM, Chen YH, et al. Burden of Metastatic Castrate Naive Prostate Cancer Patients, to Identify Men More Likely to Benefit from Early Docetaxel: further Analyses of CHAARTED and GETUGAFU15 Studies. European urology. 2018;73(6):847-55. PMID: CN-01608144. Ineligible population Pubmed 29475737.

186. Gray PJ, Lin CC, Cooperberg MR, et al. Temporal Trends and the Impact of Race, Insurance, and Socioeconomic Status in the Management of Localized Prostate Cancer. European Urology. 201701 May;71(5):72937. PMID: 613204465. No eligible outcomes reported

187. Greenberg DC, Lophatananon A, Wright KA, et al. Trends and outcome from radical therapy for primary non-metastatic prostate cancer in alpha UK population. PLoS ONE. 2015 05 Mar;10 (3) (no pagination)(e0119494). PMID: 602685034. Ineligible population

188. Grossgold E, Given R, Ruckle H, et al. Does neoadjuvant androgen deprivation therapy before primary whole gland cryoablation of the prostate affect the outcome? Urology. 2014 Feb;83(2):379-83. PMID: 24315304. Ineligible study design

189. Gu X, Gao X, Cui M, et al. Survival outcomes of radical prostatectomy and external beam radiotherapy in clinically localized high-risk prostate cancer: a population-based, propensity score matched study. Cancer Manag Res. 2018;10:1061-7. PMID: 29773955. Ineligible population

190. Gumulec J, Raudenska M, Pacik D, et al. Posttreatment urinary sarcosine as a predictor of recurrent relapses in patients with prostate cancer. Cancer Medicine. 2018 Nov;7(11):5411-9. PMID: 30209891. Ineligible population

191. Guttilla A, Bortolus R, Giannarini G, et al. Multimodal treatment for high-risk prostate cancer with high-dose intensity-modulated radiation therapy preceded or not by radical prostatectomy, concurrent intensified-dose docetaxel and long-term androgen deprivation therapy: results of a prospective phase II trial. Radiation Oncology. 2014 Jan 14;9:24. PMID: 24423462. Ineligible study design
192. Ha B, Cho KH, Lee KH, et al. Long-term results of a phase II study of hypofractionated proton therapy for prostate cancer: moderate versus extreme hypofractionation. Radiation Oncology. 2019 Jan 10;14(1):4. PMID: 30630500. Ineligible comparison

193. Habl G, Hatiboglu G, Edler L, et al. Ion Prostate Irradiation (IPI) - a pilot study to establish the safety and feasibility of primary hypofractionated irradiation of the prostate with protons and carbon ions in a raster scan technique. BMC Cancer. 2014;14:202.

PMID: CN-01117742. No eligible outcomes reported Pubmed 24641841.

194. Habl G, Uhl M, Katayama S, et al. Acute Toxicity and Quality of Life in Patients With Prostate Cancer Treated With Protons or Carbon Ions in a Prospective Randomized Phase II Study--The IPI Trial. International Journal of Radiation Oncology, Biology, Physics. 2016 May 01;95(1):435-43. PMID: 27084659. No eligible outcomes reported

195. Hajdenberg J. Radical prostatectomy reduced long-term mortality more than watchful waiting in early prostate cancer. Annals of internal medicine. 2014;160(12):Jc10. PMID: CN-00995617. Ineligible study design Pubmed 24935504.

196. Halpern JA, Sedrakyan A, Hsu WC, et al. Use, complications, and costs of stereotactic body radiotherapy for localized prostate cancer. Cancer. 201615 Aug;122(16):2496-504. PMID: 611581484. Ineligible population

197. Hamidi N, Atmaca AF, Canda AE, et al. Does Presence of a Median Lobe Affect Perioperative Complications, Oncological Outcomes and Urinary Continence Following Robotic-assisted Radical Prostatectomy? Urol. 201809 26;15(5):24855. PMID: 30178450. Ineligible comparison

198. Hamilton SN, Tyldesley S, Hamm J, et al. Incidence of second malignancies in prostate cancer patients treated with low-dose-rate brachytherapy and radical prostatectomy. International Journal of Radiation Oncology, Biology, Physics. 2014 Nov 15;90(4):93441. PMID: 25240272. No eligible outcomes reported 
199. Hammerer PG, Wirth MP. Health-Related Quality of Life in 536 Long-Term Prostate Cancer Survivors after Treatment with Leuprorelin Acetate: A Combined Retrospective and Prospective Analysis. Urol Int. 2018;100(1):72-8. PMID: 29183006. Ineligible population

200. Hamstra DA, Mariados N, Sylvester J, et al. Sexual quality of life following prostate intensity modulated radiation therapy (IMRT) with a rectal/prostate spacer: Secondary analysis of a phase 3 trial. Practical Radiation Oncology. 2018 Jan Feb;8(1):e7-e15. PMID: 28951089. Ineligible comparison

201. Hamstra DA, Mariados N, Sylvester J, et al. Continued Benefit to Rectal Separation for Prostate Radiation Therapy: Final Results of a Phase III Trial. International Journal of Radiation Oncology, Biology, Physics. 2017 04 01;97(5):976-85. PMID: 28209443. Ineligible comparison

202. Han Y, Xu J, Kim J, et al. LINE-1 methylation in peripheral blood leukocytes and clinical characteristics and prognosis of prostate cancer patients. Oncotarget. 201701 Nov;8(55):94020-7. PMID: 619144330. Ineligible intervention

203. Hansen J, Gandaglia G, Bianchi M, et al. Reassessment of 30-, 60- and 90-day mortality rates in non-metastatic prostate cancer patients treated either with radical prostatectomy or radiation therapy. Can Urol Assoc J. 2014 Jan-Feb;8(1-2):E75-80. PMID: 24554978. Insufficient follow-up time

204. Haque R, UlcickasYood M, Xu X, et al. Cardiovascular disease risk and androgen deprivation therapy in patients with localised prostate cancer: a prospective cohort study. British Journal of Cancer. 2017 Oct 10;117(8):1233-40. PMID: 29017178. Ineligible intervention

205. Harke N, Godes M, Habibzada J, et al. Postoperative patient comfort in suprapubic drainage versus transurethral catheterization following robot-assisted radical prostatectomy: a prospective randomized clinical trial. World Journal of Urology. 2017 Mar;35(3):389-94. PMID: 27334135. Ineligible comparison
206. Harris CR, Punnen S, Carroll PR. Men with low preoperative sexual function may benefit from nerve sparing radical prostatectomy. Journal of Urology. 2013 Sep;190(3):981-6. PMID: 23410984. Ineligible intervention

207. Hauck CR, Ye H, Chen PY, et al. Increasing Fractional Doses Increases the Probability of Benign PSA Bounce in Patients Undergoing Definitive HDR Brachytherapy for Prostate Cancer. International Journal of Radiation Oncology, Biology, Physics. 201705 01;98(1):108-14. PMID: 28586946. Ineligible intervention

208. Heemsbergen W, Al-Mamgani A, Slot A, et al. Long-term results of the Dutch randomized prostate cancer trial: impact of doseescalation on local, biochemical, clinical failure, and survival. Radiotherapy and oncology. 2014;110(1):104-9. PMID: CN00986381. Ineligible population Pubmed 24246414.

209. Hegemann NS, Schlesinger-Raab A, Ganswindt $\mathrm{U}$, et al. Risk of second cancer following radiotherapy for prostate cancer: A population-based analysis. Radiation Oncology. 201703 Jan;12 (1) (no pagination)(2). PMID: 614136069. Ineligible population

210. Heidenreich A, Pfister D, Brehmer B, et al. Cytoreductive radical prostatectomy for prostate cancer with minimal osseous metastases: results of a first feasibility and case control study. Der urologe Ausg A. 2015;54(1):14-21. PMID: CN-01111558. Not available in English Pubmed 25519996.

211. Helou J, D'Alimonte L, Quon H, et al. Stereotactic ablative radiotherapy in the treatment of low and intermediate risk prostate cancer: Is there an optimal dose? Radiother Oncol. 2017 06;123(3):478-82. PMID: 28433413. Ineligible comparison

212. Henderson RH, Bryant C, Hoppe BS, et al. Fiveyear outcomes from a prospective trial of image-guided accelerated hypofractionated proton therapy for prostate cancer. Acta Oncol. 2017 Jul;56(7):963-70. PMID: 28514929. Ineligible comparison 
213. Herden J, Ernstmann N, Schnell D, et al. The HAROW study: an example of outcomes research: a prospective, non-interventional study comparing treatment options in localized prostate cancer. Der urologe Ausg A. 2014;53(12):1743-52. PMID: CN01112347. Not available in English Pubmed 25412911.

214. Hervas A, Gomez-Caamano A, Casana M, et al. Adjuvant versus salvage radiotherapy in prostate cancer: multi-institutional retrospective analysis of the Spanish RECAP database. Clin Transl Oncol. 2018 Feb;20(2):193-200. PMID: 28667448. Ineligible comparison

215. Hicks BM, Klil-Drori AJ, Yin H, et al. Androgen Deprivation Therapy and the Risk of Anemia in Men with Prostate Cancer. Epidemiology. 2017 09;28(5):712-8. PMID: 28768300. Ineligible population

216. Hicks BM, Yin H, Bladou F, et al. Androgen deprivation therapy for prostate cancer and the risk of hospitalisation for communityacquired pneumonia. Thorax. 2017 07;72(7):596-7. PMID: 27986803. Ineligible population

217. Hirasawa Y, Yoshioka K, Nasu Y, et al. Impact of Surgeon and Hospital Volume on the Safety of Robot-Assisted Radical Prostatectomy: A Multi-Institutional Study Based on a National Database. Urol Int. 2017;98(3):334-42. PMID: 28253500. Insufficient follow-up time

218. Hofer MD, Meeks JJ, Cashy J, et al. Impact of increasing prevalence of minimally invasive prostatectomy on open prostatectomy observed in the national inpatient sample and national surgical quality improvement program. Journal of Endourology. 2013 Jan;27(1):102-7. PMID: 22834981. No eligible outcomes reported

219. Hoffman K, Skinner H, Pugh T, et al. Patientreported Urinary, Bowel, and Sexual Function After Hypofractionated Intensitymodulated Radiation Therapy for Prostate Cancer: results From a Randomized Trial. American journal of clinical oncology: cancer clinical trials. 2016;(no pagination). PMID: CN-01210232. Ineligible comparison
220. Hoffman KE, Voong KR, Pugh TJ, et al. Risk of late toxicity in men receiving dose-escalated hypofractionated intensity modulated prostate radiation therapy: results from a randomized trial. International Journal of Radiation Oncology, Biology, Physics. 2014 Apr 01;88(5):1074-84. PMID: 24661661. Ineligible comparison

221. Horovitz D, Feng C, Messing EM, et al. Extraperitoneal vs Transperitoneal RobotAssisted Radical Prostatectomy in the Setting of Prior Abdominal or Pelvic Surgery. Journal of Endourology. 2017 04;31(4):366-73. PMID: 28073298. Ineligible comparison

222. Hoskin PJ, Rojas AM, Ostler PJ, et al. Quality of life after radical radiotherapy for prostate cancer: longitudinal study from a randomised trial of external beam radiotherapy alone or in combination with high dose rate brachytherapy. Clin Oncol (R Coll Radiol). 2013 May;25(5):321-7. PMID: 23384799. Ineligible population

223. Hounsome L, Rowe E, Verne J, et al. Variation in usage of radical prostatectomy and radical radiotherapy for men with locally advanced prostate cancer. Journal of Clinical Urology. 201701 Jan;10(1_suppl):34-8. PMID: 614410376. No eligible outcomes reported

224. Hu J, Aprikian AG, Cury FL, et al. Comparison of Surgery and Radiation as Local Treatments in the Risk of Locoregional Complications in Men Subsequently Dying From Prostate Cancer. Clinical Genitourinary Cancer. 2017 Sep 05;05:05. PMID: 28943330. Ineligible population

225. Hu JC, Gandaglia G, Karakiewicz PI, et al. Comparative effectiveness of robot-assisted versus open radical prostatectomy cancer control. European Urology. 2014 Oct;66(4):666-72. PMID: 24602934. Ineligible population

226. Hu JC, O'Malley P, Chughtai B, et al. Comparative Effectiveness of Cancer Control and Survival after Robot-Assisted versus Open Radical Prostatectomy. Journal of Urology. 2017 01;197(1):115-21. PMID: 27720782. Ineligible population 
227. Huang H, Muscatelli S, Naslund M, et al. Evaluation of Cancer Specific Mortality with Surgery versus Radiation as Primary Therapy for Localized High Grade Prostate Cancer in Men Younger Than 60 Years. Journal of Urology. 2019 Jan;201(1):120-8. PMID: 30577404. Ineligible population

228. Huang Y, Huang H, Pan XW, et al. The prognostic value of lymphovascular invasion in radical prostatectomy: a systematic review and meta-analysis. Asian Journal of Andrology. 2016 Sep-Oct;18(5):780-5. PMID: 26459779. Ineligible study design

229. Hughes D, Camp C, O'Hara J, et al. Health resource use after robot-assisted surgery vs open and conventional laparoscopic techniques in oncology: analysis of English secondary care data for radical prostatectomy and partial nephrectomy. BJU International. 2016 06;117(6):940-7. PMID: 26696305. No eligible outcomes reported

230. Hurwitz LM, Cullen J, Kim DJ, et al. Longitudinal regret after treatment for lowand intermediate-risk prostate cancer. Cancer. 2017 Nov 01;123(21):4252-8. PMID: 28678408. No eligible outcomes reported

231. Hussain M, Fizazi K, Saad F, et al. Enzalutamide in Men with Nonmetastatic, Castration-Resistant Prostate Cancer. N Engl J Med. 2018 Jun 28;378(26):2465-74. PMID: 29949494. Ineligible population

232. Hussain M, Tangen CM, Berry DL, et al. Intermittent versus continuous androgen deprivation in prostate cancer. N Engl J Med. 2013 Apr 04;368(14):1314-25. PMID: 23550669. Ineligible comparison

233. Huynh MA, Chen MH, Wu J, et al. Influence of Comorbidity on the Risk of Mortality in Men With Unfavorable-Risk Prostate Cancer Undergoing High-Dose Radiation Therapy Alone. International Journal of Radiation Oncology, Biology, Physics. 2016 07 15;95(4):1158-67. PMID: 27209511. Ineligible comparison

234. Hwang WL, Tendulkar RD, Niemierko A, et al. Comparison Between Adjuvant and EarlySalvage Postprostatectomy Radiotherapy for Prostate Cancer With Adverse Pathological Features. JAMA Oncol. 2018 May 10;4(5):e175230. PMID: 29372236. Ineligible population
235. Hyldgard VB, Laursen KR, Poulsen J, et al. Robot-assisted surgery in a broader healthcare perspective: a difference-indifference-based cost analysis of a national prostatectomy cohort. BMJ Open. 2017 Jul 21;7(7):e015580. PMID: 28733299. No eligible outcomes reported

236. Incrocci L, Wortel RC, Alemayehu WG, et al. Hypofractionated versus conventionally fractionated radiotherapy for patients with localised prostate cancer (HYPRO): final efficacy results from a randomised, multicentre, open-label, phase 3 trial. Lancet Oncol. 2016 Aug;17(8):1061-9. PMID: 27339116. Ineligible population

237. Ishiyama H, Kamitani N, Kawamura H, et al. Nationwide multi-institutional retrospective analysis of high-dose-rate brachytherapy combined with external beam radiotherapy for localized prostate cancer: An Asian Prostate HDR-BT Consortium. Brachytherapy. 2017 May - Jun;16(3):50310. PMID: 28222973. Ineligible population

238. Jacobs BL, Yabes JG, Lopa SH, et al. The early adoption of intensity-modulated radiotherapy and stereotactic body radiation treatment among older Medicare beneficiaries with prostate cancer. Cancer. 2017 Aug 01;123(15):2945-54. PMID: 28301689. No eligible outcomes reported

239. Jacobs BL, Zhang Y, Schroeck FR, et al. Use of advanced treatment technologies among men at low risk of dying from prostate cancer. Jama. 2013 Jun 26;309(24):2587-95. PMID: 23800935. No eligible outcomes reported

240. Jacobs BL, Zhang Y, Tan HJ, et al. Hospitalization trends after prostate and bladder surgery: implications of potential payment reforms. Journal of Urology. 2013 Jan;189(1):59-65. PMID: 23164391. Ineligible comparison

241. Jafri SM, Nguyen LN, Sirls LT. Recovery of urinary function after robotic-assisted laparoscopic prostatectomy versus radical perineal prostatectomy for early-stage prostate cancer. Int Urol Nephrol. 2018 Dec;50(12):2187-91. PMID: 30328088. Ineligible study design 
242. James ND, e Bono JS, Spears MR, et al. Abiraterone for Prostate Cancer Not Previously Treated with Hormone Therapy. N Engl J Med. 201707 27;377(4):338-51. PMID: 28578639. Ineligible population

243. Jang TL, Patel N, Faiena I, et al. Comparative effectiveness of radical prostatectomy with adjuvant radiotherapy versus radiotherapy plus androgen deprivation therapy for men with advanced prostate cancer. Cancer. 2018 Oct 15;124(20):4010-22. PMID: 30252932. Ineligible population

244. Jani AB, Schreibmann E, Rossi PJ, et al. Impact of $<$ sup $>18<$ /sup $>$ F-Fluciclovine PET on Target Volume Definition for Postprostatectomy Salvage Radiotherapy: Initial Findings from a Randomized Trial. J Nucl Med. 2017 Mar;58(3):412-8. PMID: 27609792. Ineligible population

245. Jansen H, van Oort IM, van Andel G, et al. Immediate treatment vs. active-surveillance in very-low-risk prostate cancer: the role of patient-, tumour-, and hospital-related factors. Prostate Cancer and Prostatic Diseases. 2018. PMID: 624996284. No eligible outcomes reported

246. Javanmard B, Hassanzadeh Haddad A, Yaghoobi M, et al. Diode laser ablation of prostate and channel transurethral resection of prostate in patients with prostate cancer and bladder outlet obstruction symptoms. Urol. 2014 Sep 06;11(4):1788-92. PMID: 25194077. Insufficient follow-up time

247. Jeong SJ, Yeon JS, Lee JK, et al. Development and validation of nomograms to predict the recovery of urinary continence after radical prostatectomy: comparisons between immediate, early, and late continence. World Journal of Urology. 2014 Apr;32(2):437-44. PMID: 23832420. Ineligible comparison

248. Jereczek-Fossa BA, Maucieri A, Marvaso G, et al. Impact of image guidance on toxicity and tumour outcome in moderately hypofractionated external-beam radiotherapy for prostate cancer. Medical Oncology. 201901 Jan;36 (1) (no pagination)(9). PMID: 625285488. Ineligible study design
249. Jereczek-Fossa BA, Surgo A, Maisonneuve P, et al. Late toxicity of image-guided hypofractionated radiotherapy for prostate: non-randomized comparison with conventional fractionation. Radiol Med (Torino). 2019 Jan;124(1):65-78. PMID: 30219945. Ineligible study design

250. Jespersen CG, Norgaard M, Borre M. Androgen-deprivation therapy in treatment of prostate cancer and risk of myocardial infarction and stroke: a nationwide Danish population-based cohort study. European Urology. 2014 Apr;65(4):704-9. PMID: 23433805. Ineligible population

251. Jespersen CG, Norgaard M, Jacobsen JB, et al. Patient comorbidity is associated with conservative treatment of localized prostate cancer. Scandinavian Journal of Urology. 2015;49(5):366-70. PMID: 25903072. No eligible outcomes reported

252. Jhan JH, Yang YH, Chang YH, et al. Hormone therapy for prostate cancer increases the risk of Alzheimer's disease: a nationwide 4-year longitudinal cohort study. Aging Male. 2017 Mar;20(1):33-8. PMID: 28067607. Ineligible population

253. Jhan JH, Yeh HC, Chang YH, et al. New-onset diabetes after androgen-deprivation therapy for prostate cancer: A nationwide propensity score-matched four-year longitudinal cohort study. J Diabetes Complications. 2018 Jul;32(7):688-92. PMID: 29909141. No eligible outcomes reported

254. Johnson ME, Zaorsky NG, Martin JM, et al. Patient reported outcomes among treatment modalities for prostate cancer. Can J Urol. 2016 Dec;23(6):8535-45. PMID: 27995848. Ineligible study design

255. Johnson SB, Lester-Coll NH, Kelly JR, et al. Brachytherapy Boost Utilization and Survival in Unfavorable-risk Prostate Cancer. European Urology. 2017 11;72(5):738-44. PMID: 28688613. Ineligible population

256. Johnson SC, Packiam VT, Golan S, et al. The Effect of Obesity on Perioperative Outcomes for Open and Minimally Invasive Prostatectomy. Urology. 2017 Feb;100:1116. PMID: 27890683. No eligible outcomes reported 
257. Joo EY, Moon YJ, Yoon SH, et al. Comparison of Acute Kidney Injury After RobotAssisted Laparoscopic Radical Prostatectomy Versus Retropubic Radical Prostatectomy: A Propensity Score Matching Analysis. Medicine (Baltimore). 2016 Feb;95(5):e2650. PMID: 26844486. No eligible outcomes reported

258. Kachnic LA, Pugh SL, Tai P, et al. RTOG 0518: randomized phase III trial to evaluate zoledronic acid for prevention of osteoporosis and associated fractures in prostate cancer patients. Prostate Cancer Prostatic Dis. 2013 Dec;16(4):382-6. PMID: 24080992. Ineligible intervention

259. Kallidonis P, Liatsikos E. Re: long-term Results of Active Surveillance in the Goteborg Randomized, Population-based Prostate Cancer Screening Trial. European urology. 2017;(no pagination). PMID: CN-01299752. Ineligible study design

260. Kamrava M, Rwigema JC, Chung M, et al. Predictors of distant metastasis after combined HDR brachytherapy and external beam radiation for prostate cancer. Journal of Contemporary Brachytherapy. 2013;5(3):127-33. PMID: 370041766. Ineligible study design

261. Kane CJ, Liss MA. Prostate cancer: risk versus benefit of lymph node dissection during prostatectomy. Nat Rev Urol. 2013 May;10(5):262-3. PMID: 23609844. Ineligible study design

262. Kaneda T, Ohashi T, Sakayori M, et al. Plan reproducibility of intraoperatively custombuilt linked seeds compared to loose seeds for prostate brachytherapy. Journal of Contemporary Brachytherapy. 2018;10(4):291-6. PMID: 624050735. Ineligible population

263. Kao HH, Kao LT, Li IH, et al. Androgen Deprivation Therapy Use Increases the Risk of Heart Failure in Patients With Prostate Cancer: A Population-Based Cohort Study. J Clin Pharmacol. 2019 Mar;59(3):335-43. PMID: 30402905. Ineligible comparison
264. Kao LT, Lin HC, Chung SD, et al. No increased risk of dementia in patients receiving androgen deprivation therapy for prostate cancer: a 5-year follow-up study. Asian Journal of Andrology. 2017 JulAug;19(4):414-7. PMID: 27232853. Ineligible comparison

265. Kapanen M, Collan J, Beule A, et al. Commissioning of MRI-only based treatment planning procedure for external beam radiotherapy of prostate. Magn Reson Med. 2013 Jul;70(1):127-35. PMID: 22886780. No eligible outcomes reported

266. Karl A, Buchner A, Tympner C, et al. Risk and timing of biochemical recurrence in pT3aN0/Nx prostate cancer with positive surgical margin - A multicenter study. Radiother Oncol. 2015 Jul;116(1):119-24. PMID: 26138059. Ineligible population

267. Karnes RJ, Sharma V, Choeurng V, et al. Development and validation of a prostate cancer genomic signature that predicts early adt treatment response following radical prostatectomy. Clinical Cancer Research. 201815 Aug;24(16):3908-16. PMID: 623453178. Ineligible comparison

268. Karsh LI, Gross ET, Pieczonka CM, et al. Absorbable Hydrogel Spacer Use in Prostate Radiotherapy: A Comprehensive Review of Phase 3 Clinical Trial Published Data. Urology. 2018 May;115:39-44. PMID: 29174940. Ineligible intervention

269. Kasuya G, Ishikawa H, Tsuji H, et al. Cancerspecific mortality of high-risk prostate cancer after carbon-ion radiotherapy plus long-term androgen deprivation therapy. Cancer Sci. 2017 Dec;108(12):2422-9. PMID: 28921785. Ineligible comparison

270. Katayama N, Yorozu A, Maruo S, et al. Predictive factors of rectal toxicity after permanent iodine-125 seed implantation: Prospective cohort study in 2339 patients. Brachytherapy. 2016 Nov - Dec;15(6):73645. PMID: 27720311. Ineligible intervention

271. Keane FK, Chen MH, Zhang D, et al. Androgen deprivation therapy and the risk of death from prostate cancer among men with favorable or unfavorable intermediate-risk disease. Cancer. 2015 Aug 15;121(16):27139. PMID: 25925789. Ineligible study design 
272. Keane FK, D'Amico AV. Androgen deprivation therapy use and the risk of death in men treated with high dose radiation for intermediate risk prostate cancer. Cancer. 2016;122(15):2341-9. PMID: 623491121. Ineligible study design

273. Kearns JT, Faino AV, Schenk JM, et al. Continued 5alpha-Reductase Inhibitor Use after Prostate Cancer Diagnosis and the Risk of Reclassification and Adverse Pathological Outcomes in the PASS. Journal of Urology. 2019 Jan;201(1):106-11. PMID: 30076904. Ineligible comparison

274. Keating NL, Liu PH, O'Malley AJ, et al. Androgen-deprivation therapy and diabetes control among diabetic men with prostate cancer. European Urology. 2014 Apr;65(4):816-24. PMID: 23453420. No eligible outcomes reported

275. Kendal WS. Age Bias in Time From Diagnosis Comparisons of Prostate Cancer Treatment. Am J Clin Oncol. 2018 Apr;41(4):402-8. PMID: 26967329. Ineligible population

276. Khan S, Hicks V, Colditz GA, et al. The association of weight change in young adulthood and smoking status with risk of prostate cancer recurrence. Int J Cancer. 201805 15;142(10):2011-8. PMID: 29270988. Ineligible comparison

277. Khmelevsky EV, Kancheli IN, Khoroshkov VS, et al. Morbidity dynamics in proton-photon or photon radiation therapy for locally advanced prostate cancer. Reports of Practical Oncology and Radiotherapy. 2018 January;23(1):21-7. PMID: 619354360. Ineligible comparison

278. Khoder WY, Trottmann M, Stuber A, et al. Early incontinence after radical prostatectomy: a community based retrospective analysis in 911 men and implications for preoperative counseling. Urol. 2013 Oct;31(7):1006-11. PMID: 22100069. Insufficient follow-up time

279. Khosrow-Khavar F, Rej S, Yin H, et al. Androgen Deprivation Therapy and the Risk of Dementia in Patients With Prostate Cancer. Journal of Clinical Oncology. 2017 Jan 10;35(2):201-7. PMID: 27870566. Ineligible population
280. Kim DK, Lee JY, Kim KJ, et al. Effect of Androgen-Deprivation Therapy on Bone Mineral Density in Patients with Prostate Cancer: A Systematic Review and MetaAnalysis. J. 2019 Jan 18;8(1):18. PMID: 30669289. Ineligible study design

281. Kim SH, Joung JY, Kim S, et al. Comparison of bone mineral loss by combined androgen block agonist versus $\mathrm{GnRH}$ in patients with prostate cancer: A 12 month-prospective observational study. Sci. 201703 06;7:39562. PMID: 28262724. Ineligible comparison

282. Kim SH, Joung JY, Suh YS, et al. Prevalence and survival prognosis of prostate cancer in patients with end-stage renal disease: a retrospective study based on the Korea national database (2003-2010). Oncotarget. 2017 Sep 08;8(38):64250-62. PMID: 28969067. Ineligible intervention

283. Kim SP, Shah ND, Karnes RJ, et al. Hospitalization costs for radical prostatectomy attributable to robotic surgery. European Urology. 2013 Jul;64(1):11-6. PMID: 22959352. Ineligible study design

284. King CR, Collins S, Fuller D, et al. Healthrelated quality of life after stereotactic body radiation therapy for localized prostate cancer: results from a multi-institutional consortium of prospective trials. International Journal of Radiation Oncology, Biology, Physics. 2013 Dec 01;87(5):939-

45. PMID: 24119836. Ineligible comparison

285. King CR, Freeman D, Kaplan I, et al. Stereotactic body radiotherapy for localized prostate cancer: pooled analysis from a multi-institutional consortium of prospective phase II trials. Radiother Oncol. 2013 Nov;109(2):217-21. PMID: 24060175. Ineligible study design

286. King MT, Chen MH, Moran BJ, et al. Brachytherapy monotherapy may be sufficient for a subset of patients with unfavorable intermediate risk prostate cancer. Urol. 2018 04;36(4):157.e15-.e20. PMID: 29276060. Ineligible study design 
287. King MT, Yang DD, Muralidhar V, et al. A comparative analysis of overall survival between high-dose-rate and low-dose-rate brachytherapy boosts for unfavorable-risk prostate cancer. Brachytherapy. 2019. PMID: 2001445217. Ineligible comparison

288. Kinoshita H, Nakagawa K, Usui Y, et al. Highdefinition resolution three-dimensional imaging systems in laparoscopic radical prostatectomy: randomized comparative study with high-definition resolution twodimensional systems. Surgical endoscopy. 2015;29(8):2203-9. PMID: CN-01254562. Ineligible intervention Pubmed 25361650.

289. Kishan AU, Cook RR, Ciezki JP, et al. Radical Prostatectomy, External Beam Radiotherapy, or External Beam Radiotherapy With Brachytherapy Boost and Disease Progression and Mortality in Patients With Gleason Score 9-10 Prostate Cancer. Jama. 201803 06;319(9):896-905. PMID: 29509865. Ineligible population

290. Kishan AU, Wang X, Seiferheld W, et al. Association of Gleason Grade With Androgen Deprivation Therapy Duration and Survival Outcomes: A Systematic Review and Patient-Level Meta-analysis. JAMA Oncol. 2019 Jan 01;5(1):91-6. PMID: 30326032. Ineligible study design

291. Klil-Drori AJ, Yin H, Tagalakis V, et al. Androgen Deprivation Therapy for Prostate Cancer and the Risk of Venous Thromboembolism. European Urology. 2016 07;70(1):56-61. PMID: 26138040. Ineligible comparison

292. Klotz L, Loblaw A, Sugar L, et al. Active Surveillance Magnetic Resonance Imaging Study (ASIST): results of a Randomized Multicenter Prospective Trial. European urology. 2018. PMID: CN-01617417. Ineligible population

293. Klotz L, Miller K, Crawford ED, et al. Disease control outcomes from analysis of pooled individual patient data from five comparative randomised clinical trials of degarelix versus luteinising hormonereleasing hormone agonists. European Urology. 2014 Dec;66(6):1101-8. PMID: 24440304. Ineligible comparison
294. Klotz L, O'Callaghan C, Ding K, et al. Nadir testosterone within first year of androgendeprivation therapy (ADT) predicts for time to castration-resistant progression: a secondary analysis of the PR-7 trial of intermittent versus continuous ADT. Journal of clinical oncology. 2015;33(10):1151-6. PMID: CN-01070336. Ineligible population Pubmed 25732157.

295. Klotz LH, McNeill IY, Kebabdjian M, et al. A phase 3, double-blind, randomised, parallelgroup, placebo-controlled study of oral weekly alendronate for the prevention of androgen deprivation bone loss in nonmetastatic prostate cancer: the Cancer and Osteoporosis Research with Alendronate and Leuprolide (CORAL) study. European Urology. 2013 May;63(5):927-35. PMID: 23040208. Ineligible population

296. Koo KC, Cho JS, Bang WJ, et al. CancerSpecific Mortality Among Korean Men with Localized or Locally Advanced Prostate Cancer Treated with Radical Prostatectomy Versus Radiotherapy: A Multi-Center Study Using Propensity Scoring and Competing Risk Regression Analyses. Cancer Res. 2018 Jan;50(1):129-37. PMID: 28279064. Ineligible population

297. Koo KC, Tuliao P, Yoon YE, et al. Robotassisted radical prostatectomy in the Korean population: a 5-year propensity-score matched comparative analysis versus open radical prostatectomy. International Journal of Urology. 2014 Aug;21(8):781-5. PMID: 24661241. Ineligible study design

298. Kopp RP, Marshall LM, Wang PY, et al. The burden of urinary incontinence and urinary bother among elderly prostate cancer survivors. European Urology. 2013 Oct;64(4):672-9. PMID: 23587870. Ineligible comparison

299. Koulikov D, Mohler MC, Mehedint DC, et al. Low detectable prostate specific antigen after radical prostatectomy - Treat or watch? Journal of Urology. 201401 Nov;192(5):1390-6. PMID: 600402743. Ineligible comparison 
300. Kowalczyk KJ, Gu X, Nguyen PL, et al. Optimal timing of early versus delayed adjuvant radiotherapy following radical prostatectomy for locally advanced prostate cancer. Urologic Oncology: Seminars and Original Investigations. 2014 April;32(3):303-8. PMID: 52904786. Ineligible comparison

301. Kowalczyk KJ, Huang AC, Hevelone ND, et al. Effect of minimizing tension during roboticassisted laparoscopic radical prostatectomy on urinary function recovery. World Journal of Urology. 2013 Jun;31(3):515-21. PMID: 23135639. Ineligible comparison

302. Kozuka T, Nakano M, Hashimoto M, et al. Acute and late complications after hypofractionated intensity modulated radiotherapy in prostate cancer. Jpn J Radiol. 2017 May;35(5):269-78. PMID: 28281047. Ineligible study design

303. Krahn MD, Bremner KE, Zagorski B, et al. Health care costs for state transition models in prostate cancer. Med Decis Making. 2014 04;34(3):366-78. PMID: 23894082. No eligible outcomes reported

304. Krasnow RE, Rodriguez D, Nagle RT, et al. The impact of age at the time of radiotherapy for localized prostate cancer on the development of second primary malignancies. Urol. 2018 11;36(11):500.e11-.e19. PMID: 30249519. Ineligible population

305. Krauss D, Hu C, Bahary J, et al. Importance of Local Control in Early-Stage Prostate Cancer: outcomes of Patients With Positive Post-Radiation Therapy Biopsy Results Treated in RTOG 9408. International journal of radiation oncology, biology, physics. 2015;92(4):863-73. PMID: CN-01101266. Ineligible comparison Pubmed 26104939.

306. Ku JY, Lee CH, Lee JZ, et al. Comparison of functional outcomes between laparoscopic radical prostatectomy and robot-assisted laparoscopic radical prostatectomy: a propensity score-matched comparison study. Asia-Pacific Journal of Clinical Oncology. 2017 June;13(3):212-8. PMID: 612422321. Ineligible study design
307. Ku JY, Lee JZ, Ha HK. The effect of continuous androgen deprivation treatment on prostate cancer patients as compared with intermittent androgen deprivation treatment. Korean J Urol. 2015 Oct;56(10):689-94. PMID: 26495069. Ineligible population

308. Kumar A, Tandon S, Samavedi S, et al. Current status of various neurovascular bundlesparing techniques in robot-assisted radical prostatectomy. J. 2016 Sep;10(3):187-200. PMID: 27251473. Ineligible comparison

309. Kurokawa S, Umemoto Y, Mizuno K, et al. New steps of robot-assisted radical prostatectomy using the extraperitoneal approach: a propensity-score matched comparison between extraperitoneal and transperitoneal approach in Japanese patients. BMC Urology. 2017 Nov 21;17(1):106. PMID: 29162068. No eligible outcomes reported

310. Kyrdalen AE, Dahl AA, Hernes E, et al. A national study of adverse effects and global quality of life among candidates for curative treatment for prostate cancer. BJU International. 2013 February;111(2):221-32. PMID: 52050921. Ineligible population

311. Ladjevardi S, Berglund A, Varenhorst E, et al. Treatment with curative intent and survival in men with high-risk prostate cancer. A population-based study of 11380 men with serum PSA level 20-100 ng/mL. BJU International. 2013 March;111(3):381-8. PMID: 52098993. Ineligible population

312. Laing KA, Bramwell SP, McNeill A, et al. Prostate cancer in Scotland: Does geography matter? An analysis of incidence, disease characteristics and survival between urban and rural areas. Journal of Clinical Urology. 2014 May;7(3):176-84. PMID: 372993095. Ineligible study design

313. Lange JM, Trock BJ, Gulati R, et al. A Framework for Treatment Decision Making at Prostate Cancer Recurrence. Med Decis Making. 2017 11;37(8):905-13. PMID: 28564551. Ineligible intervention 
314. Lawton CAF, Lin X, Hanks GE, et al. Duration of Androgen Deprivation in Locally Advanced Prostate Cancer: Long-Term Update of NRG Oncology RTOG 9202. International Journal of Radiation Oncology, Biology, Physics. 201706 01;98(2):296303. PMID: 28463149. Ineligible intervention

315. Lee A, Becker DJ, Lederman AJ, et al. Comparison of neoadjuvant vs concurrent/adjuvant androgen deprivation in men with high-risk prostate cancer receiving definitive radiation therapy. Tumori. 2017 July-August;103(4):387-93. PMID: 618174345. Ineligible comparison

316. Lee BH, Kibel AS, Ciezki JP, et al. Are biochemical recurrence outcomes similar after radical prostatectomy and radiation therapy? Analysis of prostate cancer-specific mortality by nomogram-predicted risks of biochemical recurrence. European Urology. 201501 Feb;67(2):204-9. PMID: 600335115. Included in previous report

317. Lee DJ, Barocas DA, Zhao Z, et al. Contemporary prostate cancer radiation therapy in the United States: Patterns of care and compliance with quality measures. Practical Radiation Oncology. 2018 September - October;8(5):307-16. PMID: 2000839553. Ineligible comparison

318. Lee DJ, Zhao Z, Huang LC, et al. Racial variation in receipt of quality radiation therapy for prostate cancer. Cancer Causes Control. 2018 Oct;29(10):895-9. PMID: 30099628. No eligible outcomes reported

319. Lee JK, Sjoberg DD, Miller MI, et al. Improved Recovery of Erectile Function in Younger Men after Radical Prostatectomy: Does it Justify Immediate Surgery in Low-risk Patients? European Urology. 2018 01;73(1):33-7. PMID: 28851580. Ineligible study design

320. Lee WR, Dignam JJ, Amin MB, et al. Randomized Phase III Noninferiority Study Comparing Two Radiotherapy Fractionation Schedules in Patients With Low-Risk Prostate Cancer. Journal of Clinical Oncology. 201607 10;34(20):2325-32. PMID: 27044935. Ineligible comparison
321. Leow JJ, Chang SL, Meyer CP, et al. Robotassisted Versus Open Radical Prostatectomy: A Contemporary Analysis of an All-payer Discharge Database. European Urology. 2016 11;70(5):837-45. PMID: 26874806. No eligible outcomes reported

322. Li R, Ruckle HC, Schlaifer AE, et al. The Effect of Androgen Deprivation Therapy Before Salvage Whole-gland Cryoablation After Primary Radiation Failure in Prostate Cancer Treatment. Urology. 2015 May;85(5):1137-42. PMID: 25799176. Ineligible population

323. Liesenfeld L, Kron M, Gschwend JE, et al. Prognostic Factors for Biochemical Recurrence More than 10 Years after Radical Prostatectomy. Journal of Urology. 2017 01;197(1):143-8. PMID: 27418452. Ineligible comparison

324. Lin CC, Gray PJ, Jemal A, et al. Androgen deprivation with or without radiation therapy for clinically node-positive prostate cancer. J Natl Cancer Inst. 2015 Jul;107(7). PMID: 25957435. Ineligible population

325. Linder BJ, Boorjian SA, Umbreit EC, et al. Interaction of adjuvant androgen deprivation therapy with patient comorbidity status on overall survival after radical prostatectomy for high-risk prostate cancer. International Journal of Urology. 2013 August;20(8):798805. PMID: 52369458. Ineligible population

326. Ling DC, Karukonda P, Smith RP, et al. Declining brachytherapy utilization for highrisk prostate cancer-Can clinical pathways reverse the trend? Brachytherapy. 2018 Nov - Dec;17(6):895-8. PMID: 30217434. No eligible outcomes reported

327. Liu JM, Yu CP, Chuang HC, et al. Androgen deprivation therapy for prostate cancer and the risk of autoimmune diseases. Prostate Cancer Prostatic Dis. 2019 Jan 28;28:28. PMID: 30692587. No eligible outcomes reported 
328. Liu P, Liu L, Zu X. Re: claude Schulman, Erik Cornel, Vsevolod Matveev, et al. Intermittent Versus Continuous Androgen Deprivation Therapy in Patients with Relapsing or Locally Advanced Prostate Cancer: a Phase 3b Randomised Study (ICELAND). Eur Urol 2016;69: 720-7. European urology. 2016;(no pagination). PMID: CN-01247314. Ineligible comparison

329. Loblaw A, Pickles T, Crook J, et al. Stereotactic Ablative Radiotherapy Versus Low Dose Rate Brachytherapy or External Beam Radiotherapy: Propensity Score Matched Analyses of Canadian Data. Clin Oncol (R Coll Radiol). 2017 Mar;29(3):161-70. PMID: 27780694. Ineligible study design

330. Loeb S, Berglund A, Stattin P. Population based study of use and determinants of active surveillance and watchful waiting for low and intermediate risk prostate cancer. Journal of Urology. 2013 November;190(5):1742-9. No eligible outcomes reported

331. Loeb S, Folkvaljon Y, Robinson D, et al. Immediate versus delayed prostatectomy: Nationwide population-based study $<$ sup $>*<$ sup $>$. Scandinavian Journal of Urology. 201603 Jul;50(4):246-54. PMID: 609870741. Ineligible comparison

332. Loeb S, Zhou Q, Siebert U, et al. Active Surveillance Versus Watchful Waiting for Localized Prostate Cancer: A Model to Inform Decisions. European Urology. 2017 12;72(6):899-907. PMID: 28844371. Ineligible study design

333. Loriot Y, Supiot S, Beauval JB, et al. Management of non-metastatic castrateresistant prostate cancer: A systematic review. Cancer Treatment Reviews. 2018 November;70:223-31. PMID: 2001162770. Ineligible study design

334. Ludwig MS, Kuban DA, Strom SS, et al. Assessing the Optimum Use of AndrogenDeprivation Therapy in High-Risk Prostate Cancer Patients Undergoing External Beam Radiation Therapy. Am j. 2017 Jan;11(1):73-81. PMID: 25891393. Ineligible study design
335. Lund JA, Wibe A, Widmark A, et al. Late effects to the rectum and anus in prostate cancer patients randomized to hormonal therapy versus hormonal therapy plus radiotherapy. Journal of Gastroenterology and Hepatology Research. 2013;2(10):82732. PMID: 372721355. Ineligible study design

336. Lundstrom KJ, Folkvaljon Y, Loeb S, et al. Small bowel obstruction and abdominal pain after robotic versus open radical prostatectomy. Scandinavian Journal of Urology. 2016 Jun;50(3):155-9. PMID: 26936203. Ineligible population

337. Lu-Yao GL, Albertsen PC, Moore DF, et al. Fifteen-year Outcomes Following Conservative Management Among Men Aged 65 Years or Older with Localized Prostate Cancer. European Urology. 2015 Nov;68(5):805-11. PMID: 25800944. Ineligible comparison

338. MacDougall ND, Dean C, Muirhead R. Stereotactic Body Radiotherapy in Prostate Cancer: Is Rapidarc a Better Solution than Cyberknife? Clinical Oncology. 2014 January;26(1):4-9. PMID: 52790677. Ineligible study design

339. Magheli A, Busch J, Leva N, et al. Comparison of surgical technique (open vs. laparoscopic) on pathological and long term functional outcomes following radical prostatectomy. BMC Urology. 2014 Feb 07;14:18. PMID: 24506815. Ineligible comparison

340. Mahal BA, Aizer AA, Ziehr DR, et al. Racial disparities in prostate cancere specific mortality in men with low-risk prostate cancer. Clinical Genitourinary Cancer. 2014;12(5):e189-e95. PMID: 53143517. Ineligible comparison

341. Mahal BA, Chen MH, Renshaw AA, et al. Early Versus Delayed Initiation of Salvage Androgen Deprivation Therapy and Risk of Prostate Cancer-Specific Mortality. J. 2018 Jun;16(6):727-34. PMID: 29891524. Ineligible comparison 
342. Major T, Polgar C, Jorgo K, et al. Dosimetric comparison between treatment plans of patients treated with low-dose-rate vs. highdose-rate interstitial prostate brachytherapy as monotherapy: Initial findings of a randomized clinical trial. Brachytherapy. 2017 May - Jun;16(3):608-15. PMID: 28325472. No eligible outcomes reported

343. Majumder K, Brandberg Y, Johansson H, et al. Effect on prostate volume following neoadjuvant treatment with an androgen receptor inhibitor monotherapy versus castration plus an androgen receptor inhibitor in prostate cancer patients intended for curative radiation therapy: A randomised study. Mol. 2018 Jan;8(1):141-6. PMID: 29387407. Insufficient follow-up time

344. Majumder K, Brandberg Y, Johansson H, et al. Less satisfaction with information in patients with prostate cancer treated with surgery and salvage radiotherapy compared with patients treated with curative radiotherapy alone, despite similar healthrelated quality of life. Clinical Genitourinary Cancer. 2014 Jun;12(3):e71-82. PMID: 24445250. Ineligible study design

345. Majumder K, Nilsson S, Johansson H, et al. Higher sexual interest with androgen receptor inhibitor monotherapy than with castration plus an androgen receptor inhibitor in prostate cancer patients treated with curative radiotherapy, but otherwise small health-related quality of life differences: A randomised prospective 18month follow-up study. Eur J Cancer. 2016 09;65:43-51. PMID: 27459586. Ineligible comparison

346. Mak RH, Hunt D, Efstathiou JA, et al. Acute and late urinary toxicity following radiation in men with an intact prostate gland or after a radical prostatectomy: A secondary analysis of RTOG 94-08 and 96-01. Urol. 2016 10;34(10):430.e1-7. PMID: 27381895. Ineligible comparison

347. Marcello M, Ebert M, Haworth A, et al. Association between treatment planning and delivery factors and disease progression in prostate cancer radiotherapy: Results from the TROG 03.04 RADAR trial. Radiother Oncol. 2018 02;126(2):249-56. PMID: 29122360. Ineligible intervention
348. Margel D, Nandy I, Wilson TH, et al. Predictors of pathological progression among men with localized prostate cancer undergoing active surveillance: a sub-analysis of the REDEEM study. Journal of Urology. 2013 Dec;190(6):2039-45. PMID: 23820059. No eligible outcomes reported

349. Mariados N, Sylvester J, Shah D, et al. Hydrogel Spacer Prospective Multicenter Randomized Controlled Pivotal Trial: Dosimetric and Clinical Effects of Perirectal Spacer Application in Men Undergoing Prostate Image Guided Intensity Modulated Radiation Therapy. International Journal of Radiation Oncology, Biology, Physics. 2015 Aug 01;92(5):971-7. PMID: 26054865. Ineligible comparison

350. Marina O, Gustafson GS, Kestin LL, et al. Comparison of dose-escalated, imageguided radiotherapy vs. dose-escalated, high-dose-rate brachytherapy boost in a modern cohort of intermediate-risk prostate cancer patients. Brachytherapy. 2014 JanFeb;13(1):59-67. PMID: 23871661. Included in previous report

351. Marsh S, Walters RW, Silberstein PT. Survival Outcomes of Radical Prostatectomy Versus Radiotherapy in Intermediate-Risk Prostate Cancer: A NCDB Study. Clinical Genitourinary Cancer. 2017 Aug 09;09:09. PMID: 28869138. No eligible outcomes reported

352. Marshall DT, Ramey S, Golshayan AR, et al. Phase I trial of weekly docetaxel, total androgen blockade, and image-guided intensity-modulated radiotherapy for localized high-risk prostate adenocarcinoma. Clinical Genitourinary Cancer. 2014 Apr;12(2):80-6. PMID: 24378335. Ineligible study design

353. Martell K, Husain S, Taussky D, et al. Multicenter Evaluation of Biochemical Relapse-Free Survival Outcomes for Intraoperatively Planned Prostate Brachytherapy Using an Automated Delivery System. International Journal of Radiation Oncology, Biology, Physics. 2017 11 15;99(4):895-903. PMID: 28807532. Ineligible comparison 
354. Marvaso G, Viola A, Fodor C, et al. Radiotherapy Plus Total Androgen Block Versus Radiotherapy Plus LHRH Analog Monotherapy for Non-metastatic Prostate Cancer. Anticancer Res. 2018 05;38(5):3139-43. PMID: 29715154. Ineligible comparison

355. Mason M, Clarke N, James N, et al. Adding Celecoxib With or Without Zoledronic Acid for Hormone-Naïve Prostate Cancer: longTerm Survival Results From an Adaptive, Multiarm, Multistage, Platform, Randomized Controlled Trial. Journal of clinical oncology. 2017;35(14):1530-41. PMID: CN-01372627. Ineligible intervention Pubmed 28300506.

356. Mason M, Maldonado Pijoan X, Steidle C, et al. Neoadjuvant androgen deprivation therapy for prostate volume reduction, lower urinary tract symptom relief and quality of life improvement in men with intermediate- to high-risk prostate cancer: a randomised noninferiority trial of degarelix versus goserelin plus bicalutamide. Clin Oncol (R Coll Radiol). 2013 Mar;25(3):190-6. PMID: 23257248. Ineligible population

357. Mason MD, Parulekar WR, Sydes MR, et al. Final report of the intergroup randomized study of combined androgen-deprivation therapy plus radiotherapy versus androgendeprivation therapy alone in locally advanced prostate cancer. Journal of Clinical Oncology. 201501 Jul;33(19):2143-50. PMID: 605202602. Ineligible population

358. Masterson TA, Cheng L, Boris RS, et al. Open vs. robotic-assisted radical prostatectomy: a single surgeon and pathologist comparison of pathologic and oncologic outcomes. Urol. 2013 Oct;31(7):1043-8. PMID: 22222059. Ineligible study design

359. Matsuyama H, Matsumoto H, Nagao K, et al. Running suture versus interrupted suture for vesicourethral anastomosis in retropubic radical prostatectomy: A randomized study. International Journal of Urology. 201501 Mar;22(3):271-7. PMID: 602967112. Ineligible comparison

360. Matthes KL, Limam M, Dehler S, et al. Primary Treatment Choice Over Time and Relative Survival of Prostate Cancer Patients: Influence of Age, Grade, and Stage. Oncol Res Treat. 2017;40(9):484-9. PMID: 28813713. Ineligible study design
361. Matthew AG, Raz O, Currie KL, et al. Psychological distress and lifestyle disruption in low-risk prostate cancer patients: Comparison between active surveillance and radical prostatectomy. Journal of Psychosocial Oncology. 2018 Mar-Apr;36(2):159-74. PMID: 28613997. Ineligible study design

362. Matzkin H, Chen J, German L, et al. Comparison between preoperative and realtime intraoperative planning 125I permanent prostate brachytherapy: long-term clinical biochemical outcome. Radiation Oncology. 2013 Dec 17;8:288. PMID: 24341548. Ineligible comparison

363. Mayor S. Adding radiotherapy to hormone treatment improves survival in older men with prostate cancer. BMJ (Online). 201508 Jan;350 (no pagination)(h84). PMID: 601430457. Ineligible study design

364. McCarthy A, Shaban R, Gillespie K, et al. Cryotherapy for docetaxel-induced hand and nail toxicity: randomised control trial. Supportive care in cancer. 2014;22(5):137583. PMID: CN-00984412. Ineligible intervention Pubmed 24362908.

365. McClintock TR, von Landenberg N, Cole AP, et al. Neoadjuvant Androgen Deprivation Therapy Prior to Radical Prostatectomy: Recent Trends in Utilization and Association with Postoperative Surgical Margin Status. Annals of Surgical Oncology. 201915 Jan;26(1):297-305. PMID: 625035796. No eligible outcomes reported

366. McKay RR, Zurita AJ, Werner L, et al. A Randomized Phase II Trial of Short-Course Androgen Deprivation Therapy With or Without Bevacizumab for Patients With Recurrent Prostate Cancer After Definitive Local Therapy. Journal of Clinical Oncology. 201606 01;34(16):1913-20. PMID: 27044933. Ineligible population

367. Menon M, Dalela D, Jamil M, et al. Functional Recovery, Oncologic Outcomes and Postoperative Complications after RobotAssisted Radical Prostatectomy: An Evidence-Based Analysis Comparing the Retzius Sparing and Standard Approaches. Journal of Urology. 2018 May;199(5):12107. PMID: 29225060. Ineligible comparison 
368. Merino T, San Francisco IF, Rojas PA, et al. Intensity-modulated radiotherapy versus radical prostatectomy in patients with localized prostate cancer: long-term followup. BMC Cancer. 2013 Nov 08;13:530. PMID: 24209381. Ineligible study design

369. Merrick GS, Wallner KE, Galbreath RW, et al. Is supplemental external beam radiation therapy essential to maximize brachytherapy outcomes in patients with unfavorable intermediate-risk disease? Brachytherapy. 2016 Jan-Feb;15(1):79-84. PMID: 26525214. Ineligible comparison

370. Michalski JM, Moughan J, Purdy J, et al. Effect of Standard vs Dose-Escalated Radiation Therapy for Patients With Intermediate-Risk Prostate Cancer: The NRG Oncology RTOG 0126 Randomized Clinical Trial. JAMA Oncol. 2018 Jun 14;4(6):e180039. PMID: 29543933. Ineligible comparison

371. Michalski JM, Yan Y, Watkins-Bruner D, et al. Preliminary toxicity analysis of 3dimensional conformal radiation therapy versus intensity modulated radiation therapy on the high-dose arm of the Radiation Therapy Oncology Group 0126 prostate cancer trial. International Journal of Radiation Oncology, Biology, Physics. 2013 Dec 01;87(5):932-8. PMID: 24113055. Ineligible study design

372. Miki K, Sasaki H, Kido M, et al. A comparative study on the efficacies of gonadotropinreleasing hormone ( $\mathrm{GnRH})$ agonist and GnRH antagonist in neoadjuvant androgen deprivation therapy combined with transperineal prostate brachytherapy for localized prostate cancer. BMC Cancer. 201609 01;16:708. PMID: 27586506. Ineligible study design

373. Miller ET, Chamie K, Kwan L, et al. Impact of treatment on progression to castrationresistance, metastases, and death in men with localized high-grade prostate cancer. Cancer Medicine. 2017 01;6(1):163-72. PMID: 27997745. Ineligible study design

374. Minana B, Rodriguez-Antolin A, Gomez-Veiga $\mathrm{F}$, et al. Treatment trends for clinically localised prostate cancer. National population analysis: GESCAP group. Actas Urol Esp. 2016 May;40(4):209-16. PMID: 26723895. Ineligible population
375. Mirhadi AJ, Zhang Q, Hanks GE, et al. Effect of Long-Term Hormonal Therapy (vs ShortTerm Hormonal Therapy): A Secondary Analysis of Intermediate-Risk Prostate Cancer Patients Treated on NRG Oncology RTOG 9202. International Journal of Radiation Oncology, Biology, Physics. 2017 03 01;97(3):511-5. PMID: 28126300. Ineligible comparison

376. Mishra MV, Shen X, Den RB, et al. Patterns of care for elderly men diagnosed with favorable-risk prostate cancer from 2004 to 2008: a population-based analysis. Am J Clin Oncol. 2013 Dec;36(6):606-11. PMID: 22892435. Ineligible intervention

377. Miszczyk M, Majewski W. Hematologic Toxicity of Conformal Radiotherapy and Intensity Modulated Radiotherapy in Prostate and Bladder Cancer Patients. Asian Pac J Cancer Prev. 2018 Oct 26;19(10):2803-6. PMID: 30360609. Ineligible study design

378. Montgomery B, Tretiakova MS, Joshua AM, et al. Neoadjuvant Enzalutamide Prior to Prostatectomy. Clinical Cancer Research. 2017 May 01;23(9):2169-76. PMID: 28151719. Insufficient follow-up time

379. Montorsi F, Brock G, Stolzenburg JU, et al. Effects of tadalafil treatment on erectile function recovery following bilateral nervesparing radical prostatectomy: a randomised placebo-controlled study (REACTT). European Urology. 2014 Mar;65(3):587-96. PMID: 24169081. Ineligible intervention

380. Moore C, Robertson N, Jichi F, et al. The Effect of Dutasteride on Magnetic Resonance Imaging Defined Prostate Cancer: mAPPED-A Randomized, Placebo Controlled, Double-Blind Clinical Trial. Journal of urology. 2017;(no pagination). PMID: CN-01341628. Ineligible intervention

381. Morgan SC, Hoffman K, Loblaw DA, et al. Hypofractionated Radiation Therapy for Localized Prostate Cancer: Executive Summary of an ASTRO, ASCO and AUA Evidence-Based Guideline. Journal of Urology. 2019 Mar;201(3):528-34. PMID: 30759696. Ineligible study design 
382. Morgans A, Chen YH, Sweeney C, et al. Quality of life during treatment with chemohormonal therapy: analysis of E3805 chemohormonal androgen ablation randomized trial in prostate cancer. Journal of clinical oncology. 2018;36(11):1088-95. PMID: CN-01570674. Ineligible population

383. Morris WJ, Pickles T, Keyes M. Using a surgical prostate-specific antigen threshold of $>0.2 \mathrm{ng} / \mathrm{mL}$ to define biochemical failure for intermediate- and high-risk prostate cancer patients treated with definitive radiation therapy in the ASCENDE-RT randomized control trial. Brachytherapy. 2018 November - December;17(6):837-44. PMID: 2001115892. No eligible outcomes reported

384. Morton G, Chung HT, McGuffin M, et al. Prostate high dose-rate brachytherapy as monotherapy for low and intermediate risk prostate cancer: Early toxicity and quality-of life results from a randomized phase II clinical trial of one fraction of 19Gy or two fractions of 13.5Gy. Radiother Oncol. 2017 01;122(1):87-92. PMID: 27823821. Ineligible comparison

385. Moschini M, Sharma V, Gandaglia G, et al. Long-term utility of adjuvant hormonal and radiation therapy for patients with seminal vesicle invasion at radical prostatectomy. BJU International. 2017 07;120(1):69-75. PMID: 27753192. Ineligible population

386. Moschini M, Zaffuto E, Karakiewicz PI, et al. External Beam Radiotherapy Increases the Risk of Bladder Cancer When Compared with Radical Prostatectomy in Patients Affected by Prostate Cancer: A Populationbased Analysis. European Urology. 2019 Feb;75(2):319-28. PMID: 30293908. Ineligible population

387. Mostaghel EA, Nelson PS, Lange P, et al. Targeted androgen pathway suppression in localized prostate cancer: a pilot study. Journal of Clinical Oncology. 2014 Jan 20;32(3):229-37. PMID: 24323034. No eligible outcomes reported
388. Moteabbed M, Trofimov A, Sharp GC, et al. A Prospective Comparison of the Effects of Interfractional Variations on Proton Therapy and Intensity Modulated Radiation Therapy for Prostate Cancer. International Journal of Radiation Oncology, Biology, Physics. 2016 May 01;95(1):444-53. PMID: 26907917. No eligible outcomes reported

389. Mulhall JP, Klein EA, Slawin K, et al. A Randomized, Double-Blind, PlaceboControlled Trial to Assess the Utility of Tacrolimus (FK506) for the Prevention of Erectile Dysfunction Following Bilateral Nerve-Sparing Radical Prostatectomy. J Sex Med. 2018 Sep;15(9):1293-9. PMID: 30224019. Ineligible intervention

390. Muralidhar V, Dinh KT, Mahal BA, et al. Differential post-prostatectomy cancerspecific survival of occult T3 vs. clinical T3 prostate cancer: Implications for managing patients upstaged on prostate magnetic resonance imaging. Urologic Oncology: Seminars and Original Investigations. 2015 01 Jul;33(7):330.e19-.e25. PMID: 604433827. Ineligible study design

391. Muralidhar V, Regan MM, Werner L, et al. Duration of Androgen Deprivation Therapy for High-Risk Prostate Cancer: Application of Randomized Trial Data in a Tertiary Referral Cancer Center. Clinical Genitourinary Cancer. 2016 08;14(4):e299305. PMID: 26778006. Ineligible study design

392. Murray NP, Aedo S, Fuentealba C, et al. 10 Year Biochemical Failure Free Survival of Men with CD82 Positive Primary Circulating Prostate Cells Treated by Radical Prostatectomy. Asian Pac J Cancer Prev. 2018 Jun 25;19(6):1577-83. PMID: 29936782. Ineligible intervention

393. Murtola TJ, Syvala H, Tolonen T, et al. Atorvastatin Versus Placebo for Prostate Cancer Before Radical Prostatectomy-A Randomized, Double-blind, Placebocontrolled Clinical Trial. European Urology. 2018 12;74(6):697-701. PMID: 30031572. Insufficient follow-up time 
394. Myers SN, Ghani KR, Dunn RL, et al. Notable Outcomes and Trackable Events after Surgery: Evaluating an Uncomplicated Recovery after Radical Prostatectomy. Journal of Urology. 2016 08;196(2):399404. PMID: 26916722. No eligible outcomes reported

395. Nabid A, Carrier N, Martin AG, et al. Duration of Androgen Deprivation Therapy in Highrisk Prostate Cancer: A Randomized Phase III Trial. European Urology. 2018 October;74(4):432-41. PMID: 2000916056. Ineligible comparison

396. Nam RK, Cheung P, Herschorn S, et al. Incidence of complications other than urinary incontinence or erectile dysfunction after radical prostatectomy or radiotherapy for prostate cancer: a population-based cohort study. Lancet Oncol. 2014 Feb;15(2):223-31. PMID: 24440474. Ineligible population

397. Narita T, Koie T, Ookubo T, et al. The impact of extended lymph node dissection versus neoadjuvant therapy with limited lymph node dissection on biochemical recurrence in high-risk prostate cancer patients treated with radical prostatectomy: a multiinstitutional analysis. Medical Oncology. 2017 Jan;34(1):1. PMID: 27889880. Ineligible intervention

398. Nead KT, Gaskin G, Chester C, et al. Influence of age on androgen deprivation therapyassociated Alzheimer's disease. Sci. 201610 18;6:35695. PMID: 27752112. No eligible outcomes reported

399. Nehra A, Parker WP, Haloi R, et al. Identification of Recurrence Sites Following Post-Prostatectomy Treatment for Prostate Cancer Using $<$ sup $>11</$ sup $>$ C-Choline Positron Emission Tomography and Multiparametric Pelvic Magnetic Resonance Imaging. Journal of Urology. 2018 Mar;199(3):726-33. PMID: 28916273. Ineligible population

400. Newcomb LF, Thompson IM, Jr B, et al. Outcomes of Active Surveillance for Clinically Localized Prostate Cancer in the Prospective, Multi-Institutional Canary PASS Cohort. Journal of Urology. 2016 Feb;195(2):313-20. PMID: 26327354. No eligible outcomes reported
401. Nguyen C, Lairson DR, Swartz MD, et al. Risks of Major Long-Term Side Effects Associated with Androgen-Deprivation Therapy in Men with Prostate Cancer. Pharmacotherapy. 2018 Oct;38(10):9991009. PMID: 30080934. Ineligible population

402. Nilsson S, Cislo P, Sartor O, et al. Patientreported quality-of-life analysis of radium223 dichloride from the phase III ALSYMPCA study. Annals of oncology : official journal of the european society for medical oncology. 2016;27(5):868-74. PMID: CN-01153221. Insufficient follow-up time Pubmed 26912557.

403. Nishimura S, Ohashi T, Momma T, et al. Prostate-specific antigen nadir within 12 months as an early surrogate marker of biochemical failure and distant metastasis after low-dose-rate brachytherapy or external beam radiotherapy for localized prostate cancer. Cancer Medicine. 2018 May;7(5):1794-801. PMID: 621425524. No eligible outcomes reported

404. Niwa N, Matsumoto K, Nishiyama T, et al. Selection of patients who would not require long-term prostate-specific antigen monitoring after low-dose-rate brachytherapy. Brachytherapy. 2018 Nov Dec;17(6):899-905. PMID: 30245170. Ineligible study design

405. Nossiter J, Sujenthiran A, Charman SC, et al. Robot-assisted radical prostatectomy vs laparoscopic and open retropubic radical prostatectomy: functional outcomes 18 months after diagnosis from a national cohort study in England. British Journal of Cancer. 201802 20;118(4):489-94. PMID: 29348490. Ineligible population

406. Noweski A, Roosen A, Lebdai S, et al. Mediumterm Follow-up of Vascular-targeted Photodynamic Therapy of Localized Prostate Cancer Using TOOKAD Soluble WST-11 (Phase II Trials). European Urology Focus. 2018. PMID: 2000657359. Ineligible population 
407. Nyarangi-Dix JN, Tichy D, Hatiboglu G, et al. Complete bladder neck preservation promotes long-term post-prostatectomy continence without compromising midterm oncological outcome: analysis of a randomised controlled cohort. World Journal of Urology. 2018 Mar;36(3):349-55. PMID: 29214353. Ineligible comparison

408. Obirieze AC, Moten A, Allen D, et al. AfricanAmerican Men with Low-Risk Prostate Cancer: Modern Treatment and Outcome Trends. J Racial Ethn Health Disparities. 2015 Sep;2(3):295-302. PMID: 26863460. Ineligible comparison

409. Ocampo-Trujillo A, Carbonell-Gonzalez J, Martinez-Blanco A, et al. Pre-operative training induces changes in the histomorphometry and muscle function of the pelvic floor in patients with indication of radical prostatectomy. Actas Urol Esp. 2014 Jul-Aug;38(6):378-84. PMID: 24440083. Ineligible intervention

410. Ogaya-Pinies G, Palayapalam-Ganapathi H, Rogers T, et al. Can dehydrated human amnion/chorion membrane accelerate the return to potency after a nerve-sparing robotic-assisted radical prostatectomy? Propensity score-matched analysis. J. 2018 Jun;12(2):235-43. PMID: 28656504. Ineligible intervention

411. Ohashi T, Yorozu A, Saito S, et al. Outcomes following iodine-125 prostate brachytherapy with or without neoadjuvant androgen deprivation. Radiotherapy and Oncology. 2013 November;109(2):241-5. PMID: 52845601. Ineligible comparison

412. Ohtani M, Suto H, Nosaka T, et al. Long-Term Endoscopic Follow-Up of Patients with Chronic Radiation Proctopathy after Brachytherapy for Prostate Cancer. Diagn. 2016;2016:1414090. PMID: 27378828. Ineligible study design

413. O'Neil B, Hoffman KE, Koyama T, et al. Patient Reported Comparative Effectiveness of Contemporary Intensity Modulated Radiation Therapy Versus External Beam Radiation Therapy of the Mid 1990s for Localized Prostate Cancer. Urology Practice. 2018 November;5(6):471-9. PMID: 2001159032. Ineligible population
414. O'Neil B, Koyama T, Alvarez J, et al. The Comparative Harms of Open and Robotic Prostatectomy in Population Based Samples. Journal of Urology. 2016 Feb;195(2):321-9. PMID: 26343985. Ineligible population

415. Ong WL, Evans SM, Millar JL. Underutilisation of high-dose-rate brachytherapy boost in men with intermediate-high risk prostate cancer treated with external beam radiotherapy. J Med Imaging Radiat Oncol. 2018 Apr;62(2):256-61. PMID: 29271056. No eligible outcomes reported

416. Ong WL, Evans SM, Spelman T, et al. Comparison of oncological and healthrelated quality of life outcomes between open and robot-assisted radical prostatectomy for localised prostate cancer findings from the population-based Victorian Prostate Cancer Registry. BJU International. 2016 Oct;118(4):563-9. PMID: 26573954. Ineligible population

417. Ording AG, Horvath-Puho E, Lash TL, et al. Does comorbidity interact with prostate cancer to increase mortality? A Danish cohort study of 45326 prostate cancer patients diagnosed during 1995-2011. Acta Oncol. 2016 May;55(5):611-8. PMID: 26586474. Ineligible comparison

418. Organ M, Wood L, Wilke D, et al. Intermittent LHRH therapy in the management of castrate-resistant prostate cancer (CRPCa): results of a multi-institutional randomized prospective clinical trial. Am J Clin Oncol. 2013 Dec;36(6):601-5. PMID: 22868247. Ineligible comparison

419. Orom H, Biddle C, Underwood W, et al. Worse Urinary, Sexual and Bowel Function Cause Emotional Distress and Vice Versa in Men Treated for Prostate Cancer. Journal of Urology. 2018 June;199(6):1464-9. PMID: 2000681299. Ineligible comparison

420. Orom H, Biddle C, Underwood W, et al. Racial or Ethnic and Socioeconomic Disparities in Prostate Cancer Survivors' Prostate-specific Quality of Life. Urology. 2018 Feb;112:1327. PMID: 28842210. Ineligible comparison

421. Orom H, Underwood W, Biddle C. Emotional Distress Increases the Likelihood of Undergoing Surgery among Men with Localized Prostate Cancer. Journal of Urology. 201701 Feb;197(2):350-5. PMID: 613973712. Insufficient follow-up time 
422. Oudard S, Latorzeff I, Caty A, et al. Effect of Adding Docetaxel to Androgen-Deprivation Therapy in Patients With High-Risk Prostate Cancer With Rising Prostate-Specific Antigen Levels After Primary Local Therapy: A Randomized Clinical Trial. JAMA Oncol. 2019 Jan 31;31:31. PMID: 30703190. Ineligible population

423. Paller CJ, Zhou XC, Heath EI, et al. Muscadine Grape Skin Extract (MPX) in Men with Biochemically Recurrent Prostate Cancer: A Randomized, Multicenter, PlaceboControlled Clinical Trial. Clinical Cancer Research. 2018 Jan 15;24(2):306-15. PMID: 29113986. Ineligible population

424. Pan HY, Jiang J, Hoffman KE, et al. Comparative toxicities and cost of intensitymodulated radiotherapy, proton radiation, and stereotactic body radiotherapy among younger men with prostate cancer. Journal of Clinical Oncology. 201820 Jun;36(18):1823-30. PMID: 623257885. Ineligible population

425. Parekh A, Chen MH, D'Amico AV, et al. Identification of comorbidities that place men at highest risk of death from androgen deprivation therapy before brachytherapy for prostate cancer. Brachytherapy. 2013 SepOct;12(5):415-21. PMID: 23651926. No eligible outcomes reported

426. Park J, Yoo DS, Song C, et al. Comparison of oncological outcomes between retropubic radical prostatectomy and robot-assisted radical prostatectomy: an analysis stratified by surgical experience. World Journal of Urology. 2014 Feb;32(1):193-9. PMID: 24062092. No eligible outcomes reported

427. Parnes HL, Brawley OW, Minasian LM, et al. Phase III prostate cancer chemoprevention trials. Recent Results Cancer Res. 2014;202:73-7. PMID: 24531780. Ineligible study design

428. Parsons JK, Pinto PA, Pavlovich CP, et al. A Randomized, Double-blind, Phase II Trial of PSA-TRICOM (PROSTVAC) in Patients with Localized Prostate Cancer: The Immunotherapy to Prevent Progression on Active Surveillance Study. Eur Urol Focus. 2018 09;4(5):636-8. PMID: 30197041. Ineligible intervention
429. Pastore AL, Palleschi G, Silvestri L, et al. Prospective randomized study of radiofrequency versus ultrasound scalpels on functional outcomes of laparoscopic radical prostatectomy. Journal of Endourology. 2013 Aug;27(8):989-93. PMID: 23510321. Ineligible comparison

430. Patel SA, Chen MH, Loffredo M, et al. The impact of comorbidity and PSA doubling time on the risk of death in men experiencing PSA failure following radiation therapy with or with androgen deprivation therapy for unfavorable-risk prostate cancer. Prostate Cancer Prostatic Dis. 2017 06;20(2):234-40. PMID: 28117382. Ineligible intervention

431. Pearce SM, Pariser JJ, Karrison T, et al. Comparison of Perioperative and Early Oncologic Outcomes between Open and Robotic Assisted Laparoscopic Prostatectomy in a Contemporary Population Based Cohort. Journal of Urology. 2016 07;196(1):76-81. PMID: 26860793. Insufficient follow-up time

432. Pearlstein KA, Basak R, Chen RC. Comparative Effectiveness of Prostate Cancer Treatment Options: Limitations of Retrospective Analysis of Cancer Registry Data. International Journal of Radiation Oncology, Biology, Physics. 2018 Aug 09;09:09. PMID: 30099129. Ineligible population

433. Pearse M, Fraser-Browne C, Davis ID, et al. A Phase III trial to investigate the timing of radiotherapy for prostate cancer with highrisk features: background and rationale of the Radiotherapy -- Adjuvant Versus Early Salvage (RAVES) trial. BJU International. 2014 Mar;113 Suppl 2:7-12. PMID: 24894850. Ineligible comparison

434. Peters M, Smit Duijzentkunst DA, Westendorp $\mathrm{H}$, et al. Adaptive cone-beam CT planning improves long-term biochemical diseasefree survival for $<$ sup $>125</$ sup $>$ I prostate brachytherapy. Brachytherapy. 2017 Mar Apr;16(2):282-90. PMID: 28110899. Ineligible study design

435. Pettersson A, Robinson D, Garmo H, et al. Age at diagnosis and prostate cancer treatment and prognosis: a population-based cohort study. Ann Oncol. 201802 01;29(2):377-85. PMID: 29161337. Ineligible intervention 
436. Picardi C, Rouzaud M, Kountouri M, et al. Impact of hydrogel spacer injections on interfraction prostate motion during prostate cancer radiotherapy. Acta Oncol. 2016 Jul;55(7):834-8. PMID: 26796870. Ineligible intervention

437. Pickles T, Tyldesley S, Hamm J, et al. Brachytherapy for Intermediate-Risk Prostate Cancer, Androgen Deprivation, and the Risk of Death. International Journal of Radiation Oncology, Biology, Physics. 2018 01 01;100(1):45-52. PMID: 29029889. Ineligible population

438. Pinkawa M, Berneking V, Konig L, et al. Hydrogel injection reduces rectal toxicity after radiotherapy for localized prostate cancer. Strahlentherapie und Onkologie. 2017 Jan;193(1):22-8. PMID: 27632342. Ineligible intervention

439. Pisansky T, Hunt D, Gomella L, et al. Duration of androgen suppression before radiotherapy for localized prostate cancer: radiation therapy oncology group randomized clinical trial 9910. Journal of clinical oncology. 2015;33(4):332-9. PMID: CN-01048466. Ineligible population Pubmed 25534388.

440. Ploussard G, e la Taille A, Moulin M, et al. Comparisons of the perioperative, functional, and oncologic outcomes after robot-assisted versus pure extraperitoneal laparoscopic radical prostatectomy. European Urology. 2014 Mar;65(3):610-9. PMID: 23245815. Ineligible study design

441. Pohle M, Magheli A, Fischer T, et al. The Effect of Evolving Strategies in the Surgical Management of Organ-Confined Prostate Cancer: Comparison of Data from 2005 to 2014 in a Multicenter Setting. Adv Ther. 2017 02;34(2):576-85. PMID: 28054309. No eligible outcomes reported

442. Pokala N, Trulson JJ, Islam M. Long-term outcome following radical prostatectomy for Gleason 8-10 prostatic adenocarcinoma. World Journal of Urology. 2014 Dec;32(6):1385-92. PMID: 24510158. Ineligible population

443. Pollack A, Walker G, Horwitz EM, et al. Randomized trial of hypofractionated external-beam radiotherapy for prostate cancer. Journal of Clinical Oncology. 2013 Nov 01;31(31):3860-8. PMID: 24101042. Ineligible comparison
444. Pommier P, Chabaud S, Lagrange JL, et al. Is There a Role for Pelvic Irradiation in Localized Prostate Adenocarcinoma? Update of the Long-Term Survival Results of the GETUG-01 Randomized Study. International Journal of Radiation Oncology Biology Physics. 201615 Nov;96(4):75969. PMID: 612966874. Ineligible population

445. Pompe RS, Davis-Bondarenko H, Zaffuto E, et al. Population-Based Validation of the 2014 ISUP Gleason Grade Groups in Patients Treated With Radical Prostatectomy, Brachytherapy, External Beam Radiation, or no Local Treatment. Prostate. 2017 May;77(6):686-93. PMID: 28156003. Ineligible population

446. Potosky AL, Haque R, Cassidy-Bushrow AE, et al. 1 Effectiveness of primary androgendeprivation therapy for clinically localized prostate cancer. Journal of Clinical Oncology. 201401 May;32(13):1324-30. PMID: 373747139. Ineligible population

447. Potters L, Rana Z, Lee L, et al. Outcomes of a Dose-Escalated Stereotactic Body Radiation Phase I/II trial for Patients with Low and Intermediate-Risk Prostate Cancer. International Journal of Radiation Oncology, Biology, Physics. 2019 Feb 02;02:02. PMID: 30721721. Ineligible study design

448. Presley CJ, Raldow AC, Cramer LD, et al. A new approach to understanding racial disparities in prostate cancer treatment. Journal of Geriatric Oncology. 2013 January;4(1):1-8. PMID: 52171218. Ineligible comparison

449. Punnen S, Cowan JE, Dunn LB, et al. A longitudinal study of anxiety, depression and distress as predictors of sexual and urinary quality of life in men with prostate cancer. BJU International. 2013 Jul;112(2):E67-75. PMID: 23795800. Ineligible population

450. Quon HC, Musunuru HB, Cheung P, et al. Dose-escalated stereotactic body radiation therapy for prostate cancer: Quality-of-life comparison of two prospective trials. Frontiers in Oncology. 201629 Aug;6 (AUG) (no pagination)(185). PMID: 612505481. Ineligible comparison 
451. Rajan P, Sooriakumaran P, Nyberg T, et al. Effect of Comorbidity on Prostate CancerSpecific Mortality: A Prospective Observational Study. Journal of Clinical Oncology. 2017 Nov 01;35(31):3566-74. PMID: 28930493. Ineligible comparison

452. Rammant E, Ost P, Swimberghe M, et al. Patient- versus physician-reported outcomes in prostate cancer patients receiving hypofractionated radiotherapy within a randomized controlled trial. Strahlentherapie und onkologie. 2018;(no pagination). PMID: CN-01668560. Ineligible population

453. Ranasinghe W, e Silva D, Bandaragoda T, et al. Robotic-assisted vs. open radical prostatectomy: A machine learning framework for intelligent analysis of patientreported outcomes from online cancer support groups. Urol. 2018 12;36(12):529.e1-.e9. PMID: 30236854. Ineligible population

454. Raval AD, Madhavan S, Mattes MD, et al. Types of chronic conditions combinations and initial cancer treatment among elderly Medicare beneficiaries with localised prostate cancer. Int J Clin Pract. 2016 Jul;70(7):606-18. PMID: 27291866. No eligible outcomes reported

455. Raziee H, Moraes FY, Murgic J, et al. Improved outcomes with dose escalation in localized prostate cancer treated with precision imageguided radiotherapy. Radiother Oncol. 2017 06;123(3):459-65. PMID: 28434799. Ineligible comparison

456. Reeve BB, Chen RC, Moore DT, et al. Impact of comorbidity on health-related quality of life after prostate cancer treatment: combined analysis of two prospective cohort studies. BJU International. 2014 Dec;114(6b):E74-E81. PMID: 24588845. Ineligible population

457. Renner W, Langsenlehner U, Krenn-Pilko S, et al. BCL2 genotypes and prostate cancer survival. Strahlentherapie und Onkologie. 2017 Jun;193(6):466-71. PMID: 28396899. Ineligible intervention
458. Resnick MJ, Guzzo TJ, Cowan JE, et al. Factors associated with satisfaction with prostate cancer care: Results from Cancer of the Prostate Strategic Urologic Research Endeavor (CaPSURE). BJU International. 2013 February;111(2):213-20. PMID: 52187361. Ineligible intervention

459. Rexer H. First-line therapy for "high-risk prostate cancer". HELP - high intensity focused ultrasound (HIFU) and Eilgard in patients with high-risk prostate cancer - a prospective, randomized study (AP 62/10 of the AUO). Der urologe Ausg A. 2014;53(7):1063-4. PMID: CN-01115817. Not available in English Pubmed 24939285.

460. Rexer H, Bussar-Maatz R. Comparison of four treatment options for low-risk prostate cancer: preference-based randomized study for the evaluation of four treatment modalities in prostate cancer with low or "early intermediate" risk (PREFERE) - trial AP 65/11 of the AUO. Der urologe Ausg A. 2015;54(5):723-5. PMID: CN-01256526. Not available in English Pubmed 25802104.

461. Rexer H, Graefen M. Phase III study for local or locally advanced prostate cancer : randomized, double-blind, placebocontrolled phase 3 study of apalutamide in patients with local high-risk prostate cancer or locally advanced prostate cancer receiving primary radiotherapy (ATLAS) study AP 90/15 of the AUO. Der urologe Ausg A. 2017;56(2):243-4. PMID: CN01413459. Not available in English Pubmed 28144693.

462. Ritch CR, You C, May AT, et al. Biochemical recurrence-free survival after roboticassisted laparoscopic vs open radical prostatectomy for intermediate- and highrisk prostate cancer. Urology. 2014 Jun;83(6):1309-15. PMID: 24746665. Ineligible study design 
463. Ritter MA. Commentary on "Postoperative radiotherapy after radical prostatectomy for high-risk prostate cancer: long-term results of a randomised controlled trial (EORTC trial 22911)." Bolla M, van Poppel H, Tombal B, Vekemans K, Da Pozzo L, de Reijke TM, Verbaeys A, Bosset JF, van Velthoven R, Colombel M, van de Beek C, Verhagen P, van den Bergh A, Sternberg C, Gasser T, van Tienhoven G, Scalliet P, Haustermans K, Collette L; European Organisation for Research and Treatment of Cancer, Radiation Oncology and GenitoUrinary Groups. Department of Radiation Oncology, Centre Hospitalier Universitaire A Michallon, Grenoble, France.: Lancet 2012;380(9858):2018-27. doi: 10.1016/S0140-6736(12)61253-7. Urol. 2014 Apr;32(3):372-3. PMID: 24679462. Ineligible study design

464. Roach M, Moughan J, Lawton CAF, et al. Sequence of hormonal therapy and radiotherapy field size in unfavourable, localised prostate cancer (NRG/RTOG 9413): long-term results of a randomised, phase 3 trial. Lancet Oncol. 2018 Nov;19(11):1504-15. PMID: 30316827. Ineligible population

465. Robertson NL, Moore CM, Ambler G, et al. MAPPED study design: a 6 month randomised controlled study to evaluate the effect of dutasteride on prostate cancer volume using magnetic resonance imaging. Contemporary Clinical Trials. 2013 Jan;34(1):80-9. PMID: 23085153. Ineligible intervention

466. Roder MA, Brasso K, Rusch E, et al. Length of life gained with surgical treatment of prostate cancer: A population-based analysis. Scandinavian Journal of Urology. 201501 Aug;49(4):275-81. PMID: 605251713. Ineligible comparison

467. Rodin D, Drumm M, Clayman R, et al. Risk Factors for Disease Progression After Postprostatectomy Salvage Radiation: Longterm Results of a Single-institution Experience. Clinical Genitourinary Cancer. 2017 Aug 03;03:03. PMID: 28864223. Ineligible study design
468. Romesser PB, Pei X, Shi W, et al. ProstateSpecific Antigen (PSA) Bounce After DoseEscalated External Beam Radiation Therapy Is an Independent Predictor of PSA Recurrence, Metastasis, and Survival in Prostate Adenocarcinoma Patients. International Journal of Radiation Oncology, Biology, Physics. 201801 01;100(1):59-67. PMID: 29254782. Ineligible comparison

469. Rosenberg K. Similar Mortality Rates After Surgery or Observation of Localized Prostate Cancer. The American journal of nursing. 201701 Nov;117(11):62. PMID: 619170812. Ineligible study design

470. Rosenthal SA, Hunt D, Sartor AO, et al. A phase 3 trial of 2 years of androgen suppression and radiation therapy with or without adjuvant chemotherapy for high-risk prostate cancer: Final results of radiation therapy oncology group phase 3 randomized trial NRG oncology RTOG 9902. International Journal of Radiation Oncology Biology Physics. 201501 Oct;93(2):294302. PMID: 605265910. Ineligible intervention

471. Royce TJ, Chen MH, Wu J, et al. Surrogate End Points for All-Cause Mortality in Men With Localized Unfavorable-Risk Prostate Cancer Treated With Radiation Therapy vs Radiation Therapy Plus Androgen Deprivation Therapy: A Secondary Analysis of a Randomized Clinical Trial. JAMA Oncol. 2017 May 01;3(5):652-8. PMID: 28097317. Ineligible comparison

472. Saad F, Cella D, Basch E, et al. Effect of apalutamide on health-related quality of life in patients with non-metastatic castrationresistant prostate cancer: an analysis of the SPARTAN randomised, placebo-controlled, phase 3 trial. Lancet Oncol. 2018 Oct;19(10):1404-16. PMID: 30213449. Ineligible comparison

473. Sachdeva A, Veeratterapillay R, Voysey A, et al. Positive surgical margins and biochemical recurrence following minimally-invasive radical prostatectomy An analysis of outcomes from a UK tertiary referral centre. BMC Urology. 2017 Oct 02;17(1):91. PMID: 28969608. Ineligible intervention 
474. Safdieh J, Wong A, Weiner JP, et al. Utilization of prostate brachytherapy for low risk prostate cancer: Is the decline overstated? Journal of Contemporary Brachytherapy. 2016;8(4):289-93. PMID: 611887094. No eligible outcomes reported

475. Salji M, Jones R, Paul J, et al. Feasibility study of a randomised controlled trial to compare (deferred) androgen deprivation therapy and cryotherapy in men with localised radiationrecurrent prostate cancer. British Journal of Cancer. 2014 Jul 29;111(3):424-9. PMID: 24946001. Ineligible population

476. Salomon G. Partial gland ablation with vasculartargeted phototherapy versus active surveillance for low-risk prostate cancer: results of a randomized trial. Der urologe. 2018;(no pagination). PMID: CN-01666502. Not available in English

477. Salonen AJ, Taari K, Ala-Opas M, et al. Advanced prostate cancer treated with intermittent or continuous androgen deprivation in the randomised FinnProstate Study VII: quality of life and adverse effects. European Urology. 2013 Jan;63(1):111-20. PMID: 22857983. Ineligible population

478. Sammon JD, Karakiewicz PI, Sun M, et al. Robot-assisted versus open radical prostatectomy: the differential effect of regionalization, procedure volume and operative approach. Journal of Urology. 2013 Apr;189(4):1289-94. PMID: 23085052. Insufficient follow-up time

479. Sanford N, Chen MH, Loffredo M, et al. Duration of the anti-androgen in men undergoing 6 months of an LHRH agonist and radiation therapy for unfavorable-risk prostate cancer and the risk of death. Prostate cancer and prostatic diseases. 2016;(no pagination). PMID: CN-01248524. Ineligible study design

480. Sanguineti G, Arcidiacono F, Landoni V, et al. Macroscopic Hematuria After Conventional or Hypofractionated Radiation Therapy: Results From a Prospective Phase 3 Study. International Journal of Radiation Oncology, Biology, Physics. 201610 01;96(2):304-12. PMID: 27475670. No eligible outcomes reported
481. Sartor O, Hoskin P, Coleman R, et al. Chemotherapy following radium-223 dichloride treatment in ALSYMPCA. Prostate. 2016;76(10):905-16. PMID: CN01167524. Ineligible intervention Pubmed 27004570.

482. Sasaki H, Kido M, Miki K, et al. Results of central pathology review of prostatic biopsies in a contemporary series from a phase III, multicenter, randomized controlled trial (SHIP0804). Pathology international. 2015;65(4):177-82. PMID: $\mathrm{CN}-01255717$. Ineligible intervention Pubmed 25707702.

483. Satkunasivam R, Lo M, Stern M, et al. The role of provider characteristics in the selection of surgery or radiation for localized prostate cancer and association with quality of care indicators. American Journal of Clinical Oncology: Cancer Clinical Trials. 201801 Nov;41(11):1076-82. PMID: 624634461. No eligible outcomes reported

484. Savard J, Hervouet S, Ivers H. Prostate cancer treatments and their side effects are associated with increased insomnia. Psychooncology. 2013 Jun;22(6):1381-8. PMID: 22888075. No eligible outcomes reported

485. Sayyid RK, Alibhai SMH, Sutradhar R, et al. Population-based outcomes of men with a single negative prostate biopsy: Importance of continued follow-up among older patients. Urol. 2019 Feb 13;13:13. PMID: 30770299. Ineligible population

486. Sayyid RK, Evans A, Hersey K, et al. A Phase II, Randomized, Open-Label Study of Neoadjuvant Degarelix versus LHRH Agonist in Prostate Cancer Patients Prior to Radical Prostatectomy. Clinical Cancer Research. 201704 15;23(8):1974-80. PMID: 27756786. No eligible outcomes reported

487. Sayyid RK, Sayyid AK, Klaassen Z, et al. Testosterone Responders to Continuous Androgen Deprivation Therapy Show Considerable Variations in Testosterone Levels on Followup: Implications for Clinical Practice. Journal of Urology. 2018 Jan;199(1):251-6. PMID: 28751266. Ineligible population 
488. Scailteux LM, Vincendeau S, Balusson F, et al. Androgen deprivation therapy and cardiovascular risk: No meaningful difference between GnRH antagonist and agonists-a nationwide population-based cohort study based on 2010-2013 French Health Insurance data. Eur J Cancer. 2017 05;77:99-108. PMID: 28390298. Ineligible population

489. Schiffmann J, Haese A, Lenz J, et al. Differences in Patient Characteristics Among Men Choosing Open or RobotAssisted Radical Prostatectomy in Contemporary Practice at a European HighVolume Center. Urol Int. 2016;97(1):8-15. PMID: 26780655. Ineligible study design

490. Schiffmann J, Larcher A, Sun M, et al. Differences in Patient Characteristics among Men Choosing Open or Robot-Assisted Radical Prostatectomy in Contemporary Practice - Analysis of Surveillance, Epidemiology, and End Results Database. Urol Int. 2017;98(1):40-8. PMID: 27486887. No eligible outcomes reported

491. Schiffmann J, Lesmana H, Tennstedt P, et al. Additional androgen deprivation makes the difference: biochemical recurrence-free survival in prostate cancer patients after HDR brachytherapy and external beam radiotherapy. Strahlentherapie und Onkologie. 2015;191(4):330-7. PMID: CN01111889. Ineligible study design Pubmed 25471276.

492. Schlussel Markovic E, Buckstein M, Stone NN, et al. Outcomes and toxicities in patients with intermediate-risk prostate cancer treated with brachytherapy alone or brachytherapy and supplemental external beam radiation therapy. BJU International. 2018 05;121(5):774-80. PMID: 29319919. Ineligible study design

493. Schmid M, Hanske J, Ravi P, et al. Relationship between androgen deprivation therapy and community-acquired respiratory infections in patients with prostate cancer. International Journal of Urology. 2016 Apr;23(4):305-11. PMID: 26763083. No eligible outcomes reported
494. Schmid M, Sammon JD, Reznor G, et al. Dosedependent effect of androgen deprivation therapy for localized prostate cancer on adverse cardiac events. BJU International. 201601 Aug;118(2):221-9. PMID: 611289239. Ineligible intervention

495. Schmidt B, Eapen RS, Cowan JE, et al. Practice patterns of primary EBRT with and without ADT in prostate cancer treatment. Prostate Cancer Prostatic Dis. 2019 03;22(1):117-24. PMID: 30171230. No eligible outcomes reported

496. Schoenfeld JD, Margalit DN, Kasperzyk JL, et al. A single nucleotide polymorphism in inflammatory gene RNASEL predicts outcome after radiation therapy for localized prostate cancer. Clinical Cancer Research. 2013 Mar 15;19(6):1612-9. PMID: 23382116. Ineligible study design

497. Schreiber D, Rineer J, Weiss JP, et al. Clinical and biochemical outcomes of men undergoing radical prostatectomy or radiation therapy for localized prostate cancer. Radiation Oncology Journal. 2015;33(1):21-8. PMID: 603679497. Ineligible study design

498. Schroeck FR, Kaufman SR, Jacobs BL, et al. Receipt of best care according to current quality of care measures and outcomes in men with prostate cancer. Journal of Urology. 201501 Feb;193(2):500-4. PMID: 601149348. Ineligible comparison

499. Schroeck FR, Kaufman SR, Jacobs BL, et al. Regional variation in quality of prostate cancer care. Journal of Urology. 2014 Apr;191(4):957-62. PMID: 24144685. Ineligible population

500. Schroeck FR, Kaufman SR, Jacobs BL, et al. Adherence to performance measures and outcomes among men treated for prostate cancer. Journal of Urology. 2014 Sep;192(3):743-8. PMID: 24681332. Ineligible intervention

501. Schulman C, Cornel E, Matveev V, et al. Intermittent Versus Continuous Androgen Deprivation Therapy in Patients with Relapsing or Locally Advanced Prostate Cancer: A Phase 3b Randomised Study (ICELAND). European Urology. 2016 Apr;69(4):720-7. PMID: 26520703. Ineligible population 
502. Seisen T, Vetterlein MW, Karabon P, et al. Efficacy of Local Treatment in Prostate Cancer Patients with Clinically Pelvic Lymph Node-positive Disease at Initial Diagnosis. European Urology. 2017 Sep 07;07:07. PMID: 28890245. Ineligible population

503. Senzaki T, Fukumori T, Mori H, et al. Clinical Significance of Neoadjuvant Combined Androgen Blockade for More Than Six Months in Patients with Localized Prostate Cancer Treated with Prostate Brachytherapy. Urol Int. 2015;95(4):457-64. PMID: 26461847. Ineligible study design

504. Sfoungaristos S, Kontogiannis S, Perimenis P. Early continence recovery after preservation of maximal urethral length until the level of verumontanum during radical prostatectomy: primary oncological and functional outcomes after 1 year of followup. Biomed Res Int. 2013;2013:426208. PMID: 24163815. Ineligible comparison

505. Shah S, Young HN, Cobran EK. Comparative Effectiveness of Conservative Management Compared to Cryotherapy in Localized Prostate Cancer Patients. Am j. 2018 09;12(5):1681-91. PMID: 29877137. Insufficient follow-up time

506. Shahid N, Loblaw A, Chung HT, et al. Longterm Toxicity and Health-related Quality of Life after Single-fraction High Dose Rate Brachytherapy Boost and Hypofractionated External Beam Radiotherapy for Intermediate-risk Prostate Cancer. Clin Oncol (R Coll Radiol). 2017 Jul;29(7):41220. PMID: 28190638. Ineligible study design

507. Shaikh MP, Alite F, Wu MJ, et al. Adjuvant Radiotherapy Versus Wait-and-See Strategy for Pathologic T3 or Margin-Positive Prostate Cancer: A Meta-Analysis. Am J Clin Oncol. 2018 Aug;41(8):730-8. PMID: 28225445. Ineligible study design

508. Shaikh T, Li T, Handorf EA, et al. Long-Term Patient-Reported Outcomes From a Phase 3 Randomized Prospective Trial of Conventional Versus Hypofractionated Radiation Therapy for Localized Prostate Cancer. International Journal of Radiation Oncology, Biology, Physics. 201703 15;97(4):722-31. PMID: 28244407. Ineligible comparison
509. Shao YH, Kim S, Moore DF, et al. Cancerspecific survival after metastasis following primary radical prostatectomy compared with radiation therapy in prostate cancer patients: results of a population-based, propensity score-matched analysis. European Urology. 2014 Apr;65(4):693700. PMID: 23759328. Ineligible population

510. Shao YH, Moore DF, Shih W, et al. Fracture after androgen deprivation therapy among men with a high baseline risk of skeletal complications. BJU International. 2013 May;111(5):745-52. PMID: 23331464. Ineligible comparison

511. Sharfo AWM, Dirkx MLP, Bijman RG, et al. Late toxicity in the randomized multicenter HYPRO trial for prostate cancer analyzed with automated treatment planning. Radiotherapy and Oncology. 2018 August;128(2):349-56. PMID: 2000825115. Ineligible population

512. Sharma V, Meeks JJ. Open conversion during minimally invasive radical prostatectomy: Impact on perioperative complications and predictors from national data. Journal of Urology. 201401 Dec;192(6):1657-62. PMID: 600450054. Insufficient follow-up time

513. Sharpley C, Bitsika V, Christie D, et al. Psychological resilience aspects that mediate the depressive effects of urinary incontinence in prostate cancer survivors 10 years after treatment with radiation and hormone ablation. Journal of psychosocial oncology. 2017;35(4):438-50. PMID: CN01401150. Ineligible population Pubmed 28318448.

514. Sheng W, Kirschner-Hermanns R, Zhang H. Elderly patients aged $>=75$ years with locally advanced prostate cancer may benefit from local treatment: a populationbased propensity score-adjusted analysis. World journal of urology. 201901 Feb;37(2):317-25. PMID: 626358638. Ineligible population

515. Sheng W, Zhang H, Lu Y. Survival outcomes of locally advanced prostate cancer in patients aged $<50$ years after local therapy in the contemporary US population. Int Urol Nephrol. 2018 Aug;50(8):1435-44. PMID: 29982959. Ineligible population 
516. Sheth N, Youssef I, Osborn V, et al. Association of Nadir Prostate-specific Antigen $>0.5$ $\mathrm{ng} / \mathrm{mL}$ after Dose-escalated External Beam Radiation with Prostate Cancer-specific Endpoints. Cureus. 2018 Jun 12;10(6):e2790. PMID: 30112266. Ineligible study design

517. Shilkrut M, Merrick GS, McLaughlin PW, et al. The addition of low-dose-rate brachytherapy and androgen-deprivation therapy decreases biochemical failure and prostate cancer death compared with dose-escalated external-beam radiation therapy for highrisk prostate cancer. Cancer. $2013 \mathrm{Feb}$ 01;119(3):681-90. PMID: 22893254. Ineligible population

518. Shimer SE. Prostate cancer treatment modalities and survival outcomes: a comparative analysis of Falmouth Hospital versus Massachusetts and nationwide hospitals. $\mathrm{J}$ Registry Manag. 2013;40(2):78-83. PMID: 24002132. Ineligible population

519. Shiota M, Yokomizo A, Takeuchi A, et al. Smoking effect on secondary bladder cancer after external beam radiotherapy for prostate cancer. Jpn J Clin Oncol. 2016 Oct;46(10):952-7. PMID: 27432454. Ineligible comparison

520. Shipley WU, Seiferheld W, Lukka HR, et al. Radiation with or without Antiandrogen Therapy in Recurrent Prostate Cancer. N Engl J Med. 201702 02;376(5):417-28. PMID: 28146658. Ineligible population

521. Sierra LC, Sánchez ZD, e PCA. Quality of life in patients diagnosed of prostate cancer treated with continuous androgen deprivation therapy vs. intermittent therapy. Anales del sistema sanitario de navarra. 2015;38(2):193-201. PMID: CN-01411577. Ineligible comparison Pubmed 26486525.

522. Silberstein JL, Poon SA, Sjoberg DD, et al. Long-term oncological outcomes of a phase II trial of neoadjuvant chemohormonal therapy followed by radical prostatectomy for patients with clinically localised, highrisk prostate cancer. BJU International. 2015 01 Jul;116(1):50-6. PMID: 603925915. Ineligible study design
523. Silberstein JL, Su D, Glickman L, et al. Oncological outcomes: Open vs robotic prostatectomy. BJU International. 2013 February;111(2):206-12. PMID: 368242036. Ineligible study design

524. Slagsvold JE, Viset T, Wibe A, et al. Radiation therapy did not induce long-term changes in rectal mucosa: Results from the randomized scandinavian prostate cancer group 7 trial. International Journal of Radiation Oncology Biology Physics. 201615 Jul;95(4):126872. PMID: 610189076. Ineligible study design

525. Smith MR, Morton RA, Barnette KG, et al. Toremifene to reduce fracture risk in men receiving androgen deprivation therapy for prostate cancer. Journal of Urology. 2013 Jan;189(1 Suppl):S45-50. PMID: 23234631. Ineligible intervention

526. Smith MR, Saad F, Chowdhury S, et al. Apalutamide Treatment and Metastasis-free Survival in Prostate Cancer. N Engl J Med. 2018 Apr 12;378(15):1408-18. PMID: 29420164. Ineligible comparison

527. Song W, Park JH, Jeon HG, et al. Comparison of Oncologic Outcomes and Complications According to Surgical Approach to Radical Prostatectomy: Special Focus on the Perineal Approach. Clinical Genitourinary Cancer. 2017 08;15(4):e645-e52. PMID: 28216277. Ineligible study design

528. Sooriakumaran P, Nyberg T, Akre O, et al. Comparative effectiveness of radical prostatectomy and radiotherapy in prostate cancer: observational study of mortality outcomes. Bmj. 2014 Feb 26;348:g1502. PMID: 24574496. Ineligible population

529. Sooriakumaran P, Nyberg T, Akre O, et al. Survival Among Men at High Risk of Disseminated Prostate Cancer Receiving Initial Locally Directed Radical Treatment or Initial Androgen Deprivation Therapy. European Urology. 2017 09;72(3):345-51. PMID: 28416350. Ineligible comparison

530. Spector BL, Brooks NA, Strigenz ME, et al. Bladder Neck Contracture Following Radical Retropubic versus Robotic-Assisted Laparoscopic Prostatectomy. Current Urology. 2017 Aug;10(3):145-9. PMID: 28878598. Ineligible study design 
531. Stabile A, Orczyk C, Hosking-Jervis F, et al. Medium term oncological outcomes in a large cohort of men treated with either focal or hemi-ablation using HIFU for primary localized prostate cancer. BJU International. 2019 Feb 12;12:12. PMID: 30753756. Ineligible comparison

532. Steuber T, Jilg C, Tennstedt P, et al. Standard of Care Versus Metastases-directed Therapy for PET-detected Nodal Oligorecurrent Prostate Cancer Following Multimodality Treatment: A Multi-institutional Casecontrol Study. Eur Urol Focus. $2018 \mathrm{Mar}$ 10;10:10. PMID: 29530632. Ineligible population

533. Stinesen Kollberg K, Thorsteinsdottir T, Wilderang U, et al. Social constraints and psychological well-being after prostate cancer: A follow-up at 12 and 24 months after surgery. Psychooncology. 2018 02;27(2):668-75. PMID: 29024232. Ineligible study design

534. Stock RG, Buckstein M, Liu JT, et al. The relative importance of hormonal therapy and biological effective dose in optimizing prostate brachytherapy treatment outcomes. BJU International. 2013 Jul;112(2):E44-50. PMID: 23773225. No eligible outcomes reported

535. Stone NN, Stock RG. 15-year cause specific and all-cause survival following brachytherapy for prostate cancer: Negative impact of longterm hormonal therapy. Journal of Urology. 2014 September;192(3):754-9. PMID: 53253119. Ineligible population

536. Stone NN, Stock RG. Stage T3b prostate cancer diagnosed by seminal vesicle biopsy and treated with neoadjuvant hormone therapy, permanent brachytherapy and external beam radiotherapy. BJU International. 2019 Februaryy;123(2):277-83. PMID: 623776728. Ineligible study design

537. Stone NN, Winoker JS, Kaplan SA, et al. Factors influencing long-term urinary symptoms after prostate brachytherapy. BJU International. 2018 Nov;122(5):831-6. PMID: 29726091. Ineligible study design
538. Stoyanova R, Pahlajani NH, Egleston BL, et al. The impact of dose-escalated radiotherapy plus androgen deprivation for prostate cancer using 2 linked nomograms. Cancer. 201301 Mar;119(5):1080-8. PMID: 52273855. Ineligible population

539. Stranne J, Brasso K, Brennhovd B, et al. SPCG15: a prospective randomized study comparing primary radical prostatectomy and primary radiotherapy plus androgen deprivation therapy for locally advanced prostate cancer. Scandinavian Journal of Urology. 2018 Dec 26:1-8. PMID: 30585526. Ineligible population

540. Student V, Jr V, A G, et al. Advanced Reconstruction of Vesicourethral Support (ARVUS) during Robot-assisted Radical Prostatectomy: One-year Functional Outcomes in a Two-group Randomised Controlled Trial. European Urology. 2017 05;71(5):822-30. PMID: 27283216. No eligible outcomes reported

541. Studer UE, Whelan P, Wimpissinger F, et al. Differences in time to disease progression do not predict for cancer-specific survival in patients receiving immediate or deferred androgen-deprivation therapy for prostate cancer: final results of EORTC randomized trial 30891 with 12 years of follow-up. European Urology. 2014 Nov;66(5):829-38. PMID: 23932338. Ineligible population

542. Suardi N, Moschini M, Gallina A, et al. Nervesparing approach during radical prostatectomy is strongly associated with the rate of postoperative urinary continence recovery. BJU International. 2013 May;111(5):717-22. PMID: 22726993. Ineligible comparison

543. Sugihara T, Yasunaga H, Horiguchi H, et al. Comparisons of perioperative outcomes and costs between open and laparoscopic radical prostatectomy: a propensity-score matching analysis based on the Japanese Diagnosis Procedure Combination database. International Journal of Urology. 2013 Mar;20(3):349-53. PMID: 23320826. Ineligible study design 
544. Sujenthiran A, Nossiter J, Charman SC, et al. National Population-Based Study Comparing Treatment-Related Toxicity in Men Who Received Intensity Modulated Versus 3-Dimensional Conformal Radical Radiation Therapy for Prostate Cancer. International Journal of Radiation Oncology, Biology, Physics. 201712 01;99(5):125360. PMID: 28974414. Ineligible population

545. Sujenthiran A, Nossiter J, Parry M, et al. National cohort study comparing severe medium-term urinary complications after robot-assisted vs laparoscopic vs retropubic open radical prostatectomy. BJU International. 2018 03;121(3):445-52. PMID: 29032582. Ineligible population

546. Sujenthiran A, Nossiter J, Parry M, et al. Treatment-related toxicity in men who received Intensity-modulated versus 3Dconformal radiotherapy after radical prostatectomy: A national population-based study. Radiother Oncol. 2018 08;128(2):357-63. PMID: 29773442. Ineligible population

547. Sureda A, Fumado L, Ferrer M, et al. Healthrelated quality of life in men with prostate cancer undergoing active surveillance versus radical prostatectomy, external-beam radiotherapy, prostate brachytherapy and reference population: a cross-sectional study. Health Qual Life Outcomes. 2019 Jan 14;17(1):11. PMID: 30642340. Ineligible study design

548. Sussman R, Carvalho FLF, Harbin A, et al. Survival and secondary interventions following treatment for locally-advanced prostate cancer. Can J Urol. 2018 Oct;25(5):9516-24. PMID: 30281010. Ineligible population

549. Sutani S, Ohashi T, Sakayori M, et al. Comparison of genitourinary and gastrointestinal toxicity among four radiotherapy modalities for prostate cancer: Conventional radiotherapy, intensitymodulated radiotherapy, and permanent iodine-125 implantation with or without external beam radiotherapy. Radiother Oncol. 2015 Nov;117(2):270-6. PMID: 26318662. Ineligible study design
550. Sveistrup J, f Rosenschold PM, Deasy JO, et al. Improvement in toxicity in high risk prostate cancer patients treated with image-guided intensity-modulated radiotherapy compared to 3D conformal radiotherapy without daily image guidance. Radiation Oncology. 2014 Feb 04;9:44. PMID: 24495815. Ineligible population

551. Taira AV, Merrick GS, Butler WM, et al. Time to failure after definitive therapy for prostate cancer: Implications for importance of aggressive local treatment. Journal of Contemporary Brachytherapy. 2013;5(4):215-21. PMID: 372166277. Ineligible comparison

552. Taira AV, Merrick GS, Galbreath RW, et al. Impact of Androgen Deprivation Therapy on Overall Mortality in Prostate Brachytherapy Patients With Low Pretreatment Testosterone Levels. Am J Clin Oncol. 2018 Jul;41(7):667-73. PMID: 27740974. Ineligible study design

553. Tan HJ, Xiong S, Laviana AA, et al. Technique and outcomes of bladder neck intussusception during robot-assisted laparoscopic prostatectomy: A parallel comparative trial. Urol. 2016 12;34(12):529.e1-.e7. PMID: 27743849. Ineligible study design

554. Tanaka N, Asakawa I, Nakai Y, et al. Comparison of PSA value at last follow-up of patients who underwent low-dose rate brachytherapy and intensity-modulated radiation therapy for prostate cancer. BMC Cancer. 201725 Aug;17 (1) (no pagination)(573). PMID: 617962241. Ineligible study design

555. Tanaka N, Hirayama A, Yoneda T, et al. Trends of risk classification and primary therapy for Japanese patients with prostate cancer in Nara Uro-Oncological Research Group (NUORG)--a comparison between 20042006 and 2007-2009. BMC Cancer. 2013 Dec 10;13:588. PMID: 24325407. No eligible outcomes reported

556. Tatsugami K, Yoshioka K, Shiroki R, et al. Reality of nerve sparing and surgical margins in surgeons' early experience with robot-assisted radical prostatectomy in Japan. International Journal of Urology. 2017 03;24(3):191-6. PMID: 28122393. No eligible outcomes reported 
557. Taussky D, Bedwani S, Meissner N, et al. A comparison of early prostate-specific antigen decline between prostate brachytherapy and different fractionation of external beam radiation-Impact on biochemical failure. Brachytherapy. 2018 Mar - Apr;17(2):277-82. PMID: 29306674. Ineligible study design

558. Taussky D, Preisser F, Karakiewicz PI, et al. Impact of diabetes and metformin use on prostate cancer outcome of patients treated with radiation therapy: results from a large institutional database. Can J Urol. 2018 Oct;25(5):9509-15. PMID: 30281009. Ineligible population

559. Tay KJ, Polascik TJ, Elshafei A, et al. Propensity Score-Matched Comparison of Partial to Whole-Gland Cryotherapy for Intermediate-Risk Prostate Cancer: An Analysis of the Cryo On-Line Data Registry Data. Journal of Endourology. 2017 June;31(6):564-71. PMID: 616689162. Ineligible study design

560. Taylor KL, Hoffman RM, Davis KM, et al. Treatment Preferences for Active Surveillance versus Active Treatment among Men with Low-Risk Prostate Cancer. Cancer Epidemiol Biomarkers Prev. 2016 08;25(8):1240-50. PMID: 27257092. No eligible outcomes reported

561. Tendulkar R. Point: Early Salvage vs Adjuvant Radiotherapy for High-Risk Prostate Cancer: Serial PSA Testing With Early Salvage Radiotherapy Is a Viable Option in Most High-Risk Men. Oncology (Williston). 201710 15;31(10):750-2. PMID: 29083470. Ineligible study design

562. Thomas HR, Chen MH, D'Amico AV, et al. Association Between Androgen Deprivation Therapy and Patient-reported Depression in Men With Recurrent Prostate Cancer. Clinical Genitourinary Cancer. 2018 08;16(4):313-7. PMID: 29866496. Ineligible population

563. Thompson JE, Egger S, Bohm M, et al. Superior Biochemical Recurrence and Long-term Quality-of-life Outcomes Are Achievable with Robotic Radical Prostatectomy After a Long Learning Curve-Updated Analysis of a Prospective Single-surgeon Cohort of 2206 Consecutive Cases. European Urology. 2018 05;73(5):664-71. PMID: 29273404. Ineligible study design
564. Thompson JE, Hayen A, Landau A, et al. Medium-term oncological outcomes for extended vs saturation biopsy and transrectal vs transperineal biopsy in active surveillance for prostate cancer. BJU International. 2015 01 Jun;115(6):884-91. PMID: 604586732. Ineligible intervention

565. Thomsen FB, Brasso K, Christensen IJ, et al. Survival benefit of early androgen receptor inhibitor therapy in locally advanced prostate cancer: long-term follow-up of the SPCG-6 study. Eur J Cancer. 2015 Jul;51(10):1283-92. PMID: 25892647. Ineligible population

566. Thor M, Jackson A, Zelefsky MJ, et al. Interinstitutional analysis demonstrates the importance of lower than previously anticipated dose regions to prevent late rectal bleeding following prostate radiotherapy. Radiother Oncol. 2018 04;127(1):88-95. PMID: 29530433. Ineligible comparison

567. Tiberi D, Rodrigues G, Pickles T, et al. External validation of the ProCaRS nomograms and comparison of existing risk-stratification tools for localized prostate cancer. Can Urol Assoc J. 2017 Mar-Apr;11(3-4):94-100. PMID: 28515807. No eligible outcomes reported

568. Tienza A, Hevia M, Merino I, et al. Can low urinary tract symptoms influence postprostatectomy urinary incontinence? Minerva Urologica e Nefrologica. 2016 August;68(4):324-9. PMID: 612922230. Ineligible comparison

569. Tilki D, Preisser F, Tennstedt P, et al. Adjuvant radiation therapy is associated with better oncological outcome compared with salvage radiation therapy in patients with $\mathrm{pN} 1$ prostate cancer treated with radical prostatectomy. BJU International. 2017 05;119(5):717-23. PMID: 27743493. Ineligible comparison

570. Tinay I, Aslan G, Kural AR, et al. Pathologic Outcomes of Candidates for Active Surveillance Undergoing Radical Prostatectomy: Results from a Contemporary Turkish Patient Cohort. Urol Int. 2018;100(1):43-9. PMID: 29275406. Ineligible study design 
571. Tombal B, Cornel EB, Persad R, et al. Clinical

Outcomes and Testosterone Levels

Following Continuous Androgen

Deprivation in Patients with Relapsing or

Locally Advanced Prostate Cancer: A Post

Hoc Analysis of the ICELAND Study. Journal of Urology. 2017 Nov;198(5):1054-

60. PMID: 28552710. Ineligible comparison

572. Tomita N, Soga N, Ogura Y, et al. Effects of dose-escalated radiotherapy in combination with long-term androgen deprivation on prostate cancer. Br J Radiol. 2018 Feb;91(1083):20170431. PMID: 29166142. Ineligible comparison

573. Toren P, Wong LM, Timilshina N, et al. Active surveillance in patients with a PSA $>10$ ng/mL. Can Urol Assoc J. 2014 Sep;8(910):E702-7. PMID: 25408810. Ineligible comparison

574. Tosco L, Laenen A, Briganti A, et al. The survival impact of neoadjuvant hormonal therapy before radical prostatectomy for treatment of high-risk prostate cancer. Prostate Cancer Prostatic Dis. 2017 12;20(4):407-12. PMID: 28485390. Ineligible population

575. Tosco L, Laenen A, Gevaert T, et al. Neoadjuvant degarelix with or without apalutamide followed by radical prostatectomy for intermediate and high-risk prostate cancer: ARNEO, a randomized, double blind, placebo-controlled trial. BMC Cancer. 201804 02;18(1):354. PMID: 29606109. Ineligible comparison

576. Tozawa K, Yasui T, Umemoto Y, et al. Pitfalls of robot-assisted radical prostatectomy: a comparison of positive surgical margins between robotic and laparoscopic surgery. International Journal of Urology. 2014 Oct;21(10):976-9. PMID: 24912809. Ineligible study design

577. Trama A, Botta L, Nicolai N, et al. Prostate cancer changes in clinical presentation and treatments in two decades: an Italian population-based study. Eur J Cancer. 2016 11;67:91-8. PMID: 27620947. Ineligible population
578. Tsai HT, Pfeiffer RM, Philips GK, et al. Risks of Serious Toxicities from Intermittent versus Continuous Androgen Deprivation Therapy for Advanced Prostate Cancer: A Population Based Study. Journal of Urology. 2017 05;197(5):1251-7. PMID: 27993663. Ineligible comparison

579. Tseng YD, Paciorek AT, Martin NE, et al. Impact of national guidelines on brachytherapy monotherapy practice patterns for prostate cancer. Cancer. 201415 Mar;120(6):824-32. PMID: 52898804. No eligible outcomes reported

580. Tyson MD, Castle. Racial disparities in survival for patients with clinically localized prostate cancer adjusted for treatment effects. Mayo Clin Proc. 2014 Mar;89(3):300-7. PMID: 24582189. Included in previous report

581. Ueno S, Kitagawa Y, Onozawa M, et al. Background factors and short-term healthrelated quality of life in patients who initially underwent radical prostatectomy or androgen deprivation therapy for localized prostate cancer in a Japanese prospective observational study (J-CaP Innovative Study-1). Prostate International. 2018 Mar;6(1):7-11. PMID: 29556483. No eligible outcomes reported

582. van den Bos W, Muller BG, e la Rosette JJ. A randomized controlled trial on focal therapy for localized prostate carcinoma: hemiablation versus complete ablation with irreversible electroporation. Journal of Endourology. 2013 Mar;27(3):262-4. PMID: 23469828. Ineligible study design

583. Van Hemelrijck M, Garmo H, Holmberg L, et al. Thromboembolic events following surgery for prostate cancer. European Urology. 2013 Feb;63(2):354-63. PMID: 23021972. Insufficient follow-up time

584. van Tol-Geerdink JJ, Willem Leer J, Weijerman PC, et al. Choice between prostatectomy and radiotherapy when men are eligible for both: a randomized controlled trial of usual care vs decision aid. BJU International. 2013 Apr;111(4):564-73. PMID: 22882966. No eligible outcomes reported 
585. Vargas CE, Hartsell WF, Dunn M, et al. Hypofractionated Versus Standard Fractionated Proton-beam Therapy for Lowrisk Prostate Cancer: Interim Results of a Randomized Trial PCG GU 002. Am J Clin Oncol. 2018 Feb;41(2):115-20. PMID: 26523442. Ineligible study design

586. Venderbos L, Aluwini S, Roobol M, et al. Longterm follow-up after active surveillance or curative treatment: quality-of-life outcomes of men with low-risk prostate cancer. Quality of life research. 2017:1-11. PMID: $\mathrm{CN}-01332179$. Ineligible study design

587. Viani GA, Silva LB, Silva BB, et al. Acute toxicity profile in prostate cancer with conventional and hypofractionated treatment. Radiation Oncology. 2013 Apr 21;8:94. PMID: 23601254. Insufficient follow-up time

588. Vigneault E, Mbodji K, Magnan S, et al. Highdose-rate brachytherapy boost for prostate cancer treatment: Different combinations of hypofractionated regimens and clinical outcomes. Radiother Oncol. 2017 07;124(1):49-55. PMID: 28652094. Ineligible study design

589. Vigneault E, Morton G, Parulekar WR, et al. Randomised Phase II Feasibility Trial of Image-guided External Beam Radiotherapy With or Without High Dose Rate Brachytherapy Boost in Men with Intermediate-risk Prostate Cancer (CCTG PR15/ NCT01982786). Clin Oncol (R Coll Radiol). 2018 Sep;30(9):527-33. PMID: 29903505. No eligible outcomes reported

590. Waddle MR, Sio TT, Van Houten HK, et al. Photon and Proton Radiation Therapy Utilization in a Population of More Than 100 Million Commercially Insured Patients. International Journal of Radiation Oncology, Biology, Physics. 201712 01;99(5):107882. PMID: 28939229. No eligible outcomes reported

591. Wallander M, Axelsson KF, Lundh D, et al. Patients with prostate cancer and androgen deprivation therapy have increased risk of fractures-a study from the fractures and fall injuries in the elderly cohort (FRAILCO). Osteoporos Int. 2019 Jan;30(1):115-25. PMID: 30324413. Ineligible population
592. Wallis CJ, Mahar A, Cheung P, et al. New Rates of Interventions to Manage Complications of Modern Prostate Cancer Treatment in Older Men. European Urology. 2016 05;69(5):933-41. PMID: 26572707. No eligible outcomes reported

593. Wallis CJ, Mahar AL, Satkunasivam R, et al. Cardiovascular and Skeletal-related Events Following Localized Prostate Cancer Treatment: Role of Surgery, Radiotherapy, and Androgen Deprivation. Urology. 2016 11;97:145-52. PMID: 27502032. Ineligible population

594. Wallis CJD, Cheung P, Herschorn S, et al. Complications following surgery with or without radiotherapy or radiotherapy alone for prostate cancer. British Journal of Cancer. 201517 Mar;112(6):977-82. PMID: 605464224. Ineligible population

595. Wallis CJD, Mahar AL, Cheung P, et al. Hospitalizations to Manage Complications of Modern Prostate Cancer Treatment in Older Men. Urology. 2016 10;96:142-7. PMID: 27289026. Ineligible population

596. Wang C, Kamrava M, King C, et al. Racial Disparity in Prostate Cancer-Specific Mortality for High-Risk Prostate Cancer: A Population-Based Study. Cureus. 2017 Jan 06;9(1):e961. PMID: 28168138. Ineligible comparison

597. Wang C, King CR, Kamrava M, et al. Pattern of solid and hematopoietic second malignancy after local therapy for prostate cancer. Radiother Oncol. 2017 04;123(1):133-8. PMID: 28187996. No eligible outcomes reported

598. Wang C, Kishan AU, Kamrava M, et al. External Beam Radiation Therapy With a Brachytherapy Boost Versus Radical Prostatectomy in Gleason Pattern 5 Prostate Cancer: A Population-Based Cohort Study. International Journal of Radiation Oncology, Biology, Physics. 201708 01;98(5):104552. PMID: 28721887. Ineligible population

599. Wang EH, Yu JB, Gross CP, et al. Variation in pelvic lymph node dissection among patients undergoing radical prostatectomy by hospital characteristics and surgical approach: Results from the national cancer database. Journal of Urology. 201501 Mar;193(3):820-5. PMID: 601694064. No eligible outcomes reported 
600. Wang R, Zeidan AM, Yu JB, et al. Myelodysplastic Syndromes and Acute Myeloid Leukemia After Radiotherapy for Prostate Cancer: A Population-Based Study. Prostate. 2017 04;77(5):437-45. PMID: 27868212. No eligible outcomes reported

601. Wedde TB, Smastuen MC, Brabrand S, et al. Ten-year survival after High-Dose-Rate Brachytherapy combined with External Beam Radiation Therapy in high-risk prostate cancer: A comparison with the Norwegian SPCG-7 cohort. Radiother Oncol. 2018 Oct 30;30:30. PMID: 30389241. Ineligible study design

602. Wiegel T, Bartkowiak D, Bottke D, et al. Adjuvant radiotherapy versus wait-and-see after radical prostatectomy: 10-year followup of the ARO 96-02/AUO AP 09/95 trial. European Urology. 2014 Aug;66(2):243-50. PMID: 24680359. Ineligible population

603. Wiegel T, Bartkowiak D, Bottke D, et al. Prostate-specific antigen persistence after radical prostatectomy as a predictive factor of clinical relapse-free survival and overall survival: 10-year data of the ARO 96-02 trial. International journal of radiation oncology, biology, physics. 2015;91(2):28894. PMID: CN-01112088. Ineligible population Pubmed 25445556.

604. Wiegel T, Stöckle M, Bartkowiak D. PREFEREnce-based randomized evaluation of treatment modalities in low or early intermediate-risk prostate cancer. European urology. 2015;67(1):1-2. PMID: CN01113409. No eligible outcomes reported Pubmed 25269383.

605. Wilkins A, Mossop H, Syndikus I, et al. Hypofractionated radiotherapy versus conventionally fractionated radiotherapy for patients with intermediate-risk localised prostate cancer: 2-year patient-reported outcomes of the randomised, non-inferiority, phase 3 CHHiP trial. Lancet Oncol. 2015 Dec;16(16):1605-16. PMID: 26522334. Ineligible comparison

606. Williams SB, Duan Z, Chamie K, et al. Risk of hospitalisation after primary treatment for prostate cancer. BJU International. 2017 07;120(1):48-55. PMID: 27561186. No eligible outcomes reported
607. Williams SB, Huo J, Chamie K, et al. Discerning the survival advantage among patients with prostate cancer who undergo radical prostatectomy or radiotherapy: The limitations of cancer registry data. Cancer. 201705 01;123(9):1617-24. PMID: 28099688. Ineligible comparison

608. Wilson JM, Dearnaley DP, Syndikus I, et al. The Efficacy and Safety of Conventional and Hypofractionated High-Dose Radiation Therapy for Prostate Cancer in an Elderly Population: A Subgroup Analysis of the CHHiP Trial. International Journal of Radiation Oncology, Biology, Physics. 2018 04 01;100(5):1179-89. PMID: 29722660. Ineligible population

609. Wilt TJ. Management of low risk and low PSA prostate cancer: Long term results from the prostate cancer intervention versus observation trial. Prostate Cancer Prevention. 2014; Recent Results in Cancer Research. 202:149-69. PMID: 372701452. Included in previous report

610. Wong AT, Safdieh JJ, Rineer J, et al. A population-based analysis of contemporary patterns of care in younger men $(<60$ years old) with localized prostate cancer. Int Urol Nephrol. 2015 Oct;47(10):1629-34. PMID: 26329748. Ineligible population

611. Wong AT, Schwartz D, Osborn V, et al. Adjuvant radiation with hormonal therapy is associated with improved survival in men with pathologically involved lymph nodes after radical surgery for prostate cancer. Urologic Oncology: Seminars and Original Investigations. 201601 Dec;34(12):529.e15.e20. PMID: 613439872. Ineligible population

612. Wortel RC, Oomen-de Hoop E, Heemsbergen WD, et al. Moderate Hypofractionation in Intermediate- and High-Risk, Localized Prostate Cancer: Health-Related Quality of Life From the Randomized, Phase 3 HYPRO Trial. International Journal of Radiation Oncology Biology Physics. 2019 15 March;103(4):823-33. PMID: 2001462441. Ineligible population 
613. Wortel RC, Pos FJ, Heemsbergen WD, et al. Sexual Function After Hypofractionated Versus Conventionally Fractionated Radiotherapy for Prostate Cancer: Results From the Randomized Phase III HYPRO Trial. J Sex Med. 2016 11;13(11):1695-703. PMID: 27665195. No eligible outcomes reported

614. Wright JL, Plymate SR, Porter MP, et al. Hyperglycemia and prostate cancer recurrence in men treated for localized prostate cancer. Prostate Cancer and Prostatic Diseases. 2013 June;16(2):204-8. PMID: 52472912. No eligible outcomes reported

615. Wu CT, Yang YH, Chen PC, et al. Androgen deprivation increases the risk of fracture in prostate cancer patients: a population-based study in Chinese patients. Osteoporos Int. 2015 Sep;26(9):2281-90. PMID: 25990353. Ineligible population

616. Wu FJ, Kao LT, Sheu SY, et al. Increased risk of a herpes zoster attack in patients receiving androgen deprivation therapy for prostate cancer. Andrologia. 2018 Mar;50(2). PMID: 28786220. No eligible outcomes reported

617. Yamazaki H, Masui K, Suzuki G, et al. Radiothrerapy for Elderly Patients Aged $>=75$ Years with Clinically Localized Prostate Cancer-Is There a Role of Brachytherapy? J. 2018 Nov 08;7(11):08. PMID: 30413025. Ineligible comparison

618. Yamazaki H, Masui K, Suzuki G, et al. Highdose-rate brachytherapy monotherapy versus image-guided intensity-modulated radiotherapy with helical tomotherapy for patients with localized prostate cancer. Cancers. 201810 Sep;10 (9) (no pagination)(322). PMID: 623889630. Ineligible population

619. Yamazaki H, Masui K, Suzuki G, et al. Highdose-rate brachytherapy monotherapy versus low-dose-rate brachytherapy with or without external beam radiotherapy for clinically localized prostate cancer. Radiotherapy and Oncology. 2018. PMID: 2001252974.

Ineligible comparison
620. Yanamadala S, Chung BI, Hernandez-Boussard TM. Robot-assisted versus open radical prostatectomy utilization in hospitals offering robotics. Can J Urol. 2016 Jun;23(3):8279-84. PMID: 27347621. No eligible outcomes reported

621. Yang DD, Mahal BA, Muralidhar V, et al. Receipt of definitive therapy in elderly patients with unfavorable-risk prostate cancer. Cancer. 2017 Dec 15;123(24):483240. PMID: 28832984. Insufficient follow-up time

622. Yang DD, Muralidhar V, Mahal BA, et al. Lack of Apparent Survival Benefit With Use of Androgen Deprivation Therapy in Patients With High-risk Prostate Cancer Receiving Combined External Beam Radiation Therapy and Brachytherapy. International Journal of Radiation Oncology, Biology, Physics. 201801 01;100(1):53-8. PMID: 29254781. Ineligible population

623. Yaxley JW, Coughlin GD, Chambers SK, et al. Robot-assisted laparoscopic prostatectomy versus open radical retropubic prostatectomy: early outcomes from a randomised controlled phase 3 study. Lancet. 201609 10;388(10049):1057-66. PMID: 27474375. Insufficient follow-up time

624. Yu EY, Getzenberg RH, Coss CC, et al. Selective estrogen receptor alpha agonist GTX-758 decreases testosterone with reduced side effects of androgen deprivation therapy in men with advanced prostate cancer. European Urology. 201501 Feb;67(2):334-41. PMID: 53204511. Ineligible intervention

625. Yu JB, Cramer LD, Herrin J, et al. Stereotactic body radiation therapy versus intensitymodulated radiation therapy for prostate cancer: comparison of toxicity. Journal of Clinical Oncology. 2014 Apr 20;32(12):1195-201. PMID: 24616315. No eligible outcomes reported

626. Yu JB, Soulos PR, Herrin J, et al. Proton versus intensity-modulated radiotherapy for prostate cancer: patterns of care and early toxicity. J Natl Cancer Inst. 2013 Jan 02;105(1):25-32. PMID: 23243199. Ineligible population 
627. Yu YD, Lee M, Hong SK, et al. Impact of Variations in Prostatic Apex Shape on Apical Margin Positive Rate After Radical Prostatectomy: Robot-Assisted Laparoscopic Radical Prostatectomy vs Open Radical Prostatectomy. Journal of Endourology. 2018 01;32(1):46-53. PMID: 29212368. Ineligible population

628. Zaffuto E, Gandaglia G, Fossati N, et al. Early Postoperative Radiotherapy is Associated with Worse Functional Outcomes in Patients with Prostate Cancer. Journal of Urology. 2017 03;197(3 Pt 1):669-75. PMID: 27670915. Ineligible study design

629. Zakeri K, Rose BS, Gulaya S, et al. Competing event risk stratification may improve the design and efficiency of clinical trials: Secondary analysis of SWOG 8794. Contemporary Clinical Trials. 2013 January;34(1):74-9. PMID: 366197662. Ineligible population

630. Zapatero A, Guerrero A, Maldonado X, et al. High-dose radiotherapy with short-term or long-term androgen deprivation in localised prostate cancer (DART01/05 GICOR): a randomised, controlled, phase 3 trial. Lancet Oncol. 2015 Mar;16(3):320-7. doi: 10.1016/S1470-2045(15)70045-8. PMID: 25702876. Ineligible comparison

631. Zapatero A, Guerrero A, Maldonado X, et al. Late Radiation and Cardiovascular Adverse Effects After Androgen Deprivation and High-Dose Radiation Therapy in Prostate Cancer: Results From the DART 01/05 Randomized Phase 3 Trial. International Journal of Radiation Oncology, Biology, Physics. 201610 01;96(2):341-8. PMID: 27598804. Ineligible comparison

632. Zapatero A, Roch M, Buchser D, et al. Reduced late urinary toxicity with high-dose intensity-modulated radiotherapy using intra-prostate fiducial markers for localized prostate cancer. Clinical and Translational Oncology. 201701 Sep;19(9):1161-7. PMID: 615203330. No eligible outcomes reported

633. Zelefsky MJ, Cohen GN, Bosch WR, et al. Results from the Quality Research in Radiation Oncology (QRRO) survey: Evaluation of dosimetric outcomes for lowdose-rate prostate brachytherapy. Brachytherapy. 2013 Jan-Feb;12(1):19-24. PMID: 22819388. Ineligible study design
634. Zelefsky MJ, Poon BY, Eastham J, et al. Longitudinal assessment of quality of life after surgery, conformal brachytherapy, and intensity-modulated radiation therapy for prostate cancer. Radiother Oncol. 2016 Jan;118(1):85-91. PMID: 26780999. Ineligible study design

635. Zimmermann JS, Osieka R, Bruns T, et al. Fiveyear effectiveness of low-dose-rate brachytherapy: Comparisons with nomogram predictions in patients with nonmetastatic prostate cancer presenting significant control of intra- and periprostatic disease. Journal of Contemporary Brachytherapy. 2018;10(4):297-305. PMID: 624050738. Ineligible study design

636. Zurita AJ, Pisters LL, Wang X, et al. Integrating chemohormonal therapy and surgery in known or suspected lymph node metastatic prostate cancer. Prostate Cancer Prostatic Dis. 2015 Sep;18(3):276-80. PMID: 26171883. Ineligible study design

637. Alayed Y, Quon H, Cheung P, et al. Two versus five stereotactic ablative radiotherapy treatments for localized prostate cancer: A quality of life analysis of two prospective clinical trials. Radiother Oncol. 2019 Nov;140:105-9. doi: 10.1016/j.radonc.2019.06.018. PMID: 31265940. Ineligible comparison

638. Alexidis P, Karatzoglou S, Dragoumis D, et al. Late results of a randomized trial on the role of mild hypofractionated radiotherapy for the treatment of localized prostate cancer. J Cancer. 2020;11(5):1008-16. doi: 10.7150/jca.37825. PMID: 31956347. Ineligible comparison

639. Bryant RJ, Oxley J, Young GJ, et al. The ProtecT trial: analysis of the patient cohort, baseline risk stratification and disease progression. BJU Int. 2020 Jan 3;03:03. doi: 10.1111/bju.14987. PMID: 31900963. Ineligible comparison

640. e Crevoisier R, Bayar MA, Pommier P, et al. Daily Versus Weekly Prostate Cancer Image Guided Radiation Therapy: phase 3 Multicenter Randomized Trial. International journal of radiation oncology, biology, physics. 2018;102(5):1420-9. PMID: CN01668036. Ineligible comparison 
641. Lovegrove CE, Peters M, Guillaumier S, et al. Evaluation of functional outcomes after a second focal high-intensity focused ultrasonography (HIFU) procedure in men with primary localized, non-metastatic prostate cancer: results from the HIFU Evaluation and Assessment of Treatment (HEAT) registry. BJU Int. 2020 Jan 23;23:23. doi: 10.1111/bju.15004. PMID: 31971335. Ineligible comparison

642. Murray J, Griffin C, Gulliford S, et al. A randomised assessment of image guided radiotherapy within a phase 3 trial of conventional or hypofractionated high dose intensity modulated radiotherapy for prostate cancer. Radiother Oncol. 2020 Jan;142:62-71. doi: 10.1016/j.radonc.2019.10.017. PMID: 31767473. Ineligible comparison

643. Pasalic D, Kuban DA, Allen PK, et al. Dose Escalation for Prostate Adenocarcinoma: A Long-Term Update on the Outcomes of a Phase 3, Single Institution Randomized Clinical Trial. Int J Radiat Oncol Biol Phys. 2019 Jul 15;104(4):790-7. doi: 10.1016/j.ijrobp.2019.02.045. PMID: 30836166. Ineligible comparison

644. Rammant E, Ost P, Swimberghe M, et al. Patient- versus physician-reported outcomes in prostate cancer patients receiving hypofractionated radiotherapy within a randomized controlled trial. Strahlenther Onkol. 2019 May;195(5):393-401. doi: 10.1007/s00066-018-1395-y. PMID: 30406289. Ineligible comparison

645. Richard PO, Timilshina N, Komisarenko M, et al. The long-term outcomes of Gleason grade groups 2 and 3 prostate cancer managed by active surveillance: Results from a large population-based cohort. Can Urol Assoc J. 2020 Jan 20;20:20. doi: 10.5489/cuaj.6328. PMID: 31977306. Ineligible comparison

646. Stabile A, Orczyk C, Hosking-Jervis F, et al. Medium-term oncological outcomes in a large cohort of men treated with either focal or hemi-ablation using high-intensity focused ultrasonography for primary localized prostate cancer. BJU Int. 2019 Sep;124(3):431-40. doi: 10.1111/bju.14710. PMID: 30753756. Ineligible comparison
647. Vitzthum LK, Straka C, Sarkar RR, et al. Combined Androgen Blockade in Localized Prostate Cancer Treated With Definitive Radiation Therapy. J. 2019 Dec;17(12):1497-504. doi: 10.6004/jnccn.2019.7335. PMID: 31805534. Ineligible comparison

648. Xia L, Sperling CD, Taylor BL, et al. Associations between Hospital Volume and Outcomes of Robot-Assisted Radical Prostatectomy. J Urol. 2019 Dec 17:101097JU0000000000000698. doi: 10.1097/JU.0000000000000698. PMID: 31846391. Ineligible comparison

649. Kellokumpu-Lehtinen PL, Hjalm-Eriksson M, Thellenberg-Karlsson C, et al. Docetaxel Versus Surveillance After Radical Radiotherapy for Intermediate- or High-risk Prostate Cancer-Results from the Prospective, Randomised, Open-label Phase III SPCG-13 Trial. Eur Urol. 2019 Dec;76(6):823-30. doi: 10.1016/j.eururo.2019.08.010. PMID: 31443961. Ineligible intervention

650. Aksnessaether BY, Myklebust TA, Solberg A, et al. Second Cancers in Patients With Locally Advanced Prostate Cancer Randomized to Lifelong Endocrine Treatment With or Without Radical Radiation Therapy: Long-Term Follow-up of the Scandinavian Prostate Cancer Group7 Trial. International Journal of Radiation Oncology Biology Physics. 2020. PMID: 2004490265. Ineligible population

651. Baunacke M, Schmidt ML, Thomas C, et al. Long-term functional outcomes after robotic vs. retropubic radical prostatectomy in routine care: a 6-year follow-up of a large German health services research study. World J Urol. 2019 Sep 17;17:17. doi: 10.1007/s00345-019-02956-8. PMID: 31531690. Ineligible population

652. Dess RT, Hartman HE, Mahal BA, et al. Association of Black Race With Prostate Cancer-Specific and Other-Cause Mortality. JAMA Oncol. 2019 Jul 1;5(7):975-83. doi: 10.1001/jamaoncol.2019.0826. PMID: 31120534. Ineligible population 
653. Efstathiou E, Davis JW, Pisters L, et al. Clinical and Biological Characterisation of Localised High-risk Prostate Cancer: Results of a Randomised Preoperative Study of a Luteinising Hormone-releasing Hormone Agonist with or Without Abiraterone Acetate plus Prednisone. European Urology. 2019 October;76(4):418-24. PMID: 2002069914. Ineligible population

654. Ha US, Choi JB, Shim JI, et al. Is Primary Androgen Deprivation Therapy a Suitable Option for Asian Patients With Prostate Cancer Compared With Radical Prostatectomy? J. 2019 May 1;17(5):441-9. doi: 10.6004/jnccn.2018.7265. PMID: 31085754. Ineligible population

655. Jayadevappa R, Lee DI, Chhatre S, et al. Comparative effectiveness of treatments for high-risk prostate cancer patients. Urol. 2019 Sep;37(9):574 e11- e18. doi: 10.1016/j.urolonc.2019.06.005. PMID: 31285113. Ineligible population

656. Knipper S, Palumbo C, Pecoraro A, et al. Survival outcomes of radical prostatectomy vs. external beam radiation therapy in prostate cancer patients with Gleason Score 9-10 at biopsy: A population-based analysis. Urologic Oncology: Seminars and Original Investigations. 2019. PMID: 2003436253. Ineligible population

657. Margel D, Peer A, Ber Y, et al. Cardiovascular Morbidity in a Randomized Trial Comparing GnRH Agonist and GnRH Antagonist among Patients with Advanced Prostate Cancer and Preexisting Cardiovascular Disease. J Urol. 2019 Dec;202(6):1199-208. doi: 10.1097/JU.0000000000000384. PMID: 31188734. Ineligible population

658. Moon DH, Basak RS, Usinger DS, et al. Patientreported Quality of Life Following Stereotactic Body Radiotherapy and Conventionally Fractionated External Beam Radiotherapy Compared with Active Surveillance Among Men with Localized Prostate Cancer. Eur Urol. 2019 Sep;76(3):391-7. doi: 10.1016/j.eururo.2019.02.026. PMID: 30857758. Ineligible population
659. Mullins BT, Basak R, Broughman JR, et al. Patient-reported sexual quality of life after different types of radical prostatectomy and radiotherapy: Analysis of a population-based prospective cohort. Cancer. 2019 Oct 15;125(20):3657-65. doi: 10.1002/cncr.32288. PMID: 31256432. Ineligible population

660. Muralidhar V, Mahal BA, Butler S, et al. Combined External Beam Radiation Therapy and Brachytherapy versus Radical Prostatectomy with Adjuvant Radiation Therapy for Gleason 9-10 Prostate Cancer. J Urol. 2019 Nov;202(5):973-8. doi: 10.1097/JU.0000000000000352. PMID: 31144590. Ineligible population

661. Murthy V, Maitre P, Bhatia J, et al. Late toxicity and quality of life with prostate only or whole pelvic radiation therapy in high risk prostate cancer (POP-RT): A randomised trial. Radiother Oncol. 2020 Jan 7;145:7180. doi: 10.1016/j.radonc.2019.12.006. PMID: 31923712. Ineligible population

662. Nguyen-Nielsen M, Moller H, Tjonneland A, et al. Patient-reported outcome measures after treatment for prostate cancer: Results from the Danish Prostate Cancer Registry (DAPROCAdata). Cancer epidemiol. 2020 Feb;64:101623. doi: 10.1016/j.canep.2019.101623. PMID: 31760356. Ineligible population

663. Sandler KA, Cook RR, Ciezki JP, et al. Prostateonly Versus Whole-pelvis Radiation with or Without a Brachytherapy Boost for Gleason Grade Group 5 Prostate Cancer: A Retrospective Analysis. Eur Urol. 2020 Jan;77(1):3-10. doi: 10.1016/j.eururo.2019.03.022. PMID: 30992160. Ineligible population

664. Sargos P, Mottet N, Bellera C, et al. Long-term androgen deprivation, with or without radiotherapy, in locally-advanced prostate cancer: updated results from a phase III randomized trial. BJU international. 2019;04. PMID: 627219439. Ineligible population

665. Tang J, Zhong L, Paoli C, et al. Longitudinal Comparison of Patient-Level Outcomes and Costs Across Prostate Cancer Treatments With Urinary Problems. Am J Mens Health. 2019 Mar-Apr;13(2):1557988319835326. doi: 10.1177/1557988319835326. PMID: 30836832. Ineligible population 
666. Tharmalingam H, Tsang Y, Choudhury A, et al. External Beam Radiation Therapy (EBRT) and High-Dose-Rate (HDR) Brachytherapy for Intermediate and High-Risk Prostate Cancer: The Impact of EBRT Volume. Int J Radiat Oncol Biol Phys. 2020 Mar 1;106(3):525-33. doi: 10.1016/j.ijrobp.2019.09.044. PMID: 31610249. Ineligible population

667. Vitzthum LK, Park H, Zakeri K, et al. Risk of Pelvic Fracture With Radiation Therapy in Older Patients. Int J Radiat Oncol Biol Phys. 2020 Mar 1;106(3):485-92. doi: 10.1016/j.ijrobp.2019.10.006. PMID: 31610251. Ineligible population

668. Wang Y, Gieschen H, Greenberger M, et al. Survival After Robotic-Assisted Prostatectomy for Localized Prostate Cancer: An Epidemiologic Study. Ann Surg. 2019 Oct 28;28:28. doi: 10.1097/SLA.0000000000003637. PMID: 31663972. Ineligible population

669. Yamazaki H, Masui K, Suzuki G, et al. Effect of Androgen Deprivation Therapy on OtherCause of Mortality in Elderly Patients with Clinically Localized Prostate Cancer Treated with Modern Radiotherapy: Is There a Negative Impact? J. 2019 Mar 11;8(3):11. doi: 10.3390/jcm8030338. PMID: 30862069. Ineligible population

670. Yin M, Zhao J, Monk P, et al. Comparative effectiveness of surgery versus external beam radiation with/without brachytherapy in high-risk localized prostate cancer. Cancer Med. 2020 Jan;9(1):27-34. doi: 10.1002/cam4.2605. PMID: 31697452. Ineligible population

671. Zhang S, Zhao S, Fu X. Intensity modulated radiotherapy in combination with endocrinotherapy in the treatment of middle and advanced Prostatic Cancer. Pak. 2019 Sep-Oct;35(5):1264-9. doi: 10.12669/pjms.35.5.591. PMID: 31488990. Ineligible population

672. Alayed Y, Cheung P, Chu W, et al. Two StereoTactic ablative radiotherapy treatments for localized prostate cancer (2STAR): Results from a prospective clinical trial. Radiother Oncol. 2019 Jun;135:86-90. doi: 10.1016/j.radonc.2019.03.002. PMID: 31015175. Ineligible study design
673. Hayashi N, Osaka K, Muraoka K, et al. Outcomes of treatment for localized prostate cancer in a single institution: comparison of radical prostatectomy and radiation therapy by propensity score matching analysis. World J Urol. 2019 Dec 24;24. doi: 10.1007/s00345-019-03056-3. PMID: 31875247. Ineligible study design

674. Kent AR, Matheson B, Millar JL. Improved survival for patients with prostate cancer receiving high-dose-rate brachytherapy boost to EBRT compared with EBRT alone. Brachytherapy. 2019 May - Jun;18(3):31321. doi: 10.1016/j.brachy.2019.01.013. PMID: 30846330. Ineligible study design

675. Ling DC, Chen KS, Benoit RM, et al. LongTerm Patient-Reported Rectal Bleeding and Bowel-Related Quality of Life After Cs-131 Prostate Brachytherapy. Int J Radiat Oncol Biol Phys. 2019 Jul 1;104(3):622-30. doi: 10.1016/j.ijrobp.2019.02.056. PMID: 30853423. Ineligible study design

676. Marvaso G, Ciardo D, Gandini S, et al. Comparison of Outcomes and Toxicity Between Extreme and Moderate Radiation Therapy Hypofractionation in Localized Prostate Cancer: A Propensity Score Analysis. International Journal of Radiation Oncology Biology Physics. 201915 November;105(4):735-44. PMID: 2002822617. Ineligible study design

677. Matzkin H, Chen J, Agai R, et al. Long-term biochemical progression-free survival following brachytherapy for prostate cancer: Further insight into the role of short-term androgen deprivation and intermediate risk group subclassification. PLoS One. 2019;14(4):e0215582. doi: 10.1371/journal.pone.0215582. PMID: 31002732. Ineligible study design

678. Okegawa T, Omura S, Samejima M, et al. Laparoscopic radical prostatectomy versus robot-assisted radical prostatectomy: comparison of oncological outcomes at a single center. Prostate International. 2019. PMID: 2004421842. Ineligible study design 
679. Slevin F, Rodda SL, Bownes P, et al. A comparison of outcomes for patients with intermediate and high risk prostate cancer treated with low dose rate and high dose rate brachytherapy in combination with external beam radiotherapy. Clin Transl Radiat Oncol. 2020 Jan;20:1-8. doi: 10.1016/j.ctro.2019.10.001. PMID: 31701035. Ineligible study design

680. Stonier T, Simson N, Davis J, et al. Retziussparing robot-assisted radical prostatectomy (RS-RARP) vs standard RARP: it's time for critical appraisal. BJU Int. 2019 Jan;123(1):5-7. doi: 10.1111/bju.14468. PMID: 29959814. Ineligible study design

681. Taguchi S, Shiraishi K, Fujimura T, et al. Robot-assisted radical prostatectomy versus volumetric modulated arc therapy: Comparison of front-line therapies for localized prostate cancer. Radiother Oncol. 2019 Nov;140:62-7. doi: 10.1016/j.radonc.2019.05.015. PMID: 31176208. Ineligible study design

682. Vicier C, Feng FY, Fizazi K. Overview of Systemic Therapy Augmenting Management of High-risk Localized Prostate Cancer. Eur Urol Focus. 2019 Mar;5(2):168-70. doi: 10.1016/j.euf.2019.01.015. PMID: 30745118. Ineligible study design

683. Brand DH, Tree AC, Ostler P, et al. Intensitymodulated fractionated radiotherapy versus stereotactic body radiotherapy for prostate cancer (PACE-B): acute toxicity findings from an international, randomised, openlabel, phase 3, non-inferiority trial. Lancet Oncol. 2019 Nov;20(11):1531-43. doi: 10.1016/S1470-2045(19)30569-8. PMID: 31540791. Insufficient follow-up time

684. Sebastian NT, McElroy JP, Martin DD, et al. Survival after radiotherapy vs. radical prostatectomy for unfavorable intermediaterisk prostate cancer. Urol. 2019 Nov;37(11):813 e11- e19. doi: 10.1016/j.urolonc.2019.04.022. PMID: 31109836. Insufficient follow-up time

685. Ahlberg MS, Adami HO, Beckmann K, et al. PCASTt/SPCG-17-a randomised trial of active surveillance in prostate cancer: rationale and design. BMJ Open. 2019 Aug 22;9(8):e027860. doi: 10.1136/bmjopen2018-027860. PMID: 31444180. No eligible outcomes reported
686. Antonelli A, Palumbo C, Noale M, et al. Overview of potential determinants of radical prostatectomy versus radiation therapy in management of clinically localized prostate cancer: results from an Italian, prospective, observational study (the Pros-IT CNR study). Minerva Urol Nefrol. 2020 Jan 7;07. doi: 10.23736/S03932249.19.03637-3. PMID: 31920063. No eligible outcomes reported

687. Antonelli A, Palumbo C, Veccia A, et al. Standard vs delayed ligature of the dorsal vascular complex during robot-assisted radical prostatectomy: results from a randomized controlled trial. J. 2019 Apr;13(2):253-60. doi: 10.1007/s11701-0180847-9. PMID: 30006861. No eligible outcomes reported

688. Dinneen E, Haider A, Allen C, et al. NeuroSAFE robot-assisted laparoscopic prostatectomy versus standard robot-assisted laparoscopic prostatectomy for men with localised prostate cancer (NeuroSAFE PROOF): protocol for a randomised controlled feasibility study. BMJ Open. 2019 Jun 11;9(6):e028132. doi: 10.1136/bmjopen-2018-028132. PMID: 31189680. No eligible outcomes reported

689. Koerber SA, Katayama S, Sander A, et al. Prostate bed irradiation with alternative radio-oncological approaches (PAROS) - a prospective, multicenter and randomized phase III trial. Radiat Oncol. 2019 Jul 10;14(1):122. doi: 10.1186/s13014-0191325-x. PMID: 31291969. No eligible outcomes reported

690. Martin J, Keall P, Siva S, et al. TROG 18.01 phase III randomised clinical trial of the Novel Integration of New prostate radiation schedules with adJuvant Androgen deprivation: NINJA study protocol. BMJ Open. 2019 Aug 20;9(8):e030731. doi: 10.1136/bmjopen-2019-030731. PMID: 31434782. No eligible outcomes reported

691. Mohiuddin JJ, Narayan V, Venigalla S, et al. Variations in patterns of concurrent androgen deprivation therapy use based on dose escalation with external beam radiotherapy vs. brachytherapy boost for prostate cancer. Brachytherapy. 2019 May Jun;18(3):322-31. doi: 10.1016/j.brachy.2019.01.016. PMID: 30862436. No eligible outcomes reported 
692. Morias S, Buckley E, Beckmann K, et al. Variation in radiotherapy patterns of care in the radical treatment of South Australian men with non-metastatic prostate cancer between 2005-2015. Radiotherapy and Oncology. 2020 April;145:138-45. PMID: 2004666362. No eligible outcomes reported

693. Sanguineti G, Giannarelli D, Petrongari MG, et al. Leukotoxicity after moderately Hypofractionated radiotherapy versus conventionally fractionated dose escalated radiotherapy for localized prostate Cancer: a secondary analysis from a randomized study. Radiat Oncol. 2019 Jan 30;14(1):23. doi: 10.1186/s13014-019-1223-2. PMID: 30700317. No eligible outcomes reported

694. Shelton JB, Buffington P, Augspurger R, et al. Contemporary Management of Incident Prostate Cancer in Large Community Urology Practices in the United States. Urology. 2019 Jul;129:79-86. doi: 10.1016/j.urology.2019.01.061. PMID: 30954610. No eligible outcomes reported
695. Tissaverasinghe S, Crook J, Bachand F, et al. Dose to the dominant intraprostatic lesion using HDR vs. LDR monotherapy: A Phase II randomized trial. Brachytherapy. 2019 May - Jun;18(3):299-305. doi: 10.1016/j.brachy.2019.01.006. PMID: 30795889. No eligible outcomes reported

696. Wang C, Raldow AC, Nickols NG, et al. Underutilization of Androgen Deprivation Therapy with External Beam Radiotherapy in Men with High-grade Prostate Cancer. Eur Urol Oncol. 2019 Feb 1;01:01. doi: 10.1016/j.euo.2019.01.006. PMID: 31411981. No eligible outcomes reported 


\section{Appendix F. Watchful Waiting}

Table F-1. Risk of bias assessments for randomized controlled trials: watchful waiting comparisons

\begin{tabular}{|c|c|c|c|c|c|c|c|c|}
\hline $\begin{array}{l}\text { Intervention/ } \\
\text { Comparison } \\
\text { (Outcomes) }\end{array}$ & Author Year & $\begin{array}{l}\text { Selection } \\
\text { Bias }\end{array}$ & $\begin{array}{l}\text { Performance } \\
\text { Bias }\end{array}$ & $\begin{array}{l}\text { Detection } \\
\text { Bias }\end{array}$ & Attrition & $\begin{array}{l}\text { Reporting } \\
\text { Bias }\end{array}$ & Other Bias & $\begin{array}{l}\text { Overall } \\
\text { Rating }\end{array}$ \\
\hline $\begin{array}{l}\text { RP/WW } \\
\text { (All-cause } \\
\text { mortality, prostate- } \\
\text { specific mortality, } \\
\text { metastases) }\end{array}$ & $\begin{array}{l}\text { Bill-Axelson } \\
2014^{1} \\
\text { Bill-Axelson } \\
2018^{2}\end{array}$ & Low & Low & Medium & Low & Low & $\mathrm{NI}$ & Low \\
\hline $\begin{array}{l}\text { RP/WW } \\
\text { (All-cause } \\
\text { mortality, prostate- } \\
\text { specific mortality, } \\
\text { metastases) }\end{array}$ & Wilt $2017^{3}$ & Low & Low & Medium & Medium & Low & $\mathrm{NI}$ & Low \\
\hline
\end{tabular}

Abbreviations: $\mathrm{NI}=$ none identified; $\mathrm{RP}=$ radical prostatectomy; $\mathrm{WW}=$ watchful waiting

Table F-2. Summary risk of bias assessments for observational studies: watchful waiting

\begin{tabular}{|c|c|c|c|c|c|c|c|c|c|}
\hline $\begin{array}{l}\text { Intervention/ } \\
\text { Comparison } \\
\text { (Outcomes) }\end{array}$ & $\begin{array}{l}\text { Author, } \\
\text { Year }\end{array}$ & $\begin{array}{l}\text { Bias Due to } \\
\text { Confounding }\end{array}$ & $\begin{array}{l}\text { Bias in } \\
\text { Selection of } \\
\text { Participants }\end{array}$ & $\begin{array}{l}\text { Bias in } \\
\text { Classification } \\
\text { of } \\
\text { Interventions }\end{array}$ & $\begin{array}{l}\text { Bias Due to } \\
\text { Deviations } \\
\text { for Intended } \\
\text { Interventions }\end{array}$ & $\begin{array}{l}\text { Bias Due } \\
\text { to Missing } \\
\text { Data }\end{array}$ & $\begin{array}{l}\text { Bias in } \\
\text { Measurement } \\
\text { of Outcomes }\end{array}$ & $\begin{array}{l}\text { Bias in } \\
\text { Selection } \\
\text { of the } \\
\text { Reported } \\
\text { Result }\end{array}$ & Overall \\
\hline $\begin{array}{l}\text { WW/RT, } \\
\text { AS/ADT, RP, RT }\end{array}$ & $\begin{array}{l}\text { Herden } \\
2016^{4}\end{array}$ & Moderate & Low & Moderate & Low & Serious & Serious & Low & Serious \\
\hline \multirow[t]{2}{*}{ WW/RT, RP } & $\begin{array}{l}\text { Hoffman } \\
2017^{5}\end{array}$ & Serious & Low & Moderate & Low & Serious & Moderate & Low & Serious \\
\hline & $\begin{array}{l}\text { Lu-Yao } \\
2015^{6}\end{array}$ & Moderate & Moderate & Low & Low & $\begin{array}{l}\text { No } \\
\text { Information }\end{array}$ & Serious & Low & Serious \\
\hline $\begin{array}{l}\text { WW/RT, } \\
\text { RT+ADT }\end{array}$ & $\begin{array}{l}\text { Dell'Oglio } \\
2016^{7}\end{array}$ & Serious & Low & Low & Low & $\begin{array}{l}\text { No } \\
\text { Information }\end{array}$ & Moderate & Moderate & Serious \\
\hline
\end{tabular}

Abbreviations: ADT=androgen deprivation therapy; $\mathrm{RP}=$ radical prostatectomy; $\mathrm{RT}=$ =radiation therapy; WW=watchful waiting 
Table F-3. Characteristics of eligible studies: watchful waiting comparisons

\begin{tabular}{|c|c|c|c|c|c|}
\hline $\begin{array}{l}\text { Study } \\
\text { Design } \\
\text { Country } \\
\text { ROB }\end{array}$ & $\mathrm{N}=$ & Population & $\begin{array}{l}\text { Intervention } \\
\text { Frequency } \\
\text { Duration }\end{array}$ & $\begin{array}{l}\text { Comparison } \\
\text { Frequency } \\
\text { Duration }\end{array}$ & $\begin{array}{l}\text { Followup Time(s) } \\
\text { Outcome } \\
\text { (Instrument) }\end{array}$ \\
\hline $\begin{array}{l}\text { Bill-Axelson 2014 } \\
\text { Bill-Axelson 2018 } \\
\text { RCT } \\
\text { Scandinavia } \\
\text { (Sweden, Finland } \\
\text { and Iceland) } \\
\text { Low }\end{array}$ & 695 & $\begin{array}{l}\text { Men }<75 \text { with life } \\
\text { expectancy } \geq 10 \text { years; no } \\
\text { other cancers; clinical stage } \\
\text { T1, T2; PSA < } 50 \text { ng/mL } \\
\text { T1b: } 12 \% \\
\text { T2c: } 12 \% \\
\text { T2: } 76 \% \\
\text { Gleason sum } \\
\text { 2-4: } 13 \% \\
\text { 5-6: } 48 \% \\
\text { 7: } 23 \% \\
\text { 8-10: } 5 \% \\
\text { Age } 65 \\
\text { Race NR }\end{array}$ & Radical prostatectomy & $\begin{array}{l}\text { Watchful waiting: no } \\
\text { immediate treatment }\end{array}$ & $\begin{array}{l}18 \text { years } \\
\text { All-cause mortality } \\
\text { Prostate-specific mortality } \\
\text { Metastases } \\
29 \text { years } \\
\text { All-cause mortality } \\
\text { Prostate-specific mortality } \\
\text { Metastases }\end{array}$ \\
\hline $\begin{array}{l}\text { Wilt } 2009^{8} \\
\text { Wilt } 2017^{3} \\
\text { RCT } \\
\text { United States } \\
\text { Low }\end{array}$ & 731 & $\begin{array}{l}\text { Men age } \leq 75 \text { PSA }<50 \\
\text { ng/ml with life expectancy } \geq \\
\text { 10 years } \\
\text { Stage (T1-T2, Nx, M0) of } \\
\text { any grade diagnosed } \leq 12 \\
\text { months, bone scan negative } \\
\text { for metastatic disease } \\
\text { T1a: } 2 \% \\
\text { T1b: } 2 \% \\
\text { T1c: } 50 \% \\
\text { T2a: } 25 \% \\
\text { T2b: } 12 \% \\
\text { Gleason sum } \\
\text { 2-4: } 23 \% \\
\text { 5-6: } 51 \% \\
\text { 7: } 21 \% \\
\text { 8-10: } 7 \% \\
\text { Age } 67 \\
\text { 62\% White } \\
\text { 33\% Black } \\
\text { Median PSA: } 7.8 \mathrm{ng} / \mathrm{ml}\end{array}$ & $\begin{array}{l}\text { Radical prostatectomy } \\
\text { Surgery }\end{array}$ & $\begin{array}{l}\text { Watchful Waiting } \\
\text { No Intervention } \\
\text { Closely watching, } \\
\text { waiting and treating } \\
\text { symptoms if and when } \\
\text { cancer progresses }\end{array}$ & $\begin{array}{l}19.5 \text { years } \\
\text { All-cause mortality } \\
\text { Prostate-specific mortality } \\
\text { Erectile dysfunction } \\
\text { Incontinence }\end{array}$ \\
\hline
\end{tabular}

Abbreviations: ng/ml=nanogram per milliliter; NR=not reported; PSA=Prostate-specific antigen; RCT=randomized controlled trial; ROB=risk of bias 
Table F-4. Mortality, survival, and metastases: watchful waiting

\begin{tabular}{|c|c|c|c|c|}
\hline $\begin{array}{l}\text { Interventionl } \\
\text { Comparison }\end{array}$ & $\begin{array}{l}\text { Study } \\
\text { Design } \\
\text { (Trial/Registry) } \\
\text { Risk of Bias } \\
\end{array}$ & $\begin{array}{l}\text { Followup } \\
\text { Overall Mortality }\end{array}$ & $\begin{array}{l}\text { Followup } \\
\text { Prostate Cancer Specific Mortality }\end{array}$ & $\begin{array}{l}\text { Followup } \\
\text { Metastases }\end{array}$ \\
\hline WW/RP & $\begin{array}{l}\text { Bill-Axelson } 2014^{1} \\
\text { Bill-Axelson } 2018^{2} \\
\text { RCT } \\
\text { (SPCG-4) } \\
\text { Low }\end{array}$ & 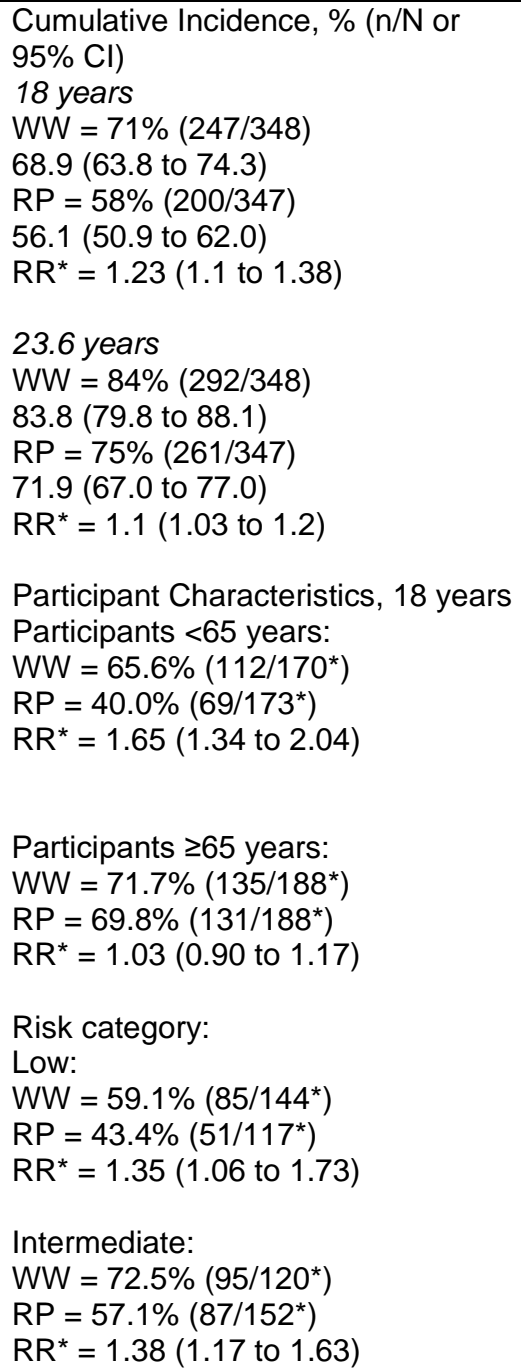 & 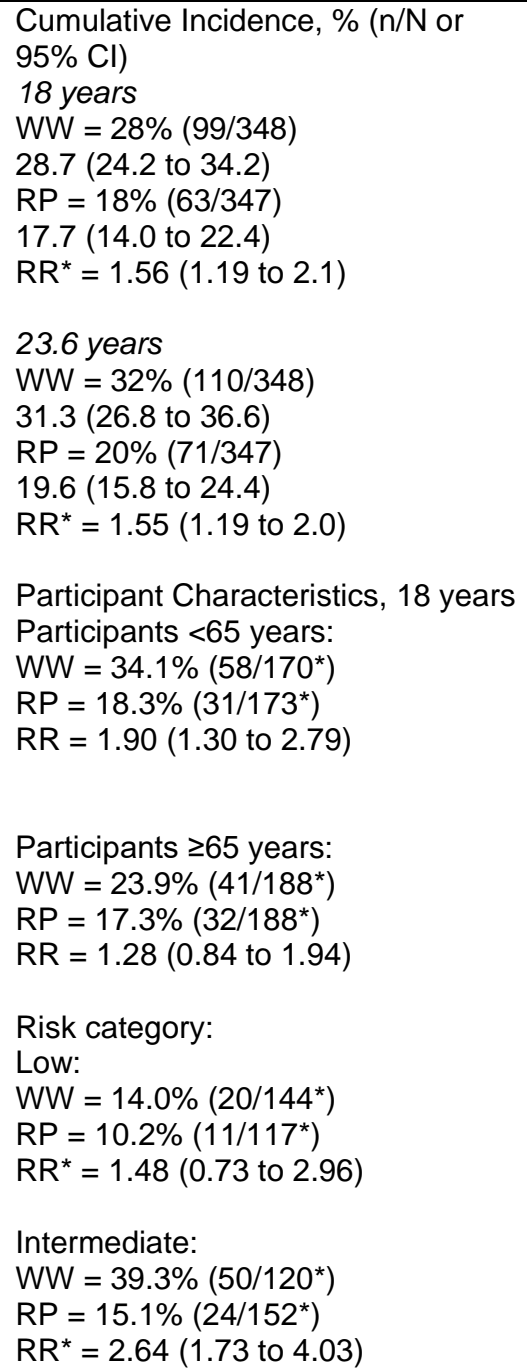 & 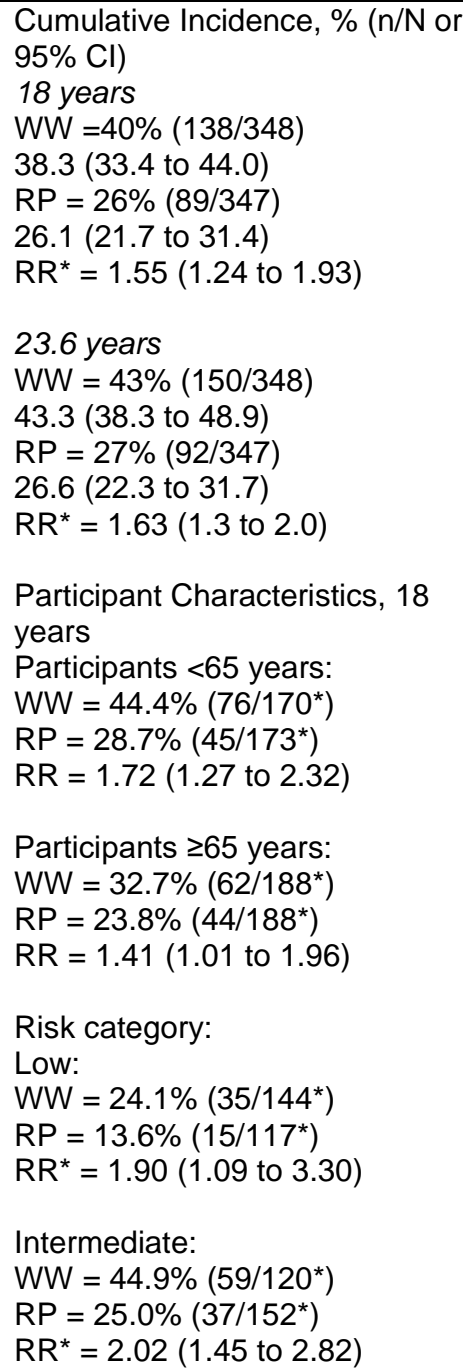 \\
\hline
\end{tabular}




\begin{tabular}{|c|c|c|c|c|}
\hline $\begin{array}{l}\text { Intervention/ } \\
\text { Comparison }\end{array}$ & $\begin{array}{l}\text { Study } \\
\text { Design } \\
\text { (Trial/Registry) } \\
\text { Risk of Bias }\end{array}$ & $\begin{array}{l}\text { Followup } \\
\text { Overall Mortality }\end{array}$ & $\begin{array}{l}\text { Followup } \\
\text { Prostate Cancer Specific Mortality }\end{array}$ & $\begin{array}{l}\text { Followup } \\
\text { Metastases }\end{array}$ \\
\hline & & $\begin{array}{l}\text { High: } \\
\text { WW }=78.8 \%\left(67 / 85^{\star}\right) \\
\text { RP }=73.3 \%\left(62 / 85^{\star}\right) \\
R^{\star}=1.08(0.91 \text { to } 1.28) \\
\text { Participant Characteristics, } 23 \text { years } \\
\text { Participants }<65 \text { years: } \\
\text { WW }=77.6 \%(129 / 166) \\
R P=62.6 \%(105 / 157) \\
R^{\star}=1.16(1.01 \text { to } 1.33) \\
\text { Participants } \geq 65 \text { years: } \\
\text { WW }=87.3 \%(163 / 182) \\
R P=79.2 \%(156 / 190) \\
R R^{\star}=1.09(1.00 \text { to } 1.19)\end{array}$ & $\begin{array}{l}\text { High: } \\
\text { WW }=35.7 \%\left(29 / 85^{\star}\right) \\
R P=33.1 \%\left(28 / 85^{\star}\right) \\
R^{\star}=1.06(0.69 \text { to } 1.62) \\
\text { Participant Characteristics, } 23 \text { years } \\
\text { Participants <65 years: } \\
W W=37.9 \%(63 / 166) \\
R P=22.8 \%(39 / 157) \\
\left.R^{\star}=1.52 \text { (1.09 to } 2.13\right) \\
\text { Participants } \geq 65 \text { years: } \\
\text { WW }=25.3 \%(47 / 182) \\
R P=16.9 \%(32 / 190) \\
\left.R R^{\star}=1.53 \text { (1.03 to } 2.29\right)\end{array}$ & $\begin{array}{l}\text { High: } \\
\text { WW }=50.8 \%\left(44 / 85^{\star}\right) \\
\mathrm{RP}=45.9 \%\left(37 / 85^{\star}\right) \\
\mathrm{RR}^{\star}=1.19(0.87 \text { to } 1.63) \\
\text { Participant Characteristics, } 23 \\
\text { years } \\
\text { Participants <65 years: } \\
\text { WW }=49.4 \%(81 / 166) \\
\mathrm{RP}=30.8 \%(48 / 157) \\
\mathrm{RR}=1.63(1.23 \text { to } 2.15) \\
\text { Participants } \geq 65 \text { years: } \\
\text { WW }=37.7 \%(69 / 182) \\
\mathrm{RP}=23.2 \%(44 / 190) \\
\mathrm{RR}=1.67(1.21 \text { to } 2.30)\end{array}$ \\
\hline
\end{tabular}




\begin{tabular}{|c|c|c|c|c|}
\hline $\begin{array}{l}\text { Intervention/ } \\
\text { Comparison }\end{array}$ & \begin{tabular}{|l|} 
Study \\
Design \\
(Trial/Registry) \\
Risk of Bias \\
\end{tabular} & $\begin{array}{l}\text { Followup } \\
\text { Overall Mortality }\end{array}$ & $\begin{array}{l}\text { Followup } \\
\text { Prostate Cancer Specific Mortality }\end{array}$ & $\begin{array}{l}\text { Followup } \\
\text { Metastases }\end{array}$ \\
\hline & \begin{tabular}{|l|} 
Wilt $2017^{3}$ \\
Wilt $2012^{9}$ \\
(median 10 years) \\
RCT \\
PIVOT \\
19.5 years \\
Low
\end{tabular} & 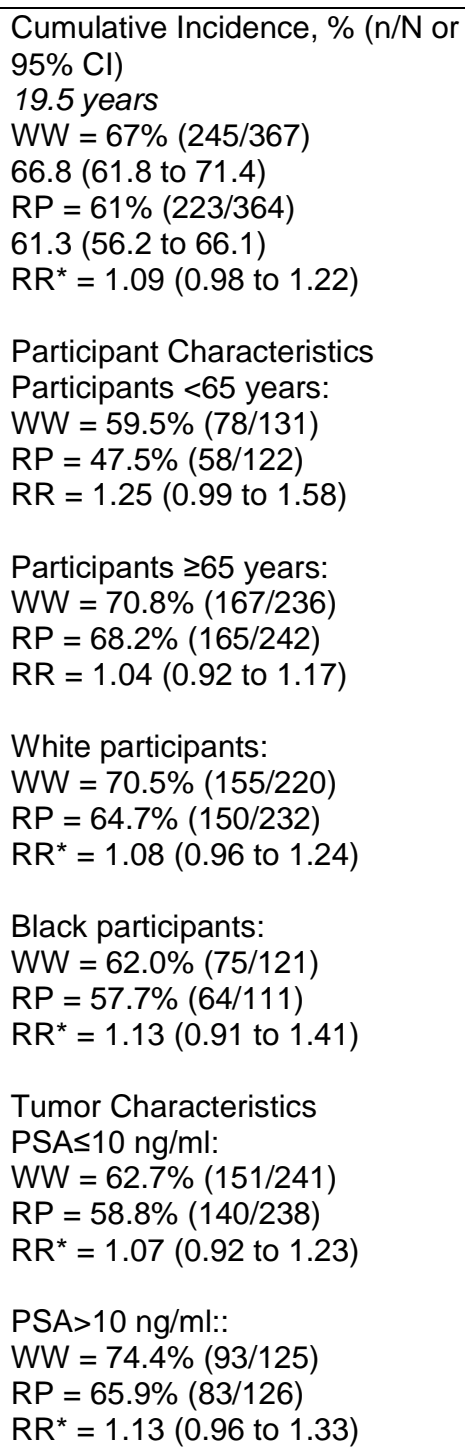 & 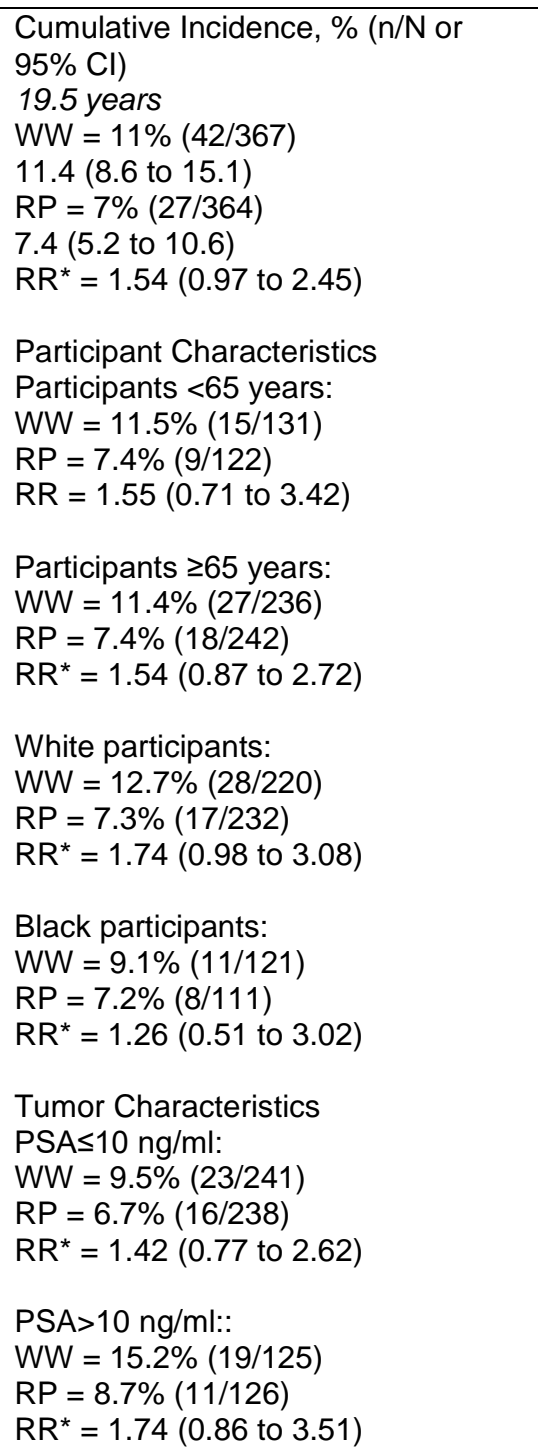 & $\begin{array}{l}\text { NR at } 19.5 \text { years } \\
\text { Bone metastases at } 10 \text { years } \\
W W=10.6 \%(39 / 367) \\
R P=4.7 \%(17 / 364) \\
\mathrm{HR}=0.40(0.22 \text { to } 0.70)\end{array}$ \\
\hline
\end{tabular}




\begin{tabular}{|c|c|c|c|c|}
\hline $\begin{array}{l}\text { Intervention/ } \\
\text { Comparison }\end{array}$ & $\begin{array}{l}\text { Study } \\
\text { Design } \\
\text { (Trial/Registry) } \\
\text { Risk of Bias }\end{array}$ & $\begin{array}{l}\text { Followup } \\
\text { Overall Mortality }\end{array}$ & $\begin{array}{l}\text { Followup } \\
\text { Prostate Cancer Specific Mortality }\end{array}$ & $\begin{array}{l}\text { Followup } \\
\text { Metastases }\end{array}$ \\
\hline & & $\begin{array}{l}\text { Risk category (locally assessed) } \\
\text { Low: } \\
\text { WW }=56.1 \% \text { (83/148) } \\
\mathrm{RP}=55.4 \%(82 / 148) \\
\left.\mathrm{RR}^{\star}=1.01 \text { (0.83 to } 1.24\right) \\
\text { Intermediate: } \\
W W=74.2 \%(89 / 120) \\
\mathrm{RP}=59.7 \%(77 / 129) \\
\mathrm{RR}^{\star}=1.25(1.05 \text { to } 1.48) \\
\text { High: } \\
W W=73.8 \%(59 / 80) \\
\mathrm{RP}=71.4 \%(59 / 77) \\
\mathrm{RR}^{\star}=0.96(0.80 \text { to } 1.15)\end{array}$ & $\begin{array}{l}\text { Risk category (locally assessed) } \\
\text { Low: } \\
\text { WW }=5.4 \%(8 / 148) \\
\mathrm{RP}=4.1 \%(6 / 148) \\
\mathrm{RR}^{\star}=1.33(0.47 \text { to } 3.75) \\
\text { Intermediate: } \\
\text { WW }=15.8 \%(19 / 120) \\
\mathrm{RP}=8.5 \%(11 / 129) \\
\mathrm{RR}^{\star}=1.85(0.92 \text { to } 3.74) \\
\text { High: } \\
\text { WW }=18.8 \%(15 / 80) \\
\mathrm{RP}=13.0 \%(10 / 77) \\
\mathrm{RR}^{\star}=1.44(0.69 \text { to } 3.02)\end{array}$ & \\
\hline
\end{tabular}

*Calculated by EPC

Abbreviations: CI=Confidence Interval; NR=Not Reported; PIVOT=Prostate Cancer Intervention Versus Observation Trial; PSA=Prostate-specific antigen; RCT=randomized controlled trial; RP=radical prostatectomy; RR=relative risk; SPCG-4=Scandinavian Prostatic Cancer Group; WW=watchful waiting 
Table F-5. Health status and quality of life: watchful waiting comparisons

\begin{tabular}{|c|c|c|c|}
\hline $\begin{array}{l}\text { Intervention/ } \\
\text { Comparison }\end{array}$ & $\begin{array}{l}\text { Study } \\
\text { Design } \\
\text { (Trial/Registry) } \\
\text { Followup } \\
\text { Risk of Bias } \\
\end{array}$ & Health Status & $\begin{array}{l}\text { Quality of Life } \\
\text { Prostate Cancer Related Quality of Life }\end{array}$ \\
\hline \multirow[t]{2}{*}{ WW/RP } & $\begin{array}{l}\text { Bill-Axelson } 2014^{1} \\
18 \text { years } \\
\text { Bill-Axelson } 2018^{2} \\
\text { RCT } \\
23.6 \text { years } \\
\text { (SPCG-4) } \\
\text { Low }\end{array}$ & NR & NR \\
\hline & $\begin{array}{l}\text { Wilt } 2017^{3} \\
\text { RCT } \\
\text { PIVOT } \\
19.5 \text { years } \\
\text { Low }\end{array}$ & NR & NR \\
\hline
\end{tabular}

Abbreviations: NR=Not Reported; PIVOT=Prostate Cancer Intervention Versus Observation Trial; RCT=randomized controlled trial; RP=radical prostatectomy; SPCG4=Scandinavian Prostatic Cancer Group; WW=watchful waiting 
Table F-6. Harms: watchful waiting comparisons

\begin{tabular}{|c|c|c|}
\hline $\begin{array}{l}\text { Intervention/ } \\
\text { Comparison }\end{array}$ & $\begin{array}{l}\text { Study } \\
\text { (Trial) } \\
\text { Followup } \\
\text { Risk of Bias }\end{array}$ & Adverse Effects \\
\hline \multirow[t]{3}{*}{ WW/RP } & $\begin{array}{l}\text { Bill-Axelson } 2014^{1} \\
\text { RCT } \\
\text { (SPCG-4) } \\
18 \text { years } \\
\text { Low }\end{array}$ & NR \\
\hline & $\begin{array}{l}\text { Bill-Axelson } 2018^{2} \\
\text { RCT } \\
\text { (SPCG-4) } \\
23.6 \text { years } \\
\text { Low }\end{array}$ & NR \\
\hline & $\begin{array}{l}\text { Wilt } 2017^{3} \\
\text { Wilt } 2012^{9} \\
\text { Personal } \\
\text { communication with } \\
\text { author } \\
\text { (median } 10 \text { years } \\
\text { RCT } \\
\text { PIVOT } \\
10 \text { years } \\
\text { Low }\end{array}$ & $\begin{array}{l}\text { Erectile dysfunction, defined as:1) able to have an erection that is insufficient for vaginal penetration or } 2) \text { unable to } \\
\text { have erection } \\
\text { WW: } 102 / 146(69.9 \%) \\
\text { RP: } 125 / 147(85.0 \%) \\
\text { Urinary incontinence, defined as }>1 \text { pad/day } \\
\text { WW: } 8 / 147(5.4 \%) \\
\text { RP: } 32 / 148(21.6 \%) \\
\text { Bowel dysfunction, defined as moderate or big problem } \\
\text { WW } 34 / 150(22.7 \%) \text { RP } 28 / 149(18.8 \%)\end{array}$ \\
\hline
\end{tabular}

Abbreviations: NR=Not Reported; PIVOT=Prostate Cancer Intervention Versus Observation Trial; RCT=randomized controlled trial; RP=radical prostatectomy; SPCG-

4=Scandinavian Prostatic Cancer Group; WW=watchful waiting Group

Table F-7. Evidence certainty: watchful waiting versus RP

\begin{tabular}{|c|c|c|c|c|c|c|c|c|c|c|c|}
\hline $\begin{array}{l}\text { Intervention/ } \\
\text { Comparison }\end{array}$ & $\begin{array}{l}\text { k= } \\
\text { Study } \\
\text { Design }\end{array}$ & $\begin{array}{l}\text { Risk of } \\
\text { Bias }\end{array}$ & $\begin{array}{l}\text { Incon- } \\
\text { sistency }\end{array}$ & $\begin{array}{l}\text { Indirect- } \\
\text { ness }\end{array}$ & $\begin{array}{l}\text { Impre- } \\
\text { cision }\end{array}$ & $\begin{array}{l}\text { Other } \\
\text { Consid- } \\
\text { erations }\end{array}$ & I & C & $\begin{array}{l}\text { Relative } \\
\text { (95\% Cl) }\end{array}$ & $\begin{array}{l}\text { Absolute } \\
(95 \% \mathrm{Cl})\end{array}$ & Certainty \\
\hline $\begin{array}{l}\text { All-cause } \\
\text { mortality } \\
20 \text { years }\end{array}$ & 2 RCTs & $\begin{array}{l}\text { Not } \\
\text { serious }\end{array}$ & Serious & $\begin{array}{l}\text { Not } \\
\text { serious }\end{array}$ & Serious & None & $\begin{array}{c}247 / 348 \\
(71 \%) \\
245 / 367 \\
(67 \%)\end{array}$ & $\begin{array}{c}200 / 347 \\
(58 \%) \\
223 / 364 \\
(61 \%)\end{array}$ & $\begin{array}{c}\text { RR } 1.23 \\
\text { (1.10 to } 1.38) \\
\text { RR } 1.09 \\
\text { (0.98 to } 1.22)\end{array}$ & $\begin{array}{c}13.3 \% \\
(6.3 \text { to } 20.4) \\
5.5 \% \\
(-1.45 \text { to } 12.4)\end{array}$ & $\stackrel{\text { Low }}{\oplus \oplus \bigcirc \bigcirc \mathrm{a}, \mathrm{b}}$ \\
\hline $\begin{array}{l}\text { All-cause } \\
\text { mortality } \\
25 \text { years }\end{array}$ & $1 \mathrm{RCT}$ & $\begin{array}{l}\text { Not } \\
\text { serious }\end{array}$ & $\begin{array}{l}\text { Not } \\
\text { serious }\end{array}$ & $\begin{array}{l}\text { Not } \\
\text { serious }\end{array}$ & Serious & None & $\begin{array}{c}292 / 348 \\
(84 \%)\end{array}$ & $\begin{array}{c}261 / 347 \\
(75 \%)\end{array}$ & $\begin{array}{c}\text { RR } 1.12 \\
\text { (1.03 to } 1.20)\end{array}$ & $\begin{array}{c}8.7 \% \\
(2.7 \text { to } 14.6)\end{array}$ & $\begin{array}{l}\text { Moderate } \\
\oplus \oplus \oplus \bigcirc^{b}\end{array}$ \\
\hline
\end{tabular}




\begin{tabular}{|c|c|c|c|c|c|c|c|c|c|c|c|}
\hline $\begin{array}{l}\text { Intervention/ } \\
\text { Comparison }\end{array}$ & $\begin{array}{l}\text { k= } \\
\text { Study } \\
\text { Design }\end{array}$ & $\begin{array}{l}\text { Risk of } \\
\text { Bias }\end{array}$ & $\begin{array}{l}\text { Incon- } \\
\text { sistency }\end{array}$ & $\begin{array}{l}\text { Indirect- } \\
\text { ness }\end{array}$ & $\begin{array}{l}\text { Impre- } \\
\text { cision }\end{array}$ & $\begin{array}{l}\text { Other } \\
\text { Consid- } \\
\text { erations }\end{array}$ & I & C & $\begin{array}{l}\text { Relative } \\
\text { (95\% Cl) }\end{array}$ & $\begin{array}{l}\text { Absolute } \\
\text { (95\% Cl) }\end{array}$ & Certainty \\
\hline $\begin{array}{l}\text { Prostate } \\
\text { Cancer Specific } \\
\text { Mortality } \\
20 \text { years }\end{array}$ & 2 RCTs & $\begin{array}{l}\text { Not } \\
\text { serious }\end{array}$ & Serious & $\begin{array}{l}\text { Not } \\
\text { serious }\end{array}$ & Serious & None & $\begin{array}{c}99 / 348 \\
(28 \%) \\
42 / 367 \\
(11 \%)\end{array}$ & $\begin{array}{c}63 / 347 \\
(18 \%) \\
27 / 364 \\
(7 \%)\end{array}$ & $\begin{array}{c}\text { RR } 1.57 \\
\text { (1.19 to } 2.07) \\
\text { RR } 1.54 \\
\text { (0.97 to } 2.45)\end{array}$ & $\begin{array}{c}10.3 \% \\
(4.05 \text { to } 16.5) \\
4 \% \\
(-0.19 \text { to } 8.25)\end{array}$ & $\stackrel{\text { Low }}{\oplus \oplus \bigcirc \bigcirc^{a, b}}$ \\
\hline $\begin{array}{l}\text { Prostate } \\
\text { Cancer Specific } \\
\text { Mortality } \\
25 \text { years }\end{array}$ & $1 \mathrm{RCT}$ & $\begin{array}{l}\text { Not } \\
\text { serious }\end{array}$ & $\begin{array}{l}\text { Not } \\
\text { serious }\end{array}$ & $\begin{array}{l}\text { Not } \\
\text { serious }\end{array}$ & Serious & None & $\begin{array}{c}110 / 348 \\
(32 \%)\end{array}$ & $\begin{array}{l}71 / 347 \\
(20 \%)\end{array}$ & $\begin{array}{c}\text { RR } 1.54 \\
\text { (1.19 to } 2.00)\end{array}$ & $\begin{array}{c}11.1 \% \\
(4.7 \text { to } 17.6)\end{array}$ & $\begin{array}{l}\text { Moderate } \\
\oplus \oplus \oplus \bigcirc^{b}\end{array}$ \\
\hline $\begin{array}{l}\text { Metastases } \\
20 \text { years }\end{array}$ & $1 \mathrm{RCT}$ & $\begin{array}{l}\text { Not } \\
\text { serious }\end{array}$ & $\begin{array}{l}\text { Not } \\
\text { serious }\end{array}$ & $\begin{array}{l}\text { Not } \\
\text { serious }\end{array}$ & Serious & None & $\begin{array}{c}138 / 348 \\
(40 \%)\end{array}$ & $\begin{array}{c}89 / 347 \\
(26 \%)\end{array}$ & $\begin{array}{c}\text { RR } 1.54 \\
\text { (1.24 to } 1.93)\end{array}$ & $\begin{array}{c}14 \% \\
(7.1 \text { to } 20.9)\end{array}$ & $\begin{array}{l}\text { Moderate } \\
\oplus \oplus \oplus \bigcirc^{b}\end{array}$ \\
\hline $\begin{array}{l}\text { Metastases } \\
25 \text { years }\end{array}$ & $1 \mathrm{RCT}$ & $\begin{array}{l}\text { Not } \\
\text { serious }\end{array}$ & $\begin{array}{l}\text { Not } \\
\text { serious }\end{array}$ & $\begin{array}{l}\text { Not } \\
\text { serious }\end{array}$ & Serious & None & $\begin{array}{c}150 / 348 \\
(43 \%)\end{array}$ & $\begin{array}{c}92 / 347 \\
(27 \%)\end{array}$ & $\begin{array}{c}\text { RR } 1.63 \\
\text { (1.3 to } 2.00)\end{array}$ & $\begin{array}{c}16.6 \% \\
\text { (9.6 to } 23.6)\end{array}$ & $\begin{array}{l}\text { Moderate } \\
\oplus \oplus \oplus \bigcirc^{b}\end{array}$ \\
\hline $\begin{array}{l}\text { Erectile } \\
\text { dysfunction } \\
10 \text { years }\end{array}$ & $1 \mathrm{RCT}$ & $\begin{array}{l}\text { Not } \\
\text { serious }\end{array}$ & $\begin{array}{l}\text { Not } \\
\text { serious }\end{array}$ & $\begin{array}{l}\text { Not } \\
\text { serious }\end{array}$ & Serious & None & $\begin{array}{c}102 / 146 \\
(70 \%)\end{array}$ & $\begin{array}{c}125 / 147 \\
(85 \%)\end{array}$ & $\begin{array}{c}\text { RR } 0.82 \\
\text { (0.72 to } 0.93)\end{array}$ & $\begin{array}{c}-15.2 \% \\
(-24.6 \text { to }-5.8)\end{array}$ & $\begin{array}{l}\text { Moderate } \\
\oplus \oplus \oplus \bigcirc^{b}\end{array}$ \\
\hline $\begin{array}{l}\text { Urinary } \\
\text { incontinence } \\
\text { (>1 pad/day) } \\
10 \text { years }\end{array}$ & $1 \mathrm{RCT}$ & $\begin{array}{l}\text { Not } \\
\text { serious }\end{array}$ & $\begin{array}{l}\text { Not } \\
\text { serious }\end{array}$ & $\begin{array}{l}\text { Not } \\
\text { serious }\end{array}$ & Serious & None & $\begin{array}{c}8 / 147 \\
(5 \%)\end{array}$ & $\begin{array}{c}32 / 148 \\
(22 \%)\end{array}$ & $\begin{array}{c}\text { RR } 0.25 \\
\text { (0.12 to } 0.53)\end{array}$ & $\begin{array}{c}-16.2 \% \\
(--23.8 \text { to }-8.6)\end{array}$ & $\begin{array}{l}\text { Moderate } \\
\oplus \oplus \oplus \bigcirc^{b}\end{array}$ \\
\hline $\begin{array}{l}\text { Bowel } \\
\text { dysfunction } \\
10 \text { years }\end{array}$ & $1 \mathrm{RCT}$ & $\begin{array}{l}\text { Not } \\
\text { serious }\end{array}$ & $\begin{array}{l}\text { Not } \\
\text { serious }\end{array}$ & $\begin{array}{l}\text { Not } \\
\text { serious }\end{array}$ & Serious & None & $\begin{array}{c}34 / 150 \\
(23 \%)\end{array}$ & $\begin{array}{c}28 / 149 \\
(19 \%)\end{array}$ & $\begin{array}{l}\text { RR } 1.21 \\
(0.77 \text { to } \\
1.88)\end{array}$ & $\begin{array}{c}3.9 \% \\
(-5.3 \text { to } 13.1)\end{array}$ & $\stackrel{\text { Low }}{\oplus \oplus \bigcirc^{c}}$ \\
\hline
\end{tabular}

Abbreviations: C=control; $\mathrm{CI}=$ confidence interval; I=intervention; $\mathrm{RCT}=$ randomized controlled trial; $\mathrm{RP}=$ radical prostatectomy

\section{Explanations}

a. Rated down one level for inconsistency

b. Rated down one level for imprecision

c. Rated down two levels for imprecision 


\section{Appendix G. Active Surveillance/Active Monitoring}

Table G-1. Risk of bias assessments for randomized controlled trials: active surveillancelactive monitoring

\begin{tabular}{|c|c|c|c|c|c|c|c|c|}
\hline $\begin{array}{l}\text { Intervention/ } \\
\text { Comparison } \\
\text { (Outcomes) } \\
\text { Follow-up Time }\end{array}$ & Author, Year & $\begin{array}{l}\text { Selection } \\
\text { Bias }\end{array}$ & $\begin{array}{l}\text { Performance } \\
\text { Bias }\end{array}$ & $\begin{array}{l}\text { Detection } \\
\text { Bias }\end{array}$ & Attrition & $\begin{array}{l}\text { Reporting } \\
\text { Bias }\end{array}$ & Other Bias & $\begin{array}{l}\text { Overall } \\
\text { Rating }\end{array}$ \\
\hline $\begin{array}{l}\text { AS/PDT } \\
\text { (QoL) } \\
24 \text { months }\end{array}$ & Azzouzi $2017^{10}$ & Low & High & Low & High & Medium & None & Medium \\
\hline $\begin{array}{l}\text { AS/PDT } \\
\text { (harms) } \\
24 \text { months } \\
\end{array}$ & Azzouzi $2017^{10}$ & Low & High & Low & Medium & Medium & None & Medium \\
\hline $\begin{array}{l}\text { AM/RT } \\
\text { (mortality/metastases) } \\
10 \text { years }\end{array}$ & $\begin{array}{l}\text { Hamdy } 2016^{11} \\
\text { Donovan } 2016^{12} \\
\text { Lane } 2016^{13} \\
\text { Neal } 2020^{14}\end{array}$ & Low & Low & Low & Low & Low & None & Low \\
\hline $\begin{array}{l}\text { AM/RT } \\
\text { (harms) } \\
10 \text { years }\end{array}$ & $\begin{array}{l}\text { Hamdy } 2016^{11} \\
\text { Donovan } 2016^{12} \\
\text { Lane } 2016^{13} \\
\text { Neal } 2020^{14}\end{array}$ & Low & Low & Low & Moderate & Low & None & Low \\
\hline
\end{tabular}

Abbreviations: AS=active surveillance; PDT=photodynamic therapy; QoL=quality of life; RP= radical prostatectomy; RT=radiation therapy 
Table G-2. Summary risk of bias assessments for observational studies: active surveillance

\begin{tabular}{|c|c|c|c|c|c|c|c|c|c|}
\hline $\begin{array}{l}\text { Intervention/ } \\
\text { Comparison } \\
\text { (Outcomes) }\end{array}$ & $\begin{array}{l}\text { Author, } \\
\text { Year }\end{array}$ & $\begin{array}{l}\text { Bias Due to } \\
\text { Confounding }\end{array}$ & $\begin{array}{l}\text { Bias in } \\
\text { Selection of } \\
\text { Participants }\end{array}$ & $\begin{array}{l}\text { Bias in } \\
\text { Classification } \\
\text { of } \\
\text { Interventions }\end{array}$ & $\begin{array}{l}\text { Bias Due to } \\
\text { Deviations } \\
\text { for Intended } \\
\text { Interventions }\end{array}$ & $\begin{array}{l}\text { Bias Due } \\
\text { to Missing } \\
\text { Data }\end{array}$ & $\begin{array}{l}\text { Bias in } \\
\text { Measurement } \\
\text { of Outcomes }\end{array}$ & $\begin{array}{l}\text { Bias in } \\
\text { Selection } \\
\text { of the } \\
\text { Reported } \\
\text { Result }\end{array}$ & Overall \\
\hline \multirow[t]{2}{*}{ AS/RP } & $\begin{array}{l}\text { Thomsen } \\
2019^{15}\end{array}$ & Moderate & Serious & Critical & $\begin{array}{l}\text { No } \\
\text { Information }\end{array}$ & Low & Low & Low & Critical \\
\hline & $\begin{array}{l}\text { Barocas } \\
2013^{16} ; \\
\text { Barocas } \\
2017^{17} ; \\
\text { Tyson } \\
2017^{18}\end{array}$ & Moderate & Low & Moderate & Low & Serious & Moderate & Low & Serious \\
\hline AS/ADT, RT, RP & $\begin{array}{l}\text { Herden } \\
2016^{4} ; \\
\text { Ansmann } \\
2018^{19} \\
\end{array}$ & Moderate & Low & Moderate & Low & Serious & Serious & Low & Serious \\
\hline AS/RP & $\begin{array}{l}\text { Tosoian } \\
2016^{20} \\
\end{array}$ & Serious & Moderate & Moderate & Serious & $\begin{array}{l}\text { No } \\
\text { Information }\end{array}$ & Moderate & Low & Critical \\
\hline
\end{tabular}

Abbreviations: ADT=androgen deprivation therapy; AM=active monitoring; AS=active surveillance; $\mathrm{RT}=$ radiation therapy; $\mathrm{RP}=$ radical prostatectomy 
Table G-3. Characteristics of eligible studies: active surveillance comparisons

\begin{tabular}{|c|c|c|c|c|c|}
\hline $\begin{array}{l}\text { Study } \\
\text { Design } \\
\text { Country } \\
\text { ROB }\end{array}$ & $\mathrm{N}=$ & Population & $\begin{array}{l}\text { Intervention } \\
\text { Frequency } \\
\text { Duration }\end{array}$ & $\begin{array}{l}\text { Comparison } \\
\text { Frequency } \\
\text { Duration }\end{array}$ & $\begin{array}{l}\text { Followup Time(s) } \\
\text { Outcome } \\
\text { (Instrument) }\end{array}$ \\
\hline $\begin{array}{l}\text { Azzouzi } 2017^{10} \\
\text { RCT } \\
10 \text { European } \\
\text { countries } \\
\text { Medium }\end{array}$ & 413 & $\begin{array}{l}\text { Adult men with low-risk } \\
\text { CLPC (cT2a) diagnosed by } \\
\text { transrectal ultrasound - } \\
\text { guided biopsy } \\
\text { Gleason pattern } 3 \\
\text { T1c: } 87 \% \\
\text { T2a: } 13 \% \\
\text { Age } 64 \\
\text { 99\% White }\end{array}$ & $\begin{array}{l}\text { Active Surveillance } \\
\text { Biopsy: 1-year intervals PSA \& } \\
\text { digital rectal exam: 3-month } \\
\text { intervals }\end{array}$ & $\begin{array}{l}\text { Photo dynamic therapy } \\
\text { Drug + laser } \\
\text { padeliporfin (IV)+laser } \\
\text { (753nm) } \\
\text { energy dose: } 200 \mathrm{~J} / \mathrm{cm} \\
\text { padeliporfin: } 10 \mathrm{~min} ; \\
\text { laser: } 22 \mathrm{~min}\end{array}$ & $\begin{array}{l}1 \text { year } \\
\text { QoL (EQ-5D) } \\
2 \text { years } \\
\text { QoL (EQ-5D) } \\
\text { Bladder dysfunction } \\
\text { Sexual dysfunction (IIEF-15) }\end{array}$ \\
\hline $\begin{array}{l}\text { Hamdy } 2016^{11-14,21} \\
\text { RCT } \\
\text { United Kingdom } \\
\text { Low }\end{array}$ & 1090 & $\begin{array}{l}\text { Men with median PSA level } \\
\text { of } 4.6 \mathrm{ng} / \text { milliliter and a } \\
\text { Gleason score of } 6(77 \%) \\
\text { T1c : } 76 \% \\
\text { T2: } 23 \% \\
\text { Gleason sum } \\
6: 78 \% \\
\text { 7: } 20 \% \\
\text { 8-10: } 2 \% \\
\text { Age } 62 \\
99 \% \text { White }\end{array}$ & $\begin{array}{l}\text { Active Monitoring: PSA at 3- } \\
\text { month intervals year 1; 6-month } \\
\text { to 1-year intervals thereafter. } \\
50 \% \text { increase previous 1-year } \\
\text { triggered review }\end{array}$ & $\begin{array}{l}\text { 3D-CRT } \\
74 \text { Gy in } 37 \text { fractions } \\
\text { With neoadjuvant ADT } \\
\text { for } 3 \text { to } 6 \text { months before } \\
\text { RT }\end{array}$ & $\begin{array}{l}5 \text { years } \\
\text { Prostate-specific mortality } \\
10 \text { years } \\
\text { Prostate-specific mortality } \\
\text { All-cause mortality } \\
\text { Metastases }\end{array}$ \\
\hline
\end{tabular}

Abbreviations: 3D-CRT=three-dimensional conformal radiation therapy; CLPC=clinically localized prostate cancer; EQ-5D=EuroQoL-5D; Gy=Gray; IIEF-15=International Index of Erectile Function; IV=intravenous; J/cm=Joules per centimeter; min=minutes; ng/ml=nanogram per milliliter; nm=nanometer; PSA=Prostate-specific antigen; $\mathrm{RCT}=$ randomized controlled trial; $\mathrm{ROB}=$ risk of bias 
Table G-4. Mortality, survival, and metastases: active surveillance

\begin{tabular}{|c|c|c|c|c|c|}
\hline $\begin{array}{l}\text { Intervention/ } \\
\text { Comparison }\end{array}$ & $\begin{array}{l}\text { Study } \\
\text { Design } \\
\text { (Trial/Registry) } \\
\text { Followup } \\
\text { Risk of Bias }\end{array}$ & $\begin{array}{l}\text { Overall Survival and } \\
\text { Mortality }\end{array}$ & $\begin{array}{l}\text { Prostate Cancer Specific } \\
\text { Survival and Mortality }\end{array}$ & $\begin{array}{l}\text { Metastatic } \\
\text { Progression Free } \\
\text { Survival }\end{array}$ & Metastases \\
\hline AS/PDT & $\begin{array}{l}\text { Azzouzi } 2017^{10} \\
\text { RCT } \\
\text { CLIN1001 PCM301 } \\
2 \text { years } \\
\text { Low }\end{array}$ & NR & NR & NR & NR \\
\hline AM/RT & $\begin{array}{l}\text { Hamdy } 2016^{11,12,14} \\
\text { RCT } \\
\text { ProtecT } \\
10 \text { years } \\
\text { Low }\end{array}$ & $\begin{array}{l}\text { AM: } 11 \%(59 / 545) \\
\text { RT: } 10 \%(55 / 545) \\
R^{*}=1.07(0.76 \text { to } 1.52)\end{array}$ & $\begin{array}{l}\text { AM: } 1.5 \%(8 / 545) \\
\text { RT: } 1 \%(4 / 545) \\
R^{*}=2.0(0.6 \text { to } 6.6)\end{array}$ & NR & $\begin{array}{l}\text { AM: } 6 \%(33 / 545) \\
\text { RT: } 3 \%(16 / 545) \\
R^{*}=2.06(1.15 \text { to } 3.7)\end{array}$ \\
\hline
\end{tabular}

*Calculated by EPC

Abbreviations: AM=active monitoring; AS=active surveillance; NR=Not Reported; PDT=photodynamic therapy; RCT=randomized controlled trial; RP=radical prostatectomy; $\mathrm{RR}=$ relative risk; $\mathrm{RT}=$ =radiation therapy

Table G-5. Health status and quality of life: active surveillance comparisons

\begin{tabular}{|l|l|l|l|}
\hline $\begin{array}{l}\text { Intervention/ } \\
\text { Comparison }\end{array}$ & $\begin{array}{l}\text { Study } \\
\text { Design } \\
\text { (Trial/Registry) } \\
\text { Followup } \\
\text { Risk of Bias }\end{array}$ & Health Status & $\begin{array}{l}\text { Quality of Life } \\
\text { Prostate Cancer Related Quality of Life }\end{array}$ \\
\hline AS/PDT & Azzouzi 201710 & NR & EQ-5D, Adjusted mean change (95\% Cl) \\
& RCT & & AS: $-3.0(-5.0,-1.0)$ \\
& CLIN1001 PCM301 & & PDT: $-2.3(-4.2,-0.4)$ \\
Difference: -0.7 & \\
& 2 years & & \\
\hline
\end{tabular}

Abbreviations: AS=active surveillance; EQ-5D=EuroQoL-5D; NR=Not Reported; PDT=photodynamic therapy; RCT=randomized controlled trial 
Table G-6. Harms: active surveillance comparisons

\begin{tabular}{|c|c|c|}
\hline $\begin{array}{l}\text { Intervention/ } \\
\text { Comparison }\end{array}$ & $\begin{array}{l}\text { Study } \\
\text { (Trial) } \\
\text { Followup } \\
\text { Risk of Bias }\end{array}$ & Adverse Effects \\
\hline AS/PDT & $\begin{array}{l}\text { Azzouzi } 2017^{10} \\
\text { RCT } \\
\text { CLIN1001 PCM301 } \\
2 \text { years } \\
\text { Low }\end{array}$ & 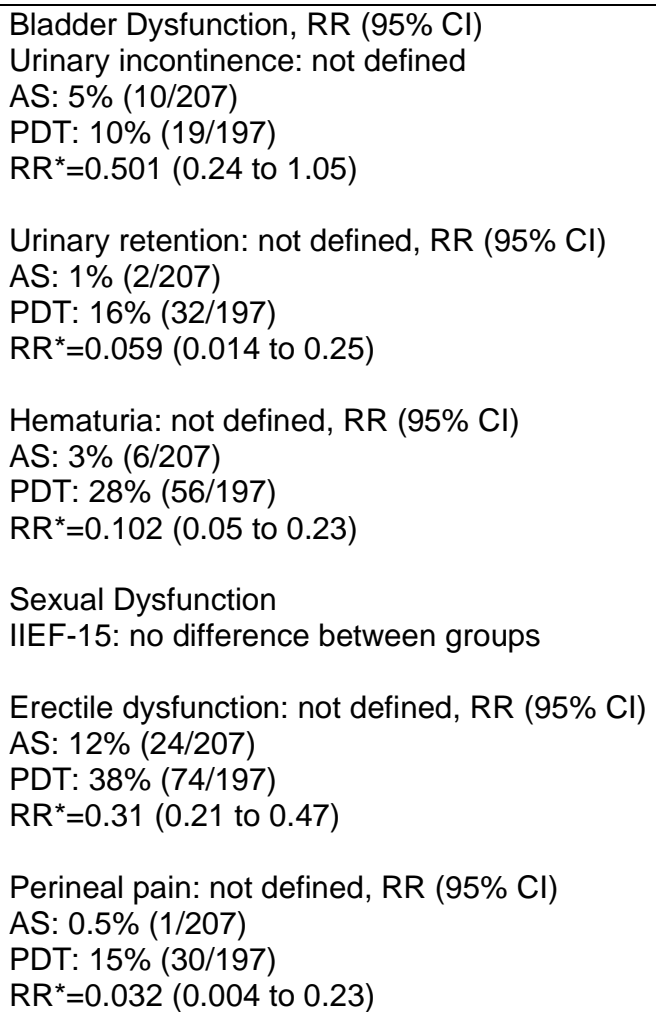 \\
\hline
\end{tabular}




\begin{tabular}{|c|c|c|}
\hline $\begin{array}{l}\text { Intervention/ } \\
\text { Comparison }\end{array}$ & $\begin{array}{l}\text { Study } \\
\text { (Trial) } \\
\text { Followup } \\
\text { Risk of Bias }\end{array}$ & Adverse Effects \\
\hline RT/AM & $\begin{array}{l}\text { Hamdy } 2016^{11-13} \\
\text { RCT } \\
\text { ProtecT } \\
6 \text { years } \\
\text { Low }\end{array}$ & $\begin{array}{l}\text { Bladder Dysfunction, } \mathrm{RR}(95 \% \mathrm{Cl}) \\
\text { Urinary incontinence: Percent of men reporting more than } 1 \text { pad per day in past } 4 \text { weeks } \\
1 \text { year } \\
\text { AM: } 4 \%(15 / 357) \\
\text { RT: } 4 \%(13 / 358) \\
\text { RR }=1.16(0.56 \text { to } 2.40) \\
6 \text { years } \\
\text { AM: } 8 \%(38 / 453) \\
\text { RT: } 4 \%(16 / 452) \\
\text { RR }=2.37(1.34 \text { to } 4.19) \\
\text { Sexual Dysfunction, RR }(95 \% \mathrm{Cl}) \\
\text { Erectile dysfunction: Men unable to have an erection or able to have an erection that is of insufficient strength for } \\
\text { vaginal penetration } \\
1 \text { year } \\
\text { AM: } 51 \%(173 / 340) \\
\text { RT: } 62 \%(219 / 351) \\
\text { RR }=0.82(0.71 \text { to } 0.93) \\
6 \text { years } \\
\text { AM: } 70 \%(318 / 452) \\
\text { RT: } 73 \%(331 / 456) \\
\text { RR }=0.97(0.89 \text { to } 1.05) \\
\text { Bowel Dysfunction, RR (95\% Cl) } \\
\text { Fecal incontinence: Percent of men reporting fecal incontinence more than once per week } \\
1 \text { year } \\
\text { AM: } 1 \%(4 / 356) \\
\text { RT: } 4 \%(14 / 358) \\
\text { RR }=0.64 \text { (0.31 to } 1.29) \\
6 \text { years } \\
\text { AM } 3 \%(12 / 462) \\
\text { RT: } 4 \%(19 / 465) \\
\text { RR }{ }^{\star}=0.64(0.31 \text { to } 1.3)\end{array}$ \\
\hline
\end{tabular}

Abbreviations: AM=active monitoring; AS=active surveillance; IIEF-15=International Index of Erectile Function; PDT=photodynamic therapy; RCT=randomized controlled trial; $\mathrm{RR}=$ relative risk; $\mathrm{RT}=$ radiation therapy 
Table G-7. Evidence certainty: active surveillance comparisons

\begin{tabular}{|c|c|c|c|c|c|c|c|c|c|c|c|}
\hline $\begin{array}{l}\text { Intervention/ } \\
\text { Comparison } \\
\text { Outcome }\end{array}$ & $\begin{array}{l}\text { k= } \\
\text { Study } \\
\text { Design }\end{array}$ & $\begin{array}{l}\text { Risk of } \\
\text { Bias }\end{array}$ & $\begin{array}{l}\text { Inconsist- } \\
\text { ency }\end{array}$ & $\begin{array}{l}\text { Indirect- } \\
\text { ness }\end{array}$ & $\begin{array}{l}\text { Impre- } \\
\text { cisions }\end{array}$ & $\begin{array}{l}\text { Other } \\
\text { Consid- } \\
\text { erations }\end{array}$ & I & C & $\begin{array}{l}\text { Relative } \\
(95 \% \mathrm{Cl})\end{array}$ & $\begin{array}{l}\text { Absolute } \\
(95 \% \mathrm{Cl})\end{array}$ & Certainty \\
\hline $\begin{array}{l}\text { AM/EBRT + } \\
\text { ADT } \\
\text { All-cause } \\
\text { mortality } \\
10 \text { years }\end{array}$ & $1 \mathrm{RCT}$ & Not serious & Not serious & Not serious & Serious & None & $\begin{array}{l}59 / 545 \\
(11 \%)\end{array}$ & $\begin{array}{l}55 / 545 \\
(10 \%)\end{array}$ & $\begin{array}{l}\text { RR } 1.07 \\
\text { (0.8 to } 1.50)\end{array}$ & $\begin{array}{l}0.7 \% \\
(-2.9 \text { to } 4.4)\end{array}$ & $\begin{array}{l}\text { Moderate } \\
\oplus \oplus \oplus \bigcirc^{\mathrm{a}}\end{array}$ \\
\hline $\begin{array}{l}\text { AM/ EBRT + } \\
\text { ADT } \\
\text { PC-specific } \\
\text { mortality } \\
10 \text { years }\end{array}$ & $1 \mathrm{RCT}$ & Not serious & Not serious & Not serious & $\begin{array}{l}\text { Very } \\
\text { serious }\end{array}$ & None & $\begin{array}{l}8 / 545 \\
(1.5 \%)\end{array}$ & $\begin{array}{l}4 / 545 \\
(0.7 \%)\end{array}$ & $\begin{array}{l}\text { Peto OR } \\
1.96 \\
(0.63 \text { to } 6.12)\end{array}$ & $\begin{array}{l}0.7 \% \\
(-0.5 \text { to } 1.9)\end{array}$ & $\begin{array}{l}\text { Low } \\
\oplus \oplus \bigcirc \bigcirc^{b}\end{array}$ \\
\hline $\begin{array}{l}\text { AM/ EBRT + } \\
\text { ADT } \\
\text { Metastases } \\
10 \text { years }\end{array}$ & $1 \mathrm{RCT}$ & Not serious & Not serious & Not serious & Serious & None & $\begin{array}{l}33 / 545 \\
(6 \%)\end{array}$ & $\begin{array}{l}16 / 545 \\
(3 \%)\end{array}$ & $\begin{array}{l}\text { RR 2.1 } \\
\text { (1.15 to 3.70) }\end{array}$ & $\begin{array}{l}3.1 \% \\
(0.67 \text { to } 5.6)\end{array}$ & $\begin{array}{l}\text { Moderate } \\
\oplus \oplus \oplus \bigcirc^{\mathrm{a}}\end{array}$ \\
\hline $\begin{array}{l}\text { AM/ EBRT + } \\
\text { ADT } \\
\text { Erectile } \\
\text { dysfunction } \\
6 \text { years }\end{array}$ & $1 \mathrm{RCT}$ & Not serious & Not serious & Not serious & $\begin{array}{l}\text { Very } \\
\text { serious }\end{array}$ & None & $\begin{array}{l}318 / 452 \\
(70 \%)\end{array}$ & \begin{tabular}{|l|}
$331 / 456$ \\
$(73 \%)$
\end{tabular} & $\begin{array}{l}\text { RR } 0.97 \\
\text { (0.89 to } 1.05)\end{array}$ & $\begin{array}{l}-2.4 \% \\
(-8.2 \text { to } 3.5)\end{array}$ & $\begin{array}{l}\text { Low } \\
\oplus \oplus \bigcirc \bigcirc^{b}\end{array}$ \\
\hline $\begin{array}{l}\text { AM/ EBRT + } \\
\text { ADT } \\
\text { Urinary } \\
\text { incontinence } \\
6 \text { years }\end{array}$ & $1 \mathrm{RCT}$ & Not serious & Not serious & Not serious & Serious & None & $\begin{array}{l}38 / 453 \\
(8 \%)\end{array}$ & $\begin{array}{l}16 / 452 \\
(4 \%)\end{array}$ & $\begin{array}{l}\text { RR } 2.37 \\
\text { (1.34 to } 4.19)\end{array}$ & $\begin{array}{l}4.8 \% \\
\text { (1.8 to } 7.9)\end{array}$ & $\begin{array}{l}\text { Moderate } \\
\oplus \oplus \oplus \bigcirc^{\mathrm{a}}\end{array}$ \\
\hline $\begin{array}{l}\text { AM/ EBRT + } \\
\text { ADT } \\
\text { Fecal } \\
\text { incontinence }\end{array}$ & $1 \mathrm{RCT}$ & Not serious & Not serious & Not serious & $\begin{array}{l}\text { Very } \\
\text { serious }\end{array}$ & None & $\begin{array}{l}12 / 462 \\
(3 \%)\end{array}$ & $\begin{array}{l}19 / 465 \\
(4 \%)\end{array}$ & $\begin{array}{l}\text { RR } 0.64 \\
\text { (0.31 to } 1.30)\end{array}$ & $\begin{array}{l}-1.5 \\
(-3.8 \text { to } 0.82)\end{array}$ & $\begin{array}{l}\text { Low } \\
\oplus \oplus \bigcirc \bigcirc^{b}\end{array}$ \\
\hline $\begin{array}{l}\text { AS/PDT } \\
\text { Urinary } \\
\text { incontinence } \\
2 \text { years }\end{array}$ & $1 \mathrm{RCT}$ & Serious & Not serious & Not serious & $\begin{array}{l}\text { Very } \\
\text { serious }\end{array}$ & None & $\begin{array}{c}10 / 207 \\
(5 \%)\end{array}$ & $\begin{array}{c}19 / 197 \\
(10 \%)\end{array}$ & $\begin{array}{c}\text { RR } 0.50 \\
(0.24 \text { to1.05) }\end{array}$ & $\begin{array}{c}-4.8 \% \\
(-9.9 \text { to } 2.4)\end{array}$ & $\begin{array}{l}\text { Insufficient } \\
\oplus \bigcirc \bigcirc \bigcirc^{b, c}\end{array}$ \\
\hline
\end{tabular}




\begin{tabular}{|c|c|c|c|c|c|c|c|c|c|c|c|}
\hline $\begin{array}{l}\text { Intervention/ } \\
\text { Comparison } \\
\text { Outcome }\end{array}$ & $\begin{array}{l}\text { k= } \\
\text { Study } \\
\text { Design }\end{array}$ & $\begin{array}{l}\text { Risk of } \\
\text { Bias }\end{array}$ & $\begin{array}{l}\text { Inconsist- } \\
\text { ency }\end{array}$ & $\begin{array}{l}\text { Indirect- } \\
\text { ness }\end{array}$ & $\begin{array}{l}\text { Impre- } \\
\text { cisions }\end{array}$ & $\begin{array}{l}\text { Other } \\
\text { Consid- } \\
\text { erations }\end{array}$ & I & C & $\begin{array}{l}\text { Relative } \\
(95 \% \mathrm{Cl})\end{array}$ & $\begin{array}{c}\text { Absolute } \\
(95 \% \mathrm{Cl})\end{array}$ & Certainty \\
\hline $\begin{array}{l}\text { AS/PDT } \\
\text { Urinary retention } \\
2 \text { years }\end{array}$ & $1 \mathrm{RCT}$ & Serious & Not serious & Not serious & Not serious & None & $\begin{array}{l}2 / 207 \\
(1 \%)\end{array}$ & $\begin{array}{l}32 / 197 \\
(16 \%)\end{array}$ & $\begin{array}{c}\text { RR } 0.06 \\
(0.01 \text { to } 0.24)\end{array}$ & $\begin{array}{c}15.3 \% \\
(-20.6 \text { to }-10)\end{array}$ & $\begin{array}{l}\text { Moderate } \\
\oplus \oplus \oplus \bigcirc^{c}\end{array}$ \\
\hline $\begin{array}{l}\text { AS/PDT } \\
\text { Erectile } \\
\text { dysfunction } \\
2 \text { years }\end{array}$ & $1 \mathrm{RCT}$ & Serious & Not serious & Not serious & Not serious & None & $\begin{array}{r}24 / 207 \\
(12 \%)\end{array}$ & $\begin{array}{l}74 / 197 \\
(38 \%)\end{array}$ & $\begin{array}{c}\text { RR } \mathbf{0 . 3 1} \\
(0.20 \text { to } 0.50)\end{array}$ & $\begin{array}{c}-26 \% \\
(-34 \text { to }-18)\end{array}$ & $\begin{array}{l}\text { Moderate } \\
\oplus \oplus \oplus \bigcirc^{c}\end{array}$ \\
\hline
\end{tabular}

Abbreviations: ADT=androgen deprivation therapy; AM=active monitoring; AS=active surveillance; $\mathrm{C}=$ control; $\mathrm{CI}=\mathrm{confidence} \mathrm{interval;} \mathrm{EBRT=external} \mathrm{beam} \mathrm{radiation} \mathrm{therapy;}$ $\mathrm{I}=$ intervention; $\mathrm{PDT}=$ photodynamic therapy; $\mathrm{RCT}=$ randomized controlled trial; $\mathrm{RR}=$ relative risk

\section{Explanations}

a. Rated down one level for imprecision

b. Rated down two levels for imprecision

c. Rated down one level for risk of bias 


\section{Appendix H. External Beam Radiation Therapy}

Table H-1. Risk of bias assessments for randomized controlled trials: external beam radiation therapy

\begin{tabular}{|c|c|c|c|c|c|c|c|c|}
\hline $\begin{array}{l}\text { Interventionl } \\
\text { Comparison } \\
\text { (Outcomes) }\end{array}$ & Author, Year & $\begin{array}{l}\text { Selection } \\
\text { Bias }\end{array}$ & $\begin{array}{l}\text { Performance } \\
\text { Bias }\end{array}$ & $\begin{array}{l}\text { Detection } \\
\text { Bias }\end{array}$ & Attrition & $\begin{array}{l}\text { Reporting } \\
\text { Bias }\end{array}$ & Other Bias & Overall \\
\hline $\begin{array}{l}\text { Extreme hypofractionated } \\
\text { PBRT/ } \\
\text { Standard PBRT }\end{array}$ & Vargas $2018^{22}$ & Low & Low & Unclear & High & Low & High & High \\
\hline $\begin{array}{l}\text { 3D-CRT + ADT/ } \\
\text { 3D-CRT + ADT + LDR-PB } \\
\text { boost }\end{array}$ & $\begin{array}{l}\text { Morris } 2017^{23} \\
\text { Rodda } 2017^{24} \\
\text { Rodda } 2017^{25} \\
\end{array}$ & Low & Low & Low & Low & $\begin{array}{l}\text { Low (High for } \\
\text { Rodda } 2017^{25}\end{array}$ & High & Medium \\
\hline $\begin{array}{l}\text { 3D-CRT/ } \\
\text { IMRT }\end{array}$ & Viani $2016^{26}$ & Low & Unclear & Unclear & High & Low & Low & Medium \\
\hline $\begin{array}{l}\text { EBRT + ADT/EBRT } \\
\text { (overall mortality/survival, } \\
\text { prostate cancer mortality, } \\
\text { distant metastasis, late toxicity, } \\
\text { quality of life) }\end{array}$ & Bolla, 2016 27 & Low & High & High & Low & Low & None & Medium \\
\hline $\begin{array}{l}\text { EBRT + ADT/EBRT } \\
\text { (overall survival/mortality, } \\
\text { distant metastasis, erectile } \\
\text { function, quality of life) }\end{array}$ & McPartlin, $2016^{28}$ & Low & Unclear & Unclear & Low & Low & None & Medium \\
\hline $\begin{array}{l}\text { EBRT + ADT/EBRT (stratified } \\
\text { results reported for } \pm \\
\text { brachytherapy) } \\
\text { (IPSS, EPIC scores, erectile } \\
\text { function, adverse } \\
\text { events/toxicity) }\end{array}$ & Vargas, $2019^{29}$ & Low & Unclear & High & High & Unclear & Yes & High \\
\hline $\begin{array}{l}\text { EBRT + ADT/EBRT } \\
\text { (overall mortality, prostate } \\
\text { cancer mortality) }\end{array}$ & $\begin{array}{l}\text { Phillips, } 2014^{30} \\
\text { McDuff, } 2018^{31} \\
\text { Giacalone, } 2016^{32}\end{array}$ & Low & Low & High & Low & High & None & Medium \\
\hline $\begin{array}{l}\text { EBRT + neoadjuvant and } \\
\text { concurrent ADT/EBRT + } \\
\text { concurrent and adjuvant ADT } \\
\text { (overall mortality, prostate } \\
\text { cancer mortality, metastasis, } \\
\text { late toxicity) }\end{array}$ & Malone $2019^{33}$ & Low & Unclear & High & Low & Low & None & Medium \\
\hline
\end{tabular}




\begin{tabular}{|c|c|c|c|c|c|c|c|c|}
\hline $\begin{array}{l}\text { Intervention/ } \\
\text { Comparison } \\
\text { (Outcomes) }\end{array}$ & Author, Year & $\begin{array}{l}\text { Selection } \\
\text { Bias }\end{array}$ & $\begin{array}{l}\text { Performance } \\
\text { Bias }\end{array}$ & $\begin{array}{l}\text { Detection } \\
\text { Bias }\end{array}$ & Attrition & $\begin{array}{l}\text { Reporting } \\
\text { Bias }\end{array}$ & Other Bias & Overall \\
\hline $\begin{array}{l}\text { Conventionally fractionated } \\
\text { EBRT/Ultra-hypofractionated } \\
\text { EBRT } \\
\text { (overall mortality, prostate } \\
\text { cancer mortality, metastasis, } \\
\text { harms) }\end{array}$ & Widmark $2019^{34}$ & Low & Unclear & High & $\begin{array}{l}\text { Low } \\
\text { (except } \\
\text { harms at } \\
\text { longer } \\
\text { follow-up) }\end{array}$ & Low & None & Medium \\
\hline
\end{tabular}

Abbreviations: 3D-CRT=3-dimensional conformal radiation therapy; ADT=androgen deprivation therapy; EBRT=external beam radiation therapy; EPIC=Expanded Prostate Cancer Index Composite; IPSS=International Prostate Symptom Score; IMRT=intensity-guided radiation therapy; LBR-PB=low dose rate prostate brachytherapy; NR=not reported; PBRT=proton beam radiation therapy

Table H-2. Summary risk of bias assessments for observational studies: external beam radiation therapy

\begin{tabular}{|c|c|c|c|c|c|c|c|c|c|}
\hline $\begin{array}{l}\text { Intervention/ } \\
\text { Comparison } \\
\text { (Outcomes) }\end{array}$ & $\begin{array}{l}\text { Author, } \\
\text { Year }\end{array}$ & $\begin{array}{l}\text { Bias Due to } \\
\text { Confounding }\end{array}$ & $\begin{array}{l}\text { Bias in } \\
\text { Selection of } \\
\text { Participants }\end{array}$ & $\begin{array}{l}\text { Bias in } \\
\text { Classification } \\
\text { of } \\
\text { Interventions }\end{array}$ & $\begin{array}{l}\text { Bias Due to } \\
\text { Deviations } \\
\text { for Intended } \\
\text { Interventions }\end{array}$ & $\begin{array}{l}\text { Bias Due } \\
\text { to Missing } \\
\text { Data }\end{array}$ & $\begin{array}{l}\text { Bias in } \\
\text { Measurement } \\
\text { of Outcomes }\end{array}$ & $\begin{array}{l}\text { Bias in } \\
\text { Selection } \\
\text { of the } \\
\text { Reported } \\
\text { Result }\end{array}$ & Overall \\
\hline $\begin{array}{l}\text { EBRT/ } \\
\text { Brachytherapy }\end{array}$ & Smith $2015^{35}$ & Serious & Serious & Moderate & $\begin{array}{l}\text { No } \\
\text { information }\end{array}$ & Low & Moderate & Low & Serious \\
\hline \multirow{2}{*}{$\begin{array}{l}\text { EBRT } \pm \\
\text { Brachytherapy }\end{array}$} & Lee $2018^{36}$ & Moderate & Critical & Moderate & Low & Moderate & Low & Low & Critical \\
\hline & $\begin{array}{l}\text { Abugharib } \\
2017^{37}\end{array}$ & Serious & Serious & Low & Low & $\begin{array}{l}\text { No } \\
\text { information }\end{array}$ & Serious & Moderate & Serious \\
\hline \multirow{2}{*}{$\begin{array}{l}\text { EBRT/ } \\
\text { Brachytherapy } \\
+ \text { EBRT }\end{array}$} & $\begin{array}{l}\text { Muralidhar } \\
2016^{38}\end{array}$ & Serious & Serious & Serious & $\begin{array}{l}\text { No } \\
\text { information }\end{array}$ & Moderate & Moderate & Moderate & Serious \\
\hline & Xiang $2015^{39}$ & Serious & Serious & Serious & $\begin{array}{l}\text { No } \\
\text { information }\end{array}$ & $\begin{array}{l}\text { No } \\
\text { information }\end{array}$ & Moderate & Moderate & Serious \\
\hline \multirow{3}{*}{$\begin{array}{l}\text { Brachytherapy/ } \\
\text { Brachytherapy } \\
+ \text { EBRT }\end{array}$} & Yang $2017^{40}$ & Serious & Moderate & Moderate & $\begin{array}{l}\text { No } \\
\text { information }\end{array}$ & Moderate & Moderate & Moderate & Serious \\
\hline & Amini $2016^{41}$ & Moderate & Moderate & Low & $\begin{array}{l}\text { No } \\
\text { information }\end{array}$ & $\begin{array}{l}\text { No } \\
\text { information }\end{array}$ & Low & Moderate & $\begin{array}{l}\text { Moderate } \\
\text { (for } \\
\text { propensity } \\
\text { score- } \\
\text { matched } \\
\text { analyses) }\end{array}$ \\
\hline & $\begin{array}{l}\text { Tward } \\
2016^{42}\end{array}$ & Serious & Serious & Moderate & $\begin{array}{l}\text { No } \\
\text { information }\end{array}$ & $\begin{array}{l}\text { No } \\
\text { information }\end{array}$ & Moderate & Moderate & Serious \\
\hline $\begin{array}{l}\text { EBRT/ } \\
\text { Brachytherapy } \\
\pm \text { EBRT }\end{array}$ & $\begin{array}{l}\text { Ashmalla } \\
2017^{43}\end{array}$ & Serious & Serious & Low & $\begin{array}{l}\text { No } \\
\text { information }\end{array}$ & $\begin{array}{l}\text { No } \\
\text { information }\end{array}$ & Moderate & Moderate & Serious \\
\hline
\end{tabular}




\begin{tabular}{|c|c|c|c|c|c|c|c|c|c|}
\hline $\begin{array}{l}\text { Intervention/ } \\
\text { Comparison } \\
\text { (Outcomes) }\end{array}$ & $\begin{array}{l}\text { Author, } \\
\text { Year }\end{array}$ & $\begin{array}{l}\text { Bias Due to } \\
\text { Confounding }\end{array}$ & $\begin{array}{l}\text { Bias in } \\
\text { Selection of } \\
\text { Participants }\end{array}$ & $\begin{array}{l}\text { Bias in } \\
\text { Classification } \\
\text { of } \\
\text { Interventions }\end{array}$ & $\begin{array}{l}\text { Bias Due to } \\
\text { Deviations } \\
\text { for Intended } \\
\text { Interventions }\end{array}$ & $\begin{array}{l}\text { Bias Due } \\
\text { to Missing } \\
\text { Data }\end{array}$ & $\begin{array}{l}\text { Bias in } \\
\text { Measurement } \\
\text { of Outcomes }\end{array}$ & $\begin{array}{l}\text { Bias in } \\
\text { Selection } \\
\text { of the } \\
\text { Reported } \\
\text { Result }\end{array}$ & Overall \\
\hline $\begin{array}{l}\text { EBRT/ } \\
\text { Brachytherapy/ } \\
\text { EBRT } \pm \\
\text { Brachytherapy }\end{array}$ & $\begin{array}{l}\text { Jackson } \\
2017^{44}\end{array}$ & Serious & Serious & Low & $\begin{array}{l}\text { No } \\
\text { information }\end{array}$ & $\begin{array}{l}\text { No } \\
\text { information }\end{array}$ & Moderate & Low & Serious \\
\hline $\begin{array}{l}\text { Brachytherapy/ } \\
\text { EBRT/ } \\
\text { Brachytherapy } \\
+ \text { EBRT }\end{array}$ & Jiang $2015^{45}$ & Moderate & Serious & Moderate & $\begin{array}{l}\text { No } \\
\text { information }\end{array}$ & $\begin{array}{l}\text { No } \\
\text { information }\end{array}$ & Moderate & Low & Serious \\
\hline $\begin{array}{l}\text { IMRT/ } \\
\text { SBRT }\end{array}$ & Ricco $2017^{46}$ & Moderate & Moderate & Low & $\begin{array}{l}\text { No } \\
\text { information }\end{array}$ & Low & Low & Moderate & Moderate \\
\hline $\begin{array}{l}\text { IMRT/ } \\
\text { SBRT/ } \\
\text { Brachytherapy }\end{array}$ & $\begin{array}{l}\text { Evans } \\
2015^{47}\end{array}$ & Critical & Serious & Moderate & $\begin{array}{l}\text { No } \\
\text { information }\end{array}$ & Moderate & Moderate & Low & Critical \\
\hline $\begin{array}{l}\text { EBRT + } \\
\text { ADT/ADT } \\
\text { (overall } \\
\text { mortality and } \\
\text { prostate } \\
\text { cancer } \\
\text { mortality) }\end{array}$ & $\begin{array}{l}\text { Bekelman, } \\
2015^{48}\end{array}$ & $\begin{array}{l}\text { Serious } \\
\text { (did not adjust } \\
\text { for PSA) }\end{array}$ & Low & Low & Low & Low & $\begin{array}{l}\text { Moderate } \\
\text { (except overall } \\
\text { mortality) }\end{array}$ & Moderate & Serious \\
\hline $\begin{array}{l}\text { EBRT + } \\
\text { adjuvant } \\
\text { ADT/EBRT + } \\
\text { neoadjuvant } \\
\text { ADT } \\
\text { (overall } \\
\text { survival and } \\
\text { distant } \\
\text { metastasis- } \\
\text { free survival) } \\
\end{array}$ & $\begin{array}{l}\text { Weller } \\
2015^{49}\end{array}$ & $\begin{array}{l}\text { Serious } \\
\text { (did not adjust } \\
\text { for age or co- } \\
\text { morbidity) }\end{array}$ & Low & Moderate & Low & Low & $\begin{array}{l}\text { Moderate } \\
\text { (except overall } \\
\text { survival) }\end{array}$ & Moderate & Serious \\
\hline $\begin{array}{l}\text { EBRT/ } \\
\text { Brachytherapy }\end{array}$ & Goy $2019^{50}$ & Moderate & Moderate & Moderate & $\begin{array}{l}\text { No } \\
\text { information }\end{array}$ & $\begin{array}{l}\text { No } \\
\text { information }\end{array}$ & Low & Moderate & Moderate \\
\hline
\end{tabular}

Abbreviations: ADT=androgen deprivation therapy; EBRT=external beam radiation therapy; IMRT=intensity-modulated radiation therapy; PSA=prostate specific antigen; SBRT=stereotactic body radiation therapy 
Table H-3. Characteristics of eligible studies: external beam radiation therapy

\begin{tabular}{|c|c|c|c|c|c|}
\hline $\begin{array}{l}\text { Study } \\
\text { Design } \\
\text { Country } \\
\text { ROB }\end{array}$ & $\mathrm{N}=$ & Population & $\begin{array}{l}\text { Intervention } \\
\text { Frequency } \\
\text { Duration }\end{array}$ & $\begin{array}{l}\text { Comparison } \\
\text { Frequency } \\
\text { Duration }\end{array}$ & $\begin{array}{l}\text { Followup Time(s) } \\
\text { Outcome } \\
\text { (Instrument) }\end{array}$ \\
\hline $\begin{array}{l}\text { Androgen } \\
\text { Suppression } \\
\text { Combined with } \\
\text { Elective Nodal and } \\
\text { Dose Escalated } \\
\text { Radiation } \\
\text { (ASCENDE-RT) } \\
\text { Trial } \\
\text { RCT } \\
\text { Morris } 2017^{23} \\
\text { Medium ROB } \\
\text { Rodda 201724 } \\
\text { Medium ROB } \\
\text { Rodda 201725 } \\
\text { High ROB } \\
\text { Canada }\end{array}$ & $\begin{array}{l}398 \\
\text { treatment } \\
\text { outcomes } \\
\text { and harms } \\
357 \text { for } \\
\text { QOL }\end{array}$ & $\begin{array}{l}\text { High or intermediate risk according to National } \\
\text { Comprehensive Cancer Network criteria; } \\
\text { Gleason sum } \geq 8 \text {; PSA > } 20 \text { ng/mL } \\
\text { T1c-T2c: } 71 \% \\
\text { T3a: } 29 \% \\
\text { Gleason sum } \\
6: 5 \% \\
\text { 7: } 54 \% \\
\text { 8-10: } 41 \% \\
\text { Age } 68 \\
\text { Race NR }\end{array}$ & $\begin{array}{l}\text { Dose escalated 3D- } \\
\text { CRT } \\
115 \text { Gy (minimal } \\
\text { peripheral dose) } \\
\text { With } 12 \text { months } \\
\text { neoadjuvant ADT } \\
\text { (depot injection and } \\
\text { oral nonsteroidal } \\
\text { antiandrogen + pelvic } \\
\text { irradiation (46 Gy in } 23 \\
\text { fractions) started } 8 \\
\text { months before RT }\end{array}$ & $\begin{array}{l}\text { 3D-CRT with low } \\
\text { dose rate prostate } \\
\text { brachytherapy (LDR- } \\
\text { PB) boost } \\
32 \text { Gy in } 16 \text { fractions } \\
\text { With } 12 \text { months } \\
\text { neoadjuvant ADT } \\
\text { (depot injection and } \\
\text { oral nonsteroidal } \\
\text { antiandrogen) + } \\
\text { pelvic irradiation (46 } \\
\text { Gy in } 23 \text { fractions) } \\
\text { started } 8 \text { months } \\
\text { before RT }\end{array}$ & $\begin{array}{l}\text { 5, } 9 \text { years } \\
\text { Mortality } \\
\text { Overall survival } \\
\text { Metastasis-free } \\
\text { survival } \\
\text { Prostate cancer- } \\
\text { specific survival } \\
\text { 2, } 5 \text { years } \\
\text { Incontinence pad } \\
\text { use } \\
\text { 1, } 5 \text { years } \\
\text { Erectile function } \\
1 \text { year } \\
\text { Health-related QOL } \\
\text { (SF36) - high ROB }\end{array}$ \\
\hline $\begin{array}{l}\text { Ricco } 2017^{46} \\
\text { Retrospective } \\
\text { database analysis } \\
\text { US } \\
\text { Medium }\end{array}$ & 5,430 & $\begin{array}{l}\text { Propensity-matched subset of the National } \\
\text { Cancer Database (2004-2013). Excluded people } \\
\text { who received other treatments, or who received } \\
\text { RT doses outside the thresholds to the right. } \\
\text { T1: } 80 \% \\
\text { T2: } 19 \% \\
\text { Gleason sum } \\
\text { 6: } 56 \% \\
\text { 7: } 38 \% \\
8: 4 \% \\
9: 1 \% \\
\text { Age } 69 \\
87 \% \text { White } \\
\text { 11\% Black }\end{array}$ & $\begin{array}{l}\text { IMRT } \\
72-86.4 \mathrm{~Gy}\end{array}$ & $\begin{array}{l}\text { SBRT } \\
35-50 \mathrm{~Gy}\end{array}$ & $\begin{array}{l}8 \text { years } \\
\text { Overall survival }\end{array}$ \\
\hline
\end{tabular}




\begin{tabular}{|c|c|c|c|c|c|}
\hline $\begin{array}{l}\text { Study } \\
\text { Design } \\
\text { Country } \\
\text { ROB }\end{array}$ & $\mathrm{N}=$ & Population & $\begin{array}{l}\text { Intervention } \\
\text { Frequency } \\
\text { Duration }\end{array}$ & $\begin{array}{l}\text { Comparison } \\
\text { Frequency } \\
\text { Duration }\end{array}$ & $\begin{array}{l}\text { Followup Time(s) } \\
\text { Outcome } \\
\text { (Instrument) }\end{array}$ \\
\hline $\begin{array}{l}\text { Amini } 2016^{41} \\
\text { Retrospective } \\
\text { database analysis } \\
\text { US } \\
\text { Medium (for } \\
\text { propensity score- } \\
\text { matched analyses) }\end{array}$ & 5,858 & $\begin{array}{l}\text { Propensity-matched subset of the National } \\
\text { Cancer Database (2004-2006). Excluded people } \\
\text { with high-risk features (>T3, Gleason 8-10, etc) } \\
\text { and people who received other surgical or } \\
\text { chemotherapy treatments. Baseline } \\
\text { characteristics NR separately for propensity- } \\
\text { matched subset. } \\
\text { T1: } 61 \% \\
\text { T2: } 39 \% \\
\text { Gleason sum } \\
\text { 6: } 27 \% \\
7: 73 \% \\
\text { Age } 69 \\
83 \% \text { White } \\
\text { 13\% Black }\end{array}$ & $\begin{array}{l}\text { Brachytherapy + EBRT } \\
\text { EBRT doses 40-50.4 } \\
\text { Gy in 1.8-2.0 Gy } \\
\text { fractionations }\end{array}$ & $\begin{array}{l}\text { Brachytherapy } \\
\text { Dose NR }\end{array}$ & $\begin{array}{l}5,7 \text { years } \\
\text { Overall survival }\end{array}$ \\
\hline $\begin{array}{l}\text { Viani } 2016^{26} \\
\text { RCT } \\
\text { Brazil } \\
\text { Medium }\end{array}$ & 215 & $\begin{array}{l}\text { Treatment-naïve men with clinically localized } \\
\text { prostate cancer; PSA>150 ng/mL } \\
\text { Ta1-T2a: } 79 \% \\
\text { T2b: } 7 \% \\
\text { T2c-T3b: } 14 \% \\
\text { Age } 72 \\
\text { Race NR }\end{array}$ & $\begin{array}{l}\text { 3D-CRT } \\
70 \text { Gy in } 25 \text { fractions } \\
\text { (single daily dose } 2.8 \\
\text { Gy) }\end{array}$ & $\begin{array}{l}\text { IMRT } \\
70 \text { Gy in } 25 \text { fractions } \\
\text { (single daily dose } \\
2.8 \text { Gy) }\end{array}$ & $\begin{array}{l}\text { 1,3 years } \\
\text { Prostate-Specific } \\
\text { Quality of Life } \\
\text { (European } \\
\text { Organization for } \\
\text { Research and } \\
\text { Treatment of Cancer } \\
\text { Quality of Life } \\
\text { Questionnaire } \\
\text { [EORTC } \\
\text { QLQPR25]) }\end{array}$ \\
\hline
\end{tabular}




\begin{tabular}{|c|c|c|c|c|c|}
\hline $\begin{array}{l}\text { Study } \\
\text { Design } \\
\text { Country } \\
\text { ROB }\end{array}$ & $\mathrm{N}=$ & Population & $\begin{array}{l}\text { Intervention } \\
\text { Frequency } \\
\text { Duration }\end{array}$ & $\begin{array}{l}\text { Comparison } \\
\text { Frequency } \\
\text { Duration }\end{array}$ & $\begin{array}{l}\text { Followup Time(s) } \\
\text { Outcome } \\
\text { (Instrument) }\end{array}$ \\
\hline $\begin{array}{l}\text { Bolla } 2016^{27} \\
\text { RCT } \\
\text { Multi-national (13 } \\
\text { countries in Europe } \\
\text { and Israel) } \\
\text { Medium }\end{array}$ & $\begin{array}{l}819 \\
\text { randomized }\end{array}$ & $\begin{array}{l}\text { Histologically confirmed prostate } \\
\text { adenocarcinoma T1b to T2a (International Union } \\
\text { Against Cancer 1997 staging criteria with PSA > } \\
10 \mathrm{ng} / \mathrm{mL} \text { or Gleason } \geq 7 \text {; no involvement of } \\
\text { pelvic lymph nodes as assessed by computed } \\
\text { tomography scan, magnetic resonance imaging, } \\
\text { or laparoscopic surgery; no clinical evidence of } \\
\text { metastatic spread; or clinical tumor stages T2b to } \\
\text { T4 and a PSA level of up to } 12.5 \text { times the upper } \\
\text { limit of the normal range; a WHO performance } \\
\text { status } \leq 2 ; \text { no previous pelvic irradiation or radical } \\
\text { prostatectomy; no previous hormonal therapy; no } \\
\text { other malignancy except adequately treated } \\
\text { basal cell carcinoma of the skin or another } \\
\text { malignancy cured for at least } 5 \text { years. } \\
\text { T1a (ineligible): } 0.1 \% \\
\text { T1b: } 3.3 \% \\
\text { T1c: } 44.8 \% \\
\text { T2a: } 50.9 \% \\
\text { T2b (ineligible): } 0.9 \% \\
\text { Gleason (sum) <6: } 11.2 \% \\
\text { Gleason (sum) 6: } 37.9 \% \\
\text { Gleason (sum) } 7: 40.9 \% \\
\text { Gleason (sum) 8-10: } 10.0 \% \\
\text { Age (median): } 70 \text { years } \\
\text { Race: NR }\end{array}$ & $\begin{array}{l}\text { EBRT (predominantly } \\
\text { 3D-CRT) plus ADT } \\
\text { 3D-CRT or IMRT was } \\
\text { performed. The } \\
\text { radiation dose was a } \\
\text { center-chosen } \\
\text { characteristic. RT was } \\
\text { delivered once per } \\
\text { day, five fractions per } \\
\text { day of } 2 \text { Gy per week } \\
\text { at a dose of } 46 \text { Gy for } \\
\text { PTV I; } 24 \text { Gy for PTV } \\
\text { II; and } 0,4, \text { or } 8 \text { Gy for } \\
\text { PTV III, depending on } \\
\text { center policy, resulting } \\
\text { in total doses of } 70, \\
74, \text { or } 78 \text { Gy, } \\
\text { respectively. Median } \\
\text { RT duration ranged } \\
\text { from } 51-57 \text { days. } \\
\text { ADT consisted of two } \\
\text { subcutaneous } \\
\text { injections of every-3 } \\
\text { months depot of LHRH } \\
\text { analog (goserelin) } \\
\text { given the first day of } \\
\text { RT, then } 3 \text { months } \\
\text { later. Flare protection } \\
\text { consisted of } 1 \text { month } \\
\text { of antiandrogen } \\
\text { (bicalutamide; } 50 \\
\text { mg/d) started } 1 \text { week } \\
\text { before the first LHRH } \\
\text { injection. }\end{array}$ & $\begin{array}{l}\text { EBRT } \\
\text { (predominantly 3D- } \\
\text { CRT) }\end{array}$ & $\begin{array}{l}\text { 10 years: } \\
\text { Distant metastasis } \\
\text { Overall survival } \\
7.2 \text { years (median } \\
\text { follow-up): } \\
\text { Prostate cancer } \\
\text { mortality } \\
\text { Distant metastases } \\
\text { Overall survival } \\
\text { From } 6 \text { months to } \\
\text { end of follow-up (7.2 } \\
\text { median): } \\
\text { Late toxicity } \\
5 \text { years: } \\
\text { Distant metastases } \\
\text { Overall survival } \\
3 \text { years: } \\
\text { Quality of life scales } \\
\text { (EORTC QLQ) } \\
1 \text { year: } \\
\text { Quality of life scales } \\
\text { (EORTC QLQ) }\end{array}$ \\
\hline
\end{tabular}




\begin{tabular}{|c|c|c|c|c|c|}
\hline $\begin{array}{l}\text { Study } \\
\text { Design } \\
\text { Country } \\
\text { ROB }\end{array}$ & $\mathrm{N}=$ & Population & $\begin{array}{l}\text { Intervention } \\
\text { Frequency } \\
\text { Duration }\end{array}$ & $\begin{array}{l}\text { Comparison } \\
\text { Frequency } \\
\text { Duration }\end{array}$ & $\begin{array}{l}\text { Followup Time(s) } \\
\text { Outcome } \\
\text { (Instrument) }\end{array}$ \\
\hline $\begin{array}{l}\text { McPartlin } 2006^{28} \\
\text { RCT } \\
\text { Canada } \\
\text { Medium }\end{array}$ & $\begin{array}{l}252 \\
\text { randomized }\end{array}$ & $\begin{array}{l}\text { Prostate carcinoma with T1b through T2 tumors, } \\
\text { Gleason scores from } 6 \text { to } 8 \text {, and prostate-specific } \\
\text { antigen (PSA) levels } \leq 20 \mathrm{ng} / \mathrm{mL} \text {. Patients who } \\
\text { had clinical T1b/T2a tumors and a Gleason score } \\
\text { of } 6 \text { were required to have PSA levels from } 10 \text { to } \\
20 \mathrm{ng} / \mathrm{mL} \text {. All patients who had PSA levels }>10 \\
\text { ng/mL had a negative bone scan within } 12 \\
\text { months of study entry. No previous hormone or } \\
\text { cytotoxic therapy was permitted before study } \\
\text { entry. All patients had an Eastern Cooperative } \\
\text { Oncology Group performance status } \leq 2 \text {, were } \\
\text { aged } \leq 80 \text { years, and had no contraindication to } \\
\text { DE-EBRT. } \\
\text { T1b-T2a: } 77.59 \% \\
\text { T2b-T2c: } 22.41 \% \\
\text { Gleason 3+3: } 12.45 \% \\
\text { Gleason 3+4: } 57.26 \% \\
\text { Gleason 4+3: } 24.90 \% \\
\text { Gleason 3+5: } 1.24 \% \\
\text { Gleason 4+4: } 3.73 \% \\
\text { Gleason 5+3: } 0.41 \% \\
\text { Age (median): } 71.4 \text { and } 70.9 \text { years in the two } \\
\text { treatment groups } \\
\text { Race: NR }\end{array}$ & $\begin{array}{l}\text { EBRT (IMRT) plus } \\
\text { bicalutamide } \\
\text { Patients received RT } \\
\text { using } 6 \text {-coplanar, } \\
\text { equally weighted } 18 \\
\text { MV beams or IMRT, } \\
\text { with daily imaging } \\
\text { using an electronic } \\
\text { portal imaging device } \\
\text { and setup verification } \\
\text { using fiducial markers. } \\
\text { From } 1999 \text { to } 2001 \text {, } \\
\text { patients received } 75.6 \\
\text { Gy in } 42 \text { fractions over } \\
8.5 \text { weeks. } \\
\text { Subsequently, the } \\
\text { dose was increased up } \\
\text { to } 79.8 \text { Gy in } 42 \\
\text { fractions and then to } \\
78 \text { Gy in } 39 \text { fractions } \\
\text { as experience with DE- } \\
\text { EBRT increased. } \\
\text { Bicalutamide was } \\
\text { given at } 150 \text { mg, } 5 \\
\text { months of neoadjuvant } \\
\text { and adjuvant starting } 3 \\
\text { months before RT. }\end{array}$ & EBRT (IMRT) & $\begin{array}{l}\text { 10 years: } \\
\text { Overall survival } \\
9.1 \text { years (median } \\
\text { follow-up): } \\
\text { Overall survival } \\
\text { Distant metastases } \\
5 \text { years: } \\
\text { Overall survival } \\
\text { >4 years: } \\
\text { Sexual function } \\
\text { (IIEF) } \\
\text { Quality of life } \\
\text { (EORTC-30) } \\
1 \text { year: } \\
\text { Sexual function } \\
\text { (IIEF) } \\
\text { Quality of life } \\
\text { (EORTC-30) } \\
\text { Timing of outcome } \\
\text { not clearly reported: } \\
\text { Late toxicity }\end{array}$ \\
\hline
\end{tabular}




\begin{tabular}{|c|c|c|c|c|c|}
\hline $\begin{array}{l}\text { Study } \\
\text { Design } \\
\text { Country } \\
\text { ROB }\end{array}$ & $\mathrm{N}=$ & Population & $\begin{array}{l}\text { Intervention } \\
\text { Frequency } \\
\text { Duration }\end{array}$ & $\begin{array}{l}\text { Comparison } \\
\text { Frequency } \\
\text { Duration }\end{array}$ & $\begin{array}{l}\text { Followup Time(s) } \\
\text { Outcome } \\
\text { (Instrument) }\end{array}$ \\
\hline $\begin{array}{l}\text { Phillips, 2014 } \\
\text { McDuff, 2018 } \\
\text { Giacalone, 2016; }{ }^{32} \\
\text { (all secondary } \\
\text { references to } \\
\text { D'Amico 200851) } \\
\text { RCT } \\
\text { US } \\
\text { Medium }\end{array}$ & $\begin{array}{l}206 \\
\text { randomized }\end{array}$ & $\begin{array}{l}\text { T1B to T2b, NX, MO adenocarcinoma of the } \\
\text { prostate. Patients with PSA of at least } 10 \mathrm{ng} / \mathrm{mL} \\
\text { (maximum } 40 \text { ng/ML) or a Gleason score of at } \\
\text { least } 7 \text { (range, 5-10). Low-risk patients were } \\
\text { ineligible unless they had radiographic evidence } \\
\text { using endorectal coil MRI of extracapsular } \\
\text { extension or seminal vesicle invasion. Patients } \\
\text { were also considered ineligible if they had a prior } \\
\text { history of malignancy except for nonmelanoma } \\
\text { skin cancer or any history of hormone therapy } \\
\text { use. All patients were required to have a } \\
\text { negative bone scan and pelvic lymph node } \\
\text { assessment using MRI or CT within } 6 \text { months of } \\
\text { randomization. Eligible patients also needed to } \\
\text { have an Eastern Cooperative Oncology Group } \\
\text { performance status of } 0 \text { or } 1 \text { (range, } 0-4 \text { ), white } \\
\text { blood cell count of at least } 3000 / \mu L, \text { hematocrit } \\
\text { of more than } 30 \%, \text { platelet count of more than } \\
\text { 100x103/ } \mu \text { L, and a life expectancy of least } 10 \\
\text { years, excluding death related to prostate cancer } \\
\text { at study entry. } \\
\text { T1b: } 1.94 \% \\
\text { T1c: } 46.12 \% \\
\text { T2a: } 22.33 \% \\
\text { T2b: } 29.61 \% \\
\text { Gleason } 5 \text { or } 6: 27.67 \% \\
\text { Gleason } 3+4: 34.95 \% \\
\text { Gleason } 4+3: 22.82 \% \\
\text { Gleason } 8-10: 14.56 \% \\
\text { Age (median): } 73 \text { and } 72 \text { years in the two } \\
\text { treatment groups } \\
\text { Race: NR }\end{array}$ & $\begin{array}{l}\text { EBRT (3D-CRT) plus } \\
\text { ADT } \\
\text { EBRT consisted of 3D- } \\
\text { CRT. Daily dose of } 1.8 \\
\text { Gy for initial } 25 \\
\text { treatments, totaling } 45 \\
\text { Gy, and } 2.0 \text { Gy for final } \\
11 \text { treatments, totaling } \\
22 \text { Gy. } \\
\text { ADT included a LHRH } \\
\text { agonist and the } \\
\text { antiandrogen } \\
\text { flutamide. }\end{array}$ & EBRT (3D-CRT) & $\begin{array}{l}\text { 16.62 years } \\
\text { Overall mortality } \\
\text { Prostate cancer } \\
\text { mortality } \\
14.26 \text { years } \\
\text { Prostate cancer } \\
\text { mortality }\end{array}$ \\
\hline
\end{tabular}




\begin{tabular}{|c|c|c|c|c|c|}
\hline $\begin{array}{l}\text { Study } \\
\text { Design } \\
\text { Country } \\
\text { ROB }\end{array}$ & $\mathrm{N}=$ & Population & $\begin{array}{l}\text { Intervention } \\
\text { Frequency } \\
\text { Duration }\end{array}$ & $\begin{array}{l}\text { Comparison } \\
\text { Frequency } \\
\text { Duration }\end{array}$ & $\begin{array}{l}\text { Followup Time(s) } \\
\text { Outcome } \\
\text { (Instrument) }\end{array}$ \\
\hline $\begin{array}{l}\text { Malone, } 2019^{33} \\
\text { RCT } \\
\text { Canada } \\
\text { Medium }\end{array}$ & $\begin{array}{l}432 \\
\text { randomized }\end{array}$ & $\begin{array}{l}\text { Men age }>18 \text { years with Eastern Cooperative } \\
\text { Oncology Group performance status }<2 \text { and } \\
\text { histologically confirmed diagnosis of } \\
\text { adenocarcinoma of the prostate, with Gleason } \\
\text { score } \leq 7 \text {, clinical tumor stage of T1b to T3a, and } \\
\text { serum PSA }<30 \mathrm{ng} / \mathrm{mL} \leq 4 \text { weeks before } \\
\text { enrollment. Patients with baseline PSA } \geq 10 \\
\text { ng/mL underwent a whole-body bone scan } \leq 12 \\
\text { weeks before study entry, whereas those with } \\
\text { PSA } \geq 20 \text { ng/mL underwent a contrast-enhanced } \\
\text { CT scan of the abdomen and pelvis performed } \leq \\
12 \text { weeks before study entry. Patients with low- } \\
\text { risk PCa (Gleason score } \leq 6, \text { T1-T2a, and PSA } \leq \\
10 \text { ng/mL) or radiologic evidence of nodal or } \\
\text { distant metastasis were excluded. Also excluded } \\
\text { were patients with active or prior malignancies, } \\
\text { except for nonmelanoma skin carcinoma within } 5 \\
\text { years of the diagnosis of PCa; those with } \\
\text { contraindications to RT, including inflammatory } \\
\text { bowel disease; and those who had received prior } \\
\text { pelvic RT, cytotoxic chemotherapy, or ADT. } \\
\text { T1b-T1c: } 46.3 \% \\
\text { T2a: } 23.8 \% \\
\text { T2b-T2c: } 28.7 \% \\
\text { T3: } 1.2 \% \\
\text { Gleason }<7: 22.9 \% \\
\text { Gleason } 7: 77.1 \% \\
\text { Age: } 69.1 \text { years } \\
\text { Race: NR }\end{array}$ & $\begin{array}{l}\text { EBRT (3D-CRT) plus } \\
\text { neoadjuvant and } \\
\text { concurrent ADT } \\
\text { A total RT dose of } 76 \\
\text { Gy in } 38 \text { fractions over } \\
7.5 \text { weeks using 3D- } \\
\text { CRT and over } 2 \\
\text { phases. In the first } \\
\text { phase, } 56 \text { Gy was } \\
\text { delivered to the } \\
\text { prostate and proximal } \\
10 \text { mm of seminal } \\
\text { vesicles in } 28 \text { fractions } \\
\text { over } 5.5 \text { weeks. An } \\
\text { additional boost of } 20 \\
\text { Gy in } 10 \text { fractions was } \\
\text { subsequently delivered } \\
\text { to the prostate alone } \\
\text { over } 2 \text { weeks. } \\
6 \text { months ADT starting } \\
4 \text { months before RT. } \\
\text { ADT comprised of an } \\
\text { oral antiandrogen } \\
\text { (bicalutamide } 50 \text { mg } \\
\text { once daily) plus } \\
\text { goserelin (10.8 mg } \\
\text { subcutaneously } \\
\text { starting } 7 \text { days after } \\
\text { bicalutamide with a } \\
\text { second injection } \\
\text { administered } 3 \text { months } \\
\text { thereafter). }\end{array}$ & $\begin{array}{l}\text { EBRT (3D-CRT) } \\
\text { plus concurrent and } \\
\text { adjuvant ADT } \\
6 \text { months ADT } \\
\text { starting } \\
\text { simultaneously with } \\
\text { RT. }\end{array}$ & $\begin{array}{l}\text { 12.17 years } \\
\text { Overall mortality } \\
\text { Prostate cancer } \\
\text { mortality } \\
\text { Distant progression } \\
10 \text { years } \\
\text { Overall survival } \\
\text { Prostate cancer } \\
\text { mortality } \\
\text { Metastatic-free } \\
\text { survival } \\
3 \text { years } \\
\text { Late toxicity }\end{array}$ \\
\hline
\end{tabular}




\begin{tabular}{|c|c|c|c|c|c|}
\hline $\begin{array}{l}\text { Study } \\
\text { Design } \\
\text { Country } \\
\text { ROB }\end{array}$ & $\mathrm{N}=$ & Population & $\begin{array}{l}\text { Intervention } \\
\text { Frequency } \\
\text { Duration }\end{array}$ & $\begin{array}{l}\text { Comparison } \\
\text { Frequency } \\
\text { Duration }\end{array}$ & $\begin{array}{l}\text { Followup Time(s) } \\
\text { Outcome } \\
\text { (Instrument) }\end{array}$ \\
\hline $\begin{array}{l}\text { Goy } 2019^{50} \\
\text { Retrospective } \\
\text { database analysis } \\
\text { US } \\
\text { Medium (for } \\
\text { propensity score- } \\
\text { matched analyses }\end{array}$ & 684 & $\begin{array}{l}\text { Men with intermediate-risk prostate cancer, } \\
\text { classified as clinical stage T2b-c, GS } 3+4 \\
\text { (group 2) or } 4+3 \text { (group 3), and/or } \\
\text { iPSA of } 10.1-20.0 \text {. The men were clinically } \\
\text { staged, with a digital rectal } \\
\text { examination for T-stage from the } 2002 \text { American } \\
\text { Joint Committee Cancer staging. } \\
\text { T1a-b: }<1 \% \\
\text { T1c: } 69 \% \\
\text { T2a: } 20 \% \\
\text { T2b: } 11 \% \\
\text { Gleason } 6(3+3): 30 \% \\
\text { Gleason } 7(3+4): 48 \% \\
\text { Gleason } 7(4+3): 22 \% \\
\text { Age (median): EBRT } 70.8 \text { years, brachytherapy } \\
65.3 \text { years } \\
49 \% \text { White } \\
25 \% \text { Black } \\
17 \% \text { Hispanic }\end{array}$ & $\begin{array}{l}\text { EBRT (3D-CRT) (+/- } \\
\text { neoadjuvant ADT) } \\
\text { Median dose to the } \\
\text { isocenter was } 75.3 \\
\text { Gray (range } 73.5-77.1) \\
\text { over } 81 / 2 \text { weeks, with } \\
94 \% \text { receiving } 75.3 \\
\text { Gray. } \\
\text { Neoadjuvant androgen } \\
\text { deprivation } \\
\text { therapy was given } \\
\text { using Leuprolide for a } \\
\text { median } 6 \text { months for } \\
59 \% \text { of the EBRT } \\
\text { patients. }\end{array}$ & $\begin{array}{l}\text { Brachytherapy (+/- } \\
\text { neoadjuvant ADT) } \\
\text { Administered as } \\
\text { lodine-125 } \\
\text { radioactive seeds } \\
\text { A minimum } \\
\text { peripheral dose of } \\
145 \text { Gray was } \\
\text { prescribed } \\
\text { Neoadjuvant } \\
\text { androgen } \\
\text { deprivation } \\
\text { therapy was given } \\
\text { using Leuprolide for } \\
\text { a median } 4 \text { months } \\
\text { in } 13 \% \text { of the } \\
\text { patients }\end{array}$ & $\begin{array}{l}10 \text { years (medians } \\
\text { of } 9.6 \text { and } 9.8 \text { years } \\
\text { for EBRT and } \\
\text { brachytherapy, } \\
\text { respectively) } \\
\text { Overall survival } \\
\text { Prostate cancer } \\
\text { mortality } \\
\text { Metastatic-free } \\
\text { survival } \\
5 \text { years: } \\
\text { Overall survival } \\
\text { Prostate cancer } \\
\text { mortality } \\
\text { Metastatic-free } \\
\text { survival }\end{array}$ \\
\hline
\end{tabular}




\begin{tabular}{|c|c|c|c|c|c|}
\hline $\begin{array}{l}\text { Study } \\
\text { Design } \\
\text { Country } \\
\text { ROB }\end{array}$ & $\mathbf{N}=$ & Population & $\begin{array}{l}\text { Intervention } \\
\text { Frequency } \\
\text { Duration }\end{array}$ & $\begin{array}{l}\text { Comparison } \\
\text { Frequency } \\
\text { Duration }\end{array}$ & $\begin{array}{l}\text { Followup Time(s) } \\
\text { Outcome } \\
\text { (Instrument) }\end{array}$ \\
\hline $\begin{array}{l}\text { Widmark, } 2019^{34} \\
\text { RCT } \\
\text { Sweden and } \\
\text { Denmark } \\
\text { Medium ROB }\end{array}$ & $\begin{array}{l}1200 \\
\text { randomized }\end{array}$ & $\begin{array}{l}\text { Participants were men up to } 75 \text { years of age with } \\
\text { histologically verified intermediate-to-high risk } \\
\text { prostate cancer and WHO performance status } \\
\text { between } 0 \text { and } 2 \text {. Intermediate-to-high-risk } \\
\text { prostate cancer was categorized according to } \\
\text { TNM classification system as T1c-T3a with no } \\
\text { evidence of lymph node involvement or distant } \\
\text { metastases with one or two of the following risk } \\
\text { factors: stage T3a, Gleason score of at least } 7 \text {, } \\
\text { or PSA of at least } 10 \text { ng/mL. The maximum PSA } \\
\text { allowed was } 20 \text { ng/mL and no ADT was } \\
\text { permitted. } \\
\text { T1c: } 51.0 \% \\
\text { T2: } 44.7 \% \\
\text { T3a: } 4.3 \% \\
\text { Gleason } 5: 0.6 \% \\
\text { Gleason } 6: 17.4 \% \\
\text { Gleason } 7: 75.5 \% \\
\text { Gleason } 8: 5.9 \% \\
\text { Gleason } 9: 0.6 \% \\
\text { Age: median } 69 \text { and } 68 \text { years in the two } \\
\text { treatment groups, respectively } \\
\text { Race: NR }\end{array}$ & $\begin{array}{l}\text { EBRT conventional } \\
\text { fractionation } \\
\text { Radiotherapy was } \\
\text { delivered with image- } \\
\text { guided 3D-CRT, IMRT, } \\
\text { or VMAT with use of } \\
\text { fiducial markers. 80\% } \\
\text { of patients received } \\
\text { 3D-CRT and 20\% } \\
\text { VMAT/IMRT. } \\
\text { No ADT was } \\
\text { permitted. } \\
\text { Patients in the } \\
\text { conventional } \\
\text { fractionation group } \\
\text { received } 78.0 \text { Gy in } 39 \\
\text { fractions } 5 \text { days per } \\
\text { week for } 8 \text { weeks. }\end{array}$ & $\begin{array}{l}\text { EBRT ultra- } \\
\text { hypofractionation } \\
\text { Patients in the ultra- } \\
\text { hypofractionation } \\
\text { arm received } 42.7 \\
\text { Gy in } 7 \text { fractions } 3 \\
\text { days per week for } \\
2.5 \text { weeks inclusive } \\
\text { of two weekends. }\end{array}$ & $\begin{array}{l}5 \text { years } \\
\text { Overall } \\
\text { mortality/survival } \\
\text { Prostate cancer } \\
\text { mortality/survival } \\
\text { Distant progression } \\
2 \text { years } \\
\text { Bowel, urinary, and } \\
\text { erectile harms } \\
1 \text { year } \\
\text { Bowel, urinary, and } \\
\text { erectile harms } \\
\text { *harms also } \\
\text { reported at longer } \\
\text { follow-up but not } \\
\text { extracted due to the } \\
\text { extent of missing } \\
\text { data }\end{array}$ \\
\hline
\end{tabular}

Abbreviations: 3D-CRT=3 dimensional conformal radiation therapy; ADT=androgen deprivation therapy; AEs=adverse effects; ASCENDE-RT=Androgen Suppression

Combined with Elective Nodal and Dose Escalated Radiation; CT=Computed tomography; DE-EBRT=dose escalated external beam radiation therapy; EBRT=external beam radiation therapy; EPIC=Expanded Prostate Cancer Index Composite; EORTC QLQPR25=European Organization for Research and Treatment of Cancer Quality of Life Questionnaire; Gy=Gray units; IIEF=International index of erectile function; IMRT=intensity modulated radiation therapy; IPSS=International Prostate Symptom Score; LDRPB=low dose rate prostate brachytherapy; LENT-SOMA= Late Effects of Normal Tissue - Somatic, Objective, Management, Analytic scale; LHRH=Luteinizing hormonereleasing hormone; MRI=Magnetic resonance imaging; ml=milliliters; ng=nanograms; NR=not reported; PBRT=proton beam radiation therapy; PCa=prostate cancer; PSA=prostate-specific antigen; QOL=quality of life; RCT=randomized controlled trial; ROB=risk of bias; RT=radiation therapy; SEER=Surveillance, Epidemiology, and End Results; SF36=short form 36 item health survey questionnaire; T=clinical T stage; US=United States; VMAT=volumetric modulated arc therapy; WHO=World Health Organization 
Table H-4. Mortality, survival, and metastases outcomes: external beam radiation therapy

\begin{tabular}{|c|c|c|c|c|}
\hline $\begin{array}{l}\text { Intervention/ } \\
\text { Comparison }\end{array}$ & $\begin{array}{l}\text { Study } \\
\text { Design } \\
\text { (Trial/Registry) } \\
\text { Followup } \\
\text { Risk of Bias }\end{array}$ & Overall Survival and Mortality & $\begin{array}{l}\text { Prostate Cancer Specific Survival and } \\
\text { Mortality }\end{array}$ & $\begin{array}{l}\text { Metastatic Progression } \\
\text { Free Survival } \\
\text { Metastases }\end{array}$ \\
\hline $\begin{array}{l}\text { 3D-CRT + } \\
\text { ADT/3D-CRT + } \\
\text { ADT + LDR-PB } \\
\text { boost }\end{array}$ & $\begin{array}{l}\text { ASCENDE-RT }{ }^{23} \text {, } \\
24 \\
\text { RCT } \\
\text { Median } 6.5 \text { years } \\
\text { Medium }\end{array}$ & $\begin{array}{l}\text { Mortality (ITT), \% (n/N) } \\
\text { 3D-CRT + ADT: 19\% (38/200) } \\
\text { LDR-PB: 15\% (30/198) } \\
\text { RR*=1.25 (95\% Cl 0.81 to 1.94) } p=0.31 \\
\text { Overall survival, KM Estimate }(95 \% \mathrm{Cl}) \\
5 \text { years } \\
\text { 3D-CRT +ADT: } 88.7(4.8) \\
\text { LDR-PB: } 91.3(4.4) \\
9 \text { years } \\
\text { 3D-CRT +ADT: } 73.6(8.4) \\
\text { LDR-PB: } 77.9(8.2)\end{array}$ & $\begin{array}{l}\text { Prostate cancer-specific mortality (ITT), \% } \\
\text { (n/N) } \\
\text { 3D-CRT + ADT: } 6 \%(11 / 200) \\
\text { LDR-PB: } 4 \%(7 / 198) \\
\text { RR*=1.56 (95\% Cl } 0.62 \text { to 3.93) } p=0.35 \\
\text { Prostate cancer-specific survival, KM } \\
\text { Estimate ( } \pm 95 \% \text { Cl) } \\
5 \text { years } \\
\text { 3D-CRT + ADT: } 97.5(2.4) \\
\text { LDR-PB: } 96.8(2.8) \\
\text { 9 years } \\
\text { 3D-CRT + ADT: } 92.1(5.6) \\
\text { LDR-PB: } 94.8(4.0)\end{array}$ & $\begin{array}{l}\text { Metastasis-free } \\
\text { survival, KM Estimate } \\
\text { ( } \pm 95 \% \text { CI) } \\
5 \text { years } \\
\text { 3D-CRT + ADT: } 92.5 \\
(4.0) \\
\text { LDR-PB: } 93.3(3.8) \\
9 \text { years } \\
\text { 3D-CRT + ADT: } 84.8 \\
\text { (7.6) } \\
\text { LDR-PB: } 88.6(5.6) \\
\text { Metastatic disease } \\
5 \text { years } \\
\text { 3D-CRT + ADT: } 9 \% \\
(18 / 200) \\
\text { LDR-PB: } 9 \%(17 / 198) \\
\text { RR*=1.05 (95\% CI } 0.56 \\
\text { to } 1.97) \text { p=0.88 }\end{array}$ \\
\hline 3D-CRT/ IMRT & $\begin{array}{l}\text { Viani } 2016^{26} \\
\text { RCT } \\
3 \text { years } \\
\text { Medium }\end{array}$ & NR & NR & NR \\
\hline $\begin{array}{l}\text { Brachytherapy + } \\
\text { EBRT/ } \\
\text { Brachytherapy }\end{array}$ & $\begin{array}{l}\text { Amini } 2016^{41} \\
\text { Observational } \\
\text { (National Cancer } \\
\text { Database) } \\
7 \text { years } \\
\text { Medium } \\
\end{array}$ & $\begin{array}{l}\text { Overall survival, KM Estimate } \\
\text { Brachytherapy + EBRT: } 85.8 \% \\
\text { Brachytherapy: } 83.1 \% \\
\text { HR=0.85 (95\% Cl } 0.75 \text { to } 0.97) \\
p=0.006\end{array}$ & NR & NR \\
\hline IMRT/SBRT & $\begin{array}{l}\text { Ricco } 2017^{46} \\
\text { Observational } \\
\text { (National Cancer } \\
\text { Database) } \\
8 \text { years } \\
\text { Medium } \\
\end{array}$ & $\begin{array}{l}\text { Overall survival, KM Estimate } \\
\text { IMRT: } 77.2 \% \\
\text { SBRT: } 79.4 \% \\
\text { Log-rank } p \text {-value } \\
\text { p=0.65 }\end{array}$ & NR & NR \\
\hline
\end{tabular}




\begin{tabular}{|c|c|c|c|c|}
\hline $\begin{array}{l}\text { Interventionl } \\
\text { Comparison }\end{array}$ & $\begin{array}{l}\text { Study } \\
\text { Design } \\
\text { (Trial/Registry) } \\
\text { Followup } \\
\text { Risk of Bias }\end{array}$ & Overall Survival and Mortality & $\begin{array}{l}\text { Prostate Cancer Specific Survival and } \\
\text { Mortality }\end{array}$ & $\begin{array}{l}\text { Metastatic Progression } \\
\text { Free Survival } \\
\text { Metastases }\end{array}$ \\
\hline \multirow[t]{2}{*}{$\begin{array}{l}\text { EBRT + } \\
\text { ADT/EBRT }\end{array}$} & $\begin{array}{l}\text { Bolla 201627 } \\
\text { RCT (EORTC } \\
\text { Trial 22991) } \\
\text { Median follow-up } \\
7.2 \text { years } \\
\text { Medium }\end{array}$ & $\begin{array}{l}\text { Overall survival } \\
5 \text { years: } \\
\text { EBRT + ADT: } 91.3 \% \text { (95\% Cl } 88.0 \text { to } \\
93.7) \\
\text { EBRT: } 88.4 \%(95 \% \mathrm{Cl} 84.7 \text { to } 91.3) \\
10 \text { years: } \\
\text { EBRT + ADT: } \sim 72 \% \text { (estimated from } \\
\text { graph) } \\
\text { EBRT: } \sim 67 \% \text { (estimated from graph) } \\
\text { Overall mortality } \\
7.2 \text { years: } \\
\text { EBRT + ADT: } 69 / 410(16.8 \%) \\
\text { EBRT: } 83 / 409(20.3 \%)\end{array}$ & $\begin{array}{l}\text { Prostate cancer mortality } \\
7.2 \text { years: } \\
\text { EBRT + ADT: } 9 / 410(2.2 \%) \\
\text { EBRT: } 16 / 409(3.9 \%)\end{array}$ & $\begin{array}{l}\text { Distant metastasis } \\
5 \text { years: } \\
\text { EBRT + ADT: } ~ 3 \% \\
\text { (estimated from graph) } \\
\text { EBRT: } \sim 7 \% \text { (estimated } \\
\text { from graph) } \\
7.2 \text { years: } \\
\text { EBRT + ADT: } 18 / 410 \\
(4.4 \%) \\
\text { EBRT: } 31 / 409(7.6 \%) \\
10 \text { years: } \\
\text { EBRT + ADT: } ~ 7 \% \\
\text { (estimated from graph) } \\
\text { EBRT: } \sim 10 \% \text { (estimated } \\
\text { from graph) }\end{array}$ \\
\hline & $\begin{array}{l}\text { McPartlin 2016 } 28 \\
\text { RCT (PMH 9907) } \\
\text { Median follow-up } \\
9.1 \text { years } \\
\text { Medium }\end{array}$ & $\begin{array}{l}\text { Overall survival } \\
5 \text { years: } \\
\text { EBRT + ADT: } 92 \% \text { (estimated from } \\
\text { graph) } \\
\text { EBRT: } ~ 96 \% \text { (estimated from graph) } \\
9.1 \text { years: } \\
\text { EBRT + ADT: } 82 \%(95 \% \mathrm{Cl} 75 \%-90 \%) \\
\text { EBRT: } 86 \%(95 \% \mathrm{Cl} 80 \%-94 \%) \\
\text { p=0.37 } \\
\text { HR [EBRT vs. EBRT + ADT] =1.33 (95\% } \\
\text { Cl 0.72-2.47) } \\
10 \text { years: } \\
\text { EBRT + ADT: } ~ 78 \% \text { (estimated from } \\
\text { graph) } \\
\text { EBRT: } ~ 85 \% \text { (estimated from graph) } \\
\text { Overall mortality } \\
9.1 \text { years: } \\
\text { EBRT + ADT: } 23 / 119(19.3 \%) \\
\text { EBRT: } 18 / 122(14.8 \%)\end{array}$ & $\mathrm{NR}$ & $\begin{array}{l}\text { Distant metastasis } \\
9.1 \text { years: } \\
\text { Among patients who had } \\
\text { biochemical relapse: } \\
\text { EBRT + ADT: } 24 / 47 \\
(51.1 \%) \\
\text { EBRT: } 35 / 51(68.6 \%)\end{array}$ \\
\hline
\end{tabular}




\begin{tabular}{|c|c|c|c|c|}
\hline $\begin{array}{l}\text { Intervention/ } \\
\text { Comparison }\end{array}$ & $\begin{array}{l}\text { Study } \\
\text { Design } \\
\text { (Trial/Registry) } \\
\text { Followup } \\
\text { Risk of Bias }\end{array}$ & Overall Survival and Mortality & $\begin{array}{l}\text { Prostate Cancer Specific Survival and } \\
\text { Mortality }\end{array}$ & $\begin{array}{l}\text { Metastatic Progression } \\
\text { Free Survival } \\
\text { Metastases }\end{array}$ \\
\hline & $\begin{array}{l}\text { Phillips, 2014;30 } \\
\text { McDuff, 2018;31 } \\
\text { Giacalone, 201632 } \\
\text { (all secondary } \\
\text { references to } \\
\text { D'Amico 200851) } \\
\text { RCT } \\
\text { (NCT00116220) } \\
\text { Median follow-up } \\
14.26 \text { to } 18.19 \\
\text { years } \\
\text { Medium }\end{array}$ & $\begin{array}{l}\text { Overall mortality } \\
\text { 16.62 years: } \\
\text { EBRT + ADT: } 76 / 102(74.5 \%) \\
\text { EBRT: } 80 / 104(76.9 \%) \\
\text { Among patients with no/minimal } \\
\text { comorbidity } \\
16.62 \text { years: } \\
\text { EBRT + ADT: } 53 / 78(67.9 \%) \\
\text { EBRT: } 57 / 79(72.2 \%) \\
\text { HRadj=0.87 (95\% Cl, } 0.57 \text { to } 1.34) \\
\text { Among patients with moderate/severe } \\
\text { comorbidity } \\
\text { 16.62 years: } \\
\text { EBRT + ADT: } 23 / 24(95.8 \%) \\
\text { EBRT: } 23 / 25(92.0 \%) \\
\text { HRadj=2.42 }(95 \% \mathrm{Cl}, 1.19 \text { to } 4.94)\end{array}$ & $\begin{array}{l}\text { Prostate cancer mortality } \\
16.62 \text { years: } \\
\text { EBRT + ADT: } 6 / 102(5.9 \%) \\
\text { EBRT: } 23 / 104(22.1 \%) \\
\\
\text { Among patients with no/minimal comorbidity } \\
14.26 \text { years: } \\
\text { EBRT + ADT: } 4 / 73(5.5 \%) \\
\text { EBRT: } 16 / 76(21.1 \%) \\
\text { HRadj [EBRT vs. EBRT + ADT]=4.12 (95\% } \\
\text { Cl, } 1.10 \text { to } 15.35) \\
16.62 \text { years: } \\
\text { EBRT + ADT: } 5 / 78(6.4 \%) \\
\text { EBRT: } 20 / 79(25.3 \%) \\
\text { Among patients with moderate/severe } \\
\text { comorbidity } \\
\text { 16.62 years: } \\
\text { EBRT + ADT: } 1 / 24(4.2 \%) \\
\text { EBRT: } 3 / 25(12.0 \%)\end{array}$ & NR \\
\hline $\begin{array}{l}\text { EBRT + } \\
\text { neoadjuvant and } \\
\text { concurrent } \\
\text { ADT/EBRT + } \\
\text { concurrent and } \\
\text { adjuvant ADT }\end{array}$ & $\begin{array}{l}\text { Malone, } 2019^{33} \\
\text { RCT } \\
\text { Median follow-up } \\
12.17 \text { years } \\
\text { Medium }\end{array}$ & $\begin{array}{l}\text { Overall mortality } \\
12.17 \text { years: } \\
\text { EBRT + neoadjuvant and concurrent } \\
\text { ADT: } 75 / 215 \text { ( } 34.9 \%) \\
\text { EBRT + concurrent and adjuvant ADT: } \\
72 / 217(33.2 \%) \\
\text { Overall survival } \\
10 \text { years: } \\
\text { EBRT + neoadjuvant and concurrent } \\
\text { ADT: } 76.4 \%(95 \% \mathrm{Cl}, 70.6 \% \text { to } 82.7 \%) \\
\text { EBRT + concurrent and adjuvant ADT: } \\
73.7 \% \text { ( } 95 \% \mathrm{Cl}, 67.6 \% \text { to } 80.2 \%) \\
\text { Stratified log-rank test, } \mathrm{p}=0.70 \\
\text { HR univariate: } 0.94(95 \% \mathrm{Cl}, 0.68 \text { to } \\
1.30) \\
\text { HR multivariate: } 1.04(95 \% \mathrm{Cl}, 0.75 \text { to } \\
1.44)\end{array}$ & $\begin{array}{l}\text { Prostate cancer mortality } \\
12.17 \text { years: } \\
\text { EBRT + neoadjuvant and concurrent ADT: } \\
7 / 215(3.3 \%) \\
\text { EBRT + concurrent and adjuvant ADT: } 7 / 217 \\
(3.2 \%) \\
10 \text { years: } \\
\text { EBRT + neoadjuvant and concurrent ADT: } \\
2 \% \\
\text { EBRT + concurrent and adjuvant ADT: } 1.9 \% \\
P=0.98\end{array}$ & $\begin{array}{l}\text { Distant progression } \\
\text { 12.17 years: } \\
\text { EBRT + neoadjuvant and } \\
\text { concurrent ADT: } 12 / 215 \\
(5.6 \%) \\
\text { EBRT + concurrent and } \\
\text { adjuvant ADT: } 9 / 217 \\
\text { (4.1\%) } \\
\text { Metastatic-free survival } \\
\text { 10 years: } \\
\text { EBRT + neoadjuvant and } \\
\text { concurrent ADT: } 94 \% \\
(95 \% \mathrm{Cl}, 90.0 \% \text { to } \\
98.3 \%) \\
\text { EBRT + concurrent and } \\
\text { adjuvant ADT: } 95.1 \% \\
(95 \% \mathrm{Cl}, 91.5 \% \text { to } \\
98.9 \%) \\
\mathrm{P}=0.60\end{array}$ \\
\hline
\end{tabular}




\begin{tabular}{|c|c|c|c|c|}
\hline $\begin{array}{l}\text { Interventionl } \\
\text { Comparison }\end{array}$ & $\begin{array}{l}\text { Study } \\
\text { Design } \\
\text { (Trial/Registry) } \\
\text { Followup } \\
\text { Risk of Bias } \\
\end{array}$ & Overall Survival and Mortality & $\begin{array}{l}\text { Prostate Cancer Specific Survival and } \\
\text { Mortality }\end{array}$ & $\begin{array}{l}\text { Metastatic Progression } \\
\text { Free Survival } \\
\text { Metastases }\end{array}$ \\
\hline $\begin{array}{l}\text { EBRT I } \\
\text { Brachytherapy }\end{array}$ & $\begin{array}{l}\text { Goy } 2019^{50} \\
\text { Observational } \\
\text { Median follow-up } \\
9.6-9.8 \text { years } \\
\text { Medium }\end{array}$ & $\begin{array}{l}\text { Overall survival (10-year propensity score } \\
\text { adjusted probability) } \\
\text { Median follow-up } 9.6-9.8 \text { years } \\
\text { EBRT + neoadjuvant ADT: } 75.5 \% \text { (95\% } \\
\text { Cl, } 71.8 \% \text { to } 79.4 \%) \\
\text { Brachytherapy: } 78.3 \% \text { (95\% Cl } 70.1 \% \text { to } \\
87.4 \%) \\
\text { Overall mortality } \\
5 \text { years } \\
\text { EBRT + neoadjuvant ADT: } 26 \% \\
\text { (150/574) } \\
\text { Unadjusted K-M probability for survival } \\
90.6 \% \\
\text { Brachytherapy: } 12 \% \text { (13/110) } \\
\text { Unadjusted K-M probability for survival } \\
98.1 \%\end{array}$ & $\begin{array}{l}\text { Overall survival (10-year propensity score } \\
\text { adjusted probability) } \\
\text { Median follow-up 9.6-9.8 years } \\
\text { EBRT + neoadjuvant ADT: } 96.2 \%(95 \% \mathrm{CI} \text {, } \\
94.3 \% \text { to } 98.1 \%) \\
\text { Brachytherapy: } 95.4 \% \text { (95\% CI } 91.1 \% \text { to } \\
100.0 \%) \\
\text { Prostate cancer-specific mortality } \\
5 \text { years } \\
\text { EBRT + neoadjuvant ADT: } 2.9 \%(16 / 574) \\
\text { Unadjusted K-M probability for survival } \\
\text { 99.2\% } \\
\text { Brachytherapy: } 2.7 \% \text { (3/110) } \\
\text { Unadjusted K-M probability for survival } \\
\text { 99.0\% }\end{array}$ & $\begin{array}{l}\text { Overall survival (10-year } \\
\text { propensity score } \\
\text { adjusted probability) } \\
\text { Median follow-up 9.6-9.8 } \\
\text { years } \\
\text { EBRT + neoadjuvant } \\
\text { ADT: } 90.6 \% \text { (95\% Cl, } \\
87.9 \% \text { to } 93.3 \%) \\
\text { Brachytherapy: } 94.1 \% \\
(95 \% \mathrm{Cl} 89.5 \% \text { to } \\
98.9 \%) \\
\text { Metastases } \\
5 \text { years } \\
\text { EBRT + neoadjuvant } \\
\text { ADT: } 7.1 \% \text { (41/574) } \\
\text { Unadjusted K-M } \\
\text { probability for survival } \\
97.8 \% \\
\text { Brachytherapy: } 6.4 \% \\
\text { (7/110) } \\
\text { Unadjusted K-M } \\
\text { probability for survival } \\
97.1 \%\end{array}$ \\
\hline
\end{tabular}




\begin{tabular}{|c|c|c|c|c|}
\hline $\begin{array}{l}\text { Intervention/ } \\
\text { Comparison }\end{array}$ & $\begin{array}{l}\text { Study } \\
\text { Design } \\
\text { (Trial/Registry) } \\
\text { Followup } \\
\text { Risk of Bias }\end{array}$ & Overall Survival and Mortality & $\begin{array}{l}\text { Prostate Cancer Specific Survival and } \\
\text { Mortality }\end{array}$ & $\begin{array}{l}\text { Metastatic Progression } \\
\text { Free Survival } \\
\text { Metastases }\end{array}$ \\
\hline $\begin{array}{l}\text { Conventional } \\
\text { fractionated } \\
\text { EBRT/ultra- } \\
\text { hypofractionated } \\
\text { EBRT }\end{array}$ & $\begin{array}{l}\text { Widmark, } 2019^{34} \\
\text { RCT } \\
\text { Median follow-up } \\
5 \text { years } \\
\text { Medium ROB }\end{array}$ & $\begin{array}{l}\text { Overall mortality } \\
\text { Median } 5 \text { years: } \\
\text { Conventionally fractionated EBRT: } \\
43 / 591 \text { ( } 7.3 \%) \\
\text { Ultra-hypofractioned EBRT: } 46 / 589 \\
(7.8 \%) \\
\text { Overall survival } \\
5 \text { years: } \\
\text { Conventionally fractionated EBRT: } 96.4 \% \\
\text { (95\% Cl, } 94.6 \text { to } 98.1) \\
\text { Ultra-hypofractioned EBRT: } 93.9 \% \text { (95\% } \\
\text { Cl, } 91.7 \text { to } 96.2) \\
\text { Unadjusted HR: } 1.11 \text { (95\% Cl, } 0.73 \text { to } \\
\text { 1.69) } \\
\text { Log-rank test, } p=0.951\end{array}$ & $\begin{array}{l}\text { Prostate cancer mortality } \\
\text { Median } 5 \text { years: } \\
\text { Conventionally fractionated EBRT: } 8 / 591 \\
(1.4 \%) \\
\text { Ultra-hypofractioned EBRT: } 11 / 589(1.9 \%) \\
\text { Prostate cancer specific survival } \\
5 \text { years: } \\
\text { Conventionally fractionated EBRT: } 99.8 \% \\
\text { (95\% Cl, } 99.5 \text { to } 100) \\
\text { Ultra-hypofractionated EBRT: } 98.2 \% \text { (95\% } \\
\text { Cl, } 96.9 \text { to } 99.6) \\
\text { Unadjusted HR: } 1.40(95 \% \mathrm{Cl}, 0.56 \text { to } 3.49) \\
\text { Log-rank test, p=0.46 }\end{array}$ & $\begin{array}{l}\text { Distant failure } \\
\text { Median } 5 \text { years: } \\
\text { Conventionally } \\
\text { fractionated EBRT: } \\
39 / 591(6.6 \%) \\
\text { Ultra-hypofractionated } \\
\text { EBRT: } 38 / 589 \text { (6.5\%) } \\
\\
\text { Distant failure (free of } \\
\text { event) } \\
5 \text { years: } \\
\text { Conventionally } \\
\text { fractionated EBRT: } \\
94.6 \% \text { (95\% Cl, } 92.5 \text { to } \\
96.8) \\
\text { Ultra-hypofractionated } \\
\text { EBRT: } 93.7 \%(95 \% \mathrm{Cl} \text {, } \\
91.5 \text { to } 96.0) \\
\text { Unadjusted HR: } 0.99 \\
\text { (95\% CI, } 0.63 \text { to } 1.54) \\
\text { Log-rank test, } \mathrm{p}=0.95\end{array}$ \\
\hline
\end{tabular}

Abbreviations: 3D-CRT=3-dimensional conformal radiation therapy; ADJ=adjusted; ADT=androgen deprivation therapy; ASCENDE-RT=Androgen Suppression Combined with Elective Nodal and Dose Escalated Radiation; CI=confidence interval; EBRT=external beam radiation therapy; EORTC=European Organization for Research and Treatment of Cancer; HR=hazard ratio; IMRT=intensity-modulated radiation therapy; IMRT=intensity-modulated radiation therapy; ITT=intent to treat; KM=Kaplan-Meier estimate; LDR$\mathrm{PB}=$ low dose rate prostate brachytherapy; NR=not reported; RCT=randomized controlled trial; ROB=risk of bias; RR=risk ratio; RT=radiation therapy; $\mathrm{SBRT}=$ stereotactic body radiation therapy 
Table H-5. Health status and quality of life outcomes: external beam radiation therapy

\begin{tabular}{|l|l|l|l|}
\hline $\begin{array}{l}\text { Intervention/ } \\
\text { Comparison }\end{array}$ & $\begin{array}{l}\text { Study } \\
\text { Design } \\
\text { (Trial/Registry) } \\
\text { Followup } \\
\text { Risk of Bias }\end{array}$ & Health Status & $\begin{array}{l}\text { Quality of Life } \\
\text { Prostate Cancer Related Quality of Life }\end{array}$ \\
\hline 3D-CRT + & $\begin{array}{l}\text { ASCENDE-RT23, } \\
\text { ADT/3D-CRT }+\end{array}$ & NR & NR \\
ADT + LDR-PB & RCT \\
boost & $\begin{array}{l}\text { Median } 6.5 \text { years } \\
\text { (RT began } 8 \\
\text { months after } \\
\text { ADT) } \\
\text { Medium }\end{array}$ & & \\
& & \\
\end{tabular}




\begin{tabular}{|c|c|c|c|}
\hline 3D-CRT/ IMRT & $\begin{array}{l}\text { Viani } 2016^{26} \\
\text { RCT } \\
3 \text { years } \\
\text { Medium }\end{array}$ & NR & $\begin{array}{l}\text { Prostate-specific Quality of Life } \\
\text { EORTC QLQPR25 items, Mean (SD): } \\
\text { 1 year (n=181/215) } \\
\text { Urinary symptoms } \\
\text { 3D-CRT: } 22 \text { (21) } \\
\text { IMRT: } 12 \text { (14) } \\
\text { p<0.001 } \\
\text { Bowel symptoms } \\
\text { 3D-CRT: } 9 \text { (17) } \\
\text { IMRT: } 4 \text { (10) } \\
\text { p=0.02 } \\
\text { Treatment-related symptoms } \\
\text { 3D-CRT: } 12 \text { (13) } \\
\text { IMRT: } 9 \text { (9) } \\
\text { p=0.048 } \\
\text { Sexual function } \\
\text { 3D-CRT: 30 (22) } \\
\text { IMRT: } 27 \text { (22) } \\
\text { p=0.42 } \\
\text { Sexual activity } \\
\text { 3D-CRT: } 56 \text { (33) } \\
\text { IMRT: } 53 \text { (35) } \\
\text { p=0.55 } \\
\text { 3 years (n=175/215) } \\
\text { Urinary symptoms } \\
\text { 3D-CRT: 14 (15) } \\
\text { IMRT: } 12 \text { (12) } \\
\text { p=0.29 } \\
\text { Bowel symptoms } \\
\text { 3D-CRT: } 4 \text { (10) } \\
\text { IMRT: } 6 \text { (12) } \\
\text { p=0.31 } \\
\text { Treatment-related symptoms } \\
\text { 3D-CRT: } 6(8) \\
\text { IMRT: } 8 \text { (8) } \\
\text { p=0.26 } \\
\text { Sexual function } \\
\text { 3D-CRT: } 25(24) \\
\text { IMRT: } 23 \text { (19) } \\
\text { p=0.43 } \\
\text { Sexual activity } \\
\text { 3D-CRT: } 64(32) \\
\text { IMRT: } 64 \text { (36) } \\
\text { p=0.90 }\end{array}$ \\
\hline
\end{tabular}




\begin{tabular}{|c|c|c|c|}
\hline $\begin{array}{l}\text { Interventionl } \\
\text { Comparison }\end{array}$ & $\begin{array}{l}\text { Study } \\
\text { Design } \\
\text { (Trial/Registry) } \\
\text { Followup } \\
\text { Risk of Bias }\end{array}$ & Health Status & $\begin{array}{l}\text { Quality of Life } \\
\text { Prostate Cancer Related Quality of Life }\end{array}$ \\
\hline $\begin{array}{l}\text { Brachytherapy + } \\
\text { EBRT/ } \\
\text { Brachytherapy }\end{array}$ & $\begin{array}{l}\text { Amini } 2016^{41} \\
\text { Observational } \\
\text { (National Cancer } \\
\text { Database) } \\
7 \text { years } \\
\text { Medium }\end{array}$ & $\mathrm{NR}$ & NR \\
\hline IMRT/SBRT & $\begin{array}{l}\text { Ricco } 2017^{46} \\
\text { Observational } \\
\text { (National Cancer } \\
\text { Database) } \\
8 \text { years } \\
\text { Medium }\end{array}$ & NR & NR \\
\hline
\end{tabular}




\begin{tabular}{|c|c|c|c|}
\hline $\begin{array}{l}\text { Intervention/ } \\
\text { Comparison }\end{array}$ & $\begin{array}{l}\text { Study } \\
\text { Design } \\
\text { (Trial/Registry) } \\
\text { Followup } \\
\text { Risk of Bias }\end{array}$ & Health Status & $\begin{array}{l}\text { Quality of Life } \\
\text { Prostate Cancer Related Quality of Life }\end{array}$ \\
\hline $\begin{array}{l}\text { EBRT }+ \\
\text { ADT/EBRT }\end{array}$ & $\begin{array}{l}\text { Bolla 201627 } \\
\text { RCT (EORTC } \\
\text { Trial 22991) } \\
\text { Median Follow-up } \\
7.2 \text { years } \\
\text { Medium }\end{array}$ & (see quality of life) & 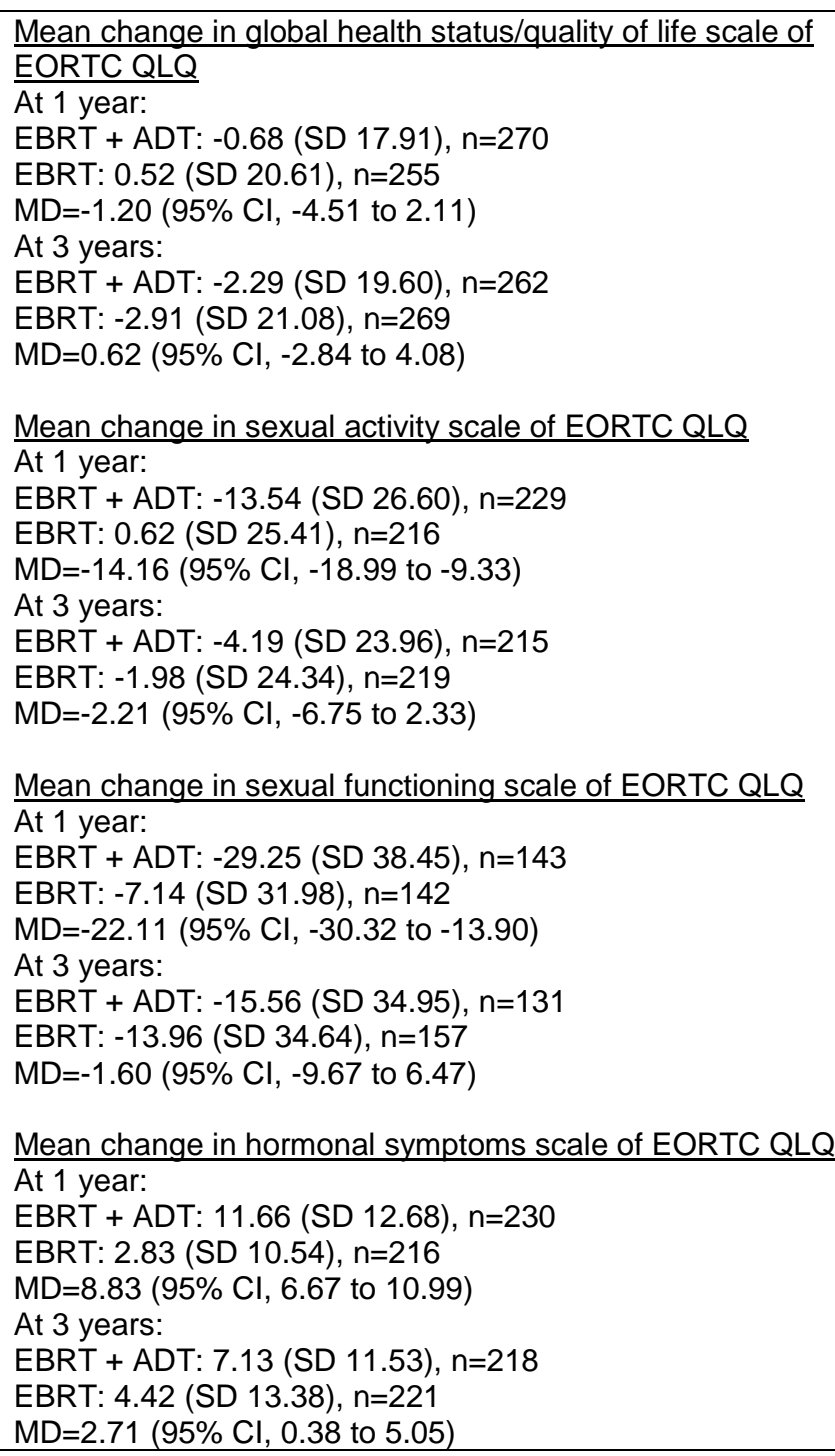 \\
\hline
\end{tabular}




\begin{tabular}{|c|c|c|c|}
\hline $\begin{array}{l}\text { Intervention/ } \\
\text { Comparison }\end{array}$ & $\begin{array}{l}\text { Study } \\
\text { Design } \\
\text { (Trial/Registry) } \\
\text { Followup } \\
\text { Risk of Bias }\end{array}$ & Health Status & $\begin{array}{l}\text { Quality of Life } \\
\text { Prostate Cancer Related Quality of Life }\end{array}$ \\
\hline & $\begin{array}{l}\text { McPartlin 2016 } \\
\text { RCT (PMH 9907) } \\
\text { Median follow-up } \\
\text { 9.1 years } \\
\text { Medium }\end{array}$ & NR & $\begin{array}{l}\text { EORTC quality of life } \\
\text { No data reported. From the article, "EORTC-30 questionnaire } \\
\text { similarly identified no marked effect of the addition of } \\
\text { bicalutamide, with stable overall QoL reported in both groups } \\
\text { through the treatment period." }\end{array}$ \\
\hline $\begin{array}{l}\text { EBRT }+ \\
\text { neoadjuvant and } \\
\text { concurrent } \\
\text { ADT/EBRT + } \\
\text { concurrent and } \\
\text { adjuvant ADT }\end{array}$ & $\begin{array}{l}\text { Malone, } 2019 \\
\text { RCT } \\
\text { Median follow-up } \\
\text { 12.17 years } \\
\text { Medium }\end{array}$ & NR & NR \\
\hline $\begin{array}{l}\text { EBRT / } \\
\text { Brachytherapy }\end{array}$ & $\begin{array}{l}\text { Goy } 2019 \\
\text { Observational } \\
\text { Median follow-up } \\
\text { 9.6-9.8 years } \\
\text { Medium }\end{array}$ & NR & $\underline{\mathrm{NR}}$ \\
\hline $\begin{array}{l}\text { Conventional } \\
\text { fractionated } \\
\text { EBRT/ultra- } \\
\text { hypofractionated } \\
\text { EBRT }\end{array}$ & $\begin{array}{l}\text { Widmark, } 2019 \\
\text { RCT } \\
\text { Median follow-up } \\
5 \text { years } \\
\text { Medium ROB }\end{array}$ & NR & NR \\
\hline
\end{tabular}

Abbreviations: 3D-CRT=3-dimensional conformal radiation therapy; ADT=Androgen deprivation therapy; ASCENDE-RT=Androgen Suppression Combined with Elective

Nodal and Dose Escalated Radiation; $\mathrm{CI}=$ confidence interval; EBRT=external beam radiation therapy; EORTC=European Organisation for Research and Treatment of Cancer;

$\mathrm{HR}=$ hazard ratio; IMRT=intensity-modulated radiation therapy; LDR-PB=low dose rate prostate brachytherapy; $\mathrm{MD}=\mathrm{Mean}$ difference; $\mathrm{NR}=$ not reported; RCT=randomized controlled trial; ROB=risk of bias; RR=risk ratio; RT=radiation therapy; QLQ=Quality of Life Questionnaire; QoL=Quality of life; SBRT=stereotactic body radiation therapy; $\mathrm{SD}=$ standard deviation 
Table H-6. Harms: external beam radiation therapy

\begin{tabular}{|c|c|c|}
\hline $\begin{array}{l}\text { Intervention/ } \\
\text { Comparison }\end{array}$ & $\begin{array}{l}\text { Study } \\
\text { Design } \\
\text { (Trial/Registry) } \\
\text { Followup } \\
\text { Risk of Bias }\end{array}$ & Adverse Effects \\
\hline $\begin{array}{l}\text { 3D-CRT + } \\
\text { ADT/3D-CRT + } \\
\text { ADT + LDR-PB } \\
\text { boost }\end{array}$ & $\begin{array}{l}\text { ASCENDE- } \\
\mathrm{RT}^{23,24} \\
\text { RCT } \\
\text { Median } 6.5 \\
\text { years (RT } \\
\text { began } 8 \\
\text { months after } \\
\text { ADT) } \\
\text { Medium }\end{array}$ & $\begin{array}{l}\text { Urinary incontinence and pad use } \\
\text { Cumulative incidence at } \mathbf{5} \text { years } \\
\text { 3D-CRT + ADT: } 16 \% \\
\text { LDR-PB: } 6 \% \\
\text { p<0.001 } \\
\text { Prevalence at } 2 \text { years } \\
\text { 3D-CRT + ADT: } 8 \% \\
\text { LDR-PB: } 1 \% \\
\text { p=0.003 } \\
\text { Prevalence at } 5 \text { years } \\
\text { 3D-CRT + ADT: } 7 \% \\
\text { LDR-PB: } 1 \% \\
\text { p=0.049 } \\
\text { Erectile function (defined as erection adequate for penetration) } \\
1 \text { year } \\
\text { 3D-CRT + ADT: } 7 \% \text { (NR/195) } \\
\text { LDR-PB: } 5 \% \text { (NR/188) } \\
\text { p=NR } \\
5 \text { years } \\
\text { 3D-CRT + ADT: } 31 \%(N R / 195) \\
\text { LDR-PB: } 34 \% \text { (NR/188) } \\
\text { p=0.60 }\end{array}$ \\
\hline 3D-CRT/IMRT & $\begin{array}{l}\text { Viani } 2016^{26} \\
\text { RCT } \\
3 \text { years } \\
\text { Medium }\end{array}$ & NR at eligible followup time \\
\hline $\begin{array}{l}\text { Brachytherapy + } \\
\text { EBRT/ } \\
\text { Brachytherapy }\end{array}$ & $\begin{array}{l}\text { Amini } 2016^{41} \\
\text { Observational } \\
\text { (National } \\
\text { Cancer } \\
\text { Database) } \\
7 \text { years } \\
\text { Medium }\end{array}$ & NR \\
\hline
\end{tabular}




\begin{tabular}{|c|c|c|}
\hline $\begin{array}{l}\text { Intervention/ } \\
\text { Comparison }\end{array}$ & $\begin{array}{l}\text { Study } \\
\text { Design } \\
\text { (Trial/Registry) } \\
\text { Followup } \\
\text { Risk of Bias }\end{array}$ & Adverse Effects \\
\hline IMRT/SBRT & $\begin{array}{l}\text { Ricco } 2017^{46} \\
\text { Observational } \\
\text { (National } \\
\text { Cancer } \\
\text { Database) } \\
8 \text { years } \\
\text { Medium } \\
\end{array}$ & NR \\
\hline \multirow[t]{2}{*}{$\begin{array}{l}\text { EBRT + } \\
\text { ADT/EBRT }\end{array}$} & $\begin{array}{l}\text { Bolla } 2016^{27} \\
\text { RCT (EORTC } \\
\text { Trial 22991) } \\
\text { Median Follow- } \\
\text { up } 7.2 \text { years } \\
\text { Medium }\end{array}$ & $\begin{array}{l}\text { Late genitourinary toxicity grade } 3 \text { to } 4 \text { (measured from } 6 \text { months until end of follow-up) } \\
\text { EBRT + ADT: } 5.9 \% \text {, calculated } 24 / 406 \text { based on safety sample size } \\
\text { EBRT: } 3.6 \% \text {, calculated } 15 / 407 \text { based on safety sample size } \\
P=.14 \\
\text { Severe impairment of sexual function (measured from } 6 \text { months until end of follow-up) } \\
\text { EBRT + ADT: } 27.0 \% \text {, calculated } 110 / 406 \text { based on safety sample size } \\
\text { EBRT: } 19.4 \% \text {, calculated } 79 / 407 \text { based on safety sample size } \\
P=.01\end{array}$ \\
\hline & $\begin{array}{l}\text { McPartlin } \\
2016^{28} \\
\text { RCT (PMH } \\
\text { 9907) } \\
\text { Median follow- } \\
\text { up 9.1 years } \\
\text { Medium }\end{array}$ & $\begin{array}{l}\text { Late genitourinary toxicity (timing of outcome not clearly reported) } \\
\text { Grade } 2 \\
\text { EBRT + ADT: } 9.6 \% \\
\text { EBRT: } 5.5 \% \\
\text { Grade } 3 \\
\text { EBRT + ADT: } 11.4 \% \\
\text { EBRT: } 11 \% \\
\text { Late gastrointestinal toxicity (timing of outcome not clearly reported) } \\
\text { Grade } 2 \\
\text { EBRT + ADT: } 3.5 \% \\
\text { EBRT: } 4.7 \% \\
\text { Grade } 3 \\
\text { EBRT + ADT: } 0 \% \\
\text { EBRT: } 0.8 \% \\
\text { IIEF } \\
\text { Not extracted due to high attrition }\end{array}$ \\
\hline
\end{tabular}




\begin{tabular}{|c|c|c|}
\hline $\begin{array}{l}\text { Intervention/ } \\
\text { Comparison }\end{array}$ & $\begin{array}{l}\text { Study } \\
\text { Design } \\
\text { (Trial/Registry) } \\
\text { Followup } \\
\text { Risk of Bias }\end{array}$ & Adverse Effects \\
\hline $\begin{array}{l}\text { EBRT + } \\
\text { neoadjuvant } \\
\text { and concurrent } \\
\text { ADT/EBRT + } \\
\text { concurrent and } \\
\text { adjuvant ADT }\end{array}$ & $\begin{array}{l}\text { Malone, } 2019 \\
\text { RCT } \\
\text { Median follow- } \\
\text { up } 12.17 \text { years } \\
\text { Medium }\end{array}$ & $\begin{array}{l}\text { Late gastrointestinal toxicity grade } \geq 3 \\
3 \text { year cumulative incidence } \\
\text { EBRT + neoadjuvant and concurrent ADT: } 2.5 \% \\
\text { EBRT + concurrent and adjuvant ADT: } 3.9 \% \\
P=0.44 \\
\text { Late genitourinary toxicity grade } \geq 3 \\
3 \text { year cumulative incidence } \\
\text { EBRT + neoadjuvant and concurrent ADT: } 2.9 \% \text {, calculated } 6 / 213 \text { based on late toxicity sample size } \\
\text { EBRT + concurrent and adjuvant ADT: } 2.9 \% \text {, calculated } 6 / 215 \text { based on late toxicity sample size } \\
P=0.82\end{array}$ \\
\hline $\begin{array}{l}\text { EBRT I } \\
\text { Brachytherapy }\end{array}$ & $\begin{array}{l}\text { Goy } 2019^{50} \\
\text { Observational } \\
\text { Median follow- } \\
\text { up 9.6-9.8 } \\
\text { years } \\
\text { Medium }\end{array}$ & NR \\
\hline $\begin{array}{l}\text { Conventional } \\
\text { fractionated } \\
\text { EBRT/ultra- } \\
\text { hypofractionated } \\
\text { EBRT }\end{array}$ & $\begin{array}{l}\text { Widmark, } \\
2019^{34} \\
\text { RCT } \\
\text { Median follow- } \\
\text { up } 5 \text { years } \\
\text { Medium ROB }\end{array}$ & $\begin{array}{l}\text { Urinary toxicity grade } \geq 1 \text { (physician evaluated with the RTOG morbidity scale) } \\
2 \text { years } \\
\text { Conventional fractionated EBRT: } 22.7 \%(113 / 497) \\
\text { Ultra-hypofractionated EBRT: } 23.6 \%(116 / 492) \\
2 \text { years cumulative incidence } \\
\text { Conventional fractionated EBRT: } 38.2 \%(95 \% \mathrm{Cl}, 34.4 \text { to } 42.4) \\
\text { Ultra-hypofractionated EBRT: } 43.1 \%(95 \% \mathrm{Cl}, 39.2 \text { to } 47.4) \\
5 \text { years } \\
\text { Data not extracted due to missing data/high attrition } \\
\text { Urinary toxicity grade } \geq 2 \text { (physician evaluated with the RTOG morbidity scale) } \\
1 \text { year } \\
\text { Conventional fractionated EBRT: } 2.5 \%(13 / 529) \\
\text { Ultra-hypofractionated EBRT: } 6.1 \%(32 / 528) \\
2 \text { years } \\
\text { Conventional fractionated EBRT: } 5.6 \%(28 / 497) \\
\text { Ultra-hypofractionated EBRT: } 5.1 \%(25 / 492) \\
2 \text { years cumulative incidence }\end{array}$ \\
\hline
\end{tabular}




\begin{tabular}{|c|c|c|}
\hline $\begin{array}{l}\text { Intervention/ } \\
\text { Comparison }\end{array}$ & $\begin{array}{l}\text { Study } \\
\text { Design } \\
\text { (Trial/Registry) } \\
\text { Followup } \\
\text { Risk of Bias }\end{array}$ & Adverse Effects \\
\hline & & $\begin{array}{l}\text { Conventional fractionated EBRT: } 9.4 \%(95 \% \mathrm{Cl}, 7.3 \text { to } 12.1) \\
\text { Ultra-hypofractionated EBRT: } 13.2 \% \text { ( } 95 \% \mathrm{Cl}, 10.7 \text { to } 16.3) \\
5 \text { years } \\
\text { Data not extracted due to missing data/high attrition } \\
\text { Urinary toxicity grade } \geq 3 \text { (physician evaluated with the RTOG morbidity scale) } \\
2 \text { years } \\
\text { Conventional fractionated EBRT: } 1.0 \%(5 / 497) \\
\text { Ultra-hypofractionated EBRT: } 0.4 \%(2 / 492) \\
2 \text { years cumulative incidence } \\
\text { Conventional fractionated EBRT: } 1.9 \%(95 \% \mathrm{Cl}, 1.1 \text { to } 3.4) \\
\text { Ultra-hypofractionated EBRT: } 3.5 \%(95 \% \mathrm{Cl}, 2.3 \text { to } 5.4) \\
5 \text { years } \\
\text { Data not extracted due to missing data/high attrition } \\
\text { Urinary problems patient-reported (symptom severity based on PCSS question) } \\
1 \text { year } \\
\text { Conventional fractionated EBRT: mean } 1.58 \text { (95\% Cl, } 1.37 \text { to } 1.78), \mathrm{n}=427 \\
\text { Ultra-hypofractionated EBRT: mean } 2.06 \text { (95\% Cl, } 1.82 \text { to } 2.30), \mathrm{n}=425 \\
\text { p=0.0036 } \\
2 \text { years } \\
\text { Only reported graphically, not significantly different (p=0.18) } \\
\text { 4+ years } \\
\text { Data not extracted due to missing data/high attrition } \\
\text { Bowel toxicity grade } \geq 1 \text { (physician evaluated with the RTOG morbidity scale) } \\
2 \text { years } \\
\text { Conventional fractionated EBRT: } 18.3 \%(91 / 496) \\
\text { Ultra-hypofractionated EBRT: } 19.2 \%(95 / 495) \\
2 \text { years cumulative incidence: } \\
\text { Conventional fractionated EBRT: } 36.5 \%(95 \% \mathrm{Cl}, 32.8 \text { to } 40.6) \\
\text { Ultra-hypofractionated EBRT: } 43.7 \%(95 \% \mathrm{Cl}, 39.8 \text { to } 48.0) \\
5 \text { years }\end{array}$ \\
\hline
\end{tabular}




\begin{tabular}{|c|c|c|}
\hline $\begin{array}{l}\text { Intervention/ } \\
\text { Comparison }\end{array}$ & $\begin{array}{l}\text { Study } \\
\text { Design } \\
\text { (Trial/Registry) } \\
\text { Followup } \\
\text { Risk of Bias }\end{array}$ & Adverse Effects \\
\hline & & $\begin{array}{l}\text { Data not extracted due to missing data/high attrition } \\
\text { Bowel toxicity grade } \geq 2 \text { (physician evaluated with the RTOG morbidity scale) } \\
2 \text { years } \\
\text { Conventional fractionated EBRT: } 3.2 \%(16 / 496) \\
\text { Ultra-hypofractionated EBRT: } 1.8 \%(9 / 495) \\
2 \text { years cumulative incidence: } \\
\text { Conventional fractionated EBRT: } 5.4 \%(95 \% \mathrm{Cl}, 3.8 \text { to } 7.6) \\
\text { Ultra-hypofractionated EBRT: } 6.3 \%(95 \% \mathrm{Cl}, 4.6 \text { to } 8.6) \\
5 \text { years } \\
\text { Data not extracted due to missing data/high attrition } \\
\text { Bowel toxicity grade } \geq 3 \text { (physician evaluated with the RTOG morbidity scale) } \\
2 \text { years } \\
\text { Conventional fractionated EBRT: } 0 \% \text { (0/496) } \\
\text { Ultra-hypofractionated EBRT: } 0.4 \% \text { ( } 2 / 495) \\
2 \text { years cumulative incidence: } \\
\text { Conventional fractionated EBRT: } 0.3 \%(95 \% \text { Cl, } 0.1 \text { to } 1.4) \\
\text { Ultra-hypofractionated EBRT: } 1.1 \% \text { (95\% Cl, } 0.5 \text { to } 2.3) \\
5 \text { years } \\
\text { Data not extracted due to missing data/high attrition } \\
\text { Bowel problems patient-reported (symptom severity based on PCSS question) } \\
1 \text { year } \\
\text { Only reported graphically, not significantly different ( } p=0.059) \\
2 \text { years } \\
\text { Only reported graphically, not significantly different ( } p=0.32) \\
4+\text { years } \\
\text { Data not extracted due to missing data/high attrition } \\
\text { Erectile function (physician recorded) } \\
1 \text { year } \\
\text { Only reported graphically, not significantly different ( } p=0.59)\end{array}$ \\
\hline
\end{tabular}




\begin{tabular}{|c|c|c|}
\hline $\begin{array}{l}\text { Intervention/ } \\
\text { Comparison }\end{array}$ & $\begin{array}{l}\text { Study } \\
\text { Design } \\
\text { (Trial/Registry) } \\
\text { Followup } \\
\text { Risk of Bias }\end{array}$ & Adverse Effects \\
\hline & & $\begin{array}{l}2 \text { years } \\
\text { Only reported graphically, not significantly different }(p=0.60) \\
3+\text { years } \\
\text { Data not extracted due to missing data/high attrition } \\
\text { Erectile problems patient-reported (symptom severity based on PCSS question) } \\
1 \text { year } \\
\text { Only reported graphically, not significantly different }(p=0.74) \\
2 \text { years } \\
\text { Only reported graphically, not significantly different }(p=0.18) \\
4+\text { years } \\
\text { Data not extracted due to missing data/high attrition }\end{array}$ \\
\hline
\end{tabular}

Abbreviations: 3D-CRT=3-dimensional conformal radiation therapy; ASCENDE-RT=Androgen Suppression Combined with Elective Nodal and Dose Escalated Radiation; $\mathrm{CI}=$ confidence interval; EBRT=external beam radiation therapy; EORTC=European Organization for Research and Treatment of Cancer; IIEF: International index of erectile function; HR=hazard ratio; IMRT=intensity-modulated radiation therapy; LDR-PB=low dose rate prostate brachytherapy; NR=not reported; PCSS=Prostate Cancer Symptom Scale; RCT=randomized controlled trial; ROB=risk of bias; RR=risk ratio; RT=radiation therapy; RTOG=Radiation Therapy Oncology Group; SBRT=stereotactic body radiation therapy; $\mathrm{SD}=$ standard deviation

Table H-7. Evidence certainty: external beam radiation therapy

\begin{tabular}{|c|c|c|c|c|c|c|c|c|c|c|c|}
\hline $\begin{array}{l}\text { Intervention/ } \\
\text { Comparison: } \\
\text { Outcome }\end{array}$ & $\begin{array}{l}\mathbf{k}= \\
\text { Study } \\
\text { Design }\end{array}$ & $\begin{array}{l}\text { Risk of } \\
\text { Bias }\end{array}$ & $\begin{array}{l}\text { Incon- } \\
\text { sistency }\end{array}$ & $\begin{array}{l}\text { Indi- } \\
\text { rect- } \\
\text { ness }\end{array}$ & $\begin{array}{l}\text { Impre- } \\
\text { cision }\end{array}$ & $\begin{array}{l}\text { Other } \\
\text { Consid- } \\
\text { erations }\end{array}$ & I & C & $\begin{array}{l}\text { Relative } \\
\text { (95\% Cl) }\end{array}$ & $\begin{array}{l}\text { Abso- } \\
\text { lute } \\
(95 \% \\
\mathrm{Cl})\end{array}$ & Certainty \\
\hline $\begin{array}{l}\text { 3D-CRT + ADT/3D- } \\
\text { CRT +ADT + LDR- } \\
\text { PB boost: } \\
\text { Mortality (Follow-up: } \\
5 \text { years) }\end{array}$ & $1 \mathrm{RCT}$ & Serious & $\begin{array}{l}\text { Not } \\
\text { serious }\end{array}$ & $\begin{array}{l}\text { Not } \\
\text { serious }\end{array}$ & Serious & None & $\begin{array}{l}38 / 200 \\
(19.0 \%)\end{array}$ & $\begin{array}{l}30 / 198 \\
(15.2 \%)\end{array}$ & $\begin{array}{l}\text { RR } 1.25 \\
\text { (0.81 to } 1.94)\end{array}$ & $\begin{array}{l}3.8 \% \\
(-3.5 \text { to } \\
11.2)\end{array}$ & $\begin{array}{l}\oplus \oplus \bigcirc \bigcirc^{a, b} \\
\text { LOW }\end{array}$ \\
\hline $\begin{array}{l}\text { 3D-CRT + ADT/3D- } \\
\text { CRT +ADT + LDR- } \\
\text { PB boost: Prostate- } \\
\text { specific mortality } \\
\text { (Follow-up: } 5 \text { years) }\end{array}$ & $1 \mathrm{RCT}$ & Serious & $\begin{array}{l}\text { Not } \\
\text { serious }\end{array}$ & $\begin{array}{l}\text { Not } \\
\text { serious }\end{array}$ & $\begin{array}{l}\text { Very } \\
\text { serious }\end{array}$ & None & $\begin{array}{l}11 / 200 \\
(5.5 \%)\end{array}$ & $\begin{array}{l}7 / 198 \\
(3.5 \%)\end{array}$ & $\begin{array}{l}\text { RR } 1.56 \\
\text { (0.62 to } 3.93 \text { ) }\end{array}$ & $\begin{array}{l}2 \% \\
(-2.1 \text { to } \\
6.0)\end{array}$ & $\begin{array}{l}\oplus \bigcirc \bigcirc \bigcirc \\
\text { INSUFFICIENT }^{\mathrm{a}, \mathrm{c}}\end{array}$ \\
\hline
\end{tabular}




\begin{tabular}{|c|c|c|c|c|c|c|c|c|c|c|c|}
\hline $\begin{array}{l}\text { Intervention/ } \\
\text { Comparison: } \\
\text { Outcome }\end{array}$ & $\begin{array}{l}\text { k= } \\
\text { Study } \\
\text { Design }\end{array}$ & $\begin{array}{l}\text { Risk of } \\
\text { Bias }\end{array}$ & $\begin{array}{l}\text { Incon- } \\
\text { sistency }\end{array}$ & $\begin{array}{l}\text { Indi- } \\
\text { rect- } \\
\text { ness }\end{array}$ & $\begin{array}{l}\text { Impre- } \\
\text { cision }\end{array}$ & $\begin{array}{l}\text { Other } \\
\text { Consid- } \\
\text { erations }\end{array}$ & 1 & C & $\begin{array}{l}\text { Relative } \\
(95 \% \mathrm{Cl})\end{array}$ & $\begin{array}{l}\text { Abso- } \\
\text { lute } \\
(95 \% \\
\mathrm{Cl})\end{array}$ & Certainty \\
\hline $\begin{array}{l}\text { 3D-CRT + ADT/3D- } \\
\text { CRT +ADT + LDR- } \\
\text { PB boost: } \\
\text { Metastatic disease } \\
\text { (Follow-up: } 5 \text { years) }\end{array}$ & $1 \mathrm{RCT}$ & Serious & $\begin{array}{l}\text { Not } \\
\text { serious }\end{array}$ & $\begin{array}{l}\text { Not } \\
\text { serious }\end{array}$ & Serious & None & $\begin{array}{l}18 / 200 \\
(9.0 \%)\end{array}$ & $\begin{array}{l}17 / 198 \\
(8.6 \%)\end{array}$ & $\begin{array}{l}\text { RR } 1.05 \\
\text { (0.56 to } 1.97)\end{array}$ & $\begin{array}{l}0.4 \% \\
(-5.1 \text { to } \\
6.0)\end{array}$ & $\begin{array}{l}\oplus \oplus \bigcirc \bigcirc \\
\text { LOW }^{a, b}\end{array}$ \\
\hline $\begin{array}{l}\text { 3D-CRT + ADT/3D- } \\
\text { CRT +ADT + LDR- } \\
\text { PB boost: } \\
\text { Urinary } \\
\text { incontinence } \\
\text { (Follow-up: } 5 \text { years) }\end{array}$ & $1 \mathrm{RCT}$ & Serious & $\begin{array}{l}\text { Not } \\
\text { serious }\end{array}$ & $\begin{array}{l}\text { Not } \\
\text { serious }\end{array}$ & Serious & $\begin{array}{l}\text { Publication } \\
\text { bias } \\
\text { strongly } \\
\text { suspected }\end{array}$ & $-/ 195$ & $-/ 188$ & not estimable & - & $\begin{array}{l}\oplus \bigcirc \bigcirc \bigcirc \\
\text { INSUFFICIENT }^{\text {a, d }}\end{array}$ \\
\hline $\begin{array}{l}\text { 3D-CRT + ADT/3D- } \\
\text { CRT + ADT + LDR- } \\
\text { PB boost: } \\
\text { Erectile function } \\
\text { (Follow-up: } 5 \text { years) }\end{array}$ & $1 \mathrm{RCT}$ & Serious & $\begin{array}{l}\text { Not } \\
\text { serious }\end{array}$ & $\begin{array}{l}\text { Not } \\
\text { serious }\end{array}$ & $\begin{array}{l}\text { Very } \\
\text { serious }\end{array}$ & $\begin{array}{l}\text { Publication } \\
\text { bias } \\
\text { strongly } \\
\text { suspected }\end{array}$ & $-/ 195$ & $-/ 188$ & not estimable & - & $\begin{array}{l}\oplus \bigcirc \bigcirc \bigcirc \\
\text { INSUFFICIENT }^{\text {a, e }}\end{array}$ \\
\hline $\begin{array}{l}\text { Brachytherapy + } \\
\text { EBRT/ } \\
\text { Brachytherapy: } \\
\text { Overall survival } \\
\text { (Follow-up: } 7 \text { years) }\end{array}$ & $\begin{array}{l}1 \\
\text { observa } \\
\text { tional }\end{array}$ & Serious & $\begin{array}{l}\text { Not } \\
\text { serious }\end{array}$ & $\begin{array}{l}\text { Not } \\
\text { serious }\end{array}$ & $\begin{array}{l}\text { Not } \\
\text { serious }\end{array}$ & $\begin{array}{l}\text { Publication } \\
\text { bias } \\
\text { strongly } \\
\text { suspected }\end{array}$ & $-/ 2929$ & $-/ 2929$ & not estimable & - & $\begin{array}{l}\oplus \bigcirc \bigcirc \bigcirc \\
\text { INSUFFICIENT }^{f, g}\end{array}$ \\
\hline $\begin{array}{l}\text { IMRT/SBRT: } \\
\text { Overall survival } \\
\text { (Follow-up: } 8 \text { years) }\end{array}$ & $\begin{array}{l}1 \\
\text { observa } \\
\text {-tional }\end{array}$ & Serious & $\begin{array}{l}\text { Not } \\
\text { serious }\end{array}$ & $\begin{array}{l}\text { Not } \\
\text { serious }\end{array}$ & $\begin{array}{l}\text { Very } \\
\text { serious }\end{array}$ & $\begin{array}{l}\text { Publication } \\
\text { bias } \\
\text { strongly } \\
\text { suspected }\end{array}$ & $-/ 2715$ & $-/ 2715$ & not estimable & - & $\begin{array}{l}\oplus \bigcirc \bigcirc \bigcirc \\
\text { INSUFFICIENT } \\
f, g, h\end{array}$ \\
\hline $\begin{array}{l}\text { EBRT plus ADT vs. } \\
\text { EBRT: Overall } \\
\text { mortality-5.9 to } 9.1 \\
\text { years }\end{array}$ & 5 RCTs & $\begin{array}{l}\text { Not } \\
\text { serious }\end{array}$ & $\begin{array}{l}\text { Not } \\
\text { serious }\end{array}$ & $\begin{array}{l}\text { Not } \\
\text { serious }\end{array}$ & Serious & None & $\begin{array}{l}587 / 2150 \\
(27.3 \%)\end{array}$ & $\begin{array}{l}615 / 1897 \\
(32.4 \%)\end{array}$ & $\begin{array}{l}\text { RR } 0.86 \\
\text { (0.69 to 1.06) }\end{array}$ & $\begin{array}{l}-3.7 \% \\
(-9.8 \text { to } \\
2.4)\end{array}$ & $\begin{array}{l}\oplus \oplus \oplus \bigcirc \\
\text { MODERATE }\end{array}$ \\
\hline $\begin{array}{l}\text { EBRT plus ADT vs. } \\
\text { EBRT: Prostate } \\
\text { cancer mortality- } 7.2 \\
\text { to } 9.1 \text { years }\end{array}$ & 3 RCTs & Serious & $\begin{array}{l}\text { Not } \\
\text { serious }\end{array}$ & $\begin{array}{l}\text { Not } \\
\text { serious }\end{array}$ & Serious & None & $\begin{array}{l}53 / 1499 \\
(3.5 \%)\end{array}$ & $\begin{array}{l}104 / 1505 \\
(6.9 \%)\end{array}$ & $\begin{array}{l}\text { Peto OR } 0.51 \\
(0.37 \text { to } 0.70)\end{array}$ & $\begin{array}{l}-3.4 \% \\
(-4.95 \\
\text { to }-1.8)\end{array}$ & $\begin{array}{l}\oplus \oplus \bigcirc \bigcirc \\
L O W b, j\end{array}$ \\
\hline
\end{tabular}




\begin{tabular}{|c|c|c|c|c|c|c|c|c|c|c|c|}
\hline $\begin{array}{l}\text { Intervention/ } \\
\text { Comparison: } \\
\text { Outcome }\end{array}$ & $\begin{array}{l}\mathbf{k}= \\
\text { Study } \\
\text { Design }\end{array}$ & $\begin{array}{l}\text { Risk of } \\
\text { Bias }\end{array}$ & $\begin{array}{l}\text { Incon- } \\
\text { sistency }\end{array}$ & $\begin{array}{l}\text { Indi- } \\
\text { rect- } \\
\text { ness }\end{array}$ & $\begin{array}{l}\text { Impre- } \\
\text { cision }\end{array}$ & $\begin{array}{l}\text { Other } \\
\text { Consid- } \\
\text { erations }\end{array}$ & I & C & $\begin{array}{l}\text { Relative } \\
\text { (95\% Cl) }\end{array}$ & $\begin{array}{l}\text { Abso- } \\
\text { lute } \\
(95 \% \\
\mathrm{Cl})\end{array}$ & Certainty \\
\hline $\begin{array}{l}\text { EBRT plus ADT vs. } \\
\text { EBRT: Metastasis-5 } \\
\text { to } 10 \text { years }\end{array}$ & 4 RCTs & Serious & $\begin{array}{l}\text { Not } \\
\text { serious }\end{array}$ & $\begin{array}{l}\text { Not } \\
\text { serious }\end{array}$ & Serious & None & $\begin{array}{l}284 / 2461 \\
(11.5 \%)\end{array}$ & $\begin{array}{l}289 / 2203 \\
(13.1 \%)\end{array}$ & $\begin{array}{l}\text { RR } 0.83 \\
\text { (0.71 to } 0.97)\end{array}$ & $\begin{array}{l}-2.3 \% \\
(-4.1 \text { to } \\
-0.4)\end{array}$ & $\begin{array}{l}\oplus \oplus \bigcirc \bigcirc \\
L O W b, j\end{array}$ \\
\hline $\begin{array}{l}\text { EBRT plus ADT vs. } \\
\text { EBRT: Severe } \\
\text { impairment of } \\
\text { sexual function } \\
\text { based on late } \\
\text { toxicity scores- } \\
\text { measured from six } \\
\text { months until end of } \\
\text { follow-up ( } 7.2 \text { years) }\end{array}$ & $1 \mathrm{RCT}$ & Serious & $\begin{array}{l}\text { Not } \\
\text { serious }\end{array}$ & $\begin{array}{l}\text { Not } \\
\text { serious }\end{array}$ & Serious & None & $\begin{array}{l}110 / 406 \\
(27.0 \%)\end{array}$ & $\begin{array}{l}79 / 407 \\
(19.4 \%)\end{array}$ & $\begin{array}{l}\text { RR } 1.40 \\
(1.08 \text { to } 1.80)\end{array}$ & $\begin{array}{l}7.7 \% \\
(1.9 \text { to } \\
13.5)\end{array}$ & $\begin{array}{l}\oplus \oplus \bigcirc \bigcirc \\
\mathrm{LOW}^{\mathrm{b}, \mathrm{j}}\end{array}$ \\
\hline $\begin{array}{l}\text { EBRT plus ADT vs. } \\
\text { EBRT: sexual } \\
\text { function impotence } \\
\text { grade } 2-4 \text { ( } 4.5 \\
\text { years) }\end{array}$ & $1 \mathrm{RCT}$ & Serious & $\begin{array}{l}\text { Not } \\
\text { serious }\end{array}$ & $\begin{array}{l}\text { Not } \\
\text { serious }\end{array}$ & $\begin{array}{l}\text { Very } \\
\text { serious }\end{array}$ & None & $\begin{array}{l}32 / 98 \\
(32.7 \%)\end{array}$ & $\begin{array}{l}28 / 103 \\
(27.2 \%)\end{array}$ & $\begin{array}{l}\text { RR } 1.20 \\
\text { (0.79 to } 1.84)\end{array}$ & $\begin{array}{l}5.5 \% \\
(-7.2 \text { to } \\
18.1)\end{array}$ & $\begin{array}{l}\oplus \bigcirc \bigcirc \bigcirc \\
\text { INSUFFICIENT }^{\mathrm{c}, \mathrm{j}}\end{array}$ \\
\hline $\begin{array}{l}\text { EBRT plus ADT vs. } \\
\text { EBRT: urinary } \\
\text { incontinence } \\
\text { (stress) grade } 2-4 \\
\text { (4.5 years) }\end{array}$ & $1 \mathrm{RCT}$ & Serious & $\begin{array}{l}\text { Not } \\
\text { serious }\end{array}$ & $\begin{array}{l}\text { Not } \\
\text { serious }\end{array}$ & $\begin{array}{l}\text { Very } \\
\text { serious }\end{array}$ & None & $\begin{array}{l}6 / 98 \\
(6.1 \%)\end{array}$ & $\begin{array}{l}7 / 103 \\
(6.8 \%)\end{array}$ & $\begin{array}{l}\text { RR } 0.90 \\
(0.31 \text { to } 2.59)\end{array}$ & $\begin{array}{l}-0.7 \% \\
(-7.5 \text { to } \\
6.1)\end{array}$ & $\begin{array}{l}\oplus \bigcirc \bigcirc \bigcirc \\
\text { INSUFFICIENTT, } \mathrm{j}^{\mathrm{c}}\end{array}$ \\
\hline $\begin{array}{l}\text { EBRT plus ADT vs. } \\
\text { EBRT: rectal } \\
\text { bleeding grade } 2-4 \\
\text { (4.5 years) }\end{array}$ & $1 \mathrm{RCT}$ & Serious & $\begin{array}{l}\text { Not } \\
\text { serious }\end{array}$ & $\begin{array}{l}\text { Not } \\
\text { serious }\end{array}$ & $\begin{array}{l}\text { Very } \\
\text { serious }\end{array}$ & None & $\begin{array}{l}19 / 98 \\
(19.4 \%)\end{array}$ & $\begin{array}{l}20 / 103 \\
(19.4 \%)\end{array}$ & $\begin{array}{l}\text { RR } 1.00 \\
\text { (0.57 to } 1.75)\end{array}$ & $\begin{array}{l}0.0 \% \\
(-11.0 \\
\text { to } 10.9)\end{array}$ & $\begin{array}{l}\oplus \bigcirc \bigcirc \bigcirc \\
\text { INSUFFICIENTT, } \mathrm{j}^{\mathrm{c}}\end{array}$ \\
\hline $\begin{array}{l}\text { EBRT plus } \\
\text { neoadjuvant and } \\
\text { concurrent ADT vs. } \\
\text { EBRT plus } \\
\text { concurrent and } \\
\text { adjuvant ADT: } \\
\text { Overall mortality- } \\
12.2 \text { years }\end{array}$ & $1 \mathrm{RCT}$ & $\begin{array}{l}\text { Not } \\
\text { serious }\end{array}$ & $\begin{array}{l}\text { Not } \\
\text { serious }\end{array}$ & $\begin{array}{l}\text { Not } \\
\text { serious }\end{array}$ & $\begin{array}{l}\text { Very } \\
\text { serious }\end{array}$ & None & $\begin{array}{l}75 / 215 \\
(34.9 \%)\end{array}$ & $\begin{array}{l}72 / 217 \\
(33.2 \%)\end{array}$ & $\begin{array}{l}\text { RR } 1.05 \\
\text { (0.81 to } 1.37 \text { ) }\end{array}$ & $\begin{array}{l}1.7 \% \\
(-7.2 \% \\
\text { to } \\
10.6 \%)\end{array}$ & $\begin{array}{l}\oplus \oplus \bigcirc \bigcirc \\
\mathrm{LOW}^{\mathrm{c}}\end{array}$ \\
\hline
\end{tabular}




\begin{tabular}{|c|c|c|c|c|c|c|c|c|c|c|c|}
\hline $\begin{array}{l}\text { Intervention/ } \\
\text { Comparison: } \\
\text { Outcome }\end{array}$ & $\begin{array}{l}\text { k= } \\
\text { Study } \\
\text { Design }\end{array}$ & $\begin{array}{l}\text { Risk of } \\
\text { Bias }\end{array}$ & $\begin{array}{l}\text { Incon- } \\
\text { sistency }\end{array}$ & $\begin{array}{l}\text { Indi- } \\
\text { rect- } \\
\text { ness }\end{array}$ & $\begin{array}{l}\text { Impre- } \\
\text { cision }\end{array}$ & $\begin{array}{l}\text { Other } \\
\text { Consid- } \\
\text { erations }\end{array}$ & I & C & $\begin{array}{l}\text { Relative } \\
(95 \% \mathrm{Cl})\end{array}$ & $\begin{array}{l}\text { Abso- } \\
\text { lute } \\
(95 \% \\
\mathrm{Cl})\end{array}$ & Certainty \\
\hline $\begin{array}{l}\text { EBRT plus } \\
\text { neoadjuvant and } \\
\text { concurrent ADT vs. } \\
\text { EBRT plus } \\
\text { concurrent and } \\
\text { adjuvant ADT: } \\
\text { Prostate cancer } \\
\text { mortality-12.2 years }\end{array}$ & $1 \mathrm{RCT}$ & Serious & $\begin{array}{l}\text { Not } \\
\text { serious }\end{array}$ & $\begin{array}{l}\text { Not } \\
\text { serious }\end{array}$ & Serious & None & $\begin{array}{l}7 / 215 \\
(3.3 \%)\end{array}$ & $\begin{array}{l}7 / 217 \\
(3.2 \%)\end{array}$ & $\begin{array}{l}\text { Peto OR } \\
1.01 \\
(0.35 \text { to } 2.93)\end{array}$ & $\begin{array}{l}0 \% \\
(-3.3 \% \\
\text { to } \\
3.4 \%)\end{array}$ & $\begin{array}{l}\oplus \bigoplus \bigcirc \bigcirc \\
\operatorname{LOW}^{b, j}\end{array}$ \\
\hline $\begin{array}{l}\text { EBRT plus } \\
\text { neoadjuvant and } \\
\text { concurrent ADT vs. } \\
\text { EBRT plus } \\
\text { concurrent and } \\
\text { adjuvant ADT: } \\
\text { Metastasis distant } \\
\text { progression-12.2 } \\
\text { years }\end{array}$ & $1 \mathrm{RCT}$ & Serious & $\begin{array}{l}\text { Not } \\
\text { serious }\end{array}$ & $\begin{array}{l}\text { Not } \\
\text { serious }\end{array}$ & $\begin{array}{l}\text { Very } \\
\text { serious }\end{array}$ & None & $\begin{array}{l}12 / 215 \\
(5.6 \%)\end{array}$ & $\begin{array}{l}9 / 217 \\
(4.1 \%)\end{array}$ & $\begin{array}{l}\text { Peto OR } \\
1.36 \\
(0.57 \text { to } 3.27)\end{array}$ & $\begin{array}{l}1.4 \% \\
(-2.6 \% \\
\text { to } \\
5.5 \%)\end{array}$ & $\begin{array}{l}\oplus \bigcirc \bigcirc \bigcirc \\
\text { INSUFFICIENT c, j }\end{array}$ \\
\hline $\begin{array}{l}\text { EBRT plus } \\
\text { neoadjuvant and } \\
\text { concurrent ADT vs. } \\
\text { EBRT plus } \\
\text { concurrent and } \\
\text { adjuvant ADT: Late } \\
\text { genitourinary } \\
\text { toxicity grade } \geq 3-3 \\
\text { years }\end{array}$ & $1 \mathrm{RCT}$ & Serious & $\begin{array}{l}\text { Not } \\
\text { serious }\end{array}$ & $\begin{array}{l}\text { Not } \\
\text { serious }\end{array}$ & Serious & None & $\begin{array}{l}6 / 213 \\
(2.8 \%)\end{array}$ & $\begin{array}{l}6 / 215 \\
(2.8 \%)\end{array}$ & $\begin{array}{l}\text { Peto OR } \\
1.01 \\
(0.32 \text { to } 3.18)\end{array}$ & $\begin{array}{l}0 \% \\
(-3.1 \% \\
\text { to } \\
3.2 \%)\end{array}$ & $\begin{array}{l}\oplus \oplus \bigcirc \bigcirc \\
L O W b, j\end{array}$ \\
\hline $\begin{array}{l}\text { EBRT / } \\
\text { Brachytherapy } \\
\text { Overall survival- } \\
\text { median } 10 \text { years }\end{array}$ & 1 Obs & $\begin{array}{l}\text { Very } \\
\text { serious }\end{array}$ & $\begin{array}{l}\text { Not } \\
\text { serious }\end{array}$ & $\begin{array}{l}\text { Not } \\
\text { serious }\end{array}$ & Serious & None & $\begin{array}{l}\text { KM est. } \\
75.5 \%(\mathrm{Cl} \\
71.8 \text { to } \\
79.4)\end{array}$ & $\begin{array}{l}\text { KM est. } \\
78.3 \%(\mathrm{Cl} \\
70.1 \text { to } \\
87.4)\end{array}$ & NA & $\begin{array}{l}\sim-2.8 \% \\
\text { (not } \\
\text { estimab } \\
\text { le) }\end{array}$ & $\begin{array}{l}\oplus \bigcirc \bigcirc \bigcirc \\
\text { INSUFFICIENT k,I }\end{array}$ \\
\hline $\begin{array}{l}\text { EBRT / } \\
\text { Brachytherapy }\end{array}$ & 1 Obs & $\begin{array}{l}\text { Very } \\
\text { serious }\end{array}$ & $\begin{array}{l}\text { Not } \\
\text { serious }\end{array}$ & $\begin{array}{l}\text { Not } \\
\text { serious }\end{array}$ & Serious & None & $\begin{array}{l}\text { KM est. } \\
96.2 \%(\mathrm{Cl} \\
94.3 \text { to } \\
98.1)\end{array}$ & $\begin{array}{l}\text { KM est. } \\
95.4 \%(\mathrm{Cl} \\
91.1 \text { to } \\
100)\end{array}$ & NA & $\begin{array}{l}\sim 0.8 \% \\
\text { (not } \\
\text { estimab } \\
\text { le) }\end{array}$ & $\begin{array}{l}\oplus \bigcirc \bigcirc \bigcirc \\
\text { INSUFFICIENT k,I }\end{array}$ \\
\hline
\end{tabular}




\begin{tabular}{|c|c|c|c|c|c|c|c|c|c|c|c|}
\hline $\begin{array}{l}\text { Intervention/ } \\
\text { Comparison: } \\
\text { Outcome }\end{array}$ & $\begin{array}{l}\text { k= } \\
\text { Study } \\
\text { Design }\end{array}$ & $\begin{array}{l}\text { Risk of } \\
\text { Bias }\end{array}$ & $\begin{array}{l}\text { Incon- } \\
\text { sistency }\end{array}$ & $\begin{array}{l}\text { Indi- } \\
\text { rect- } \\
\text { ness }\end{array}$ & $\begin{array}{l}\text { Impre- } \\
\text { cision }\end{array}$ & $\begin{array}{l}\text { Other } \\
\text { Consid- } \\
\text { erations }\end{array}$ & I & C & $\begin{array}{l}\text { Relative } \\
(95 \% \mathrm{Cl})\end{array}$ & $\begin{array}{l}\text { Abso- } \\
\text { lute } \\
(95 \% \\
\mathrm{Cl})\end{array}$ & Certainty \\
\hline $\begin{array}{l}\text { EBRT / } \\
\text { Brachytherapy }\end{array}$ & 1 Obs & $\begin{array}{l}\text { Very } \\
\text { serious }\end{array}$ & $\begin{array}{l}\text { Not } \\
\text { serious }\end{array}$ & $\begin{array}{l}\text { Not } \\
\text { serious }\end{array}$ & Serious & None & $\begin{array}{l}\text { KM est. } \\
90.6 \%(\mathrm{Cl} \\
87.9 \text { to } \\
93.3)\end{array}$ & $\begin{array}{l}\text { KM est. } \\
94.1 \%(\mathrm{Cl} \\
89.5 \text { to } \\
98.9)\end{array}$ & NA & $\begin{array}{l}\sim-3.5 \% \\
\text { (not } \\
\text { estimab } \\
\text { le) }\end{array}$ & $\begin{array}{l}\oplus \bigcirc \bigcirc \bigcirc \\
\text { INSUFFICIENT k,I }\end{array}$ \\
\hline $\begin{array}{l}\text { Conventionally } \\
\text { fractionated } \\
\text { EBRT/ultra- } \\
\text { hypofractionated } \\
\text { EBRT: overall } \\
\text { mortality-5-year } \\
\text { follow-up }\end{array}$ & $1 \mathrm{RCT}$ & $\begin{array}{l}\text { Not } \\
\text { serious }\end{array}$ & $\begin{array}{l}\text { Not } \\
\text { serious }\end{array}$ & $\begin{array}{l}\text { Not } \\
\text { serious }\end{array}$ & Serious & None & $\begin{array}{l}7.3 \% \\
(43 / 591)\end{array}$ & $\begin{array}{l}7.8 \% \\
(46 / 589)\end{array}$ & $\begin{array}{l}\text { RR } 0.93 \\
\text { (0.63 to 1.39) }\end{array}$ & $\begin{array}{l}-0.5 \% \\
(-3.5 \text { to } \\
2.5)\end{array}$ & $\begin{array}{l}\oplus \oplus \oplus \bigcirc \\
\text { MODERATE } b\end{array}$ \\
\hline $\begin{array}{l}\text { Conventionally } \\
\text { fractionated } \\
\text { EBRT/ultra- } \\
\text { hypofractionated } \\
\text { EBRT: prostate } \\
\text { cancer mortality-5- } \\
\text { year follow-up }\end{array}$ & $1 \mathrm{RCT}$ & Serious & $\begin{array}{l}\text { Not } \\
\text { serious }\end{array}$ & $\begin{array}{l}\text { Not } \\
\text { serious }\end{array}$ & Serious & None & $\begin{array}{l}1.4 \% \\
(8 / 591)\end{array}$ & $\begin{array}{l}1.9 \% \\
(11 / 589)\end{array}$ & $\begin{array}{l}\text { Peto OR } 0.72 \\
(0.29 \text { to } 1.79)\end{array}$ & $\begin{array}{l}-0.5 \% \\
(-2.0 \text { to } \\
0.9)\end{array}$ & $\begin{array}{l}\oplus \oplus \bigcirc \bigcirc \\
L O W b, j\end{array}$ \\
\hline $\begin{array}{l}\text { Conventionally } \\
\text { fractionated } \\
\text { EBRT/ultra- } \\
\text { hypofractionated } \\
\text { EBRT: metastasis- } \\
\text { 5-year-follow-up }\end{array}$ & $1 \mathrm{RCT}$ & Serious & $\begin{array}{l}\text { Not } \\
\text { serious }\end{array}$ & $\begin{array}{l}\text { Not } \\
\text { serious }\end{array}$ & Serious & None & $\begin{array}{l}6.6 \% \\
(39 / 591)\end{array}$ & $\begin{array}{l}6.5 \% \\
(38 / 589)\end{array}$ & $\begin{array}{l}\text { RR } 1.02 \\
\text { (0.66 to 1.58) }\end{array}$ & $\begin{array}{l}0.1 \% \\
(-2.7 \text { to } \\
3.0)\end{array}$ & $\begin{array}{l}\bigoplus \bigoplus \bigcirc \bigcirc \\
L^{\circ} W^{b, j}\end{array}$ \\
\hline $\begin{array}{l}\text { Conventionally } \\
\text { fractionated } \\
\text { EBRT/ultra- } \\
\text { hypofractionated } \\
\text { EBRT: urinary } \\
\text { toxicity grade } \geq 2 \\
\text { based on RTOG } \\
\text { morbidity scale-1 } \\
\text { and } 2 \text { year follow-up }\end{array}$ & $1 \mathrm{RCT}$ & Serious & $\begin{array}{l}\text { Not } \\
\text { serious }\end{array}$ & $\begin{array}{l}\text { Not } \\
\text { serious }\end{array}$ & Serious & None & $\begin{array}{l}\frac{1 \text { year }}{2.5 \%} \\
(13 / 529) \\
\frac{2 \text { years }}{5.6 \%} \\
(28 / 497)\end{array}$ & $\begin{array}{l}\frac{1 \text { year }}{6.1 \%} \\
(32 / 528) \\
\frac{2 \text { years }}{5.1 \%} \\
(25 / 492)\end{array}$ & $\begin{array}{l}\frac{1 \text { year }}{\text { RR } 0.41} \\
(0.22 \text { to } 0.76) \\
\frac{2 \text { years }}{\text { RR } 1.11} \\
(0.66 \text { to } 1.87)\end{array}$ & $\begin{array}{l}\frac{1 \text { year }}{-3.6 \%} \\
(-6.0 \text { to } \\
-1.2) \\
2 \text { years } \\
0.6 \% \\
(-2.3 \text { to } \\
3.4)\end{array}$ & $\begin{array}{l}\oplus \oplus \bigcirc \bigcirc \\
L^{\circ} W^{b, j}\end{array}$ \\
\hline
\end{tabular}




\begin{tabular}{|c|c|c|c|c|c|c|c|c|c|c|c|}
\hline $\begin{array}{l}\text { Intervention/ } \\
\text { Comparison: } \\
\text { Outcome }\end{array}$ & $\begin{array}{l}\text { k= } \\
\text { Study } \\
\text { Design }\end{array}$ & $\begin{array}{l}\text { Risk of } \\
\text { Bias }\end{array}$ & $\begin{array}{l}\text { Incon- } \\
\text { sistency }\end{array}$ & $\begin{array}{l}\text { Indi- } \\
\text { rect- } \\
\text { ness }\end{array}$ & $\begin{array}{l}\text { Impre- } \\
\text { cision }\end{array}$ & $\begin{array}{l}\text { Other } \\
\text { Consid- } \\
\text { erations }\end{array}$ & I & C & $\begin{array}{l}\text { Relative } \\
(95 \% \mathrm{Cl})\end{array}$ & $\begin{array}{l}\text { Abso- } \\
\text { lute } \\
(95 \% \\
\mathrm{Cl})\end{array}$ & Certainty \\
\hline $\begin{array}{l}\text { Conventionally } \\
\text { fractionated } \\
\text { EBRT/ultra- } \\
\text { hypofractionated } \\
\text { EBRT: Bowel } \\
\text { toxicity grade } \geq 2 \\
\text { based on RTOG } \\
\text { morbidity scale-2- } \\
\text { year followup }\end{array}$ & $1 \mathrm{RCT}$ & Serious & $\begin{array}{l}\text { Not } \\
\text { serious }\end{array}$ & $\begin{array}{l}\text { Not } \\
\text { serious }\end{array}$ & Serious & None & $\begin{array}{l}3.2 \% \\
(16 / 496)\end{array}$ & $\begin{array}{l}1.8 \% \\
(9 / 495)\end{array}$ & $\begin{array}{l}\text { Peto OR } 1.77 \\
(0.80 \text { to } 3.92)\end{array}$ & $\begin{array}{l}1.4 \% \\
(-0.5 \text { to } \\
3.4)\end{array}$ & $\begin{array}{l}\oplus \oplus \bigcirc \bigcirc \\
L^{b} W^{b, j}\end{array}$ \\
\hline $\begin{array}{l}\text { Conventionally } \\
\text { fractionated } \\
\text { EBRT/ultra- } \\
\text { hypofractionated } \\
\text { EBRT: Erectile } \\
\text { function-1 and 2- } \\
\text { year follow-up }\end{array}$ & $1 \mathrm{RCT}$ & Serious & $\begin{array}{l}\text { Not } \\
\text { serious }\end{array}$ & $\begin{array}{l}\text { Not } \\
\text { serious }\end{array}$ & $\begin{array}{l}\text { Very } \\
\text { serious }\end{array}$ & None & NR & NR & not estimable & $\begin{array}{l}\text { Not } \\
\text { significa } \\
\text { ntly } \\
\text { different } \\
(p=0.59 \\
-0.60)\end{array}$ & $\begin{array}{l}\oplus \bigcirc \bigcirc \bigcirc \\
\text { INSUFFICIENT j,m }\end{array}$ \\
\hline
\end{tabular}

Abbreviations: 3D-CRT=3-dimensional conformal radiation therapy; $\mathrm{C}=$ comparison; $\mathrm{CI}=$ confidence interval; EBRT=external beam radiation therapy; $\mathrm{I}=$ intervention;

$\mathrm{KM}=$ Kaplan-Meier estimate, propensity score adjusted; LDR-PB=low-dose rate prostate brachytherapy; NR=not reported; RCT=randomized controlled trial; RR=risk ratio;

RTOG=Radiation Therapy Oncology Group

Explanations:

a Downgraded for study limitations (14 people received wrong intervention, 15 people received no intervention).

b. Downgraded for one level imprecision (confidence interval overlapped threshold for small unimportant effect).

c. Downgraded for two levels imprecision (very wide CIs).

d. Downgraded for $\ldots . p=0.049$. Percentages reported without numerators or CIs.

e. $p$-value NR at 1 year. $p$-value at 5 years $\mathrm{p}=0.60$. No CI reported.

f. Residual confounding bias expected after adjustment; some selection and reporting bias

g. Very little reported for the propensity-score matched analyses.

h. CI NR; p-value not significant.

i. No CI or relative effect estimate reported.

j. Rated down one level for risk of bias: outcomes except all-cause mortality could be influenced by lack of blinding

k. Rated down two levels for risk of bias (observational study)

l. Rated down one level for imprecision (unable to estimate based on data presented)

m. Rated down two levels for imprecision (difficult to interpret based on graphical display of data only) 


\section{Appendix I. Radical Prostatectomy}

Table I-1. Risk of bias assessments for randomized controlled trials: radical prostatectomy

\begin{tabular}{|c|c|c|c|c|c|c|c|c|}
\hline $\begin{array}{l}\text { Intervention/ } \\
\text { Comparison } \\
\text { (Outcomes) }\end{array}$ & Author, Year & $\begin{array}{l}\text { Selection } \\
\text { Bias }\end{array}$ & $\begin{array}{l}\text { Performance } \\
\text { Bias }\end{array}$ & $\begin{array}{l}\text { Detection } \\
\text { Bias }\end{array}$ & Attrition & $\begin{array}{l}\text { Reporting } \\
\text { Bias }\end{array}$ & Other Bias & Overall \\
\hline $\begin{array}{l}\text { RP/AM } \\
\text { (mortality, metastases, } \\
\text { QoL, harms) }\end{array}$ & $\begin{array}{l}\text { Hamdy } 2016^{11} \\
\text { Donovan } \\
2016^{12} \\
\text { Lane } 2016^{13}\end{array}$ & Low & Low & Low & $\begin{array}{l}\text { Low for mortality } \\
\text { Moderate for harms (15- } \\
17 \%)\end{array}$ & Low & None & Low \\
\hline $\begin{array}{l}\mathrm{RP} / \mathrm{RT}+\mathrm{ADT} \\
\text { (mortality, metastases, } \\
\text { QoL, harms) }\end{array}$ & $\begin{array}{l}\text { Hamdy } 2016^{11} \\
\text { Donovan } \\
2016^{12} \\
\text { Lane } 2016^{13}\end{array}$ & Low & Low & Low & $\begin{array}{l}\text { Low for mortality } \\
\text { Moderate for harms (15- } \\
17 \%)\end{array}$ & Low & None & Low \\
\hline $\begin{array}{l}\text { RP + ADT/ RT + High- } \\
\text { dose brachytherapy + } \\
\text { ADT } \\
\text { (mortality, metastases, } \\
\text { QoL, harms) }\end{array}$ & $\begin{array}{l}\text { Lennernäs } \\
2015^{52}\end{array}$ & Low & Low & Unclear & $\begin{array}{l}\text { Low for mortality } \\
\text { Moderate-high for } \\
\text { harms } \\
(38 \%)\end{array}$ & $\begin{array}{l}\text { Unclear n's for } \\
\text { harms } \\
\text { outcomes }\end{array}$ & None & Moderate \\
\hline $\begin{array}{l}\text { Laparoscopic } \\
\text { RP/RARP } \\
\text { (QoL, harms) }\end{array}$ & $\begin{array}{l}\text { Porpiglia } \\
2018^{53}\end{array}$ & Low & $\begin{array}{l}\text { Single } \\
\text { surgeon } \\
\text { performed all } \\
\text { procedures }\end{array}$ & Unclear & Low & Low & None & Moderate \\
\hline $\begin{array}{l}\text { RP vs. HIFU } \\
\text { (urinary and fecal } \\
\text { incontinence,erectile } \\
\text { dysfunction) }\end{array}$ & Hamdy $2018^{54}$ & Low & Low & High & Unclear & Low & None & Medium \\
\hline
\end{tabular}

Abbreviations: ADT=androgen deprivation therapy; AM=active monitoring; HIFU=high intensity focused ultrasound; QoL=quality of life; RARP=robotic-assisted radical

prostatectomy; RCT=randomized controlled trial; $\mathrm{ROB}=$ =risk of bias; $\mathrm{RP}=$ radical prostatectomy; $\mathrm{RT}=$ =radiation therapy

Table I-2. Summary risk of bias assessments for observational studies: radical prostatectomy comparisons

\begin{tabular}{|l|l|l|l|l|l|l|l|}
\hline $\begin{array}{l}\text { Inter- } \\
\text { vention/ } \\
\text { Comparison } \\
\text { (Outcomes) }\end{array}$ & Author, Year & $\begin{array}{l}\text { Bias Due to } \\
\text { Confounding }\end{array}$ & $\begin{array}{l}\text { Bias in } \\
\text { Selection of } \\
\text { Participants } \\
\text { Into the } \\
\text { Study }\end{array}$ & $\begin{array}{l}\text { Bias in } \\
\text { Classification } \\
\text { of } \\
\text { Interventions }\end{array}$ & $\begin{array}{l}\text { Bias Due to } \\
\text { Deviations } \\
\text { From } \\
\text { Intended } \\
\text { Interventions }\end{array}$ & $\begin{array}{l}\text { Bias Due } \\
\text { to Missing } \\
\text { Data }\end{array}$ & $\begin{array}{l}\text { Bias in } \\
\text { Measurement } \\
\text { of Outcomes }\end{array}$ \\
\hline $\begin{array}{l}\text { RARP/ Open } \\
\text { RRP }\end{array}$ & $\begin{array}{l}\text { Sooriakumaran } \\
2018^{55}\end{array}$ & Moderate & $\begin{array}{l}\text { Moderate } \\
\text { Selection } \\
\text { Reported } \\
\text { Result }\end{array}$ & Low & Low & Low & Moderate \\
\hline $\begin{array}{l}\text { RARP/ Open } \\
\text { RRP }\end{array}$ & Loeb $2016^{56}$ & Serious & Serious & Critical & Low & Low & Moderate \\
\hline
\end{tabular}




\begin{tabular}{|c|c|c|c|c|c|c|c|c|c|}
\hline $\begin{array}{l}\text { Inter- } \\
\text { vention/ } \\
\text { Comparison } \\
\text { (Outcomes) }\end{array}$ & Author, Year & $\begin{array}{l}\text { Bias Due to } \\
\text { Confounding }\end{array}$ & $\begin{array}{l}\text { Bias in } \\
\text { Selection of } \\
\text { Participants } \\
\text { Into the } \\
\text { Study }\end{array}$ & $\begin{array}{l}\text { Bias in } \\
\text { Classification } \\
\text { of } \\
\text { Interventions }\end{array}$ & $\begin{array}{l}\text { Bias Due to } \\
\text { Deviations } \\
\text { From } \\
\text { Intended } \\
\text { Interventions }\end{array}$ & $\begin{array}{l}\text { Bias Due } \\
\text { to Missing } \\
\text { Data }\end{array}$ & $\begin{array}{l}\text { Bias in } \\
\text { Measurement } \\
\text { of Outcomes }\end{array}$ & $\begin{array}{l}\text { Bias in } \\
\text { Selection } \\
\text { of the } \\
\text { Reported } \\
\text { Result }\end{array}$ & Overall \\
\hline $\begin{array}{l}\text { RARP/ Open } \\
\text { RP }\end{array}$ & $\begin{array}{l}\text { Herlemann } \\
2018^{57}\end{array}$ & Moderate & Serious & Moderate & Moderate & Moderate & Moderate & Moderate & Serious \\
\hline $\begin{array}{l}\text { RP } \\
\text { (aggregate) } \\
\text { /BT }\end{array}$ & Chang $2017^{58}$ & Moderate & Serious & Moderate & Low & Low & Serious & Moderate & Serious \\
\hline $\begin{array}{l}\text { RP/ADT } \\
\text { (quality of } \\
\text { life) }\end{array}$ & $\begin{array}{l}\text { Herden, 2016 } \\
\text { Weissbach, } \\
2016^{59}\end{array}$ & Moderate & Low & Moderate & Low & Serious & Serious & Low & Serious \\
\hline $\begin{array}{l}\text { RP } \\
\text { (aggregate) } \\
\text { /Low-dose } \\
\text { BT/AS/EBRT }\end{array}$ & $\begin{array}{l}\text { Hoffman } \\
2020^{60}\end{array}$ & Moderate & Moderate & Serious & Moderate & Moderate & Moderate & Moderate & Serious \\
\hline RP/FLA & Zheng $2019^{61}$ & Moderate & Moderate & Critical & Moderate & Moderate & Moderate & Moderate & Critical \\
\hline RP/EBRT & Knipper $2019^{62}$ & Critical & Critical & Serious & $\begin{array}{l}\text { No } \\
\text { Information }\end{array}$ & $\begin{array}{l}\text { No } \\
\text { Information }\end{array}$ & Low & Low & Critical \\
\hline
\end{tabular}

Abbreviations: ADT=androgen deprivation therapy; BT=brachytherapy; EBRT=external beam radiation therapy; FLA=focal laser ablation; RCT=randomized controlled trial; $\mathrm{RARP}=$ robotic-assisted radical prostatectomy; $\mathrm{ROB}=$ =risk of bias; $\mathrm{RP}=$ radical prostatectomy; $\mathrm{RRP}=$ retropubic radical prostatectomy 
Table I-3. Characteristics of eligible studies: radical prostatectomy comparisons

\begin{tabular}{|c|c|c|c|c|c|}
\hline $\begin{array}{l}\text { Study } \\
\text { Design } \\
\text { Country } \\
\text { ROB }\end{array}$ & $\mathrm{N}=$ & Population & $\begin{array}{l}\text { Intervention } \\
\text { Frequency } \\
\text { Duration }\end{array}$ & $\begin{array}{l}\text { Comparison } \\
\text { Frequency } \\
\text { Duration }\end{array}$ & $\begin{array}{l}\text { Followup Time(s) } \\
\text { Outcome } \\
\text { (Instrument) }\end{array}$ \\
\hline $\begin{array}{l}\text { Hamdy } 2016^{11,12,} \\
21 \\
\text { ProtecT trial } \\
\text { RCT } \\
\text { UK } \\
\text { Low }\end{array}$ & $\begin{array}{l}1098 \\
\text { (exclude. } \\
\text { AM arm) }\end{array}$ & $\begin{array}{l}\text { Inclusion criteria } \\
\text { Men with clinically localized } \\
\text { prostate cancer aged } 50-69 \\
\text { years, with a PSA } \geq 3.0 \mu g / L \text { to } \\
<20.0 \mu \mathrm{g} / \mathrm{L} \text { without a previous } \\
\text { malignancy (apart from skin } \\
\text { cancer), renal transplant or on } \\
\text { renal dialysis, major CVD or } \\
\text { respiratory comorbidities, } \\
\text { bilateral hip replacement, or an } \\
\text { estimated life expectancy }<10 \\
\text { years. Men with a PSA } \geq 10 \mu \mathrm{L} / \mathrm{L} \\
\text { or a Gleason score }>7 \text { points } \\
\text { underwent an isotope bone } \\
\text { scan to exclude metastatic } \\
\text { disease. } \\
\text { Clinical Stage (\%) } \\
\text { T1c } 76 \% \\
\text { T2 } 24 \% \\
\text { Gleason score }(\%) \\
677 \% \\
721 \% \\
8-102 \% \\
\text { Age (median) } \\
62 \\
\text { Race }(\%) \\
\text { White } 99 \%\end{array}$ & $\begin{array}{l}\text { Radical prostatectomy } \\
\text { predominant approach } \\
\text { was open retropubic } \\
\text { radical prostatectomy. } \\
\text { Participants with a } \\
\text { baseline PSA } \geq 10 \mu \mathrm{g} / \mathrm{L} \\
\text { or a biopsy Gleason } \\
\text { score } \geq 7 \text { points } \\
\text { received bilateral lymph } \\
\text { adenectomy. } \\
\text { Postoperatively, } \\
\text { PSA levels were } \\
\text { measured every } 3 \\
\text { months for the first year, } \\
\text { every } 6 \text { months for } 2 \\
\text { years, and then yearly. }\end{array}$ & $\begin{array}{l}\text { Radiation therapy } \\
\text { (EBRT+ADT) } \\
\text { External beam 3D } \\
\text { conformal radiation } \\
\text { therapy, } \\
\text { Dose } \\
74 \text { Gy in } 37 \text { fractions } \\
\text { Neoadjuvant androgen } \\
\text { suppression was given for } \\
\text { 3-6 months before and } \\
\text { concomitantly with 3D- } \\
\text { conformal radiation } \\
\text { therapy. }\end{array}$ & $\begin{array}{l}10 \text { years } \\
\text { All-cause mortality } \\
\text { Prostate-cancer-specific } \\
\text { mortality } \\
6 \text { years } \\
\text { Urinary incontinence } \\
\text { Erectile dysfunction } \\
\text { Fecal incontinence and bloody } \\
\text { stools } \\
\text { SF-12 physical and mental } \\
\text { health subscales and the } \\
\text { EORTC QLQ-C30 } \\
5 \text { years } \\
\text { Prostate-cancer-specific } \\
\text { mortality } \\
1 \text { year } \\
\text { Urinary incontinence } \\
\text { Erectile dysfunction } \\
\text { Fecal incontinence and bloody } \\
\text { stools } \\
\text { SF-12 physical and mental } \\
\text { health subscales } \\
\text { EORTC QLQ-C30 }\end{array}$ \\
\hline
\end{tabular}




\begin{tabular}{|c|c|c|c|c|c|}
\hline $\begin{array}{l}\text { Study } \\
\text { Design } \\
\text { Country } \\
\text { ROB }\end{array}$ & $\mathrm{N}=$ & Population & $\begin{array}{l}\text { Intervention } \\
\text { Frequency } \\
\text { Duration }\end{array}$ & $\begin{array}{l}\text { Comparison } \\
\text { Frequency } \\
\text { Duration }\end{array}$ & $\begin{array}{l}\text { Followup Time(s) } \\
\text { Outcome } \\
\text { (Instrument) }\end{array}$ \\
\hline $\begin{array}{l}\text { Hamdy } 2016^{11,12,} \\
{ }^{21} \text { ProtecT trial } \\
\text { RCT } \\
\text { UK } \\
\text { Low }\end{array}$ & $\begin{array}{l}1090 \\
\text { (exclude. } \\
\text { RT arm) }\end{array}$ & $\begin{array}{l}\text { Inclusion criteria } \\
\text { Men with clinically localized } \\
\text { prostate cancer aged } 50-69 \\
\text { years, with a PSA } \geq 3.0 \mu g / L \text { to } \\
<20.0 \mu g / L \text { without a previous } \\
\text { malignancy (apart from skin } \\
\text { cancer), renal transplant or on } \\
\text { renal dialysis, major CVD or } \\
\text { respiratory comorbidities, } \\
\text { bilateral hip replacement, or an } \\
\text { estimated life expectancy }<10 \\
\text { years. Men with a PSA } \geq 10 \mu g / L \\
\text { or a Gleason score }>7 \text { points } \\
\text { underwent an isotope bone } \\
\text { scan to exclude metastatic } \\
\text { disease. } \\
\text { Clinical Stage (\%) } \\
\text { T1c } 77 \% \\
\text { T2 } 23 \% \\
\text { Gleason score (\%) } \\
677 \% \\
720 \% \\
8-102 \% \\
\text { Age (median) } \\
62 \\
\text { Race (\%) } \\
\text { White } 99 \%\end{array}$ & $\begin{array}{l}\text { Radical prostatectomy } \\
\text { predominant approach } \\
\text { was open retropubic } \\
\text { radical prostatectomy. } \\
\text { Participants with a } \\
\text { baseline PSA } \geq 10 \mu \mathrm{g} / \mathrm{L} \\
\text { or a biopsy Gleason } \\
\text { score } \geq 7 \text { points } \\
\text { received bilateral lymph } \\
\text { adenectomy. } \\
\text { Postoperatively, } \\
\text { PSA levels were } \\
\text { measured every } 3 \\
\text { months for the first year, } \\
\text { every } 6 \text { months for } 2 \\
\text { years, and then yearly. }\end{array}$ & $\begin{array}{l}\text { Active monitoring } \\
\text { PSA levels measured and } \\
\text { reviewed every } 3 \text { months } \\
\text { in the first year and twice } \\
\text { yearly thereafter. } \\
\text { Changes in PSA levels } \\
\text { were assessed at each } \\
\text { visit, and a rise } \geq 50 \% \\
\text { during the previous } 12 \\
\text { months triggered repeat } \\
\text { testing within } 6-9 \text { weeks. } \\
\text { If the PSA levels were } \\
\text { persistently raised, or the } \\
\text { patient had any other } \\
\text { concerns, a review } \\
\text { appointment was made } \\
\text { with the center urologist } \\
\text { for discussion of further } \\
\text { tests including re-biopsy } \\
\text { and all relevant } \\
\text { management options. }\end{array}$ & $\begin{array}{l}10 \text { years } \\
\text { All-cause mortality } \\
\text { Prostate-cancer-specific } \\
\text { mortality } \\
6 \text { years } \\
\text { Urinary incontinence } \\
\text { Erectile dysfunction } \\
\text { Fecal incontinence and bloody } \\
\text { stools } \\
\text { SF-12 physical and mental } \\
\text { health subscales and the } \\
\text { EORTC QLQ-C30 } \\
5 \text { years } \\
\text { Prostate-cancer-specific } \\
\text { mortality } \\
1 \text { year } \\
\text { Urinary incontinence } \\
\text { Erectile dysfunction } \\
\text { Fecal incontinence and bloody } \\
\text { stools } \\
\text { SF-12 physical and mental } \\
\text { health subscales } \\
\text { EORTC QLQ-C30 }\end{array}$ \\
\hline
\end{tabular}




\begin{tabular}{|c|c|c|c|c|c|}
\hline $\begin{array}{l}\text { Study } \\
\text { Design } \\
\text { Country } \\
\text { ROB }\end{array}$ & $\mathrm{N}=$ & Population & $\begin{array}{l}\text { Intervention } \\
\text { Frequency } \\
\text { Duration }\end{array}$ & $\begin{array}{l}\text { Comparison } \\
\text { Frequency } \\
\text { Duration }\end{array}$ & $\begin{array}{l}\text { Followup Time(s) } \\
\text { Outcome } \\
\text { (Instrument) }\end{array}$ \\
\hline $\begin{array}{l}\text { Lennernäs } \\
2015^{52} \\
\text { RCT } \\
\text { Sweden } \\
\text { Moderate }\end{array}$ & 89 & $\begin{array}{l}\text { Inclusion criteria } \\
\text { Men with clinically localized/ } \\
\text { locally advanced prostate } \\
\text { cancer clinical category T1b - } \\
\text { T3a, N0, M0 and a PSA value } \geq \\
50 \text { ng/ml } \\
\text { Clinical Stage (\%) } \\
\text { T1 39\% } \\
\text { T2 37\% } \\
\text { T3 8\% } \\
\text { Gleason score (\%) } \\
\text { NR } \\
\text { Unknown 16\% } \\
\text { Age (median) } \\
64 \text { (RP) and } 66 \text { (RT) } \\
\text { Race (\%) } \\
\text { NR }\end{array}$ & $\begin{array}{l}\text { Radical prostatectomy } \\
\text { recommended RP } \\
\text { approach was the nerve } \\
\text { sparing method } \\
\text { Lymphadenectomy was } \\
\text { conducted in participants } \\
\text { with stage T1b-T2 PC } \\
\text { and PSA } \geq 20 \text { ng/ml and } \\
\text { in all those with either T3 } \\
\text { tumors, irrespective of } \\
\text { grades, or grade } 3 \\
\text { tumors irrespective of } \\
\text { stages. } \\
\text { All patients were treated } \\
\text { with neoadjuvant ADT } \\
\text { that continued for six } \\
\text { months. }\end{array}$ & $\begin{array}{l}\text { High-dose radiation } \\
\text { therapy } \\
\text { Combined EBRT ( } 25 \times 2 \\
\text { Gy) and high-dose } \\
\text { brachytherapy ( } 2 \text { × } 10 \mathrm{~Gy} \text {; } \\
\text { minimum radiation dose } \\
\text { was } 10 \mathrm{~Gy}) \text {. } \\
\text { Clinical target volume } \\
\text { comprised the tumor and } \\
\text { the entire prostate gland } \\
\text { All patients were treated } \\
\text { with neoadjuvant ADT that } \\
\text { continued for six months. }\end{array}$ & $\begin{array}{l}10 \text { years } \\
\text { All-cause mortality } \\
\text { Prostate-cancer-specific } \\
\text { mortality } \\
2 \text { years } \\
\text { Urinary incontinence } \\
\text { Erectile dysfunction } \\
\text { Fecal incontinence and bloody } \\
\text { stools } \\
\text { EORTC QLQ-C30 }\end{array}$ \\
\hline $\begin{array}{l}\text { Porpiglia } 2018^{53} \\
\text { RCT } \\
\text { Italy } \\
\text { Moderate }\end{array}$ & 120 & $\begin{array}{l}\text { Inclusion criteria } \\
\text { Men with clinically staged T1- } \\
\text { T2N0M0 aged 40-75 years. } \\
\text { Clinical Stage (\%) } \\
\text { T1-T2 100\% } \\
\text { Gleason score (\%) } \\
2-650 \% \\
743 \% \\
8-107 \% \\
\text { Age } \\
64 \\
\text { Race (\%) } \\
\text { NR }\end{array}$ & $\begin{array}{l}\text { Laparoscopic radical } \\
\text { prostatectomy, using } \\
\text { transperitoneal } \\
\text { anterograde technique. } \\
\text { Bilateral nerve-sparing } \\
\text { procedure and extended } \\
\text { pelvic lymph-node } \\
\text { dissection performed } \\
\text { when indicated. }\end{array}$ & $\begin{array}{l}\text { Robot-assisted radical } \\
\text { prostatectomy, using } \\
\text { transperitoneal } \\
\text { anterograde technique. } \\
\text { Bilateral nerve-sparing } \\
\text { procedure and extended } \\
\text { pelvic lymph-node } \\
\text { dissection performed } \\
\text { when indicated. }\end{array}$ & $\begin{array}{l}5 \text { years } \\
\text { Urinary incontinence } \\
\text { Erectile function (potency) } \\
\text { Expanded Prostate Cancer Index } \\
\text { Composite questionnaire - } \\
\text { Patient satisfaction and health } \\
\text { status }\end{array}$ \\
\hline
\end{tabular}




\begin{tabular}{|c|c|c|c|c|c|}
\hline $\begin{array}{l}\text { Study } \\
\text { Design } \\
\text { Country } \\
\text { ROB }\end{array}$ & $\mathrm{N}=$ & Population & $\begin{array}{l}\text { Intervention } \\
\text { Frequency } \\
\text { Duration }\end{array}$ & $\begin{array}{l}\text { Comparison } \\
\text { Frequency } \\
\text { Duration }\end{array}$ & $\begin{array}{l}\text { Followup Time(s) } \\
\text { Outcome } \\
\text { (Instrument) }\end{array}$ \\
\hline $\begin{array}{l}\text { Sooriakumaran } \\
2018^{55} \\
\text { Observational } \\
\text { Sweden } \\
\text { Moderate }\end{array}$ & $\begin{array}{l}2545, \\
1702 \\
\text { potent at } \\
\text { baseline }\end{array}$ & $\begin{array}{l}\text { Inclusion criteria } \\
\text { men aged }<75 \text { years, PC staged } \\
\text { clinically as T1-T3, PSA<20 } \\
\text { ng/ml, no previous malignancy, } \\
\text { and no signs of distant } \\
\text { metastases. Men who were } \\
\text { preoperatively potent ( } \mathrm{n}=1702 \\
\text { using the same definition as for } \\
\text { post-operative potency given } \\
\text { below) } \\
\text { were included in the erectile } \\
\text { function analyses. } \\
\text { Clinical Stage (\%) } \\
\text { T1 } 63 \% \\
\text { T2 34\% } \\
\text { T3 3\% } \\
\text { Gleason score (\%) } \\
\leq 793 \% \\
\geq 87 \% \\
\text { Age (median) } \\
63.3-63.5 \\
\text { Race } \\
\text { NR }\end{array}$ & $\begin{array}{l}\text { Robot-assisted } \\
\text { laparoscopic radical } \\
\text { prostatectomy } \\
\text { Bilateral to no nerve- } \\
\text { sparing procedures } \\
\text { were performed when } \\
\text { indicated. }\end{array}$ & $\begin{array}{l}\text { Open retropubic radical } \\
\text { prostatectomy } \\
\text { Bilateral to no nerve- } \\
\text { sparing procedures } \\
\text { were performed when } \\
\text { indicated. }\end{array}$ & $\begin{array}{l}2 \text { years } \\
\text { Erectile function (potency) } \\
\end{array}$ \\
\hline
\end{tabular}




\begin{tabular}{|c|c|c|c|c|c|}
\hline $\begin{array}{l}\text { Study } \\
\text { Design } \\
\text { Country } \\
\text { ROB }\end{array}$ & $\mathrm{N}=$ & Population & $\begin{array}{l}\text { Intervention } \\
\text { Frequency } \\
\text { Duration }\end{array}$ & $\begin{array}{l}\text { Comparison } \\
\text { Frequency } \\
\text { Duration }\end{array}$ & $\begin{array}{l}\text { Followup Time(s) } \\
\text { Outcome } \\
\text { (Instrument) }\end{array}$ \\
\hline $\begin{array}{l}\text { Hamdy } 2018^{54} \\
\text { RCT } \\
\text { UK ( } 5 \text { centers) } \\
\text { Medium }\end{array}$ & 82 & $\begin{array}{l}\text { Adult men with unilateral, } \\
\text { clinically significant intermediate } \\
\text { risk PC, Gleason score of } 7 \text { or } \\
\text { high-volume Gleason score of } 6 \text {, } \\
\text { PSA } \leq 20 \text { ng/ml, clinical stage } \\
\leq \text { T2b, life expectancy } \geq 10 \text { years, } \\
\text { be fit, eligible and normally } \\
\text { destined for radical surgery, } \\
\text { have no concomitant cancer, no } \\
\text { previous treatment of PC, } \\
\text { proficiency in English language } \\
\text { RP Arm } \\
\text { T1c: } 1 / 41(2.4 \%) \\
\text { T2: } 12 / 41(29.3 \%) \\
\text { T2a: } 22 / 41(53.7 \%) \\
\text { T2b: } 5 / 41(12.2 \%) \\
\text { T2c: } 1 / 41 \text { (2.4\%) } \\
\text { Gleason score } 3+4: 32(78 \%) \\
\text { Gleason score } 4+3: 8(19.5 \%) \\
\text { High volume } 6: 1(2.4 \%) \\
\text { Age (median) } 65.5 \\
\text { White: } 40 / 41(97.6 \%) \\
\text { HIFU Arm } \\
\text { T1c: } 0 \\
\text { T2: } 11 / 41(26.8 \%) \\
\text { T2a: } 26 / 41(63.4 \%) \\
\text { T2b: } 2 / 41(4.9 \%) \\
\text { T2c: } 1 / 41(2.4 \%) \\
\text { Gleason score } 3+4: 39(95 \%) \\
\text { Gleason score } 4+3: 8(19.5 \%) \\
\text { High volume } 6: 1(2.4 \%) \\
\text { Age (median) } 66.4 \\
\text { White: } 39 / 41(95.1 \%)\end{array}$ & $\begin{array}{l}\text { Conventional open, } \\
\text { laparoscopic or robot- } \\
\text { assisted RP }\end{array}$ & HIFU & $\begin{array}{l}12 \text { months } \\
\text { Urinary incontinence } \\
\text { Fecal incontinence } \\
\text { Erectile dysfunction }\end{array}$ \\
\hline
\end{tabular}

Abbreviations: ADT=androgen deprivation therapy; AM=active monitoring; CVD=cardiovascular disease; EBRT=external beam radiation therapy; EORTC QLQ-C30=European Organization for Research and Treatment of Cancer Quality-of-Life Questionnaire-Core 30 module; Gy=Gray units; HIFU=high-intensity focused ultrasound; NR=not reported; PC=prostate cancer; PSA=prostate specific antigen; ROB=risk of bias; RP=radical prostatectomy; RT=radiation therapy; RCT=randomized controlled trial; SF12=short form 12 item health survey questionnaire; UK=United Kingdom; $\mu \mathrm{g} / \mathrm{L}=$ micrograms per liter 
Table I-4. Mortality, survival, and metastases outcomes: radical prostatectomy comparisons

\begin{tabular}{|c|c|c|c|c|}
\hline $\begin{array}{l}\text { Intervention/ } \\
\text { Comparison }\end{array}$ & $\begin{array}{l}\text { Study } \\
\text { Design } \\
\text { (Trial/Registry) } \\
\text { Followup } \\
\text { Risk of Bias }\end{array}$ & Overall Survival and Mortality & $\begin{array}{l}\text { Prostate Cancer Specific Survival and } \\
\text { Mortality }\end{array}$ & $\begin{array}{l}\text { Metastases or Metastatic } \\
\text { Progression Free Survival } \\
\text { (define) }\end{array}$ \\
\hline $\begin{array}{l}\text { RPI } \\
\text { AM }\end{array}$ & $\begin{array}{l}\text { Hamdy } 2016^{11} \\
\text { ProtecT } \\
\text { RCT } \\
\text { Low }\end{array}$ & $\begin{array}{l}\text { All-cause Mortality at } 10 \text { years } \\
\text { (median) } \\
\text { RP: } 9.9 \%(55 / 553) 10.1(95 \% \mathrm{Cl} \\
7.8 \text { to } 13.2) \text { deaths per } 1000 \\
\text { person-years } \\
\text { RT: } 10.8 \%(59 / 545) 10.9(95 \% \mathrm{Cl} \\
8.5 \text { to } 14.1) \text { deaths per } 1000 \\
\text { person-years } \\
\text { RR=0.92 }(95 \% \mathrm{Cl} 0.65 \text { to } 1.30) ; \\
\text { ARD }=-0.9(95 \% \mathrm{Cl}-4.5 \text { to } 2.7)\end{array}$ & $\begin{array}{l}\text { Prostate Cancer Specific Survival at } 5 \\
\text { years } \\
\text { RP: } 100 \% \\
\text { AS: } 99 \% \\
\text { Prostate Cancer Mortality at } 10 \text { years } \\
\text { RP: } 0.9 \%(5 / 553) 0.9(95 \% \mathrm{Cl} 0.4 \text { to } 2.2) \\
\text { deaths per } 1000 \text { person-years } \\
\text { AM: } 1.5 \%(8 / 545) 1.5 \text { (95\% Cl } 0.7 \text { to } 3.0) \\
\text { deaths per } 1000 \text { person-years } \\
\text { P=0.48 between groups (+AS) } \\
\text { HR }=0.63(95 \% \mathrm{Cl} 0.21 \text { to } 1.93) \\
\text { Peto OR }=0.62(95 \% \mathrm{Cl} 0.21 \text { to } 1.84) \\
\text { ARD }=-0.6 \%(95 \% \mathrm{Cl}-1.8 \text { to } 0.7)\end{array}$ & $\begin{array}{l}\text { RP: } 2.4 \%(13 / 553) 2.4(95 \% \mathrm{Cl} 1.4 \\
\text { to } 4.2) \text { metastatic disease per } 1000 \\
\text { person-years } \\
\text { AM: } 6.1 \%(33 / 545) 6.3(95 \% \mathrm{Cl} 4.5 \\
\text { to } 8.8) \text { metastatic disease per } 1000 \\
\text { person-years } \\
\text { Peto OR=0.40 (95\% Cl } 0.22 \text { to } \\
0.72) \\
\text { ARD }=-4 \%(95 \% \mathrm{Cl}-6.1 \text { to }-1.3)\end{array}$ \\
\hline $\begin{array}{l}\text { RPI } \\
\text { EBRT+ADT }\end{array}$ & $\begin{array}{l}\text { Hamdy } 2016^{11} \\
\text { ProtecT } \\
\text { RCT } \\
\text { Low }\end{array}$ & $\begin{array}{l}\frac{\text { All-cause Mortality at } 10 \text { years }}{\text { (median) }} \\
\text { RP: } 9.9 \%(55 / 553) 10.1(95 \% \mathrm{Cl} \\
7.8 \text { to } 13.2) \text { deaths per } 1000 \\
\text { person-years } \\
\text { RT: } 10.1 \%(55 / 545) 10.3(95 \% \mathrm{Cl} \\
7.9 \text { to } 13.4) \text { deaths per } 1000 \\
\text { person-years } \\
\text { RR=0.99 }(95 \% \mathrm{Cl} 0.69 \text { to } 1.41) \\
\text { ARD }=-0.1(95 \% \mathrm{Cl}-3.7-3.4)\end{array}$ & $\begin{array}{l}\text { Prostate Cancer Specific Survival at } 5 \\
\text { years } \\
\text { RP: } 100 \% \\
\text { RT: } 100 \% \\
\text { Prostate Cancer Mortality at } 10 \text { years } \\
\text { RP: } 0.9 \%(5 / 553) 0.9(95 \% \mathrm{Cl} 0.4 \text { to } 2.2) \\
\text { deaths per } 1000 \text { person-years } \\
\text { EBRT+ADT: } 0.7 \%(4 / 545) 0.7(95 \% \mathrm{Cl} \\
0.3 \text { to } 2.0) \text { deaths per } 1000 \text { person-years } \\
\text { P=0.48 between groups (+AS) } \\
\text { HR }=1.25(95 \% \mathrm{Cl} 0.33 \text { to } 4.55) \\
\text { Peto OR }=1.23(95 \% \mathrm{Cl} 0.33 \text { to } 4.58) \\
\text { ARD=0.2\% }(95 \% \mathrm{Cl}-0.9,1.2)\end{array}$ & $\begin{array}{l}\text { RP: } 2.4 \%(13 / 553) 2.4(95 \% \mathrm{Cl} 1.4 \\
\text { to } 4.2) \text { metastatic disease per } 1000 \\
\text { person-years } \\
\text { EBRT+ADT: } 2.9 \%(16 / 545) 3.0 \\
\text { ( } 95 \% \mathrm{Cl} 1.9 \text { to } 4.9) \text { metastatic } \\
\text { disease per } 1000 \text { person-years } \\
\text { Peto OR=0.80 (95\% Cl } 0.38 \text { to } \\
1.67) \\
\text { ARD }=-0.6 \%(95 \% \mathrm{Cl}-2.5 \text { to } 1.3)\end{array}$ \\
\hline $\begin{array}{l}\text { RP + ADT/ } \\
\text { High-dose RT } \\
\text { (EBRT and } \\
\text { BT) + ADT }\end{array}$ & $\begin{array}{l}\text { Lennernäs } 2015^{52} \\
\text { RCT } \\
\text { Moderate }\end{array}$ & $\begin{array}{l}\text { All-cause Mortality at } 10 \text { years } \\
\text { RP + ADT: } 26.7 \%(12 / 45) \\
\text { High-dose RT + ADT: } 20.5 \% \text { (9/44) } \\
\text { RR=1.30 (95\% Cl } 0.61 \text { to } 2.78) \\
\text { ARD }=6.2 \%(95 \% \mathrm{Cl}-11.4 \text { to } 23.8)\end{array}$ & $\begin{array}{l}\text { Prostate Cancer Mortality at } 10 \text { years } \\
\text { RP + ADT: } 13.3 \%(6 / 45) \\
\text { High-dose RT +ADT: } 4.5 \%(2 / 44) \\
\text { Peto OR }=2.89(95 \% \mathrm{Cl} 0.68 \text { to } 12.27) \\
\text { ARD }=8.8 \%(95 \% \mathrm{Cl}-2.9 \text { to } 20.52)\end{array}$ & NR \\
\hline
\end{tabular}




\begin{tabular}{|c|c|c|c|c|}
\hline $\begin{array}{l}\text { Intervention/ } \\
\text { Comparison }\end{array}$ & $\begin{array}{l}\text { Study } \\
\text { Design } \\
\text { (Trial/Registry) } \\
\text { Followup } \\
\text { Risk of Bias }\end{array}$ & Overall Survival and Mortality & $\begin{array}{l}\text { Prostate Cancer Specific Survival and } \\
\text { Mortality }\end{array}$ & $\begin{array}{l}\text { Metastases or Metastatic } \\
\text { Progression Free Survival } \\
\text { (define) }\end{array}$ \\
\hline LRP/RARP & $\begin{array}{l}\text { Porpiglia } 2018^{53} \\
\text { RCT } \\
\text { Moderate }\end{array}$ & NR & NR & NR \\
\hline $\begin{array}{l}\text { RALRP/ } \\
\text { Open RRP }\end{array}$ & $\begin{array}{l}\text { Sooriakumaran } \\
2018^{55} \\
\text { Observational } \\
\text { Moderate }\end{array}$ & NR & NR & NR \\
\hline RP vs. HIFU & $\begin{array}{l}\text { Hamdy } 2018^{54} \\
\text { RCT } \\
12 \text { months } \\
\text { Medium }\end{array}$ & NR & NR & NR \\
\hline
\end{tabular}

Abbreviations: ADT=androgen deprivation therapy; ARD=absolute risk difference; $\mathrm{AM}=$ active monitoring; $\mathrm{BT}=$ brachytherapy; $\mathrm{CI}=\mathrm{confidence} \mathrm{interval;} \mathrm{EBRT=external} \mathrm{beam}$ radiation therapy; HIFU=high intensity focused ultrasound; LRP=laparoscopic radical prostatectomy; NR=not reported; OR=odds ratio; RALRP=robotic-assisted laparoscopic radical prostatectomy; RARP=robotic-assisted radical prostatectomy; $\mathrm{RP}=$ radical prostatectomy; $\mathrm{RR}=$ risk ratio; $\mathrm{RRP}=$ retropubic radical prostatectomy; $\mathrm{RT}=$ radiation therapy; RCT=randomized controlled trial 
Table I-5. Health status and quality of life outcomes: radical prostatectomy comparisons

\begin{tabular}{|c|c|c|c|}
\hline $\begin{array}{l}\text { Intervention/ } \\
\text { Comparison }\end{array}$ & $\begin{array}{l}\text { Study } \\
\text { Design } \\
\text { (Trial/Registry) } \\
\text { Followup } \\
\text { Risk of Bias }\end{array}$ & Health Status & $\begin{array}{l}\text { Quality of Life } \\
\text { Prostate Cancer Related Quality of Life }\end{array}$ \\
\hline $\begin{array}{l}\mathrm{RP} / \\
\mathrm{AM}\end{array}$ & $\begin{array}{l}\text { Hamdy } 2016^{11,12} \\
\text { ProtecT } \\
\text { RCT } \\
\text { Low }\end{array}$ & NR & $\begin{array}{l}\text { SF-12: Physical health subscale (mean [SD]) } \\
\text { At } 12 \text { months } \\
\text { RP: } 49.9 \text { (8.5); } n=447 \\
\text { AM: } 49.9 \text { (9.1); } n=453 \\
\text { At } 72 \text { months } \\
\text { RP: } 48.8 \text { (9.1); } n=428 \\
\text { AM: } 46.9 \text { (10.6); } n=428 \\
\text { SF-12: Mental health subscale (mean [SD]) } \\
\text { At } 12 \text { months } \\
\text { RP: } 53.7 \text { (8.3); } n=447 \\
\text { AM: } 53.6 \text { (8.1); } n=453 \\
\text { At } 72 \text { months } \\
\text { RP: } 53.5 \text { (8.3); } n=428 \\
\text { AM: } 53.0 \text { (8.8); } n=428 \\
\text { Cancer-specific quality of life: EORTC-QLQ-C30 at } 5 \text { years, } \\
\text { Global health status (mean [SD]) } \\
\text { RP: } 78.4 \text { (17.7); } n=386 \\
\text { AM: } 76.8 \text { (17.6); } n=394\end{array}$ \\
\hline
\end{tabular}




\begin{tabular}{|c|c|c|c|}
\hline $\begin{array}{l}\text { Intervention/ } \\
\text { Comparison }\end{array}$ & $\begin{array}{l}\text { Study } \\
\text { Design } \\
\text { (Trial/Registry) } \\
\text { Followup } \\
\text { Risk of Bias }\end{array}$ & Health Status & $\begin{array}{l}\text { Quality of Life } \\
\text { Prostate Cancer Related Quality of Life }\end{array}$ \\
\hline $\begin{array}{l}\text { RPI } \\
\text { EBRT+ADT }\end{array}$ & $\begin{array}{l}\text { Hamdy } 2016^{11,12} \\
\text { ProtecT } \\
\text { RCT } \\
\text { Low }\end{array}$ & NR & $\begin{array}{l}\text { SF-12: Physical health subscale (mean [SD]) } \\
\text { At } 12 \text { months } \\
\text { RP: } 49.9 \text { (8.5); } n=447 \\
\text { RT: } 50.2 \text { (8.6); } n=440 \\
\text { At } 72 \text { months } \\
\text { RP: } 48.8 \text { (9.1); } n=428 \\
\text { RT: } 48.4 \text { (9.4); } n=428 \\
\\
\text { SF-12: Mental health subscale (mean [SD]) } \\
\text { At } 12 \text { months } \\
\text { RP: } 53.7 \text { (8.3); } n=447 \\
\text { RT: } 53.3 \text { (8.5); } n=440 \\
\text { At } 72 \text { months } \\
\text { RP: } 53.5 \text { (8.3); } n=428 \\
\text { RT: } 53.8 \text { (7.8); } n=428 \\
\text { Cancer-specific quality of life: EORTC-QLQ-C30 at } 5 \text { years, } \\
\text { Global health status (mean [SD]) } \\
\text { RP: } 78.4(17.7) ; n=386 \\
\text { RT: } 77.4 \text { (19.0); } n=400\end{array}$ \\
\hline
\end{tabular}




\begin{tabular}{|c|c|c|c|}
\hline $\begin{array}{l}\text { Interventionl } \\
\text { Comparison }\end{array}$ & $\begin{array}{l}\text { Study } \\
\text { Design } \\
\text { (Trial/Registry) } \\
\text { Followup } \\
\text { Risk of Bias } \\
\end{array}$ & Health Status & $\begin{array}{l}\text { Quality of Life } \\
\text { Prostate Cancer Related Quality of Life }\end{array}$ \\
\hline $\begin{array}{l}\text { RP +ADT/ } \\
\text { High-dose RT } \\
\text { (EBRT and BT) } \\
+ \text { ADT }\end{array}$ & $\begin{array}{l}\text { Lennernäs } 2015^{52} \\
\text { RCT } \\
\text { Moderate }\end{array}$ & NR & $\begin{array}{l}\text { EORTC QLQ-C33 subscales } \\
\text { Global quality of life at } 12 \text { months } \\
\text { RP + ADT: } 77 \text { (16); } n=31 \\
\text { High-dose RT + ADT: } 76 \text { (22); } n=24 \\
\text { Global quality of life at } 24 \text { months } \\
\text { RP + ADT: } 77 \text { (21); } n=31 \\
\text { High-dose RT + ADT: } 75 \text { (20); } n=24 \\
\text { Physical functioning at } 12 \text { months } \\
\text { RP + ADT: } 96 \text { (9); n=33 } \\
\text { High-dose RT + ADT: } 94 \text { (14); } n=25 \\
\text { Physical functioning at } 24 \text { months } \\
\text { RP + ADT: } 96 \text { (12); } n=33 \\
\text { High-dose RT + ADT: } 94 \text { (17); } n=25 \\
\text { Emotional functioning at } 12 \text { months } \\
\text { RP + ADT: } 89 \text { (15); } n=33 \\
\text { High-dose RT + ADT: } 86 \text { (19); } n=25 \\
\text { Emotional functioning at } 24 \text { months } \\
\text { RP + ADT: } 88 \text { (16); } n=33 \\
\text { High-dose RT + ADT: } 87 \text { (17); } n=25\end{array}$ \\
\hline LRP/RARP & $\begin{array}{l}\text { Porpiglia } 2018^{53} \\
\text { RCT } \\
\text { Moderate }\end{array}$ & $\begin{array}{l}\text { Health status, self-rated as excellent, very good, } \\
\text { or good (from the Expanded Prostate Cancer } \\
\text { Index Composite (EPIC) questionnaire) at } 5 \\
\text { years } \\
\text { LRP: } 86 \%(50 / 58) \\
\text { RARP: } 100 \%(57 / 57), P=.003\end{array}$ & NR \\
\hline $\begin{array}{l}\text { RALRP/ } \\
\text { Open RRP }\end{array}$ & $\begin{array}{l}\text { Sooriakumaran } \\
2018^{55} \\
\text { Observational } \\
\text { Moderate }\end{array}$ & NR & NR \\
\hline RP vs. HIFU & $\begin{array}{l}\text { Hamdy } 2018^{54} \\
\text { RCT } \\
12 \text { months } \\
\text { Medium }\end{array}$ & NR & NR \\
\hline
\end{tabular}

Abbreviations: ADT=androgen deprivation therapy; AM=active monitoring; BT=brachytherapy; EBRT=external beam radiation therapy; EORTC QLQ-C30=European Organization for Research and Treatment of Cancer Quality-of-Life Questionnaire-Core 30 module; HIFU=high intensity focused ultrasound; LRP=laparoscopic radical prostatectomy; $\mathrm{NR}=$ not reported; RALRP=robotic-assisted laparoscopic radical prostatectomy; $\mathrm{RARP}=$ robotic-assisted radical prostatectomy; RP=radical prostatectomy; $\mathrm{RRP}=$ retropubic radical prostatectomy; RT=radiation therapy; RCT=randomized controlled trial; $\mathrm{SD}=$ standard deviation; SF12=short form 12 item health survey questionnaire 
Table I-6. Harms: radical prostatectomy comparisons

\begin{tabular}{|c|c|c|}
\hline $\begin{array}{l}\text { Intervention/ } \\
\text { Comparison }\end{array}$ & $\begin{array}{l}\text { Study } \\
\text { (Trial) } \\
\text { Followup } \\
\text { Risk of Bias } \\
\end{array}$ & Common Treatment Related Side Effects \\
\hline $\begin{array}{l}\text { RP/ } \\
\text { AM }\end{array}$ & $\begin{array}{l}\text { Hamdy 2016; } \\
\text { Donovan 2016; Lane } \\
\text { 2016 } \\
\text { ProtecT } \\
\text { RCT } \\
\text { Low }\end{array}$ & 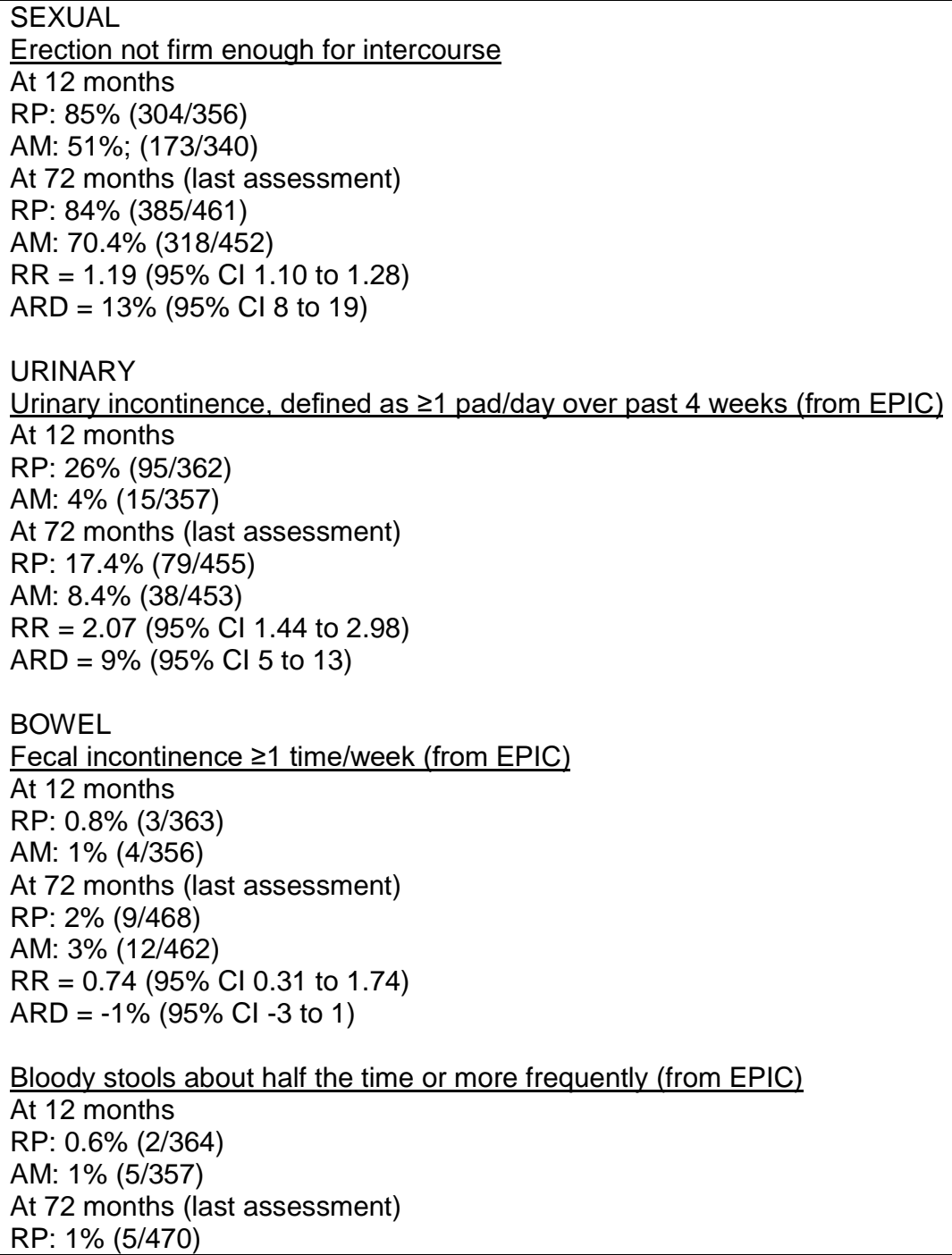 \\
\hline
\end{tabular}




\begin{tabular}{|l|l|l|}
\hline $\begin{array}{l}\text { Interventionl } \\
\text { Comparison }\end{array}$ & $\begin{array}{l}\text { Study } \\
\text { (Trial) } \\
\text { Followup } \\
\text { Risk of Bias }\end{array}$ & Common Treatment Related Side Effects \\
\hline & & $\begin{array}{l}\text { AM: } 1 \%(6 / 465) \\
\text { RR }=0.82(95 \% \mathrm{Cl} 0.25 \text { to } 2.68) \\
\text { ARD }=0 \%(95 \% \mathrm{Cl}-2 \text { to } 1)\end{array}$ \\
\hline
\end{tabular}




\begin{tabular}{|c|c|c|}
\hline $\begin{array}{l}\mathrm{RP} / \\
\text { EBRT+ADT }\end{array}$ & $\begin{array}{l}\text { Hamdy 2016; } \\
\text { Donovan 2016; Lane } \\
2016^{11-13} \\
\text { ProtecT } \\
\text { RCT } \\
\text { Low }\end{array}$ & 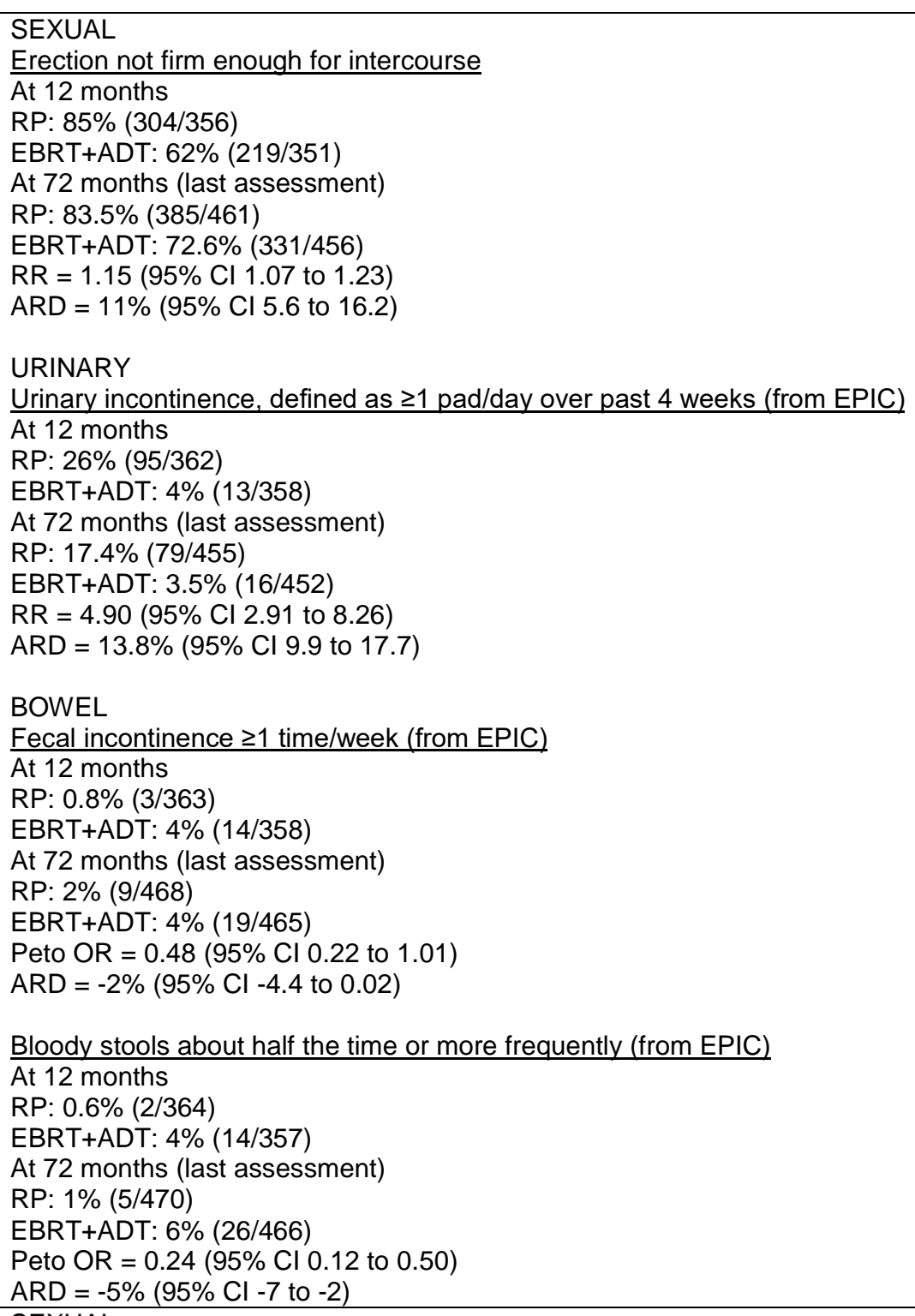 \\
\hline $\begin{array}{l}\text { RP +ADT/ High- } \\
\text { dose RT (EBRT and } \\
\text { BT) +ADT }\end{array}$ & $\begin{array}{l}\text { Lennernäs } 2015^{52} \\
\text { RCT } \\
\text { Moderate }\end{array}$ & $\begin{array}{l}\text { SEXUAL } \\
\text { Grade } 3 \text { (quite a bit)-4 (very much) Erectile dysfunction at } 24 \text { months: } \\
\text { RP + ADT: } 89 \%(33 / 37)\end{array}$ \\
\hline
\end{tabular}




\begin{tabular}{|c|c|c|}
\hline $\begin{array}{l}\text { Intervention/ } \\
\text { Comparison }\end{array}$ & $\begin{array}{l}\text { Study } \\
\text { (Trial) } \\
\text { Followup } \\
\text { Risk of Bias }\end{array}$ & Common Treatment Related Side Effects \\
\hline & & 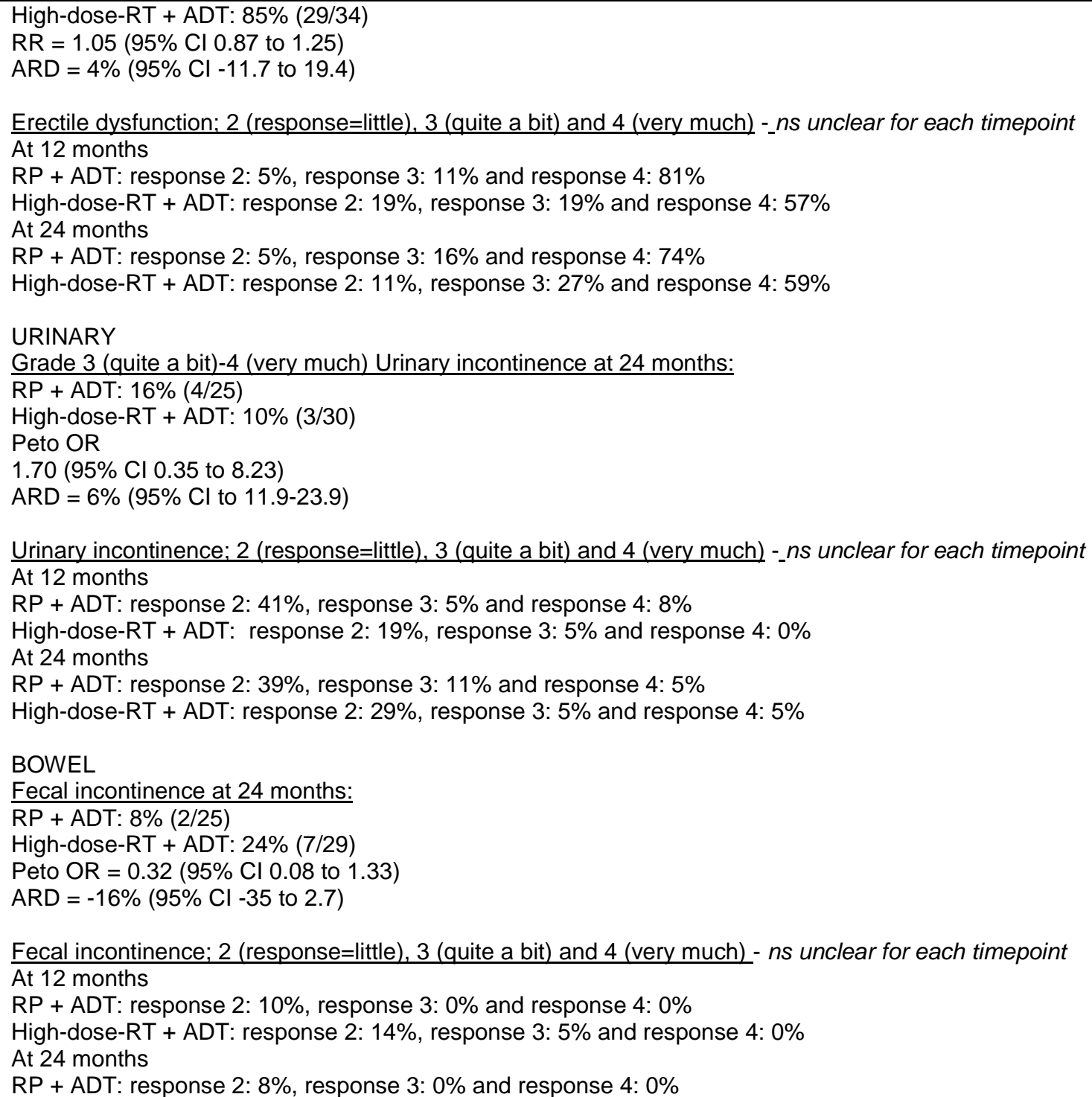 \\
\hline
\end{tabular}




\begin{tabular}{|c|c|c|}
\hline $\begin{array}{l}\text { Intervention/ } \\
\text { Comparison }\end{array}$ & $\begin{array}{l}\text { Study } \\
\text { (Trial) } \\
\text { Followup } \\
\text { Risk of Bias }\end{array}$ & Common Treatment Related Side Effects \\
\hline & & $\begin{array}{l}\text { High-dose-RT + ADT: response 2: } 24 \% \text {, response } 3: 0 \% \text { and response } 4: 0 \% \\
\text { Bloody stools; } 2 \text { (response=little), } 3 \text { (quite a bit) and } 4 \text { (very much)- ns unclear for each timepoint } \\
\text { At } 12 \text { months } \\
\text { RP + ADT: response 2: } 8 \% \text {, response } 3: 0 \% \text { and response } 4: 0 \% \\
\text { High-dose-RT + ADT: response 2: } 8 \% \text {, response } 3: 3 \% \text { and response } 4: 0 \% \\
\text { At } 24 \text { months } \\
\text { RP + ADT: response 2: } 3 \% \text {, response } 3: 3 \% \text { and response } 4: 0 \% \\
\text { High-dose-RT + ADT: response 2: } 15 \% \text {, response } 3: 3 \% \text { and response } 4: 3 \%\end{array}$ \\
\hline LRP/RARP & $\begin{array}{l}\text { Porpiglia } 2018^{53} \\
\text { RCT } \\
\text { Moderate }\end{array}$ & $\begin{array}{l}\text { SEXUAL (Patients who underwent nerve-sparing only) } \\
\text { Erectile dysfunction, an erection not sufficient for penetration } \\
\text { At } 12 \text { months } \\
\text { LRP: } 45.7 \%(16 / 35) \\
\text { RARP: } 20 \%(7 / 35) \\
\text { At } 60 \text { months } \\
\text { LRP: } 48.6 \%(17 / 35) \\
\text { RARP: } 25.7 \%(9 / 35) \\
\text { ARD }=22.9 \%(95 \% \mathrm{Cl} 0.9 \text { to } 44.9) \\
\text { URINARY } \\
\text { Urinary incontinence defined as } \geq 1 \text { pad/day or use of } \geq 1 \text { safety pad/day (from EPIC) } \\
\text { At } 12 \text { months } \\
\text { LRP: } 16.7 \%(10 / 60) \\
\text { RARP: } 5 \%(3 / 60) \\
\text { At } 60 \text { months } \\
\text { LRP: } 15.5 \%(9 / 58) \\
\text { RARP: } 3.5 \%(2 / 57) \\
\text { ARD }=12 \%(95 \% \mathrm{Cl} 1.5 \text { to } 22.5) \\
\text { BOWEL } \\
\text { NR }\end{array}$ \\
\hline $\begin{array}{l}\text { RALRP/ } \\
\text { Open RRP }\end{array}$ & $\begin{array}{l}\text { Sooriakumaran } \\
2018^{55} \\
\text { Observational } \\
\text { Moderate }\end{array}$ & $\begin{array}{l}\text { SEXUAL } \\
\text { Postoperative erectile dysfunction was defined as when a patient answered they could not achieve a stiff } \\
\text { erection at any time or an erection stiff enough for intercourse at any time } \\
\text { At } 12 \text { months } \\
\text { RARP: } 57 \% \\
\text { Open RRP: } 69 \% \\
\text { At } 24 \text { months } \\
\text { RARP: } 49 \% \\
\text { Open RRP: } 61 \%\end{array}$ \\
\hline
\end{tabular}




\begin{tabular}{|l|l|l|}
\hline $\begin{array}{l}\text { Intervention/ } \\
\text { Comparison }\end{array}$ & $\begin{array}{l}\text { Study } \\
\text { (Trial) } \\
\text { Followup } \\
\text { Risk of Bias }\end{array}$ & Common Treatment Related Side Effects \\
\hline RP vs. HIFU & $\begin{array}{l}\text { Hamdy } 2018^{54} \\
\text { RCT } \\
12 \text { months } \\
\text { Medium }\end{array}$ & $\begin{array}{l}\text { Urinary incontinence (need to use absorbent pad at least once per day) } \\
\text { RP } \sim 58 \% \\
\text { HIFU 0\% }\end{array}$ \\
& $\begin{array}{l}\text { Fecal incontinence (half of the time or more within last } 4 \text { weeks of questionnaire) } \\
\text { RP } \sim 50 \% \\
\text { HIFU } ~\end{array}$ \\
& $\begin{array}{l}\text { Erectile dysfunction } \\
\text { RP } \sim 22 \% \\
\text { HIFU } ~\end{array}$ \\
&
\end{tabular}

Abbreviations: ADT=androgen deprivation therapy; $\mathrm{ARD}=$ absolute risk difference; $\mathrm{AM}=$ active monitoring; $\mathrm{BT}=$ brachytherapy; $\mathrm{EBRT}=\mathrm{external}$ beam radiation therapy; EPIC=Expanded Prostate Cancer Index Composite questionnaire; HIFU=high-intensity focused ultrasound; LRP=laparoscopic radical prostatectomy; NR=not reported; OR=odds ratio; RALRP=robotic-assisted laparoscopic radical prostatectomy; RARP=robotic-assisted radical prostatectomy; $\mathrm{RP}=$ radical prostatectomy; $\mathrm{RR}=$ risk ratio; $\mathrm{RRP}=$ retropubic radical prostatectomy; $\mathrm{RT}=$ radiation therapy; $\mathrm{RCT}=$ randomized controlled trial; 
Table I-7. Evidence certainty: radical prostatectomy comparisons

\begin{tabular}{|c|c|c|c|c|c|c|c|c|c|c|c|c|}
\hline $\begin{array}{l}\text { Intervention/ } \\
\text { Comparison }\end{array}$ & Outcome & $\begin{array}{l}\text { k= } \\
\text { Study } \\
\text { Design }\end{array}$ & $\begin{array}{l}\text { Risk of } \\
\text { Bias }\end{array}$ & $\begin{array}{l}\text { Inconsis- } \\
\text { tency }\end{array}$ & $\begin{array}{l}\text { Indirect- } \\
\text { ness }\end{array}$ & Imprecision & Other & I & C & $\begin{array}{l}\text { Relative } \\
\text { (95\% Cl) }\end{array}$ & $\begin{array}{l}\text { Abso- } \\
\text { lute } \\
\text { (95\% } \\
\text { CI) }\end{array}$ & Certainty \\
\hline \multirow[t]{6}{*}{ RP vs. AM } & $\begin{array}{l}\text { All-cause } \\
\text { mortality } \\
10 \text { years }\end{array}$ & $1 \mathrm{RCT}$ & Low & $\begin{array}{l}\text { Single } \\
\text { study }\end{array}$ & Direct & Imprecise & None & $\begin{array}{l}(55 / 553) \\
10 \%\end{array}$ & $\begin{array}{l}(59 / 545) \\
11 \%\end{array}$ & $\begin{array}{l}\text { RR } 0.92 \\
(0.65 \text { to } \\
1.30)\end{array}$ & $\begin{array}{l}-0.9 \% \\
(-4.5 \text { to } \\
2.7)\end{array}$ & $\begin{array}{l}\text { Moderate }{ }^{a} \\
\oplus \oplus \oplus \bigcirc\end{array}$ \\
\hline & $\begin{array}{l}\text { PC-specific } \\
\text { mortality } \\
10 \text { years }\end{array}$ & $1 \mathrm{RCT}$ & Low & $\begin{array}{l}\text { Single } \\
\text { study }\end{array}$ & Direct & $\begin{array}{l}\text { Very } \\
\text { imprecise }\end{array}$ & None & $\begin{array}{l}(5 / 553) \\
0.9 \%\end{array}$ & $\begin{array}{l}(8 / 545) \\
1.5 \%\end{array}$ & $\begin{array}{l}\text { Peto OR } \\
0.62 \\
(0.20 \text { to } \\
1.87)\end{array}$ & $\begin{array}{l}-0.6 \% \\
(-1.8 \text { to } \\
0.7)\end{array}$ & $\begin{array}{l}\text { Low }^{b} \\
\oplus \oplus \bigcirc \bigcirc\end{array}$ \\
\hline & $\begin{array}{l}\text { Metastases } \\
10 \text { years }\end{array}$ & $1 \mathrm{RCT}$ & Low & $\begin{array}{l}\text { Single } \\
\text { study }\end{array}$ & Direct & Imprecise & None & $\begin{array}{l}(13 / 553) \\
2.4 \%\end{array}$ & $\begin{array}{l}(33 / 545) \\
6.4 \%\end{array}$ & $\begin{array}{l}\text { Peto OR } \\
0.40 \\
(0.22 \text { to } \\
0.72)\end{array}$ & $\begin{array}{l}-4.0 \% \\
(-6.1 \text { to } \\
-1.3)\end{array}$ & $\begin{array}{l}\text { Moderate }{ }^{a} \\
\oplus \oplus \oplus \bigcirc\end{array}$ \\
\hline & $\begin{array}{l}\text { ED } \\
6 \text { years }\end{array}$ & $1 \mathrm{RCT}$ & $\begin{array}{l}\text { Moderate } \\
\text { (attrition) }\end{array}$ & $\begin{array}{l}\text { Single } \\
\text { study }\end{array}$ & Direct & Precise & None & $\begin{array}{l}(385 / 461) \\
83.5 \%\end{array}$ & $\begin{array}{l}(318 / 452 \\
)_{70.4 \%}\end{array}$ & $\begin{array}{l}\text { RR } 1.19 \\
(1.10 \text { to } \\
1.28)\end{array}$ & $\begin{array}{l}13 \% \\
(8 \text { to } \\
19)\end{array}$ & $\begin{array}{l}\text { Moderate } \\
\oplus \oplus \oplus \bigcirc^{\circ}\end{array}$ \\
\hline & $\begin{array}{l}\text { Urinary } \\
\text { Incontinence } \\
6 \text { years }\end{array}$ & $1 \mathrm{RCT}$ & $\begin{array}{l}\text { Moderate } \\
\text { (attrition) }\end{array}$ & $\begin{array}{l}\text { Single } \\
\text { study }\end{array}$ & Direct & Precise & None & $\begin{array}{l}(79 / 455) \\
17.4 \%\end{array}$ & $\begin{array}{l}(38 / 453) \\
8.4 \%\end{array}$ & $\begin{array}{l}\text { RR } 2.07 \\
(1.44 \text { to } \\
2.98)\end{array}$ & $\begin{array}{l}9 \% \\
(5 \text { to } \\
13)\end{array}$ & $\begin{array}{l}\text { Moderate } \\
\oplus \oplus \oplus \bigcirc^{\circ}\end{array}$ \\
\hline & $\begin{array}{l}\text { Fecal } \\
\text { incontinence } \\
6 \text { years }\end{array}$ & $1 \mathrm{RCT}$ & $\begin{array}{l}\text { Moderate } \\
\text { (attrition) }\end{array}$ & $\begin{array}{l}\text { Single } \\
\text { study }\end{array}$ & Direct & Imprecise & None & $\begin{array}{c}(9 / 468) \\
1.9 \%\end{array}$ & $\begin{array}{c}(12 / 462) \\
2.6 \%\end{array}$ & $\begin{array}{l}\text { Peto OR } \\
0.74 \\
(0.31 \text { to } \\
1.75)\end{array}$ & $\begin{array}{l}-0.7 \% \\
(-2.6 \text { to } \\
1.2)\end{array}$ & $\begin{array}{l}\text { Low } \\
\oplus \oplus \bigcirc \bigcirc a, c\end{array}$ \\
\hline \multirow[t]{2}{*}{$\begin{array}{l}\text { RP vs. } \\
\text { EBRT+ADT }\end{array}$} & $\begin{array}{l}\text { All-cause } \\
\text { mortality } \\
10 \text { years }\end{array}$ & $1 \mathrm{RCT}$ & Low & $\begin{array}{l}\text { Single } \\
\text { study }\end{array}$ & Direct & Imprecise & None & $\begin{array}{c}(55 / 553) \\
10 \%\end{array}$ & $\begin{array}{c}(55 / 545) \\
10 \%\end{array}$ & $\begin{array}{l}\text { RR } 0.99 \\
(0.69 \text { to } \\
1.04)\end{array}$ & $\begin{array}{l}-0.1 \% \\
(-3.7 \text { to } \\
3.4)\end{array}$ & $\begin{array}{l}\text { Moderate }^{a} \\
\oplus \oplus \oplus \bigcirc\end{array}$ \\
\hline & $\begin{array}{l}\text { PC-specific } \\
\text { mortality } \\
10 \text { years }\end{array}$ & $1 \mathrm{RCT}$ & Low & $\begin{array}{l}\text { Single } \\
\text { study }\end{array}$ & Direct & $\begin{array}{l}\text { Very } \\
\text { imprecise }\end{array}$ & None & $\begin{array}{c}(5 / 553) \\
0.9 \%\end{array}$ & $\begin{array}{c}(4 / 545) \\
0.7 \%\end{array}$ & $\begin{array}{l}\text { Peto OR } \\
1.23 \\
(0.33 \text { to } \\
4.58)\end{array}$ & $\begin{array}{l}0.2 \% \\
(-0.9 \text { to } \\
1.2)\end{array}$ & $\begin{array}{l}\text { Low }^{\mathrm{b}} \\
\oplus \oplus \bigcirc \bigcirc\end{array}$ \\
\hline
\end{tabular}




\begin{tabular}{|c|c|c|c|c|c|c|c|c|c|c|c|c|}
\hline $\begin{array}{l}\text { Intervention/ } \\
\text { Comparison }\end{array}$ & Outcome & $\begin{array}{l}\text { k= } \\
\text { Study } \\
\text { Design }\end{array}$ & $\begin{array}{l}\text { Risk of } \\
\text { Bias }\end{array}$ & $\begin{array}{l}\text { Inconsis- } \\
\text { tency }\end{array}$ & $\begin{array}{l}\text { Indirect- } \\
\text { ness }\end{array}$ & Imprecision & Other & I & C & $\begin{array}{l}\text { Relative } \\
(95 \% \mathrm{Cl})\end{array}$ & $\begin{array}{l}\text { Abso- } \\
\text { lute } \\
(95 \% \\
\text { Cl) }\end{array}$ & Certainty \\
\hline & $\begin{array}{l}\text { Metastases } \\
10 \text { years }\end{array}$ & $1 \mathrm{RCT}$ & Low & $\begin{array}{l}\text { Single } \\
\text { study }\end{array}$ & Direct & Imprecise & None & $\begin{array}{c}(13 / 553) \\
2.4 \%\end{array}$ & $\begin{array}{c}(16 / 545) \\
2.9 \%\end{array}$ & $\begin{array}{l}\text { Peto OR } \\
0.80 \\
(0.38 \text { to } \\
1.67)\end{array}$ & $\begin{array}{l}-0.6 \% \\
-2.5 \text { to } \\
1.3)\end{array}$ & $\begin{array}{l}\text { Low }^{b} \\
\oplus \oplus \bigcirc \bigcirc\end{array}$ \\
\hline & $\begin{array}{l}\text { ED } \\
6 \text { years }\end{array}$ & $1 \mathrm{RCT}$ & $\begin{array}{l}\text { Moderate } \\
\text { (attrition) }\end{array}$ & $\begin{array}{l}\text { Single } \\
\text { study }\end{array}$ & Direct & Precise & None & $\begin{array}{c}(385 / 461) \\
83.5 \%\end{array}$ & $\begin{array}{c}(331 / 456 \\
) \\
72.6 \%\end{array}$ & $\begin{array}{l}\text { RR 1.15 } \\
(1.07 \text { to } \\
1.23)\end{array}$ & $\begin{array}{l}11 \% \\
(6 \text { to } \\
16)\end{array}$ & $\begin{array}{l}\text { Moderate } \\
\oplus \oplus \oplus \bigcirc c\end{array}$ \\
\hline & $\begin{array}{l}\text { Urinary } \\
\text { Incontinence } \\
6 \text { years }\end{array}$ & $1 \mathrm{RCT}$ & $\begin{array}{l}\text { Moderate } \\
\text { (attrition) }\end{array}$ & $\begin{array}{l}\text { Single } \\
\text { study }\end{array}$ & Direct & Precise & None & $\begin{array}{c}(79 / 455) \\
17.4 \%\end{array}$ & $\begin{array}{c}(16 / 452) \\
3.5 \%\end{array}$ & $\begin{array}{l}\text { RR } 4.90 \\
(2.91 \text { to } \\
8.26)\end{array}$ & $\begin{array}{c}14 \% \\
(10 \text { to } \\
18)\end{array}$ & $\begin{array}{l}\text { Moderate } \\
\oplus \oplus \bigcirc \bigcirc c\end{array}$ \\
\hline & $\begin{array}{l}\text { Fecal } \\
\text { incontinence } \\
6 \text { years }\end{array}$ & $1 \mathrm{RCT}$ & $\begin{array}{l}\text { Moderate } \\
\text { (attrition) }\end{array}$ & $\begin{array}{l}\text { Single } \\
\text { study }\end{array}$ & Direct & Imprecise & None & $\begin{array}{c}(9 / 468) \\
1.9 \%\end{array}$ & $\begin{array}{c}(19 / 465) \\
4.1 \%\end{array}$ & \begin{tabular}{|l} 
Peto OR \\
0.48 \\
$(0.22$ to \\
$1.01)$
\end{tabular} & $\begin{array}{c}-2.2 \\
(-4.4 \text { to } \\
0.02)\end{array}$ & $\begin{array}{l}\text { Low } \\
\oplus \oplus \bigcirc \bigcirc a, c\end{array}$ \\
\hline \multirow{5}{*}{$\begin{array}{l}\text { RP plus ADT } \\
\text { vs. EBRT } \\
\text { plus High- } \\
\text { dose BT plus } \\
\text { ADT }\end{array}$} & $\begin{array}{l}\text { All-cause } \\
\text { mortality } \\
10 \text { years }\end{array}$ & $1 \mathrm{RCT}$ & Moderate & $\begin{array}{l}\text { Single } \\
\text { study }\end{array}$ & Direct & $\begin{array}{l}\text { Very } \\
\text { imprecise }\end{array}$ & None & $\begin{array}{l}(12 / 45) \\
26.7 \%\end{array}$ & $\begin{array}{l}(9 / 44) \\
20.4 \%\end{array}$ & $\begin{array}{l}\text { RR } 1.30 \\
(0.61 \text { to } \\
2.78)\end{array}$ & $\begin{array}{c}6.2 \% \\
(-11.4 \\
\text { to } 23.8)\end{array}$ & $\begin{array}{l}\text { Insufficient } \\
\oplus \bigcirc \bigcirc \bigcirc a, b\end{array}$ \\
\hline & $\begin{array}{l}\text { PC-specific } \\
\text { mortality } \\
10 \text { years }\end{array}$ & $1 \mathrm{RCT}$ & Moderate & $\begin{array}{l}\text { Single } \\
\text { study }\end{array}$ & Direct & $\begin{array}{l}\text { Very } \\
\text { imprecise }\end{array}$ & None & $\begin{array}{l}(6 / 45) \\
13.3 \%\end{array}$ & $\begin{array}{l}(2 / 44) \\
4.5 \%\end{array}$ & $\begin{array}{l}\text { Peto OR } \\
2.89 \\
(0.68 \text { to } \\
12.27)\end{array}$ & $\begin{array}{c}8.8 \% \\
(-2.9 \text { to } \\
20.5)\end{array}$ & $\begin{array}{l}\text { Insufficient } \\
\oplus \bigcirc \bigcirc \bigcirc \text { a, b }\end{array}$ \\
\hline & $\begin{array}{l}\text { Urinary } \\
\text { Incontinence } \\
2 \text { years }\end{array}$ & $1 \mathrm{RCT}$ & High & $\begin{array}{l}\text { Single } \\
\text { study }\end{array}$ & Direct & $\begin{array}{l}\text { Very } \\
\text { imprecise }\end{array}$ & None & $\begin{array}{c}(4 / 25) \\
16 \%\end{array}$ & $\begin{array}{l}(3 / 30) \\
10 \%\end{array}$ & \begin{tabular}{|l} 
Peto OR \\
1.70 \\
$(0.35$ to \\
$8.23)$
\end{tabular} & $\begin{array}{c}6 \% \\
(-11.9 \\
\text { to } 23.9)\end{array}$ & $\begin{array}{l}\text { Insufficient }{ }^{\mathrm{a}, \mathrm{b}} \\
\oplus \bigcirc \bigcirc \bigcirc\end{array}$ \\
\hline & $\begin{array}{l}\text { Fecal } \\
\text { incontinence } \\
2 \text { years }\end{array}$ & $1 \mathrm{RCT}$ & High & $\begin{array}{l}\text { Single } \\
\text { study }\end{array}$ & Direct & $\begin{array}{l}\text { Very } \\
\text { imprecise }\end{array}$ & None & $\begin{array}{c}(2 / 25) \\
8 \%\end{array}$ & $\begin{array}{c}(7 / 29) \\
24 \%\end{array}$ & $\begin{array}{l}\text { Peto OR } \\
0.32 \\
(0.08 \text { to } \\
1.33\end{array}$ & $\begin{array}{c}-16.1 \% \\
(-35 \text { to } \\
27)\end{array}$ & $\begin{array}{l}\text { Insufficient } \\
\oplus \bigcirc \bigcirc \bigcirc\end{array}$ \\
\hline & $\begin{array}{l}\text { ED } \\
60 \text { months }\end{array}$ & $1 \mathrm{RCT}$ & Medium & $\begin{array}{l}\text { Single } \\
\text { study }\end{array}$ & Direct & Imprecise & None & $\begin{array}{c}(17 / 35) \\
49 \%\end{array}$ & $\begin{array}{c}(9 / 35) \\
26 \%\end{array}$ & $\begin{array}{l}\text { RR } 1.89 \\
(0.98 \text { to } \\
3.65)\end{array}$ & $\begin{array}{c}23 \% \\
(1 \text { to } \\
45)\end{array}$ & $\begin{array}{l}\text { Low }^{\text {a,d }} \\
\oplus \oplus \bigcirc \bigcirc\end{array}$ \\
\hline
\end{tabular}




\begin{tabular}{|c|c|c|c|c|c|c|c|c|c|c|c|c|}
\hline $\begin{array}{l}\text { Intervention/ } \\
\text { Comparison }\end{array}$ & Outcome & $\begin{array}{l}\text { k= } \\
\text { Study } \\
\text { Design }\end{array}$ & $\begin{array}{l}\text { Risk of } \\
\text { Bias }\end{array}$ & $\begin{array}{l}\text { Inconsis- } \\
\text { tency }\end{array}$ & $\begin{array}{l}\text { Indirect- } \\
\text { ness }\end{array}$ & Imprecision & Other & I & C & $\begin{array}{l}\text { Relative } \\
(95 \% \mathrm{Cl})\end{array}$ & $\begin{array}{l}\text { Abso- } \\
\text { lute } \\
(95 \% \\
\text { Cl) }\end{array}$ & Certainty \\
\hline \multirow[t]{2}{*}{$\begin{array}{l}\text { LRP vs. } \\
\text { RARP }\end{array}$} & $\begin{array}{l}\text { Urinary } \\
\text { Incontinence- } \\
60 \text { months }\end{array}$ & $1 \mathrm{RCT}$ & Medium & $\begin{array}{l}\text { Single } \\
\text { study }\end{array}$ & Direct & Imprecise & None & $\begin{array}{l}(9 / 58) \\
15.5 \%\end{array}$ & $\begin{array}{l}(2 / 57) \\
3.5 \%\end{array}$ & $\begin{array}{l}\text { Peto OR } \\
3.96 \\
(1.15 \text { to } \\
13.65)\end{array}$ & $\begin{array}{l}12 \% \\
(1.5 \text { to } \\
22.5)\end{array}$ & $\begin{array}{l}\text { Low }^{\text {a,b }} \\
\oplus \oplus \bigcirc \bigcirc\end{array}$ \\
\hline & $\begin{array}{l}\text { ED } \\
2 \text { years }\end{array}$ & $1 \mathrm{RCT}$ & Moderate & $\begin{array}{l}\text { Single } \\
\text { study }\end{array}$ & Direct & Imprecise & None & $\begin{array}{c}(33 / 37) \\
90 \%\end{array}$ & $\begin{array}{c}(29 / 34) \\
85 \%\end{array}$ & $\begin{array}{l}\text { RR } 1.05 \\
(0.87 \text { to } \\
1.25)\end{array}$ & $\begin{array}{c}4 \% \\
(-12 \text { to } \\
19)\end{array}$ & $\begin{array}{l}\text { Low }^{\mathrm{a}, \mathrm{d}} \\
\oplus \oplus \bigcirc \bigcirc\end{array}$ \\
\hline $\begin{array}{l}\text { RALRP vs. } \\
\text { Open RRP }\end{array}$ & ED & 1 Obs. & Medium & $\begin{array}{l}\text { Single } \\
\text { study }\end{array}$ & Direct & $\begin{array}{l}\text { Unclear, } \\
\text { data not } \\
\text { presented in } \\
\text { usable } \\
\text { manner }\end{array}$ & None & Unclear & Unclear & - & $\begin{array}{c}-12 \% \\
\text { (CI NA) }\end{array}$ & $\begin{array}{l}\text { Insufficient } \mathrm{c}, \mathrm{d} \\
\oplus \bigcirc \bigcirc \bigcirc\end{array}$ \\
\hline \multirow[t]{3}{*}{ RP vs. HIFU } & $\begin{array}{l}\text { Urinary } \\
\text { Incontinence- } \\
12 \text { months }\end{array}$ & $1 \mathrm{RCT}$ & Medium & $\begin{array}{l}\text { Single } \\
\text { study }\end{array}$ & Direct & $\begin{array}{l}\text { Unclear, } \\
\text { data not } \\
\text { presented in } \\
\text { usable } \\
\text { manner }\end{array}$ & None & $58 \%$ & $0 \%$ & - & $\begin{array}{l}-58 \% \\
\text { (CI NA) }\end{array}$ & $\begin{array}{l}\text { Insufficient }{ }^{\mathrm{c}, \mathrm{d}} \\
\oplus \bigcirc \bigcirc \bigcirc\end{array}$ \\
\hline & $\begin{array}{l}\text { ED } \\
12 \text { months }\end{array}$ & $1 \mathrm{RCT}$ & Medium & $\begin{array}{l}\text { Single } \\
\text { study }\end{array}$ & Direct & $\begin{array}{l}\text { Unclear, } \\
\text { data not } \\
\text { presented in } \\
\text { usable } \\
\text { manner }\end{array}$ & None & $50 \%$ & $20 \%$ & - & $\begin{array}{l}-30 \% \\
\text { (CI NA) }\end{array}$ & $\begin{array}{l}\text { Insufficient }{ }^{\mathrm{c}, \mathrm{d}} \\
\oplus \bigcirc \bigcirc \bigcirc\end{array}$ \\
\hline & $\begin{array}{l}\text { Fecal } \\
\text { incontinence } \\
12 \text { months }\end{array}$ & $1 \mathrm{RCT}$ & Medium & $\begin{array}{l}\text { Single } \\
\text { study }\end{array}$ & Direct & $\begin{array}{l}\text { Unclear, } \\
\text { data not } \\
\text { presented in } \\
\text { usable } \\
\text { manner }\end{array}$ & None & $22 \%$ & $15 \%$ & - & $\begin{array}{l}-7 \% \\
\text { (CI NA) }\end{array}$ & $\begin{array}{l}\text { Insufficient }{ }^{\mathrm{c}, \mathrm{d}} \\
\oplus \bigcirc \bigcirc \bigcirc\end{array}$ \\
\hline
\end{tabular}

Abbreviations: $\mathrm{ADT}=$ androgen deprivation therapy; $\mathrm{AM}=$ active monitoring; $\mathrm{BT}=$ brachytherapy; $\mathrm{CI}=$ confidence interval; EBRT=external beam radiation therapy; $\mathrm{ED}=\mathrm{erectile}$ dysfunction; LRP=laparoscopic radical prostatectomy; $\mathrm{OR}=$ odds ratio; $\mathrm{PC}=$ prostate cancer; RALRP=robotic-assisted laparoscopic radical prostatectomy; RARP=robotic-assisted radical prostatectomy; $\mathrm{RP}=$ radical prostatectomy; $\mathrm{RR}=$ risk ratio; $\mathrm{RRP}=$ retropubic radical prostatectomy; $\mathrm{RT}=$ radiation therapy

Explanations

a. Rated down by one level for imprecision

b. Rated down by two levels for imprecision and sparse data.

c. Rated down by one level for risk of bias

d. Rated down one level for unknown precision 


\section{Appendix J. Comparisons From Past Reports}

The following is a list of the comparisons which were analyzed in the 2016 evidence report commissioned by AUA which we did not identify any additional studies of low or moderate risk of bias (ROB) published after this report.*

- Transperitoneal robot assisted laparoscopic radical prostatectomy (RALRP) vs. Extraperitoneal RALRP: 1 randomized controlled trial (RCT).

- Androgen deprivation therapy (ADT) vs. ADT plus Docetaxel and Estramustine: 1 RCT.

- External beam radiation therapy (EBRT) vs. Cryotherapy: 1 RCT and 1 non-RCT.

- Radical retropubic prostatectomy (RRP) vs. brachytherapy (BT): 1 RCT and 2 nonRCTs.

- ADT vs. ADT plus EBRT: 1 RCT.

- RRP vs. radical perineal prostatectomy (RPP): 1 RCT.

- Three-dimensional conformal radiotherapy (3D-CRT) conventional dose vs. 3D-CRT high dose: 2 RCTs.

- Hypofractionated radiation therapy (RT) vs. conventionally-fractionated RT: 2 RCTs.

- $\quad$ EBRT vs. EBRT plus BT: 1 RCT and 5 non-RCTs.

- $\quad$ ADT plus radical prostatectomy (RP) vs. RP alone: 4 RCTs and 3 non-RCTs.

- ADT plus standard of care (SOC) (watchful waiting [WW]) vs. SOC (WW): 1 RCT and 7 non-RCTs.

- ADT plus SOC (either RP or RT) versus SOC (either RP or RT): 1 RCT.

- BT conventional dose vs. BT low dose: 1 RCT.

- ADT short-term plus RT vs. ADT long-term plus RT: 2 RCTs.

- RP vs. RALRP: 1 non-RCT.

- ADT short duration vs. ADT long duration: 1 non-RCT.

- RP vs. BT: 10 non-RCTs.

- RRP vs. 3D-CRT: 2 non-RCTs.

- 3D-CRT vs. BT: 3 non-RCTs.

- ADT vs. RP: 5 non-RCTs.

- Intensity modulated radiation therapy (IMRT) BT vs. IMRT: 1 non-RCT.

- EBRT vs. Observation: 3 non-RCTs.

- BT with ADT/HT (hormone therapy) versus BT alone: 4 non-RCTs.

- Laparoscopic radical prostatectomy (LRP) vs. RALRP: 1 non-RCT.

- BT plus EBRT vs. BT plus ADT: 2 non-RCTs.

- 3D-CRT vs. conservative management: 1 non-RCT.

- IMRT vs. conservative management: 1 non-RCT.

- Proton Beam vs. conservative management: 1 non-RCT.

- BT alone vs. conservative management: 1 non-RCT.

- Proton Beam vs. 3D-CRT: 2 non-RCTs.

- IMRT vs. Proton Beam: 2 non-RCTs.

- IMRT vs. BT: 2 non-RCTs.

- BT vs. Cryotherapy: 3 non-RCTs.

- EBRT vs. ADT: 1 non-RCT. 
- HIFU (high-intensity focused ultrasound) vs. HIFU plus ADT: 1 non-RCT.

- RT vs. expectant management (EM)/WW: 2 non-RCTs.

- Cryotherapy vs. RP: 2 non-RCTs.

- Cryotherapy vs. RT: 1 non-RCT.

- Cryotherapy vs. EM: 1 non-RCT.

- Cryotherapy vs. ADT: 1 non-RCT.

- RT vs. ADT: 2 non-RCTs.

- RRP vs. Cryotherapy: 1 non-RCT.

- RALRP vs. BT: 1 non-RCT.

- RALRP vs. Cryotherapy: 1 non-RCT.

- 3D-CRT vs. EBRT plus BT: 1 non-RCT.

- IMRT vs. EBRT plus BT: 1 non-RCT.

- RP vs. RP plus RT: 2 non-RCTs.

- RP plus RT vs. EBRT: 1 non-RCT.

- RP plus RT vs. EBRT plus BT: 1 non-RCT.

- RP plus RT vs. BT: 1 non-RCT.

- RP plus RT vs. RT: 1 non-RCT.

- BT vs. RT: 1 non-RCT.

- RT vs. EBRT: 1 non-RCT.

- RT vs. EBRT plus BT: 1 non-RCT.

- RP vs. RP plus EBRT: 2 non-RCTs.

- RP plus EBRT vs. BT: 2 non-RCTs.

- RP plus EBRT vs. EBRT: 2 non-RCTs.

- RP plus EBRT vs. BT plus EBRT: 2 non-RCTs.

- RP plus EBRT vs. Cryotherapy: 1 non-RCT.

- BT plus EBRT vs. Cryotherapy: 1 non-RCT.

- WW vs. RP plus RT: 1 non-RCT.

- WW vs. RP plus ADT: 1 non-RCT.

- WW vs. RT plus ADT: 1 non-RCT.

- WW vs. RP plus RT plus ADT: 1 non-RCT.

- RP vs. RP plus RT plus ADT: 1 non-RCT.

- RT vs. RP plus ADT: 1 non-RCT.

- RT vs. RP plus RT plus ADT: 1 non-RCT.

- RP plus RT vs. ADT: 1 non-RCT.

- RP plus RT vs. RP plus ADT: 1 non-RCT.

- RP plus RT vs. RT plus ADT: 1 non-RCT.

- RP plus RT vs. RP plus RT plus ADT: 1 non-RCT.

- ADT vs. RP plus ADT: 1 non-RCT.

- ADT vs. RT plus ADT: 1 non-RCT.

- ADT vs. RP plus RT plus ADT: 1 non-RCT.

- RP plus ADT vs. RT plus ADT: 1 non-RCT.

- RP plus ADT vs. RP plus RT plus ADT: 1 non-RCT.

- RT vs. RP plus RT plus ADT: 1 non-RCT.

- RP vs. BT plus ADT: 1 non-RCT. 
- RP vs. BT plus EBRT plus ADT: 1 non-RCT.

- RP plus ADT vs. RP plus EBRT: 1 non-RCT.

- RP plus ADT vs. BT: 1 non-RCT.

- RP plus ADT vs. BT plus ADT: 1 non-RCT.

- RP plus ADT vs. BT plus EBRT: 1 non-RCT.

- RP plus ADT vs. EBRT: 1 non-RCT.

- RP plus ADT vs. EBRT plus ADT: 1 non-RCT.

- RP plus EBRT vs. BT plus ADT: 1 non-RCT.

- RP plus EBRT vs. BT plus EBRT plus ADT: 1 non-RCT.

- RP plus EBRT vs. EBRT plus ADT: 1 non-RCT.

- BT vs. BT plus EBRT plus ADT: 1 non-RCT.

- BT vs. EBRT plus ADT: 1 non-RCT.

- BT plus ADT vs. EBRT: 1 non-RCT.

- BT plus ADT vs. EBRT plus ADT: 1 non-RCT.

- BT plus EBRT vs. BT plus EBRT plus ADT: 1 non-RCT.

- BT plus EBRT vs. EBRT plus ADT: 1 non-RCT.

- BT plus EBRT plus ADT vs. EBRT: 1 non-RCT.

*The 2016 evidence report included several comparisons that we would have excluded, such as comparisons of ADT duration, select EBRT techniques/doses, and chemotherapy. 


\section{Appendix K. Ongoing RCTs for CLPC or Locally Advanced PC}

Table K-1. Ongoing RCTs of nonpharmacological interventions for CLPC or locally advanced PC with large planned enrollment ( $\mathrm{n}>300)$

\begin{tabular}{|c|c|c|c|}
\hline Cos & Interventions & $\begin{array}{l}\text { Planned Completion } \\
\text { Planned Enrollment } \\
\text { Countries Conducted }\end{array}$ & NCT Number \\
\hline $\begin{array}{l}\text { Evaluation of Four Treatment Modalities in } \\
\text { Prostate Cancer With Low or "Early } \\
\text { Intermediate" Risk }\end{array}$ & $\begin{array}{l}\text { Radical prostatectomy vs. } \\
\text { percutaneous radiation therapy vs. } \\
\text { permanent seed implantation radiation vs. } \\
\text { active surveillance }\end{array}$ & $\begin{array}{l}\text { December } 2030 \\
N=7600 \\
\text { Germany }\end{array}$ & NCT01717677 \\
\hline $\begin{array}{l}\text { Intensity-Modulated Radiation Therapy in } \\
\text { Treating Patients With Localized Prostate } \\
\text { Cancer }\end{array}$ & $\begin{array}{l}\text { Conventional radiotherapy ( } 74 \text { Gy delivered in } 37 \\
\text { fractions) vs. } \\
\text { hypofractionated radiation therapy ( } 60 \text { Gy in } 20 \text { fractions) } \\
\text { vs. } \\
\text { hypofractionated radiation therapy ( } 57 \text { Gy in } 19 \text { fractions) }\end{array}$ & $\begin{array}{l}\text { June } 2021 \\
\mathrm{~N}=3216 \\
\text { England, Ireland, } \\
\text { Scotland, Wales }\end{array}$ & NCT00392535 \\
\hline $\begin{array}{l}\text { Androgen-Deprivation Therapy and } \\
\text { Radiation Therapy in Treating Patients With } \\
\text { Prostate Cancer }\end{array}$ & $\begin{array}{l}\text { Radiation therapy vs. } \\
\text { Whole-pelvic radiotherapy }\end{array}$ & $\begin{array}{l}\text { July } 2031 \\
\mathrm{~N}=2592 \\
\text { United States, Canada, } \\
\text { Hong Kong, Israel, } \\
\text { Singapore, Switzerland }\end{array}$ & NCT01368588 \\
\hline $\begin{array}{l}\text { Comparative Health Research Outcomes of } \\
\text { NOvel Surgery in Prostate Cancer }\end{array}$ & $\begin{array}{l}\text { A: Radical therapy (radiotherapy or prostatectomy } \\
\text { [radiotherapy can be external beam or brachytherapy]) vs. } \\
\text { Focal therapy (either high intensity focused ultrasound or } \\
\text { cryotherapy) } \\
\text { B: Focal therapy vs. } \\
\text { focal therapy after finasteride } 5 \mathrm{Mg} \text { for } 12 \text { weeks vs. } \\
\text { focal therapy after Bicalutamide } 50 \mathrm{Mg} \text { for } 12 \text { weeks }\end{array}$ & $\begin{array}{l}\text { May } 2027 \\
\mathrm{~N}=2450 \\
\text { England }\end{array}$ & NCT04049747 \\
\hline $\begin{array}{l}\text { Prostate Advances in Comparative } \\
\text { Evidence }\end{array}$ & $\begin{array}{l}\text { A: Laproscopic prostatectomy vs. } \\
\text { prostate stereotactic body radiotherapy } \\
\text { B: Conventionally fractionated prostate radiotherapy vs. } \\
\text { prostate stereotactic body radiotherapy }\end{array}$ & $\begin{array}{l}\text { September } 2026 \\
\mathrm{~N}=1716 \\
\text { England }\end{array}$ & NCT01584258 \\
\hline $\begin{array}{l}\text { Role of Lymph node Dissection in Men With } \\
\text { Prostate Cancer Treated With Radical } \\
\text { Prostatectomy }\end{array}$ & $\begin{array}{l}\text { lymph node dissection vs. } \\
\text { standardized surgical technique without extensive lymph } \\
\text { node dissection }\end{array}$ & $\begin{array}{l}\text { October } 2025 \\
\text { N=1610 } \\
\text { Germany }\end{array}$ & NCT04269512 \\
\hline
\end{tabular}




\begin{tabular}{|c|c|c|c|}
\hline Title & Interventions & $\begin{array}{l}\text { Planned Completion } \\
\text { Planned Enrollment } \\
\text { Countries Conducted }\end{array}$ & NCT Number \\
\hline $\begin{array}{l}\text { Surgery Versus Radiotherapy for Locally } \\
\text { Advanced Prostate Cancer }\end{array}$ & $\begin{array}{l}\text { Prostatectomy/surgery vs. } \\
\text { radiotherapy with adjuvant androgen deprivation therapy }\end{array}$ & $\begin{array}{l}\text { December } 2027 \\
\mathrm{~N}=1200 \\
\text { Denmark, Finland, } \\
\text { Norway, Sweden }\end{array}$ & NCT02102477 \\
\hline $\begin{array}{l}\text { Extended Pelvic Lymph Node Dissection vs. } \\
\text { no Pelvic Lymph Node Dissection at Radical } \\
\text { Prostatectomy for intermediate-and High- } \\
\text { risk Prostate Cancer }\end{array}$ & $\begin{array}{l}\text { Radical prostatectomy followed by extended pelvic lymph } \\
\text { node dissection vs. } \\
\text { radical prostatectomy only }\end{array}$ & $\begin{array}{l}\text { December } 2038 \\
\mathrm{~N}=900 \\
\text { Switzerland }\end{array}$ & NCT03921996 \\
\hline $\begin{array}{l}\text { Prostate Cancer Patients Treated With } \\
\text { Alternative Radiation Oncology Strategies }\end{array}$ & $\begin{array}{l}\text { Hypofractionated radiotherapy with photons vs. } \\
\text { hypofractionated radiotherapy with protons vs. } \\
\text { normofractionated radiotherapy with photons }\end{array}$ & $\begin{array}{l}\text { January } 2028 \\
\mathrm{~N}=897 \\
\text { Germany }\end{array}$ & NCT04083937 \\
\hline $\begin{array}{l}\text { Laparoscopic and Robot-Assisted Radical } \\
\text { Prostatectomy - a Comparative Study }\end{array}$ & $\begin{array}{l}\text { Robot-assisted laparoscopic prostatectomy vs. } \\
\text { conventional radical laparoscopic prostatectomy }\end{array}$ & $\begin{array}{l}\text { June } 2020 \\
\mathrm{~N}=782 \\
\text { Germany }\end{array}$ & NCT03682146 \\
\hline $\begin{array}{l}\text { Stereotactic Body Radiation Therapy or } \\
\text { Intensity-Modulated Radiation Therapy in } \\
\text { Treating Patients With Stage IIA-B Prostate } \\
\text { Cancer }\end{array}$ & $\begin{array}{l}\text { Intensity-Modulated Radiation Therapy vs. Stereotactic } \\
\text { Body Radiation Therapy }\end{array}$ & $\begin{array}{l}\text { December } 2030 \\
\mathrm{~N}=622 \\
\text { United States, Canada, } \\
\text { Ireland }\end{array}$ & NCT03367702 \\
\hline $\begin{array}{l}\text { Radical Prostatectomy Versus Radical } \\
\text { Radiotherapy for Locally Advanced Prostate } \\
\text { Cancer }\end{array}$ & $\begin{array}{l}\text { Radical Prostatectomy vs. } \\
\text { Radical Radiotherapy }\end{array}$ & $\begin{array}{l}\text { December } 2026 \\
\mathrm{~N}=600 \\
\text { China }\end{array}$ & NCT04093375 \\
\hline $\begin{array}{l}\text { Study on the Role of Hormonal Treatment } \\
\text { for Two Dosage Levels of Prostate } \\
\text { Radiation Therapy Versus Prostate } \\
\text { Radiation Therapy Alone }\end{array}$ & $\begin{array}{l}\text { Androgen blockade for } 6 \text { months plus radiotherapy } 70 \text { Gy } \\
\text { vs. } \\
\text { androgen blockage for } 6 \text { months plus radiotherapy } 76 \text { Gy } \\
\text { vs. } \\
\text { Radiotherapy alone with } 76 \text { Gy }\end{array}$ & $\begin{array}{l}\text { December } 2020 \\
\mathrm{~N}=600 \\
\text { Canada }\end{array}$ & NCT00223145 \\
\hline $\begin{array}{l}\text { Radiation Therapy in Treating Patients } \\
\text { Receiving Hormone Therapy for Prostate } \\
\text { Cancer (GETUG-AFU 18) }\end{array}$ & $\begin{array}{l}80 \text { Gy of 3-dimensional conformal radiation therapy or } \\
\text { intensity-modulated radiation therapy vs. } \\
70 \text { Gy of 3-dimensional conformal radiation therapy or } \\
\text { intensity-modulated radiation therapy }\end{array}$ & $\begin{array}{l}\text { October } 2026 \\
\mathrm{~N}=500 \\
\text { France }\end{array}$ & NCT00967863 \\
\hline
\end{tabular}




\begin{tabular}{|c|c|c|c|}
\hline Title & Interventions & $\begin{array}{l}\text { Planned Completion } \\
\text { Planned Enrollment } \\
\text { Countries Conducted }\end{array}$ & NCT Number \\
\hline $\begin{array}{l}\text { Prostate Cancer Active Surveillance Trigger } \\
\text { Trial (PCASTT-UK): Comparing Current } \\
\text { Practice for Men With Prostate Cancer on } \\
\text { Active Surveillance to an Active Surveillance } \\
\text { Protocol With Standardised Triggers for } \\
\text { Transitioning to Curative Treatment }\end{array}$ & $\begin{array}{l}\text { Current practice for active surveillance vs. standardized } \\
\text { triggers for treatment }\end{array}$ & $\begin{array}{l}\text { December } 2030 \\
\mathrm{~N}=500 \\
\text { England, Sweden }\end{array}$ & NCT04029714 \\
\hline $\begin{array}{l}\text { Neoadjuvant Chemo-hormonal Therapy } \\
\text { Combined With Radical Prostatectomy for } \\
\text { Locally Advanced Prostate Cancer }\end{array}$ & $\begin{array}{l}\text { Neoadjuvant chemotherapy combined with hormone } \\
\text { therapy and radical prostatectomy with extended lymph } \\
\text { node dissection vs. } \\
\text { neoadjuvant hormonal therapy and radical prostatectomy } \\
\text { with extended lymph node dissection vs. } \\
\text { radical prostatectomy with extended lymph node } \\
\text { dissection }\end{array}$ & $\begin{array}{l}\text { December } 2024 \\
\mathrm{~N}=475 \\
\text { China }\end{array}$ & NCT04220398 \\
\hline $\begin{array}{l}\text { Radiation Hypofractionation Via Extended } \\
\text { Versus Accelerated Therapy (HEAT) For } \\
\text { Prostate Cancer }\end{array}$ & $\begin{array}{l}\text { Extended hypofractionation radiotherapy vs. accelerated } \\
\text { hypofractionation radiotherapy }\end{array}$ & $\begin{array}{l}\text { March } 2023 \\
\mathrm{~N}=456 \\
\text { United States, } \\
\text { Australia, Italy }\end{array}$ & NCT01794403 \\
\hline $\begin{array}{l}\text { Evaluating the Effects of Frozen Section } \\
\text { Technology on Oncological and Functional } \\
\text { Outcomes at Radical Prostatectomy. }\end{array}$ & $\begin{array}{l}\text { NeuroSAFE robotic assisted radical prostatectomy vs. } \\
\text { standard robotic assisted radical prostatectomy }\end{array}$ & $\begin{array}{l}\text { June } 2022 \\
\mathrm{~N}=454 \\
\text { England, Scotland, }\end{array}$ & NCT03317990 \\
\hline $\begin{array}{l}\text { Comparison of Irreversible Electroporation } \\
\text { and Radical Prostatectomy in Treating } \\
\text { Prostate Cancer }\end{array}$ & $\begin{array}{l}\text { Irreversible electroporation vs. } \\
\text { radical prostatectomy }\end{array}$ & $\begin{array}{l}\text { September } 2027 \\
N=438 \\
\text { China }\end{array}$ & NCT04278261 \\
\hline $\begin{array}{l}\text { Proton Therapy vs. Intensity Modulated } \\
\text { Radiation Therapy for Low or Intermediate } \\
\text { Risk Prostate Cancer }\end{array}$ & $\begin{array}{l}\text { Proton beam therapy vs. } \\
\text { intensity modulated radiation therapy }\end{array}$ & $\begin{array}{l}\text { December } 2026 \\
\mathrm{~N}=400 \\
\text { United States }\end{array}$ & NCT01617161 \\
\hline $\begin{array}{l}\text { Hypofractionated, Dose Escalation } \\
\text { Radiotherapy for High Risk Adenocarcinoma } \\
\text { of the Prostate }\end{array}$ & $\begin{array}{l}\text { Hypofractionation radiation vs. } \\
\text { conventional radiation }\end{array}$ & $\begin{array}{l}\text { January } 2023 \\
\mathrm{~N}=329 \\
\text { Canada }\end{array}$ & NCT01444820 \\
\hline $\begin{array}{l}\text { Early Deep Venous Complex Ligation and } \\
\text { Urinary Continence Recovery After Robot- } \\
\text { Assisted Radical Prostatectomy }\end{array}$ & $\begin{array}{l}\text { Early deep venous complex ligation during robot-assisted } \\
\text { radical prostatectomy vs. } \\
\text { standard technique }\end{array}$ & $\begin{array}{l}\text { August } 2020 \\
\mathrm{~N}=312 \\
\text { Italy }\end{array}$ & NCT03368378 \\
\hline
\end{tabular}




\section{Appendix L. References}

1. Bill-Axelson A, Holmberg L, Garmo H, et al. Radical prostatectomy or watchful waiting in early prostate cancer. N Engl J Med. 2014 Mar 06;370(10):932-42. PMID: 24597866.

2. Bill-Axelson A, Holmberg L, Garmo H, et al. Radical Prostatectomy or Watchful Waiting in Prostate Cancer - 29-Year Follow-up. N Engl J Med. 201812 13;379(24):2319-29. PMID: 30575473.

3. Wilt TJ, Jones KM, Barry MJ, et al. Follow-up of Prostatectomy versus Observation for Early Prostate Cancer. N Engl J Med. 201707 13;377(2):132-42. PMID: 28700844.

4. Herden J, Ansmann L, Ernstmann N, et al. The Treatment of Localized Prostate Cancer in Everyday Practice in Germany. Dtsch. 2016 May 13;113(19):329-36. PMID: 27232362.

5. Hoffman RM, Lo M, Clark JA, et al. Treatment Decision Regret Among Long-Term Survivors of Localized Prostate Cancer: Results From the Prostate Cancer Outcomes Study. Journal of Clinical Oncology. 2017 Jul 10;35(20):2306-14. PMID: 28493812.

6. Lu-Yao GL, Kim S, Moore DF, et al. Primary radiotherapy vs conservative management for localized prostate cancer - A populationbased study. Prostate Cancer and Prostatic Diseases. 201501 Dec;18(4):317-24. PMID: 604966551.

7. Dell'Oglio P, Boehm K, Trudeau V, et al. Survival After Conservative Management Versus External Beam Radiation Therapy in Elderly Patients With Localized Prostate Cancer. International Journal of Radiation Oncology Biology Physics. 201601 Dec;96(5):103745. PMID: 611451436.

8. Wilt TJ, Brawer MK, Barry MJ, et al. The Prostate cancer Intervention Versus Observation Trial:VA/NCI/AHRQ Cooperative Studies Program \#407 (PIVOT): design and baseline results of a randomized controlled trial comparing radical prostatectomy to watchful waiting for men with clinically localized prostate cancer. Contemp Clin Trials. 2009 Jan;30(1):81-7. doi: https://dx.doi.org/10.1016/j.cct.2008.08.002. PMID: 18783735.
9. Wilt TJ. The Prostate Cancer Intervention Versus Observation Trial: VA/NCI/AHRQ Cooperative Studies Program \#407 (PIVOT): design and baseline results of a randomized controlled trial comparing radical prostatectomy with watchful waiting for men with clinically localized prostate cancer. J Natl Cancer Inst Monogr. 2012 Dec;2012(45):184-90. doi: https://dx.doi.org/10.1093/jncimonographs/l gs041. PMID: 23271771.

10. Azzouzi AR, Vincendeau S, Barret E, et al. Padeliporfin vascular-targeted photodynamic therapy versus active surveillance in men with low-risk prostate cancer (CLIN1001 PCM301): an open-label, phase 3, randomised controlled trial. Lancet Oncol. 2017 Feb;18(2):181-91. doi: 10.1016/S1470-2045(16)30661-1. PMID: 28007457.

11. Hamdy FC, Donovan JL, Lane JA, et al. 10-Year Outcomes after Monitoring, Surgery, or Radiotherapy for Localized Prostate Cancer. N Engl J Med. 201610 13;375(15):1415-24. PMID: 27626136.

12. Donovan JL, Hamdy FC, Lane JA, et al. PatientReported Outcomes after Monitoring, Surgery, or Radiotherapy for Prostate Cancer. N Engl J Med. 2016 Oct 13;375(15):1425-37. doi: https://dx.doi.org/10.1056/NEJMoa1606221. PMID: 27626365.

13. Lane A, Metcalfe C, Young GJ, et al. Patientreported outcomes in the ProtecT randomized trial of clinically localized prostate cancer treatments: study design, and baseline urinary, bowel and sexual function and quality of life. BJU Int. 2016 Dec;118(6):869-79. doi: 10.1111/bju.13582. PMID: 27415448.

14. Neal DE, Metcalfe C, Donovan JL, et al. Tenyear Mortality, Disease Progression, and Treatment-related Side Effects in Men with Localised Prostate Cancer from the ProtecT Randomised Controlled Trial According to Treatment Received. Eur Urol. 2020 Mar;77(3):320-30. doi: 10.1016/j.eururo.2019.10.030. PMID: 31771797. 
15. Thomsen FB, Roder MA, Jakobsen H, et al. Active Surveillance Versus Radical Prostatectomy in Favorable-risk Localized Prostate Cancer. Clin Genitourin Cancer. 2019 Aug;17(4):e814-e21. doi: 10.1016/j.clgc.2019.05.005. PMID: 31196798.

16. Barocas DA, Chen V, Cooperberg M, et al. Using a population-based observational cohort study to address difficult comparative effectiveness research questions: The CEASAR study. Journal of Comparative Effectiveness Research. 2013 July;2(4):44560. doi: http://dx.doi.org/10.2217/cer.13.34. PMID: 369311498.

17. Barocas DA, Alvarez J, Resnick MJ, et al. Association Between Radiation Therapy, Surgery, or Observation for Localized Prostate Cancer and Patient-Reported Outcomes After 3 Years. Jama. 201703 21;317(11):1126-40. PMID: 28324093.

18. Tyson MD, Alvarez J, Koyama T, et al. Racial Variation in Patient-Reported Outcomes Following Treatment for Localized Prostate Cancer: Results from the CEASAR Study. European Urology. 2017 08;72(2):307-14. PMID: 27816300.

19. Ansmann L, Winter N, Ernstmann N, et al. Health-related quality of life in active surveillance and radical prostatectomy for low-risk prostate cancer: a prospective observational study (HAROW - Hormonal therapy, Active Surveillance, Radiation, Operation, Watchful Waiting). BJU Int. 2018 Sep;122(3):401-10. PMID: 29603553.

20. Tosoian JJ, Sundi D, Trock BJ, et al. Pathologic Outcomes in Favorable-risk Prostate Cancer: Comparative Analysis of Men Electing Active Surveillance and Immediate Surgery. European Urology. 2016 Apr;69(4):576-81. PMID: 26456680.

21. Lane JA, Donovan JL, Davis M, et al. Active monitoring, radical prostatectomy, or radiotherapy for localised prostate cancer: study design and diagnostic and baseline results of the ProtecT randomised phase 3 trial. Lancet Oncology. 2014

Sep;15(10):1109-18. doi: https://dx.doi.org/10.1016/S14702045(14)70361-4. PMID: 25163905.
22. Vargas C, Schmidt M, Jr H, et al. Initial toxicity, quality-of-life outcomes, and dosimetric impact in a randomized phase 3 trial of hypofractionated versus standard fractionated proton therapy for low-risk prostate cancer. Advances in radiation oncology. 2018;3(3):322-30. PMID: CN01611807.

23. Morris WJ, Tyldesley S, Rodda S, et al. Androgen Suppression Combined with Elective Nodal and Dose Escalated Radiation Therapy (the ASCENDE-RT Trial): An Analysis of Survival Endpoints for a Randomized Trial Comparing a LowDose-Rate Brachytherapy Boost to a DoseEscalated External Beam Boost for Highand Intermediate-risk Prostate Cancer. International Journal of Radiation Oncology, Biology, Physics. 201706 01;98(2):275-85. PMID: 28262473.

24. Rodda S, Tyldesley S, Morris WJ, et al. ASCENDE-RT: An Analysis of TreatmentRelated Morbidity for a Randomized Trial Comparing a Low-Dose-Rate Brachytherapy Boost with a Dose-Escalated External Beam Boost for High- and Intermediate-Risk Prostate Cancer. International Journal of Radiation Oncology, Biology, Physics. 2017 06 01;98(2):286-95. PMID: 28433432.

25. Rodda S, Morris WJ, Hamm J, et al. ASCENDERT: An Analysis of Health-Related Quality of Life for a Randomized Trial Comparing Low-Dose-Rate Brachytherapy Boost With Dose-Escalated External Beam Boost for High- and Intermediate-Risk Prostate Cancer. International Journal of Radiation Oncology, Biology, Physics. 201707 01;98(3):581-9. PMID: 28581398.

26. Viani GA, Viana BS, Martin JE, et al. Intensitymodulated radiotherapy reduces toxicity with similar biochemical control compared with 3-dimensional conformal radiotherapy for prostate cancer: A randomized clinical trial. Cancer. 2016 Jul 01;122(13):2004-11. PMID: 27028170.

27. Bolla M, Maingon P, Carrie C, et al. Short Androgen Suppression and Radiation Dose Escalation for Intermediate- and High-Risk Localized Prostate Cancer: Results of EORTC Trial 22991. Journal of Clinical Oncology. 201605 20;34(15):1748-56. PMID: 26976418. 
28. McPartlin AJ, Glicksman R, Pintilie M, et al. PMH 9907: Long-term outcomes of a randomized phase 3 study of short-term bicalutamide hormone therapy and doseescalated external-beam radiation therapy for localized prostate cancer. Cancer. 2016 Aug 15;122(16):2595-603. PMID: 27219522.

29. Vargas CE, Alam NB, Terk M, et al. Initial results of a randomized phase III trial of high dose image guided radiation with or without androgen deprivation therapy for intermediate-risk prostate cancer. Cancer Treatment and Research Communications. 201901 Jan;19 (no pagination)(100119). PMID: 2001573236.

30. Phillips JG, Chen MH, Zhang D, et al. Percent positive biopsy cores and the risk of death from prostate cancer in men with unfavorable-risk prostate cancer. Journal of Radiation Oncology. 201401 Sep;3(3):30712. PMID: 603283685.

31. McDuff SGR, Chen MH, Renshaw AA, et al. Impact of time to testosterone rebound and comorbidity on the risk of cause-specific mortality in men with unfavorable-risk prostate cancer. Cancer. $2018 \mathrm{Apr}$ 01;124(7):1391-9. PMID: 29338073.

32. Giacalone NJ, Wu J, Chen MH, et al. Prostatespecific antigen failure and risk of death within comorbidity subgroups among men with unfavorable-risk prostate cancer treated in a randomized trial. Journal of Clinical Oncology. 201601 Nov;34(31):3781-6. PMID: 612965014.

33. Malone S, Roy S, Eapen L, et al. Sequencing of Androgen-Deprivation Therapy With External-Beam Radiotherapy in Localized Prostate Cancer: A Phase III Randomized Controlled Trial. J Clin Oncol. 2020 Feb 20;38(6):593-601. doi: 10.1200/JCO.19.01904. PMID: 31829912.

34. Widmark A, Gunnlaugsson A, Beckman L, et al. Ultra-hypofractionated versus conventionally fractionated radiotherapy for prostate cancer: 5-year outcomes of the HYPO-RT-PC randomised, non-inferiority, phase 3 trial. Lancet. 2019 Aug 3;394(10196):385-95. doi: 10.1016/S01406736(19)31131-6. PMID: 31227373.
35. Smith GD, Pickles T, Crook J, et al. Brachytherapy improves biochemical failure-free survival in low- and intermediate-risk prostate cancer compared with conventionally fractionated external beam radiation therapy: A propensity score matched analysis. International Journal of Radiation Oncology Biology Physics. 2015 01 Mar;91(3):505-16. PMID: 601554874.

36. Lee DJ, Barocas DA, Zhao Z, et al. Comparison of Patient-reported Outcomes After External Beam Radiation Therapy and Combined External Beam With Low-dose-rate Brachytherapy Boost in Men With Localized Prostate Cancer. International Journal of Radiation Oncology, Biology, Physics. 2018 Sep 01;102(1):116-26. PMID: 30102188.

37. Abugharib AE, Dess RT, Soni PD, et al. External beam radiation therapy with or without lowdose-rate brachytherapy: Analysis of favorable and unfavorable intermediate-risk prostate cancer patients. Brachytherapy. 2017 Jul - Aug;16(4):782-9. PMID: 28499487.

38. Muralidhar V, Xiang M, Orio PF, et al. Brachytherapy boost and cancer-specific mortality in favorable high-risk versus other high-risk prostate cancer. J. 2016 Feb;8(1):1-6. PMID: 26985191.

39. Xiang M, Nguyen PL. Significant association of brachytherapy boost with reduced prostate cancer-specific mortality in contemporary patients with localized, unfavorable-risk prostate cancer. Brachytherapy. 2015 NovDec;14(6):773-80. PMID: 26489921.

40. Yang DD, Muralidhar V, Nguyen PL, et al. Lack of Benefit From the Addition of External Beam Radiation Therapy to Brachytherapy for Intermediate- and High-risk Prostate Cancer. International Journal of Radiation Oncology, Biology, Physics. 201711 15;99(4):904-11. PMID: 29063853.

41. Amini A, Jones BL, Jackson MW, et al. Survival outcomes of combined external beam radiotherapy and brachytherapy vs. brachytherapy alone for intermediate-risk prostate cancer patients using the National Cancer Data Base. Brachytherapy. 2016 Mar-Apr;15(2):136-46. PMID: 26825856. 
42. Tward JD, Jarosek S, Chu H, et al. Time Course and Accumulated Risk of Severe Urinary Adverse Events After High- Versus LowDose-Rate Prostate Brachytherapy With or Without External Beam Radiation Therapy. International Journal of Radiation Oncology, Biology, Physics. 201608 01;95(5):144353. PMID: 27325475.

43. Ashamalla H, Guirguis A, McCool K, et al. Brachytherapy improves outcomes in young men ( $<=60$ years) with prostate cancer: A SEER analysis. Brachytherapy. 201701 Mar;16(2):323-9. PMID: 614251483.

44. Jackson MW, Amini A, Jones BL, et al. Prostate brachytherapy, either alone or in combination with external beam radiation, is associated with longer overall survival in men with favorable pathologic Group 4 (Gleason score 8) prostate cancer. Brachytherapy. 2017 July;16(4):790-6. PMID: 615636725.

45. Jiang R, Tomaszewski JJ, Ward KC, et al. The burden of overtreatment: comparison of toxicity between single and combined modality radiation therapy among low risk prostate cancer patients. The Canadian journal of urology. 201501 Feb;22(1):764855. PMID: 607086080.

46. Ricco A, Hanlon A, Lanciano R. Propensity score matched comparison of intensity modulated radiation therapy vs stereotactic body radiation therapy for localized prostate cancer: A survival analysis from the national cancer database. Frontiers in Oncology. 201731 Aug;7 (AUG) (no pagination)(185). PMID: 618033192.

47. Evans JR, Zhao S, Daignault S, et al. Patientreported quality of life after stereotactic body radiotherapy (SBRT), intensity modulated radiotherapy (IMRT), and brachytherapy. Radiother Oncol. 2015 Aug;116(2):179-84. PMID: 26276528.

48. Bekelman JE, Mitra N, Handorf EA, et al. Effectiveness of androgen-deprivation therapy and radiotherapy for older men with locally advanced prostate cancer. Journal of Clinical Oncology. 201501 Mar;33(7):71622. PMID: 602911329.
49. Weller MA, Kupelian PA, Reddy CA, et al. Adjuvant versus neoadjuvant androgen deprivation with radiotherapy for prostate cancer: Does sequencing matter? Clinical Genitourinary Cancer. 201501 Jun;13(3):e183-e9. PMID: 602262362.

50. Goy BW, Burchette R, Soper MS, et al. Ten-Year Treatment Outcomes of Radical Prostatectomy Vs External Beam Radiation Therapy Vs Brachytherapy for 1503 Patients With Intermediate-risk Prostate Cancer. Urology. 2020 Feb;136:180-9. doi: 10.1016/j.urology.2019.09.040. PMID: 31704459.

51. D'Amico AV, Chen MH, Renshaw AA, et al. Androgen suppression and radiation vs radiation alone for prostate cancer: a randomized trial. Jama. 2008 Jan 23;299(3):289-95. doi: https://dx.doi.org/10.1001/jama.299.3.289. PMID: 18212313.

52. Lennernas B, Majumder K, Damber JE, et al. Radical prostatectomy versus high-dose irradiation in localized/locally advanced prostate cancer: A Swedish multicenter randomized trial with patient-reported outcomes. Acta Oncologica. 201501 Jun;54(6):875-81. PMID: 604399742.

53. Porpiglia F, Fiori C, Bertolo R, et al. Five-year Outcomes for a Prospective Randomised Controlled Trial Comparing Laparoscopic and Robot-assisted Radical Prostatectomy. Eur Urol Focus. 2018 01;4(1):80-6. PMID: 28753822.

54. Hamdy FC, Elliott D, le Conte S, et al. Partial ablation versus radical prostatectomy in intermediate-risk prostate cancer: the PART feasibility RCT. Health Technol Assess. 2018 09;22(52):1-96. PMID: 30264692.

55. Sooriakumaran P, Pini G, Nyberg T, et al. Erectile Function and Oncologic Outcomes Following Open Retropubic and Robotassisted Radical Prostatectomy: Results from the LAParoscopic Prostatectomy Robot Open Trial. European Urology. 2018 04;73(4):618-27. PMID: 28882327.

56. Loeb S, Meyer CP, Krasnova A, et al. Risk of Small Bowel Obstruction After RobotAssisted vs Open Radical Prostatectomy. Journal of Endourology. 2016 12;30(12):1291-5. PMID: 27615204. 
57. Herlemann A, Cowan JE, Carroll PR, et al.

Community-based Outcomes of Open versus

Robot-assisted Radical Prostatectomy.

European Urology. 2018 02;73(2):215-23.

PMID: 28499617.

58. Chang P, Regan MM, Ferrer M, et al. Relief of Urinary Symptom Burden after Primary Prostate Cancer Treatment. Journal of Urology. 2017 02;197(2):376-84. PMID: 27593476.

59. Weissbach L, Stuerzebecher S, Mumperow E, et al. HAROW: the first comprehensive prospective observational study comparing treatment options in localized prostate cancer. World J Urol. 2016 May;34(5):6417. PMID: 26373955.

60. Hoffman KE, Penson DF, Zhao Z, et al. PatientReported Outcomes Through 5 Years for Active Surveillance, Surgery, Brachytherapy, or External Beam Radiation With or Without Androgen Deprivation Therapy for Localized Prostate Cancer. Jama. 2020 Jan 14;323(2):149-63. doi: 10.1001/jama.2019.20675. PMID: 31935027.

61. Zheng X, Jin K, Qiu S, et al. Focal Laser Ablation Versus Radical Prostatectomy for Localized Prostate Cancer: Survival Outcomes From a Matched Cohort. Clin Genitourin Cancer. 2019 Dec;17(6):464-9 e3. doi: 10.1016/j.clgc.2019.08.008. PMID: 31594734.

62. Knipper S, Pecoraro A, Palumbo C, et al. A 25year Period Analysis of Other-cause Mortality in Localized Prostate Cancer. Clin Genitourin Cancer. 2019 Oct;17(5):395-401. doi: 10.1016/j.clgc.2019.07.008. PMID: 31416752. 